\title{
Nuclear Data Sheets for $\mathrm{A}=42^{*}$
}

\section{JUN CHEN" AND BALRAJ SINGH}

\author{
Department of Physics and Astronomy \\ McMaster University, Hamilton, ON L8S 4M1, Canada \\ "Present address: NSCL, MSU, East Lansing, MI 48824, USA \\ (Received September 28, 2012; Revised April 30, 2016)
}

Abstract: The experimental data are evaluated for known nuclides of mass number A=42 ( $\mathrm{Al}, \mathrm{Si}, \mathrm{P}, \mathrm{S}, \mathrm{Cl}, \mathrm{Ar}, \mathrm{K}$, $\mathrm{Ca}, \mathrm{Sc}, \mathrm{Ti}, \mathrm{V}, \mathrm{Cr})$. Detailed evaluated level properties and related information are presented, including adopted values of level and $\gamma$-ray energies, decay data (energies, intensities and placement of radiations), and other spectroscopic data. This work supersedes earlier full evaluations of A=42 published by $2001 \mathrm{Si} 10$ and 1990En08 (also 1998En04 update).

No excited states are known in ${ }^{42} \mathrm{Al},{ }^{42} \mathrm{P},{ }^{42} \mathrm{~V}$ and ${ }^{42} \mathrm{Cr}$, and structure information for ${ }^{42} \mathrm{Si}$ and ${ }^{42} \mathrm{~S}$ is quite limited. There are no decay schemes available for the decay of ${ }^{42} \mathrm{Al},{ }^{42} \mathrm{Si},{ }^{42} \mathrm{P},{ }^{42} \mathrm{~V}$ and ${ }^{42} \mathrm{Cr}$, while the decay schemes of ${ }^{42} \mathrm{Cl}$ and ${ }^{42} \mathrm{Ti}$ are incomplete in view of scarcity of data, and large gap between their Q-values and the highest energy levels populated in corresponding daughter nuclei. Structures of ${ }^{42} \mathrm{Ca},{ }^{42} \mathrm{~K},{ }^{42} \mathrm{Sc}$ and ${ }^{42} \mathrm{Ar}$ nuclides remain the most extensively studied via many different nuclear reactions and decays.

Cutoff Date: Literature available up to May 31, 2016 has been considered.

General Policies and Organization of Material: see http://www.nndc.bnl.gov/nds/NDSPolicies.pdf.

Acknowledgments: The evaluator thanks McMaster undergraduate students S. Geraedts, M. Birch, E. Thiagalingam and M. Lee for 12 compiled datasets available in the XUNDL database from publications after the 2001-NDS update of $\mathrm{A}=42$. We also thank JoAnn Totans (BNL) for sending copies of some secondary reports, and reviewer of this work for several useful comments.

General Comments: The statistical analysis of $\gamma$-ray data and deduced level schemes is carried out through computer codes available at NNDC, BNL website: www.nndc.bnl.gov. The direct feedings to excited states in $\beta^{-}$ and $\varepsilon$ decays have generally been computed from $\mathrm{I}(\gamma+\mathrm{ce})$ intensity balances at each level; the associated log $f t$ values are calculated using the LOGFT code. All $\mathrm{Q}$ values have been adopted from 2012Wa38. Static magnetic dipole and electric quadruple moments have been adopted from $2014 \mathrm{StZZ}$ and $2013 \mathrm{StZZ}$, respectively, when available. In cases where weighted averaging procedures have been used, the assigned uncertainty is generally not lower than the lowest in the input dataset. Nuclear charge radii have been adopted from $2013 \mathrm{An} 02$

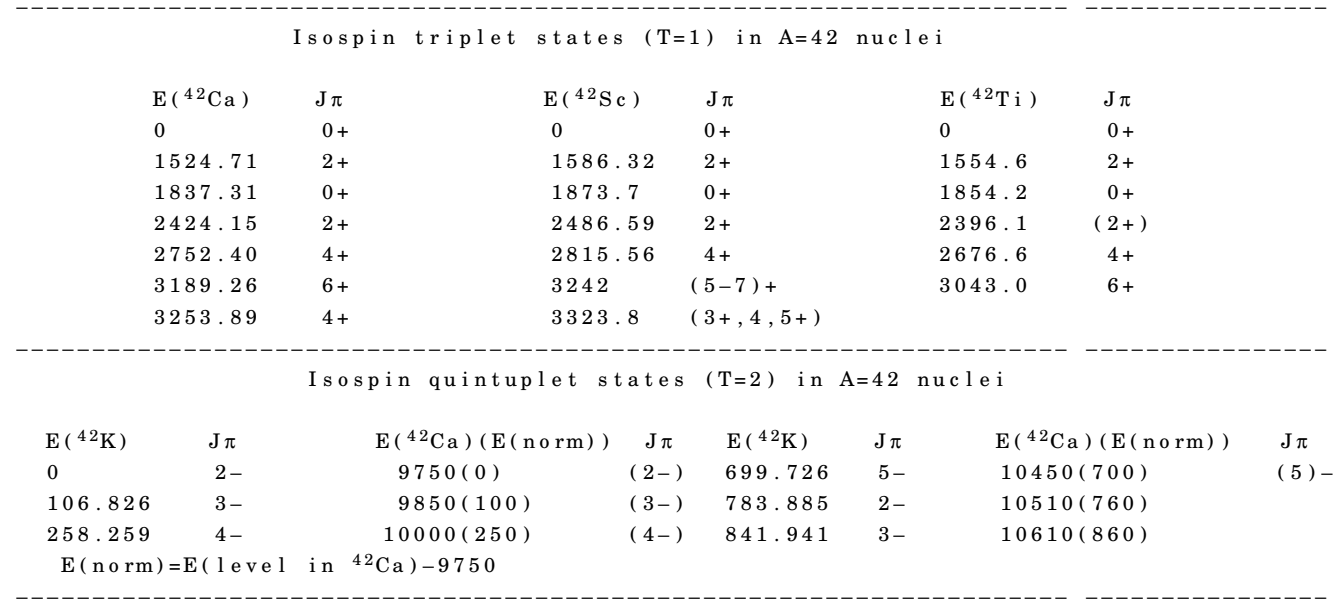

* Work supported by Office of Nuclear Physics, Office of Science, U.S. Department of Energy. 
Index for $\mathrm{A}=42$

\begin{tabular}{|c|c|c|c|c|c|}
\hline Nuclide & Data Type & Page & Nuclide & Data Type & Page \\
\hline & Skeleton Scheme for $A=42$ & 4 & ${ }^{42} \mathrm{Ca}$ & ${ }^{42} \mathrm{Ca}\left(\alpha, \alpha^{\prime}\right)$ & 133 \\
\hline${ }^{42} \mathrm{Al}$ & Adopted Levels & 6 & & ${ }^{42} \mathrm{Ca}\left({ }^{16} \mathrm{O},{ }^{16} \mathrm{O}^{\prime}\right)$ & 135 \\
\hline \multirow[t]{3}{*}{${ }^{42} \mathrm{Si}$} & Adopted Levels, Gammas & 7 & & Coulomb Excitation & 135 \\
\hline & ${ }^{9} \mathrm{Be}\left({ }^{44} \mathrm{~S},{ }^{42} \mathrm{Si} \gamma\right)$ & 8 & & ${ }^{43} \mathrm{Ca}(\mathrm{p}, \mathrm{d})$ & 136 \\
\hline & $\mathrm{C}\left({ }^{44} \mathrm{~S},{ }^{42} \mathrm{Si} \gamma\right)$ & 9 & & ${ }^{43} \mathrm{Ca}(\mathrm{d}, \mathrm{t})$ & 136 \\
\hline${ }^{42} \mathrm{P}$ & Adopted Levels & 10 & & ${ }^{43} \mathrm{Ca}\left({ }^{3} \mathrm{He}, \alpha\right)$ & 138 \\
\hline \multirow[t]{5}{*}{${ }^{42} \mathrm{~S}$} & Adopted Levels, Gammas & 11 & & ${ }^{44} \mathrm{Ca}(\mathrm{p}, \mathrm{t})$ & 140 \\
\hline & ${ }^{42} \mathrm{P} \beta^{-}$Decay $(48.5 \mathrm{~ms})$ & 11 & & ${ }^{45} \operatorname{Sc}(p, \alpha),(\operatorname{pol} p, \alpha)$ & 141 \\
\hline & ${ }^{43} \mathrm{P} \beta^{-} \mathrm{n}$ Decay $(36.5 \mathrm{~ms})$ & 12 & & ${ }^{46} \mathrm{Ti}\left(\mathrm{d},{ }^{6} \mathrm{Li}\right)$ & 141 \\
\hline & ${ }^{9} \mathrm{Be}\left({ }^{48} \mathrm{Ca}, \mathrm{X} \gamma\right)$ & 12 & & ${ }^{96} \mathrm{Zr}\left({ }^{40} \mathrm{Ca},{ }^{42} \mathrm{Ca} \gamma\right)$ & 142 \\
\hline & Coulomb Excitation & 12 & ${ }^{42} \mathrm{Sc}$ & Adopted Levels, Gammas & 143 \\
\hline \multirow[t]{4}{*}{${ }^{42} \mathrm{Cl}$} & Adopted Levels, Gammas & 13 & & ${ }^{42} \mathrm{Ti} \varepsilon$ Decay $(208.65 \mathrm{~ms})$ & 152 \\
\hline & ${ }^{42} \mathrm{~S} \beta^{-}$Decay $(1.016 \mathrm{~s})$ & 14 & & ${ }^{24} \mathrm{Mg}\left({ }^{24} \mathrm{Mg}, \propto \mathrm{pn} \gamma\right) \ldots$ & 153 \\
\hline & ${ }^{43} \mathrm{~S} \beta^{-} \mathrm{n}$ Decay $(265 \mathrm{~ms})$ & 16 & & ${ }^{39} \mathrm{~K}(\alpha, \mathrm{n}),(\alpha, \mathrm{n} \gamma)$ & 156 \\
\hline & ${ }^{208} \mathrm{~Pb}\left({ }^{40} \mathrm{Ar}, \mathrm{X} \gamma\right)$ & 17 & & ${ }^{40} \mathrm{Ca}\left({ }^{3} \mathrm{He}, \mathrm{p}\right)$ & 158 \\
\hline \multirow[t]{7}{*}{${ }^{42} \mathrm{Ar}$} & Adopted Levels, Gammas & 18 & & ${ }^{40} \mathrm{Ca}\left({ }^{3} \mathrm{He}, \mathrm{p} \gamma\right)$ & 159 \\
\hline & ${ }^{42} \mathrm{Cl} \beta^{-}$Decay $(6.8 \mathrm{~s})$ & 20 & & ${ }^{40} \mathrm{Ca}(\alpha, \mathrm{d})$ & 162 \\
\hline & ${ }^{40} \operatorname{Ar}(t, p)$ & 23 & & ${ }^{40} \mathrm{Ca}\left({ }^{12} \mathrm{C},{ }^{10} \mathrm{~B}\right)$ & 163 \\
\hline & ${ }^{40} \operatorname{Ar}(t, p \gamma)$ & 23 & & ${ }^{41} \mathrm{Ca}(\mathrm{p}, \gamma)$ & 163 \\
\hline & ${ }^{42} \operatorname{Ar}\left(p, p^{\prime}\right)$ & 25 & & ${ }^{41} \mathrm{Ca}\left({ }^{3} \mathrm{He}, \mathrm{d}\right)$ & 166 \\
\hline & ${ }^{208} \mathrm{~Pb}\left({ }^{40} \mathrm{Ar}, \mathrm{X} \gamma\right)$ & 25 & & ${ }^{42} \mathrm{Ca}(\mathrm{p}, \mathrm{n})$ & 168 \\
\hline & $\mathrm{Pb}\left({ }^{43} \mathrm{Ar}, \mathrm{n} \gamma\right)$ & 26 & & ${ }^{42} \mathrm{Ca}\left({ }^{3} \mathrm{He}, \mathrm{t}\right)$ & 168 \\
\hline \multirow[t]{11}{*}{${ }^{42} \mathrm{~K}$} & Adopted Levels, Gammas & 27 & & ${ }^{42} \mathrm{Ca}\left({ }^{6} \mathrm{Li},{ }^{6} \mathrm{He}\right)$ & 172 \\
\hline & ${ }^{42} \mathrm{Ar} \beta^{-}$Decay $(32.9$ y) & 38 & & $\mathrm{~Pb}\left({ }^{16} \mathrm{O}, \mathrm{X} \gamma\right)$ & 172 \\
\hline & ${ }^{9} \mathrm{Be}\left({ }^{36} \mathrm{~S}, 2 \mathrm{np} \gamma\right),\left({ }^{37} \mathrm{P}, 4 \mathrm{n} \gamma\right)$ & 39 & ${ }^{42} \mathrm{Ti}$ & Adopted Levels, Gammas & 173 \\
\hline & ${ }^{26} \mathrm{Mg}\left({ }^{18} \mathrm{O}, \mathrm{np} \gamma\right)$ & 40 & & ${ }^{43} \mathrm{Cr}$ \&p Decay $(21.2 \mathrm{~ms})$ & 175 \\
\hline & ${ }^{27} \mathrm{Al}\left({ }^{18} \mathrm{O}, \mathrm{n} 2 \mathrm{p} \gamma\right) \ldots$ & 41 & & ${ }^{45} \mathrm{Fe} \varepsilon 3 \mathrm{p}$ Decay $(2.45 \mathrm{~ms})$ & 176 \\
\hline & ${ }^{40} \operatorname{Ar}(\alpha, \mathrm{d})$ & 41 & & ${ }^{40} \mathrm{Ca}\left({ }^{3} \mathrm{He}, \mathrm{n}\right)$ & 177 \\
\hline & ${ }^{41} \mathrm{~K}(\mathrm{n}, \gamma) \mathrm{E}=$ thermal & 42 & & ${ }^{40} \mathrm{Ca}\left({ }^{3} \mathrm{He}, \mathrm{n} \gamma\right)$ & 178 \\
\hline & ${ }^{41} \mathrm{~K}(\mathrm{n}, \gamma)$ : Resonance & 50 & & ${ }^{40} \mathrm{Ca}\left({ }^{12} \mathrm{C},{ }^{10} \mathrm{Be}\right)$ & 179 \\
\hline & ${ }^{41} \mathrm{~K}(\mathrm{~d}, \mathrm{p}),(\mathrm{d}, \mathrm{p} \gamma)$ & 52 & & ${ }^{42} \mathrm{Ca}\left(\pi^{+}, \pi^{-}\right)$ & 179 \\
\hline & ${ }^{42} \mathrm{Ca}\left(\mathrm{t},{ }^{3} \mathrm{He}\right)$ & 55 & ${ }^{42} \mathrm{~V}$ & Adopted Levels & 180 \\
\hline & ${ }^{43} \mathrm{Ca}\left(\mathrm{d},{ }^{3} \mathrm{He}\right)$ & 56 & ${ }^{42} \mathrm{Cr}$ & Adopted Levels & 181 \\
\hline
\end{tabular}




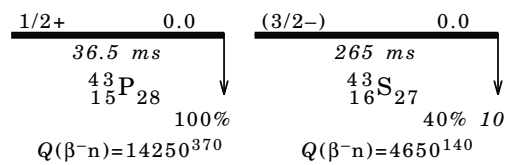

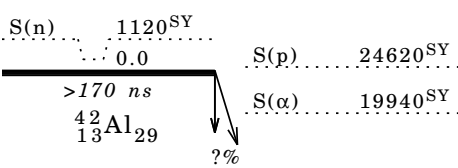

$Q^{-}=24280^{\mathrm{SY}}$
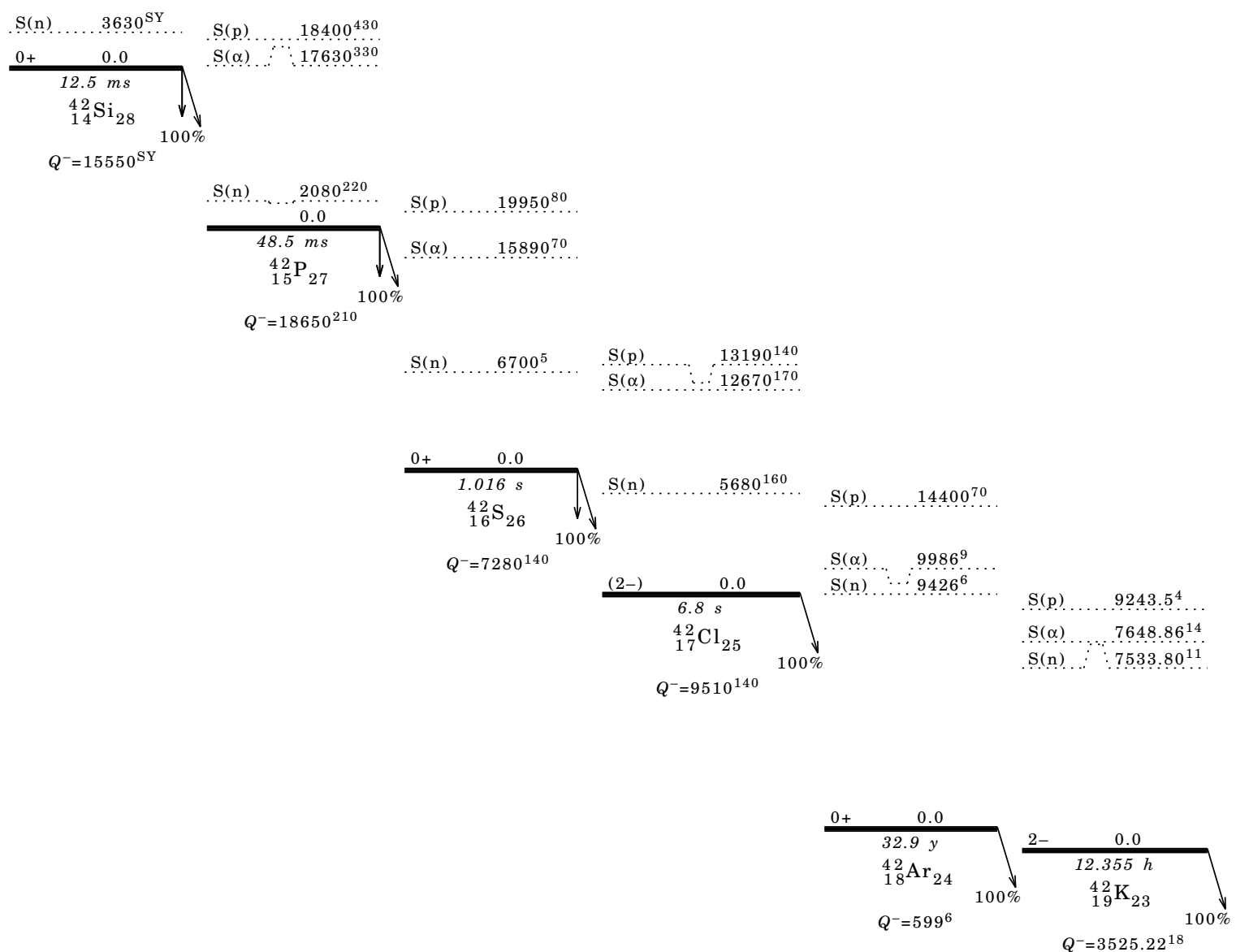
Skeleton Scheme for $A=42$ (continued)

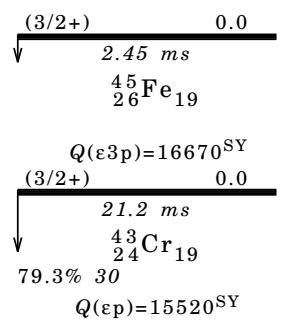

S(n) $\ldots 20340^{\mathrm{CA}}$

$\mathrm{S}(\alpha) \ldots 6850^{\mathrm{SY}}$

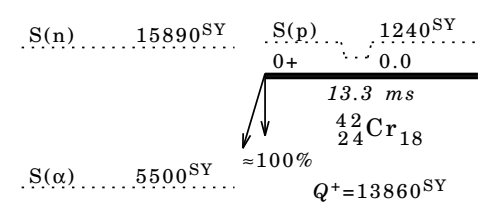

$\mathrm{S}(\mathrm{n}) \ldots \ldots \ldots+17478^{28}$

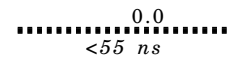

${ }_{23}^{42} \mathrm{~V}_{19}$

$Q^{+}=17490^{\mathrm{SY}}$
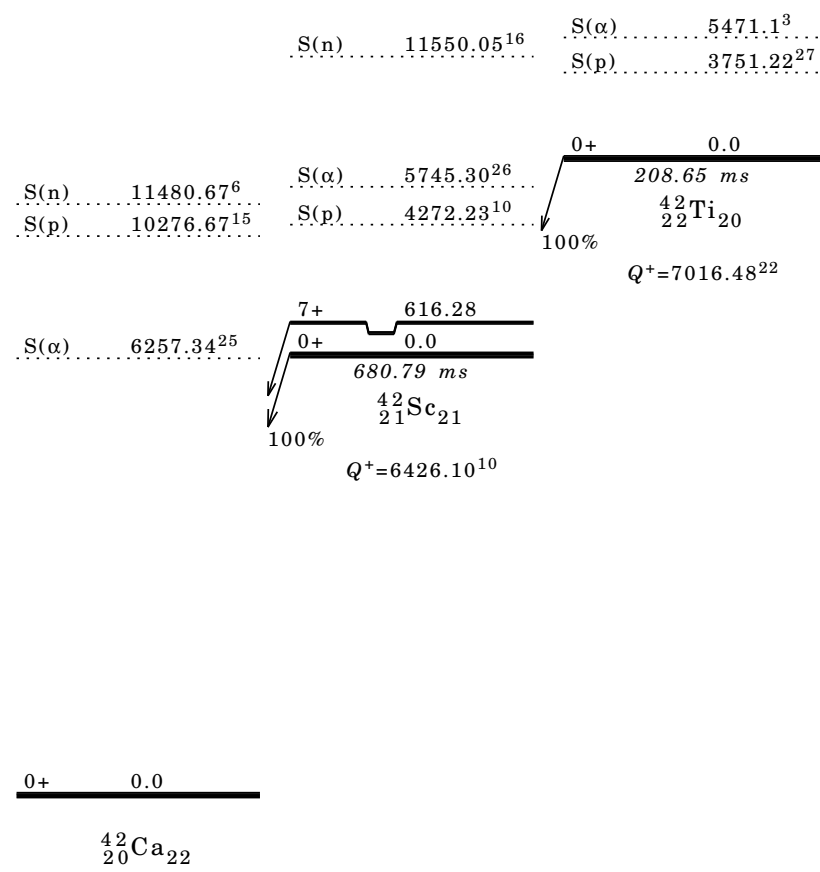

\begin{tabular}{|c|c|c|c|c|}
\hline \multicolumn{5}{|c|}{ Ground-State and Isomeric-Level Properties } \\
\hline Nuclide & Level & $\mathrm{J} \pi$ & $\mathrm{T}_{1 / 2}$ & Decay Modes \\
\hline${ }^{42} \mathrm{Al}$ & 0.0 & & $>170 \mathrm{~ns}$ & $\% \beta^{-}=? ; \% \beta^{-} \mathrm{n}=? ; \% \beta^{-} 2 \mathrm{n}=?$ \\
\hline${ }^{42} \mathrm{Si}$ & 0.0 & $0+$ & $12.5 \mathrm{~ms} 35$ & $\% \beta^{-}=100 ; \% \beta^{-} \mathrm{n}=? ; \% \beta^{-} 2 \mathrm{n}=?$ \\
\hline${ }^{42} \mathrm{P}$ & 0.0 & & $48.5 \mathrm{~ms} 15$ & $\% \beta^{-}=100 ; \% \beta^{-} \mathrm{n}=5020 ; \% \beta^{-} 2 \mathrm{n}=$ ? \\
\hline${ }^{42} \mathrm{~S}$ & 0.0 & $0+$ & $1.016 \mathrm{~s} 15$ & $\% \beta^{-}=100 ; \% \beta^{-} \mathrm{n}<4$ \\
\hline${ }^{42} \mathrm{Cl}$ & 0.0 & $(2-)$ & $6.8 \mathrm{~s} 3$ & $\% \beta^{-}=100$ \\
\hline${ }^{42} \mathrm{Ar}$ & 0.0 & $0+$ & 32.9 y 11 & $\% \beta^{-}=100$ \\
\hline${ }^{42} \mathrm{~K}$ & 0.0 & $2-$ & $12.355 \mathrm{~h} 7$ & $\% \beta^{-}=100$ \\
\hline${ }^{42} \mathrm{Ca}$ & 0.0 & $0+$ & stable & \\
\hline \multirow[t]{2}{*}{${ }^{42} \mathrm{Sc}$} & 0.0 & $0+$ & $680.79 \mathrm{~ms} 28$ & $\% \varepsilon+\% \beta^{+}=100$ \\
\hline & 616.28 & $7+$ & $61.7 \mathrm{~s} 4$ & $\% \varepsilon+\% \beta^{+}=100$ \\
\hline${ }^{42} \mathrm{Ti}$ & 0.0 & $0+$ & $208.65 \mathrm{~ms} 80$ & $\% \varepsilon+\% \beta^{+}=100$ \\
\hline${ }^{42} \mathrm{~V}$ & 0.0 & & $<55 \mathrm{~ns}$ & $\% \mathrm{p}=?$ \\
\hline${ }^{42} \mathrm{Cr}$ & 0.0 & $0+$ & $13.3 \mathrm{~ms} 10$ & $\% \varepsilon+\% \beta^{+} \approx 100 ; \% \varepsilon p=94.450$ \\
\hline${ }^{43} \mathrm{P}$ & 0.0 & $1 / 2+$ & $36.5 \mathrm{~ms} 15$ & $\% \beta^{-} \mathrm{n}=100$ \\
\hline${ }^{43} \mathrm{~S}$ & 0.0 & $(3 / 2-)$ & $265 \mathrm{~ms} 15$ & $\% \beta^{-} \mathrm{n}=40 \quad 10 ; \ldots$ \\
\hline${ }^{43} \mathrm{Cr}$ & 0.0 & $(3 / 2+)$ & $21.2 \mathrm{~ms} 7$ & $\% \varepsilon p=79.3 \quad 30 ; \ldots$ \\
\hline${ }^{45} \mathrm{Fe}$ & 0.0 & $(3 / 2+)$ & $2.45 \mathrm{~ms} 23$ & $\% \varepsilon 3 p=3.3 \quad 15 ; \ldots$ \\
\hline
\end{tabular}




\section{Adopted Levels}

$\mathrm{Q}\left(\beta^{-}\right)=24280 S Y ; \mathrm{S}(\mathrm{n})=1120 S Y ; \mathrm{S}(\mathrm{p})=24360 C A ; \mathrm{Q}(\alpha)=-22760 C A \quad 2012 \mathrm{Wa38,1997Mo25}$.

Estimated uncertainties (syst,2012Wa38): $\Delta \mathrm{Q}\left(\beta^{-}\right)=780, \Delta \mathrm{S}(\mathrm{p})=1034, \Delta \mathrm{S}(\mathrm{n})=840$.

$\mathrm{S}(\mathrm{p})$ and $\mathrm{Q}(\alpha)$ from $1997 \mathrm{Mo} 25 ; \mathrm{Q}\left(\beta^{-}\right)$and $\mathrm{S}(\mathrm{n})$ from $2012 \mathrm{Wa38}$. Theoretical $\mathrm{S}(\mathrm{n})=-2610$ (1997Mo25) differs much from 1120840 (syst,2012Wa38).

$\mathrm{S}(2 \mathrm{n})=3280780, \mathrm{Q}\left(\beta^{-} \mathrm{n}\right)=20650700 \quad($ syst,2012Wa38). Theoretical $\mathrm{S}(2 \mathrm{p})=53610$ (1997Mo25).

2007Ba71: $\mathrm{W}\left({ }^{48} \mathrm{Ca}, \mathrm{X} \gamma\right) \mathrm{E}=141 \mathrm{MeV} /$ nucleon beam from the National Superconducting Cyclotron Laboratory (NSCL). The

fragments were separated with the A1900 fragment separator. Isotopic identification by multiple $\Delta \mathrm{E}$ signals,

magnetic rigidity, total energy and time of flight analysis. Detectors: plastic scintillators, parallel-plate avalanche counters (PPACs) and silicon PIN diodes.

In $2007 \mathrm{Ba} 71$, a total of 23 events were assigned to ${ }^{42} \mathrm{Al}$, establishing particle stability of this nuclide.

\section{${ }^{42} \mathrm{Al}$ Levels}

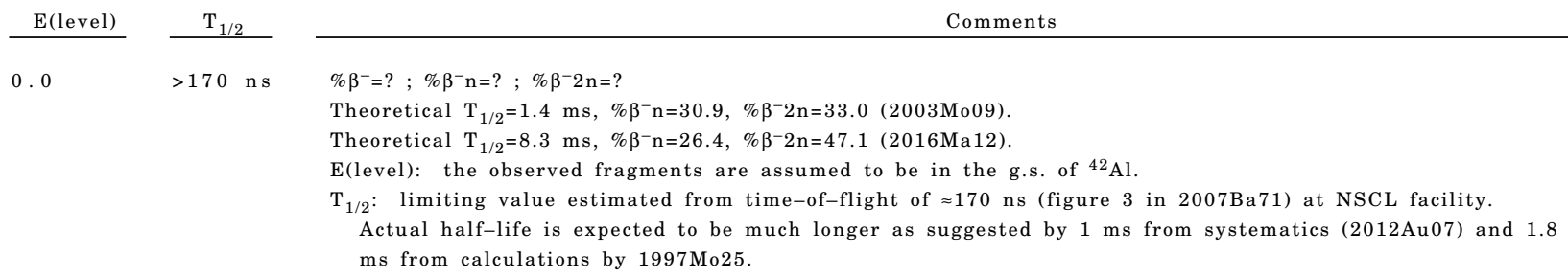




\section{Adopted Levels, Gammas}

$\mathrm{Q}\left(\beta^{-}\right)=15550 S Y ; \mathrm{S}(\mathrm{n})=3630 \mathrm{SY} ; \mathrm{S}(\mathrm{p})=24620 \mathrm{SY} ; \mathrm{Q}(\alpha)=-19940 \mathrm{SY} \quad 2012 \mathrm{Wa} 38$.

Estimated uncertainties (syst,2012Wa38): $\Delta \mathrm{Q}\left(\beta^{-}\right)=550, \Delta \mathrm{S}(\mathrm{n})=620, \Delta \mathrm{S}(\mathrm{p})=780, \Delta \mathrm{Q}(\alpha)=710 \quad(2012 \mathrm{Wa38})$.

$\mathrm{S}(2 \mathrm{n})=5010550, \mathrm{~S}(2 \mathrm{p})=46620 \quad 780, \mathrm{Q}\left(\beta^{-} \mathrm{n}\right)=13470510 \quad($ syst, $2012 \mathrm{Wa} 38)$.

1990Le03: ${ }^{42} \mathrm{Si}$ identified and produced in ${ }^{64} \mathrm{Ni}\left({ }^{48} \mathrm{Ca}, \mathrm{X}\right)$ reaction at $\mathrm{E}=44 \mathrm{MeV} /$ nucleon, followed by a measurement of fragment spectra versus atomic number.

$2004 \mathrm{Gr} 20$ (also $2004 \mathrm{Gr} 28,2003 \mathrm{Gr} 22$ ): ${ }^{42} \mathrm{Si}$ produced in ${ }^{9} \mathrm{Be}\left({ }^{48} \mathrm{Ca}, \mathrm{X}\right.$ ) at $\mathrm{E}=60 \mathrm{MeV} / \mathrm{nucleon}$, LISE 3 spectrometer at GANIL, isotopic identification by energy loss, time-of-flight and magnetic rigidities, double-sided Si strip (DSSD) detectors for residues. Measured $(\beta)$ (residues) time correlations and half-life using scintillation detectors for $\beta$ rays. Detailed QRPA calculations. Predictions of Gamow-Teller strengths, I $\beta$ and $\% \beta^{-} n$ for spherical, prolate $(\varepsilon=+0.3)$ and oblate $(\varepsilon=-0.3)$ shapes. The measured half-life suggests deformed shape when compared with QRPA calculations.

2006Fr13 (also 2005Fr19), 2006 GrZZ: see ${ }^{9} \mathrm{Be}\left({ }^{44} \mathrm{~S}, \mathrm{X} \gamma\right.$ ) data-set.

1999YoZW, from fragmentation of ${ }^{48} \mathrm{Ca}$ beam using ${ }^{9} \mathrm{Be}\left({ }^{48} \mathrm{Ca}, \mathrm{X}\right)$ and ${ }^{181} \mathrm{Ta}\left({ }^{48} \mathrm{Ca}, \mathrm{X}\right)$ reactions at $70 \mathrm{MeV} / \mathrm{nucleon}$; measured

$\mathrm{T}_{1 / 2}$ and $\% \beta^{-} \mathrm{n}$; according to the authors, the results are preliminary.

Mass measurement: mass excess $=15160580$ (2007 Ju03).

Recent theory papers: 2016Li07, 2011Li47, 2011Ka03, 2010Sm02 and 2010Ga15 (shell-model calculations), $2010 \mathrm{Ha} 33$

(quadrupole moment), 2009Co21 (half-life), 2009No01 and 2009Zi01 (B(E2)).

$$
\begin{gathered}
\frac{{ }^{42} \mathrm{Si} \text { Levels }}{\text { Cross Reference (XREF) Flags }} \\
\text { A }{ }^{9} \mathrm{Be}\left({ }^{44} \mathrm{~S},{ }^{42} \mathrm{Si} \gamma\right) \\
\text { B } \left.{ }^{44} \mathrm{~S},{ }^{42} \mathrm{Si} \gamma\right)
\end{gathered}
$$

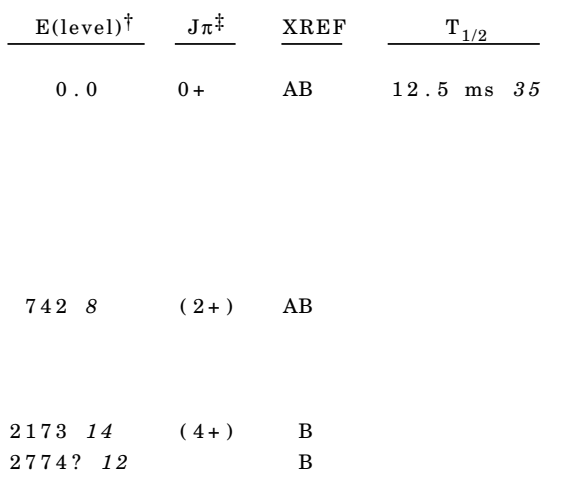

Comments

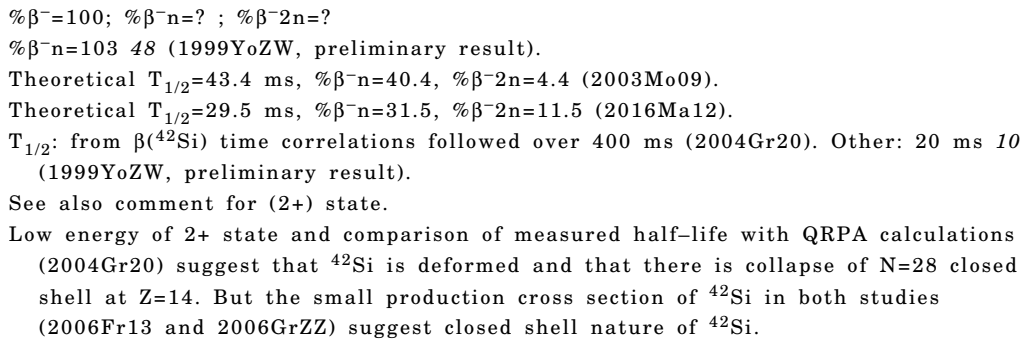

\begin{tabular}{|c|c|c|}
\hline E(level) & $\mathrm{E} \gamma^{\dagger}$ & Comments \\
\hline 742 & 7428 & $\mathrm{E} \gamma: 77019$ from ${ }^{9} \mathrm{Be}\left({ }^{44} \mathrm{~S},{ }^{42} \mathrm{Si} \gamma\right)$. \\
\hline 2173 & 143111 & \\
\hline $2774 ?$ & $2032 \neq 9$ & E $\gamma$ : possible partial feeding of the $2+$ state at $742 \mathrm{keV}(2012 \mathrm{Ta} 20)$. \\
\hline
\end{tabular}

$\dagger$ From a least-square fit to $\gamma$ energies.

$\ddagger$ From systematics of even-even nuclei. 
Adopted Levels, Gammas (continued)

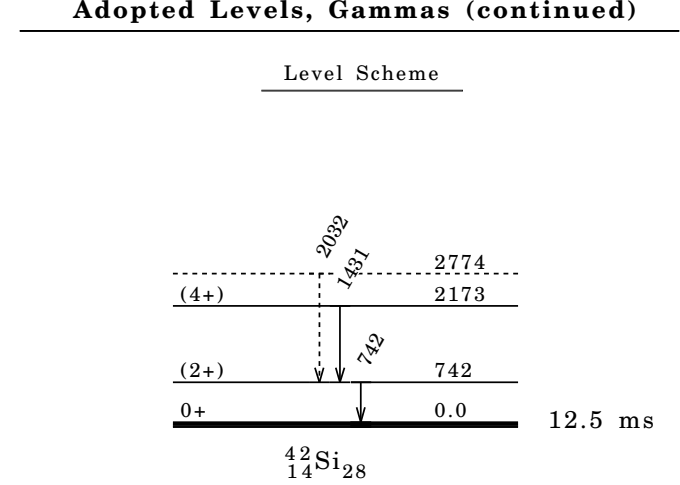

\section{${ }^{9} \mathrm{Be}\left({ }^{44} \mathrm{~S},{ }^{42} \mathrm{Si} \gamma\right) \quad 2007 \mathrm{Ba} 47,2006 \mathrm{Fr} 13$}

$2007 \mathrm{Ba} 47$ (also $2006 \mathrm{GrZZ}$ ): determination of first $2+$ state in ${ }^{9} \mathrm{Be}\left({ }^{44} \mathrm{~S},{ }^{42} \mathrm{Si} \gamma\right.$ ) reaction. The $39 \mathrm{MeV} / \mathrm{nucleon}{ }^{44} \mathrm{~S}$ beam from fragmentation of ${ }^{48} \mathrm{Ca}$ beam at $60 \mathrm{MeV} /$ nucleon in the SISSI device at GANIL facility. Isotopic identification by energy loss and time-of-flight method. The nuclei produced in the second fragmentation were transmitted through the SPEG spectrometer, where a total of 132 nuclei were observed corresponding to a cross section of $0.80 \mathrm{mb} 10$ in $2007 \mathrm{Ba}_{7}(0.102 \mathrm{mb} 26$ in $2006 \mathrm{GrZZ})$. The $\gamma$ ray detector array was $4 \pi$ Chateau de Crystal consisting of $74 \mathrm{BaF}_{2}$ detectors.

2006 Fr13 (also $2005 \mathrm{Fr} 19$ ): $\mathrm{E}\left({ }^{44} \mathrm{~S}\right)=98.6 \mathrm{MeV} /$ nucleon secondary beam produced from fragmentation of ${ }^{48} \mathrm{Ca}$ beam at 140 $\mathrm{MeV} /$ nucleon with a ${ }^{9} \mathrm{Be}$ target. Fragments were separated by A1900 separator at NSCL, MSU facility. The ${ }^{44} \mathrm{~S}$ beam impinged another ${ }^{9} \mathrm{Be}$ target and the residues were analyzed by $\mathrm{S}-800$ spectrograph. The knockout residues were identified by time-of-flight, energy loss measurement, position and angle information. The $\gamma$ rays were detected in coincidence with knockout residues of ${ }^{42} \mathrm{Si}$ using SeGA array of highly-segmented HPGe detectors.

Total cross section for ${ }^{42} \mathrm{Si}=0.12 \mathrm{mb} 2$ in comparison with $0.32 \mathrm{mb}$ from theoretical predictions, or $0.16 \mathrm{mb}$ if suppression factor of $\approx 0.5$ is used for two-nucleon shell-model strength. Calculations also predict $90 \%$ of the cross section for the population of the g.s., the contribution from the excited states to the total cross section is expected to be small (2006Fr13).

No discrete $\gamma$ rays observed in this reaction $(2006 \mathrm{Fr} 13)$.

The results suggest $\mathrm{Z}=14$ subshell closure and ${ }^{42} \mathrm{Si}$ as a doubly-magic nucleus (2006 Fr13).

\section{${ }^{42} \mathrm{Si}$ Levels}

\begin{tabular}{|c|c|c|}
\hline E(level) & $\mathrm{J} \pi$ & Comments \\
\hline $\begin{array}{lll}770 & 19\end{array}$ & $0+$ & $\begin{array}{l}\text { Low energy of } 2+\text { state and comparison of measured half-life with QRPA calculations }(2004 \mathrm{Gr} 20) \text { suggest that } \\
{ }^{42} \mathrm{Si} \text { is deformed and that there is collapse of } \mathrm{N}=28 \text { closed shell at } \mathrm{Z}=14 \text {. But the small production cross } \\
\text { section of }{ }^{42} \mathrm{Si} \text { in both studies }(2006 \mathrm{Fr} 13 \text { and } 2007 \mathrm{Ba} 47) \text { suggest closed shell nature of }{ }^{42} \mathrm{Si} \text {. } \\
\mathrm{E}(\text { level): from } 2007 \mathrm{Ba} 47 \text {. }\end{array}$ \\
\hline & & $\gamma\left({ }^{42} \mathrm{Si}\right)$ \\
\hline $\mathrm{E} \gamma$ & $\mathrm{E}$ (level) & Comments \\
\hline
\end{tabular}

$77019 \quad 770 \quad \mathrm{E} \gamma:$ from $2007 \mathrm{Ba} 47, \gamma$ observed in coincidence with ${ }^{42} \mathrm{Si}$ nuclei. 
Two-proton removal reaction.

2012Ta20: $\mathrm{E}=385 \mathrm{MeV} /$ nucleon ${ }^{48} \mathrm{Ca}$ primary beam with an average intensity of 70 pnA was produced at the RIBF facility at RIKEN and incident on a $15-\mathrm{mm}$-thick rotating beryllium target. A secondary ${ }^{44} \mathrm{~S}$ beam was analyzed by the BigRIPS fragment separator and accelerated to an energy of $210 \mathrm{MeV} /$ nucleon with an intensity of $4 \times 10^{4} \mathrm{pps}$. The secondary target was a $2.54 \mathrm{~g} / \mathrm{cm}^{2}$ carbon foil. Reaction products were analyzed by the ZeroDegree spectrometer and identified using the energy loss (ionization chamber), magnetic rigidity and time-of-flight (plastic scintillators); $\gamma$ rays were detected by the DALI2 array of $186 \mathrm{NaI}(\mathrm{Tl})$ detectors surrounding the reaction target (20\% efficiency, FWHM $=10 \%$ at $\mathrm{E} \gamma=1 \mathrm{MeV}$ ). Measured $\mathrm{E} \gamma, \mathrm{I} \gamma$, particle- $\gamma$-coin. Deduced levels, J $\pi$, rapid deformation development of Si isotopes. Comparison with shell-model calculations.

Total cross section for ${ }^{42} \mathrm{Si}=0.12 \mathrm{mb} 2$ for $\mathrm{E}=210 \mathrm{MeV} / \mathrm{nucleon}$.

${ }^{42} \mathrm{Si}$ Levels

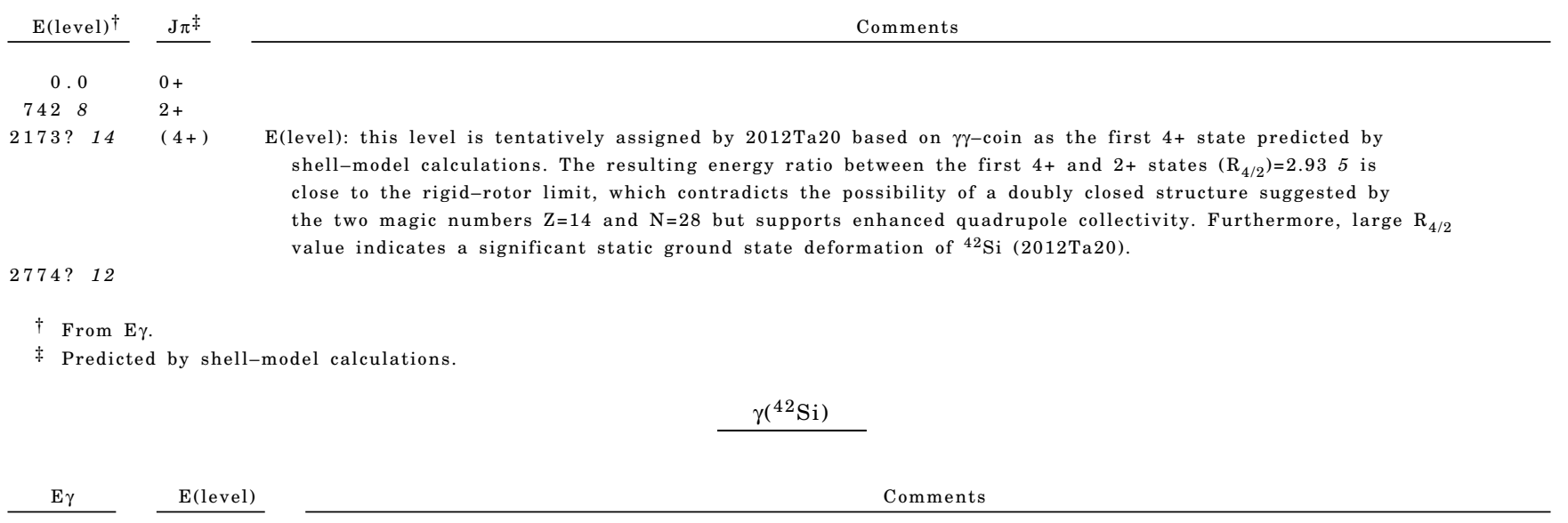

$\begin{array}{lll}742 & 8 & 742\end{array}$

$143111 \quad 2173$ ?

$2032^{\dagger} 9 \quad 2774$ ?

x $2357 \quad 15$

$\dagger$ Placement of transition in the level scheme is uncertain.

$x \quad \gamma$ ray not placed in level scheme.
E $\gamma$ : placement of this $\gamma$-ray is based on $\gamma \gamma$-coin with the $742 \gamma$-ray. Also yield of this $\gamma$-ray is consistent with $100 \%$ feeding of the $(2+)$ state.

E $\gamma$ : possible partial feeding of the $2+$ state at $742 \mathrm{keV}(2012 \mathrm{Ta} 20)$. 


\section{Adopted Levels}

$\mathrm{Q}\left(\beta^{-}\right)=18650210 ; \mathrm{S}(\mathrm{n})=2080220 ; \mathrm{S}(\mathrm{p})=18400 \quad 430 ; \mathrm{Q}(\alpha)=-17.63 \times 10^{3} 33 \quad 2012 \mathrm{Wa} 38$.

$\mathrm{S}(2 \mathrm{n})=7060240, \mathrm{~S}(2 \mathrm{p})=41540550 \quad(\mathrm{syst}), \mathrm{Q}\left(\beta^{-} \mathrm{n}\right)=11950210 \quad(2012 \mathrm{Wa} 38)$.

${ }^{42} \mathrm{P}$ identified in ${ }^{181} \mathrm{Ta}\left({ }^{48} \mathrm{Ca}, \mathrm{X}\right)$ reaction at $55 \mathrm{MeV} /$ nucleon $(1989 \mathrm{Le} 16),{ }^{9} \mathrm{Be}\left({ }^{48} \mathrm{Ca}, \mathrm{X}\right) \mathrm{E}=212 \mathrm{MeV} / \mathrm{nucleon}(1979 \mathrm{We} 10)$.

Mass measurements: 2000Sa21, 2001Sa72, 2007Ju03.

1989Le16: ${ }^{181} \mathrm{Ta}\left({ }^{48} \mathrm{Ca}, \mathrm{X}\right)$ reaction $\mathrm{E}=55 \mathrm{MeV} /$ nucleon; measured $\mathrm{T}_{1 / 2}$ and $\% \beta^{-} \mathrm{n}$. Other: $1999 \mathrm{YoZW}$, from fragmentation of

${ }^{48} \mathrm{Ca}$ beam using ${ }^{9} \mathrm{Be}\left({ }^{48} \mathrm{Ca}, \mathrm{X}\right)$ and ${ }^{181} \mathrm{Ta}\left({ }^{48} \mathrm{Ca}, \mathrm{X}\right)$ reactions at $70 \mathrm{MeV} /$ nucleon; preliminary values of $\mathrm{T}_{1 / 2}$ and $\% \beta^{-} \mathrm{n}$

reported.

2006Kh08: $\mathrm{Si}\left({ }^{42} \mathrm{P}, \mathrm{X}\right) \mathrm{E}=30-65 \mathrm{MeV} /$ nucleon; measured energy-integrated reaction $\sigma$; deduced mean radius.

Others: $1989 \mathrm{MuZU}, 2003 \mathrm{Gr} 22$.

${ }^{42} \mathrm{P}$ Levels

E(level)

0.0

$\mathrm{T}_{1 / 2}$

$48.5 \mathrm{~ms} 15$
Comments

$\% \beta^{-}=100 ; \% \beta^{-} \mathrm{n}=5020(1989 \mathrm{Le} 16) ; \% \beta^{-} 2 \mathrm{n}=$ ?

Theoretical $\mathrm{T}_{1 / 2}=42.1 \mathrm{~ms}, \% \beta^{-} \mathrm{n}=36.3, \% \beta^{-} 2 \mathrm{n}=12.8 \quad(2003$ Mo09).

Theoretical $\mathrm{T}_{1 / 2}=219 \mathrm{~ms}, \% \beta^{-} \mathrm{n}=20.6, \% \beta^{-} 2 \mathrm{n}=16.3 \quad(2016 \mathrm{Ma} 12)$.

$\mathrm{T}_{1 / 2}$ : From 2004Gr20 ( $\beta$-decay). Others: $110 \mathrm{~ms}+40-20$ (1989Le16, $\beta$-decay).

Mean radius $\mathrm{r}^{2}{ }_{0}=1.25 \mathrm{fm}^{2} 10(2006 \mathrm{Kh} 08)$. 


\section{Adopted Levels, Gammas}

$\mathrm{Q}\left(\beta^{-}\right)=7280 \quad 140 ; \mathrm{S}(\mathrm{n})=6700 \quad 5 ; \mathrm{S}(\mathrm{p})=19950 \quad 80 ; \mathrm{Q}(\alpha)=-15890 \quad 70 \quad 2012 \mathrm{Wa} 38$.

$\mathrm{S}(2 \mathrm{n})=109435, \mathrm{~S}(2 \mathrm{p})=37650230, \mathrm{Q}\left(\beta^{-} \mathrm{n}\right)=160070 \quad(2012 \mathrm{Wa} 38)$.

${ }^{42} \mathrm{~S}$ identified and produced by $1975 \mathrm{WiZG}$ using ${ }^{48} \mathrm{Ca}\left(\alpha,{ }^{10} \mathrm{C}\right)$ at $\mathrm{E}=110 \mathrm{MeV}$.

Mass measurements: mass excess=-17637.9 27 (2009Ri12), -17700 130 (2000Sa21) (weighted average of -18060330 (2000Sa21) and -17640140 from another study quoted by $2000 \mathrm{Sa} 21), 1991 \mathrm{Zh} 24$.

${ }^{42} \mathrm{~S}$ produced by fragmentation of ${ }^{48} \mathrm{Ca}$ beam $(2009 \mathrm{Ri} 12,2002 \mathrm{Az} 02,1998 \mathrm{WiZX}, 1998 \mathrm{WiZV}) ;{ }^{64} \mathrm{Ni}\left({ }^{48} \mathrm{Ca}, \mathrm{X}\right) \mathrm{E}=60 \mathrm{MeV} / \mathrm{nucleon}$ (1995So03); $\mathrm{Th}(\mathrm{p}, \mathrm{X}) \mathrm{E}=800 \mathrm{MeV}$ (1991Zh24); ${ }^{238} \mathrm{U}\left({ }^{40} \mathrm{Ar}, \mathrm{X}\right) \mathrm{E}=263 \mathrm{MeV}$ (1979Au03); ${ }^{9} \mathrm{Be}\left({ }^{48} \mathrm{Ca}, \mathrm{X}\right) \mathrm{E}=212 \mathrm{MeV}(1979 \mathrm{We} 10)$. Cross-section measurements: 2006Kh08, 2006Ro34.

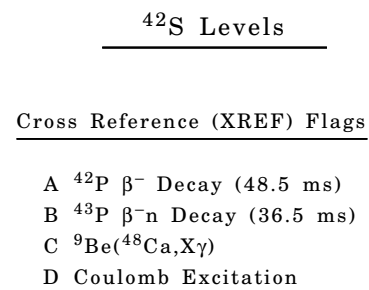

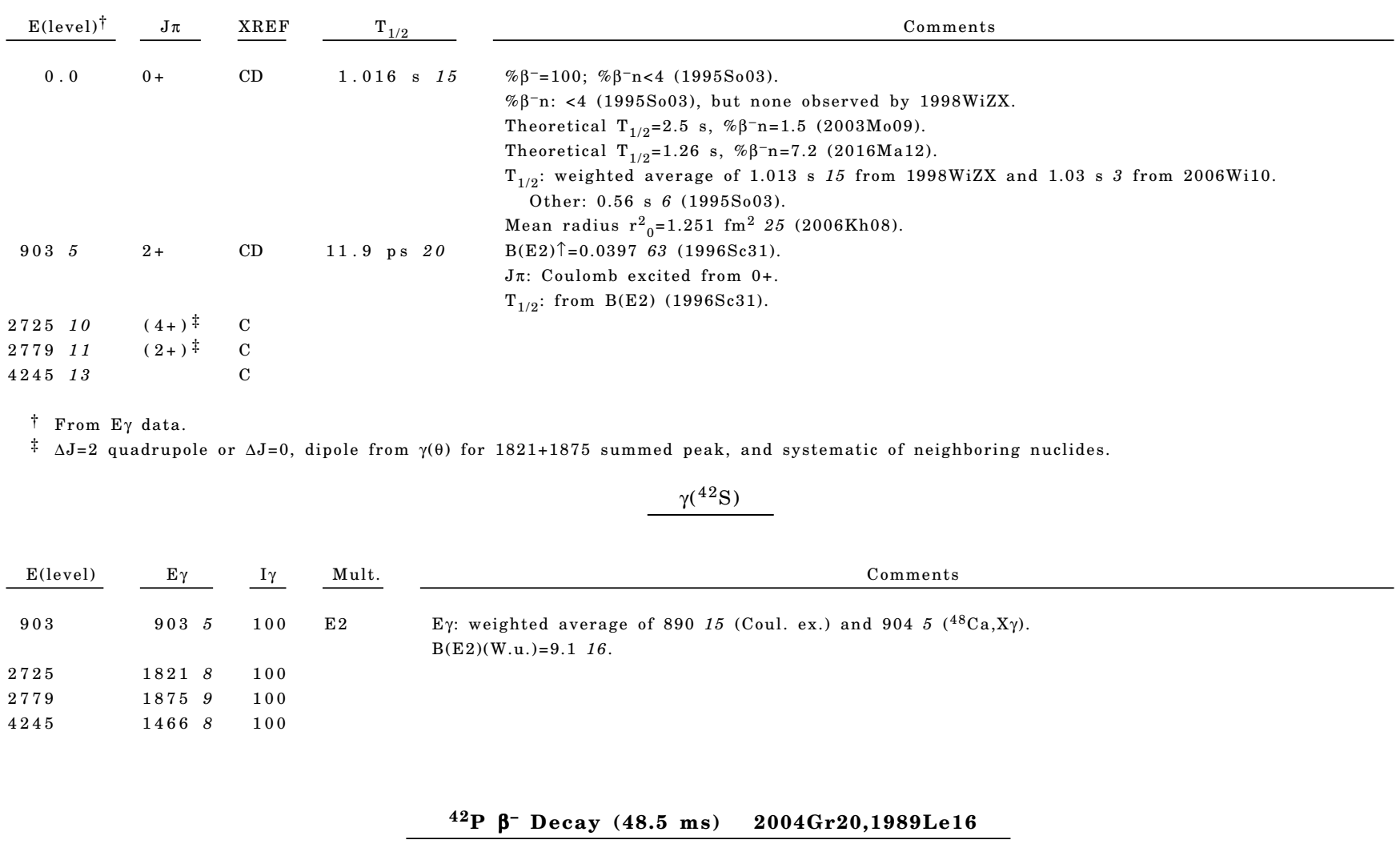

Parent ${ }^{42} \mathrm{P}: \mathrm{E}=0 ; \mathrm{J} \pi=? ; \mathrm{T}_{1 / 2}=48.5 \mathrm{~ms} 15 ; \mathrm{Q}$ (g.s.)=18650 210;\% $\beta^{-}$decay $=100$.

${ }^{42} \mathrm{P}-\mathrm{T}_{1 / 2}$ : From $2004 \mathrm{Gr} 20$. Other: $110 \mathrm{~ms}+40-20$ (1989Le16).

${ }^{42} \mathrm{P}-\mathrm{Q}\left(\beta^{-}\right)$: From $2012 \mathrm{Wa} 38$.

No decay details known. ${ }^{42} \mathrm{P}$ also decays to ${ }^{41} \mathrm{~S}$ by beta-delayed neutrons $\left(\% \beta^{-} \mathrm{n}=5020,1989\right.$ Le 16). 
Parent ${ }^{43} \mathrm{P}: \mathrm{E}=0 ; \mathrm{J} \pi=1 / 2+; \mathrm{T}_{1 / 2}=36.5 \mathrm{~ms} 15 ; \mathrm{Q}$ (g.s.) $=14250370 ; \% \beta^{-} \mathrm{n}$ decay $=100$.

${ }^{43} \mathrm{P}-\mathrm{J}_{,} \mathrm{T}_{1 / 2}$ : From ${ }^{43} \mathrm{P}$ Adopted Levels in the ENSDF database (March 2015 update).

${ }^{43} \mathrm{P}-\mathrm{Q}\left(\beta^{-} \mathrm{n}\right)$ : From $2012 \mathrm{Wa} 38$.

${ }^{43} \mathrm{P}-\% \beta^{-} \mathrm{n}$ decay: $\% \beta^{-} \mathrm{n}=100$ for ${ }^{43} \mathrm{p}$ decay (1995So03). Other: 1999YoZW.

$\% \beta^{-} n=100$ (1995So03). Other: 1999YoZW.

No information is available about population of levels in ${ }^{42} \mathrm{~S}$ from this decay.

\section{${ }^{9} \mathrm{Be}\left({ }^{48} \mathrm{Ca}, \mathrm{X} \gamma\right) \quad 2002 \mathrm{So14,2002Az02,2002Gu08}$}

2002So14,2002Az02,2002Az01,2002Gu08: E=60 MeV/nucleon ${ }^{48} \mathrm{Ca}$ beam was produced at GANIL. A $2.77 \mathrm{mg} / \mathrm{cm}{ }^{2} \mathrm{Be}$ target.

Fragments were analyzed with the SPEG spectrometer and $\gamma$-rays were detected by an array $25 \%$ efficiency at 1.33

$\mathrm{MeV}$ ) of $74 \mathrm{BaF}_{2}$ crystals ( $\mathrm{FWHM}=200 \mathrm{keV}$ at $1 \mathrm{MeV}, 500 \mathrm{keV}$ at $3 \mathrm{MeV}$ ) and three segmented Ge clover detectors (0.12\%

efficiency at $1.3 \mathrm{MeV}, \mathrm{FWHM}=30 \mathrm{keV}$ at $1.3 \mathrm{MeV}$ ). Measured $\mathrm{E} \gamma, \mathrm{I} \gamma, \gamma(\theta), \gamma \gamma$-coin. Deduced levels, J $\pi$, branchings.

Comparison with shell-model calculations.

${ }^{42} \mathrm{~S}$ Levels

\begin{tabular}{|c|c|c|c|}
\hline $\mathrm{E}(\text { level })^{\dagger}$ & $\mathrm{J} \pi$ & & Comments \\
\hline 0.0 & $0+$ & & \\
\hline $904 \quad 5$ & $2+$ & $\mathrm{J} \pi$ : from Adopted Levels. & \\
\hline $2725 \quad 10$ & $(4+) \neq$ & & \\
\hline $2779 \quad 11$ & $(2+)^{\ddagger}$ & & \\
\hline $4245 \quad 13$ & & & \\
\hline
\end{tabular}

$\gamma\left({ }^{42} \mathrm{~S}\right)$

\begin{tabular}{|c|c|c|c|}
\hline $\mathrm{E} \gamma$ & & E(level) & $\mathrm{I} \gamma$ \\
\hline 904 & 5 & 904 & 100 \\
\hline 1466 & 8 & 4245 & 30 \\
\hline 1821 & 8 & 2725 & 70 \\
\hline 1875 & 9 & 2779 & 30 \\
\hline
\end{tabular}

\section{Coulomb Excitation 1996Sc31}

1996Sc31: ${ }^{197} \mathrm{Au}\left({ }^{42} \mathrm{~S},{ }^{42} \mathrm{~S}^{\prime}\right) \mathrm{E}=1.7 \mathrm{GeV} .{ }^{42} \mathrm{~S}$ beam was obtained from fragmentation of ${ }^{48} \mathrm{Ca}$ beam with a ${ }^{9} \mathrm{Be}$ target. Measured $\gamma$-particle coincidence following Coulomb excitation using position-sensitive NaI detector array.

\section{${ }^{42} \mathrm{~S}$ Levels}

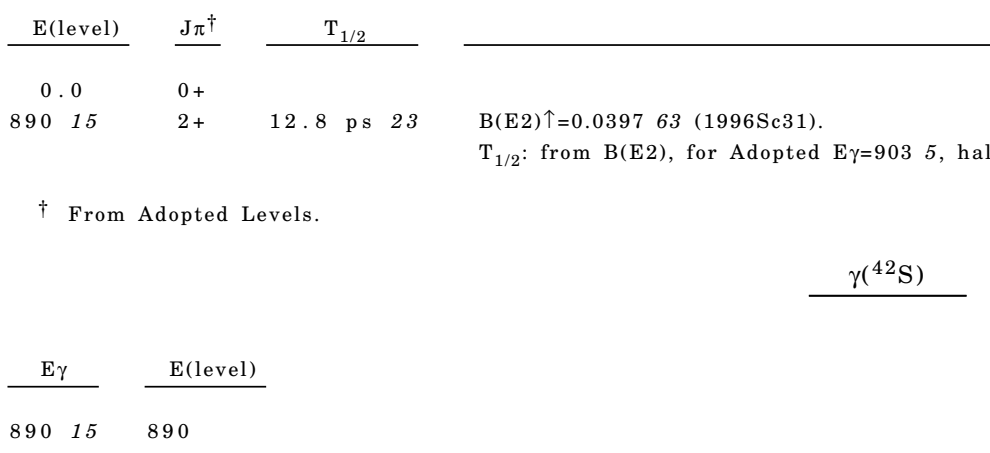




\section{Adopted Levels, Gammas}

$\mathrm{Q}\left(\beta^{-}\right)=9510140 ; \mathrm{S}(\mathrm{n})=5680 \quad 160 ; \mathrm{S}(\mathrm{p})=13190 \quad 140 ; \mathrm{Q}(\alpha)=-12.67 \times 10^{3} \quad 17 \quad 2012 \mathrm{Wa} 38$. $\mathrm{S}(2 \mathrm{n})=13500150, \mathrm{~S}(2 \mathrm{p})=31420 \quad 180, \mathrm{Q}\left(\beta^{-} \mathrm{n}\right)=80 \quad 140 \quad(2012 \mathrm{Wa} 38)$

1971Ar32: ${ }^{42} \mathrm{Cl}$ produced and identified in ${ }^{232} \mathrm{Th}\left({ }^{40} \mathrm{Ar}, \mathrm{X}\right)$ reaction at $290 \mathrm{MeV}$, measured fragment yield. 1981Vo04: ${ }^{42} \mathrm{Cl}$ produced in $\mathrm{U}, \mathrm{Nb}(\mathrm{p}, \mathrm{X})$ at $600 \mathrm{MeV}$, measured half-life. 1998WiZX, 1998WiZV, 1989Mi03, 1981HuZT: studies of ${ }^{42} \mathrm{Cl} \beta$ decay. Mass measurements: 2015Xu14, 1991Zh24, 1990Tu01.

Cross-section and radius measurements: 2006Kh08, 2006Ro34, 1999Ai02. 1991Wa19: shell-model calculations, levels, binding energy, $\beta$-decay rates.

\section{${ }^{42} \mathrm{Cl}$ Levels \\ $\underline{\text { Cross Reference (XREF) Flags }}$
A ${ }^{42} \mathrm{~S} \beta^{-}$Decay $(1.016 \mathrm{~s})$
B ${ }^{43} \mathrm{~S} \beta^{-}$n Decay $(265 \mathrm{~ms})$ \\ C ${ }^{208} \mathrm{~Pb}\left({ }^{40} \mathrm{Ar}, \mathrm{X} \gamma\right)$}

\begin{tabular}{|c|c|c|}
\hline $\mathrm{E}(\text { level })^{\dagger}$ & $\mathrm{J} \pi$ & XREF \\
\hline 0.0 & $(2-)^{\ddagger}$ & A $\mathrm{C}$ \\
\hline 118.196 & $(3-)^{\ddagger}$ & $\mathrm{A}$ \\
\hline $522 ? \quad 1$ & $(4-) \ddagger$ & $\mathrm{C}$ \\
\hline $588.97 \quad 6$ & $(1-, 2,3+) \S$ & A \\
\hline $639.44 \quad 9$ & $(1-, 2,3+) \S$ & A \\
\hline 841.916 & $(1-, 2,3+) \S$ & A \\
\hline $1025.82 \quad 23$ & $(1-, 2,3+) \S$ & A \\
\hline $1267.49 \quad 7$ & $1+$ & A \\
\hline $1385.69 \quad 18$ & & A \\
\hline 1550.429 & & A \\
\hline $1576.65 ? 20$ & & A \\
\hline $1684.22 ? \quad 11$ & & A \\
\hline 1710.928 & & A \\
\hline $1834.87 ? \quad 13$ & & A \\
\hline 2123.318 & $1+$ & A \\
\hline $2221.03 ? \quad 12$ & & A \\
\hline $2241.17 \quad 25$ & $(1+)$ & A \\
\hline $2403.2 ? \quad 4$ & & A \\
\hline $3029.71 \quad 11$ & $1+$ & $\mathrm{A}$ \\
\hline
\end{tabular}

Comments

$\% \beta^{-}=100$.

$\mathrm{J} \pi:(3-)$ in $2006 \mathrm{Wi} 10$.

$\mathrm{T}_{1 / 2}$ : from $1981 \mathrm{Vo} 04$.

Mean radius $\mathrm{r}_{0}^{2}=1.304 \mathrm{fm}^{2} 54(2006 \mathrm{Kh} 08)$.

$\mathrm{J} \pi:(2-)$ in $2006 \mathrm{Wi} 10$.

$\mathrm{J} \pi: \log f t=4.99$ from $0+$

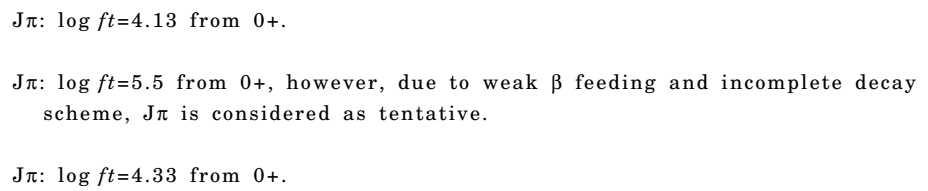

$\dagger$ From least-squares fit to $\mathrm{E} \gamma$ data.

† From shell-model calculations (2013Sz02).

$\S$ Inferred from a comparison with the decay scheme of ${ }^{40} \mathrm{~S}(2006 \mathrm{Wi10})$.

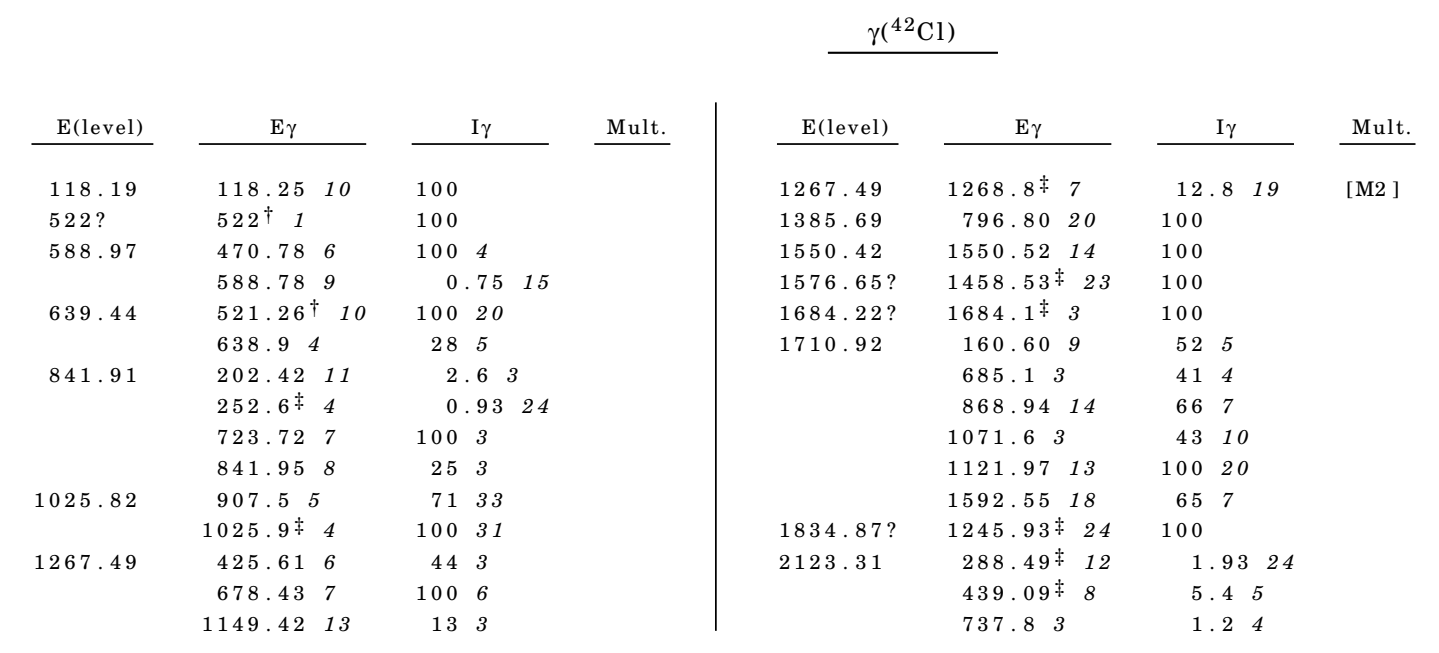

Continued on next page (footnotes at end of table) 
Adopted Levels, Gammas (continued)

\begin{tabular}{|c|c|c|c|c|c|c|c|c|}
\hline \multirow[b]{3}{*}{$\mathrm{E}$ (level) } & & \multirow{3}{*}{\multicolumn{2}{|c|}{$\mathrm{I} \gamma$}} & \multicolumn{4}{|c|}{ Adopted Leveis, Gammas (continued) } & \\
\hline & & & & & $\gamma\left({ }^{42} \mathrm{Cl}\right)$ & ntinued) & \multirow{2}{*}{\multicolumn{2}{|c|}{$\mathrm{I} \gamma$}} \\
\hline & $\mathrm{E} \gamma$ & & & Mult. & $\mathrm{E}$ (level) & $\mathrm{E} \gamma$ & & \\
\hline \multirow[t]{4}{*}{2123.31} & $1281.44 \quad 12$ & 100 & 4 & & 3029.71 & $808.55^{\ddagger} 15$ & 16 & 326 \\
\hline & $1534.26 \quad 11$ & & $0 \quad 16$ & & & $1193.1 \neq 9$ & 18 & 3 \\
\hline & $2004.93 \quad 16$ & 52 & $\begin{array}{ll}0 & 19\end{array}$ & & & $1318.88 \quad 11$ & 100 & 4 \\
\hline & 2123.95 & & 95 & [ M2 ] & & $1453.2 \neq 3$ & 14 & $4 \quad 19$ \\
\hline $2221.03 ?$ & 670.55 末 10 & 100 & & & & 2187.24 & 29 & 3 \\
\hline \multirow[t]{2}{*}{2241.17} & 1399.13 & 100 & 11 & & & 2440.63 & 26 & 3 \\
\hline & 1652.44 & 45 & 15 & & & $2911.7 \quad 5$ & 20 & 3 \\
\hline $2403.2 ?$ & $1814.2 \ddagger 4$ & 100 & & & & & & \\
\hline
\end{tabular}

$\dagger$ Note two $\gamma$ rays of nearly the same energy.

¥ Placement of transition in the level scheme is uncertain.

\section{${ }^{42} S \beta^{-}$Decay $(1.016$ s) $2006 W i 10$}

Parent ${ }^{42} \mathrm{~S}: \mathrm{E}=0 ; \mathrm{J} \pi=0+; \mathrm{T}_{1 / 2}=1.016$ s $15 ; \mathrm{Q}$ (g.s. $)=7280140 ; \% \beta^{-}$decay $=100$.

${ }^{42} \mathrm{~S}-\mathrm{T}_{1 / 2}$ : From ${ }^{42} \mathrm{~S}$ Adopted Levels.

${ }^{42} \mathrm{~S}-\mathrm{Q}\left(\beta^{-}\right)$: From $2012 \mathrm{Wa} 38$.

2006Wi10: ${ }^{42} \mathrm{~S}$ isotope produced by fragmentation of a ${ }^{48} \mathrm{Ca}$ beam at $70 \mathrm{MeV} / \mathrm{nucleon}$ hitting a ${ }^{9} \mathrm{Be}$ target. The fragments

were separated by A1200 fragment separator at NSCL, Michigan facility. Measured E $\gamma, \mathrm{I} \gamma, \gamma \gamma, \beta, \beta \gamma$ coin using two

Ge detectors for $\gamma$ rays and a plastic scintillator for $\beta$ rays. Comparisons with Shell-model calculations.

1998WiZX: reported preliminary result of $2006 \mathrm{Wi10}$; measured $\mathrm{T}_{1 / 2}$, E $\gamma, \mathrm{I} \gamma, \gamma \gamma$-coin, neutrons. No delayed neutrons

were observed by $1998 \mathrm{WiZX}$; $1995 \mathrm{So} 03$ reported $\% \beta^{-} \mathrm{n}<4$.

$2013 \mathrm{Sz} 02$ refer to a study of this decay by D. O'Donnell, Ph.D. thesis, University of the West Scotland (2008).

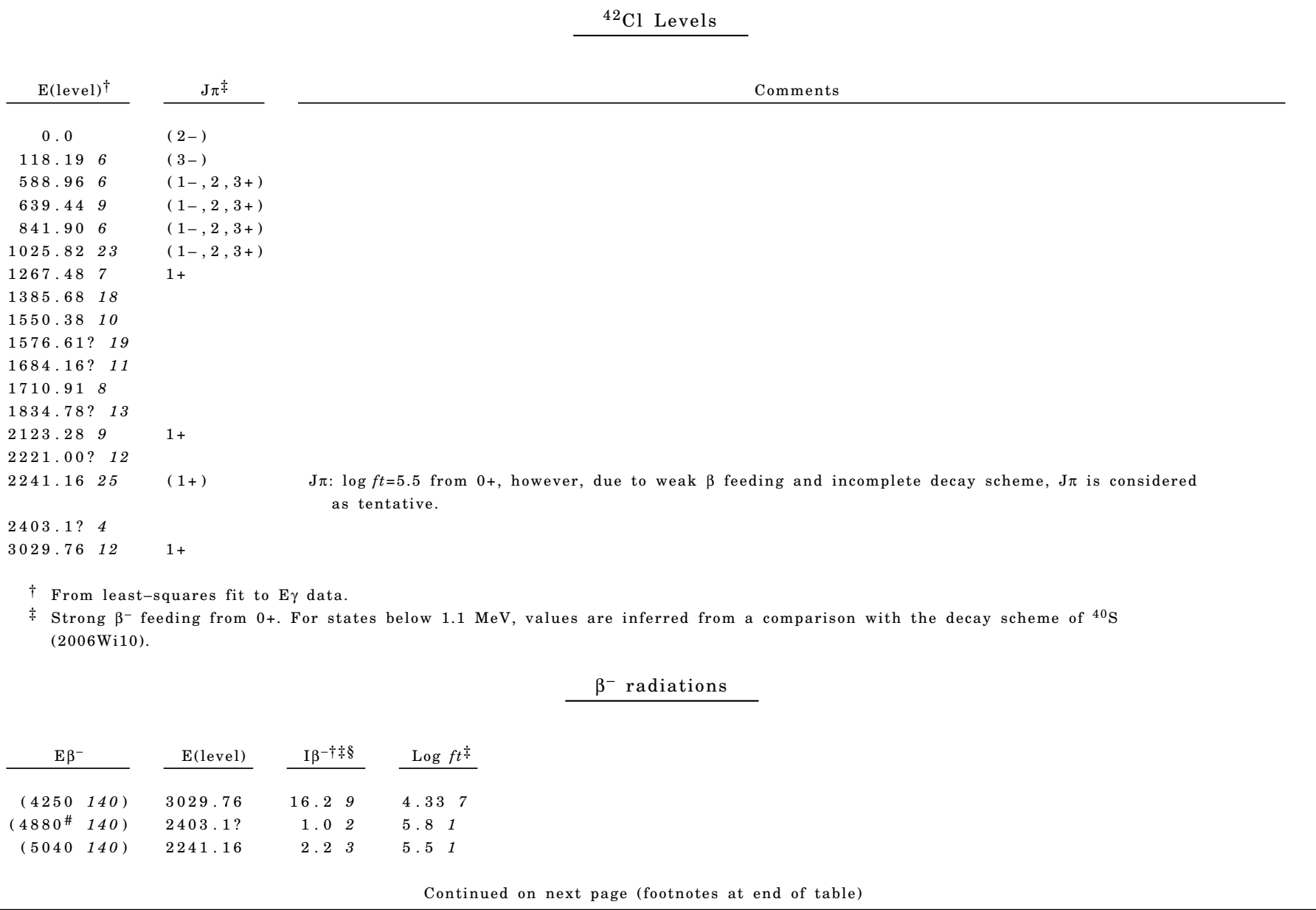


${ }^{42} S \beta^{-}$Decay (1.016 s) $2006 W i 10$ (continued)

$\beta^{-}$radiations (continued)

\begin{tabular}{|c|c|c|c|c|}
\hline \multicolumn{2}{|c|}{$\mathrm{E} \beta^{-}$} & $\mathrm{E}$ (level) & $I \beta^{-\dagger \dagger \S}$ & $\log f t^{末}$ \\
\hline ( 5160 & $140)$ & 2123.28 & $\begin{array}{ll}63 & 3\end{array}$ & $4.13 \quad 6$ \\
\hline ( 5570 & $140)$ & 1710.91 & 1.68 & $5.9 \quad 2$ \\
\hline ( 5890 & $140)$ & 1385.68 & $0.7 \quad 2$ & 6.42 \\
\hline ( $6 \begin{array}{llll} & 0 & 1 & 0\end{array}$ & $140)$ & 1267.48 & $17.6 \quad 11$ & 4.99 \\
\hline
\end{tabular}

$\dagger$ From intensity balance.

¥ Due to large $Q$ value, it is possible that there are unobserved levels above the currently known level at 3030 , thus the $\beta$-feedings and $\log f t$ values are considered as limits only. However, $\beta$ transitions with large feedings (>15\%) are considered as allowed.

$\S$ Absolute intensity per 100 decays.

\# Existence of this branch is questionable.

$\gamma\left({ }^{42} \mathrm{Cl}\right)$

I $\gamma$ normalization: $\Sigma($ I $\gamma$ to g.s. $)=100$, assuming zero feeding to g.s.

\begin{tabular}{|c|c|c|c|c|c|c|c|}
\hline $\mathrm{E} \gamma^{\dagger}$ & $\mathrm{E}($ level) & $\mathrm{I} \gamma^{\ddagger}$ & Mult. & $\mathrm{E} \gamma^{\dagger}$ & E(level) & $\mathrm{I} \gamma^{\ddagger}$ & Mult. \\
\hline $118.25 \quad 10$ & 118.19 & $100 \quad 5$ & & 1071.63 & 1710.91 & $1.3 \quad 3$ & \\
\hline $160.60 \quad 9$ & 1710.91 & $1.57 \quad 15$ & & $1121.97 \quad 13$ & 1710.91 & $3.0 \quad 6$ & \\
\hline $202.42 \quad 11$ & 841.90 & $0.96 \quad 11$ & & $1149.42 \quad 13$ & 1267.48 & $1.7 \quad 4$ & \\
\hline 252.64 & 841.90 & $0.35 \quad 9$ & & $1193.1 \S 9$ & 3029.76 & $1.6^{\dagger} 3$ & \\
\hline $288.49 \S \quad 12$ & 2123.28 & $0.72 \quad 9$ & & $1245.93 \S 24$ & $1834.78 ?$ & $1.08 \quad 18$ & \\
\hline 425.616 & 1267.48 & $5.7 \quad 4$ & & $1268.8 \S 7$ & 1267.48 & $1.65^{\dagger} 24$ & [M2 ] \\
\hline $439.09 \S \quad 8$ & 2123.28 & $2.00 \quad 17$ & & $1281.44 \quad 12$ & 2123.28 & $37.3 \quad 14$ & \\
\hline $470.78 \quad 6$ & 588.96 & $33.5 \quad 12$ & & $1318.88 \quad 11$ & 3029.76 & 9.14 & \\
\hline $521.26 \quad 10$ & 639.44 & $2.0 \quad 4$ & & 1399.13 & 2241.16 & $1.76 \quad 19$ & \\
\hline $588.78 \quad 9$ & 588.96 & $0.25 \quad 5$ & & $1453.2 \S 3$ & 3029.76 & $1.31 \quad 17$ & \\
\hline $638.9 \quad 4$ & 639.44 & 0.559 & & $1458.53 \S 23$ & $1576.61 ?$ & $1.21 \quad 20$ & \\
\hline $670.55 \S \quad 10$ & $2221.00 ?$ & $1.84 \quad 16$ & & $1534.26 \quad 11$ & 2123.28 & 12.76 & \\
\hline $678.43 \quad 7$ & 1267.48 & $12.9 \quad 7$ & & $1550.52 \quad 14$ & 1550.38 & $2.85 \quad 23$ & \\
\hline 685.13 & 1710.91 & $1.22 \quad 13$ & & $1592.55 \quad 18$ & 1710.91 & $1.94 \quad 22$ & \\
\hline $723.72 \quad 7$ & 841.90 & $37.6 \quad 12$ & & 1652.44 & 2241.16 & $0.80 \quad 26$ & \\
\hline $737.8 \quad 3$ & 2123.28 & $0.45 \quad 13$ & & $1684.1 \S 3$ & $1684.16 ?$ & $1.63 \quad 19$ & \\
\hline $796.80 \quad 20$ & 1385.68 & 1.33 & & $1814.2 \S 4$ & $2403.1 ?$ & $1.20 \quad 19$ & \\
\hline $808.55 \S \quad 15$ & 3029.76 & $1.48 \quad 24$ & & $2004.93 \quad 16$ & 2123.28 & $19.4 \quad 7$ & \\
\hline 841.958 & 841.90 & $9.4 \quad 12$ & & $2123.9 \S 5$ & 2123.28 & $1.47^{\dagger} \quad 17$ & [M2 ] \\
\hline $868.94 \quad 14$ & 1710.91 & $1.98 \quad 22$ & & 2187.24 & 3029.76 & $2.6 \quad 3$ & \\
\hline $907.5 \quad 5$ & 1025.82 & $0.39 \quad 18$ & & $2440.6 \quad 3$ & 3029.76 & $2.4 \quad 3$ & \\
\hline 1025.94 & 1025.82 & $0.55 \quad 17$ & & 2911.75 & 3029.76 & $1.8 \quad 3$ & \\
\hline \multicolumn{8}{|c|}{$\dagger$ From 1998WiZX only. } \\
\hline \multicolumn{8}{|c|}{ ¥ For absolute intensity per 100 decays, multiply by 0.874 . } \\
\hline$\S$ Placement & & the level sch & & & & & \\
\hline
\end{tabular}


${ }^{42} \mathrm{~S} \beta^{-}$Decay (1.016 s) $2006 \mathrm{Wi10}$ (continued)

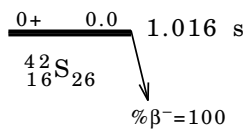

$Q^{-}$(g.s.) $=7280^{140}$

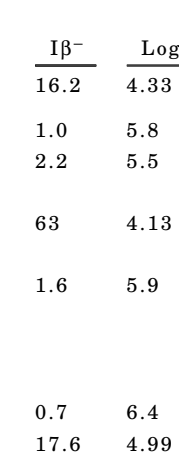

Decay Scheme

Intensities: $\mathrm{I}(\gamma+\mathrm{ce})$ per 100 parent decays

3029.76

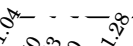

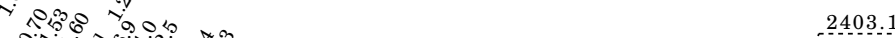

in

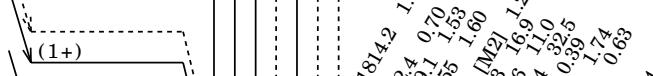

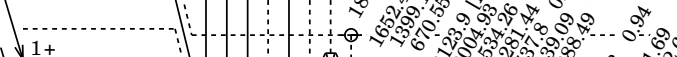

$1+$

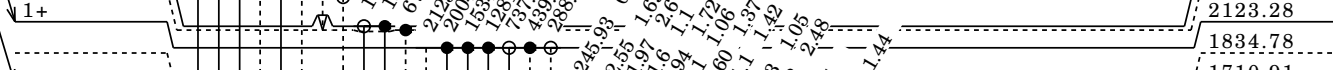



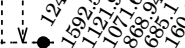

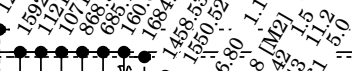

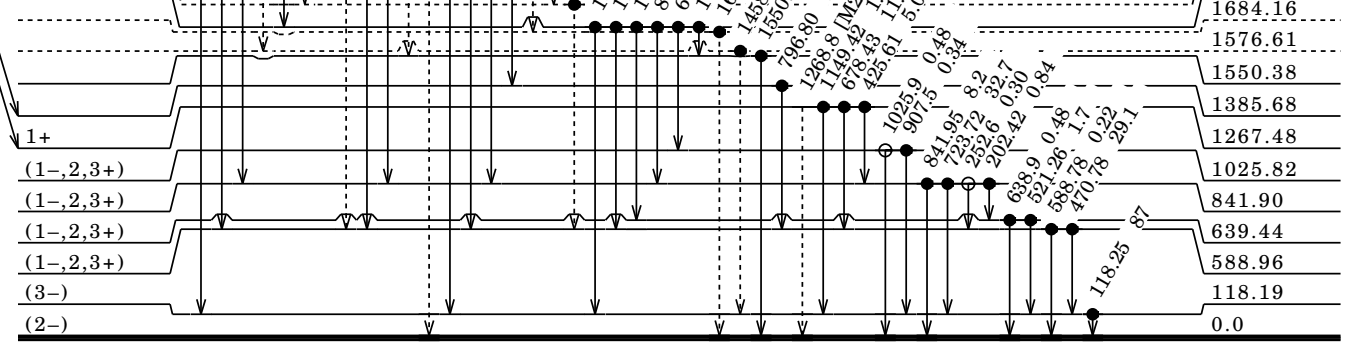

${ }_{17}^{42} \mathrm{Cl}_{25}$

${ }^{43} \mathrm{~S} \beta^{-} \mathrm{n}$ Decay $(265 \mathrm{~ms}) \quad 1989 \mathrm{Le} 16$

Parent ${ }^{43} \mathrm{~S}: \mathrm{E}=0 ; \mathrm{J} \pi=(3 / 2-) ; \mathrm{T}_{1 / 2}=265 \mathrm{~ms} 15 ; \mathrm{Q}$ (g.s.) $=4650140 ; \% \beta^{-} \mathrm{n}$ decay=40 10

${ }^{43} \mathrm{~S}-\mathrm{J}_{,} \mathrm{T}_{1 / 2}$ : From Adopted Levels of ${ }^{43} \mathrm{~S}$ in the ENSDF database (March 2015 update).

${ }^{43} \mathrm{~S}-\mathrm{Q}\left(\beta^{-} \mathrm{n}\right)$ : From $2012 \mathrm{Wa} 38$.

${ }^{43} \mathrm{~S}-\% \beta^{-} \mathrm{n}$ decay: $\% \beta^{-} \mathrm{n}=4010$ for ${ }^{43} \mathrm{~s}$ decay (1989Le16).

$\% \beta^{-} \mathrm{n}=40 \quad 10$ (1989Le16).

No information is available about population of levels in ${ }^{42} \mathrm{Cl}$ from this decay. 


\section{${ }^{208} \mathrm{~Pb}\left({ }^{40} \mathrm{Ar}, \mathrm{X} \gamma\right) \quad 2013 \mathrm{Sz} 02$}

2013Sz02 (also 2013Sz01): transfer channel: 1p removal and 3n addition $\mathrm{E}\left({ }^{40} \mathrm{Ar}\right)=255 \mathrm{MeV}$ provided by the ECR ion source and accelerated by the superconducting ALPI-Linac accelerator of the Laboratory Nazionali di Legnaro. Target $=300 \mu \mathrm{g} / \mathrm{cm}^{2}{ }^{208} \mathrm{~Pb}$. Measured fragments, E $\gamma$, I $\gamma$, time-of-flight, energy loss, $\gamma \gamma-$, (fragment) $\gamma-\mathrm{coin}$ using the Clara array and magnetic spectrometer Prisma. Deduced level, J, $\pi$. Comparison with large-scale shell-model calculations

\section{${ }^{42} \mathrm{Cl}$ Levels}

\begin{tabular}{|c|c|}
\hline $\mathrm{E}$ (level) & $\mathrm{J} \pi^{\dagger}$ \\
\hline 0.0 & $(2-)$ \\
\hline
\end{tabular}

$\dagger$ From 2013Sz02 based on shell-model calculations.

\begin{tabular}{|c|c|c|}
\hline $\mathrm{E} \gamma$ & E(level) & $\mathrm{I} \gamma^{\ddagger}$ \\
\hline $\mathrm{x}_{117^{\dagger}}$ & & \\
\hline $\mathrm{x}_{23} 0^{\dagger}$ & & \\
\hline $\mathrm{x}_{275^{\dagger}}$ & & \\
\hline$\times 400^{\dagger}$ & & \\
\hline $522 \quad 1$ & $522 ?$ & $2.0 \quad 4$ \\
\hline
\end{tabular}

$\dagger$ Weak $\gamma$ observed. Authors claim corresponding $\gamma$ (except the $230 \gamma$ ) present in ${ }^{42} \mathrm{~S} \beta^{-}$decay from the work on $\beta$ decay by D. O'Donnell, Ph.D. thesis, University of the West Scotland (2008).

\# Effective number of counts after taking into account detector efficiency. I $\gamma$ from 2013 Sz 02 divided by 10 .

$x \quad \gamma$ ray not placed in level scheme. 


\section{Adopted Levels, Gammas}

$\mathrm{Q}\left(\beta^{-}\right)=5996 ; \mathrm{S}(\mathrm{n})=9426 \quad 6 ; \mathrm{S}(\mathrm{p})=14400 \quad 70 ; \mathrm{Q}(\alpha)=-998692012 \mathrm{Wa} 38$.

$\mathrm{S}(2 \mathrm{n})=155256, \mathrm{~S}(2 \mathrm{p})=261637(2012 \mathrm{Wa} 38)$.

${ }^{42} \mathrm{Ar}$ identified and produced by $1952 \mathrm{Ka} 44$ in successive thermal neutron capture in ${ }^{40} \mathrm{Ar}$, estimated half-life from its decay to ${ }^{42} \mathrm{~K}$.

Mass measurements: mass excess $=-34422.758(2001 \mathrm{He} 29)$.

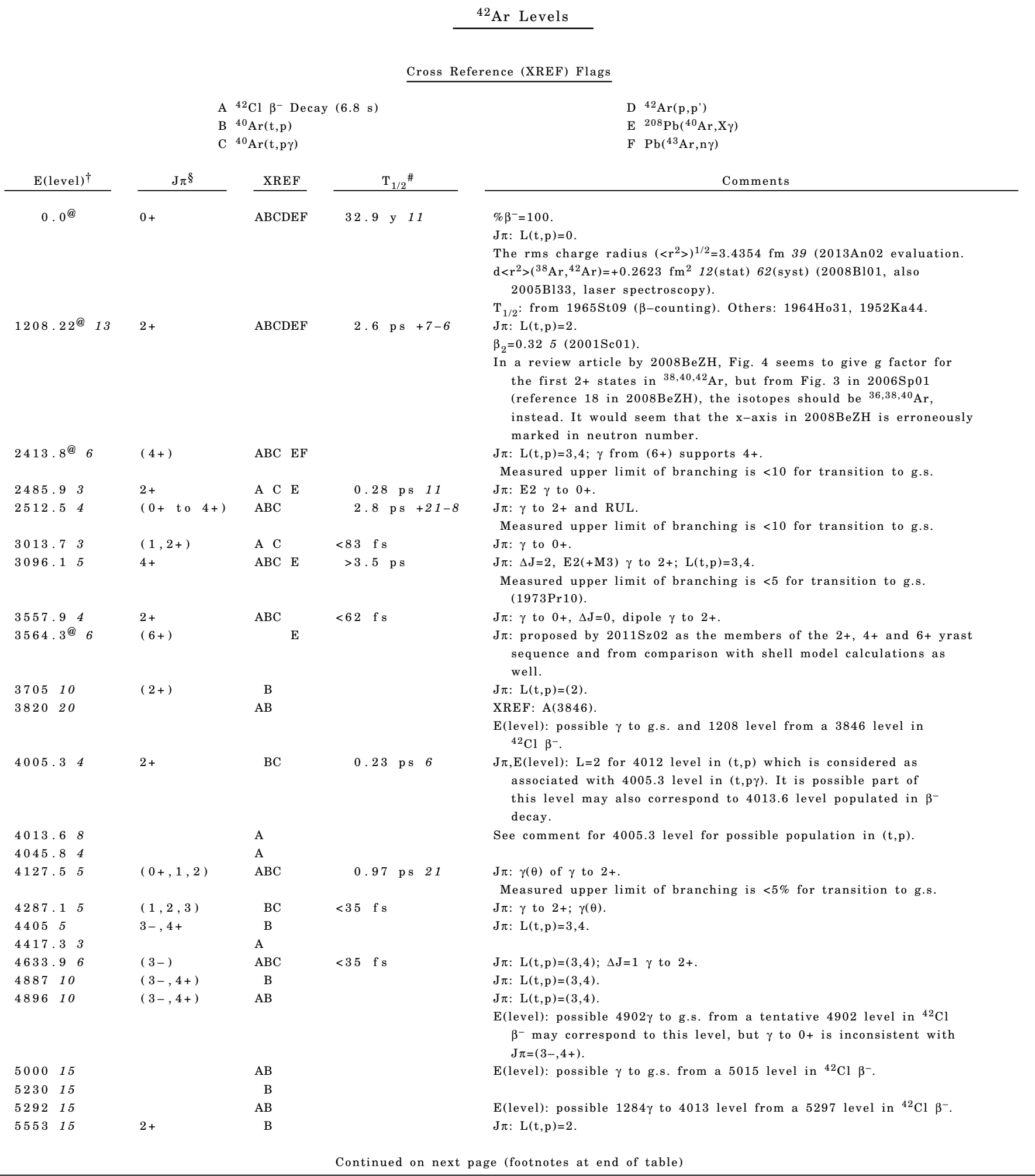


Adopted Levels, Gammas (continued)

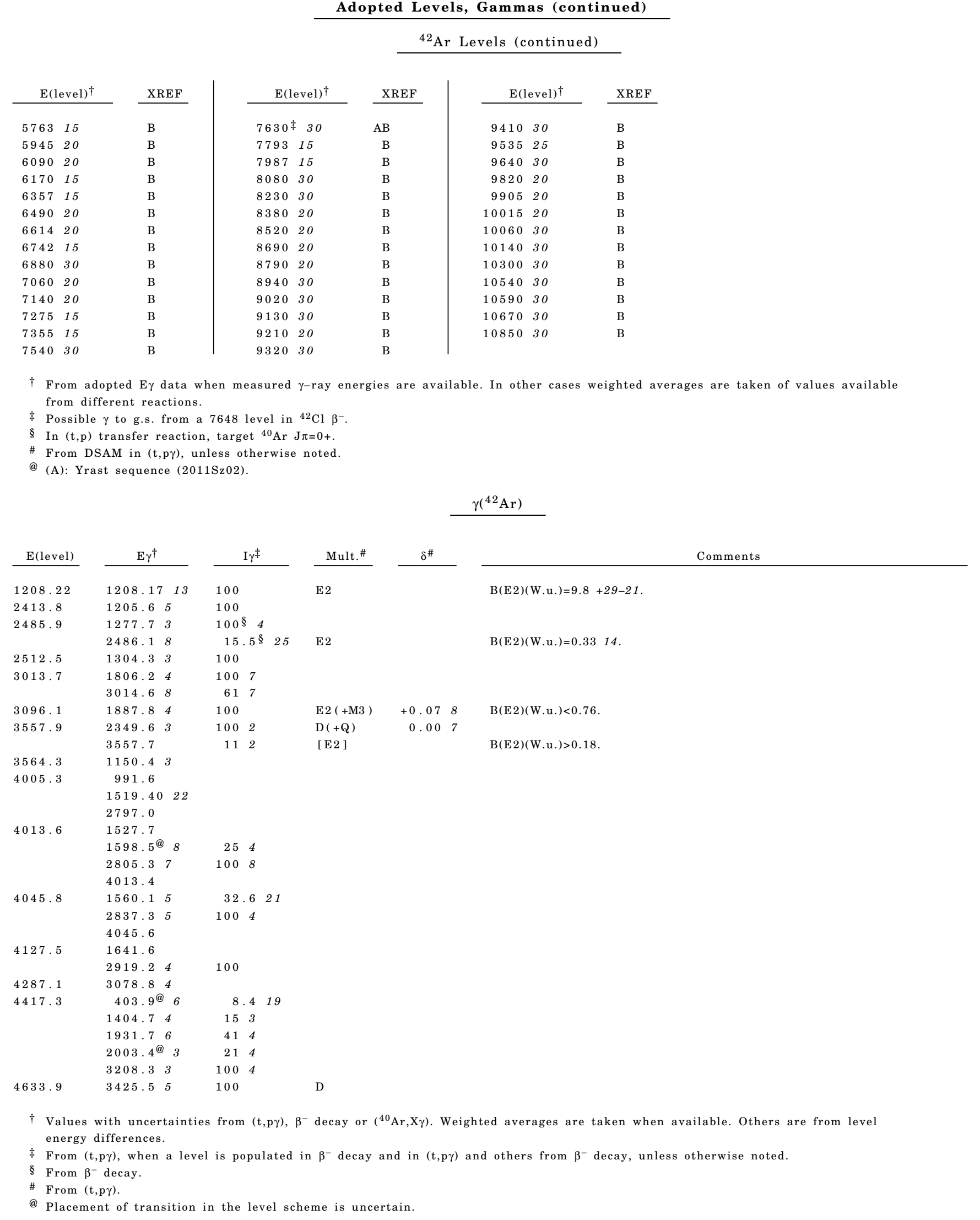


Adopted Levels, Gammas (continued)

\begin{tabular}{|c|c|}
\hline & $\begin{array}{l}\text { A) Yrast } \\
\text { equence } \\
011 \mathrm{Sz02} \text { ) }\end{array}$ \\
\hline$(6+)$ & 3564.3 \\
\hline$(4+)$ & $\downarrow \quad 2413.8$ \\
\hline $2+$ & 1208.22 \\
\hline $0+$ & $v \quad 0.0$ \\
\hline
\end{tabular}

\section{${ }^{42}$ Cl $\beta^{-}$Decay $(6.8$ s) $1981 \mathrm{HuZT}, 1998 \mathrm{WiZX}$}

Parent ${ }^{42} \mathrm{Cl}: \mathrm{E}=0 ; \mathrm{J} \pi=(2-) ; \mathrm{T}_{1 / 2}=6.8$ s $3 ; \mathrm{Q}$ (g.s. $)=9510140 ; \% \beta^{-}$decay $=100$.

${ }^{42} \mathrm{Cl}-\mathrm{J}, \mathrm{T}_{1 / 2}$ : From Adopted Levels of ${ }^{42} \mathrm{Cl}$.

${ }^{42} \mathrm{Cl}-\mathrm{Q}\left(\beta^{-}\right)$: From $2012 \mathrm{Wa} 38$.

1998WiZX: measured $\mathrm{E} \gamma, \mathrm{I} \gamma, \gamma \gamma$.

1981HuZT, 1989Mi03: measured E $\gamma, \mathrm{I} \gamma, \gamma \gamma$. $1989 \mathrm{Mi03}$ give $\beta \gamma$ coin results.

\begin{tabular}{|c|c|c|c|c|}
\hline $\mathrm{E}(\text { level })^{\dagger}$ & $\mathrm{J} \pi \ddagger$ & $\mathrm{E}(\text { level })^{\dagger}$ & $\mathrm{J} \pi \ddagger$ & $\mathrm{E}(\text { level })^{\dagger}$ \\
\hline 0.0 & $0+$ & 4181 ?§ & & $4827 ? \S$ \\
\hline $1207.7 \quad 6$ & $2+$ & $4196 ? \S$ & & $4902 ? \S$ \\
\hline 2413.48 & $(4+)$ & 4266 ?§ & & 4936 ?§ \\
\hline $2485.0 \quad 7$ & $2+$ & 4313 ? & & 4941 ?§ \\
\hline 2511 & & $4352 ? \S$ & & $5015 ? \S$ \\
\hline 3013.110 & $(1,2+)$ & $4416.5 \quad 6$ & & $5260 ? \S$ \\
\hline $3094.9 \quad 9$ & $4+$ & 4527 ? & & $5282 ? \S$ \\
\hline 3556 & $2+$ & $4566 ? \S$ & & $5297 ? \S$ \\
\hline 3846 & & 4607 ?§ & & $5506 ? \S$ \\
\hline $4012.9 \quad 7$ & & 4619 ?§ & & $5529 ? \S$ \\
\hline $4045.1 \quad 7$ & & 4633 & $(3-)$ & $5792 ? \S$ \\
\hline $4057 ? \S$ & & $4735 ? \S$ & & $6412 ? \S$ \\
\hline 4131 & & $4812 ? \S$ & & $7648 ? \S$ \\
\hline
\end{tabular}

$\dagger$ From 1981HuZT, except for levels where E $\gamma$ values are quoted with uncertainties, which are from 1998WiZX.

From Adopted Levels.

$\S$ Most levels above $4000 \mathrm{keV}$, reported only by $1981 \mathrm{HuZT}$, are considered as tentative in view of a comment by $1989 \mathrm{Mi} 03$ that the level scheme proposed earlier (1981HuZT) has been revised by taking account of source impurities. However, this revision is not available in the literature. The tentative levels are not included in the Adopted Levels, Gammas dataset.

\section{$\beta^{-}$radiations}

\begin{tabular}{|c|c|c|c|c|}
\hline \multicolumn{2}{|c|}{$\mathrm{E} \beta^{-}$} & $\mathrm{E}$ (level) & $\mathrm{I} \beta^{-}$ & $\log f t$ \\
\hline ( 1860 & $140)$ & $7648 ?$ & & \\
\hline ( 3100 & $140)$ & $6412 ?$ & & \\
\hline ( 3720 & $140)$ & $5792 ?$ & & \\
\hline ( 3980 & $140)$ & $5529 ?$ & & \\
\hline$\left(\begin{array}{llll}4 & 0 & 0 & 0\end{array}\right.$ & $140)$ & $5506 ?$ & & \\
\hline ( 4210 & $140)$ & $5297 ?$ & & \\
\hline ( 4230 & $140)$ & $5282 ?$ & & \\
\hline$(4250$ & $140)$ & $5260 ?$ & & \\
\hline ( 4500 & $140)$ & $5015 ?$ & & \\
\hline ( 4570 & $140)$ & $4941 ?$ & & \\
\hline ( 4570 & $140)$ & $4936 ?$ & & \\
\hline ( 4610 & $140)$ & $4902 ?$ & & \\
\hline ( 4680 & $140)$ & $4827 ?$ & & \\
\hline$(4700$ & $140)$ & $4812 ?$ & & \\
\hline ( 4780 & $140)$ & $4735 ?$ & & \\
\hline
\end{tabular}

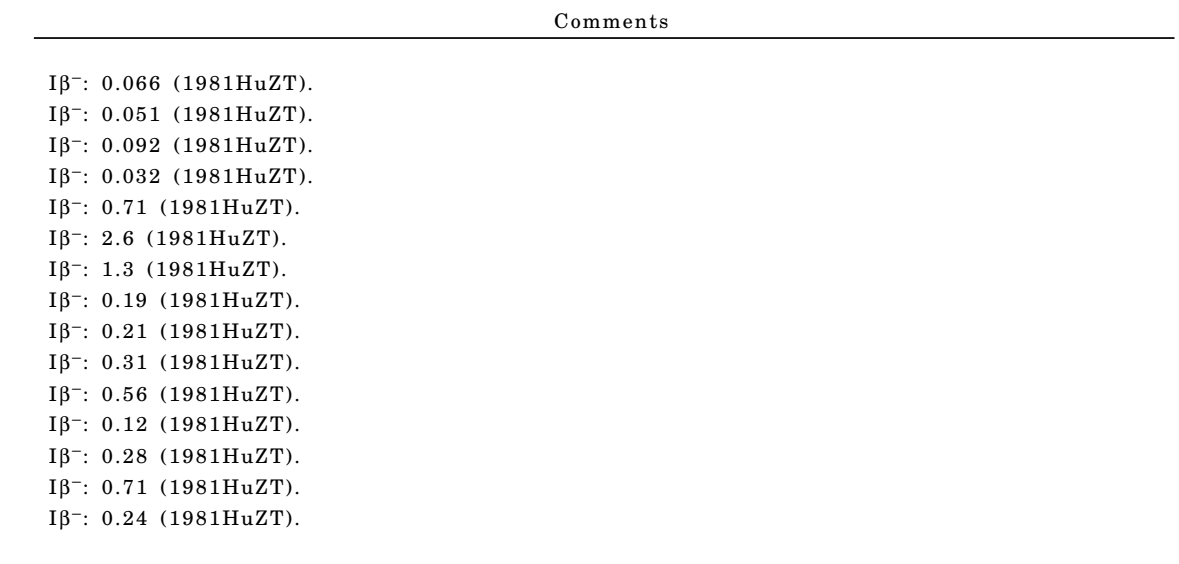

Continued on next page (footnotes at end of table) 
${ }^{42} \mathrm{Cl} \beta^{-}$Decay $(6.8$ s) $1981 \mathrm{HuZT}, 1998 W i Z X$ (continued)

$\beta^{-}$radiations (continued)

\begin{tabular}{|c|c|c|c|c|}
\hline \multicolumn{2}{|c|}{$\mathrm{E} \beta^{-}$} & \multirow{2}{*}{$\begin{array}{l}\text { E(level) } \\
4633\end{array}$} & \multirow[t]{2}{*}{$\mathrm{I} \beta^{-}$} & \multirow[t]{2}{*}{$\log f t$} \\
\hline ( 4880 & $140)$ & & & \\
\hline ( 4890 & $140)$ & $4619 ?$ & & \\
\hline$(4900$ & $140)$ & $4607 ?$ & & \\
\hline ( 4940 & $140)$ & $4566 ?$ & & \\
\hline ( 4980 & $140)$ & $4527 ?$ & & \\
\hline ( 5090 & $140)$ & 4416.5 & 17 & $\approx 5.5$ \\
\hline ( 5160 & $140)$ & $4352 ?$ & & \\
\hline ( 5200 & $140)$ & $4313 ?$ & & \\
\hline ( 5240 & $140)$ & $4266 ?$ & & \\
\hline ( 5310 & $140)$ & $4196 ?$ & & \\
\hline ( 5330 & $140)$ & $4181 ?$ & & \\
\hline ( 5380 & $140)$ & 4131 & & \\
\hline ( 5450 & $140)$ & $4057 ?$ & & \\
\hline ( 5460 & $140)$ & 4045.1 & 26 & $\approx 5.4$ \\
\hline ( 55000 & $140)$ & 4012.9 & & \\
\hline ( 5660 & $140)$ & 3846 & & \\
\hline ( 5950 & $140)$ & 3556 & & \\
\hline ( 6420 & $140)$ & 3094.9 & & \\
\hline 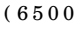 & $140)$ & 3013.1 & & \\
\hline 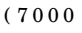 & $140)$ & 2511 & & \\
\hline ( 7030 & $140)$ & 2485.0 & & \\
\hline$(7100$ & $140)$ & 2413.4 & 19 & $\approx 6.1$ \\
\hline 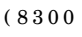 & $140)$ & 1207.7 & & \\
\hline ( 9510 & $140)$ & 0.0 & & \\
\hline
\end{tabular}

I $\beta^{-}: 0.50(1981 \mathrm{HuZT})$.

I $\beta^{-}: 0.06(1981 \mathrm{HuZT})$.

$\mathrm{I} \beta^{-}: 2.64(1981 \mathrm{HuZT})$.

$\mathrm{I} \beta^{-}: 0.19(1981 \mathrm{HuZT})$.

I $\beta^{-}: 0.04(1981 \mathrm{HuZT})$.

I $\beta^{-}$: from 1989Mi03. Others: 15 (1998WiZX), 11.6 (1981HuZT).

$\mathrm{E}\left(\beta^{-}\right)=39591400 \quad(1989 \mathrm{Mi03})$.

I $\beta^{-}: 0.10(1981 \mathrm{HuZT})$.

$\mathrm{I} \beta^{-}: 0.09(1981 \mathrm{HuZT})$.

I $\beta^{-}: 0.59(1981 \mathrm{HuZT})$.

I $\beta^{-}: 0.21(1981 \mathrm{HuZT})$.

$\mathrm{I} \beta^{-}: 0.20(1981 \mathrm{HuZT})$.

I $\beta^{-}: 1.17(1981 \mathrm{HuZT})$.

I $\beta^{-}: 0.12(1981 \mathrm{HuZT})$.

I $\beta^{-}$: from 1989Mi03. Others: 19 (1998WiZX), 17.6 (1981HuZT).

$\mathrm{E}\left(\beta^{-}\right)=46761052(1989 \mathrm{Mi03})$.

I $\beta^{-}: 4$ (1998WiZX), 2.41 (1981HuZT).

$\mathrm{I} \beta^{-}: 1.14(1981 \mathrm{HuZT})$.

$\mathrm{I} \beta^{-}: 0.14(1981 \mathrm{HuZT})$.

$\mathrm{I} \beta^{-}: 1.52(1981 \mathrm{HuZT})$.

I $\beta^{-}: 1.97(1981 \mathrm{HuZT})$.

I $\beta^{-}: 0.12(1981 \mathrm{HuZT})$.

I $\beta^{-}: 6$ (1998WiZX), 3.7 (1981HuZT).

I $\beta^{-}$: from 1989Mi03. Other: 4 (1998WiZX).

$\beta \gamma$ coin: $\mathrm{E}\left(\beta^{-}\right)=7021640,7479230(1989 \mathrm{Mi03})$.

I $\beta^{-}:<5 \%$ for g.s. +1208 level (1989Mi03). Other: 30 (1998WiZX). I $\beta=27$ (1981HuZT) is negated in the later work (1989Mi03).

$\mathrm{I} \beta^{-}:<5 \%$ for g.s. +1208 level (1989Mi03). I $\beta=20(1981 \mathrm{HuZT})$ is negated in the later work (1989Mi03).

\begin{tabular}{|c|c|c|c|c|c|c|c|}
\hline & & & & $\gamma\left({ }^{42} \mathrm{Ar}\right.$ & & & \\
\hline $\mathrm{E} \gamma^{\dagger}$ & E(level) & $\mathrm{I} \gamma \S$ & $\mathrm{E} \gamma^{\dagger}$ & E(level) & $\mathrm{I} \gamma \S$ & $\mathrm{E} \gamma^{\dagger}$ & E(level) \\
\hline $403.9 \ddagger 6$ & 4416.5 & $0.9 \quad 2$ & $2003.4^{@} 3$ & 4416.5 & 2.34 & 4313 & $4313 ?$ \\
\hline 604 & $5506 ?$ & & 2349 & 3556 & & 4352 & $4352 ?$ \\
\hline 767 & $4812 ?$ & & 2485.18 & 2485.0 & $2.5 \quad 4$ & 4527 & $4527 ?$ \\
\hline $1205.7 \quad 5$ & 2413.4 & $9.0^{\#} 8$ & 2639 & 3846 & & 4566 & $4566 ?$ \\
\hline $1207.7 \quad 6$ & 1207.7 & 100.06 & $2805.3 \quad 7$ & 4012.9 & 5.14 & 4607 & $4607 ?$ \\
\hline 1256 & $4812 ?$ & & $2837.3 \quad 5$ & 4045.1 & $19.0 \quad 7$ & 4619 & $4619 ?$ \\
\hline 1269 & $5282 ?$ & & 2924 & 4131 & & 4735 & $4735 ?$ \\
\hline $1277.3 \quad 5$ & 2485.0 & 16.16 & 3013 & 3013.1 & & 4812 & $4812 ?$ \\
\hline 1284 & $5297 ?$ & & 3059 & $4266 ?$ & & 4827 & $4827 ?$ \\
\hline 1304 & 2511 & & $3208.3 \quad 3$ & 4416.5 & 10.74 & 4902 & $4902 ?$ \\
\hline $1404.7 \ddagger 4$ & 4416.5 & 1.63 & 3359 & $4566 ?$ & & 4936 & $4936 ?$ \\
\hline 1528 & 4012.9 & & 3426 & 4633 & & 4941 & $4941 ?$ \\
\hline 1560.15 & 4045.1 & $6.0 \quad 4$ & 3556 & 3556 & & 5015 & $5015 ?$ \\
\hline 1572 & $4057 ?$ & & 3846 & 3846 & & 5260 & $5260 ?$ \\
\hline $1598.5^{@} 8$ & 4012.9 & 1.32 & 4013 & 4012.9 & & 5506 & $5506 ?$ \\
\hline 1646 & 4131 & & 4045 & 4045.1 & & 5529 & $5529 ?$ \\
\hline 1781 & $4266 ?$ & & 4057 & $4057 ?$ & & 5792 & $5792 ?$ \\
\hline 1805.4 & 3013.1 & 1.63 & 4181 & $4181 ?$ & & 6412 & $6412 ?$ \\
\hline $1887.2 \quad 7$ & 3094.9 & 1.93 & 4196 & $4196 ?$ & & 7648 & $7648 ?$ \\
\hline $1931.7 \quad 6$ & 4416.5 & $4.4 \quad 4$ & 4266 & $4266 ?$ & & & \\
\hline
\end{tabular}

$\dagger$ From 1998WiZX for E $\gamma$ data quoted with uncertainty. All other E $\gamma$ values are differences of level energies as given by $1981 \mathrm{HuZT}$.

$\ddagger$ From 1998WiZX only.

$\S$ From 1998WiZX.

\# Value of 0.98 in 1998 WiZX seems a misprint, in view of in-out intensity balance and $\beta^{-}$feeding shown in the level scheme of 1998 WiZX. On the other hand, \% I $\beta=19$ (1989Mi03) would imply a much larger intensity or the 1205.7 component of the $1206-1208$ doublet.

(a) Placement of transition in the level scheme is uncertain. 
$Q^{-}$(g.s.) $=951 \beta^{140}$

\begin{tabular}{c}
$Q^{-}$(g.s.) $=951$ \\
$I^{-} \quad \log f t$ \\
\hline
\end{tabular}<smiles>C1#CCCCC#C1</smiles>

26

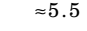

dx

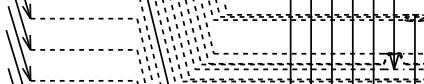

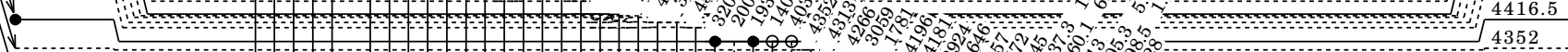

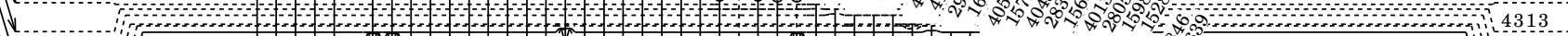
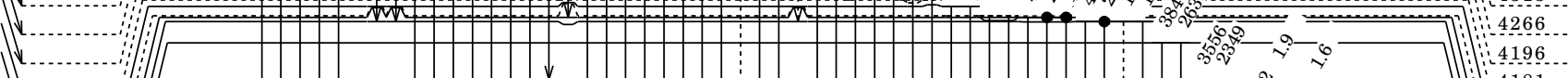

$\approx 5.4$

$\sqrt{1}$

|


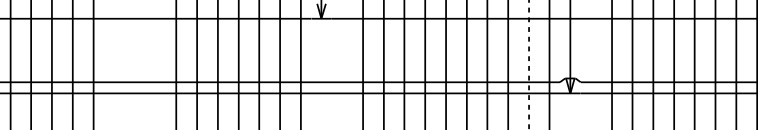

$2+$

$4+$

$(1,2+)$

$2+$

$\frac{2+}{2+}$
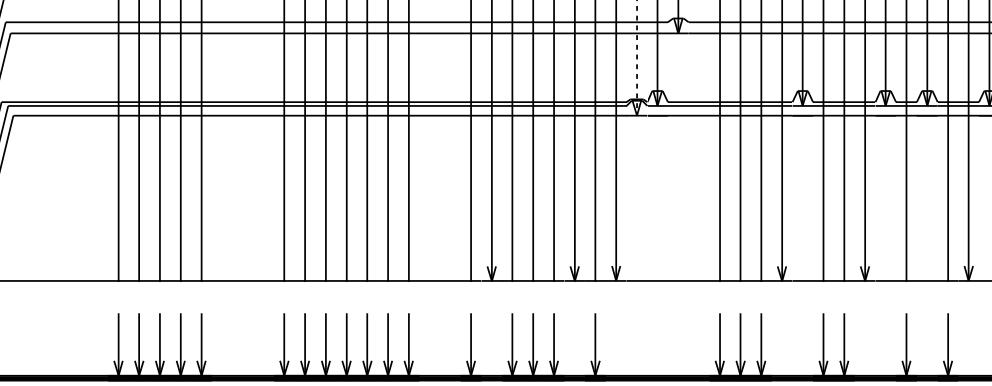

${ }_{18}^{42} \mathrm{Ar}_{24}$ 


\section{${ }^{40} \mathrm{Ar}(\mathrm{t}, \mathrm{p}) \quad \mathbf{1 9 7 5 F 1 0 8}$}

Target ${ }^{40} \mathrm{Ar} \mathrm{J} \pi=0+$.

$1975 \mathrm{Fl} 08$ (also 1973Ca13, 1961Ja07): E=20 MeV triton beam was produced from the Los Alamos three-stage electrostatic accelerator facility. Gas target of natural argon, effective thickness of $60 \mu \mathrm{g} / \mathrm{cm}^{2}$. Protons were momentum analyzed in a broad range spectrograph and detected in nuclear emulsions along the focal plane, FWHM=35 keV, at angles from $12.5^{\circ}$ to $57.5^{\circ}$. Measured $\sigma\left(\mathrm{E}_{\mathrm{p}}, \theta\right)$. Deduced levels, J $\pi$, L, DWBA analysis.

Other: $1974 \mathrm{WaYT}$

\begin{tabular}{|c|c|c|c|c|}
\hline E(level) & $L^{*}$ & $\mathrm{~d} \sigma / \mathrm{d} \Omega(\mathrm{mb} / \mathrm{sr})(\max , \mathrm{c} . \mathrm{m}.) \S$ & E(level) & $\mathrm{d} \sigma / \mathrm{d} \Omega(\mathrm{mb} / \mathrm{sr})(\max , \mathrm{c} . \mathrm{m} .)^{\S}$ \\
\hline 0.0 & 0 & $0.31^{\#}$ & $7355 \quad 15$ & \\
\hline $1208 \quad 5$ & 2 & 0.31 & $7540 \quad 30$ & 0.16 \\
\hline $2415 \quad 15$ & $4,3^{\mathrm{a}}$ & 0.041 & $7630^{\dagger} 30$ & \\
\hline $2520 ? \quad 10$ & & 0.026 & $7793 \quad 15$ & \\
\hline 30925 & $4,3^{\mathrm{a}}$ & 0.11 & $7987 \quad 15$ & \\
\hline $3563 \quad 5$ & & 0.90 & $8080^{\dagger} 30$ & \\
\hline $3705 \quad 10$ & $(2)$ & 0.085 & $8230 \dagger 30$ & $0.23 \&$ \\
\hline $3820 \quad 20$ & & $0.047^{\#}$ & $8380 \quad 20$ & 0.35 \\
\hline $4012 \quad 10$ & 2 & 0.31 & $8520^{\dagger} 20$ & \\
\hline $4130 \quad 15$ & & 0.034 & $8690 \quad 20$ & 0.34 \\
\hline $4296 \quad 5$ & & 0.22 & $8790 \quad 20$ & \\
\hline $4405 \quad 5$ & 3,4 & 0.25 & $8940^{\dagger} 30$ & 0.30 \\
\hline $4642 \quad 10$ & $(3,4)$ & 0.21 & $9020 \quad 30$ & \\
\hline $4896 \quad 10$ & $(3,4)$ & 0.12 & $9130^{\dagger} 30$ & \\
\hline $5000 \quad 15$ & & 0.23 & $9210 \quad 20$ & \\
\hline $5230^{\dagger} 15$ & & $0.16^{@}$ & $9320^{\dagger} 30$ & \\
\hline $5292^{\dagger} \quad 15$ & & $0.16^{@}$ & $9410 \quad 30$ & \\
\hline $5553 \quad 15$ & 2 & 0.20 & $9535 \quad 25$ & \\
\hline $5763 \quad 15$ & & 0.20 & $9640^{\dagger} 30$ & \\
\hline $5945 \quad 20$ & & $0.23^{\#}$ & $9820 \quad 20$ & \\
\hline $6090 \quad 20$ & & $0.13^{\#}$ & $9905 \quad 20$ & \\
\hline $6170 \quad 15$ & & & $10015^{\dagger} 20$ & \\
\hline $6357^{\dagger} \quad 15$ & & & $10060 \quad 30$ & \\
\hline $6490^{\dagger} 20$ & & & $10140 \quad 30$ & \\
\hline $6614 \quad 20$ & & 0.21 & $10300^{\dagger} 30$ & \\
\hline $6742 \quad 15$ & & 0.24 & $10540 \quad 30$ & \\
\hline $6880 \quad 30$ & & & $10590^{\dagger} 30$ & \\
\hline $7060^{\dagger} 20$ & & & $10670 \quad 30$ & \\
\hline $7140 \quad 20$ & & & $10850^{\dagger} 30$ & \\
\hline $7275^{\dagger} \quad 15$ & & 0.36 & & \\
\hline \multicolumn{5}{|c|}{$\dagger$ Probable doublet. } \\
\hline \multicolumn{5}{|c|}{$\begin{array}{l}\text { ¥ As implied by } \mathrm{J} \pi \text { values given in table } 3 \text { of } 1975 \mathrm{Fl08} \text {. } 1975 \mathrm{Fl08} \text { give experimental and calculated (DWBA) } \sigma(\theta) \text { distribution curves } \\
\text { for the following levels also, but no specific } \mathrm{L} \text { assignments were made based on these data: } 3563(\mathrm{~L}=2), 4130(\mathrm{~L}=0), 4296(\mathrm{~L}=2) \text {, } \\
5000(\mathrm{~L}=1), 5763(\mathrm{~L}=2), 5945(\mathrm{~L}=2), 6090(\mathrm{~L}=3), 6614(\mathrm{~L}=3), 6742(\mathrm{~L}=3), 7275(\mathrm{~L}=3), 8230(\mathrm{~L}=5), 8380(\mathrm{~L}=3) \text {. }\end{array}$} \\
\hline \multicolumn{5}{|c|}{$\S$ At $12.5^{\circ}$, unless otherwise stated. } \\
\hline \multicolumn{5}{|c|}{ \# At $20^{\circ}$. } \\
\hline \multicolumn{5}{|c|}{ @ For $5230+5292$. } \\
\hline \multicolumn{5}{|c|}{ \& At $27.5^{\circ}$. } \\
\hline a $\mathrm{L}=4$ is & & 050 & & \\
\hline
\end{tabular}

\section{${ }^{40} \operatorname{Ar}(t, p \gamma) \quad 1974 F i 01,1973 \operatorname{Pr} 10$}

1974Fi01: E=2.8 MeV triton beam was produced from the Lockheed 3-MV Van de Graaff accelerator. A solid target of natural argon at a temperature of $15 \mathrm{~K}$. Protons were detected in an annular silicon surface barrier detector and $\gamma$-rays by a $20-\mathrm{cm}^{3} \mathrm{Ge}(\mathrm{Li})$ detector at $30^{\prime}$ and $120^{\prime}$. Measured proton spectrum, E $\gamma$, I $\gamma$. Deduced levels, $\gamma$ branching ratios, $\mathrm{T}_{1 / 2}$ by DSAM.

1973Pr10: E=2.9 MeV beam from the Lockheed Van de Graaff accelerator. Target of a gas cell of natural argon $6.3 \mathrm{~mm}$ thick. Protons were detected by silicon annular detector (FWHM=120 keV) and $\gamma$-rays were detected by five NaI(Tl) detectors at $5^{\circ}, 35^{\circ}, 45^{\circ}, 60^{\circ}$ and $90^{\circ}$ with respect to the beam axis and a $20 \mathrm{~cm}^{3} \mathrm{Ge}(\mathrm{Li})$ detector. Measured $\mathrm{E} \gamma$ $\mathrm{I} \gamma, \mathrm{p} \gamma(\theta)$. Deduced levels, J $\pi$, mixing ratios, branching ratios.

Other: 1973BaXX. 
${ }^{40} \operatorname{Ar}(t, p \gamma) \quad 1974 F i 01,1973 P r 10$ (continued)

${ }^{42}$ Ar Levels

\begin{tabular}{|c|c|c|c|c|c|c|}
\hline \multicolumn{3}{|c|}{$\mathrm{E}(\text { level })^{\dagger}$} & $\mathrm{J} \pi^{\# @}$ & \multicolumn{3}{|c|}{$\mathrm{T}_{1 / 2} \S$} \\
\hline \multicolumn{3}{|c|}{0.0} & $0+$ & \multirow{3}{*}{$2.6 \mathrm{p}$} & \multirow{3}{*}{ os } & \multirow{3}{*}{$+7-6$} \\
\hline \multirow{2}{*}{\multicolumn{3}{|c|}{$\begin{array}{ll}1208.22 & 13 \\
2415.2 \ddagger & 10\end{array}$}} & $2+$ & & & \\
\hline & & & $(4+)$ & & & \\
\hline \multicolumn{3}{|c|}{$2486.8 \quad 4$} & $2+$ & \multicolumn{3}{|c|}{$0.28 \mathrm{ps} 11$} \\
\hline \multicolumn{3}{|c|}{$2512.5 \quad 4$} & $(0+$ to $4+)$ & \multicolumn{3}{|c|}{$2.8 \mathrm{ps}+21-8$} \\
\hline \multicolumn{3}{|c|}{3014.64} & $(1,2+)$ & \multicolumn{3}{|c|}{$<83$ f s } \\
\hline \multicolumn{3}{|c|}{$3096.5 \quad 5$} & $4+$ & \multicolumn{3}{|c|}{$>3.5 \mathrm{ps}$} \\
\hline \multicolumn{3}{|c|}{3557.93} & $2+$ & \multicolumn{3}{|c|}{$<62 \mathrm{fs}$} \\
\hline \multicolumn{3}{|c|}{4006.34} & $2+$ & \multicolumn{3}{|c|}{$\begin{array}{rl}<2 & 1 \mathrm{~s} \\
0.23 \mathrm{ps} & \end{array}$} \\
\hline \multicolumn{3}{|c|}{$4127.5 \quad 5$} & $(0+, 1,2)$ & \multicolumn{3}{|c|}{$0.97 \mathrm{ps} 21$} \\
\hline \multicolumn{3}{|c|}{4287.15} & $(1,2,3)$ & \multicolumn{3}{|c|}{$<35 \mathrm{f} \mathrm{s}$} \\
\hline \multicolumn{3}{|c|}{4633.96} & $(3-)$ & \multicolumn{3}{|l|}{$<35$ fs } \\
\hline \multicolumn{7}{|c|}{$\dagger$ From least-squares fit to $\mathrm{E} \gamma$ data. } \\
\hline \multicolumn{7}{|c|}{$\ddagger$ From $1973 \operatorname{Pr} 10$} \\
\hline \multicolumn{7}{|c|}{$\S$ From DSAM (1974Fi01). } \\
\hline \multicolumn{7}{|c|}{ \# From Adopted Levels, unless otherwise noted. } \\
\hline
\end{tabular}

$\gamma\left({ }^{42} \mathrm{Ar}\right)$

Values of $\mathrm{A}_{2}$ and $\mathrm{A}_{4}$ are from $1973 \operatorname{Pr} 10$.

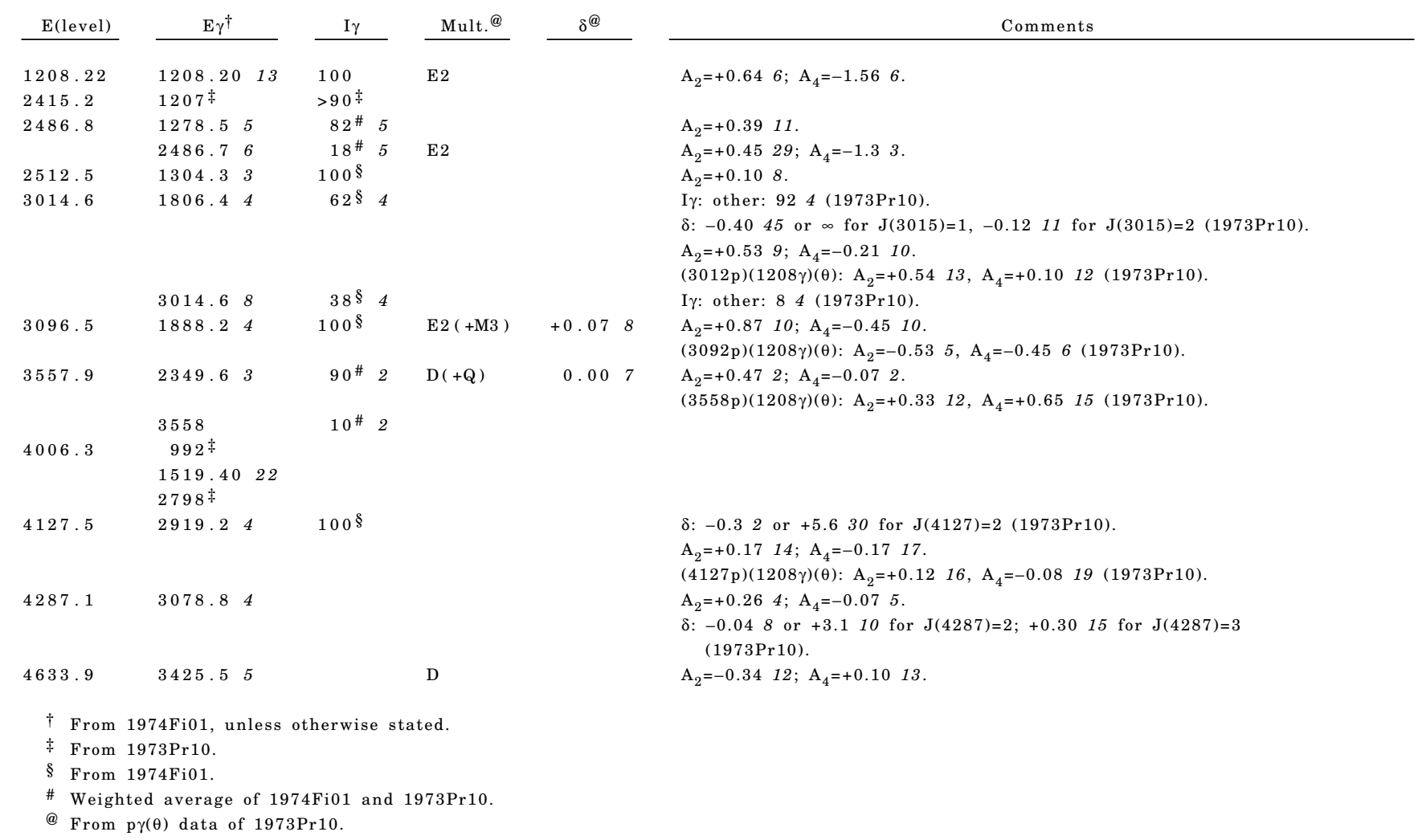




\section{${ }^{40} \mathrm{Ar}(\mathrm{t}, \mathrm{p} \gamma) \quad 1974 \mathrm{Fi} 01,1973 \operatorname{Pr} 10($ continued $)$}

Level Scheme

Intensities: \% photon branching from each level

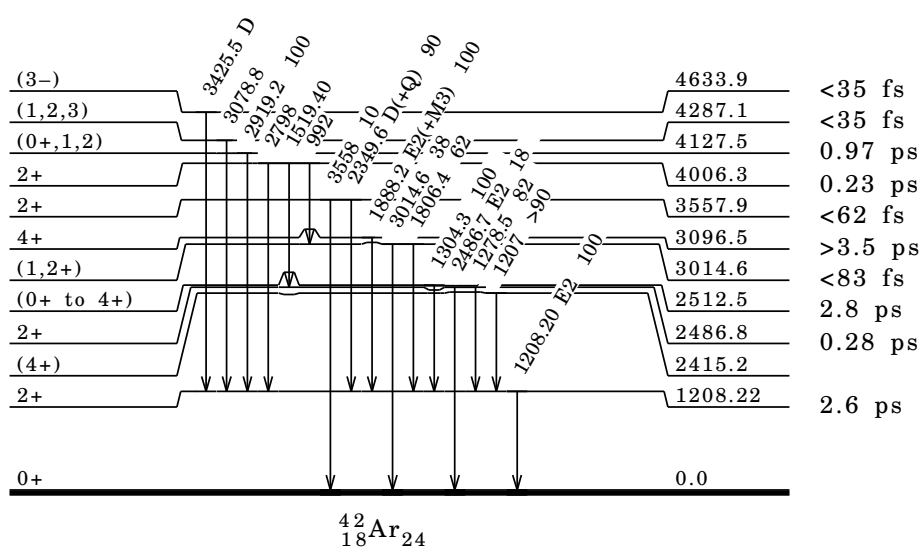

\section{${ }^{42} \mathrm{Ar}\left(\mathbf{p}, \mathbf{p}^{\prime}\right) \quad 2001 \mathrm{Sc01}$}

2001Sc01: $\mathrm{E}=33 \mathrm{MeV} /$ nucleon ${ }^{42} \mathrm{Ar}$ beam was produced from via fragmentation of a $70 \mathrm{MeV} / \mathrm{nucleon}{ }^{48} \mathrm{Ca}$ beam in a ${ }^{9} \mathrm{Be}$ target at the National Superconducting Cyclotron Laboratory. $\left(\mathrm{CH}_{2}\right)_{n}$ target. Protons were detected by silicon $\Delta \mathrm{E}-\mathrm{E}$ telescopes. Measured energies and $\sigma(\theta)$. Deduced $\beta_{2}$ for first $2+$ state in ${ }^{42} \mathrm{Ar}$ from DWBA calculations and experimental cross sections.

\section{${ }^{42} \mathrm{Ar}$ Levels}

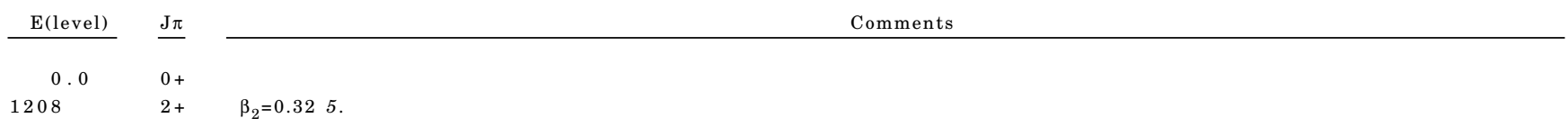

\section{${ }^{208} \mathrm{~Pb}\left({ }^{40} \mathrm{Ar}, \mathrm{X} \gamma\right) \quad 2011 \mathrm{Sz} 02$}

2011Sz02 (also 2013Sz01): E=255 MeV ${ }^{40} \mathrm{Ar}$ beam from the superconducting ALPI accelerator of the Laboratory Nazionali di Legnaro. Target $=300 \mu \mathrm{g} / \mathrm{cm}^{2}{ }^{208} \mathrm{~Pb}$. Projectile-like fragments were identified by spectrometer Prisma by $\Delta \mathrm{E}-\mathrm{E}$ and time-of-flight (TOF) measurements and $\gamma$ rays were detected by the Clara array, consisting of 24 HPGe clover-type detectors (photo-peak efficiency $3 \%$ at $1.33 \mathrm{MeV}$, FWHM=0.6\%-0.9\%). Measured E $\gamma$, I $\gamma$, (fragment) $\gamma$ coincidence. Deduced levels, J $\pi$. Comparison with shell model calculations.

\section{${ }^{42}$ Ar Levels}

$\mathrm{E}($ level $) \dagger$

$$
0.0^{\#}
$$

$1208.0 \# 10 \quad 2+$

$2413.0^{\#} 15 \quad(4+) \S$

$2485.511 \quad 2+$

$3095.6 \quad 12 \quad 4+$

$3563.5^{\#} \quad 15 \quad(6+) \S$

$\dagger$ From least-squares fit to $\mathrm{E} \gamma$ data.

$\ddagger$ From Adopted Level, unless otherwise noted.

$\S$ Proposed by $2011 \mathrm{Sz} 02$ as the members of the $2+, 4+$ and $6+$ yrast sequence and from comparison with shell model calculations as well.

\# (A): yrast sequence $(2011 \mathrm{Sz} 02)$. 
${ }^{208} \mathrm{~Pb}\left({ }^{40} \mathrm{Ar}, \mathrm{X} \gamma\right) \quad 2011 \mathrm{Sz} 02$ (continued)

$\gamma\left({ }^{42} \mathrm{Ar}\right)$

\begin{tabular}{cccc}
\multicolumn{2}{c}{$\mathrm{E} \gamma$} & & E(level) \\
\cline { 1 - 1 } 1150.4 & 3 & & 3563.5 \\
1205 & 1 & & 2413.0 \\
1208 & 1 & & 1208.0 \\
1277.5 & 3 & & 2485.5 \\
1887.5 & 6 & & 3095.6
\end{tabular}

\section{$\operatorname{Pb}\left({ }^{43} A r, n \gamma\right) \quad 1997 W a 25$}

Neutron removal from ${ }^{43} \mathrm{Ar}$ beam with a $\mathrm{Pb}$ target (acting as an anvil), leading to population of states in ${ }^{42} \mathrm{Ar}$. 1997Wa25: ${ }^{43} \mathrm{Ar}$ was produced via fragmentation of $330 \mathrm{MeV} /$ nucleon ${ }^{50} \mathrm{Ti}$ beam on a ${ }^{9} \mathrm{Be}$ target at GSI. Fragments were selected by the fragment separator FRS and identified by energy-loss $\Delta \mathrm{E}$ and time-of-flight (TOF) before the 0.9 $\mathrm{g} / \mathrm{cm}^{2}$ secondary $\mathrm{Pb}$ target. Projectile-like reaction products were identified by the $\mathrm{B} \rho-\mathrm{TOF}-\Delta \mathrm{E}$ method using the large magnetic spectrometer ALADIN, $\gamma$-rays were detected by the Darmstadt-Heidelberg Crystal Ball spectrometer (CB) and neutrons were detected by the LAND detector. Measured E $\gamma, \gamma \gamma$-coin, n $\gamma$-coin and cross section (100 mb 20 ).

${ }^{42} \mathrm{Ar}$ Levels

E(level)

0.0

1208

2414

$\gamma\left({ }^{42} \mathrm{Ar}\right)$

$\mathrm{E} \gamma^{\dagger} \quad \mathrm{E}($ level $)$

$1206 \quad 2414$

$1208 \quad 1208$

$\dagger$ Rounded energies from Adopted Gammas. 


\section{Adopted Levels, Gammas}

$\mathrm{Q}\left(\beta^{-}\right)=3525.2218 ; \mathrm{S}(\mathrm{n})=7533.8011 ; \mathrm{S}(\mathrm{p})=9243.5 \quad 4 ; \mathrm{Q}(\alpha)=-7648.86 \quad 14 \quad 2012 \mathrm{Wa} 38$.

$\mathrm{S}(2 \mathrm{n})=17629.17 \quad 12, \mathrm{~S}(2 \mathrm{p})=2204030 \quad(2012 \mathrm{Wa} 38)$.

${ }^{42} \mathrm{~K}$ produced and identified by $1950 \mathrm{Sa} 03$ using ${ }^{41} \mathrm{~K}(\mathrm{~d}, \mathrm{p})$ reaction.

2014Pa45, 2014Kr04: $\mathrm{E}(\mathrm{p})=1.4 \mathrm{GeV}$ incident on $\mathrm{UC}_{\mathrm{x}}$ target at ISOLDE-CERN facility. Fragments diffused out of target and surface ionized, then accelerated to $40 \mathrm{kV}$, followed by mass separation and bunched by gas-filled Paul Trap (ISCOOL). Hyperfine structure was studied by collinear laser spectroscopy using COLLAPS setup at ISOLDE-CERN. Measured hyperfine spectra. Deduced spin, magnetic moment, rms charge radius. Comparison with shell-model calculations. $2014 \mathrm{~Pa} 45$ present measured hyperfine parameters, hyperfine structure anomalies, spins and magnetic moments, while $2014 \mathrm{Kr} 04$ present measured $\mathrm{rms}$ radii, isotope shifts and spin determination. Other hyperfine structure measurement: $1982 \mathrm{To} 02$.

\section{${ }^{42} \mathrm{~K}$ Levels \\ Cross Reference (XREF) Flags}

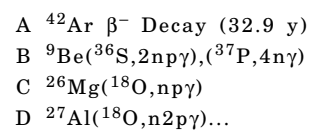

E ${ }^{40} \operatorname{Ar}(\alpha, d)$

F ${ }^{41} \mathrm{~K}(\mathrm{n}, \gamma) \quad \mathrm{E}=$ thermal

G ${ }^{41} \mathrm{~K}(\mathrm{n}, \gamma)$ : Resonance

H ${ }^{41} \mathrm{~K}(\mathrm{~d}, \mathrm{p}),(\mathrm{d}, \mathrm{p} \gamma)$ $\begin{array}{ll}\text { I } & { }^{42} \mathrm{Ca}\left(\mathrm{t},{ }^{3} \mathrm{He}\right) \\ \mathrm{J} & { }^{43} \mathrm{Ca}\left(\mathrm{d},{ }^{3} \mathrm{He}\right) \\ \mathrm{K} & { }^{44} \mathrm{Ca}\left(\mathrm{p},{ }^{3} \mathrm{He}\right) \\ \mathrm{L} & { }^{44} \mathrm{Ca}(\mathrm{d}, \alpha),(\text { pol } d, \alpha)\end{array}$

$\frac{\mathrm{E}(\text { level })^{\dagger}}{0.0} \frac{\mathrm{J} \pi \S}{2-}$

XREF

ABCDEF HI JKL

$\frac{\mathrm{T}_{1 / 2} \ddagger}{12.355 \mathrm{~h} 7}$

$12.355 \mathrm{~h} 7$

$\begin{array}{rll}106.826 & 5 & 3- \\ 258.259 & 6 & 4- \\ & & \\ & & \\ 638.726 & 7 & 3- \\ 681.942 & 8 & (2,3) \\ 699.086 & 14 & 5- \\ 783.885 & 11 & 2- \\ 841.941 & 7 & 3- \\ 1110.748 & 9 & 3+ \\ 1143.594 & 11 & 4+ \\ 1197.903 & 10 & 4- \\ 1254.821 & 11 & 2-\end{array}$

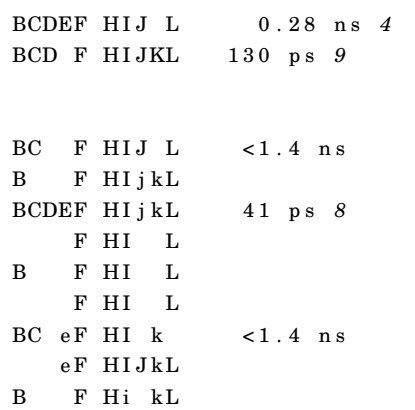

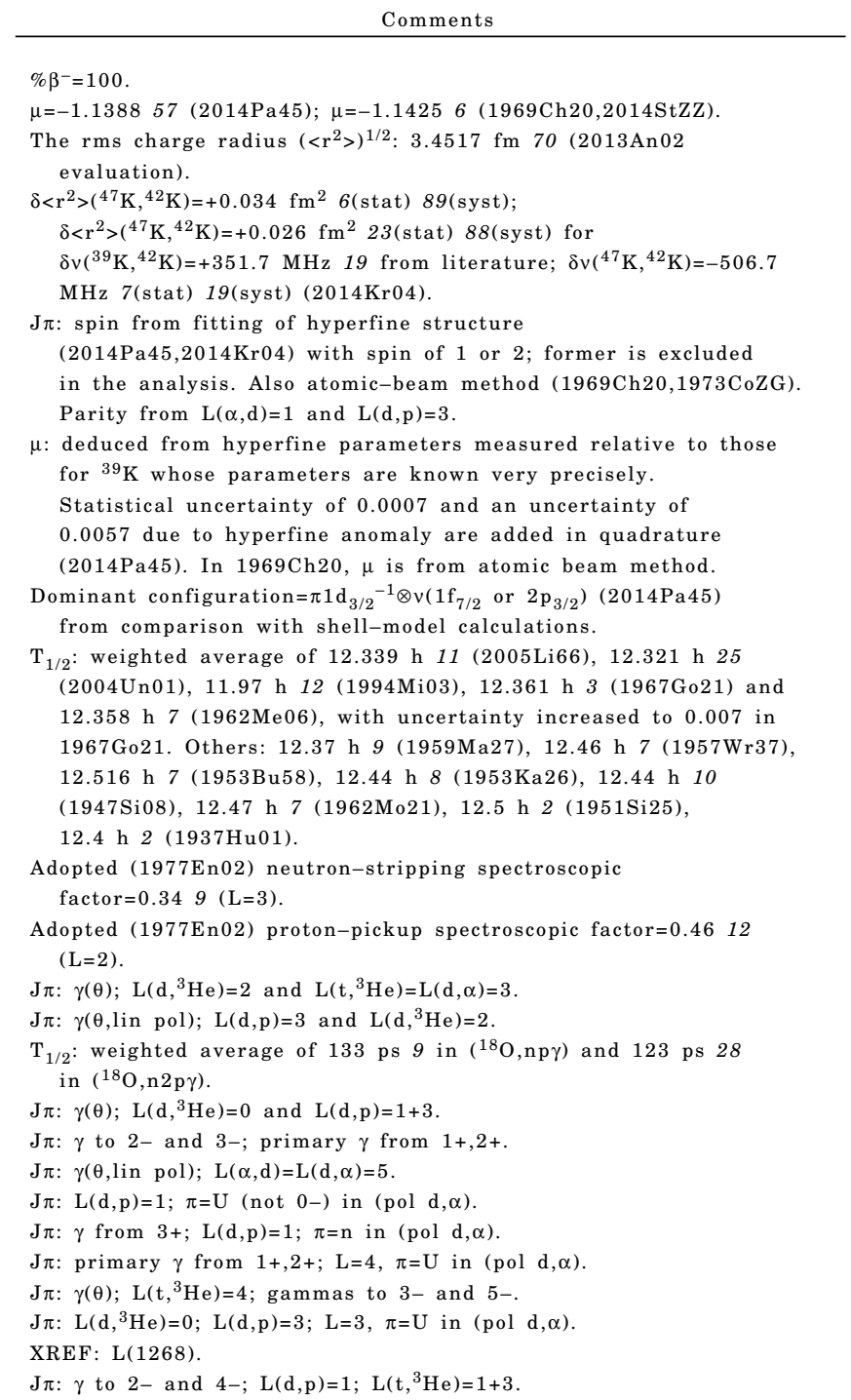


Adopted Levels, Gammas (continued)

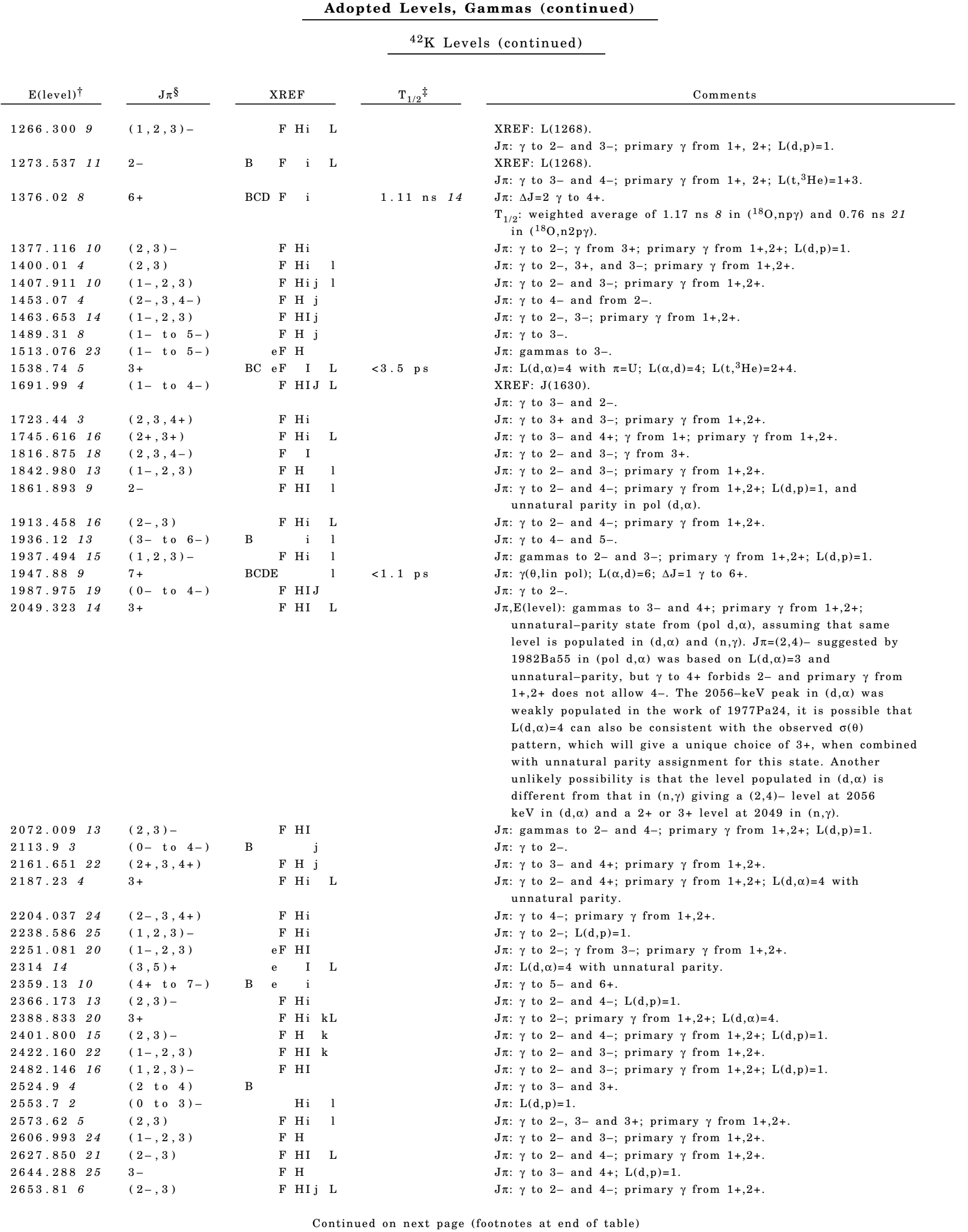


Adopted Levels, Gammas (continued)

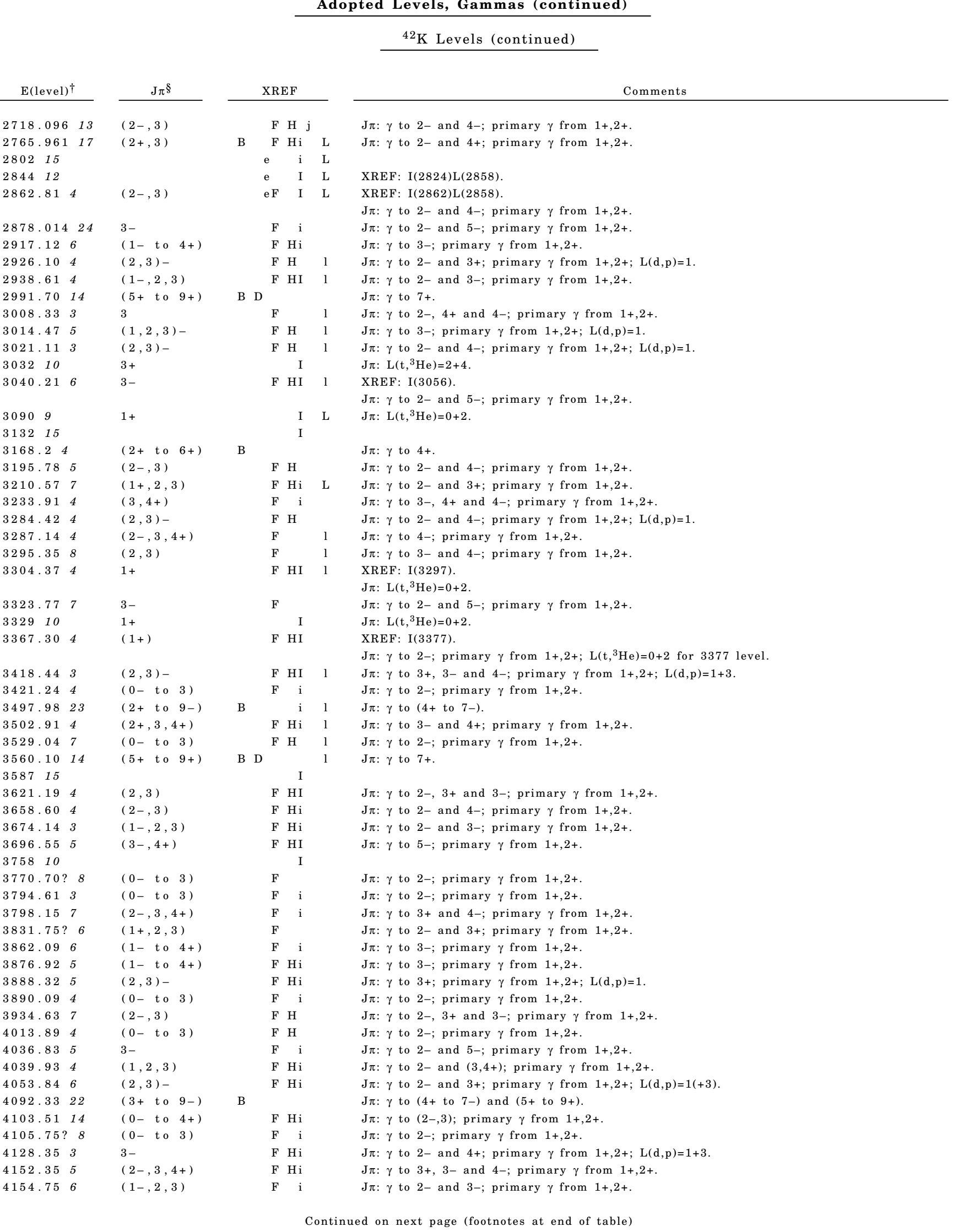


Adopted Levels, Gammas (continued)

\author{
${ }^{42} \mathrm{~K}$ Levels (continued)
}

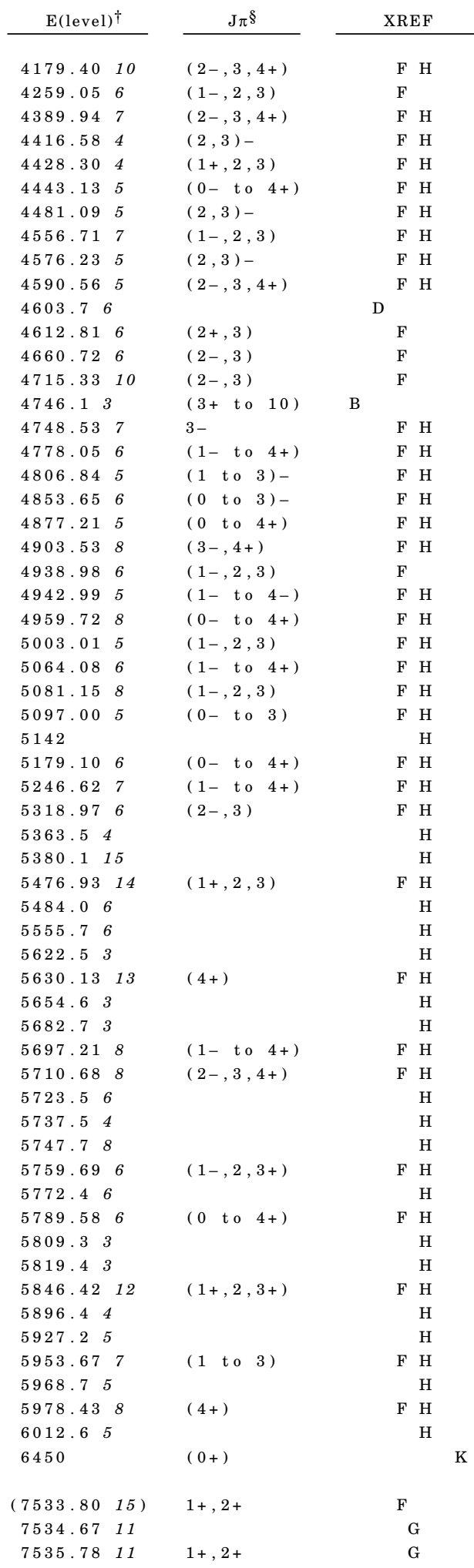

Comments

$\mathrm{J} \pi: \gamma$ to $4-;$ primary $\gamma$ from $1+, 2+$.

$\mathrm{J} \pi: \gamma$ to $2-$ and $3-$; primary $\gamma$ from $1+, 2+$.

$\mathrm{J} \pi: \gamma$ to $3+$ and 4-; primary $\gamma$ from $1+, 2+$.

$\mathrm{J} \pi: \gamma$ to $2-$ and $4-;$ primary $\gamma$ from $1+, 2+; \mathrm{L}(\mathrm{d}, \mathrm{p})=1+3$.

$\mathrm{J} \pi: \gamma$ to $2-$ and $3+$; primary $\gamma$ from $1+, 2+$.

$\mathrm{J} \pi: \gamma$ to $(2,3)-;$ primary $\gamma$ from $1+, 2+$.

$\mathrm{J} \pi: \gamma$ to $2-, 3+$ and $3-$; primary $\gamma$ from $1+, 2+; \mathrm{L}(\mathrm{d}, \mathrm{p})=1$.

$\mathrm{J} \pi: \gamma$ to $2-$ and $3-$; primary $\gamma$ from $1+, 2+$.

$\mathrm{J} \pi: \gamma$ to $3+; \mathrm{L}(\mathrm{d}, \mathrm{p})=1$.

$\mathrm{J} \pi: \gamma$ to $3+, 3-$ and $4-$; primary $\gamma$ from $1+, 2+$.

$\mathrm{J} \pi: \gamma$ to $2-$ and $4+$; primary $\gamma$ from $1+, 2+$.

$\mathrm{J} \pi: \gamma$ to $2-$ and 4-; primary $\gamma$ from $1+, 2+$.

$\mathrm{J} \pi: \gamma$ to $2-$ and 4-; primary $\gamma$ from $1+, 2+$.

$\mathrm{J} \pi: \gamma$ to $(5+$ to $9+)$ and $(2+$ to $9-)$.

$\mathrm{J} \pi: \gamma$ to $2-$ and $5-$; primary $\gamma$ from $1+, 2+$.

$\mathrm{J} \pi: \gamma$ to $3-$; primary $\gamma$ from $1+, 2+$.

$\mathrm{J} \pi: \gamma$ to $2-$; primary $\gamma$ from $1+, 2+; \mathrm{L}(\mathrm{d}, \mathrm{p})=1$.

$\mathrm{J} \pi: \gamma$ to $2-;$ primary $\gamma$ from $1+, 2+; \mathrm{L}(\mathrm{d}, \mathrm{p})=1$.

$\mathrm{J} \pi$ : primary $\gamma$ from $1+, 2+$.

$\mathrm{J} \pi: \gamma$ to $4-$ and 5-; primary $\gamma$ from $1+, 2+$.

$\mathrm{J} \pi: \gamma$ to $2-$ and $3-$; primary $\gamma$ from $1+, 2+$.

$\mathrm{J} \pi: \gamma$ to $3-;$ primary $\gamma$ from $1+, 2+$.

$\mathrm{J} \pi: \gamma$ to $(2,3)-;$ primary $\gamma$ from $1+, 2+$.

$\mathrm{J} \pi$ : $\gamma$ to $2-$ and $3-$; primary $\gamma$ from $1+, 2+$.

$\mathrm{J} \pi: \gamma$ to $3-;$ primary $\gamma$ from $1+, 2+$.

$\mathrm{J} \pi: \gamma$ to $2-$ and $3-$; primary $\gamma$ from $1+, 2+$.

$\mathrm{J} \pi: \gamma$ to $2-;$ primary $\gamma$ from $1+, 2+$.

$\mathrm{J} \pi: \gamma$ to $(2-, 3)$; primary $\gamma$ from $1+, 2+$.

$\mathrm{J} \pi: \gamma$ to $3-;$ primary $\gamma$ from $1+, 2+$.

$\mathrm{J} \pi: \gamma$ to $2-$ and 4-; primary $\gamma$ from $1+, 2+$.

$\mathrm{J} \pi: \gamma$ to $2-$ and $3+$; primary $\gamma$ from $1+, 2+$.

$\mathrm{J} \pi$ : $\gamma$ to $6+$; weak primary $\gamma$ from $1+, 2+$.

$\mathrm{J} \pi: \gamma$ to $3-$; primary $\gamma$ from $1+, 2+$.

$\mathrm{J} \pi$ : $\gamma$ to $4-$; primary $\gamma$ from $1+, 2+$.

$\mathrm{J} \pi: \gamma$ to $1+$ and $3-;$ primary $\gamma$ from $1+, 2+$.

$\mathrm{J} \pi$ : primary $\gamma$ from $1+, 2+$.

$\mathrm{J} \pi: \gamma$ to $1+$ and $3+$; primary $\gamma$ from $1+, 2+$.

$\mathrm{J} \pi: \gamma$ to $2-;$ primary $\gamma$ from $1+, 2+$.

$\mathrm{J} \pi: \gamma$ to $3-$ and $6+$; weak primary $\gamma$ from $1+, 2+$.

K $\quad \mathrm{T}=3$.

$\mathrm{J} \pi, \mathrm{T}$ : probable IAS of ${ }^{42} \mathrm{Ar}$ g.s.

$\mathrm{J} \pi$ : s-wave neutron capture in $3 / 2+$ g.s. of ${ }^{41} \mathrm{~K}$.

Continued on next page (footnotes at end of table) 
Adopted Levels, Gammas (continued)

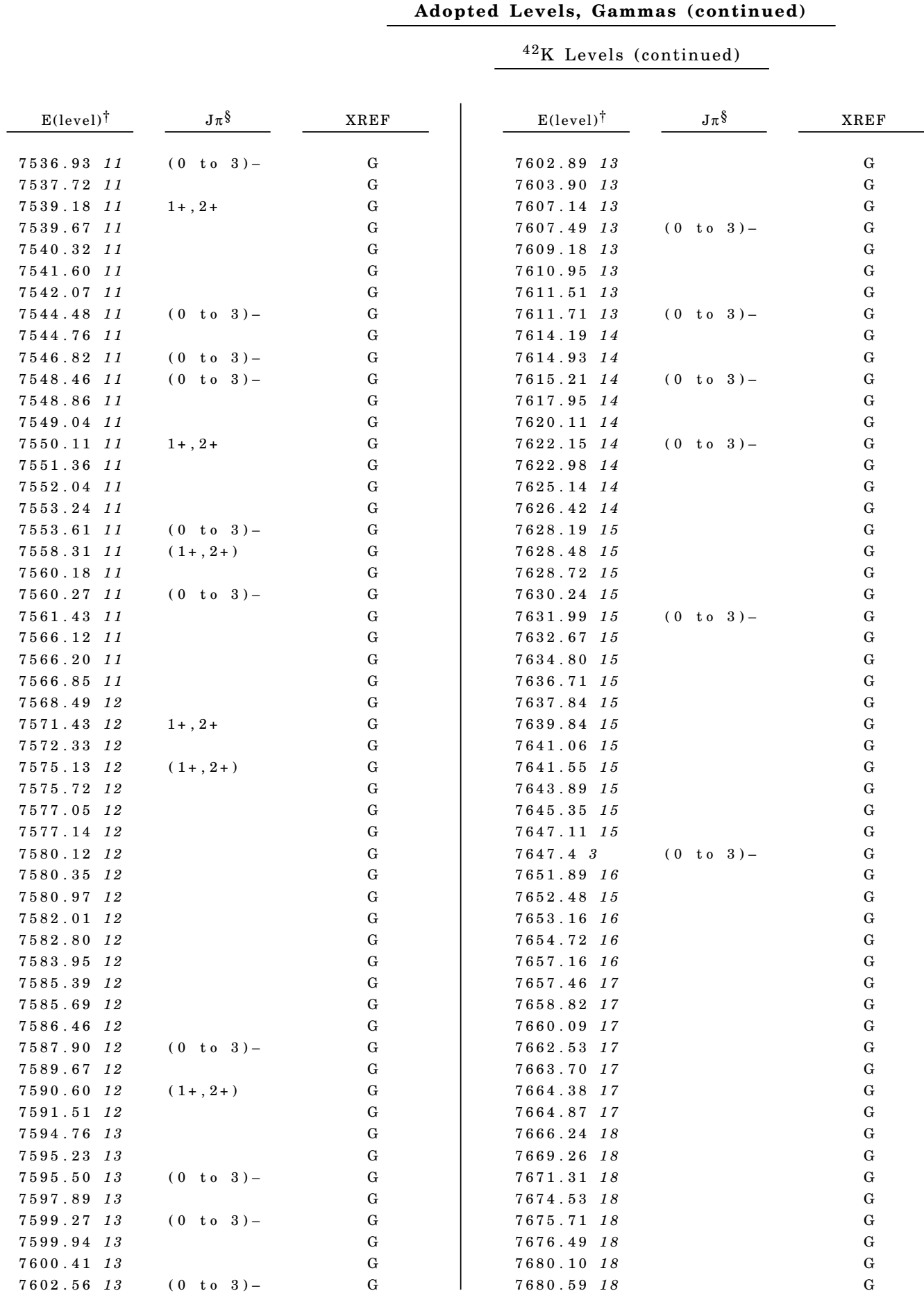

$\dagger$ From least-squares adjustment to measured E $\gamma$ data when such data are available. Otherwise weighted averages of available level energies are taken. Uncertainties for neutron resonances include only uncertainty for S(n) and statistical uncertainties for neutron energies.

† From recoil-distance method. Values are from $\left({ }^{18} \mathrm{O}, \mathrm{np} \gamma\right)(1977 \mathrm{Ek} 01)$, unless otherwise stated.

$\S$ When L-transfer arguments are used, the target spin-parity is $\mathrm{J} \pi=3 / 2+$ for ${ }^{41} \mathrm{~K}(\mathrm{~d}, \mathrm{p})$; $\mathrm{J} \pi=0+$ for ${ }^{42} \mathrm{~K}\left(\mathrm{t},{ }^{3} \mathrm{He}\right),{ }^{44} \mathrm{Ca}\left(\mathrm{p},{ }^{3} \mathrm{He}\right)$ and ${ }^{44} \mathrm{Ca}(\mathrm{d}, \alpha) ; \mathrm{J} \pi=7 / 2-$ for ${ }^{43} \mathrm{Ca}\left(\mathrm{d},{ }^{3} \mathrm{He}\right)$. When levels are only seen in $(\mathrm{n}, \gamma):$ Resonance, J $\pi$ values are from $\mathrm{s}-\mathrm{wave}$ or $\mathrm{p}-\mathrm{wave}$ assignment in the analysis of neutron-resonance data $(2006 \mathrm{MuZX})$. When assigning $J \pi$ to a level based on $\gamma$ transitions from this level to a level of known $\mathrm{J} \pi$, evaluators consider transitions to be E1, M1 or E2. 
Adopted Levels, Gammas (continued)

\begin{tabular}{|c|c|c|c|c|c|c|}
\hline \multirow{2}{*}{$\frac{\mathrm{E}(\text { level })}{106.826}$} & $\mathrm{E} \gamma^{\dagger}$ & \multicolumn{2}{|r|}{$\mathrm{I} \gamma^{\dagger}$} & \multirow{2}{*}{$\frac{\text { Mult. }{ }^{\ddagger}}{\text { M1 ( +E 2) }}$} & \multirow{2}{*}{$\frac{\delta^{\ddagger}}{+0.013}$} & Comments \\
\hline & $106.817 \quad 7$ & 100 & & & & $\mathrm{~B}(\mathrm{M} 1)(\mathrm{W} . \mathrm{u})=.0.06510 ; \mathrm{B}(\mathrm{E} 2)(\mathrm{W} . \mathrm{u})<$.12 . \\
\hline 258.259 & 151.4313 & 100 & & $\mathrm{M} 1(+\mathrm{E} 2)$ & $-0.008 \quad 16$ & $\mathrm{~B}(\mathrm{M} 1)(\mathrm{W} . \mathrm{u})=.0.0494 ; \mathrm{B}(\mathrm{E} 2)(\mathrm{W} . \mathrm{u})<2.1$. \\
\hline \multirow[t]{3}{*}{638.726} & $380.473 \quad 13$ & 44 & 9 & $(\mathrm{M} 1+\mathrm{E} 2)$ & & \\
\hline & $531.908 \quad 15$ & 100 & 16 & & & \\
\hline & $638.717 \quad 14$ & 60 & 9 & $\mathrm{D}$ & & \\
\hline \multirow[t]{2}{*}{681.942} & $575.04 \quad 5$ & & .72 & & & \\
\hline & $681.932 \quad 12$ & 100 & .02 & & & \\
\hline \multirow[t]{2}{*}{699.086} & $440.854 \quad 18$ & 100 & 2 & $\mathrm{M} 1+\mathrm{E} 2$ & +0.1028 & $\mathrm{~B}(\mathrm{M} 1)(\mathrm{W} . \mathrm{u})=.0.005912 ; \mathrm{B}(\mathrm{E} 2)($ W.u. $)=0.9424$. \\
\hline & $592.23 \S 6$ & & $.6 \S 3$ & [E2 ] & & $\mathrm{B}(\mathrm{E} 2)(\mathrm{W} . \mathrm{u})=0.9620$. \\
\hline 783.885 & $783.903 \quad 14$ & 100 & & & & \\
\hline \multirow[t]{2}{*}{841.941} & $735.099 \quad 14$ & 16 & 2 & & & \\
\hline & $841.895 \quad 13$ & 100 & 15 & & & \\
\hline \multirow[t]{3}{*}{1110.748} & $268.793 \quad 8$ & 90 & 20 & & & \\
\hline & $428.71 \quad 5$ & 10 & 2 & & & \\
\hline & $1110.767 \quad 16$ & 100 & 15 & & & \\
\hline \multirow[t]{4}{*}{1143.594} & $301.50 \quad 14$ & 4 & $.5 \quad 15$ & & & \\
\hline & $444.525 \quad 19$ & 38 & 8 & $\mathrm{D}$ & & \\
\hline & $504.828 \quad 16$ & 100 & 8 & $\mathrm{D}$ & & \\
\hline & $1036.83 \quad 3$ & 9 & .115 & & & \\
\hline \multirow[t]{4}{*}{1197.903} & $559.23 \quad 3$ & 23 & 4 & & & \\
\hline & $939.605 \quad 17$ & 100 & 15 & & & \\
\hline & $1091.088 \quad 18$ & 67 & 11 & & & \\
\hline & $1197.99 \quad 3$ & 15 & 3 & & & \\
\hline \multirow[t]{4}{*}{1254.821} & $413.16 \quad 16$ & 2 & .67 & & & \\
\hline & $616.118 \quad 14$ & 100 & 13 & & & \\
\hline & $996.511 \quad 16$ & 62 & 9 & & & \\
\hline & $1254.80 \quad 3$ & 34 & 6 & & & \\
\hline \multirow[t]{3}{*}{1266.300} & $584.40 \quad 4$ & 12 & .722 & & & \\
\hline & $627.551 \quad 15$ & 42 & 6 & & & \\
\hline & $1266.305 \quad 15$ & 100 & 16 & & & \\
\hline 1273.537 & $431.566 \quad 16$ & 100 & 15 & & & \\
\hline & $634.65 \quad 6$ & & .25 & & & \\
\hline & $1015.233 \quad 24$ & 21 & 3 & & & \\
\hline & $1166.808 \quad 25$ & 10 & .317 & & & \\
\hline 1376.02 & 232.32 & 12 & 35 & $\mathrm{E} 2(+\mathrm{M} 3)$ & -0.043 & $\mathrm{E} \gamma, \mathrm{I} \gamma:$ weighted average from $\left({ }^{18} \mathrm{O}, \mathrm{np} \gamma\right)$ and $\left({ }^{36} \mathrm{~S}, 2 \mathrm{np} \gamma\right)$ \\
\hline & $676.87 \quad 11$ & 100 & 3 & $\mathrm{E} 1+\mathrm{M} 2$ & $+0.025 \quad 11$ & $\begin{array}{l}\mathrm{B}(\mathrm{E} 2)(\mathrm{W} . \mathrm{u} .)=9.513 \\
\mathrm{I} \gamma: \text { from }\left({ }^{18} \mathrm{O}, \mathrm{np} \gamma\right) \text { and }\left({ }^{36} \mathrm{~S}, 2 \mathrm{np} \gamma\right) \\
\mathrm{B}(\mathrm{E} 1)(\mathrm{W} \cdot \mathrm{u} .)=1.45 \times 10^{-6} 20 ; \mathrm{B}(\mathrm{M} 2)(\mathrm{W} . \mathrm{u} .)=0.0098\end{array}$ \\
\hline 1377.116 & $695.189 \quad 17$ & 30 & 5 & & & \\
\hline & $1377.065 \quad 16$ & 100 & 5 & & & \\
\hline 1400.01 & $289.21 \quad 9$ & 37 & 9 & & & \\
\hline & 761.318 & 9 & $.8 \quad 17$ & & & \\
\hline & $1399.78 \quad 9$ & 100 & 15 & & & \\
\hline 1407.911 & $725.96 \quad 3$ & 5 & .28 & & & \\
\hline & $1301.008 \quad 20$ & 17 & 3 & & & \\
\hline & $1407.898 \quad 16$ & 100 & 17 & & & \\
\hline 1453.07 & $198.33 \quad 9$ & 100 & 27 & & & \\
\hline & $1195.02 \quad 13$ & 8 & .916 & & & \\
\hline 1463.653 & $621.727 \quad 16$ & 100 & 15 & & & \\
\hline & $1463.59 \quad 3$ & 19 & 3 & & & \\
\hline 1489.31 & $851.01 \quad 20$ & 100 & 17 & & & \\
\hline & 1382.23 & 69 & 17 & & & \\
\hline 1513.076 & $671.149 \quad 23$ & 100 & 29 & & & \\
\hline & $830.89 \quad 9$ & 17 & 3 & & & \\
\hline 1538.74 & $395.16 \quad 7$ & 100 & & $\mathrm{D}$ & & \\
\hline 1691.99 & $437.24 \quad 18$ & 37 & 10 & & & \\
\hline & $1585.10 \quad 4$ & 100 & 10 & & & \\
\hline 1723.44 & $612.65 \quad 5$ & 100 & 17 & & & \\
\hline & $881.33 \quad 11$ & 4 & .79 & & & \\
\hline & $1084.48 \quad 12$ & 4 & .89 & & & \\
\hline & $1616.84 \quad 11$ & 7 & $.4 \quad 12$ & & & \\
\hline 1745.616 & $602.046 \quad 22$ & 88 & 14 & & & \\
\hline & 903.63521 & 100 & 14 & & & \\
\hline
\end{tabular}


Adopted Levels, Gammas (continued)

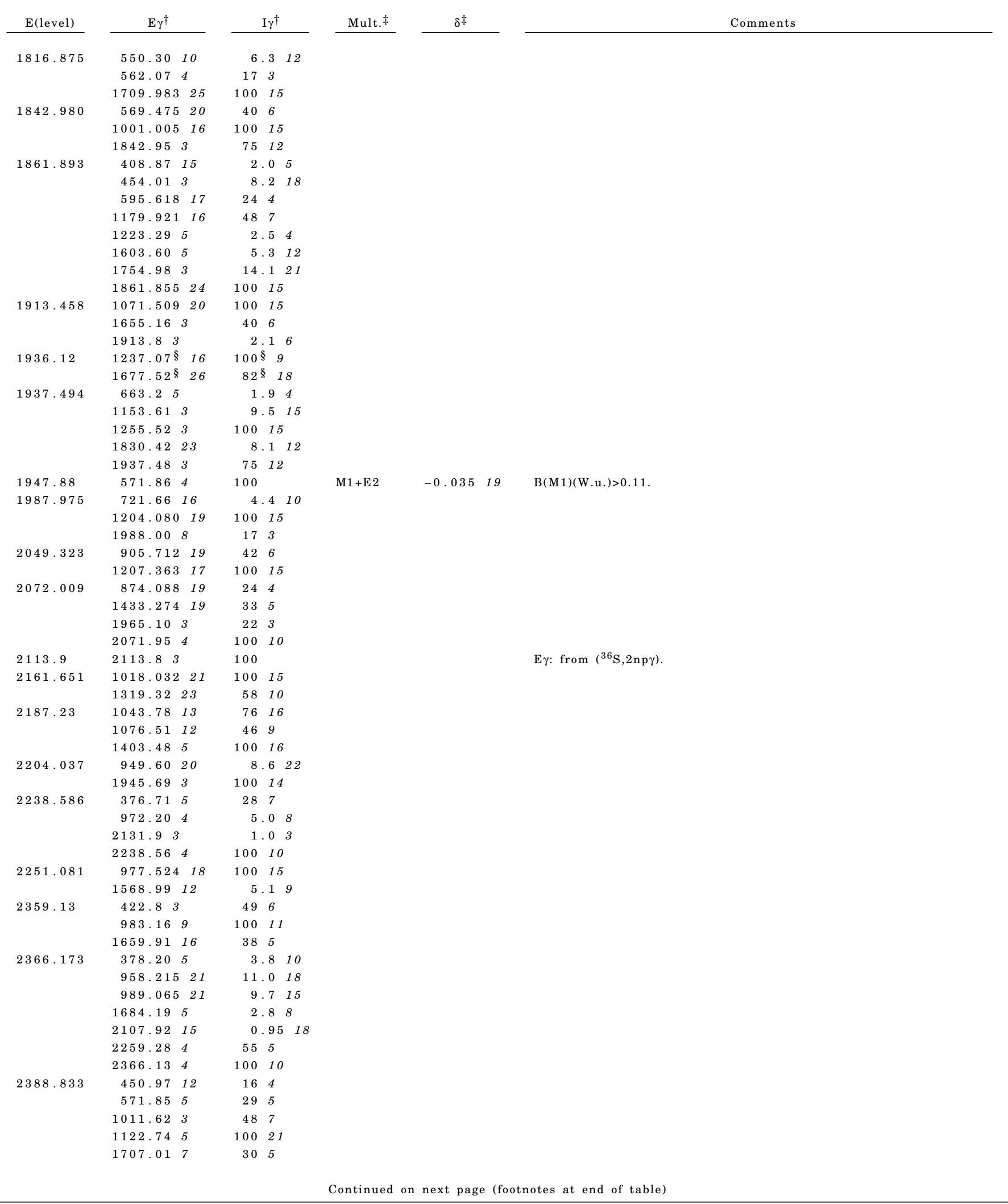


Adopted Levels, Gammas (continued)

\begin{tabular}{|c|c|c|c|c|c|c|}
\hline \multirow{2}{*}{$\frac{\text { E(level) }}{2388.833}$} & $\mathrm{E} \gamma^{\dagger}$ & $\mathrm{I} \gamma^{\dagger}$ & \multirow{2}{*}{$\frac{\mathrm{E}(\text { level })}{2878.014}$} & $\mathrm{E} \gamma^{\dagger}$ & \multicolumn{2}{|c|}{$\mathrm{I} \gamma^{\dagger}$} \\
\hline & $2281.90 \quad 10$ & $16.7 \quad 23$ & & $2094.05 \quad 11$ & 38 & 5 \\
\hline & $2388.81 \quad 5$ & $100 \quad 11$ & & $2179.04 \quad 8$ & 100 & 11 \\
\hline \multirow[t]{9}{*}{2401.800} & $678.34 \quad 6$ & 4.17 & & $2195.95 \quad 10$ & 58 & 7 \\
\hline & $912.69 \quad 23$ & $0.61 \quad 16$ & & $2770.75 \quad 13$ & 20 & 3 \\
\hline & $993.863 \quad 23$ & $9.7 \quad 16$ & & $2878.10 \quad 16$ & 13. & $3 \quad 22$ \\
\hline & $1128.20 \quad 3$ & 4.37 & 2917.12 & $1661.8 \quad 4$ & 45 & 7 \\
\hline & $1135.40 \quad 7$ & $2.0 \quad 3$ & & $2810.15 \quad 6$ & 100 & 10 \\
\hline & $1147.24 \quad 22$ & $13.5 \quad 19$ & 2926.10 & $537.17 \quad 14$ & 13 & 3 \\
\hline & $2143.43 \quad 5$ & $21.3 \quad 23$ & & $1473.00 \quad 7$ & 20 & 3 \\
\hline & $2294.98 \quad 3$ & $100 \quad 10$ & & $1671.20 \quad 11$ & 13 & 3 \\
\hline & $2401.72 \quad 5$ & $11.0 \quad 10$ & & 2926.015 & 100 & 9 \\
\hline \multirow[t]{5}{*}{2422.160} & $579.30 \quad 6$ & $7.2 \quad 12$ & 2938.61 & $1121.70 \quad 5$ & 100 & 14 \\
\hline & $1740.20 \quad 3$ & $28 \quad 4$ & & $1485.60 \quad 8$ & 26 & 5 \\
\hline & $1783.51 \quad 12$ & $27 \quad 4$ & & $2096.60 \quad 12$ & 28 & 5 \\
\hline & 2315.194 & $100 \quad 10$ & & $2938.38 \quad 6$ & 79 & 12 \\
\hline & $2422.20 \quad 11$ & $5.0 \quad 6$ & 2991.70 & $632.68 \quad 20$ & 65 & 7 \\
\hline \multirow[t]{6}{*}{2482.146} & $544.68 \quad 21$ & $2.9 \quad 8$ & & $1043.79 \quad 14$ & 100 & 9 \\
\hline & $620.244 \quad 16$ & $100 \quad 15$ & 3008.33 & $846.59 \quad 5$ & 23 & 4 \\
\hline & 1640.184 & $9.6 \quad 14$ & & $1810.51 \quad 10$ & 16 & 3 \\
\hline & $1800.15 \quad 4$ & 224 & & $1864.68 \quad 5$ & 29 & 5 \\
\hline & $2375.35 \quad 8$ & $13.1 \quad 14$ & & $2166.38 \quad 6$ & 100 & 10 \\
\hline & 2482.2120 & $4.2 \quad 7$ & & $3008.28 \quad 9$ & 32 & $1 \quad 19$ \\
\hline \multirow[t]{2}{*}{2524.9} & 986.14 & $100 \quad 27$ & 3014.47 & $612.64 \quad 5$ & 49 & 7 \\
\hline & 1682.95 & $59 \quad 15$ & & $1501.85 \quad 21$ & 12 & 3 \\
\hline \multirow[t]{3}{*}{2573.62} & $1034.90 \quad 7$ & $29 \quad 5$ & & $2172.71 \quad 25$ & 6.8 & $8 \quad 15$ \\
\hline & 2466.696 & $100 \quad 11$ & & $2332.50 \quad 11$ & 18 & $0 \quad 22$ \\
\hline & $2573.54 \quad 16$ & $54 \quad 14$ & & $2907.35 \quad 14$ & 100 & 10 \\
\hline \multirow[t]{4}{*}{2606.993} & $861.34 \quad 6$ & $43 \quad 7$ & 3021.11 & $817.05 \quad 5$ & 19 & 3 \\
\hline & $1229.89 \quad 3$ & $100 \quad 15$ & & $1766.26 \quad 3$ & 100 & 14 \\
\hline & $1352.02 \quad 7$ & $92 \quad 14$ & & $2762.47 \quad 10$ & 12. & $4 \quad 14$ \\
\hline & $1968.08 \quad 9$ & $38 \quad 6$ & & $3021.07 \quad 8$ & 63 & 3 \\
\hline \multirow[t]{6}{*}{2627.850} & 766.219 & $14 \quad 3$ & 3040.21 & $618.20 \quad 8$ & 31 & 5 \\
\hline & $1361.535 \quad 23$ & $100 \quad 14$ & & $2341.09 \quad 19$ & 10 & $8 \quad 16$ \\
\hline & $1785.95 \quad 6$ & $\begin{array}{ll}60 & 9\end{array}$ & & $3039.92 \quad 8$ & 100 & 8 \\
\hline & 2369.93 & 495 & 3168.2 & 1054.33 & 100 & 22 \\
\hline & $2520.73 \quad 9$ & 314 & & $2024.5 \quad 6$ & 61 & 17 \\
\hline & $2627.74 \quad 10$ & $26 \quad 3$ & 3195.78 & $1787.5 \quad 3$ & 67 & 12 \\
\hline \multirow[t]{6}{*}{2644.288} & $706.98 \quad 8$ & $11.8 \quad 20$ & & $1818.32 \quad 23$ & 54 & 8 \\
\hline & $920.81 \quad 6$ & $14.4 \quad 24$ & & $1998.11 \quad 11$ & 26 & 5 \\
\hline & $1500.37 \quad 13$ & $15 \quad 3$ & & 2411.93 & 26 & 8 \\
\hline & $1962.29 \quad 3$ & $100 \quad 16$ & & $2556.94 \quad 7$ & 54 & 6 \\
\hline & 2006.03 & $6.6 \quad 20$ & & $3195.59 \quad 8$ & 100 & 5 \\
\hline & $2537.36 \quad 7$ & $29 \quad 3$ & 3210.57 & $1802.57 \quad 11$ & 40 & 6 \\
\hline 2653.81 & 2395.536 & $100 \quad 10$ & & 3210.468 & 100 & 5 \\
\hline & $2653.56 \quad 10$ & $26 \quad 3$ & 3233.91 & $1391.4 \quad 3$ & 100 & 17 \\
\hline 2718.096 & $856.20 \quad 3$ & $20 \quad 3$ & & $1833.88 \quad 5$ & 64 & 10 \\
\hline & $1340.942 \quad 23$ & $47 \quad 7$ & & $2035.91 \quad 10$ & 72 & 10 \\
\hline & $1451.745 \quad 19$ & $100 \quad 15$ & & $2089.8 \quad 4$ & 58 & 7 \\
\hline & $1520.28 \quad 4$ & 234 & & $3126.83 \quad 10$ & 55 & 3 \\
\hline & $1934.16 \quad 3$ & $38 \quad 6$ & 3284.42 & $1080.17 \quad 10$ & 8 & $0 \quad 14$ \\
\hline 2765.961 & $283.78 \quad 12$ & 113 & & 2086.535 & 80 & 8 \\
\hline & $828.462 \quad 16$ & $76 \quad 11$ & & $2500.45 \quad 5$ & 100 & 10 \\
\hline & $1622.53 \quad 7$ & 5.28 & & $3177.25 \quad 16$ & 38 & $2 \quad 22$ \\
\hline & $2083.94 \quad 4$ & 849 & 3287.14 & $1909.90 \quad 5$ & 20 & 3 \\
\hline & $2765.80 \quad 4$ & $100 \quad 10$ & & $2648.38 \quad 6$ & 44 & 5 \\
\hline 2862.81 & $1454.53 \quad 12$ & $100 \quad 15$ & & $3028.89 \quad 7$ & 100 & 5 \\
\hline & $1485.60 \quad 8$ & $6.6 \quad 11$ & 3295.35 & $2184.45 \quad 14$ & 100 & 14 \\
\hline & $1596.62 \quad 23$ & 213 & & $3188.9 \quad 3$ & 20 & 3 \\
\hline & $1665.14 \quad 14$ & 3.15 & & $3295.16 \quad 11$ & 76 & 6 \\
\hline & 2020.836 & $44 \quad 4$ & 3304.37 & 1558.723 & 100 & 15 \\
\hline 2878.014 & $806.00 \quad 3$ & $100 \quad 17$ & & $2192.79 \quad 23$ & 22 & 4 \\
\hline & $964.50 \quad 9$ & $26 \quad 5$ & & $2622.61 \quad 16$ & 21 & 4 \\
\hline & $1470.09 \quad 6$ & $42 \quad 7$ & 3323.77 & $2624.65 \quad 19$ & 20 & 3 \\
\hline
\end{tabular}


Adopted Levels, Gammas (continued)

\begin{tabular}{|c|c|c|c|c|c|c|c|c|}
\hline \multirow{2}{*}{$\frac{E(\text { level) }}{3323.77}$} & $\mathrm{E} \gamma^{\dagger}$ & \multicolumn{2}{|c|}{$\mathrm{I} \gamma^{\dagger}$} & \multirow{2}{*}{$\begin{array}{c}\mathrm{E}(\text { level }) \\
3831.75 ?\end{array}$} & $\mathrm{E} \gamma^{\dagger}$ & \multicolumn{3}{|c|}{$\mathrm{I} \gamma^{\dagger}$} \\
\hline & $3065.38 \quad 19$ & 11. & $5 \quad 14$ & & $3831.32 \quad 11$ & 95 & 6 & \\
\hline & $3216.47 \quad 19$ & 21 & 3 & 3862.09 & $923.66 \quad 19$ & 15 & 3 & \\
\hline & $3323.69 \quad 9$ & 100 & 3 & & 2116.84 & 64 & 13 & \\
\hline \multirow[t]{5}{*}{3367.30} & $1990.20 \quad 9$ & 90 & 14 & & $2138.42 \quad 10$ & 59 & 7 & \\
\hline & 2101.2621 & 28 & 6 & & $2372.68 \quad 11$ & 39 & 5 & \\
\hline & $2113.09 \quad 23$ & 34 & 8 & & $2485.3 \quad 3$ & 15 & 3 & \\
\hline & 2685.178 & 89 & 10 & & $3180.00 \quad 19$ & 100 & 21 & \\
\hline & $3367.20 \quad 8$ & 100 & 13 & & $3224.0 \quad 3$ & & 4 & 16 \\
\hline \multirow[t]{9}{*}{3418.44} & $811.49 \quad 9$ & & $6 \quad 16$ & & $3755.06 \quad 9$ & 73 & 4 & \\
\hline & $1368.70 \quad 23$ & 34 & 5 & 3876.92 & $2413.34 \quad 17$ & 21 & 4 & \\
\hline & $1430.74 \quad 16$ & & 89 & & $2476.79 \quad 6$ & 44 & 4 & \\
\hline & $1574.5 \quad 3$ & 21 & 5 & & $3034.90 \quad 7$ & 100 & 5 & \\
\hline & $2163.29 \quad 11$ & 17 & 221 & & $3769.86 \quad 11$ & 14 & & 8 \\
\hline & $2220.57 \quad 5$ & 100 & 10 & 3888.32 & 1684.175 & 100 & 27 & \\
\hline & $2307.39 \quad 10$ & 12 & $4 \quad 14$ & & 1900.499 & 45 & 7 & \\
\hline & $2779.63 \quad 6$ & 32 & 4 & & $2480.28 \quad 14$ & 56 & 8 & \\
\hline & $3160.04 \quad 8$ & 60 & 3 & & $3206.66 \quad 22$ & 17 & & 18 \\
\hline \multirow[t]{3}{*}{3421.24} & $1217.32 \quad 14$ & 11. & 323 & 3890.09 & $951.11 \quad 14$ & 48 & 10 & \\
\hline & 1604.365 & 34 & 8 & & $1027.03 \quad 16$ & 24 & 5 & \\
\hline & $3421.09 \quad 8$ & 100 & 6 & & $1976.64 \quad 5$ & 100 & 15 & \\
\hline 3497.98 & $1138.78 \quad 24$ & 100 & & & $3889.93 \quad 10$ & 98 & 6 & \\
\hline \multirow[t]{6}{*}{3502.91} & 1565.475 & 24 & 4 & 3934.63 & $275.93 \quad 12$ & 100 & 28 & \\
\hline & $2050.02 \quad 10$ & 28 & 3 & & $2823.40 \quad 19$ & 16 & & 22 \\
\hline & $2125.60 \quad 13$ & 14 & $8 \quad 24$ & & $3150.87 \quad 11$ & 31 & 7 & 22 \\
\hline & $2359.17 \quad 6$ & 100 & 9 & & $3827.56 \quad 12$ & 25 & & 17 \\
\hline & $2660.49 \quad 17$ & 7. & $9 \quad 12$ & 4013.89 & $2550.12 \quad 11$ & 7 & 1 & 10 \\
\hline & 2820.8420 & 9 & $4 \quad 12$ & & 2606.43 & 23 & 2 & 23 \\
\hline \multirow[t]{4}{*}{3529.04} & $2065.16 \quad 11$ & 93 & 13 & & $2740.08 \quad 6$ & 37 & 4 & \\
\hline & $2262.79 \quad 16$ & 43 & 7 & & $2747.66 \quad 7$ & 16 & 6 & 18 \\
\hline & $2745.19 \quad 10$ & 71 & 9 & & 4013.779 & 100 & 6 & \\
\hline & $3528.78 \quad 17$ & 100 & 20 & 4036.83 & $2781.84 \quad 5$ & 100 & 12 & \\
\hline 3560.10 & $1612.19 \quad 11$ & 100 & & & $3338.36 \quad 23$ & 13 & & 12 \\
\hline \multirow[t]{3}{*}{3621.19} & 1571.824 & 100 & 16 & & 4037.23 & & 9 & 9 \\
\hline & $1759.28 \quad 6$ & 35 & 5 & 4039.93 & $806.00 \quad 3$ & 100 & 17 & \\
\hline & $3514.40 \quad 12$ & 23. & $2 \quad 16$ & & $1673.71 \quad 11$ & 29 & 5 & \\
\hline \multirow[t]{6}{*}{3658.60} & $2119.72 \quad 14$ & 16. & $7 \quad 24$ & & 2632.038 & 56 & 6 & \\
\hline & 2384.998 & 41 & 3 & & $4039.64 \quad 15$ & 23 & 9 & 22 \\
\hline & $2392.14 \quad 6$ & 100 & 9 & 4053.84 & $1665.14 \quad 14$ & 18 & 3 & \\
\hline & $2403.84 \quad 7$ & 54 & 6 & & $2787.39 \quad 7$ & 100 & 10 & \\
\hline & 2460.93 & 68 & 7 & & $3269.86 \quad 16$ & 13 & & 12 \\
\hline & $3399.5 \quad 3$ & 30. & $9 \quad 18$ & & $4053.64 \quad 12$ & 28 & & 18 \\
\hline 3674.14 & 1602.084 & 100 & 17 & 4092.33 & $1100.67 \quad 18$ & 100 & 10 & \\
\hline & $1812.50 \quad 18$ & 16 & 3 & & $1732.6 \quad 6$ & 36 & 11 & \\
\hline & 2832.136 & 92 & 9 & 4103.51 & $2115.55 \quad 18$ & 94 & 21 & \\
\hline & $2991.87 \quad 10$ & 30 & 4 & & $2189.90 \quad 19$ & 100 & 17 & \\
\hline 3696.55 & $1329.81 \quad 16$ & 14 & 3 & $4105.75 ?$ & $3321.65 \quad 9$ & 100 & 3 & \\
\hline & $2243.25 \quad 9$ & 44 & 5 & & $4105.75 \quad 12$ & 33 & 3 & \\
\hline & $2319.70 \quad 8$ & 65 & 7 & 4128.35 & $1877.13 \quad 6$ & 44 & 6 & \\
\hline & 2585.6120 & & $6 \quad 23$ & & $1941.22 \quad 7$ & 12 & & 20 \\
\hline & $2997.69 \quad 24$ & 9 & $0 \quad 18$ & & $2266.55 \quad 7$ & 30 & 3 & \\
\hline & $3014.6 \quad 3$ & 100 & 6 & & $2381.8 \quad 3$ & 36 & 4 & \\
\hline & $3589.45 \quad 9$ & 98 & 5 & & $2720.05 \quad 25$ & 60 & 6 & \\
\hline $3770.70 ?$ & $2504.34 \quad 13$ & 40 & 5 & & $2751.5 \quad 4$ & 37 & 4 & \\
\hline & $2987.07 \quad 17$ & 32 & 4 & & $2854.71 \quad 10$ & 10 & 9 & 12 \\
\hline & $3088.47 \quad 11$ & 100 & 9 & & 2862.028 & 46 & 6 & \\
\hline 3794.61 & $1881.10 \quad 3$ & 100 & 16 & & $2984.32 \quad 12$ & 12 & 3 & 14 \\
\hline & $3010.54 \quad 8$ & 48 & 3 & & 3285.64 & & 7 & 12 \\
\hline & $3112.50 \quad 9$ & 39 & 3 & & $3489.45 \quad 8$ & 100 & 5 & \\
\hline & $3794.82 \quad 12$ & 10 & 88 & & $4127.93 \quad 14$ & 9 & 1 & 6 \\
\hline 3798.15 & $2397.83 \quad 9$ & 100 & 12 & 4152.35 & $1213.51 \quad 13$ & 12 & 1 & 23 \\
\hline & $2687.54 \quad 11$ & 68 & 8 & & $1763.39 \quad 7$ & 20 & 3 & \\
\hline & $3539.88 \quad 13$ & 35 & 3 & & $3310.40 \quad 8$ & 100 & 5 & \\
\hline $3831.75 ?$ & 1644.556 & 100 & 15 & & $3893.97 \quad 9$ & 45 & 1 & 23 \\
\hline
\end{tabular}


Adopted Levels, Gammas (continued)

\begin{tabular}{|c|c|c|c|c|c|c|c|c|}
\hline \multirow{2}{*}{$\begin{array}{r}\text { E(level) } \\
4154.75\end{array}$} & $\mathrm{E} \gamma^{\dagger}$ & \multicolumn{2}{|c|}{$\mathrm{I} \gamma^{\dagger}$} & \multirow{2}{*}{$\begin{array}{c}\text { E(level) } \\
4612.81\end{array}$} & $\mathrm{E} \gamma^{\dagger}$ & \multicolumn{3}{|c|}{$\mathrm{I} \gamma^{\dagger}$} \\
\hline & $2691.07 \quad 7$ & 100 & 10 & & $3346.01 \quad 23$ & 12 & 6 & 17 \\
\hline & $3312.74 \quad 10$ & 60 & 4 & & $3468.99 \quad 9$ & 100 & 6 & \\
\hline & $4154.22 \quad 12$ & 36. & $5 \quad 22$ & & $4612.3 \quad 6$ & & 8 & 11 \\
\hline 4179.40 & $2666.13 \quad 11$ & 100 & 14 & 4660.72 & $986.45 \quad 8$ & 100 & 17 & \\
\hline & $2981.69 \quad 19$ & 36 & 6 & & $3252.93 \quad 13$ & 75 & 7 & \\
\hline 4259.05 & $2566.86 \quad 9$ & 100 & 11 & & $3394.24 \quad 12$ & 69 & 5 & \\
\hline & $3417.07 \quad 16$ & 45 & 5 & & $4402.48 \quad 21$ & 88 & 10 & \\
\hline & $3576.98 \quad 11$ & 69 & 6 & 4715.33 & $2898.28 \quad 11$ & 100 & 13 & \\
\hline & $4258.95 \quad 13$ & 47 & 3 & & 4033.44 & 56 & 6 & \\
\hline 4389.94 & $2023.76 \quad 11$ & 100 & 12 & & $4457.3 \quad 3$ & 19 & 3 & \\
\hline & $2981.69 \quad 19$ & 36 & 5 & & $4714.7 \quad 5$ & 11 & 3 & \\
\hline & $3278.85 \quad 23$ & 43 & 7 & 4746.1 & $1248.00 \quad 27$ & 100 & 14 & \\
\hline & $3707.96 \quad 12$ & 52 & 4 & & $1754.4 \quad 3$ & 88 & 15 & \\
\hline & $4131.35 \quad 23$ & 28 & 3 & 4748.53 & 2030.278 & 100 & 11 & \\
\hline 4416.58 & $2014.36 \quad 19$ & 62 & 7 & & $2561.53 \quad 12$ & 23 & 3 & \\
\hline & $2027.35 \quad 19$ & 16 & 3 & & 4048.93 & 39 & 3 & \\
\hline & $2554.93 \quad 12$ & 29 & 4 & & $4747.9 \quad 4$ & & 6 & 19 \\
\hline & $2599.42 \quad 8$ & 46 & 5 & 4778.05 & $2012.09 \quad 9$ & 100 & 11 & \\
\hline & $2952.86 \quad 6$ & 77 & 8 & & $2706.03 \quad 12$ & 25 & 4 & \\
\hline & 3161.668 & 100 & 14 & & 2961.119 & 49 & 6 & \\
\hline & $3574.58 \quad 20$ & 12 . & $7 \quad 18$ & & $3935.39 \quad 19$ & 12 & 0 & 14 \\
\hline & $3777.70 \quad 9$ & 30 & 3 & & $4095.81 \quad 18$ & 13 & & 14 \\
\hline & $4158.8 \quad 3$ & 4 & 19 & 4806.84 & 1572.93 & 40 & 10 & \\
\hline & $4309.58 \quad 12$ & 22. & $3 \quad 14$ & & $2179.04 \quad 8$ & 59 & 7 & \\
\hline & $4416.31 \quad 10$ & 99 & 5 & & $2199.61 \quad 13$ & 21 & 3 & \\
\hline 4428.30 & $925.71 \quad 24$ & 59 & 9 & & $2602.76 \quad 10$ & 19 & 6 & 23 \\
\hline & 1565.475 & 36 & 6 & & $2945.07 \quad 14$ & 22 & 3 & \\
\hline & $2490.65 \quad 7$ & 78 & 8 & & $3342.50 \quad 20$ & 6 & 3 & 7 \\
\hline & $2964.63 \quad 6$ & 76 & 8 & & $3551.83 \quad 10$ & 23 & 3 & 13 \\
\hline & 3161.828 & 100 & 14 & & $4022.8 \quad 4$ & 100 & 5 & \\
\hline & $3317.11 \quad 19$ & & 69 & & $4124.2 \quad 3$ & 4 & & 7 \\
\hline 4443.13 & 1155.998 & 66 & 11 & 4853.65 & $2487.42 \quad 7$ & 75 & 8 & \\
\hline & $2076.90 \quad 5$ & 100 & 16 & & $4853.29 \quad 11$ & 100 & 5 & \\
\hline & $2455.04 \quad 12$ & 56 & 7 & 4877.21 & 1509.924 & 55 & 9 & \\
\hline 4481.09 & $2493.18 \quad 14$ & 21 & 3 & & $3185.09 \quad 9$ & 53 & 3 & \\
\hline & $2664.15 \quad 12$ & 33 & 4 & & $3468.99 \quad 9$ & 100 & 6 & \\
\hline & $3370.05 \quad 8$ & 100 & 14 & 4903.53 & $3450.39 \quad 13$ & 100 & 13 & \\
\hline & $3638.65 \quad 16$ & 11 & $4 \quad 10$ & & $4203.80 \quad 23$ & 11 & & 14 \\
\hline & $3842.41 \quad 12$ & 20 . & $5 \quad 14$ & & $4645.03 \quad 11$ & 54 & 3 & \\
\hline & $4374.04 \quad 10$ & 71 & 4 & 4938.98 & $2060.99 \quad 18$ & 29 & 6 & \\
\hline & $4481.16 \quad 18$ & 19. & $0 \quad 19$ & & $2700.18 \quad 10$ & 50 & 6 & \\
\hline 4556.71 & $3092.86 \quad 9$ & 57 & 4 & & $3025.54 \quad 9$ & 100 & 6 & \\
\hline & $3283.44 \quad 18$ & 38 & 3 & & $4938.54 \quad 13$ & 41 & 3 & \\
\hline & $3290.62 \quad 23$ & 10. & $0 \quad 15$ & 4942.99 & 1052.883 & 100 & 16 & \\
\hline & $3714.28 \quad 17$ & 7 & 98 & & $1921.61 \quad 15$ & 16 & 3 & \\
\hline & $3874.7 \quad 4$ & 100 & 13 & & $4101.15 \quad 17$ & 13 & 0 & 12 \\
\hline 4576.23 & 1658.949 & 34 & 6 & 4959.72 & $2887.15 \quad 22$ & 24 & 5 & \\
\hline & $2094.05 \quad 11$ & 48 & 6 & & $3446.56 \quad 10$ & 100 & 6 & \\
\hline & $2325.11 \quad 12$ & 37 & 5 & & $3582.50 \quad 15$ & 39 & 3 & \\
\hline & $2504.34 \quad 13$ & 26 & 4 & 5003.01 & $2076.86 \quad 5$ & 100 & 12 & \\
\hline & $3112.50 \quad 9$ & 100 & 6 & & $2580.79 \quad 9$ & 37 & 5 & \\
\hline & $3465.26 \quad 10$ & 56 & 4 & & $3280.8 \quad 7$ & & .5 & 25 \\
\hline 4590.56 & $2188.20 \quad 18$ & 33 & 6 & & 4218.7125 & & 0 & 10 \\
\hline & $2774.0 \quad 3$ & 21. & 223 & & $4895.7 \quad 3$ & & 0 & 10 \\
\hline & $3077.33 \quad 10$ & 23. & $8 \quad 16$ & 5064.08 & $3220.89 \quad 9$ & 84 & 4 & \\
\hline & $3126.83 \quad 10$ & 31 . & $8 \quad 19$ & & 3790.369 & 100 & 6 & \\
\hline & $3479.73 \quad 8$ & 100 & 5 & & $4425.20 \quad 10$ & 86 & 5 & \\
\hline & $4331.5 \quad 4$ & 7 . & 17 & 5081.15 & $3814.8 \quad 3$ & 34 & 8 & \\
\hline & $4483.7 \quad 3$ & 11. & $3 \quad 13$ & & $4399.00 \quad 18$ & 36 & 3 & \\
\hline 4603.7 & 1043.65 & & & & $4442.06 \quad 11$ & 100 & 5 & \\
\hline 4612.81 & $1985.20 \quad 12$ & 23 & 4 & & $5080.94 \quad 13$ & 78 & 4 & \\
\hline & $2408.82 \quad 11$ & 35 & 5 & 5097.00 & $1812.50 \quad 18$ & 28 & 5 & \\
\hline & $2450.82 \quad 14$ & 50 & 9 & & $2523.30 \quad 8$ & 100 & 11 & \\
\hline
\end{tabular}


Adopted Levels, Gammas (continued)

\begin{tabular}{|c|c|c|c|c|c|c|}
\hline E(level) & $\mathrm{E} \gamma^{\dagger}$ & & $\mathrm{I} \gamma^{\dagger}$ & E(level) & $\mathrm{E} \gamma^{\dagger}$ & $\mathrm{I} \gamma^{\dagger}$ \\
\hline \multirow[t]{3}{*}{5097.00} & $2695.13 \quad 8$ & 70 & 8 & $(7533.80)$ & $2436.65 \quad 5$ & $5.0 \quad 5$ \\
\hline & $2730.74 \quad 11$ & 33 & 4 & & 2452.368 & $3.5 \quad 5$ \\
\hline & $5096.55 \quad 15$ & 22 & $.0 \quad 15$ & & $2469.81 \quad 6$ & $4.5 \quad 5$ \\
\hline \multirow[t]{2}{*}{5179.10} & $1891.92 \quad 5$ & 100 & 16 & & $2530.77 \quad 6$ & $5.0 \quad 6$ \\
\hline & $3265.39 \quad 16$ & 34 & 3 & & $2574.57 \quad 20$ & 1.86 \\
\hline \multirow[t]{4}{*}{5246.62} & $3258.0 \quad 3$ & 15 & 3 & & 2590.816 & $3.0 \quad 3$ \\
\hline & 3501.73 & & $.0 \quad 15$ & & 2594.94 & $1.95 \quad 23$ \\
\hline & $3869.12 \quad 10$ & 61 & 4 & & $2629.79 \quad 10$ & $1.89 \quad 21$ \\
\hline & $4404.62 \quad 11$ & 100 & 7 & & $2656.43 \quad 7$ & $1.94 \quad 20$ \\
\hline \multirow[t]{6}{*}{5318.97} & 1900.499 & 54 & 9 & & $2680.15 \quad 4$ & 9.49 \\
\hline & $2441.02 \quad 13$ & 45 & 7 & & $2726.90 \quad 4$ & $16.8 \quad 17$ \\
\hline & 3080.13 & 21 & 3 & & $2755.7 \quad 5$ & $2.33 \quad 24$ \\
\hline & $4120.72 \quad 11$ & 100 & 6 & & $2784.8 \quad 3$ & $1.73 \quad 18$ \\
\hline & $4534.97 \quad 13$ & 49 & 3 & & $2817.7 \quad 3$ & $1.48 \quad 15$ \\
\hline & $4636.57 \quad 20$ & 17 & .622 & & $2873.6 \quad 4$ & 3.54 \\
\hline \multirow[t]{4}{*}{5476.93} & $2710.0 \quad 4$ & 27 & 9 & & 2919.96 & $3.8 \quad 11$ \\
\hline & $3659.96 \quad 16$ & 100 & 9 & & $2943.15 \quad 5$ & $6.5 \quad 6$ \\
\hline & $4366.0 \quad 4$ & 21 & 6 & & $2957.39 \quad 5$ & 5.56 \\
\hline & 4693.14 & 27 & 6 & & $2977.04 \quad 5$ & $8.2 \quad 8$ \\
\hline \multirow[t]{3}{*}{5630.13} & $2334.63 \quad 17$ & 100 & 15 & & $3052.69 \quad 7$ & 12.16 \\
\hline & $4140.4 \quad 3$ & 23 & 4 & & $3090.55 \quad 9$ & $3.23 \quad 18$ \\
\hline & 4254.0920 & 38 & 4 & & $3105.49 \quad 7$ & $13.0 \quad 6$ \\
\hline \multirow[t]{4}{*}{5697.21} & $3624.83 \quad 14$ & 13 & .211 & & $3117.04 \quad 7$ & 19.19 \\
\hline & $4233.87 \quad 22$ & & $.5 \quad 11$ & & $3144.36 \quad 11$ & $4.27 \quad 21$ \\
\hline & $4319.91 \quad 10$ & 100 & 5 & & $3274.47 \quad 9$ & $1.82 \quad 11$ \\
\hline & 5057.63 & & .811 & & 3354.229 & $2.52 \quad 14$ \\
\hline \multirow[t]{4}{*}{5710.68} & $1606.95 \quad 6$ & 100 & 16 & & $3379.07 \quad 8$ & $3.26 \quad 18$ \\
\hline & $1673.71 \quad 11$ & 63 & 11 & & 3381.318 & $9.8 \quad 5$ \\
\hline & $3459.36 \quad 11$ & 72 & 5 & & $3405.34 \quad 7$ & $31.4 \quad 15$ \\
\hline & $5452.25 \quad 16$ & & $.7 \quad 24$ & & $3427.93 \quad 8$ & $3.52 \quad 21$ \\
\hline \multirow[t]{6}{*}{5759.69} & $2338.49 \quad 8$ & 100 & 11 & & $3429.87 \quad 8$ & $3.79 \quad 23$ \\
\hline & $2341.09 \quad 19$ & 27 & 4 & & $3479.73 \quad 8$ & $4.71 \quad 24$ \\
\hline & $2455.04 \quad 12$ & 46 & 6 & & $3493.77 \quad 9$ & $2.42 \quad 14$ \\
\hline & $2881.44 \quad 24$ & 14 & 3 & & 3496.678 & $7.2 \quad 4$ \\
\hline & 2896.23 & 20 & 5 & & $3519.73 \quad 8$ & 221 \\
\hline & $4270.35 \quad 16$ & 17 & .116 & & $3599.03 \quad 8$ & 5. $14 \quad 26$ \\
\hline \multirow[t]{2}{*}{5789.58} & $1749.63 \quad 5$ & 100 & 16 & & $3643.46 \quad 8$ & $5.09 \quad 29$ \\
\hline & $3801.25 \quad 17$ & 12 & .214 & & $3645.26 \quad 10$ & $2.29 \quad 18$ \\
\hline \multirow[t]{3}{*}{5846.42} & $2542.11 \quad 13$ & 100 & 13 & & $3656.63 \quad 8$ & 11.16 \\
\hline & 3080.13 & 42 & 7 & & $3671.70 \quad 8$ & 13.26 \\
\hline & $4734.5 \quad 4$ & 18 & 5 & & 3701.919 & $1.77 \quad 9$ \\
\hline \multirow[t]{5}{*}{5953.67} & $2332.50 \quad 11$ & 50 & 6 & & $3735.52 \quad 9$ & $3.05 \quad 17$ \\
\hline & $2450.82 \quad 14$ & 61 & 12 & & $3738.94 \quad 8$ & $11.7 \quad 6$ \\
\hline & $3346.01 \quad 23$ & 15 & .520 & & $3763.03 \quad 10$ & $1.55 \quad 8$ \\
\hline & $3881.34 \quad 20$ & 10 & $.8 \quad 14$ & & 3837.278 & 14.58 \\
\hline & $4698.54 \quad 11$ & 100 & 6 & & $3859.48 \quad 9$ & $7.9 \quad 5$ \\
\hline \multirow[t]{5}{*}{5978.43} & $3350.64 \quad 14$ & 100 & 12 & & $3875.05 \quad 9$ & 12.18 \\
\hline & $3816.80 \quad 14$ & 52 & 5 & & $3912.28 \quad 9$ & $5.30 \quad 27$ \\
\hline & $4465.4 \quad 3$ & & $.8 \quad 17$ & & $4005.79 \quad 23$ & $1.58 \quad 9$ \\
\hline & 4601.3121 & 14 & $.8 \quad 17$ & & 4030.709 & 3.9120 \\
\hline & $5339.22 \quad 14$ & 43 & 3 & & $4112.20 \quad 9$ & $8.9 \quad 5$ \\
\hline \multirow[t]{12}{*}{$(7533.80)$} & $1555.50 \quad 4$ & & $.80 \quad 27$ & & $4115.15 \quad 9$ & $26.1 \quad 14$ \\
\hline & 1580.323 & & .58 & & $4166.20 \quad 10$ & $4.86 \quad 24$ \\
\hline & $1687.08 \quad 10$ & & $.64 \quad 11$ & & $4209.94 \quad 10$ & $3.42 \quad 18$ \\
\hline & $1744.03 \quad 4$ & & .23 & & $4229.38 \quad 11$ & $3.18 \quad 17$ \\
\hline & $1774.09 \quad 5$ & & .34 & & $4238.31 \quad 11$ & $3.32 \quad 17$ \\
\hline & 1823.183 & & .28 & & $4246.39 \quad 12$ & $1.82 \quad 11$ \\
\hline & $1836.55 \quad 4$ & & .13 & & $4249.24 \quad 10$ & $13.8 \quad 8$ \\
\hline & 1903.419 & & $.77 \quad 12$ & & 4299.719 & $7.4 \quad 4$ \\
\hline & $2056.85 \quad 12$ & & $.95 \quad 14$ & & $4322.90 \quad 9$ & $7.0 \quad 4$ \\
\hline & 2215.1120 & & $.7 \quad 5$ & & 4337.719 & 12.66 \\
\hline & $2287.12 \quad 5$ & & .08 & & $4493.41 \quad 10$ & $6.6 \quad 3$ \\
\hline & $2354.55 \quad 8$ & & $.0 \quad 2$ & & $4512.50 \quad 9$ & 19.19 \\
\hline
\end{tabular}


Adopted Levels, Gammas (continued)

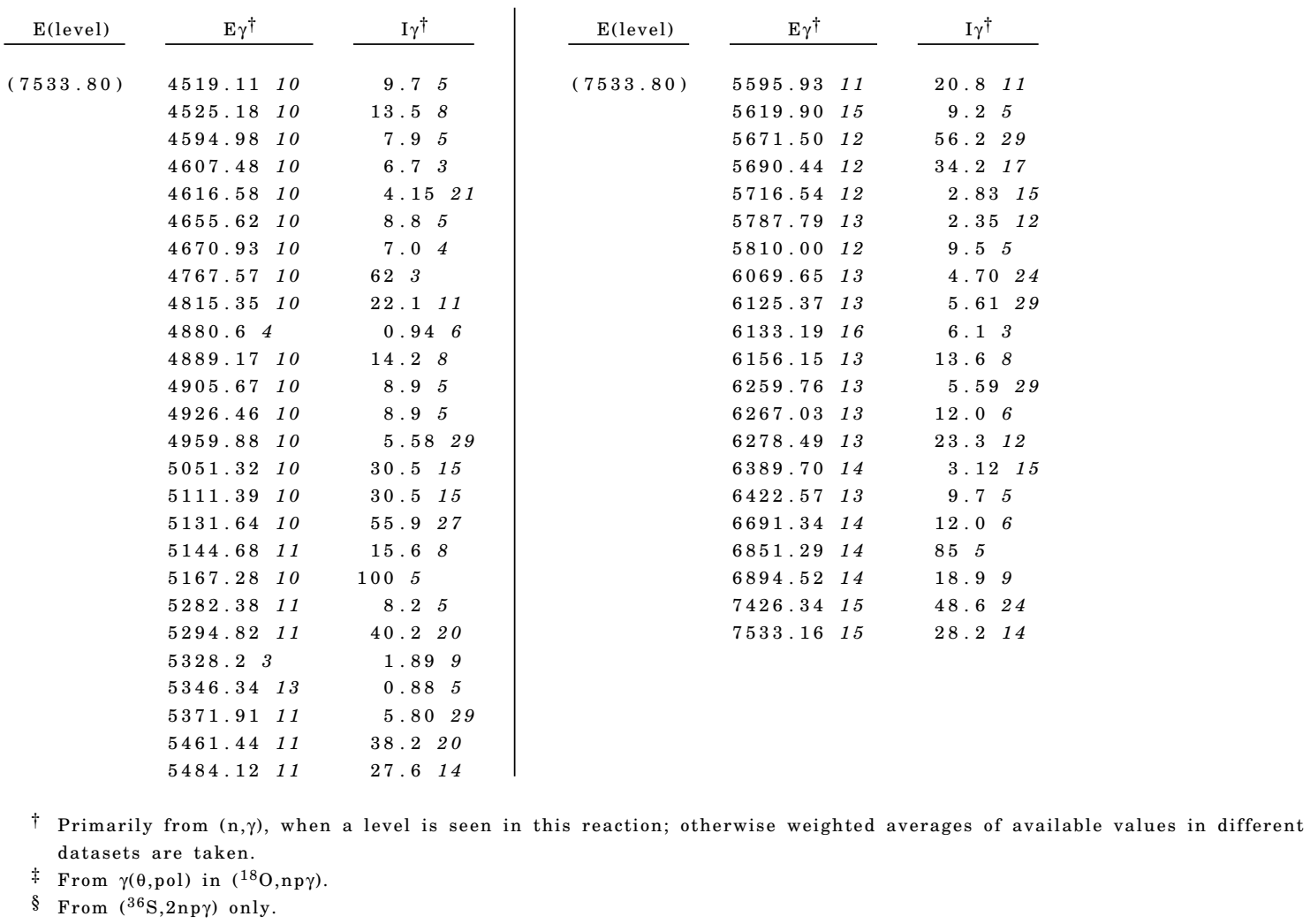

\section{${ }^{42}$ Ar $\beta^{-}$Decay $(32.9$ y) 1965 St09}

Parent ${ }^{42} \mathrm{Ar}: \mathrm{E}=0 ; \mathrm{J} \pi=0+; \mathrm{T}_{1 / 2}=32.9$ y $11 ; \mathrm{Q}$ (g.s. $)=5996 ; \% \beta^{-}$decay $=100$

${ }^{42} \mathrm{Ar}-\mathrm{T}_{1 / 2}$ : From Adopted Levels of ${ }^{42} \mathrm{Ar}$.

${ }^{42} \mathrm{Ar}-\mathrm{Q}\left(\beta^{-}\right)$: From $2012 \mathrm{Wa} 38$.

1965St09: measured $\mathrm{T}_{1 / 2}$ of ${ }^{42} \mathrm{Ar}$.

Others:

1964Ho31: measured cross section for production and $\mathrm{T}_{1 / 2}$.

1961Ja07: production of ${ }^{42} \mathrm{Ar}$ isotope.

1952Ka44: identification of ${ }^{42} \mathrm{Ar}$ and $\mathrm{T}_{1 / 2}$.

1990Mi05: measured $\beta \gamma$ coin for ${ }^{42} \mathrm{~K}$ decay. ${ }^{42} \mathrm{~K}$ isotope obtained from ${ }^{42} \mathrm{Ar}$ decay.

${ }^{42} \mathrm{~K}$ Levels

$\frac{\mathrm{E}(\text { level })}{0.0} \quad \frac{\mathrm{J} \pi^{\dagger}}{2-}$
$\quad$ From Adopted Levels.

$\beta^{-}$radiations

$\frac{\mathrm{E} \beta^{-}}{(5996)} \frac{\mathrm{E} \text { (level) }}{0.0} \quad \frac{\mathrm{I} \beta^{-\dagger}}{100} \frac{\log f t}{9.32^{1 \mathrm{u}} 17}$

$\dagger$ Absolute intensity per 100 decays. 


\section{BE(36S,2NPG),(37P,4NG) 1998Mo16,2005IdZZ}

1998Mo16: $\mathrm{E}\left({ }^{36} \mathrm{~S}\right)=90,100,110 \mathrm{MeV}{ }^{36} \mathrm{~S}$ beam was produced from the TANDEM accelerator of the University and Technical University Munich. Target of beryllium evaporated onto gold backing. $\gamma$-rays were detected with Compton-suppressed HPGe detectors. Measured E $\gamma, \mathrm{I} \gamma, \gamma \gamma-\mathrm{coin}, \mathrm{p} \gamma$-coin, recoil- $\gamma$ coin. Deduced levels, branching ratios.

2005IdZZ: $\mathrm{E}\left({ }^{37} \mathrm{P}\right) \approx 6 \mathrm{MeV} /$ nucleon secondary beam from ${ }^{9} \mathrm{Be}\left({ }^{40} \mathrm{Ar}, \mathrm{X}\right), \mathrm{E}=63 \mathrm{MeV} /$ nucleon primary reaction using $\mathrm{RIPS}$ separator at RIKEN facility. Measured E $\gamma$ using GRAPE array of 14 segmented Ge detectors. Gamma rays of 151 , 440 and $570 \mathrm{keV}$ were seen. Deduced 107,258 and $699 \mathrm{keV}$ levels in ${ }^{42} \mathrm{~K}$ from $106-151-440$ cascade. It is not clear to the evaluators whether the $570 \gamma$ is the same as the 571.9 transition from the 1947 level since no $676.8 \gamma$ in cascade with $571.9 \gamma$ is not seen by $2005 \mathrm{IdZZ}$.

All data are from ${ }^{9} \mathrm{Be}\left({ }^{36} \mathrm{~S}, 2 \mathrm{np} \gamma\right)$ (1998Mo16), unless otherwise stated.

\begin{tabular}{|c|c|c|c|c|c|}
\hline \multirow[b]{2}{*}{$\mathrm{E}(\text { level })^{\dagger}$} & \multirow[b]{2}{*}{$\mathrm{J} \pi$} & \multirow[b]{2}{*}{$\mathrm{E}(\text { level })^{\dagger}$} & \multirow[b]{2}{*}{$\mathrm{J} \pi \ddagger$} & vels & \multirow[b]{2}{*}{$\mathrm{J} \pi \ddagger$} \\
\hline & & & & $\mathrm{E}(\text { level })^{\dagger}$ & \\
\hline 0.0 & $2-$ & 1254.73 & $2-$ & $2524.7 \quad 4$ & $(2,3,4)$ \\
\hline $106.80 \quad 4$ & $3-$ & $1273.29 \quad 13$ & $(2-, 3,4+)$ & $2765.9 \quad 6$ & $(2+, 3)$ \\
\hline $258.26 \quad 5$ & $4-$ & $1375.81 \quad 7$ & $6+$ & $2991.51 \quad 13$ & $(5+$ to $9+)$ \\
\hline $638.63 \quad 4$ & $3-$ & 1538.629 & $3+$ & 3168.14 & $(2+$ to $6+)$ \\
\hline $682.10 \quad 11$ & $(2,3)$ & $1936.06 \quad 14$ & & $3497.81 \quad 23$ & $(2+$ to $9-)$ \\
\hline $699.03 \quad 5$ & $5-$ & $1947.68 \quad 8$ & $7+$ & $3559.90 \quad 14$ & 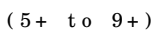 \\
\hline $841.82 \quad 10$ & $3-$ & $2113.8 \quad 3$ & & $4092.14 \quad 22$ & $\left(\begin{array}{cc}3+\text { to } & 9-)\end{array}\right.$ \\
\hline $1143.46 \quad 5$ & $4+$ & $2358.97 \quad 10$ & $(4+$ to $7-)$ & $4745.9 \quad 3$ & $(3+$ to 10$)$ \\
\hline
\end{tabular}

$\dagger$ From least-squares fit to E $\gamma$ data.

$\ddagger$ From Adopted Levels.

\begin{tabular}{|c|c|c|c|c|c|}
\hline \multirow[b]{2}{*}{$\mathrm{E} \gamma$} & \multirow[b]{2}{*}{$\mathrm{E}($ level) } & \multirow[b]{2}{*}{$\mathrm{I} \gamma^{\#}$} & \multirow[b]{2}{*}{$\mathrm{E} \gamma$} & $\left({ }^{42} \mathrm{~K}\right)$ & \multirow[b]{2}{*}{$\mathrm{I} \gamma^{\#}$} \\
\hline & & & & $\mathrm{E}($ level) & \\
\hline $106.83 \S 5$ & 106.80 & $100.0 \quad 17$ & 986.14 & 2524.7 & $0.94^{@} 25$ \\
\hline $151.48^{\ddagger} 4$ & 258.26 & 103.520 & $1015.5 \quad 4$ & 1273.29 & $0.91^{@ 25}$ \\
\hline $232.4 \quad 4$ & 1375.81 & $5.95 \quad 24$ & $1043.79 \quad 14$ & 2991.51 & $2.74 \quad 25$ \\
\hline $380.39 \quad 5$ & 638.63 & $3.93 \quad 15$ & $1054.3 \quad 3$ & 3168.1 & $0.67^{@} 15$ \\
\hline $395.16 \quad 7$ & 1538.62 & $3.55 \quad 19$ & $1100.67 \quad 18$ & 4092.14 & $2.06^{@} 20$ \\
\hline $422.8 \quad 3$ & 2358.97 & $2.3 \quad 3$ & $1138.78 \quad 24$ & 3497.81 & $1.24^{@} 17$ \\
\hline $431.44 \quad 9$ & 1273.29 & $1.73 \quad 26$ & $1237.07 \quad 16$ & 1936.06 & $2.80^{@} 24$ \\
\hline $440.78^{\ddagger} 4$ & 699.03 & $77.4 \quad 11$ & $1248.00 \quad 27$ & 4745.9 & $1.13^{@} 16$ \\
\hline $444.43 \quad 5$ & 1143.46 & 5. $12 \quad 26$ & $\mathrm{x} 1291.46$ & & $0.45^{@} 13$ \\
\hline $504.83 \quad 4$ & 1143.46 & $12.41 \quad 28$ & $\mathrm{x} 1296.6 \quad 6$ & & $0.51^{@} 13$ \\
\hline $531.80 \quad 6$ & 638.63 & $8.8 \quad 5$ & $\mathrm{x}_{1511.25}$ & & $0.65^{@} 16$ \\
\hline${ }^{x} 542.42 \quad 23$ & & $5.59 \quad 25$ & $1612.19 \quad 11$ & 3559.90 & $2.55 \quad 26$ \\
\hline $571.86 \neq 4$ & 1947.68 & $7.7 \quad 23$ & $1659.91 \quad 16$ & 2358.97 & $1.78 \quad 24$ \\
\hline $592.23 \quad 6$ & 699.03 & $3.58 \quad 21$ & $1677.52 \quad 26$ & 1936.06 & $2.3^{@} 5$ \\
\hline $616.09 \quad 29$ & 1254.7 & 2.43 & 1682.95 & 2524.7 & $0.55^{@} 14$ \\
\hline $632.68 \quad 20$ & 2991.51 & $1.79 \quad 20$ & $1732.6 \quad 6$ & 4092.14 & $0.74^{@} 22$ \\
\hline $638.60 \quad 5$ & 638.63 & $5.7 \quad 3$ & $1754.4 \quad 3$ & 4745.9 & $1.00^{@ 17}$ \\
\hline $676.77 \quad 4$ & 1375.81 & $47.8 \quad 7$ & $\mathrm{x} 1919.94$ & & $0.73^{@} 20$ \\
\hline $682.09 \quad 11$ & 682.10 & $1.72 \quad 16$ & $2024.5 \quad 6$ & 3168.1 & $0.41^{@} 11$ \\
\hline $\mathrm{x} 735.7^{\dagger} 3$ & & $0.79^{@} 14$ & $2083.7 \quad 5$ & 2765.9 & $0.71^{@} 14$ \\
\hline $841.78 \quad 10$ & 841.82 & $4.7 \quad 4$ & $2113.8 \quad 3$ & 2113.8 & $2.1^{@} 3$ \\
\hline $983.16 \quad 9$ & 2358.97 & $4.7 \quad 5$ & & & \\
\hline
\end{tabular}

$\dagger$ Placement from 842 level as proposed by $1985 \mathrm{Kr} 06$ in $(\mathrm{n}, \gamma)$ is discarded since this $\gamma$ is not observed in coincidence with $107 \gamma$.

$\ddagger$ This $\gamma$ seen in ${ }^{9} \mathrm{Be}\left({ }^{37} \mathrm{P}, 4 \mathrm{n} \gamma\right)(2005 \mathrm{IdZZ})$.

$\S$ The $106.8 \gamma$ was not seen in $2005 \mathrm{IdZZ}$.

\# For $\mathrm{E}\left({ }^{36} \mathrm{~S}\right)=90 \mathrm{MeV}$, unless otherwise stated.

(a) From recoil-gated spectrum at $\mathrm{E}\left({ }^{36} \mathrm{~S}\right)=100 \mathrm{MeV}$.

$x \quad \gamma$ ray not placed in level scheme. 


\section{${ }^{26} \mathrm{Mg}\left({ }^{18} \mathrm{O}, \mathrm{np} \gamma\right) \quad 1977 \mathrm{Ek01}$}

1977Ek01 (also 1975Eg01): $\mathrm{E}=25,34 \mathrm{MeV}{ }^{18} \mathrm{O}$ beam was produced from the Utrecht EN tandem accelerator. Target of 180 $\mu \mathrm{g} / \mathrm{cm}^{2}$ enriched ${ }^{26} \mathrm{Mg}(99.42 \%)$ evaporated on a $27 \mu \mathrm{m} \mathrm{Ni}$ backing. $\gamma$-rays were detected with Compton-suppression $\mathrm{Ge}(\mathrm{Li})$ detectors of $25 \%$ efficiency and a three-crystal Ge(Li) Compton polarimeter. Measured E $\gamma, \mathrm{I} \gamma, \gamma \gamma-\mathrm{coin}, \gamma(\theta)$, $\gamma \gamma(\theta), \gamma\left(\right.$ lin pol). Deduced levels, J $\pi$, branching ratios, $\mathrm{T}_{1 / 2}$ by Recoil-distance method (RDM).

Other: $1975 \mathrm{Wa} 04$. See ${ }^{27} \mathrm{Al}\left({ }^{18} \mathrm{O}, \mathrm{n} 2 \mathrm{p} \gamma\right)$ dataset.

\section{${ }^{42} \mathrm{~K}$ Levels}

\begin{tabular}{|c|c|c|}
\hline $\mathrm{E}(\text { level })^{\dagger}$ & $\mathrm{J} \pi \S$ & $\mathrm{T}_{1 / 2}{ }^{\ddagger}$ \\
\hline 0.0 & $2-\#$ & \\
\hline $106.780 \quad 20$ & $3-$ & 284 ps 42 \\
\hline 258.1310 & $4-$ & 133 ps 9 \\
\hline $638.45 \quad 12$ & $3-$ & $<1.4 \mathrm{n} \mathrm{s}$ \\
\hline $698.82 \quad 12$ & $5-$ & $41 \mathrm{ps} 8$ \\
\hline $1143.20 \quad 16$ & $4+$ & $<1.4 \mathrm{n} \mathrm{s}$ \\
\hline $1375.51 \quad 16$ & $6+$ & $1.17 \mathrm{~ns}$ \\
\hline 1538.24 & $(3,5)$ & $<3.5 \mathrm{ps}$ \\
\hline $1947.2 \quad 3$ & $7+$ & $<1.1 \mathrm{ps}$ \\
\hline
\end{tabular}

$\dagger$ From least-squares fit to $\mathrm{E} \gamma$ data.

$\ddagger$ Recoil-distance method (1975Eg01).

$\S$ From $\gamma(\theta)$ and $\gamma($ lin pol) in 1977Ek01, unless otherwise noted.

\# From Adopted Levels.

$\mathrm{A}_{2}, \mathrm{~A}_{4}$ and Pol values are from 1977Ek01.

\begin{tabular}{|c|c|c|c|c|c|c|c|}
\hline \multicolumn{2}{|l|}{$\mathrm{E} \gamma$} & $\mathrm{E}($ level) & \multicolumn{2}{|l|}{$\mathrm{I} \gamma^{\ddagger}$} & \multirow{2}{*}{$\frac{\text { Mult. } \S}{D(+Q)}$} & $\delta \S$ & Comments \\
\hline $106.78^{\dagger}$ & 2 & 106.780 & $>110$ & & & +0.013 & $\begin{array}{l}A_{2}=-0.15318 ; A_{4}=+0.02218 \\
I \gamma: \text { sum of feeding intensities. }\end{array}$ \\
\hline $151.33^{\dagger}$ & 12 & 258.13 & 1003 & & $\mathrm{M} 1$ ( +E 2 ) & $-0.008 \quad 16$ & $\begin{array}{l}\mathrm{A}_{2}=-0.259 \\
\mathrm{Pol}=-0.30 \quad 11\end{array}$ \\
\hline 232.32 & & 1375.51 & 7.1 & 3 & $\mathrm{E} 2(+\mathrm{M} 3)$ & $-0.04 \quad 3$ & $\mathrm{~A}_{2}=+0.345 ; \mathrm{A}_{4}=-0.165$ \\
\hline 380.32 & & 638.45 & 4.2 & 2 & $(\mathrm{M} 1+\mathrm{E} 2)$ & & $\mathrm{A}_{2}=-0.3516 ; \mathrm{A}_{4}=+0.2917$ \\
\hline $395.0 \quad 3$ & & 1538.2 & 7.5 & 13 & $\mathrm{D}$ & & $\mathrm{A}_{2}=-0.406$ \\
\hline $440.68^{\dagger}$ & 12 & 698.82 & 853 & & $\mathrm{M} 1+\mathrm{E} 2$ & +0.1028 & $\begin{array}{l}\mathrm{A}_{2}=-0.0808 ; \mathrm{A}_{4}=-0.0169 \\
\mathrm{Pol}=-0.464\end{array}$ \\
\hline $444.4 \quad 3$ & & 1143.20 & 5.1 & 2 & $\mathrm{D}$ & & $\mathrm{A}_{2}=-0.185$ \\
\hline 504.72 & & 1143.20 & 5.3 & 3 & $\mathrm{D}$ & & $\mathrm{A}_{2}=-0.386$ \\
\hline 531.72 & & 638.45 & 7.0 & 4 & & & $\mathrm{~A}_{2}=+0.288$ \\
\hline 571.72 & & 1947.2 & 374 & & $\mathrm{M} 1+\mathrm{E} 2$ & $-0.035 \quad 19$ & $\begin{array}{l}\mathrm{A}_{2}=-0.36 \quad 12 \\
\mathrm{Pol}=-0.36 \quad 6\end{array}$ \\
\hline 592.12 & & 698.82 & 3.2 & 9 & & & I $\gamma$ : from $\mathrm{I} \gamma(592) / \mathrm{I} \gamma(441)=3.610 / 96.410$ \\
\hline 638.42 & & 638.45 & 7.0 & 5 & $\mathrm{D}$ & & $\mathrm{A}_{2}=-0.3212 ; \mathrm{A}_{4}=-0.1614$ \\
\hline $676.69^{\dagger}$ & 12 & 1375.51 & 58.6 & 18 & $\mathrm{E} 1+\mathrm{M} 2$ & +0.02511 & $\begin{array}{l}\mathrm{A}_{2}=-0.21610 ; \mathrm{A}_{4}=-0.01611 . \\
\mathrm{Pol}=+0.394 .\end{array}$ \\
\hline
\end{tabular}

$\dagger$ From 1975Eg01, used as calibration line by $1977 \mathrm{Ek} 01$.

¥ For $34 \mathrm{MeV}$, unless otherwise stated.

$\S$ From $\gamma(\theta)$ and $\gamma($ lin pol) in $1977 \mathrm{Ek} 01$ 


\section{${ }^{27} \mathrm{Al}\left({ }^{18} \mathrm{O}, \mathrm{n} 2 \mathrm{p} \gamma\right),{ }^{26} \mathrm{Mg}\left({ }^{18} \mathrm{O}, \mathrm{np} \gamma\right),{ }^{26} \mathrm{Mg}\left({ }^{19} \mathrm{~F}, \mathrm{n} 2 \mathrm{p} \gamma\right) \quad 1975 \mathrm{Wa04}, 19750101$}

1975Wa04(also 19750101): E=20-60 MeV. Two Ge(Li) detectors. Measured $\gamma \gamma$-coin, $\gamma(\theta), \gamma($ lin pol). Deduced levels, J $\pi$, branching ratios, lifetimes by recoil-distance method (RDM).

\section{${ }^{42} \mathrm{~K}$ Levels}

\begin{tabular}{|c|c|c|}
\hline $\mathrm{E}(\text { level })^{\dagger}$ & $\mathrm{J} \pi^{\ddagger}$ & $\mathrm{T}_{1 / 2}^{\#}$ \\
\hline 0.0 & $2-\S$ & \\
\hline $106.85 \quad 15$ & $3-\S$ & \\
\hline $258.09 \quad 18$ & $4-$ & $123 \mathrm{ps} 28$ \\
\hline 698.93 & $5-$ & $<14$ ps \\
\hline 1375.94 & $6+$ & $0.76 \mathrm{~ns} 21$ \\
\hline 1947.95 & $7+$ & $\leq 0.52 \mathrm{~ns}^{@}$ \\
\hline \multicolumn{3}{|l|}{$2991.5 ? \quad 7$} \\
\hline \multicolumn{3}{|l|}{3560.15} \\
\hline \multicolumn{3}{|l|}{$4603.7 ? \quad 7$} \\
\hline \multicolumn{3}{|c|}{ From least-squares fit to $\mathrm{E} \gamma$ data. } \\
\hline \multicolumn{3}{|c|}{ From $\gamma(\theta)$ and $\gamma($ lin pol) in 19750101 , unless otherwise noted. } \\
\hline \multicolumn{3}{|c|}{$\S$ From Adopted Levels. } \\
\hline \multicolumn{3}{|c|}{ From recoil-distance method (RDM). } \\
\hline \multicolumn{3}{|c|}{ (a) Quoted by $1975 \mathrm{Wa} 04$ as $\leq 0.52 \mathrm{~ns} 14$. } \\
\hline
\end{tabular}

$\mathrm{A}_{2}, \mathrm{~A}_{4}$ and Pol values are from 19750101.

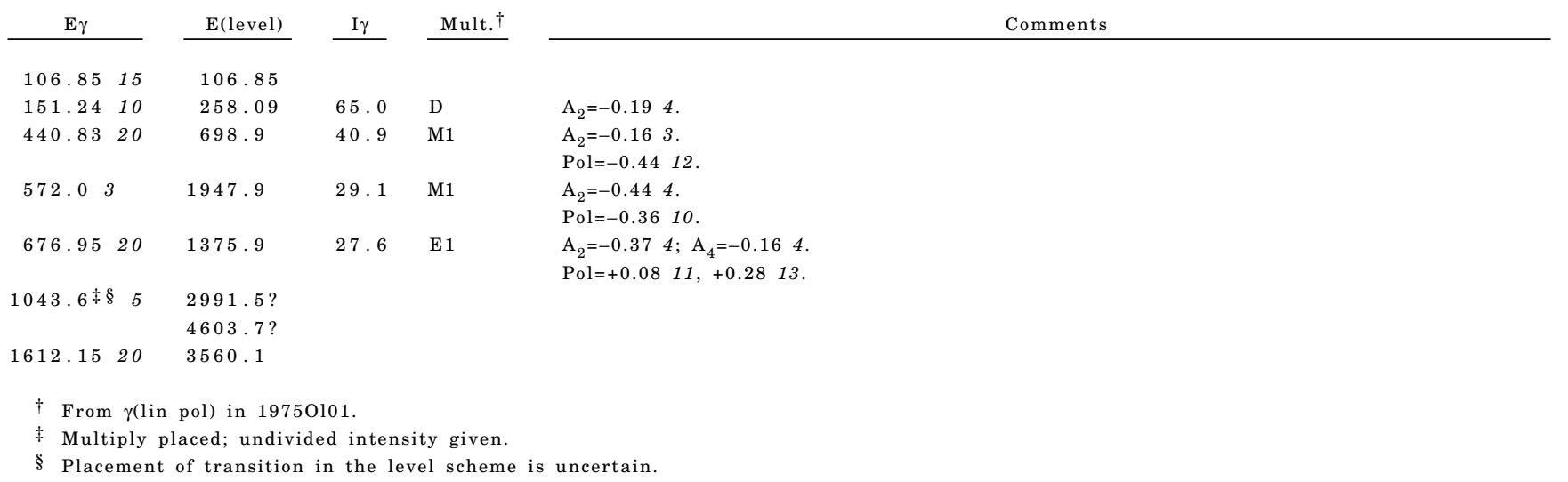

\section{${ }^{40} \operatorname{Ar}(\alpha, d) \quad 1976 D e 24$}

Target ${ }^{40} \mathrm{Ar} \mathrm{J} \pi=0+$

1976De24: E=34.3 MeV alpha beam was produced from the Princeton azimuthally varying field cyclotron. Natural Argon target. Deuterons were detected in a freon cooled $\Delta \mathrm{E}-\mathrm{E}$ silicon detector telescope followed by a third detector in anti-coincidence, FWHM=110 keV. Measured $\sigma\left(\mathrm{E}_{\mathrm{d}}, \theta\right)$. Deduced levels, L from DWBA analysis.

1966Ri04: $\mathrm{E}=44 \mathrm{MeV}$ alpha beam was produced from the Berkeley 88-in. spiral ridge cyclotron. A counter telescope of two lithium drifted silicon crystals. Measured deuteron spectra. FWHM $\approx 200 \mathrm{keV}$.

\section{${ }^{42} \mathrm{~K}$ Levels}

\begin{tabular}{|c|c|c|}
\hline E(level) & $\mathrm{L}$ & $\mathrm{d} \sigma / \mathrm{dW}(\mu \mathrm{b} / \mathrm{sr})^{\dagger}$ \\
\hline 0.0 & 1 & 32 \\
\hline 107 末 & & 15 \\
\hline $700 末$ & 5 & 150 \\
\hline $1170 \quad 50$ & & 50 \\
\hline 153450 & 4 & 300 \\
\hline $1950 \S 50$ & 6 & 700 \\
\hline
\end{tabular}


${ }^{40} \mathrm{Ar}(\alpha, d) \quad 1976 \mathrm{De} 24$ (continued)

${ }^{42} \mathrm{~K}$ Levels (continued)

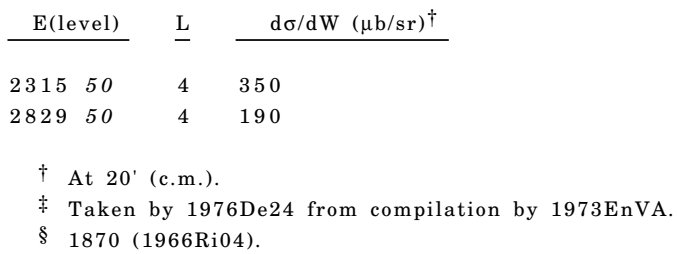

${ }^{41} K(n, \gamma)$ E=thermal $1985 K r 06$

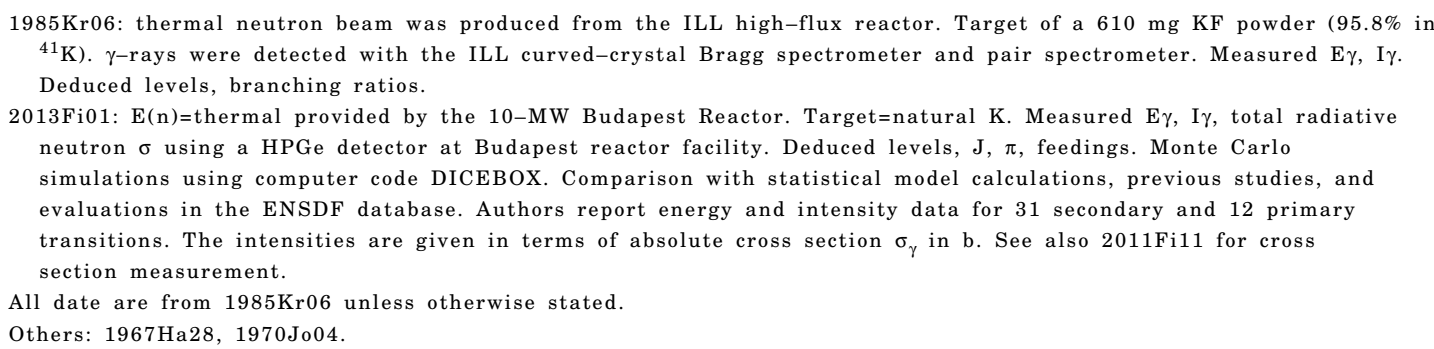

\begin{tabular}{|c|c|}
\hline $\mathrm{E}(\text { level })^{\dagger}$ & $\mathrm{J} \pi \S$ \\
\hline 0.0 & $2-$ \\
\hline $106.828 \quad 5$ & $3-$ \\
\hline $258.260 \quad 6$ & $4-$ \\
\hline $638.726 \quad 7$ & $3-$ \\
\hline $681.942 \quad 8$ & $(2,3)$ \\
\hline $699.087 \quad 15$ & $5-$ \\
\hline $783.885 \quad 11$ & $2-$ \\
\hline $841.937 \quad 7$ & $3-$ \\
\hline $1110.744 \quad 9$ & $3+$ \\
\hline $1143.593 \quad 11$ & $4+$ \\
\hline $1197.904 \quad 10$ & $4-$ \\
\hline $1254.821 \quad 10$ & $2-$ \\
\hline $1266.300 \quad 9$ & $(1,2,3)-$ \\
\hline $1273.517 \quad 12$ & $2-$ \\
\hline $1376.10 \quad 9$ & $6+$ \\
\hline $1377.121 \quad 10$ & $(2,3)-$ \\
\hline 1400.034 & $(2,3)$ \\
\hline $1407.912 \quad 10$ & $(1-, 2,3)$ \\
\hline 1453.054 & $(2-, 3,4-)$ \\
\hline $1463.649 \quad 14$ & $(1-, 2,3)$ \\
\hline $1489.30 \quad 8$ & $(1-$ to $5-)$ \\
\hline 1513.06922 & $(1-$ to $5-)$ \\
\hline $1538.74 \quad 7$ & $3+$ \\
\hline $1692.00 \quad 4$ & $\left(\begin{array}{lll}1-\mathrm{to} & 4-)\end{array}\right.$ \\
\hline $1723.44 \quad 3$ & $(2,3,4+)$ \\
\hline $1745.613 \quad 16$ & $(2+, 3+)$ \\
\hline $1816.879 \quad 18$ & $(2,3,4-)$ \\
\hline $1842.971 \quad 13$ & $(1-, 2,3)$ \\
\hline $1861.893 \quad 9$ & $2-$ \\
\hline $1913.460 \quad 16$ & $(2-, 3)$ \\
\hline $1937.492 \quad 15$ & $(1,2,3)-$ \\
\hline $1987.979 \quad 19$ & $(0-$ to $4-)$ \\
\hline $2049.320 \quad 14$ & $3+$ \\
\hline $2072.009 \quad 12$ & $(2,3)-$ \\
\hline $2161.645 \quad 22$ & $(2+, 3,4+)$ \\
\hline
\end{tabular}

$\mathrm{J} \pi:(2,3)-$ in $2013 \mathrm{Fi} 01$

$\mathrm{J} \pi:(2-, 3,4+)$ in $201 \mathrm{Fi} 01$ 
${ }^{41} \mathrm{~K}(\mathrm{n}, \gamma)$ E=thermal $1985 \mathrm{Kr} \mathbf{0 6}$ (continued)

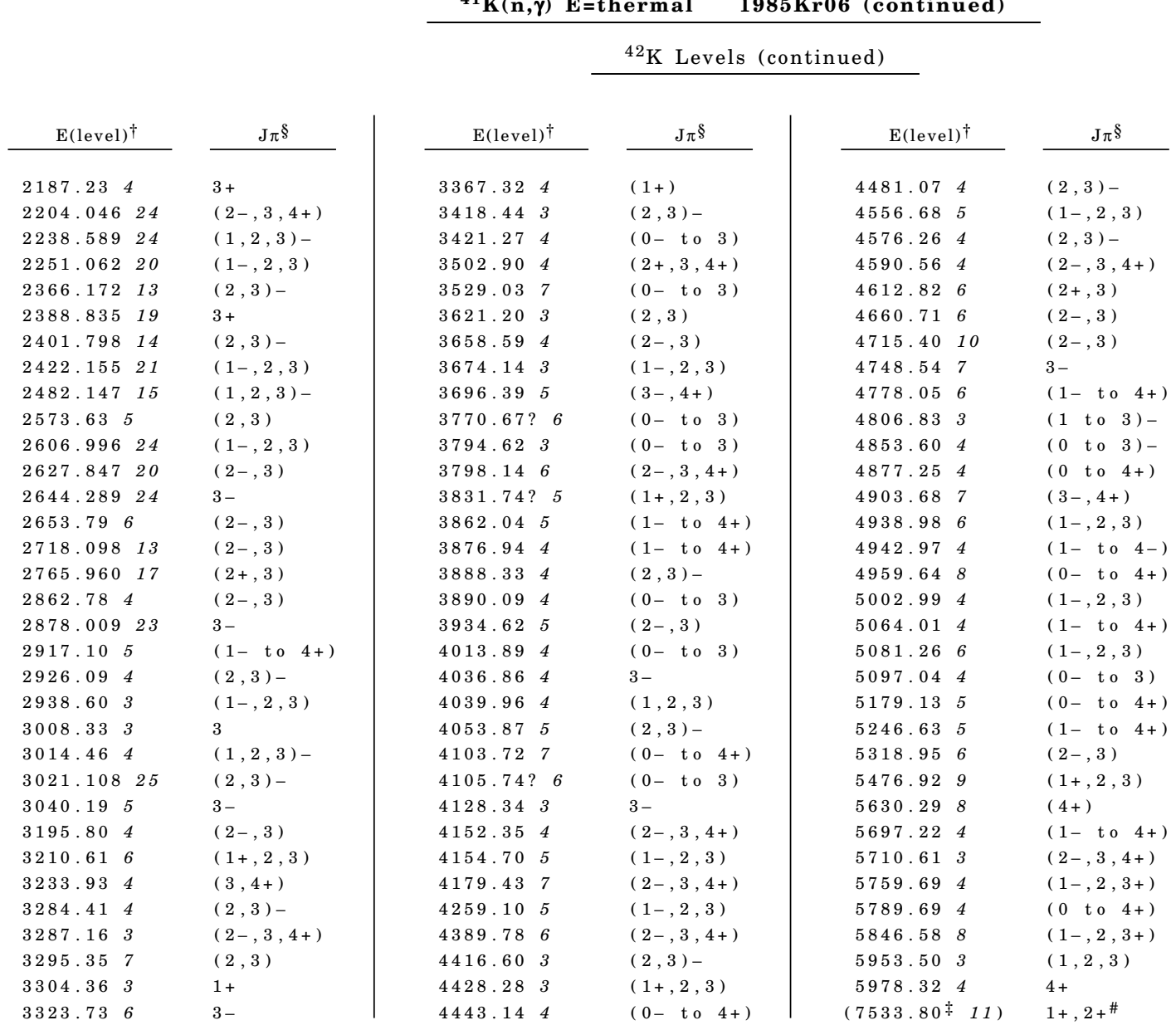

$\dagger$ From least-squares fit to $\mathrm{E} \gamma$ data. The evaluators increase the uncertainties to $0.5 \mathrm{keV}$ for fitting purpose for the following $\gamma$-rays in order to get a reasonable reduced $\chi^{2}=2.3: 1166.808,1606.95,2319.70$ and 4005.79

† From $2012 \mathrm{Wa} 38$. Observed de-excitation intensity is $89 \%$ of g.s. feeding.

$\S$ From Adopted Levels.

\# $\mathrm{s}$-wave capture in ${ }^{41} \mathrm{~K}$ g.s. $(\mathrm{J} \pi=3 / 2+)$.

$\gamma\left({ }^{42} \mathrm{~K}\right)$

Thermal capture $\sigma_{\mathrm{n}}=1.463(2006 \mathrm{MuZX})$.

I $\gamma$ normalization: 0.957 from $\Sigma($ I $\gamma$ to g.s. $)=100$. Thermal capture $\sigma_{n}=1.463(2006 \mathrm{MuZX})$.

\begin{tabular}{|c|c|c|c|c|c|}
\hline $\mathrm{E} \gamma^{\dagger}$ & E(level) & $\mathrm{I} \gamma^{\ddagger} \#$ & $\mathrm{E} \gamma^{\dagger}$ & E(level) & $\mathrm{I} \gamma^{\ddagger} \#$ \\
\hline $106.817 \quad 7$ & 106.828 & 337 & $437.24 \quad 18$ & 1692.00 & $0.048 \quad 14$ \\
\hline 151.4313 & 258.260 & $10.7 \quad 22$ & $440.854 \quad 18$ & 699.087 & $1.26 \quad 25$ \\
\hline 198.339 & 1453.05 & $0.37 \quad 10$ & $444.525 \quad 19$ & 1143.593 & $0.92 \quad 19$ \\
\hline $268.793 \quad 8$ & 1110.744 & 1.84 & $450.97 \quad 12$ & 2388.835 & $0.069 \quad 17$ \\
\hline $275.93 \quad 12$ & 3934.62 & $0.18 \quad 5$ & 454.013 & 1861.893 & $0.28 \quad 6$ \\
\hline $283.78 \quad 12$ & 2765.960 & 0.174 & $504.828 \quad 16$ & 1143.593 & 2.44 \\
\hline 289.219 & 1400.03 & $0.22 \quad 5$ & $531.908 \quad 15$ & 638.726 & 5.79 \\
\hline $301.50 \quad 14$ & 1143.593 & $0.13 \quad 3$ & $537.17 \quad 14$ & 2926.09 & $0.044 \quad 10$ \\
\hline 376.715 & 2238.589 & $0.61 \quad 14$ & $544.68 \quad 21$ & 2482.147 & $0.030 \quad 8$ \\
\hline $378.20 \quad 5$ & 2366.172 & 0.154 & $550.30 \quad 10$ & 1816.879 & $0.062 \quad 12$ \\
\hline $380.473 \quad 13$ & 638.726 & $2.5 \quad 5$ & $559.23 \quad 3$ & 1197.904 & $0.26 \quad 4$ \\
\hline 395.24 & 1538.74 & $0.14 \quad 3$ & $562.07 \quad 4$ & 1816.879 & 0.17 \\
\hline $408.87 \quad 15$ & 1861.893 & $0.067 \quad 18$ & $569.475 \quad 20$ & 1842.971 & $0.60 \quad 9$ \\
\hline $413.16 \quad 16$ & 1254.821 & $0.059 \quad 17$ & $571.85 \quad 5$ & 2388.835 & 0.12720 \\
\hline 428.715 & 1110.744 & $0.20 \quad 4$ & $575.04 \quad 5$ & 681.942 & $0.117 \quad 19$ \\
\hline $431.566 \quad 16$ & 1273.517 & 2.96 & $579.30 \quad 6$ & 2422.155 & $0.104 \quad 17$ \\
\hline
\end{tabular}


${ }^{41} \mathrm{~K}(\mathrm{n}, \gamma)$ E=thermal $1985 \mathrm{Kr} \mathbf{} \quad$ (continued)

\begin{tabular}{|c|c|c|c|c|c|}
\hline \multirow{3}{*}{$E \gamma^{\dagger}$} & \multirow[b]{3}{*}{ E(level) } & \multirow[b]{3}{*}{$\mathrm{I} \gamma^{\ddagger} \#$} & & & \\
\hline & & & \multicolumn{2}{|c|}{$\gamma\left({ }^{42} \mathrm{~K}\right)$ (continued) } & \multirow[b]{2}{*}{$\mathrm{I} \gamma \neq \#$} \\
\hline & & & $\mathrm{E} \gamma^{\dagger}$ & E(level) & \\
\hline $584.40 \quad 4$ & 1266.300 & $0.47 \quad 8$ & $1043.78 \quad 13$ & 2187.23 & $0.068 \quad 14$ \\
\hline $595.618 \quad 17$ & 1861.893 & $0.83 \quad 12$ & $1052.88 \quad 3$ & 4942.97 & $0.162 \quad 25$ \\
\hline $602.046 \quad 22$ & 1745.613 & $0.38 \quad 6$ & 1071.50920 & 1913.460 & 0.538 \\
\hline $612.64 \quad 5$ & 3014.46 & $0.20 \quad 3$ & $1076.51 \quad 12$ & 2187.23 & 0.0418 \\
\hline $612.65 \quad 5$ & 1723.44 & $0.81 \quad 14$ & $1080.17 \quad 10$ & 3284.41 & $0.041 \quad 7$ \\
\hline $616.118 \quad 14$ & 1254.821 & 2.34 & $1084.48 \quad 12$ & 1723.44 & $0.039 \quad 7$ \\
\hline $618.20 \quad 8$ & 3040.19 & $0.095 \quad 16$ & $1091.088 \quad 18$ & 1197.904 & $0.77 \quad 12$ \\
\hline $620.244 \quad 16$ & 2482.147 & $1.05 \quad 16$ & $1110.767 \quad 16$ & 1110.744 & $2.0 \quad 3$ \\
\hline $621.727 \quad 16$ & 1463.649 & $1.34 \quad 20$ & $1121.70 \quad 5$ & 2938.60 & $0.24 \quad 7$ \\
\hline $627.551 \quad 15$ & 1266.300 & $1.56 \quad 23$ & $1122.74 \quad 5$ & 2388.835 & $0.44 \quad 9$ \\
\hline $634.65 \quad 6$ & 1273.517 & $0.094 \quad 15$ & $1128.20 \quad 3$ & 2401.798 & $0.133 \quad 20$ \\
\hline $638.717 \quad 14$ & 638.726 & $3.4 \quad 5$ & $1135.40 \quad 7$ & 2401.798 & $0.063 \quad 10$ \\
\hline 663.25 & 1937.492 & 0.0378 & $1147.24 \quad 22$ & 2401.798 & $0.42 \quad 6$ \\
\hline $671.149 \quad 23$ & 1513.069 & $0.30 \quad 5$ & 1153.613 & 1937.492 & $0.19 \quad 3$ \\
\hline $676.87 \quad 11$ & 1376.10 & $0.075 \quad 14$ & 1155.998 & 4443.14 & $0.066 \quad 11$ \\
\hline $678.34 \quad 6$ & 2401.798 & $0.128 \quad 21$ & $1166.808 \quad 25$ & 1273.517 & $0.30 \quad 5$ \\
\hline $681.932 \quad 12$ & 681.942 & $15.5 \quad 23$ & $1179.921 \quad 16$ & 1861.893 & $1.64 \quad 25$ \\
\hline $695.189 \quad 17$ & 1377.121 & $0.92 \quad 14$ & $1195.02 \quad 13$ & 1453.05 & $0.033 \quad 6$ \\
\hline $706.98 \quad 8$ & 2644.289 & $0.059 \quad 10$ & $1197.99 \quad 3$ & 1197.904 & $0.17 \quad 3$ \\
\hline $721.66 \quad 16$ & 1987.979 & $0.030 \quad 7$ & $1204.080 \quad 19$ & 1987.979 & $0.68 \quad 10$ \\
\hline $725.96 \quad 3$ & 1407.912 & $0.157 \quad 24$ & $1207.363 \quad 17$ & 2049.320 & $1.44 \quad 22$ \\
\hline $735.099 \quad 14$ & 841.937 & 2.13 & $1213.51 \quad 13$ & 4152.35 & 0.0428 \\
\hline 761.318 & 1400.03 & $0.058 \quad 10$ & $1217.32 \quad 14$ & 3421.27 & $0.060 \quad 12$ \\
\hline 766.219 & 2627.847 & $0.049 \quad 9$ & 1223.295 & 1861.893 & $0.084 \quad 13$ \\
\hline $783.903 \quad 14$ & 783.885 & $2.8 \quad 4$ & $1229.89 \quad 3$ & 2606.996 & $0.157 \quad 24$ \\
\hline $806.00^{@} 3$ & 2878.009 & $0.18^{@} 3$ & $1254.80 \quad 3$ & 1254.821 & $0.79 \quad 13$ \\
\hline & 4039.96 & $0.18^{@} 3$ & 1255.523 & 1937.492 & $2.0 \quad 3$ \\
\hline 811.499 & 3418.44 & $0.050 \quad 9$ & $1266.305 \quad 15$ & 1266.300 & $3.7 \quad 6$ \\
\hline $817.05 \quad 5$ & 3021.108 & $0.093 \quad 15$ & 1301.00820 & 1407.912 & 0.518 \\
\hline $828.462 \quad 16$ & 2765.960 & $1.22 \quad 18$ & $1319.32 \quad 23$ & 2161.645 & $0.23 \quad 4$ \\
\hline $830.89 \quad 9$ & 1513.069 & 0.0529 & $1329.81 \quad 16$ & 3696.39 & $0.030 \quad 6$ \\
\hline $841.895 \quad 13$ & 841.937 & $13.2 \quad 20$ & $1340.942 \quad 23$ & 2718.098 & $0.35 \quad 5$ \\
\hline $846.59 \quad 5$ & 3008.33 & $0.095 \quad 15$ & $1352.02 \quad 7$ & 2606.996 & $0.144 \quad 22$ \\
\hline 851.0120 & 1489.30 & 0.0216 & $1361.535 \quad 23$ & 2627.847 & $0.35 \quad 5$ \\
\hline $856.20 \quad 3$ & 2718.098 & $0.147 \quad 23$ & $1368.70 \quad 23$ & 3418.44 & $0.20 \quad 3$ \\
\hline $861.34 \quad 6$ & 2606.996 & $0.067 \quad 11$ & $1377.065 \quad 16$ & 1377.121 & 3.15 \\
\hline $874.088 \quad 19$ & 2072.009 & 0.528 & 1382.23 & 1489.30 & $0.014 \quad 4$ \\
\hline $881.33 \quad 11$ & 1723.44 & $0.038 \quad 7$ & $1391.4 \quad 3$ & 3233.93 & $0.18 \quad 3$ \\
\hline 903.63521 & 1745.613 & $0.43 \quad 6$ & $1399.78 \quad 9$ & 1400.03 & $0.59 \quad 9$ \\
\hline $905.712 \quad 19$ & 2049.320 & 0.619 & 1403.485 & 2187.23 & $0.090 \quad 14$ \\
\hline 912.6923 & 2401.798 & $0.019 \quad 5$ & $1407.898 \quad 16$ & 1407.912 & $3.0 \quad 5$ \\
\hline 920.816 & 2644.289 & $0.072 \quad 12$ & $1430.74 \quad 16$ & 3418.44 & $0.028 \quad 5$ \\
\hline $923.66 \quad 19$ & 3862.04 & $0.028 \quad 6$ & $1433.274 \quad 19$ & 2072.009 & $0.71 \quad 11$ \\
\hline $925.71 \quad 24$ & 4428.28 & 0.12920 & $1451.745 \quad 19$ & 2718.098 & $0.74 \quad 11$ \\
\hline x934.486 25 & & $0.24 \quad 4$ & $1454.53 \quad 12$ & 2862.78 & $0.95 \quad 14$ \\
\hline $939.605 \quad 17$ & 1197.904 & $1.15 \quad 17$ & 1463.593 & 1463.649 & $0.25 \quad 4$ \\
\hline $949.60 \quad 20$ & 2204.046 & $0.036 \quad 9$ & 1470.096 & 2878.009 & $0.075 \quad 12$ \\
\hline $951.11 \quad 14$ & 3890.09 & $0.051 \quad 10$ & $1473.00 \quad 7$ & 2926.09 & $0.067 \quad 11$ \\
\hline $958.215 \quad 21$ & 2366.172 & $0.43 \quad 7$ & $1485.60^{@ 8} 8$ & 2862.78 & $0.063^{@} 10$ \\
\hline $964.50 \quad 9$ & 2878.009 & 0.0478 & & 2938.60 & $0.063^{@ 10}$ \\
\hline $972.20 \quad 4$ & 2238.589 & $0.108 \quad 17$ & $1500.37 \quad 13$ & 2644.289 & $0.073 \quad 15$ \\
\hline $977.524 \quad 18$ & 2251.062 & $0.78 \quad 12$ & $1501.85 \quad 21$ & 3014.46 & $0.048 \quad 12$ \\
\hline $986.45 \quad 8$ & 4660.71 & $0.059 \quad 10$ & 1509.924 & 4877.25 & $0.101 \quad 16$ \\
\hline $989.065 \quad 21$ & 2366.172 & $0.38 \quad 6$ & $1520.28 \quad 4$ & 2718.098 & $0.17 \quad 3$ \\
\hline $993.863 \quad 23$ & 2401.798 & $0.30 \quad 5$ & $1555.50 \quad 4$ & $(7533.80)$ & $0.119 \quad 18$ \\
\hline $996.511 \quad 16$ & 1254.821 & $1.43 \quad 21$ & 1558.723 & 3304.36 & $0.20 \quad 3$ \\
\hline $1001.005 \quad 16$ & 1842.971 & $1.49 \quad 22$ & $1565.47^{@} 5$ & 3502.90 & $0.080^{@ 12}$ \\
\hline 1011.623 & 2388.835 & 0.213 & & 4428.28 & $0.080^{@ 12}$ \\
\hline $1015.233 \quad 24$ & 1273.517 & 0.619 & $1568.99 \quad 12$ & 2251.062 & $0.040 \quad 7$ \\
\hline 1018.03221 & 2161.645 & $0.40 \quad 6$ & $1571.82 \quad 4$ & 3621.20 & 0.193 \\
\hline $1027.03 \quad 16$ & 3890.09 & $0.025 \quad 5$ & $1572.9 \quad 3$ & 4806.83 & $0.12 \quad 3$ \\
\hline $1034.90 \quad 7$ & 2573.63 & $0.081 \quad 13$ & $1574.5 \quad 3$ & 3418.44 & $0.12 \quad 3$ \\
\hline $1036.83 \quad 3$ & 1143.593 & $0.20 \quad 3$ & 1580.323 & $(7533.80)$ & $0.30 \quad 5$ \\
\hline
\end{tabular}


${ }^{41} \mathrm{~K}(\mathrm{n}, \gamma)$ E=thermal $1985 \mathrm{Kr} \mathbf{} \quad$ (continued)

\begin{tabular}{|c|c|c|c|c|c|}
\hline $\mathrm{E} \gamma^{\dagger}$ & E(level) & $\mathrm{I} \gamma^{\ddagger} \#$ & $\mathrm{E} \gamma^{\dagger}$ & $\mathrm{E}$ (level) & $I \gamma^{\ddagger} \#$ \\
\hline 1585.104 & 1692.00 & $0.127 \quad 19$ & ${ }^{\mathrm{x}} 1948.897$ & & $0.087 \quad 14$ \\
\hline $1596.62 \quad 23$ & 2862.78 & $0.20 \quad 3$ & $1962.29 \quad 3$ & 2644.289 & $0.50 \quad 8$ \\
\hline 1602.084 & 3674.14 & $0.24 \quad 4$ & $1965.10 \quad 3$ & 2072.009 & $0.48 \quad 7$ \\
\hline $1603.60 \quad 5$ & 1861.893 & $0.18 \quad 4$ & $1968.08 \quad 9$ & 2606.996 & $0.060 \quad 10$ \\
\hline 1604.365 & 3421.27 & $0.18 \quad 4$ & $1976.64 \quad 5$ & 3890.09 & $0.106 \quad 16$ \\
\hline $1606.95 \quad 6$ & 5710.61 & $0.083 \quad 13$ & $1985.20 \quad 12$ & 4612.82 & $0.042 \quad 7$ \\
\hline $1616.84 \quad 11$ & 1723.44 & $0.060 \quad 10$ & $1988.00 \quad 8$ & 1987.979 & $0.112 \quad 17$ \\
\hline $1622.53 \quad 7$ & 2765.960 & $0.084 \quad 13$ & $1990.20 \quad 9$ & 3367.32 & $0.088 \quad 14$ \\
\hline 1640.184 & 2482.147 & $0.101 \quad 15$ & $1998.11 \quad 11$ & 3195.80 & $0.067 \quad 12$ \\
\hline $1644.55 \quad 6$ & $3831.74 ?$ & $0.066 \quad 10$ & 2006.03 & 2644.289 & $0.033 \quad 10$ \\
\hline 1655.163 & 1913.460 & 0.213 & $2012.09 \quad 9$ & 4778.05 & $0.142 \quad 16$ \\
\hline $1658.94 \quad 9$ & 4576.26 & 0.0498 & $2014.36 \quad 19$ & 4416.60 & $0.136 \quad 15$ \\
\hline 1661.84 & 2917.10 & $0.083 \quad 13$ & 2020.836 & 2862.78 & 0.424 \\
\hline $1665.14^{@} 14$ & 2862.78 & $0.029^{@ 5} 5$ & $2023.76 \quad 11$ & 4389.78 & $0.075 \quad 9$ \\
\hline & 4053.87 & $0.029^{@} 5$ & $2027.35 \quad 19$ & 4416.60 & $0.035 \quad 6$ \\
\hline $1671.20 \quad 11$ & 2926.09 & $0.045 \quad 8$ & 2030.278 & 4748.54 & $0.163 \quad 17$ \\
\hline $1673.71^{@} 11$ & 4039.96 & $0.052^{@ 9} 9$ & $\mathrm{x}_{2} 034.26 \quad 7$ & & $0.25 \quad 3$ \\
\hline & 5710.61 & $0.052^{@} 9$ & $2035.91 \quad 10$ & 3233.93 & $0.129 \quad 17$ \\
\hline 1684.175 & 3888.33 & 0.113 & $2050.02 \quad 10$ & 3502.90 & $0.093 \quad 11$ \\
\hline 1684.195 & 2366.172 & 0.113 & $2056.85 \quad 12$ & $(7533.80)$ & 0.0639 \\
\hline $1687.08 \quad 10$ & $(7533.80)$ & 0.0427 & $2060.99 \quad 18$ & 4938.98 & $0.034 \quad 7$ \\
\hline $1707.01 \quad 7$ & 2388.835 & 0.13120 & $2065.16 \quad 11$ & 3529.03 & $0.086 \quad 12$ \\
\hline $1709.983 \quad 25$ & 1816.879 & $0.98 \quad 15$ & $2071.95 \quad 4$ & 2072.009 & $2.16 \quad 22$ \\
\hline $\mathrm{x}_{1735.05 \quad 3}$ & & $0.27 \quad 4$ & $2076.86 \quad 5$ & 5002.99 & $0.201 \quad 24$ \\
\hline $1740.20 \quad 3$ & 2422.155 & $0.40 \quad 6$ & $2076.90 \quad 5$ & 4443.14 & $0.100 \quad 16$ \\
\hline 1744.034 & $(7533.80)$ & $0.146 \quad 22$ & $2083.94 \quad 4$ & 2765.960 & 1.3514 \\
\hline 1749.635 & 5789.69 & $0.147 \quad 23$ & 2086.535 & 3284.41 & 0.414 \\
\hline $1754.98 \quad 3$ & 1861.893 & $0.48 \quad 7$ & 2089.84 & 3233.93 & $0.104 \quad 12$ \\
\hline $1759.28 \quad 6$ & 3621.20 & $0.066 \quad 10$ & $2094.05^{@ 11}$ & 2878.009 & $0.069^{@} 9$ \\
\hline $1763.39 \quad 7$ & 4152.35 & $0.068 \quad 11$ & & 4576.26 & $0.069^{@} 9$ \\
\hline $1766.26 \quad 3$ & 3021.108 & $0.49 \quad 7$ & $2096.60 \quad 12$ & 2938.60 & 0.0658 \\
\hline $1774.09 \quad 5$ & $(7533.80)$ & $0.155 \quad 24$ & $2101.26 \quad 21$ & 3367.32 & $0.027 \quad 6$ \\
\hline $1783.51 \quad 12$ & 2422.155 & $0.39 \quad 6$ & $2107.92 \quad 15$ & 2366.172 & $0.037 \quad 7$ \\
\hline $1785.95 \quad 6$ & 2627.847 & 0.213 & 2113.0923 & 3367.32 & 0.033 \\
\hline $1787.5 \quad 3$ & 3195.80 & $0.17 \quad 3$ & $2115.55 \quad 18$ & 4103.72 & $0.100 \quad 22$ \\
\hline${ }^{x} 1797.26 \quad 15$ & & $0.069 \quad 12$ & 2116.84 & 3862.04 & $0.122 \quad 24$ \\
\hline 1800.154 & 2482.147 & $0.23 \quad 4$ & $2119.72 \quad 14$ & 3658.59 & $0.055 \quad 8$ \\
\hline $1802.57 \quad 11$ & 3210.61 & $0.088 \quad 14$ & ${ }^{x} 2122.20 \quad 10$ & & $0.089 \quad 11$ \\
\hline $1810.51 \quad 10$ & 3008.33 & $0.069 \quad 11$ & $2125.60 \quad 13$ & 3502.90 & 0.049 \\
\hline $1812.50^{@ 18}$ & 3674.14 & $0.037^{@} 7$ & $\mathrm{x}_{2} 128.43 \quad 10$ & & $0.081 \quad 10$ \\
\hline & 5097.04 & $0.037^{@} 7$ & $2131.9 \quad 3$ & 2238.589 & $0.021 \quad 7$ \\
\hline $1818.32 \quad 23$ & 3195.80 & $0.138 \quad 21$ & $2138.42 \quad 10$ & 3862.04 & $0.112 \quad 13$ \\
\hline $1823.18 \quad 3$ & $(7533.80)$ & $0.34 \quad 5$ & $2143.43 \quad 5$ & 2401.798 & $0.66 \quad 7$ \\
\hline $1830.42 \quad 23$ & 1937.492 & $0.161 \quad 24$ & $\mathrm{x}_{2} 148.80 \quad 9$ & & $0.165 \quad 18$ \\
\hline $1833.88 \quad 5$ & 3233.93 & $0.115 \quad 18$ & ${ }^{x} 2156.34 \quad 8$ & & $0.194 \quad 20$ \\
\hline $1836.55 \quad 4$ & $(7533.80)$ & $0.138 \quad 21$ & $2163.29 \quad 11$ & 3418.44 & $0.100 \quad 12$ \\
\hline $1842.95 \quad 3$ & 1842.971 & $1.12 \quad 17$ & $2166.38 \quad 6$ & 3008.33 & 0.424 \\
\hline $1861.855 \quad 24$ & 1861.893 & $3.4 \quad 5$ & 2172.7125 & 3014.46 & $0.028 \quad 6$ \\
\hline $1864.68 \quad 5$ & 3008.33 & $0.123 \quad 19$ & $2179.04^{@ 8} 8$ & 2878.009 & $0.179^{@} 20$ \\
\hline $\mathrm{x}_{1872.97 \quad 18}$ & & 0.0215 & & 4806.83 & $0.179^{@ 20}$ \\
\hline $1877.13 \quad 6$ & 4128.34 & $0.22 \quad 3$ & $2184.45 \quad 14$ & 3295.35 & $0.067 \quad 9$ \\
\hline $1881.10 \quad 3$ & 3794.62 & $0.37 \quad 6$ & $2188.20 \quad 18$ & 4590.56 & $0.104 \quad 19$ \\
\hline $1891.92 \quad 5$ & 5179.13 & $0.089 \quad 14$ & $2189.90 \quad 19$ & 4103.72 & $0.106 \quad 18$ \\
\hline $1900.49^{@ 9} 9$ & 3888.33 & $0.049^{@ 8}$ & $2192.79 \quad 23$ & 3304.36 & $0.044 \quad 8$ \\
\hline & 5318.95 & $0.049^{@ 8}$ & $2195.95 \quad 10$ & 2878.009 & $0.105 \quad 12$ \\
\hline 1903.419 & $(7533.80)$ & 0.0518 & $2199.61 \quad 13$ & 4806.83 & $0.060 \quad 9$ \\
\hline $1909.90 \quad 5$ & 3287.16 & $0.083 \quad 13$ & 2215.1120 & $(7533.80)$ & $0.18 \quad 3$ \\
\hline 1913.83 & 1913.460 & 0.0113 & $2220.57 \quad 5$ & 3418.44 & $0.58 \quad 6$ \\
\hline $1921.61 \quad 15$ & 4942.97 & $0.026 \quad 5$ & $2238.56 \quad 4$ & 2238.589 & $2.17 \quad 22$ \\
\hline 1934.163 & 2718.098 & $0.28 \quad 4$ & ${ }^{x} 2240.565$ & & $0.38 \quad 4$ \\
\hline $1937.48 \quad 3$ & 1937.492 & $1.50 \quad 23$ & $2243.25 \quad 9$ & 3696.39 & $0.098 \quad 11$ \\
\hline 1941.227 & 4128.34 & $0.064 \quad 10$ & $2259.28 \quad 4$ & 2366.172 & $2.14 \quad 21$ \\
\hline 1945.693 & 2204.046 & $0.42 \quad 6$ & $2262.79 \quad 16$ & 3529.03 & $0.040 \quad 6$ \\
\hline
\end{tabular}


${ }^{41} \mathrm{~K}(\mathrm{n}, \gamma)$ E=thermal $1985 \mathrm{Kr} \mathbf{} \quad$ (continued)

\begin{tabular}{|c|c|c|c|c|c|}
\hline \multirow{3}{*}{$\mathrm{E} \gamma^{\dagger}$} & \multirow[b]{3}{*}{ E(level) } & \multirow[b]{3}{*}{$\mathrm{I} \gamma^{\ddagger} \#$} & & & \\
\hline & & & \multicolumn{2}{|c|}{$\gamma\left({ }^{42} \mathrm{~K}\right)$ (continued) } & \multirow[b]{2}{*}{$\mathrm{I} \gamma^{\ddagger} \#$} \\
\hline & & & $\mathrm{E} \gamma^{\dagger}$ & $\mathrm{E}($ level) & \\
\hline $2266.55 \quad 7$ & 4128.34 & $0.151 \quad 16$ & $2556.94 \quad 7$ & 3195.80 & $0.137 \quad 15$ \\
\hline $2281.90 \quad 10$ & 2388.835 & $0.075 \quad 9$ & $2561.53 \quad 12$ & 4748.54 & $0.038 \quad 5$ \\
\hline 2287.125 & $(7533.80)$ & $0.46 \quad 5$ & $2566.86 \quad 9$ & 4259.10 & $0.089 \quad 10$ \\
\hline $2294.98 \quad 3$ & 2401.798 & 3.13 & $2573.54 \quad 16$ & 2573.63 & $0.15 \quad 4$ \\
\hline${ }^{x} 2298.878$ & & $0.125 \quad 13$ & 2574.5720 & $(7533.80)$ & 0.124 \\
\hline $2307.39 \quad 10$ & 3418.44 & $0.072 \quad 8$ & $2580.79 \quad 9$ & 5002.99 & $0.074 \quad 9$ \\
\hline 2315.194 & 2422.155 & $1.45 \quad 14$ & 2585.6120 & 3696.39 & $0.019 \quad 5$ \\
\hline $2319.70 \quad 8$ & 3696.39 & $0.144 \quad 15$ & 2590.816 & $(7533.80)$ & 0.19720 \\
\hline $2325.11 \quad 12$ & 4576.26 & $0.053 \quad 7$ & $2594.9 \quad 4$ & $(7533.80)$ & $0.129 \quad 15$ \\
\hline \multirow[t]{2}{*}{$2332.50^{@} 11$} & 3014.46 & $0.074^{@} 9$ & $2599.42 \quad 8$ & 4416.60 & $0.102 \quad 11$ \\
\hline & 5953.50 & $0.074^{@} 9$ & $2602.76 \quad 10$ & 4806.83 & $0.059 \quad 7$ \\
\hline $2334.63 \quad 17$ & 5630.29 & $0.047 \quad 7$ & 2606.43 & 4013.89 & $0.169 \quad 17$ \\
\hline $2338.49 \quad 8$ & 5759.69 & $0.123 \quad 13$ & $\mathrm{x} 2617.489$ & & $0.083 \quad 9$ \\
\hline \multirow[t]{2}{*}{$2341.09^{@ 19}$} & 3040.19 & $0.033^{@ 5} 5$ & $2622.61 \quad 16$ & 3304.36 & $0.042 \quad 7$ \\
\hline & 5759.69 & $0.033^{@ 5} 5$ & $2624.65 \quad 19$ & 3323.73 & $0.042 \quad 6$ \\
\hline 2354.558 & $(7533.80)$ & $0.132 \quad 14$ & $2627.74 \quad 10$ & 2627.847 & $0.091 \quad 11$ \\
\hline $2359.17 \quad 6$ & 3502.90 & $0.33 \quad 3$ & $2629.79 \quad 10$ & $(7533.80)$ & $0.125 \quad 14$ \\
\hline 2366.134 & 2366.172 & 3.94 & $2632.03 \quad 8$ & 4039.96 & $0.101 \quad 11$ \\
\hline $2369.9 \quad 3$ & 2627.847 & $0.171 \quad 18$ & $2648.38 \quad 6$ & 3287.16 & $0.181 \quad 19$ \\
\hline $2372.68 \quad 11$ & 3862.04 & $0.075 \quad 9$ & $2653.56 \quad 10$ & 2653.79 & $0.055 \quad 7$ \\
\hline $2375.35 \quad 8$ & 2482.147 & $0.138 \quad 15$ & $2656.43 \quad 7$ & $(7533.80)$ & $0.128 \quad 13$ \\
\hline $2381.8 \quad 3$ & 4128.34 & $0.179 \quad 18$ & $2660.49 \quad 17$ & 3502.90 & $0.026 \quad 4$ \\
\hline 2384.998 & 3658.59 & $0.135 \quad 14$ & $2664.15 \quad 12$ & 4481.07 & $0.070 \quad 9$ \\
\hline 2388.815 & 2388.835 & $0.44 \quad 5$ & $2666.13 \quad 11$ & 4179.43 & $0.074 \quad 10$ \\
\hline $2392.14 \quad 6$ & 3658.59 & $0.33 \quad 3$ & ${ }^{x} 2672.02 \quad 14$ & & $0.038 \quad 6$ \\
\hline 2395.536 & 2653.79 & $0.212 \quad 22$ & $\mathrm{x}_{2} 678.09 \quad 10$ & & $0.102 \quad 12$ \\
\hline $2397.83 \quad 9$ & 3798.14 & $0.096 \quad 11$ & $2680.15 \quad 4$ & $(7533.80)$ & 0.626 \\
\hline 2401.725 & 2401.798 & $0.34 \quad 3$ & $2685.17 \quad 8$ & 3367.32 & $0.087 \quad 10$ \\
\hline $2403.84 \quad 7$ & 3658.59 & $0.177 \quad 19$ & $2687.54 \quad 11$ & 3798.14 & $0.065 \quad 8$ \\
\hline $2408.82 \quad 11$ & 4612.82 & $0.064 \quad 9$ & $2691.07 \quad 7$ & 4154.70 & $0.137 \quad 14$ \\
\hline 2411.93 & 3195.80 & $0.067 \quad 19$ & 2695.138 & 5097.04 & $0.093 \quad 10$ \\
\hline $2413.34 \quad 17$ & 3876.94 & $0.104 \quad 21$ & $2700.18 \quad 10$ & 4938.98 & $0.059 \quad 7$ \\
\hline $2422.20 \quad 11$ & 2422.155 & $0.072 \quad 9$ & $2706.03 \quad 12$ & 4778.05 & $0.035 \quad 5$ \\
\hline $2436.65 \quad 5$ & $(7533.80)$ & $0.33 \quad 3$ & $2710.0 \quad 4$ & 5476.92 & $0.009 \quad 3$ \\
\hline $2441.02 \quad 13$ & 5318.95 & 0.0416 & $\mathrm{x}_{2} 717.29 \quad 7$ & & $0.137 \quad 14$ \\
\hline \multirow[t]{2}{*}{$2450.82^{@} 14$} & 4612.82 & $0.091^{@} 17$ & $2720.05 \quad 25$ & 4128.34 & $0.30 \quad 3$ \\
\hline & 5953.50 & $0.091^{@} \quad 17$ & $2726.90 \quad 4$ & $(7533.80)$ & $1.11 \quad 11$ \\
\hline $2452.36 \quad 8$ & $(7533.80)$ & $0.23 \quad 3$ & $2730.74 \quad 11$ & 5097.04 & $0.044 \quad 5$ \\
\hline \multirow[t]{2}{*}{$2455.04^{@} 12$} & 4443.14 & $0.056^{@ 7} 7$ & $2740.08 \quad 6$ & 4013.89 & $0.27 \quad 3$ \\
\hline & 5759.69 & $0.056^{@} 7$ & $2745.19 \quad 10$ & 3529.03 & $0.065 \quad 8$ \\
\hline 2460.93 & 3658.59 & $0.224 \quad 23$ & $2747.66 \quad 7$ & 4013.89 & $0.121 \quad 13$ \\
\hline 2466.696 & 2573.63 & $0.28 \quad 3$ & 2751.54 & 4128.34 & $0.184 \quad 19$ \\
\hline $2469.81 \quad 6$ & $(7533.80)$ & $0.30 \quad 3$ & $2755.7 \quad 5$ & $(7533.80)$ & $0.154 \quad 16$ \\
\hline $2476.79 \quad 6$ & 3876.94 & $0.211 \quad 21$ & $2762.47 \quad 10$ & 3021.108 & $0.061 \quad 7$ \\
\hline $2480.28 \quad 14$ & 3888.33 & 0.0629 & $2765.80 \quad 4$ & 2765.960 & $1.61 \quad 16$ \\
\hline 2482.2120 & 2482.147 & $0.044 \quad 7$ & $2770.75 \quad 13$ & 2878.009 & $0.036 \quad 5$ \\
\hline $2485.3 \quad 3$ & 3862.04 & $0.028 \quad 6$ & $2774.0 \quad 3$ & 4590.56 & $0.066 \quad 7$ \\
\hline $2487.42 \quad 7$ & 4853.60 & $0.153 \quad 16$ & $2779.63 \quad 6$ & 3418.44 & $0.187 \quad 20$ \\
\hline $2490.65 \quad 7$ & 4428.28 & $0.171 \quad 18$ & $2781.84 \quad 5$ & 4036.86 & 0.354 \\
\hline $2493.18 \quad 14$ & 4481.07 & $0.043 \quad 6$ & $2784.8 \quad 3$ & $(7533.80)$ & $0.114 \quad 12$ \\
\hline $2500.45 \quad 5$ & 3284.41 & $0.51 \quad 5$ & $2787.39 \quad 7$ & 4053.87 & $0.165 \quad 17$ \\
\hline \multirow[t]{2}{*}{$2504.34^{@} 13$} & $3770.67 ?$ & $0.038^{@} 5$ & $\mathrm{x}_{2} 789.74 \quad 6$ & & $0.27 \quad 3$ \\
\hline & 4576.26 & $0.038^{@} 5$ & $2810.15 \quad 6$ & 2917.10 & $0.183 \quad 19$ \\
\hline $\mathrm{x}_{2508.15 \quad 8}$ & & $0.097 \quad 10$ & ${ }^{x} 2814.13 \quad 20$ & & $0.023 \quad 4$ \\
\hline $2520.73 \quad 9$ & 2627.847 & $0.110 \quad 12$ & $2817.7 \quad 3$ & $(7533.80)$ & $0.098 \quad 10$ \\
\hline $2523.30 \quad 8$ & 5097.04 & $0.132 \quad 14$ & $2820.84 \quad 20$ & 3502.90 & 0.0314 \\
\hline${ }^{x} 2526.25 \quad 7$ & & $0.180 \quad 19$ & $2823.40 \quad 19$ & 3934.62 & $0.029 \quad 4$ \\
\hline $2530.77 \quad 6$ & $(7533.80)$ & 0.334 & ${ }^{\mathrm{x}} 2827.25 \quad 9$ & & $0.070 \quad 8$ \\
\hline${ }^{\mathrm{x}} 2533.179$ & & $0.090 \quad 10$ & $2832.13 \quad 6$ & 3674.14 & 0.22122 \\
\hline $2537.36 \quad 7$ & 2644.289 & $0.146 \quad 16$ & ${ }^{\mathrm{x}} 2846.09 \quad 5$ & & $0.33 \quad 3$ \\
\hline $2542.11 \quad 13$ & 5846.58 & $0.045 \quad 6$ & $2854.71 \quad 10$ & 4128.34 & $0.054 \quad 6$ \\
\hline $2550.12 \quad 11$ & 4013.89 & $0.052 \quad 7$ & $x_{2} 860.49 \quad 10$ & & 0.16122 \\
\hline $2554.93 \quad 12$ & 4416.60 & $0.064 \quad 9$ & $2862.02 \quad 8$ & 4128.34 & $0.23 \quad 3$ \\
\hline
\end{tabular}

Continued on next page (footnotes at end of table) 
${ }^{41} \mathrm{~K}(\mathrm{n}, \gamma)$ E=thermal $1985 \mathrm{Kr} \mathbf{} \quad$ (continued)

\begin{tabular}{|c|c|c|c|c|c|}
\hline \multirow{3}{*}{$\mathrm{E} \gamma^{\dagger}$} & \multirow[b]{3}{*}{ E(level) } & \multirow[b]{3}{*}{$\mathrm{I} \gamma^{\ddagger} \#$} & & & \\
\hline & & & \multicolumn{2}{|c|}{$\gamma\left({ }^{42} \mathrm{~K}\right)$ (continued) } & \multirow[b]{2}{*}{$\mathrm{I} \gamma^{\ddagger} \#$} \\
\hline & & & $\mathrm{E} \gamma^{\dagger}$ & E(level) & \\
\hline 2873.64 & $(7533.80)$ & $0.233 \quad 24$ & $3220.89 \quad 9$ & 5064.01 & $0.138 \quad 7$ \\
\hline $2878.10 \quad 16$ & 2878.009 & $0.024 \quad 4$ & $3224.0 \quad 3$ & 3862.04 & $0.016 \quad 3$ \\
\hline $2881.44 \quad 24$ & 5759.69 & $0.017 \quad 4$ & $\mathrm{x} 3226.97 \quad 10$ & & $0.079 \quad 5$ \\
\hline $2887.15 \quad 22$ & 4959.64 & $0.016 \quad 3$ & ${ }^{\mathrm{x}} 3234.13 \quad 12$ & & $0.040 \quad 3$ \\
\hline $2896.2 \quad 3$ & 5759.69 & $0.025 \quad 6$ & ${ }^{x} 3238.49 \quad 11$ & & $0.075 \quad 6$ \\
\hline $2898.28 \quad 11$ & 4715.40 & $0.064 \quad 8$ & x $3240.73 \quad 8$ & & $0.180 \quad 10$ \\
\hline $2907.35 \quad 14$ & 3014.46 & $0.41 \quad 4$ & $3252.93 \quad 13$ & 4660.71 & $0.044 \quad 4$ \\
\hline $\mathrm{x}_{2913.569}$ & & $0.061 \quad 7$ & $3258.0 \quad 3$ & 5246.63 & $0.020 \quad 4$ \\
\hline 2919.96 & $(7533.80)$ & $0.25 \quad 7$ & $3265.39 \quad 16$ & 5179.13 & $0.030 \quad 3$ \\
\hline 2926.015 & 2926.09 & $0.34 \quad 3$ & $3269.86 \quad 16$ & 4053.87 & 0.0222 \\
\hline $2938.38 \quad 6$ & 2938.60 & $0.190 \quad 19$ & $3274.47 \quad 9$ & $(7533.80)$ & $0.120 \quad 7$ \\
\hline $2943.15 \quad 5$ & $(7533.80)$ & $0.43 \quad 4$ & $3278.85 \quad 23$ & 4389.78 & $0.032 \quad 5$ \\
\hline $2945.07 \quad 14$ & 4806.83 & $0.067 \quad 10$ & $3280.8 \quad 7$ & 5002.99 & $0.019 \quad 5$ \\
\hline $2952.86 \quad 6$ & 4416.60 & $0.170 \quad 17$ & $3283.44 \quad 18$ & 4556.68 & $0.090 \quad 8$ \\
\hline 2957.395 & $(7533.80)$ & $0.36 \quad 4$ & 3285.64 & 4128.34 & $0.043 \quad 6$ \\
\hline 2961.119 & 4778.05 & 0.0698 & ${ }^{\mathrm{x}} 3287.91 \quad 16$ & & $0.076 \quad 7$ \\
\hline 2964.636 & 4428.28 & $0.167 \quad 17$ & $3290.62 \quad 23$ & 4556.68 & $0.024 \quad 3$ \\
\hline $2977.04 \quad 5$ & $(7533.80)$ & $0.54 \quad 5$ & $3295.16 \quad 11$ & 3295.35 & 0.0514 \\
\hline $2981.69^{@} 19$ & 4179.43 & $0.027^{@} 4$ & $3310.40 \quad 8$ & 4152.35 & $0.348 \quad 18$ \\
\hline & 4389.78 & $0.027^{@} 4$ & $3312.74 \quad 10$ & 4154.70 & $0.082 \quad 6$ \\
\hline $2984.32 \quad 12$ & 4128.34 & $0.061 \quad 7$ & $3317.11 \quad 19$ & 4428.28 & 0.0192 \\
\hline $2987.07 \quad 17$ & $3770.67 ?$ & $0.030 \quad 4$ & $3321.65 \quad 9$ & $4105.74 ?$ & $0.189 \quad 12$ \\
\hline $2991.87 \quad 10$ & 3674.14 & 0.0719 & $3323.69 \quad 9$ & 3323.73 & $0.210 \quad 12$ \\
\hline $2997.69 \quad 24$ & 3696.39 & $0.020 \quad 4$ & $3338.36 \quad 23$ & 4036.86 & $0.047 \quad 4$ \\
\hline $3008.28 \quad 9$ & 3008.33 & 0.1358 & $3342.50 \quad 20$ & 4806.83 & 0.0192 \\
\hline $3010.54 \quad 8$ & 3794.62 & $0.176 \quad 10$ & $3346.01^{@ 23}$ & 4612.82 & $0.023^{@} 3$ \\
\hline 3014.63 & 3696.39 & $0.222 \quad 12$ & & 5953.50 & $0.023^{@} 3$ \\
\hline $3021.07 \quad 8$ & 3021.108 & $0.308 \quad 16$ & $3350.64 \quad 14$ & 5978.32 & $0.115 \quad 14$ \\
\hline $3025.54 \quad 9$ & 4938.98 & $0.119 \quad 7$ & $3354.22 \quad 9$ & $(7533.80)$ & $0.166 \quad 9$ \\
\hline $3028.89 \quad 7$ & 3287.16 & $0.407 \quad 21$ & ${ }^{\mathrm{x}} 3366.79 \quad 11$ & & $0.075 \quad 6$ \\
\hline $3034.90 \quad 7$ & 3876.94 & $0.484 \quad 24$ & $3367.20 \quad 8$ & 3367.32 & $0.098 \quad 13$ \\
\hline $3039.92 \quad 8$ & 3040.19 & $0.305 \quad 23$ & $3370.05 \quad 8$ & 4481.07 & 0.213 \\
\hline $3052.69 \quad 7$ & $(7533.80)$ & $0.80 \quad 4$ & $3379.07 \quad 8$ & $(7533.80)$ & $0.215 \quad 12$ \\
\hline $3065.38 \quad 19$ & 3323.73 & $0.024 \quad 3$ & 3381.318 & $(7533.80)$ & $0.65 \quad 3$ \\
\hline $3077.33 \quad 10$ & 4590.56 & $0.074 \quad 5$ & $3394.24 \quad 12$ & 4660.71 & 0.0413 \\
\hline $3080.1^{@} 3$ & 5318.95 & $0.019^{@ 3}$ & $3399.5 \quad 3$ & 3658.59 & $0.102 \quad 6$ \\
\hline & 5846.58 & $0.019^{@ 3}$ & $3405.34 \quad 7$ & $(7533.80)$ & $2.07 \quad 10$ \\
\hline $3088.47 \quad 11$ & $3770.67 ?$ & $0.095 \quad 8$ & x $3410.66 \quad 13$ & & $0.036 \quad 3$ \\
\hline $3090.55 \quad 9$ & $(7533.80)$ & $0.213 \quad 12$ & ${ }^{\mathrm{x}} 3414.36 \quad 9$ & & $0.126 \quad 7$ \\
\hline $3092.86 \quad 9$ & 4556.68 & $0.136 \quad 9$ & $3417.07 \quad 16$ & 4259.10 & $0.040 \quad 4$ \\
\hline $3105.49 \quad 7$ & $(7533.80)$ & $0.86 \quad 4$ & 3421.098 & 3421.27 & $0.53 \quad 3$ \\
\hline${ }^{x} 3110.298$ & & $0.291 \quad 16$ & $3427.93 \quad 8$ & $(7533.80)$ & $0.232 \quad 14$ \\
\hline $3112.50^{@} 9$ & 3794.62 & $0.145^{@} 9$ & $3429.87 \quad 8$ & $(7533.80)$ & $0.250 \quad 15$ \\
\hline & 4576.26 & $0.145^{@} 9$ & ${ }^{\mathrm{x}} 3439.12 \quad 10$ & & 0.0714 \\
\hline $3117.04 \quad 7$ & $(7533.80)$ & $1.26 \quad 6$ & $3446.56 \quad 10$ & 4959.64 & $0.066 \quad 4$ \\
\hline x $3120.98 \quad 16$ & & $0.037 \quad 3$ & $3450.39 \quad 13$ & 4903.68 & $0.142 \quad 18$ \\
\hline x $3123.85 \quad 10$ & & $0.095 \quad 6$ & $\mathrm{x} 3455.18 \quad 8$ & & $0.203 \quad 11$ \\
\hline $3126.83^{@} 10$ & 3233.93 & $0.099^{@} 6$ & $3459.36 \quad 11$ & 5710.61 & $0.060 \quad 4$ \\
\hline & 4590.56 & $0.099^{@ 6}$ & $3465.26 \quad 10$ & 4576.26 & 0.0815 \\
\hline $3144.36 \quad 11$ & $(7533.80)$ & $0.282 \quad 14$ & $3468.99^{@} 9$ & 4612.82 & $0.182^{@} 10$ \\
\hline $3150.87 \quad 11$ & 3934.62 & $0.057 \quad 4$ & & 4877.25 & $0.182^{@} 10$ \\
\hline $3160.04 \quad 8$ & 3418.44 & $0.346 \quad 19$ & ${ }^{\mathrm{x}} 3474.278$ & & $0.219 \quad 11$ \\
\hline $3161.66 \quad 8$ & 4416.60 & $0.22 \quad 3$ & $3479.73^{@} 8$ & 4590.56 & $0.311^{@} 16$ \\
\hline $3161.82 \quad 8$ & 4428.28 & $0.22 \quad 3$ & & $(7533.80)$ & $0.311^{@} 16$ \\
\hline $3177.25 \quad 16$ & 3284.41 & $0.195 \quad 11$ & ${ }^{x} 3483.49 \quad 15$ & & $0.027 \quad 3$ \\
\hline $3180.00 \quad 19$ & 3862.04 & 0.194 & $3489.45 \quad 8$ & 4128.34 & $0.496 \quad 25$ \\
\hline${ }^{\mathrm{x}} 3181.20 \quad 16$ & & $0.18 \quad 4$ & 3493.779 & $(7533.80)$ & $0.160 \quad 9$ \\
\hline $3185.09 \quad 9$ & 4877.25 & $0.096 \quad 5$ & 3496.678 & $(7533.80)$ & $0.472 \quad 24$ \\
\hline 3188.93 & 3295.35 & 0.0132 & 3501.73 & 5246.63 & 0.0122 \\
\hline 3195.598 & 3195.80 & $0.255 \quad 13$ & $3514.40 \quad 12$ & 3621.20 & $0.044 \quad 3$ \\
\hline $3206.66 \quad 22$ & 3888.33 & 0.0192 & $3519.73 \quad 8$ & $(7533.80)$ & $1.45 \quad 7$ \\
\hline $3210.46 \quad 8$ & 3210.61 & $0.219 \quad 11$ & x $3524.37 \quad 10$ & & $0.109 \quad 7$ \\
\hline $3216.47 \quad 19$ & 3323.73 & $0.044 \quad 6$ & $3528.78 \quad 17$ & 3529.03 & $0.092 \quad 18$ \\
\hline
\end{tabular}

Continued on next page (footnotes at end of table) 
${ }^{41} \mathrm{~K}(\mathrm{n}, \gamma)$ E=thermal $1985 \mathrm{Kr} \mathbf{} \quad$ (continued)

\begin{tabular}{|c|c|c|c|c|c|}
\hline $\mathrm{E} \gamma^{\dagger}$ & E(level) & $\mathrm{I} \gamma^{\ddagger} \#$ & $\mathrm{E} \gamma^{\dagger}$ & E(level) & $I \gamma^{\ddagger} \#$ \\
\hline $3539.88 \quad 13$ & 3798.14 & $0.034 \quad 3$ & 4037.23 & 4036.86 & $0.024 \quad 3$ \\
\hline${ }^{\mathrm{x}} 3548.45 \quad 20$ & & $0.033 \quad 3$ & $4039.64 \quad 15$ & 4039.96 & $0.043 \quad 4$ \\
\hline $3551.83 \quad 10$ & 4806.83 & $0.070 \quad 4$ & 4048.93 & 4748.54 & $0.063 \quad 4$ \\
\hline${ }^{\mathrm{x}} 3565.36 \quad 9$ & & 0.1528 & $4053.64 \quad 12$ & 4053.87 & $0.047 \quad 3$ \\
\hline $3574.58 \quad 20$ & 4416.60 & $0.028 \quad 4$ & ${ }^{\mathrm{x}} 4057.92 \quad 12$ & & $0.055 \quad 4$ \\
\hline $3576.98 \quad 11$ & 4259.10 & 0.0615 & $\mathrm{x}_{4064.34 \quad 11}$ & & 0.065 \\
\hline $3582.50 \quad 15$ & 4959.64 & $0.026 \quad 2$ & $4095.81 \quad 18$ & 4778.05 & $0.019 \quad 2$ \\
\hline $3589.45 \quad 9$ & 3696.39 & $0.218 \quad 11$ & $4101.15 \quad 17$ & 4942.97 & 0.0212 \\
\hline x $3594.07 \quad 15$ & & 0.0262 & $4105.75 \quad 12$ & $4105.74 ?$ & $0.062 \quad 4$ \\
\hline $3599.03 \quad 8$ & $(7533.80)$ & $0.339 \quad 17$ & $4112.20 \quad 9$ & $(7533.80)$ & $0.59 \quad 3$ \\
\hline${ }^{\mathrm{x}} 3610.11 \quad 10$ & & 0.1016 & $4115.15 \quad 9$ & $(7533.80)$ & 1.729 \\
\hline $3624.83 \quad 14$ & 5697.22 & $0.025 \quad 2$ & $4120.72 \quad 11$ & 5318.95 & 0.0915 \\
\hline $3638.65 \quad 16$ & 4481.07 & $0.024 \quad 2$ & 4124.23 & 4806.83 & 0.0142 \\
\hline $3643.46 \quad 8$ & $(7533.80)$ & $0.336 \quad 19$ & $4127.93 \quad 14$ & 4128.34 & $0.045 \quad 3$ \\
\hline $3645.26 \quad 10$ & $(7533.80)$ & $0.151 \quad 12$ & $4131.35 \quad 23$ & 4389.78 & 0.021 \\
\hline 3656.638 & $(7533.80)$ & $0.73 \quad 4$ & $4140.4 \quad 3$ & 5630.29 & 0.0112 \\
\hline $3659.96 \quad 16$ & 5476.92 & $0.033 \quad 3$ & $4154.22 \quad 12$ & 4154.70 & 0.050 \\
\hline $3671.70 \quad 8$ & $(7533.80)$ & $0.87 \quad 4$ & $4158.8 \quad 3$ & 4416.60 & $0.009 \quad 2$ \\
\hline${ }^{x} 3688.20 \quad 8$ & & $0.332 \quad 17$ & $4166.20 \quad 10$ & $(7533.80)$ & $0.321 \quad 16$ \\
\hline${ }^{x} 3698.33 \quad 15$ & & $0.042 \quad 3$ & ${ }^{x} 4173.09 \quad 12$ & & $0.060 \quad 4$ \\
\hline 3701.919 & $(7533.80)$ & $0.117 \quad 6$ & ${ }^{\mathrm{x}} 4182.98 \quad 15$ & & 0.0252 \\
\hline $3707.96 \quad 12$ & 4389.78 & $0.039 \quad 3$ & $4203.80 \quad 23$ & 4903.68 & 0.0162 \\
\hline $3714.28 \quad 17$ & 4556.68 & 0.0192 & $4209.94 \quad 10$ & $(7533.80)$ & $0.226 \quad 12$ \\
\hline x $3727.98 \quad 11$ & & $0.057 \quad 3$ & $\mathrm{x} 4212.71 \quad 13$ & & $0.055 \quad 4$ \\
\hline × $3732.40 \quad 14$ & & $0.044 \quad 3$ & $4218.71 \quad 25$ & 5002.99 & 0.0122 \\
\hline 3735.529 & $(7533.80)$ & $0.201 \quad 11$ & $4229.38 \quad 11$ & $(7533.80)$ & 0.210 \\
\hline $3738.94 \quad 8$ & $(7533.80)$ & $0.77 \quad 4$ & $4233.87 \quad 22$ & 5697.22 & $0.018 \quad 2$ \\
\hline${ }^{\mathrm{x}} 3752.52 \quad 13$ & & 0.0514 & $4238.31 \quad 11$ & $(7533.80)$ & $0.219 \quad 11$ \\
\hline $3755.06 \quad 9$ & 3862.04 & 0.1398 & $4246.39 \quad 12$ & $(7533.80)$ & $0.120 \quad 7$ \\
\hline${ }^{\mathrm{x}} 3759.14 \quad 12$ & & $0.040 \quad 3$ & $4249.24 \quad 10$ & $(7533.80)$ & 0.915 \\
\hline $3763.03 \quad 10$ & $(7533.80)$ & $0.102 \quad 5$ & $4254.09 \quad 20$ & 5630.29 & $0.018 \quad 2$ \\
\hline $3769.86 \quad 11$ & 3876.94 & $0.069 \quad 4$ & $4258.95 \quad 13$ & 4259.10 & $0.042 \quad 3$ \\
\hline $3777.70 \quad 9$ & 4416.60 & $0.067 \quad 7$ & $4270.35 \quad 16$ & 5759.69 & 0.0212 \\
\hline $3790.36 \quad 9$ & 5064.01 & 0.1649 & $\mathrm{x} 4294.4124$ & & $0.014 \quad 2$ \\
\hline $3794.82 \quad 12$ & 3794.62 & $0.040 \quad 3$ & $4299.71 \quad 9$ & $(7533.80)$ & $0.490 \quad 25$ \\
\hline $3801.25 \quad 17$ & 5789.69 & 0.0182 & $4309.58 \quad 12$ & 4416.60 & $0.049 \quad 3$ \\
\hline $3814.8 \quad 3$ & 5081.26 & $0.026 \quad 6$ & $4319.91 \quad 10$ & 5697.22 & $0.190 \quad 10$ \\
\hline $3816.80 \quad 14$ & 5978.32 & $0.060 \quad 6$ & $4322.90 \quad 9$ & $(7533.80)$ & 0.46023 \\
\hline $3827.56 \quad 12$ & 3934.62 & $0.045 \quad 3$ & $4331.5 \quad 4$ & 4590.56 & 0.0222 \\
\hline $3831.32 \quad 11$ & $3831.74 ?$ & $0.063 \quad 4$ & $4337.71 \quad 9$ & $(7533.80)$ & $0.83 \quad 4$ \\
\hline $3837.27 \quad 8$ & $(7533.80)$ & $0.96 \quad 5$ & $4366.0 \quad 4$ & 5476.92 & $0.007 \quad 2$ \\
\hline $3842.41 \quad 12$ & 4481.07 & $0.043 \quad 3$ & ${ }^{\mathrm{x}} 4371.43 \quad 10$ & & $0.228 \quad 12$ \\
\hline $3859.48 \quad 9$ & $(7533.80)$ & $0.52 \quad 3$ & $4374.04 \quad 10$ & 4481.07 & 0.149 \\
\hline $\mathrm{x}_{3862.89 \quad 9}$ & & $0.173 \quad 9$ & $4399.00 \quad 18$ & 5081.26 & 0.027 \\
\hline $3869.12 \quad 10$ & 5246.63 & $0.082 \quad 5$ & $4402.48 \quad 21$ & 4660.71 & $0.052 \quad 6$ \\
\hline $3874.7 \quad 4$ & 4556.68 & $0.24 \quad 3$ & $4404.62 \quad 11$ & 5246.63 & $0.134 \quad 9$ \\
\hline $3875.05 \quad 9$ & $(7533.80)$ & $0.80 \quad 5$ & ${ }^{x} 4410.83 \quad 15$ & & $0.025 \quad 2$ \\
\hline $3881.34 \quad 20$ & 5953.50 & 0.0162 & $4416.31 \quad 10$ & 4416.60 & $0.217 \quad 11$ \\
\hline $3889.93 \quad 10$ & 3890.09 & $0.104 \quad 6$ & $4425.20 \quad 10$ & 5064.01 & 0.1418 \\
\hline $3893.97 \quad 9$ & 4152.35 & $0.157 \quad 8$ & ${ }^{\mathrm{x}} 4432.87 \quad 15$ & & 0.0312 \\
\hline × 3901.549 & & $0.166 \quad 9$ & $4442.06 \quad 11$ & 5081.26 & $0.076 \quad 4$ \\
\hline $3912.28 \quad 9$ & $(7533.80)$ & $0.350 \quad 18$ & $4457.3 \quad 3$ & 4715.40 & 0.0122 \\
\hline${ }^{\mathrm{x}} 3914.57 \quad 18$ & & $0.040 \quad 5$ & $4465.4 \quad 3$ & 5978.32 & $0.009 \quad 2$ \\
\hline $3935.39 \quad 19$ & 4778.05 & $0.017 \quad 2$ & $\mathrm{x} 4472.85 \quad 14$ & & 0.0262 \\
\hline${ }^{\mathrm{x}} 3943.94 \quad 10$ & & $0.142 \quad 7$ & $4481.16 \quad 18$ & 4481.07 & $0.040 \quad 4$ \\
\hline x $3952.66 \quad 10$ & & $0.169 \quad 9$ & $4483.7 \quad 3$ & 4590.56 & 0.035 \\
\hline ×3971.64 15 & & 0.0222 & $4493.41 \quad 10$ & $(7533.80)$ & 0.43522 \\
\hline 4005.7923 & $(7533.80)$ & $0.104 \quad 6$ & ${ }^{\mathrm{x}} 4504.02 \quad 12$ & & $0.073 \quad 5$ \\
\hline 4013.779 & 4013.89 & $0.73 \quad 4$ & $4512.50 \quad 9$ & $(7533.80)$ & $1.26 \quad 6$ \\
\hline $\mathrm{x} 4020.19 \quad 13$ & & $0.034 \quad 2$ & $4519.11 \quad 10$ & $(7533.80)$ & $0.64 \quad 3$ \\
\hline 4022.84 & 4806.83 & $0.301 \quad 15$ & $4525.18 \quad 10$ & $(7533.80)$ & $0.89 \quad 5$ \\
\hline $4030.70 \quad 9$ & $(7533.80)$ & $0.258 \quad 13$ & $4534.97 \quad 13$ & 5318.95 & $0.045 \quad 3$ \\
\hline $4033.4 \quad 4$ & 4715.40 & $0.036 \quad 4$ & ${ }^{x} 4568.52 \quad 11$ & & $0.107 \quad 6$ \\
\hline
\end{tabular}




\begin{tabular}{|c|c|c|c|c|c|c|}
\hline \multirow[b]{3}{*}{$\mathrm{E} \gamma^{\dagger}$} & \multirow[b]{3}{*}{$\mathrm{E}($ level) } & \multicolumn{2}{|r|}{${ }^{41} \mathbf{K}(n, \gamma)$} & $E=$ thermal & \multicolumn{2}{|c|}{$1985 \mathrm{Kr} 06$ (continued) } \\
\hline & & & & \multicolumn{2}{|c|}{$\gamma\left({ }^{42} \mathrm{~K}\right)$ (continued) } & \multirow[b]{2}{*}{$I \gamma^{\ddagger} \#$} \\
\hline & & $\mathrm{I} \gamma^{\ddagger} \#$ & & $\mathrm{E} \gamma^{\dagger}$ & $\mathrm{E}($ level $)$ & \\
\hline $\mathrm{x}_{4581.67 \quad 22}$ & & $0.015 \quad 2$ & & $5282.38 \quad 11$ & $(7533.80)$ & $0.54 \quad 3$ \\
\hline $4594.98 \quad 10$ & $(7533.80)$ & $0.52 \quad 3$ & & $5294.82 \quad 11$ & $(7533.80)$ & $2.65 \quad 13$ \\
\hline 4601.3121 & 5978.32 & $0.017 \quad 2$ & & ×5316.7124 & & $0.013 \quad 2$ \\
\hline $4607.48 \quad 10$ & $(7533.80)$ & $0.440 \quad 22$ & 22 & 5328.23 & $(7533.80)$ & $0.125 \quad 6$ \\
\hline $4612.3 \quad 6$ & 4612.82 & $0.007 \quad 2$ & & $5339.22 \quad 14$ & 5978.32 & $0.049 \quad 3$ \\
\hline $4616.58 \quad 10$ & $(7533.80)$ & $0.274 \quad 14$ & 14 & $5346.34 \quad 13$ & $(7533.80)$ & $0.058 \quad 3$ \\
\hline $4636.57 \quad 20$ & 5318.95 & $0.016 \quad 2$ & & $5371.91 \quad 11$ & $(7533.80)$ & $0.383 \quad 19$ \\
\hline $4645.03 \quad 11$ & 4903.68 & $0.077 \quad 4$ & & $\times 5405.1723$ & & $0.013 \quad 1$ \\
\hline $4655.62 \quad 10$ & $(7533.80)$ & $0.58 \quad 3$ & & $5452.25 \quad 16$ & 5710.61 & 0.0282 \\
\hline $4670.93 \quad 10$ & $(7533.80)$ & $0.465 \quad 23$ & & $5461.44 \quad 11$ & $(7533.80)$ & $2.52 \quad 13$ \\
\hline $\mathrm{x}_{4675.94 \quad 15}$ & & $0.027 \quad 2$ & & $5484.12 \quad 11$ & $(7533.80)$ & 1.829 \\
\hline${ }^{\times} 4686.38 \quad 11$ & & $0.081 \quad 4$ & & $5595.93 \quad 11$ & $(7533.80)$ & $1.37 \quad 7$ \\
\hline 4693.14 & 5476.92 & 0.0092 & & $5619.90 \S 15$ & $(7533.80)$ & $0.61 \S 3$ \\
\hline $4698.54 \quad 11$ & 5953.50 & $0.148 \quad 8$ & & $5671.50 \quad 12$ & $(7533.80)$ & $3.71 \quad 19$ \\
\hline$\times 4709.1922$ & & $0.014 \quad 2$ & & $5690.44 \quad 12$ & $(7533.80)$ & $2.26 \quad 11$ \\
\hline $4714.7 \quad 5$ & 4715.40 & $0.007 \quad 2$ & & $5716.54 \quad 12$ & $(7533.80)$ & $0.187 \quad 10$ \\
\hline $\mathrm{x}_{4731.14 \quad 17}$ & & 0.0202 & & $5787.79 \quad 13$ & $(7533.80)$ & $0.155 \quad 8$ \\
\hline $4734.5 \quad 4$ & 5846.58 & $0.008 \quad 2$ & & $5810.00 \quad 12$ & $(7533.80)$ & $0.63 \quad 3$ \\
\hline${ }^{\mathrm{x}} 4745.37 \quad 23$ & & $0.023 \quad 3$ & & $\mathrm{x} 5828.6924$ & & $0.011 \quad 1$ \\
\hline $4747.9 \quad 4$ & 4748.54 & $0.014 \quad 3$ & & x 5840.0121 & & $0.013 \quad 1$ \\
\hline $4767.57 \quad 10$ & $(7533.80)$ & $4.11 \quad 21$ & & $\mathrm{x}_{5852.66 \quad 16}$ & & $0.030 \quad 2$ \\
\hline $4815.35 \quad 10$ & $(7533.80)$ & $1.46 \quad 7$ & & $\times 5858.05$ & & $0.005 \quad 1$ \\
\hline${ }^{\times} 4826.89 \quad 16$ & & $0.030 \quad 2$ & & ×5959.4218 & & $0.022 \quad 1$ \\
\hline${ }^{\mathrm{x}} 4830.94 \quad 17$ & & $0.027 \quad 2$ & & ${ }^{x} 6047.74$ & & $0.005 \quad 1$ \\
\hline $4853.29 \quad 11$ & 4853.60 & $0.205 \quad 10$ & 10 & $6069.65 \quad 13$ & $(7533.80)$ & $0.310 \quad 16$ \\
\hline$\times 4861.33 \quad 11$ & & $0.165 \quad 9$ & & $6125.37 \quad 13$ & $(7533.80)$ & $0.370 \quad 19$ \\
\hline $4880.6 \quad 4$ & $(7533.80)$ & 0.0624 & & $6133.19 \quad 16$ & $(7533.80)$ & $0.400 \quad 20$ \\
\hline $4889.17 \quad 10$ & $(7533.80)$ & $0.94 \quad 5$ & & $6156.15 \quad 13$ & $(7533.80)$ & $0.90 \quad 5$ \\
\hline $4895.7 \quad 3$ & 5002.99 & 0.0122 & & $6259.76 \quad 13$ & $(7533.80)$ & $0.369 \quad 19$ \\
\hline $4905.67 \quad 10$ & $(7533.80)$ & $0.59 \quad 3$ & & $6267.03 \quad 13$ & $(7533.80)$ & $0.79 \quad 4$ \\
\hline $4926.46 \quad 10$ & $(7533.80)$ & $0.59 \quad 3$ & & $6278.49 \quad 13$ & $(7533.80)$ & $1.54 \quad 8$ \\
\hline $4938.54 \quad 13$ & 4938.98 & $0.049 \quad 3$ & & $6389.70 \quad 14$ & $(7533.80)$ & $0.206 \quad 10$ \\
\hline $4959.88 \quad 10$ & $(7533.80)$ & $0.368 \quad 19$ & 19 & $6422.57 \quad 13$ & $(7533.80)$ & $0.64 \quad 3$ \\
\hline$\times 4985.71 \quad 14$ & & $0.042 \quad 3$ & & $6691.34 \quad 14$ & $(7533.80)$ & $0.79 \quad 4$ \\
\hline${ }^{\mathrm{x}} 5031.31 \quad 12$ & & 0.1218 & & $6851.29 \quad 14$ & $(7533.80)$ & $5.6 \quad 3$ \\
\hline $5051.32 \quad 10$ & $(7533.80)$ & $2.01 \quad 10$ & & $6894.52 \quad 14$ & $(7533.80)$ & $1.25 \quad 6$ \\
\hline 5057.63 & 5697.22 & 0.0132 & & $7426.34 \quad 15$ & $(7533.80)$ & $3.21 \quad 16$ \\
\hline $5080.94 \quad 13$ & 5081.26 & 0.0593 & & $7533.16 \quad 15$ & $(7533.80)$ & $1.86 \quad 9$ \\
\hline $5096.55 \quad 15$ & 5097.04 & 0.0292 & & & & \\
\hline $5111.39 \quad 10$ & $(7533.80)$ & $2.01 \quad 10$ & & & & \\
\hline $5131.64 \quad 10$ & $(7533.80)$ & $3.69 \quad 18$ & & & & \\
\hline $5144.68 \quad 11$ & $(7533.80)$ & $1.03 \quad 5$ & & & & \\
\hline $5167.28 \quad 10$ & $(7533.80)$ & $6.6 \quad 3$ & & & & \\
\hline$\times 5227.9 \quad 7$ & & $0.005 \quad 2$ & & & & \\
\hline$\times 5239.8522$ & & 0.0172 & & & & \\
\hline
\end{tabular}

$\dagger$ Systematic uncertainties of $5 \mathrm{ppm}$ below $1.5 \mathrm{MeV}$ energy, $10 \mathrm{ppm}$ between $1.5 \mathrm{MeV}$ and $3.0 \mathrm{MeV}$, and $20 \mathrm{ppm}$ above $3 \mathrm{MeV}$ photon energy are included.

$¥$ Intensities are per 100 neutron captures. Systematic uncertainties of $20 \%$ below $0.5 \mathrm{MeV}, 15 \%$ between $0.5 \mathrm{MeV}$ and $2 \mathrm{MeV}, 10 \%$ between $2.0 \mathrm{MeV}$ and $3.0 \mathrm{MeV}$, and $5 \%$ above $3 \mathrm{MeV}$ are included. To obtain absolute cross section on the scale given in 2013Fi01, multiply listed $I \gamma$ value by an approximate factor of 0.0150 . The obtained values of cross sections have been compared with corresponding ones in $2013 \mathrm{Fi} 01$ and they are in a good agreement within uncertainties, except for a few $\gamma$ rays: $454.01,830.89$ and 1121.70 , for which the values from $2013 \mathrm{Fi} 01$ is significantly higher.

$\S$ From E(transition $)=5620.30$, branching $=0.65 \% 3$ as listed in table 3 of $1985 \mathrm{Kr} 06$.

\# Absolute intensity per 100 neutron captures.

(1) Multiply placed; undivided intensity given.

$\mathrm{x} \gamma$ ray not placed in level scheme. 


\section{${ }^{41} \mathrm{~K}(\mathrm{n}, \gamma)$ : Resonance $2006 \mathrm{MuZX}$}

${ }^{41} \mathrm{~K}$ g.s. J $\pi=3 / 2+$

2006MuZX: Compilation of thermal neutron induced $\sigma$ and resonance parameter data for nuclei of $Z=1-100$.

Others: 1970StZZ, $1973 \mathrm{Si} 32$ (also $1971 \mathrm{SiYI})(\mathrm{E}=0-400 \mathrm{keV}), 1972 \mathrm{Ki} 24,1984 \mathrm{Ma} 40(\mathrm{E}=11-9850 \mathrm{eV}, 2.6-2000 \mathrm{keV})$.

\section{${ }^{42} \mathrm{~K}$ Levels}

$2 \mathrm{~g} \Gamma_{\mathrm{n}}=(2 \mathrm{~J}+1) \Gamma_{\mathrm{n}}$

All resonance parameters including resonance neutron energies, $J \pi, L, g \Gamma_{n}$ and $\Gamma_{\gamma}$ are directly adopted from the compilation in $2006 \mathrm{MuZX}$, unless otherwise indicated.

\begin{tabular}{|c|c|c|c|c|c|}
\hline \multicolumn{2}{|c|}{$\mathrm{E}(\text { level })^{\dagger}$} & $2 \mathrm{~g} \Gamma_{\mathrm{n}}$ & \multirow{2}{*}{$\frac{\mathrm{L}}{0}$} & $\mathrm{E}_{\mathrm{n}}(\mathrm{lab})(\mathrm{keV})$ & Comments \\
\hline $7532.9 ?$ & & & & -0.946 & $\Gamma_{\gamma}=(0.94) \mathrm{eV}$ \\
\hline 7534.67 & 11 & & & $0.8960 \quad 9$ & $\mathrm{~g} \Gamma_{\mathrm{n}} \Gamma_{\gamma} / \Gamma(\mathrm{meV})=5.173$ \\
\hline 7535.78 & 11 & $4.18 \mathrm{eV} \quad 4$ & 0 & $2.030 \quad 2$ & $\begin{array}{l}\mathrm{g} \Gamma_{\mathrm{n}} \Gamma_{\gamma} / \Gamma(\mathrm{meV})=198.811 \\
\Gamma_{\gamma}=0.352 \mathrm{eV} 4\end{array}$ \\
\hline 7536.93 & 11 & $0.24 \mathrm{eV} 3$ & 1 & $3.203 \quad 3$ & $\mathrm{~g} \Gamma_{\mathrm{n}} \Gamma_{\gamma} / \Gamma(\mathrm{meV})=112.43$ \\
\hline 7537.72 & 11 & & & 4.0124 & $\mathrm{~g} \Gamma_{\mathrm{n}} \Gamma_{\gamma} / \Gamma(\mathrm{meV})=89.03$ \\
\hline 7539.18 & 11 & $92.2 \mathrm{eV} 18$ & 0 & $5.514 \quad 6$ & $\begin{array}{l}\mathrm{g} \Gamma_{\mathrm{n}} \Gamma_{\gamma} / \Gamma(\mathrm{meV})=26026 \\
\Gamma_{\gamma}=0.70 \mathrm{eV} 7\end{array}$ \\
\hline 7539.67 & 11 & & & 6.0126 & $\mathrm{~g} \Gamma_{\mathrm{n}} \Gamma_{\gamma} / \Gamma(\mathrm{meV})=86.47$ \\
\hline 7540.32 & 11 & & & $6.679 \quad 7$ & $\mathrm{~g} \Gamma_{\mathrm{n}} \Gamma_{\gamma} / \Gamma(\mathrm{meV})=3.2020$ \\
\hline 7541.60 & 11 & & & $7.989 \quad 8$ & $\mathrm{~g} \Gamma_{\mathrm{n}} \Gamma_{\gamma} / \Gamma(\mathrm{meV})=21.76$ \\
\hline 7542.07 & 11 & & & $8.475 \quad 9$ & $\mathrm{~g} \Gamma_{\mathrm{n}} \Gamma_{\gamma} / \Gamma(\mathrm{meV})=117.74$ \\
\hline 7544.48 & 11 & & 1 & $10.940 \quad 10$ & $\mathrm{~g} \Gamma_{\mathrm{n}} \Gamma_{\gamma} / \Gamma(\mathrm{meV})=96.324$ \\
\hline 7544.76 & 11 & & & $11.230 \quad 10$ & $\mathrm{~g} \Gamma_{\mathrm{n}} \Gamma_{\gamma} / \Gamma(\mathrm{meV})=9.66$ \\
\hline 7546.82 & 11 & $5.9 \mathrm{eV} 12$ & 1 & $13.341 \quad 13$ & $\begin{array}{l}\mathrm{g} \Gamma_{\mathrm{n}} \Gamma_{\gamma} / \Gamma(\mathrm{meV})=214.022 \\
\Gamma_{\gamma}=0.369 \mathrm{eV} 4\end{array}$ \\
\hline 7548.46 & 11 & $4.8 \mathrm{eV} 12$ & 1 & $15.022 \quad 15$ & $\begin{array}{l}\mathrm{g} \Gamma_{\mathrm{n}} \Gamma_{\gamma} / \Gamma(\mathrm{meV})=137.623 \\
\Gamma_{\gamma}=0.389 \mathrm{eV} 7\end{array}$ \\
\hline 7548.86 & 11 & & & $15.430 \quad 15$ & $\mathrm{~g} \Gamma_{\mathrm{n}} \Gamma_{\gamma} / \Gamma(\mathrm{meV})=14.09$ \\
\hline 7549.04 & 11 & & & $15.620 \quad 15$ & $\mathrm{~g} \Gamma{ }_{\mathrm{n}} \Gamma_{\gamma} / \Gamma(\mathrm{meV})=135.321$. \\
\hline 7550.11 & 11 & $256 \mathrm{eV} 11$ & 0 & $16.710 \quad 17$ & $\begin{array}{l}\mathrm{g} \Gamma_{\mathrm{n}} \Gamma_{\gamma} / \Gamma(\mathrm{meV})=29312 \\
\Gamma_{\gamma}=0.78 \mathrm{eV} 3\end{array}$ \\
\hline 7551.36 & 11 & & & $17.990 \quad 17$ & $\mathrm{~g} \Gamma_{\mathrm{n}} \Gamma_{\gamma} / \Gamma(\mathrm{meV})=1443$ \\
\hline 7552.04 & 11 & & & $18.690 \quad 18$ & $\mathrm{~g} \Gamma_{\mathrm{n}} \Gamma_{\gamma} / \Gamma(\mathrm{meV})=11.011$ \\
\hline 7553.24 & 11 & & & $19.920 \quad 20$ & $\mathrm{~g} \Gamma_{\mathrm{n}} \Gamma_{\gamma} / \Gamma(\mathrm{meV})=3013$ \\
\hline 7553.61 & 11 & $32.6 \mathrm{eV} 8$ & 1 & $20.298 \quad 20$ & $\begin{array}{l}\mathrm{g} \Gamma_{\mathrm{n}} \Gamma_{\gamma} / \Gamma(\mathrm{meV})=2664 \\
\Gamma_{\gamma}=0.722 \mathrm{eV} 12\end{array}$ \\
\hline 7558.31 & 11 & $110 \mathrm{eV} 21$ & {$[0]$} & $25.118 \quad 25$ & $\begin{array}{l}\mathrm{g} \Gamma_{\mathrm{n}} \Gamma_{\gamma} / \Gamma(\mathrm{meV})=1627 . \\
\Gamma_{\gamma}=1.30 \mathrm{eV} 5 .\end{array}$ \\
\hline 7560.18 & 11 & & & $27.03 \quad 3$ & $\mathrm{~g} \Gamma_{\mathrm{n}} \Gamma_{\gamma} / \Gamma(\mathrm{meV})=463$. \\
\hline 7560.27 & 11 & $23.0 \mathrm{eV} 8$ & 1 & 27.123 & $\begin{array}{l}\mathrm{g} \Gamma_{\mathrm{n}} \Gamma_{\gamma} / \Gamma(\mathrm{meV})=2556 \\
\Gamma_{\gamma}=0.297 \mathrm{eV} 8\end{array}$ \\
\hline 7561.43 & 11 & & & 28.313 & $\mathrm{~g} \Gamma_{\mathrm{n}} \Gamma_{\gamma} / \Gamma(\mathrm{meV})=43.524$ \\
\hline 7566.12 & 11 & & & $33.12 \quad 3$ & $\mathrm{~g} \Gamma_{\mathrm{n}} \Gamma_{\gamma} / \Gamma(\mathrm{meV})=1405$ \\
\hline 7566.20 & 11 & & & $33.20 \quad 3$ & $\mathrm{~g} \Gamma_{\mathrm{n}} \Gamma_{\gamma} / \Gamma(\mathrm{meV})=2316$. \\
\hline 7566.85 & 11 & $3.42 \mathrm{eV} 22$ & & $33.86 \quad 3$ & $\mathrm{~g} \Gamma_{\mathrm{n}} \Gamma_{\gamma} / \Gamma(\mathrm{meV})=1878$. \\
\hline 7568.49 & 12 & & & $35.54 \quad 4$ & $\mathrm{~g} \Gamma_{\mathrm{n}} \Gamma_{\gamma} / \Gamma(\mathrm{meV})=945$ \\
\hline 7571.43 & 12 & $830 \quad \mathrm{eV} \quad 15$ & 0 & $38.56 \quad 4$ & $\begin{array}{l}\mathrm{g} \Gamma_{\mathrm{n}} \Gamma_{\gamma} / \Gamma(\mathrm{meV})=94241 \\
\Gamma_{\gamma}=1.51 \mathrm{eV} 7\end{array}$ \\
\hline 7572.33 & 12 & & & $39.48 \quad 4$ & $\mathrm{~g} \Gamma_{\mathrm{n}} \Gamma_{\gamma} / \Gamma(\mathrm{meV})=285$ \\
\hline 7575.13 & 12 & $270 \mathrm{eV} \quad 130$ & {$[0]$} & $42.35 \quad 4$ & $\begin{array}{l}\mathrm{g} \Gamma_{\mathrm{n}} \Gamma_{\gamma} / \Gamma \quad(\mathrm{meV})=57019 \\
\Gamma_{\gamma}=0.92 \mathrm{eV} 3 .\end{array}$ \\
\hline 7575.72 & 12 & & & $42.95 \quad 4$ & $\mathrm{~g} \Gamma_{\mathrm{n}} \Gamma_{\gamma} / \Gamma(\mathrm{meV})=1336$ \\
\hline 7577.05 & 12 & & & 44.324 & $\mathrm{~g} \Gamma_{\mathrm{n}} \Gamma_{\gamma} / \Gamma(\mathrm{meV})=1237$. \\
\hline 7577.14 & 12 & & & 44.414 & $\mathrm{~g} \Gamma_{\mathrm{n}} \Gamma_{\gamma} / \Gamma(\mathrm{meV})=1667$. \\
\hline 7580.12 & 12 & & & $47.46 \quad 5$ & $\mathrm{~g} \Gamma_{\mathrm{n}} \Gamma_{\gamma} / \Gamma(\mathrm{meV})=455$ \\
\hline 7580.35 & 12 & & & $47.70 \quad 5$ & $\mathrm{~g} \Gamma_{\mathrm{n}} \Gamma_{\gamma} / \Gamma(\mathrm{meV})=3339$. \\
\hline 7580.97 & 12 & & & $48.33 \quad 5$ & $\mathrm{~g} \Gamma_{\mathrm{n}} \Gamma_{\gamma} / \Gamma(\mathrm{meV})=1267$. \\
\hline 7582.01 & 12 & & & $49.40 \quad 5$ & $\mathrm{~g} \Gamma_{\mathrm{n}} \Gamma_{\gamma} / \Gamma(\mathrm{meV})=2719$. \\
\hline 7582.80 & 12 & & & 50.215 & $\mathrm{~g} \Gamma_{\mathrm{n}} \Gamma_{\gamma} / \Gamma(\mathrm{meV})=1548$. \\
\hline 7583.95 & 12 & & & $51.39 \quad 5$ & $\mathrm{~g} \Gamma_{\mathrm{n}} \Gamma_{\gamma} / \Gamma(\mathrm{meV})=837$ \\
\hline 7585.39 & 12 & & & $52.86 \quad 5$ & $\mathrm{~g} \Gamma_{\mathrm{n}} \Gamma_{\gamma} / \Gamma(\mathrm{meV})=2389$. \\
\hline 7585.69 & 12 & & & $53.17 \quad 5$ & $\mathrm{~g} \Gamma_{\mathrm{n}} \Gamma_{\gamma} / \Gamma(\mathrm{meV})=306$. \\
\hline 7586.46 & 12 & & & $53.96 \quad 5$ & $\mathrm{~g} \Gamma_{\mathrm{n}} \Gamma_{\gamma} / \Gamma \quad(\mathrm{meV})=2209$ \\
\hline 7587.90 & 12 & $80 \quad \mathrm{eV} \quad 24$ & 1 & $55.43 \quad 6$ & $\begin{array}{l}\mathrm{g} \Gamma_{\mathrm{n}} \Gamma_{\gamma} / \Gamma(\mathrm{meV})=21011 \\
\Gamma_{\gamma}=0.242 \mathrm{eV} 13 .\end{array}$ \\
\hline
\end{tabular}


${ }^{41} \mathrm{~K}(\mathrm{n}, \gamma)$ : Resonance $2006 \mathrm{MuZX}$ (continued)

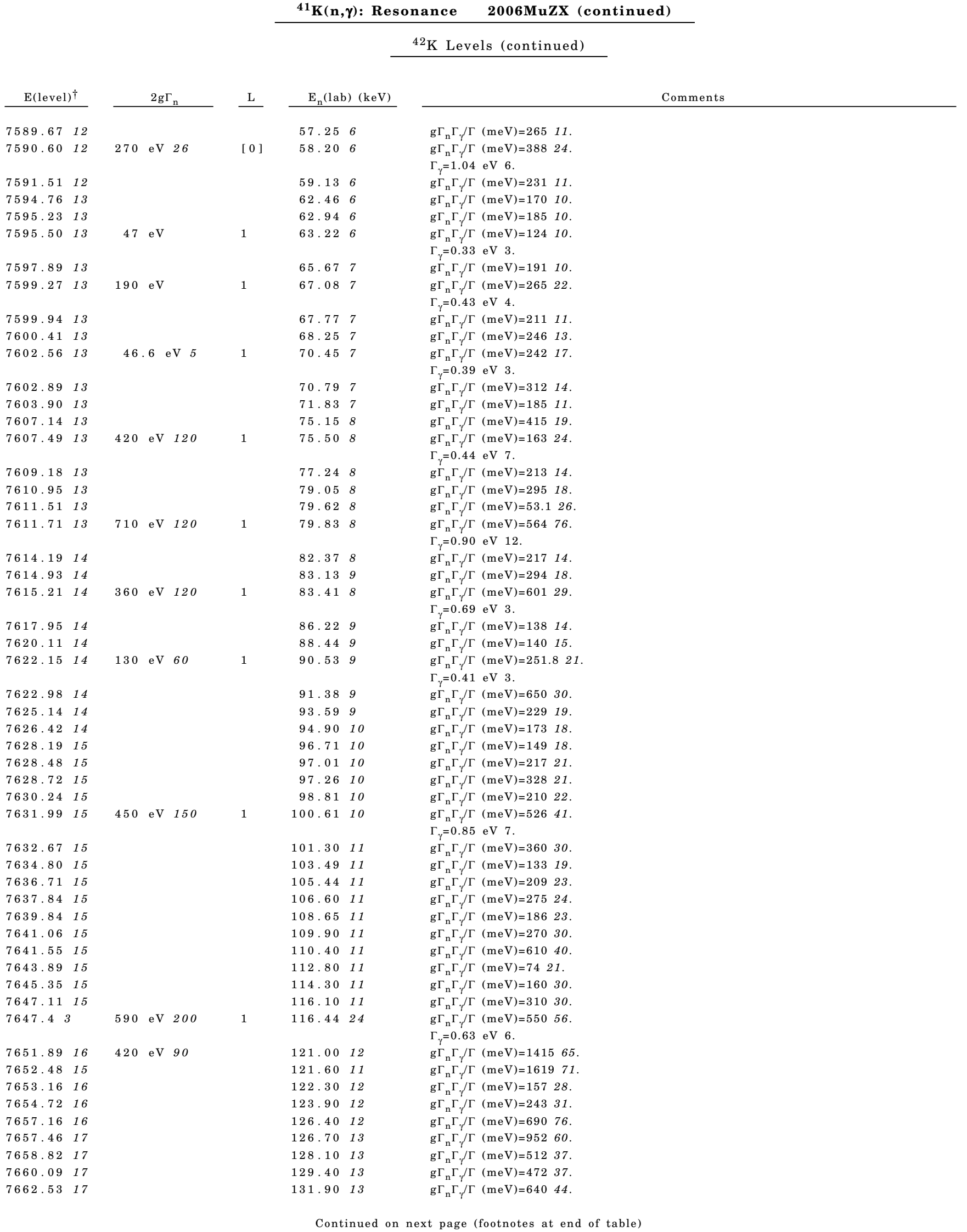


${ }^{41} K(n, \gamma):$ Resonance $\quad 2006 M u Z X$ (continued)

${ }^{42} \mathrm{~K}$ Levels (continued)

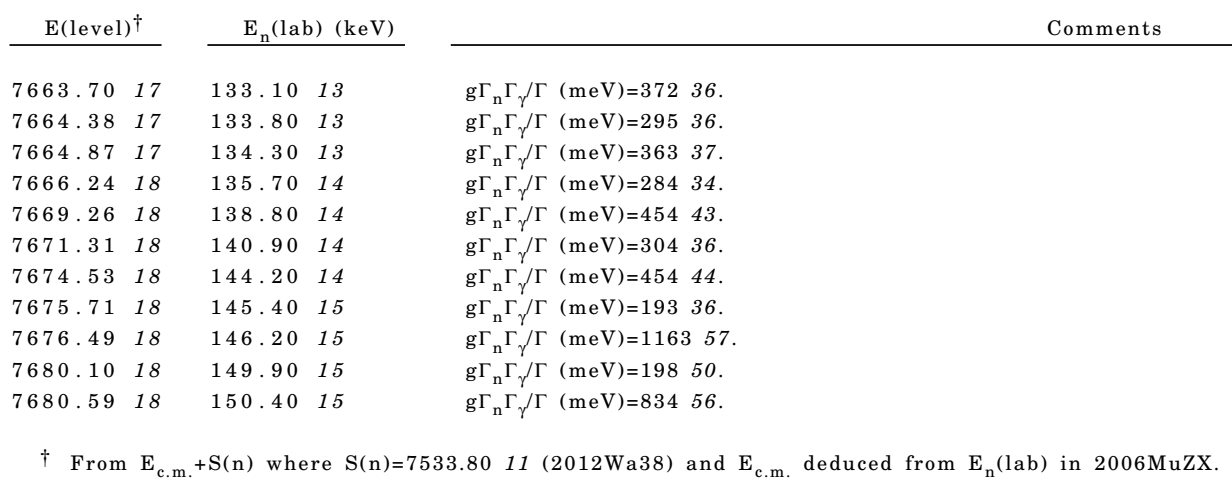

${ }^{41} \mathrm{~K}(\mathrm{~d}, \mathrm{p}),(\mathrm{d}, \mathrm{p} \gamma) \quad 1985 \mathrm{Kr} 06,1978 \mathrm{Li} 27$

Target ${ }^{41} \mathrm{~K} J \pi=3 / 2+$.

1985Kr06: (d,p) E=20 MeV deuteron beam was produced from the TU Munchen Tandem Accelerator with intensity of $1.5 \mu \mathrm{A}$ Target of a $30 \mu \mathrm{g} / \mathrm{cm}^{2} 95.8 \%$ enriched $\mathrm{KF}$ evaporated onto a $3.3 \mu \mathrm{g} / \mathrm{cm}^{2}$ carbon backing. Protons were momentum analyzed with a Q3D magnetic spectrometer and detected by a proportional counter in the focal plane, FWHM=4.5-5.5 keV. Measured $\sigma\left(\mathrm{E}_{\mathrm{p}}, \theta\right)$. Deduced levels.

1978Li27: (d,p) E=10 MeV deuteron beam was produced from a tandem Van de Graaff accelerator. A $60 \mu \mathrm{g} / \mathrm{cm}^{2} \mathrm{thickness}$ target of $\mathrm{KI}\left(98 \%\right.$ enriched in $\left.{ }^{41} \mathrm{~K}\right)$ evaporated onto a carbon backing. Protons were momentum analyzed with a broad-range magnetic spectrograph and detected in a nuclear emulsion, FWHM=35 keV. Measured $\sigma\left(E_{p}, \theta\right)$. Deduced levels, J $\pi$, L, spectroscopic factors from DWBA analysis.

1972Sp04: $(\mathrm{d}, \mathrm{p} \gamma) \mathrm{E}=6 \mathrm{MeV}$ deuteron beam was produced from the University of Iowa Van de Graaff accelerator. A 800 $\mu \mathrm{g} / \mathrm{cm}^{2}$ target of $\mathrm{KCl}\left(95 \%\right.$ enrichment in ${ }^{41} \mathrm{~K}$ ) evaporated onto a $400 \mu \mathrm{g} / \mathrm{cm}^{2}$ gold backing. $\gamma$-rays were detected with $\mathrm{NaI}(\mathrm{Tl})$ crystals. Measured $(151 \gamma)(107 \gamma)(\theta)$. Limits on mixing ratios deduced for $107 \gamma$ and $151 \gamma$. 1970Fr10: $(\mathrm{d}, \mathrm{p} \gamma) \mathrm{E}=3.5,3.7 \mathrm{MeV} .1 \mathrm{mg} / \mathrm{cm}^{2} \mathrm{KI}$ target. Ge(Li) detector. Measured $\mathrm{E} \gamma, \mathrm{p} \gamma-\mathrm{coin}$.

1969Ly02: (d,p) E=12 MeV beam from the Heidelberg tandem. Target of $\mathrm{KNO}_{3}$ on a carbon backing. A broad-range magnetic spectrograph. Measured $\sigma\left(\mathrm{E}_{\mathrm{p}}, \theta\right)$. Deduced levels, $\mathrm{J} \pi, \mathrm{L}$ and spectroscopic factors from DWBA analysis. The authors report 8 groups up to $1260 \mathrm{keV}$.

1958MoZZ: E=6 MeV. Measured proton spectra.

1950Sa03: $\mathrm{E}=3.90 \mathrm{MeV}$ deuteron was produced from the Yale cyclotron. Natural potassium target $\left(93.3 \%{ }^{39} \mathrm{~K}, 0.01 \%{ }^{40} \mathrm{~K}\right.$, and $6.7 \%{ }^{41} \mathrm{~K}$ ). Protons were detected by a proportional counter. Measured proton yields. Deduced levels.

\begin{tabular}{|c|c|}
\hline $\begin{array}{l}\text { Cross } \\
\text { Level }\end{array}$ & $\begin{array}{c}\text { section data from } 1978 \mathrm{Li} 27 \\
\mathrm{~d} \sigma / \mathrm{d} \Omega(\max )(\mathrm{mb} / \mathrm{sr})\end{array}$ \\
\hline 0 & 0.71 \\
\hline 107 & 0.82 \\
\hline 256 & 0.80 \\
\hline 632 & 0.28 \\
\hline 693 & 1.91 \\
\hline 780 & 0.91 \\
\hline 840 & 0.04 (from $1969 \mathrm{Ly} 02$ only) \\
\hline 1249 & 1.72 \\
\hline 1371 & 0.57 \\
\hline 1848 & 3.28 \\
\hline 1926 & 2.32 \\
\hline 2057 & 1.40 \\
\hline 2223 & 0.87 \\
\hline 2354 & 3.93 \\
\hline 2390 & 4.80 \\
\hline 2465 & 2.64 \\
\hline 2544 & 0.61 \\
\hline 2632 & 4.07 \\
\hline 2710 & \\
\hline 2749 & \\
\hline 2900 & \\
\hline 2916 & 2.22 \\
\hline
\end{tabular}

Continued on next page (footnotes at end of table) 
${ }^{41} \mathrm{~K}(\mathrm{~d}, \mathrm{p}),(\mathrm{d}, \mathrm{p} \gamma) \quad 1985 \mathrm{Kr} 06,1978 \mathrm{Li27}$ (continued)

\begin{tabular}{|c|c|}
\hline 3004 & 0.42 \\
\hline 3193 & 0.72 \\
\hline 3271 & 2.16 \\
\hline 3355 & 0.33 \\
\hline 3402 & 2.29 \\
\hline 3635 & 0.97 \\
\hline 3766 & 0.14 \\
\hline 3873 & 0.59 \\
\hline 3919 & 0.21 \\
\hline 4002 & 1.89 \\
\hline 4039 & 1.08 \\
\hline 4117 & 3.41 \\
\hline 4407 & 3.01 \\
\hline 4466 & 1.36 \\
\hline 4560 & 3.10 \\
\hline 4791 & 1.60 \\
\hline 4839 & 1.39 \\
\hline Un c & in level energy $=15 \mathrm{keV}$ \\
\hline
\end{tabular}

${ }^{42} \mathrm{~K}$ Levels

\begin{tabular}{|c|c|c|c|}
\hline $\mathrm{E}(\text { level })^{\dagger}$ & $L^{\S}$ & $(2 \mathrm{~J}+1) \mathrm{C}^{2} \mathrm{~S} \S$ & Comments \\
\hline 0.0 & 3 & 3.0 & $(2 \mathrm{~J}+1) \mathrm{C}^{2} \mathrm{~S}: 1.7(1969 \mathrm{Ly} 02)$ \\
\hline $106.77 \quad 22$ & $1+3$ & $0.16,2.7$ & $\begin{array}{l}\mathrm{L}: 90 \% \mathrm{~L}=3,10 \% \mathrm{~L}=1 \\
(2 \mathrm{~J}+1) \mathrm{C}^{2} \mathrm{~S}: 0.12,1.5(1969 \mathrm{Ly} 02)\end{array}$ \\
\hline $258.27 \quad 16$ & 3 & 3.3 & $\begin{array}{l}\mathrm{J} \pi: 2,3 \text { deduced from }(151 \gamma)(107 \gamma)(\theta)(1972 \mathrm{Sp} 04) \\
(2 \mathrm{~J}+1) \mathrm{C}^{2} \mathrm{~S}: 1.9(1969 \mathrm{Ly} 02)\end{array}$ \\
\hline 638.65 & $1+3$ & $0.1,0.2$ & $\begin{array}{l}\mathrm{J} \pi: 3,4 \text { deduced from }(151 \gamma)(107 \gamma)(\theta)(1972 \mathrm{Sp} 04) \\
(2 \mathrm{~J}+1) \mathrm{C}^{2} \mathrm{~S}: 0.02,0.18(1969 \mathrm{Ly} 02)\end{array}$ \\
\hline $\begin{array}{l}682.04 \\
699.12 \quad 15\end{array}$ & & & Lo $(2 \mathrm{~J}+1) \mathrm{C}^{2} \mathrm{~S} \cdot$ for 693 oroun in $1978 \mathrm{Li} 27$ \\
\hline 699.1215 & 3 & 8.1 & $\begin{array}{l}\mathrm{L},(2 \mathrm{~J}+1) \mathrm{C}^{2} \mathrm{~S}: \text { for } 693 \text { group in } 1978 \mathrm{Li} 27 \\
(2 \mathrm{~J}+1) \mathrm{C}^{2} \mathrm{~S}: 4.26(1969 \mathrm{Ly} 02) \text {. }\end{array}$ \\
\hline $783.77 \quad 17$ & $1^{\#}$ & $0.09^{\#}$ & \\
\hline 841.74 & $1^{\#}$ & $0.02^{\#}$ & \\
\hline 1111.011 & & & \\
\hline $1144.0 \quad 3$ & & & \\
\hline 1198.33 & $3 \&$ & & \\
\hline $1254.71 \quad 17$ & 1 & 0.51 & $\begin{array}{l}\mathrm{L},(2 \mathrm{~J}+1) \mathrm{C}^{2} \mathrm{~S} \text { : for } 1249 \text { group in } 1978 \mathrm{Li} 27 \text {. Also } \mathrm{L}=1 \text { quoted by } 1982 \mathrm{Ba} 55 \text { from a priv comm from } \\
\text { Summers-Gill (at McMaster). } \\
(2 \mathrm{~J}+1) \mathrm{C}^{2} \mathrm{~S}: 0.4 \text { for } 1260 \text { group }(1969 \mathrm{Ly} 02) \text {. }\end{array}$ \\
\hline $1266.18 \quad 18$ & $1^{\&}$ & & \\
\hline $1377.7 \quad 4$ & 1 & 0.20 & $\mathrm{~L},(2 \mathrm{~J}+1) \mathrm{C}^{2} \mathrm{~S}$ : for 1371 group in $1978 \mathrm{Li} 27$. \\
\hline 1399.09 & & & \\
\hline 1407.79 & & & \\
\hline 1452.03 & & & \\
\hline 1463.13 & & & \\
\hline 1489.43 & & & \\
\hline 1513.1920 & & & \\
\hline 1692.13 & & & \\
\hline 1723.75 & & & \\
\hline 1745.96 & & & \\
\hline $1787 ? \quad 12$ & & & $\begin{array}{l}\text { E(level): } 1776 \text { ( } 1958 \mathrm{MoZZ} \text { ) adjusted to } 1787 \text { by } 1978 \mathrm{En} 02 \text {. This level is considered as } \\
\text { uncertain (evaluators) since it is not reported in any other study. }\end{array}$ \\
\hline 1843.63 & & & \\
\hline $\begin{array}{l}1861.8916 \\
1913.74\end{array}$ & 1 & 0.90 & $\mathrm{~L},(2 \mathrm{~J}+1) \mathrm{C}^{2} \mathrm{~S}$ : for 1848 group in $1978 \mathrm{Li} 27$. \\
\hline $1937.43 \quad 16$ & 1 & 0.77 & $\mathrm{~L},(2 \mathrm{~J}+1) \mathrm{C}^{2} \mathrm{~S}$ : for 1926 group in $1978 \mathrm{Li} 27$ \\
\hline $\begin{array}{l}1988.14 \quad 19 \\
2052.07\end{array}$ & & & \\
\hline $2071.92 \quad 15$ & 1 & 0.38 & $\mathrm{~L},(2 \mathrm{~J}+1) \mathrm{C}^{2} \mathrm{~S}$ : for 2057 group in $1978 \mathrm{Li} 27$. \\
\hline $2160.7 \quad 7$ & & & \\
\hline 2186.37 & & & \\
\hline 2205.04 & & & \\
\hline $\begin{array}{l}2238.6024 \\
2250.9 \quad 16\end{array}$ & 1 & 0.22 & $\mathrm{~L},(2 \mathrm{~J}+1) \mathrm{C}^{2} \mathrm{~S}$ : for 2223 group in $1978 \mathrm{Li} 27$. \\
\hline
\end{tabular}


${ }^{41} \mathrm{~K}(\mathrm{~d}, \mathrm{p}),(\mathrm{d}, \mathrm{p} \gamma) \quad 1985 \mathrm{Kr} 06,1978 \mathrm{Li} 27$ (continued)

${ }^{42} \mathrm{~K}$ Levels (continued)

\begin{tabular}{|c|c|c|c|}
\hline $\mathrm{E}(\text { level })^{\dagger}$ & $L \S$ & $(2 \mathrm{~J}+1) \mathrm{C}^{2} \mathrm{~S} \S$ & Comments \\
\hline $2366.18 \quad 16$ & 1 & 1.1 & $\mathrm{~L},(2 \mathrm{~J}+1) \mathrm{C}^{2} \mathrm{~S}$ : for 2354 group in $1978 \mathrm{Li} 27$ \\
\hline $2388.8 \quad 3$ & & & \\
\hline $2401.78 \quad 16$ & 1 & 1.3 & $\mathrm{~L},(2 \mathrm{~J}+1) \mathrm{C}^{2} \mathrm{~S}$ : for 2390 group in $1978 \mathrm{Li} 27$. \\
\hline $2422.13 \quad 19$ & & & \\
\hline $2455.4 ? \quad 6$ & & & \\
\hline $2482.15 \quad 14$ & 1 & 0.68 & $\mathrm{~L},(2 \mathrm{~J}+1) \mathrm{C}^{2} \mathrm{~S}$ : for 2465 group in $1978 \mathrm{Li} 27$. \\
\hline $2553.65 \quad 23$ & 1 & 0.15 & $\mathrm{~L},(2 \mathrm{~J}+1) \mathrm{C}^{2} \mathrm{~S}$ : for 2544 group in $1978 \mathrm{Li} 27$. \\
\hline $2573.8 \quad 3$ & & & \\
\hline 2607.43 & & & \\
\hline $2627.67 \quad 22$ & & & \\
\hline $2644.22 \quad 21$ & 1 & 1.0 & $\mathrm{~L},(2 \mathrm{~J}+1) \mathrm{C}^{2} \mathrm{~S}$ : for 2632 group in $1978 \mathrm{Li} 27$. \\
\hline 2652.6925 & & & \\
\hline 2718.5120 & & & \\
\hline $2765.74 \quad 18$ & & & \\
\hline $2917.0 \quad 4$ & & & \\
\hline $2923.6 \quad 11$ & 1 & 0.54 & $\mathrm{~L},(2 \mathrm{~J}+1) \mathrm{C}^{2} \mathrm{~S}$ : for 2916 group in $1978 \mathrm{Li} 27$. \\
\hline $2938.3 \quad 3$ & & & \\
\hline 3014.23 & $1^{@}$ & $0.82^{@}$ & \\
\hline 3021.1620 & $1^{@}$ & $0.82^{@}$ & \\
\hline 3040.45 & & & \\
\hline $3193.9 \quad 4$ & & & \\
\hline $3211.6 \quad 3$ & 1 & 0.17 & $\mathrm{~L},(2 \mathrm{~J}+1) \mathrm{C}^{2} \mathrm{~S}$ : for 3193 group in $1978 \mathrm{Li} 27$ \\
\hline $3284.4 \quad 5$ & 1 & 0.50 & $\mathrm{~L},(2 \mathrm{~J}+1) \mathrm{C}^{2} \mathrm{~S}$ : for 3271 group in $1978 \mathrm{Li} 27$. \\
\hline 3304.16 & & & \\
\hline 3368.75 & & & \\
\hline $3418.34 \quad 24$ & $1+3$ & $0.50,0.32$ & $\begin{array}{l}\mathrm{L}: 80 \% \mathrm{~L}=1,20 \% \mathrm{~L}=3 \text {. } \\
\mathrm{L},(2 \mathrm{~J}+1) \mathrm{C}^{2} \mathrm{~S}: \text { for } 3402 \text { group in } 1978 \mathrm{Li} 27 .\end{array}$ \\
\hline 3505.34 & & & \\
\hline $3527.9 \quad 4$ & & & \\
\hline $3620.4 \quad 3$ & & & \\
\hline 3658.9123 & & & \\
\hline $3674.3 \quad 3$ & & & \\
\hline $3696.02 \quad 22$ & & & \\
\hline $3875.9 \quad 10$ & & & \\
\hline 3888.6322 & 1 & 0.13 & $\mathrm{~L},(2 \mathrm{~J}+1) \mathrm{C}^{2} \mathrm{~S}$ : for 3873 group in $1978 \mathrm{Li} 27$. \\
\hline $3934.0 \quad 5$ & & & \\
\hline 4013.24 & & & \\
\hline 4040.14 & & & \\
\hline 4054.23 & $1(+3)$ & $0.20,0.74$ & $\begin{array}{l}\mathrm{L} \text { : indication of } \approx 50 \% \mathrm{~L}=3 \text {. } \\
\mathrm{L},(2 \mathrm{~J}+1) \mathrm{C}^{2} \mathrm{~S} \text { : for } 4039 \text { group in } 1978 \mathrm{Li} 27 \text {. }\end{array}$ \\
\hline $4102.4 \quad 4$ & & & \\
\hline $4128.47 \quad 23$ & $1+3$ & $0.68,0.57$ & $\mathrm{~L},(2 \mathrm{~J}+1) \mathrm{C}^{2} \mathrm{~S}$ : for 4117 group in $1978 \mathrm{Li} 27$. \\
\hline $4153.0 \quad 8$ & & & \\
\hline $4179.7 \quad 3$ & & & \\
\hline $4389.5 \quad 5$ & & & \\
\hline $4416.67 \quad 18$ & $1+3$ & $0.59,0.41$ & $\mathrm{~L},(2 \mathrm{~J}+1) \mathrm{C}^{2} \mathrm{~S}$ : for 4407 group in $1978 \mathrm{Li} 27$. \\
\hline $4428.00 \quad 21$ & & & \\
\hline $4443.3 \quad 3$ & & & \\
\hline $4480.87 \quad 24$ & 1 & 0.28 & $\mathrm{~L},(2 \mathrm{~J}+1) \mathrm{C}^{2} \mathrm{~S}$ : for 4466 group in $1978 \mathrm{Li} 27$. \\
\hline $4556.55 \quad 20$ & & & \\
\hline $4576.29 \quad 20$ & 1 & 0.63 & $\mathrm{~L},(2 \mathrm{~J}+1) \mathrm{C}^{2} \mathrm{~S}$ : for 4560 group in $1978 \mathrm{Li} 27$. \\
\hline 4590.14 & & & \\
\hline $4749.0 \quad 4$ & & & \\
\hline $4776.7 \quad 5$ & & & \\
\hline $4806.87 \quad 17$ & 1 & 0.32 & $\mathrm{~L},(2 \mathrm{~J}+1) \mathrm{C}^{2} \mathrm{~S}$ : for 4791 group in $1978 \mathrm{Li} 27$. \\
\hline $4853.65 \quad 16$ & 1 & 0.27 & $\mathrm{~L},(2 \mathrm{~J}+1) \mathrm{C}^{2} \mathrm{~S}$ : for 4839 group in $1978 \mathrm{Li} 27$ \\
\hline $4878.6 \quad 3$ & & & \\
\hline 4902.14 & & & \\
\hline 4942.4725 & & & \\
\hline 4959.45 & & & \\
\hline $4974.1 ? \quad 6$ & & & \\
\hline $5003.10 \quad 24$ & & & \\
\hline 5061.95 & & & \\
\hline
\end{tabular}


${ }^{41} \mathrm{~K}(\mathrm{~d}, \mathrm{p}),(\mathrm{d}, \mathrm{p} \gamma) \quad 1985 \mathrm{Kr} 06,1978 \mathrm{Li27}$ (continued)

${ }^{42} \mathrm{~K}$ Levels (continued)

\begin{tabular}{|c|c|c|}
\hline $\mathrm{E}(\text { level })^{\dagger}$ & $\mathrm{E}(\text { level })^{\dagger}$ & $\mathrm{E}(\text { level })^{\dagger}$ \\
\hline 5080.14 & $5622.5 \quad 3$ & $5809.3 \quad 3$ \\
\hline 5096.95 & $5629.6 \quad 6$ & $5819.4 \quad 3$ \\
\hline 5142.94 & $5654.6 \quad 3$ & $5847.6 \quad 4$ \\
\hline 5176.311 & $5682.7 \quad 3$ & $5871.0 ? \quad 7$ \\
\hline $5246.39 \quad 19$ & $5699.0 \quad 3$ & $5896.4 \quad 4$ \\
\hline $5265.5 ? 9$ & $5709.4 \quad 5$ & 5927.25 \\
\hline $5318.8 \quad 4$ & $5723.5 \quad 6$ & $5951.6 \quad 13$ \\
\hline 5363.54 & $5737.5 \quad 4$ & 5968.75 \\
\hline 5380.115 & $5747.7 \quad 8$ & 5978.54 \\
\hline $5477.4 \quad 13$ & $5759.75 \quad 21$ & 6012.65 \\
\hline $5484.0 \quad 6$ & $5772.4 \quad 6$ & \\
\hline $5555.7 \quad 6$ & 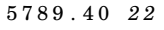 & \\
\hline
\end{tabular}

$\dagger$ From 1985Kr06, uncertainties are statistical only.

$\S$ From 1978Li27, unless otherwise stated.

\# From 1969Ly02.

(a) For $3014+3021$. Energy of this group was 3004 group in $1978 \mathrm{Li} 27$.

\& Quoted by 1982Ba55 (priv comm from Summers-Gill at McMaster).

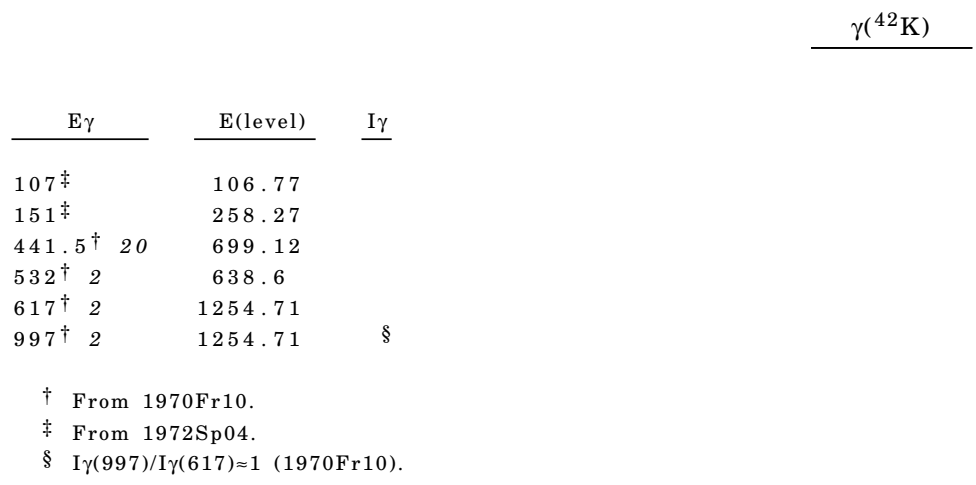

${ }^{42} \mathrm{Ca}\left(\mathrm{t},{ }^{3} \mathrm{He}\right) \quad 1985 \mathrm{Aj03}$

Target ${ }^{42} \mathrm{Ca} \mathrm{J} \pi=0+$.

1985Aj03: E=25 MeV triton beam was produced from the Los Alamos three-stage Van de Graaff facility. ${ }^{3} \mathrm{He}$ particles were analyzed with a Q3D spectrometer and detected by a position-sensitive detector. Measured $\sigma(\theta)$. Deduced levels, J $\pi$, L from CCBA analysis.

${ }^{42} \mathrm{~K}$ Levels

\begin{tabular}{|c|c|c|c|c|c|}
\hline $\mathrm{E}($ level) & $\mathrm{J} \pi^{\dagger}$ & Assumed L-transfer $\ddagger$ & $\mathrm{E}($ level) & $\mathrm{J} \pi^{\dagger}$ & Assumed L-transfer \\
\hline 0.0 & $2-$ & $1+3$ & $1413 \quad 10$ & & \\
\hline 1095 & $3-$ & 3 & $1469 \S \quad 10$ & & \\
\hline 2628 & $4-$ & $3+5$ & $1539 \quad 15$ & $3+$ & $2+4$ \\
\hline $640^{\&}$ & & & $1688 \quad 15$ & 3 & \\
\hline $680 ? \mathrm{a}$ & & & $1735 \quad 15$ & & \\
\hline $\begin{array}{l}702 \quad 8 \\
780 \&\end{array}$ & $5-$ & 5 & $\begin{array}{l}1810 \S 20 \\
1860 ? \mathrm{a}\end{array}$ & & \\
\hline 8418 & & & $1926 \quad 15$ & $3-$ & 3 \\
\hline $1107 \quad 15$ & & & $1956 \quad 15$ & $(4-)$ & $3+5$ \\
\hline $1145 \quad 10$ & $4+$ & 4 & $1990 ?^{\mathrm{a}}$ & & \\
\hline $1201 \quad 10$ & $2-, 3-$ & & 206720 & 3 & \\
\hline $1259 \# 20$ & $2-@$ & $1+3^{@}$ & $2088 \quad 20$ & $2-$ & $1+3$ \\
\hline $1277^{\#} \quad 15$ & $2-@$ & $1+3^{@}$ & $2193 \quad 15$ & $3+, 4+$ & \\
\hline $1380^{\&}$ & & & 221920 & $2,3+$ & \\
\hline
\end{tabular}


${ }^{42} \mathrm{Ca}\left(\mathrm{t},{ }^{3} \mathrm{He}\right) \quad 1985 \mathrm{Aj03}$ (continued)

\begin{tabular}{|c|c|c|c|c|c|c|}
\hline \multirow[b]{3}{*}{$\mathrm{E}($ level) } & \multirow{3}{*}{\multicolumn{2}{|c|}{ Assumed L-transfer }} & \multicolumn{4}{|c|}{${ }^{42} \mathrm{Ca}\left(\mathrm{t},{ }^{3} \mathrm{He}\right) \quad 1985 \mathrm{Aj} 03$ (continued) } \\
\hline & & & \multicolumn{3}{|c|}{${ }^{42} \mathrm{~K}$ Levels (continued) } & \multirow[b]{2}{*}{ d L-transfer $\ddagger$} \\
\hline & & & $\mathrm{E}($ level) & $\mathrm{J} \pi^{\dagger}$ & Assumed & \\
\hline & $3225 \S 20$ & & & \\
\hline & & & $3297 \quad 10$ & $1+$ & $0+2$ & \\
\hline $\begin{array}{ll}2328 & 15 \\
2373 & 15\end{array}$ & & & $3329 \quad 10$ & $1+$ & $0+2$ & \\
\hline 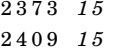 & $3+$ & $2+4$ & $3377 \quad 10$ & $1+$ & $0+2$ & \\
\hline $2434 \quad 15$ & & & $3425 \S \quad 15$ & 2 & & \\
\hline $2496 \quad 15$ & & & $3503 \quad 15$ & & & \\
\hline $2570 \S \quad 15$ & & & $3587 \quad 15$ & & & \\
\hline \multirow{2}{*}{$\begin{array}{ll}2626 & 15 \\
2662 \S & 15\end{array}$} & & & $3628 \S 15$ & & & \\
\hline & & & $3666 \quad 10$ & & & \\
\hline $2750 \quad 15$ & & & $3698 \quad 10$ & & & \\
\hline $2780 \quad 15$ & & & $3758 \quad 10$ & & & \\
\hline $2824 \quad 15$ & & & $3793 \S 15$ & & & \\
\hline \multirow{3}{*}{$\begin{array}{ll}2862 & 10 \\
2895 & 10 \\
2955 \S & 15\end{array}$} & $1+$ & $0+2$ & $3860 \quad 15$ & & & \\
\hline & 3 & & $3887 \quad 15$ & & & \\
\hline & & & $4042 \S \quad 15$ & & & \\
\hline \multirow{2}{*}{$\begin{array}{ll}3032 & 10 \\
3056 & 15\end{array}$} & $3+$ & $2+4$ & 412115 & & & \\
\hline & & & $4150 \S \quad 15$ & & & \\
\hline $3097 \S 15$ & $1+$ & $0+2$ & & & & \\
\hline \multicolumn{3}{|l|}{$3132 \S \quad 15$} & & & & \\
\hline \multicolumn{7}{|c|}{$\begin{array}{l}\dagger \text { From comparison of } \sigma(\theta) \text { with theoretical CCBA calculations, using assumed L-transfers. For some of the levels, these } \\
\text { assignments differ from those in the Adopted Levels. See Adopted Levels for details. }\end{array}$} \\
\hline \multicolumn{7}{|c|}{ L-transfer assumed in theoretical CCBA calculations. } \\
\hline \multicolumn{7}{|c|}{$\S$ Unresolved multiplet. } \\
\hline \multicolumn{7}{|c|}{ \# 1259 and 1277 are not well resolved. } \\
\hline \multicolumn{7}{|c|}{ @ Common value for 1259 and 1277 levels. } \\
\hline \multicolumn{7}{|c|}{ \& Rounded value from Adopted Levels. Weak group in $\left(\mathrm{t},{ }^{3} \mathrm{He}\right)$} \\
\hline a Round & d value $f$ & om Adopted Levels. Not obs & erved in $\left(t,{ }^{3} \mathrm{He}\right)$, but & contril & on of weak gr & roup is not excluded. \\
\hline
\end{tabular}

\section{${ }^{43} \mathrm{Ca}\left(\mathrm{d},{ }^{3} \mathrm{He}\right) \quad 1969$ Yno1}

Target ${ }^{43} \mathrm{Ca} J \pi=7 / 2-$.

1969Yn01: $\mathrm{E}=22 \mathrm{MeV}$ deuteron beam was produced from the Argonne cyclotron. Enriched calcium target $\left(81.1 \%\right.$ in $\left.{ }^{43} \mathrm{Ca}\right)$.

Reaction products were detected with a $\Delta \mathrm{E}-\mathrm{E}$ telescope of surface-barrier detectors, FWHM=70-130 keV. Measured $\sigma(\theta)$. Deduced levels, L, spectroscopic factors from DWBA analysis.

Other: 1975BrZB (also 1973BrXY).

${ }^{42} \mathrm{~K}$ Levels

\begin{tabular}{|c|c|c|c|}
\hline $\mathrm{E}($ level) & $\mathrm{L}$ & $\mathrm{C}^{2} \mathrm{~S}^{\dagger}$ & Comments \\
\hline 0.0 & 2 & 0.36 & \\
\hline $104 \quad 40$ & 2 & 0.70 & \\
\hline 25240 & 2 & 0.92 & \\
\hline 630 & 0 & & $\mathrm{E}($ level),L: from $1973 \mathrm{BrXY}$ \\
\hline 68040 & 2 & 1.07 & $\begin{array}{l}\mathrm{E} \text { (level), L, } \mathrm{C}^{2} \mathrm{~S} \text { : this level is associated with } 699,5 \text { - level in Adopted dataset. May contain } \\
\text { contribution from } 639,3 \text { - level. }\end{array}$ \\
\hline $1200 \quad 40$ & 0 & 0.53 & E(level): probably a doublet in $1973 \mathrm{BrXY}$ \\
\hline $1460 \quad 40$ & $(0)$ & 0.08 & \\
\hline 163040 & & & \\
\hline 201040 & 0 & 0.27 & \\
\hline 213040 & 0 & 0.27 & \\
\hline $2700 \quad 40$ & & & $\mathrm{~L}: \sigma(\theta)$ fitted to $\mathrm{L}=2$ and 0 , with $\mathrm{L}=2$ fit somewhat preferred. \\
\hline
\end{tabular}




\section{${ }^{44} \mathrm{Ca}\left(\mathrm{p},{ }^{3} \mathrm{He}\right) \quad 1973 \mathrm{Du} 02$}

1973Du02: $\mathrm{E}=56 \mathrm{MeV}$ proton beam produced by the Grenoble variable energy cyclotron with intensities of 100 to $600 \mathrm{nA}$. Targets of self-supported calcium metal foils of about $1 \mathrm{mg} / \mathrm{cm}^{3}$. Reaction products were detected in a $\Delta \mathrm{E}-\mathrm{E}$ silicon counter telescope, FWHM $\approx 150 \mathrm{keV}$. Measured $\sigma(\theta)$. Deduced levels.

\section{${ }^{42} \mathrm{~K}$ Levels}

\begin{tabular}{|c|c|c|}
\hline $\mathrm{E}(\text { level })^{\dagger}$ & $\mathrm{J} \pi$ & Comments \\
\hline $0.0 \ddagger$ & & \\
\hline $260 \ddagger$ & & \\
\hline 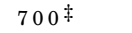 & & \\
\hline 1200 末 & & \\
\hline 2400 末 & & \\
\hline 6450 & $(0+)^{\S}$ & $\mathrm{T}=3$ \\
\hline
\end{tabular}

$\dagger$ Read from figure 5 of $1973 \mathrm{Du} 02$. Except for the analog state at 6450 , all other peak structures are unresolved multiplets.

‡ $\mathrm{T}=2$ proposed by $1973 \mathrm{Du} 02$ for group of states between ground state and 2400 level.

\section{${ }^{44} \mathrm{Ca}(\mathrm{d}, \alpha),(\operatorname{pol} \mathrm{d}, \alpha) \quad 1977 \mathrm{~Pa} 24,1982 \mathrm{Ba55}$}

Target ${ }^{44} \mathrm{Ca} \mathrm{J} \pi=0+$.

1977Pa24: $(\mathrm{d}, \alpha) \mathrm{E}=11 \mathrm{MeV}$ deuteron beam produced at a tandem Van de Graaff accelerator. Enriched calcium target $\left(98.6 \%\right.$ in $\left.{ }^{44} \mathrm{Ca}\right)$. Reaction products were momentum analyzed with a broad-range magnetic spectrograph of Browne-Buechner type and detected with a nuclear emulsion, FWHM=30 keV. Measured $\sigma\left(E_{\alpha}, \theta\right)$. Deduced levels, J $\pi$, L from DWBA analysis.

1982Ba55: (pol d, $\alpha$ ) E=7-8.25 MeV polarized deuteron beam produced at the Lamb-shift, polarized-ion source and FN tandem accelerator at McMaster University. Target of metallic calcium enriched to $98.5 \%$ in ${ }^{44} \mathrm{Ca}$ on thin carbon backings. $\alpha$-particles were momentum analyzed with an Engel split-pole magnetic spectrograph and detected by a position-sensitive, gas-filled, proportional counter in its focal plane, FWHM=25 keV. Measured $\sigma\left(\mathrm{E}_{\alpha}, \theta\right)$, $\mathrm{T}_{20}$ at $4^{\circ}$. Deduced levels, $J \pi$.

1974Fr10: $(\mathrm{d}, \alpha) \mathrm{E}=80.2 \mathrm{MeV}$ deuteron beam produced at the Orsay synchrocyclotron. $\alpha$-particles were analyzed with an magnetic spectrometer and detected by a position-sensitive detector. Measured $\sigma\left(\mathrm{E}_{\alpha}, \theta\right)$. Deduced levels, J $\pi$ from DWBA analysis for a 1910 group.

1974Le08 (also 1973LeYI): (d, $\alpha) \mathrm{E}=4.0 \mathrm{MeV}$ deuteron beam produced at the Virginia Polytechnic Institute and State University. Target of $\mathrm{CaCO}_{3}\left(98.55 \%\right.$ in $\left.{ }^{44} \mathrm{Ca}\right) . \alpha$-particles were detected with silicon surface-barrier detectors, FWHM=30-40 keV. Measured $\sigma\left(\mathrm{E}_{\alpha}, \theta\right)$. Deduced levels, L-transfers for nine states up to 1260 from DWBA analysis.

${ }^{42} \mathrm{~K}$ Levels

\begin{tabular}{|c|c|c|c|c|}
\hline $\mathrm{E}(\text { level })^{\dagger}$ & $\mathrm{J} \pi \ddagger$ & $L^{\S}$ & $\mathrm{d} \sigma / \mathrm{dW}(\max )(\mu \mathrm{b} / \mathrm{sr})^{\mathrm{c}}$ & Comments \\
\hline 0.0 & $2-\#$ & $1+3$ & 80 & $\mathrm{~L}: 40 \%(\mathrm{~L}=1)+60 \%(\mathrm{~L}=3)$ \\
\hline $103 \quad 15$ & & 3 & 30 & \\
\hline $253 \quad 15$ & $4-\#$ & $3+5$ & 32 & $\mathrm{~L}: 80 \%(\mathrm{~L}=3)+20 \%(\mathrm{~L}=5)$. \\
\hline $636 \quad 25$ & & & $<2$ & \\
\hline 682 & not $0-\#$ & & & $\mathrm{E}$ (level),J $\pi$ : from $1982 \mathrm{Ba} 55$, level not given in $1977 \mathrm{~Pa} 24$. \\
\hline $691 \quad 15$ & $5-@$ & 5 & 43 & \\
\hline $783^{\&} 4$ & $2-\#$ & & $<2$ & $\mathrm{~J} \pi$ : from $1982 \mathrm{Ba} 55$ \\
\hline $839 \quad 25$ & $(1-, 3-)^{@}$ & ( 1 ) & 7 & $\begin{array}{l}\mathrm{L}: \text { from } 1974 \mathrm{Le} 08 . \\
\mathrm{J} \pi: 1982 \mathrm{Ba} 55 \text { give } 3-.\end{array}$ \\
\hline $1108 \quad 15$ & $3+\# \mathrm{~b}$ & $4 \mathrm{~b}$ & 160 & $\mathrm{~L}: 1$ (1974Le08). \\
\hline $1201 \& 3$ & $4-\#$ & 3 & 75 & \\
\hline $1268 \quad 15$ & $(2,4)-\# b$ & $3^{b}$ & 80 & $\mathrm{~J} \pi$ : $1982 \mathrm{Ba} 55$ give $2-$, probably based on $\mathrm{L}=1$ from $1974 \mathrm{Le} 08$. \\
\hline 140315 & $(1,3)+\#$ & 2 & 30 & \\
\hline $1536 \quad 15$ & $(3,5)+\#$ & 4 & 70 & \\
\hline $1698^{\mathrm{a}} 15$ & & & 33 & \\
\hline $1749^{\mathrm{a}} 15$ & & & 27 & \\
\hline 1862 & $2-\#$ & & & $\mathrm{E}$ (level),J $\pi$ : from $1982 \mathrm{Ba} 55$, level not given in $1977 \mathrm{~Pa} 24$. \\
\hline $1916^{\mathrm{a}} \quad 15$ & & 6 & 31 & L: from $1974 \mathrm{Fr} 10$ for a 1910 group. \\
\hline $1944^{\mathrm{a}} 15$ & & & & \\
\hline
\end{tabular}


${ }^{44} \mathrm{Ca}(\mathrm{d}, \alpha),(\operatorname{pol} \mathrm{d}, \alpha) \quad 1977 \mathrm{Pa24}, 1982 \mathrm{Ba55}$ (continued)

${ }^{42} \mathrm{~K}$ Levels (continued)

$\frac{\mathrm{E}(\mathrm{level})^{\dagger}}{2056^{\&} 4} \frac{\mathrm{J} \pi^{\ddagger}}{(2,4)-^{\#}} \frac{\mathrm{L}^{\S}}{3} \frac{\mathrm{d} \sigma / \mathrm{dW}(\max )(\mu \mathrm{b} / \mathrm{sr})^{\mathrm{c}}}{60}$

$\mathrm{J} \pi, \mathrm{L}: 3+$ in Adopted Levels based on $\gamma$ decays and feeding, and $\pi=$ unnatural in ( $p o l d, \alpha)(1982 B a 55)$. $J \pi=(2,4)-$ was assigned by $1982 \mathrm{Ba} 55$ from $\mathrm{L}(\mathrm{d}, \alpha)=3$ in $1977 \mathrm{~Pa} 24$ and unnatural parity state in their work. The 2056-keV peak was weakly populated in the work of $1977 \mathrm{~Pa} 24$, and in evaluators' opinion $\mathrm{L}(\mathrm{d}, \alpha)=4$ can also satisfy the angular distribution pattern in $1977 \mathrm{~Pa} 24$, which can give $3+$. Other possibility is that level populated in $(d, \alpha)$ is different from that

$\begin{array}{lrllr}2186^{\&} & 4 & (3,5)+\# & 4 & 220 \\ 2314^{\&} 4 & (3,5)+\# & 4 & 30 \\ 2394 & 15 & & 4 & 35 \\ 2553 & 15 & & & 32 \\ 2625 & 15 & & & 7 \\ 2664 & 15 & & & 9 \\ 2750 & 15 & & (2) & 35 \\ 2802 & 15 & & (2) & 33 \\ 2858 & 15 & & & 37 \\ 3021 & 15 & & (3) & 37 \\ 3093 & 15 & & (2) & 36 \\ 3217 & 15 & & (1+3) & 62 \\ 3303 & 15 & & (1) & 35 \\ 3400^{\mathrm{a}} & 15 & & & 42 \\ 3502^{\mathrm{a}} & 15 & & & \end{array}$
in $(\mathrm{n}, \gamma)$.

$\dagger$ From 1977Pa24, unless otherwise stated.

¥ From L-transfer and (pol d, $\alpha$ ) data. $J \pi=0-, 1-, 2$ - for $L=1 ; J \pi=1+, 2+, 3+$ for $\mathrm{L}=2$; $J \pi=2-, 3-, 4-$ for $L=3 ; J \pi=3+, 4+, 5+$ for $L=4$; $\mathrm{J} \pi=4-, 5-, 6-$ for $\mathrm{L}=5 ; \mathrm{J} \pi=5+, 6+, 7+$ for $\mathrm{L}=6 ; 2-$ for $\mathrm{L}=1+3 ; 4-$ for $\mathrm{L}=3+5$. Specification of natural or unnatural parity state further limits $J \pi$.

$\S$ From 1977Pa24, unless otherwise stated.

\# Unnatural-parity state, not including $\mathrm{J} \pi=0-$, from (pol d, $\alpha$ ) (1982Ba55).

(a) Natural-parity state, not including $J \pi=0+$, from (pol d, $\alpha$ ) (1982Ba55)

\& From 1982Ba55.

a Closely-spaced levels not fully resolved.

b 1 in $1974 \mathrm{Le} 08$ gives $\mathrm{J} \pi=2-$.

c From $1977 \mathrm{~Pa} 24$ 


\section{Adopted Levels, Gammas}

$\mathrm{Q}\left(\beta^{-}\right)=-6426.1010 ; \mathrm{S}(\mathrm{n})=11480.676 ; \mathrm{S}(\mathrm{p})=10276.67 \quad 15 ; \mathrm{Q}(\alpha)=-6257.3425 \quad 2012 \mathrm{Wa} 38$

$\mathrm{S}(2 \mathrm{n})=19843.4915, \mathrm{~S}(2 \mathrm{p})=18085.2915(2012 \mathrm{Wa} 38)$.

Identification of stable ${ }^{42} \mathrm{Ca}$ by F.W. Aston, Nature 133, 684 (1934) through mass spectrographic studies.

${ }^{42} \mathrm{Ca}(\mathrm{n}, \mathrm{n})$ : 1989Ra06: $\mathrm{E}=$ thermal. Measured Bragg diffraction patterns, deduced scattering lengths.

${ }^{42} \mathrm{Ca}\left({ }^{3} \mathrm{He},{ }^{3} \mathrm{He}\right)$ : 1971Ra35: $\mathrm{E}=13.0 \mathrm{MeV}$; 1973Mo13: $\mathrm{E}=28 \mathrm{MeV}$. Measured $\sigma(\theta)$.

${ }^{42} \mathrm{Ca}\left({ }^{48} \mathrm{Ti},{ }^{48} \mathrm{Ti}\right): 1990 \mathrm{Vo} 07,1988 \mathrm{Br} 02: \mathrm{E}=240-725 \mathrm{MeV}$. Measured $\sigma(\theta)$, DWBA analysis.

Hyperfine structure and isotope-shift measurements: $2000 \mathrm{Mu} 17,2015 \mathrm{Go} 24$.

Population of levels in decays/reactions labeled with XREF=Y

${ }^{42} \mathrm{~K} \quad \beta^{-}(12.355 \mathrm{~h}): 0,1525,1837,2424,2752,3447$

${ }^{42} \mathrm{Sc} \varepsilon$ decay $(680.79 \mathrm{~ms}): 0,1525,1837$

${ }^{42} \mathrm{Sc} \varepsilon$ decay $(61.7 \mathrm{~s}): 0,1525,2424,2752,3189$

${ }^{38} \mathrm{Ar}\left({ }^{6} \mathrm{Li}, \mathrm{d}\right): 0,1525, \quad 1837,2424,2752,3300,3654,4443,4448,5866,6016+6020,6313+6390,6516,6716+6720$

${ }^{40} \mathrm{Ar}\left({ }^{3} \mathrm{He}, \mathrm{n}\right): 0,1525,1837,2424,3300(?), 3392,3654,9270,10205,14700$

${ }^{40} \mathrm{Ca}(\mathrm{t}, \mathrm{p} \gamma): 0,1520,2420,3890,5850,6020,6520,6700,6820$

${ }^{40} \mathrm{Ca}\left(\alpha,{ }^{2} \mathrm{He}\right): 0,1530,2750,3190,3660,4830,5380,7280,8810,9080,9330,9600,9870,10160$

${ }^{40} \mathrm{Ca}\left({ }^{14} \mathrm{C},{ }^{12} \mathrm{C}\right),\left({ }^{12} \mathrm{C},{ }^{10} \mathrm{C}\right),: 0,1700,2800,3500,4800$

${ }^{40} \mathrm{Ca}\left({ }^{96} \mathrm{Zr},{ }^{94} \mathrm{Zr}\right): 0,5866$

${ }^{41} \mathrm{Ca}(\mathrm{n}, \gamma) \quad \mathrm{E}=\mathrm{t}$ hermal : $0, \quad 1524.7,2424.2,2752.4,3253.9,3446.9,3954.4,3999.7,4690.1,4759.7, \quad 5017.1, \quad 11480.7$

${ }^{42} \mathrm{Ca}(\gamma, \gamma): 0,1525$

${ }^{42} \mathrm{Ca}\left(\pi+, \pi+^{\prime}\right),\left(\pi-, \pi-^{\prime}\right): 0,1520,2420,3440,4104,4680,6300$

${ }^{42} \mathrm{Ca}\left(\mathrm{d}, \mathrm{d}^{\prime}\right): 0,1524,1835,2423,2749,3445$

${ }^{42} \mathrm{Ca}\left({ }^{16} \mathrm{O},{ }^{16} \mathrm{O}^{\prime}\right): 0,1525,1837,2424,2752,3254,3447,4100,4449,4690,4971$

Coulomb excitation: 0,1525

${ }^{45} \mathrm{Sc}(\mathrm{p}, \alpha),(\mathrm{pol} \mathrm{p}, \alpha): 0,1525,1837,2424,2752,3190,3254,3954$,

4100,4117

${ }^{46} \mathrm{Ti}\left(\mathrm{d},{ }^{6} \mathrm{Li}\right): 0,1525,1837$

${ }^{96} \mathrm{Zr}\left({ }^{40} \mathrm{Ca},{ }^{42} \mathrm{Ca} \gamma\right): 0,1525, \quad 1837,2424,2752,3189,3254$,

$3447,3654,3954,4443,4760,5017,5866$

Some recent theoretical structure references (levels, B(E2), etc.): 2016Wo02 (shape coexistence), 2012Ca13, $2012 \mathrm{Ca} 27,2012 \mathrm{Ha} 26$.

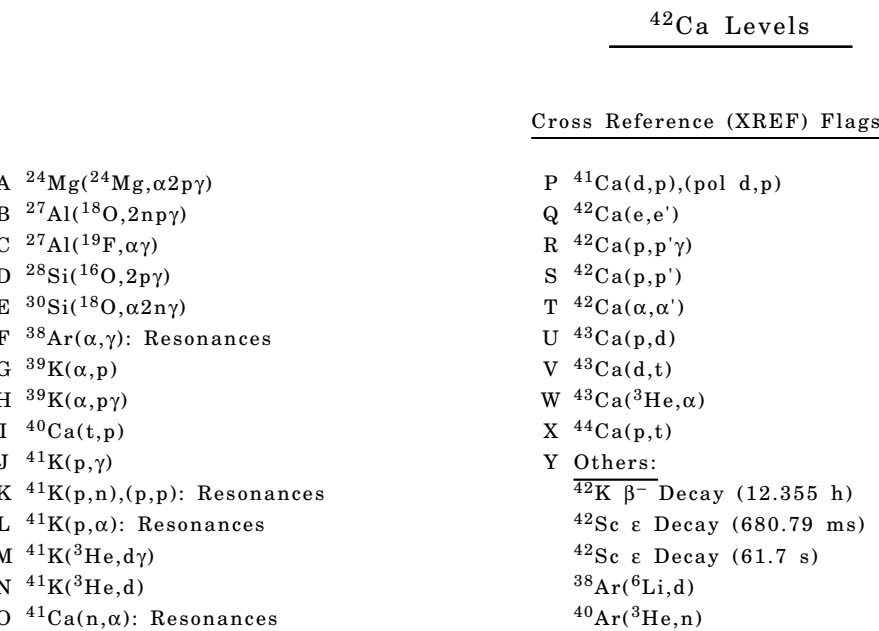

E(level) ${ }^{\dagger}$

$\mathrm{J} \pi \S$

0.0
XREF

ABC E GHIJ MN PQRSTUVWXY
$0+$
$\mathrm{T}_{1 / 2} \ddagger$

$\mathrm{stable}$
${ }^{40} \mathrm{Ca}(\mathrm{t}, \mathrm{p} \gamma)$

${ }^{40} \mathrm{Ca}\left(\alpha,{ }^{2} \mathrm{He}\right)$

${ }^{40} \mathrm{Ca}\left({ }^{14} \mathrm{C},{ }^{12} \mathrm{C}\right),\left({ }^{12} \mathrm{C},{ }^{10} \mathrm{C}\right) .$.

${ }^{40} \mathrm{Ca}\left({ }^{96} \mathrm{Zr},{ }^{94} \mathrm{Zr}\right)$

${ }^{41} \mathrm{Ca}(\mathrm{n}, \gamma) \quad \mathrm{E}=$ thermal

${ }^{42} \mathrm{Ca}(\gamma, \gamma)$

${ }^{42} \mathrm{Ca}\left(\pi^{+}, \pi^{+\prime}\right),\left(\pi^{-}, \pi^{-+}\right)$

${ }^{42} \mathrm{Ca}\left(\mathrm{d}, \mathrm{d}^{\prime}\right)$

${ }^{42} \mathrm{Ca}\left({ }^{16} \mathrm{O},{ }^{16} \mathrm{O}^{\prime}\right)$

Coulomb Excitation

${ }^{45} \operatorname{Sc}(\mathrm{p}, \alpha),($ pol $\mathrm{p}, \alpha)$

${ }^{46} \mathrm{Ti}\left(\mathrm{d},{ }^{6} \mathrm{Li}\right)$

${ }^{96} \mathrm{Zr}\left({ }^{40} \mathrm{Ca},{ }^{42} \mathrm{Ca} \gamma\right)$
Comments

The rms charge radius $\left(<\mathrm{r}^{2}>\right)^{1 / 2}$ : $3.5081 \mathrm{fm} 21$ (2013An02 evaluation). $\mathrm{J} \pi: \mathrm{L}(\mathrm{t}, \mathrm{p})=\mathrm{L}(\mathrm{p}, \mathrm{t})=0$ from $0+$ Adopted (1977En02) neutron-stripping spectroscopic factor $=1.62(\mathrm{~L}=3)$.

Adopted (1977En02) neutron-pickup spectroscopic factor $=0.586(\mathrm{~L}=3)$.

Adopted (1977En02) proton-stripping spectroscopic factor $=3.24(\mathrm{~L}=2)$. 
Adopted Levels, Gammas (continued)

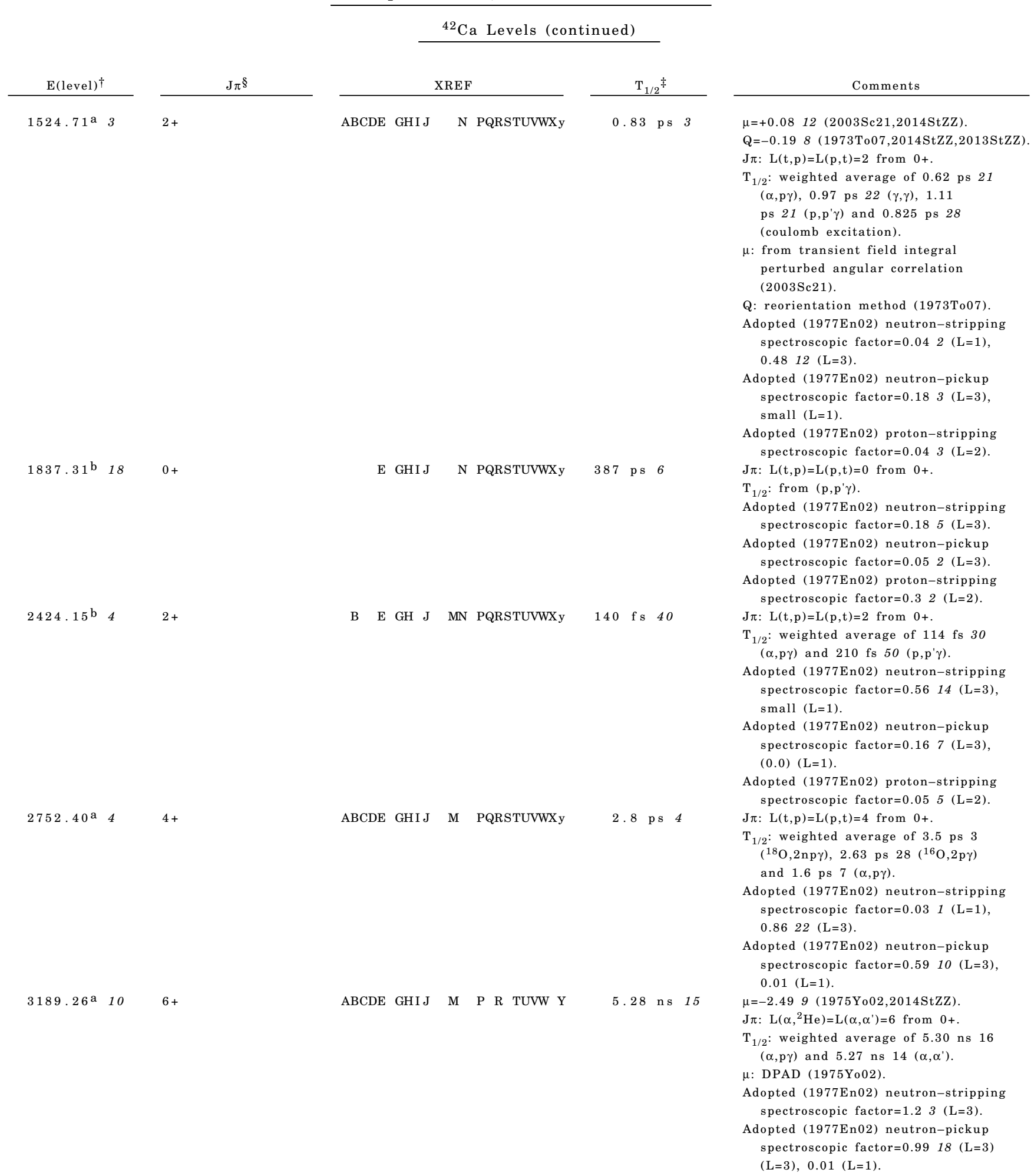


Adopted Levels, Gammas (continued)

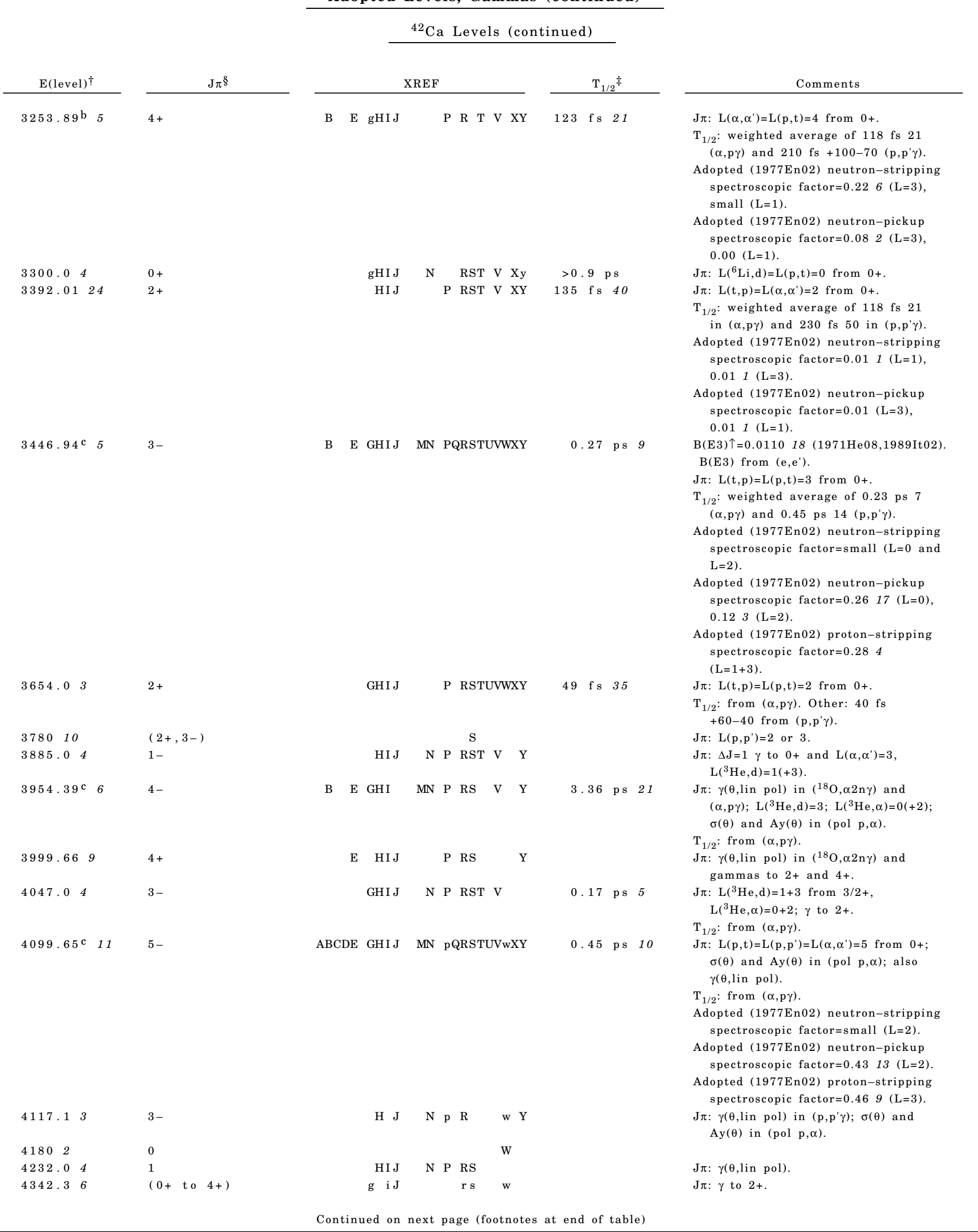


Adopted Levels, Gammas (continued)

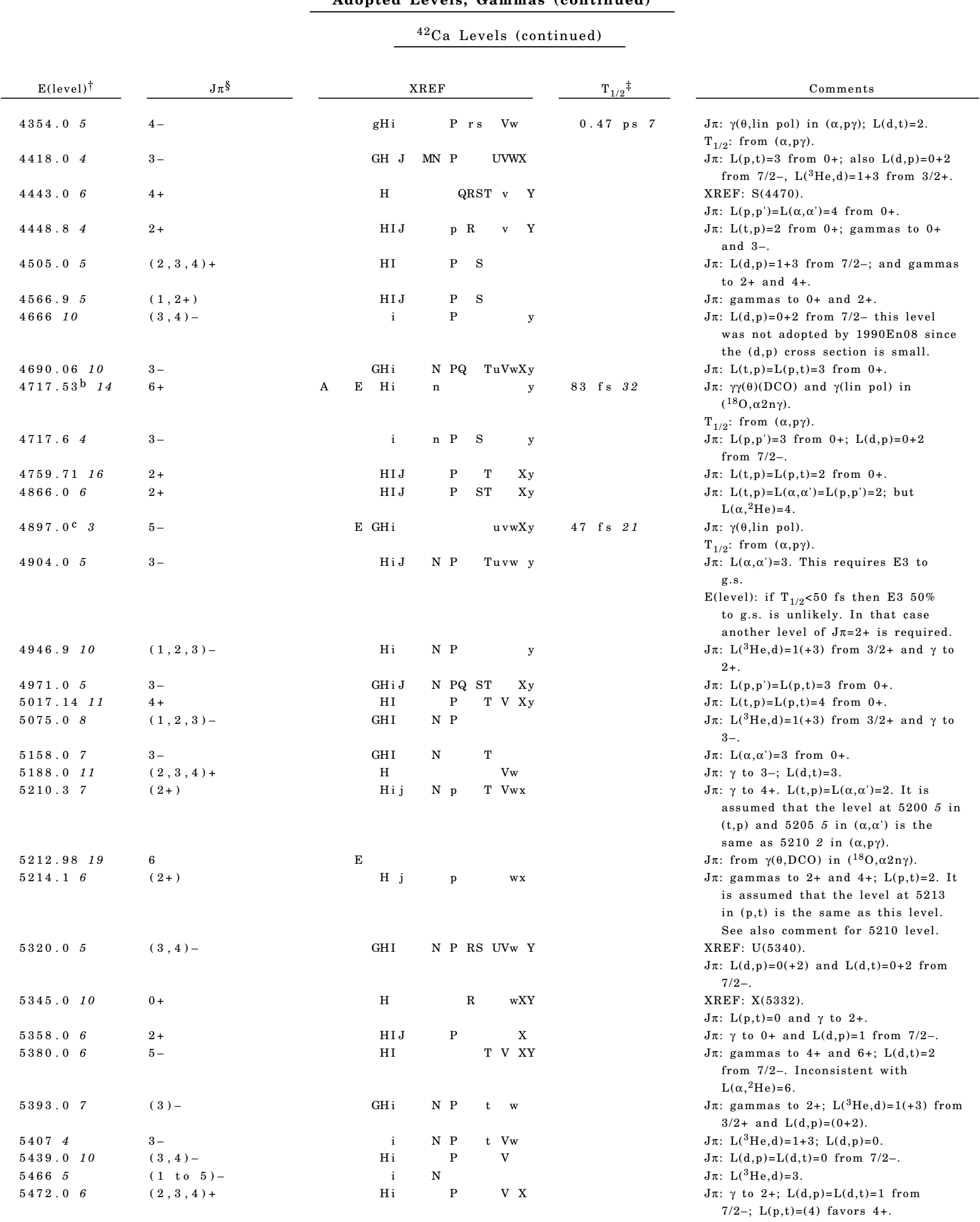

Continued on next page (footnotes at end of table) 
Adopted Levels, Gammas (continued)

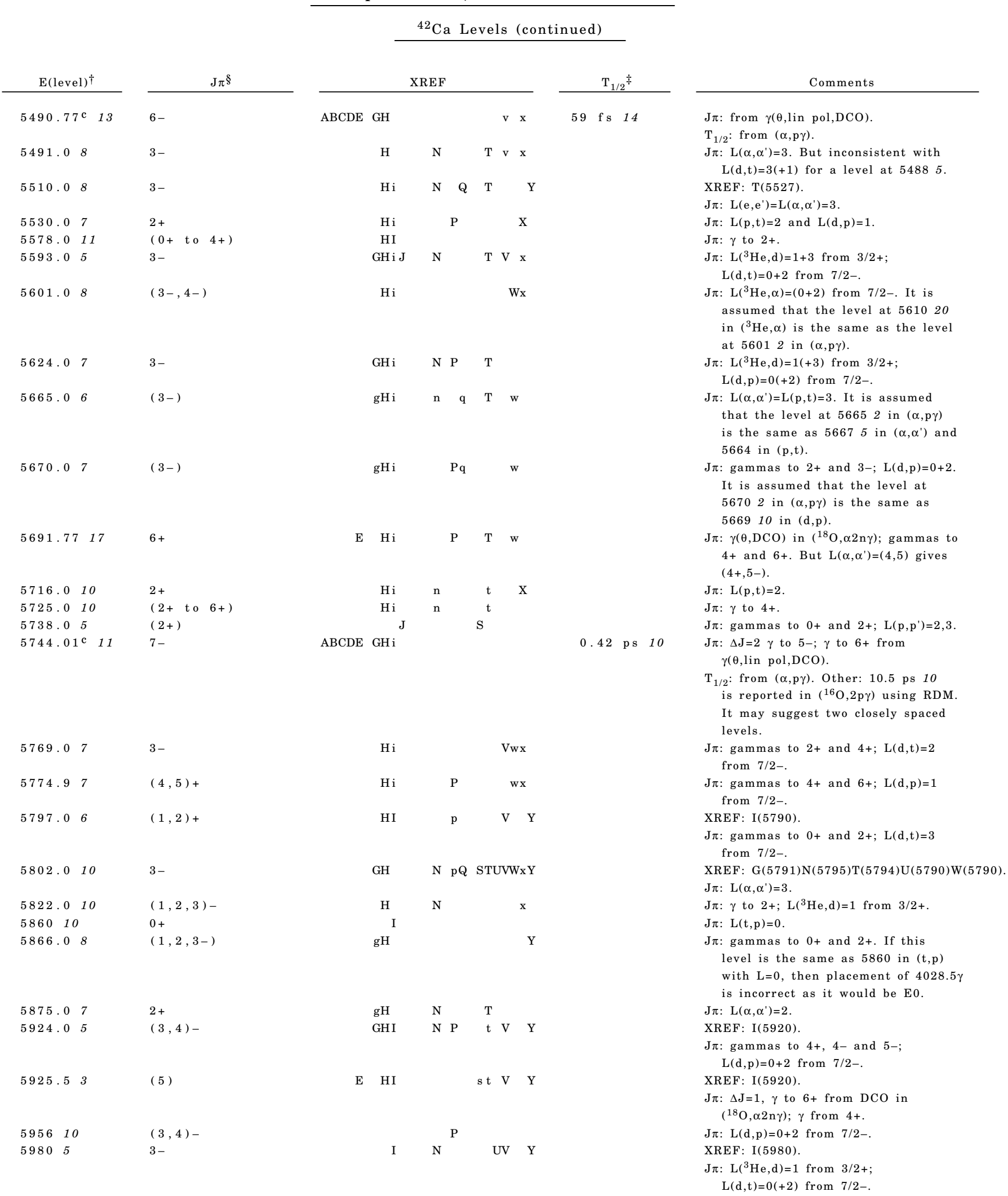


Adopted Levels, Gammas (continued)

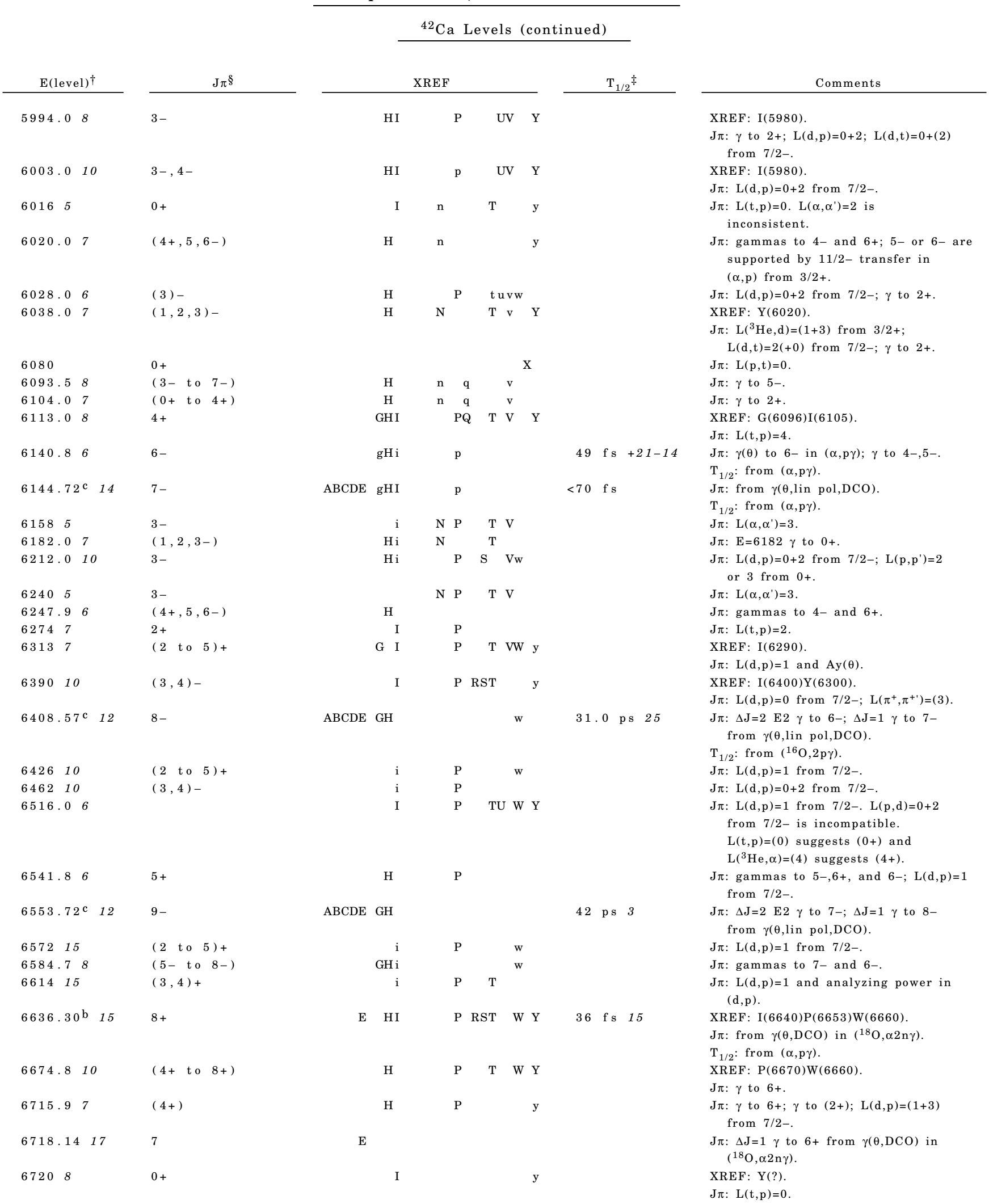


Adopted Levels, Gammas (continued)

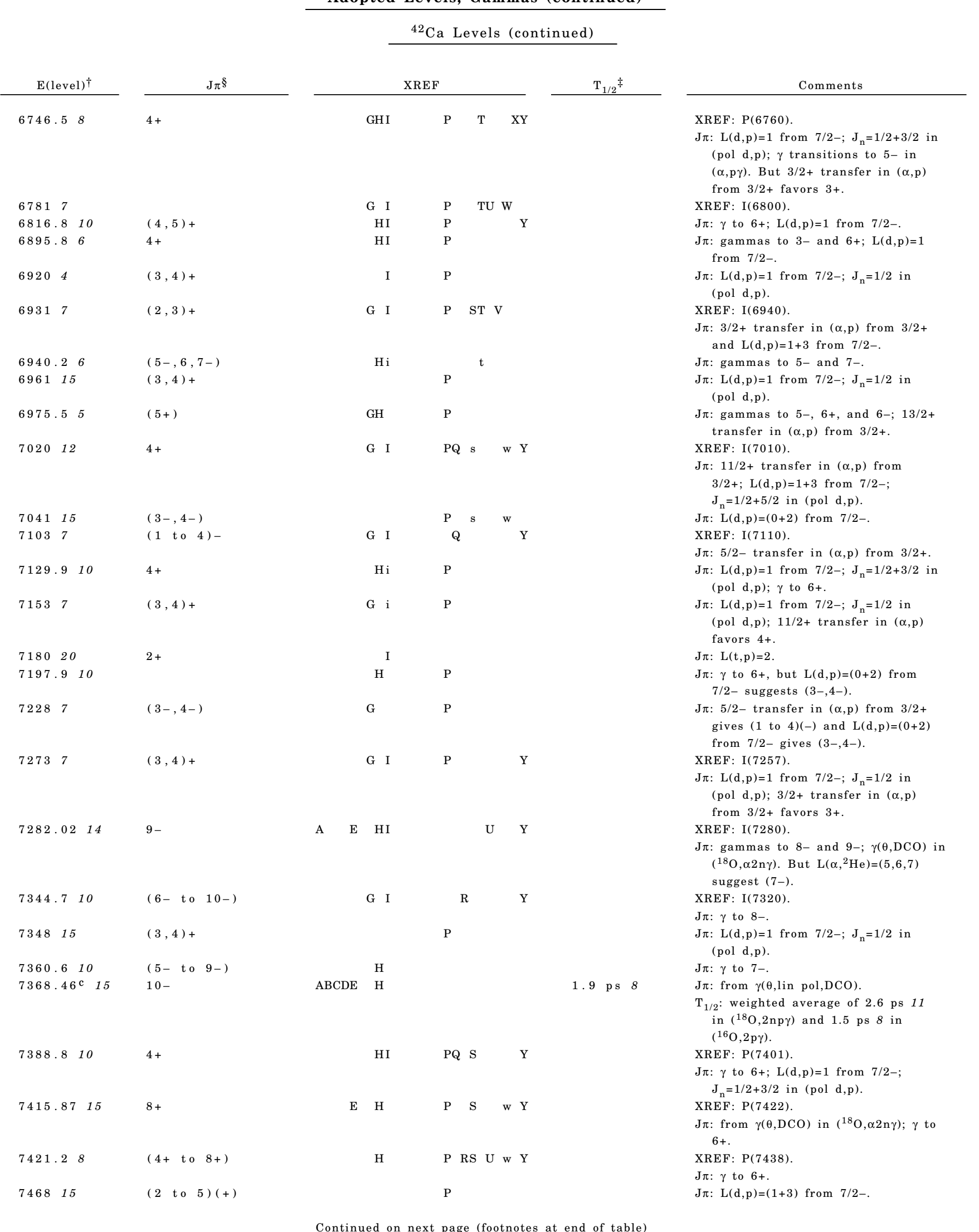


Adopted Levels, Gammas (continued)

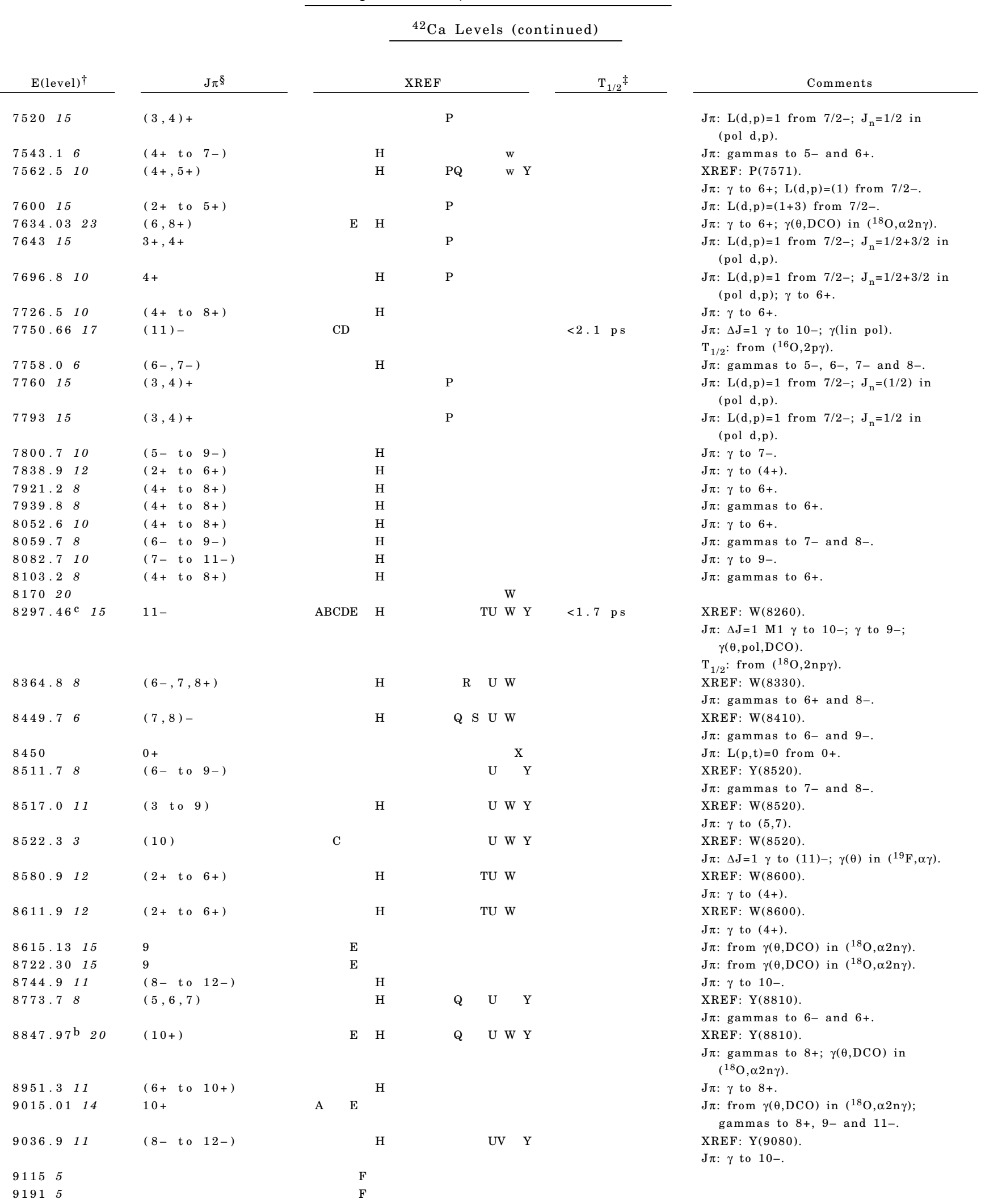


Adopted Levels, Gammas (continued)

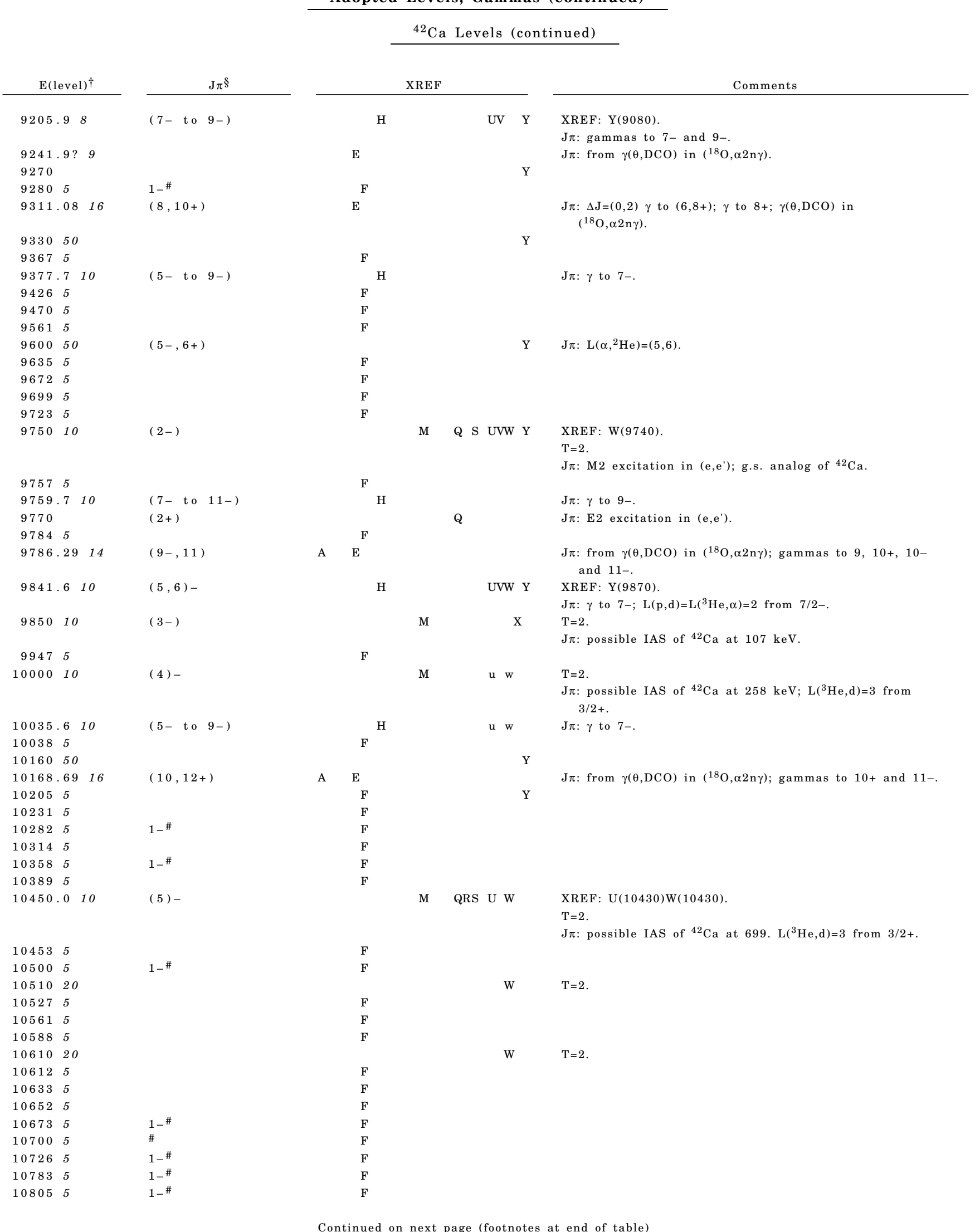


Adopted Levels, Gammas (continued)

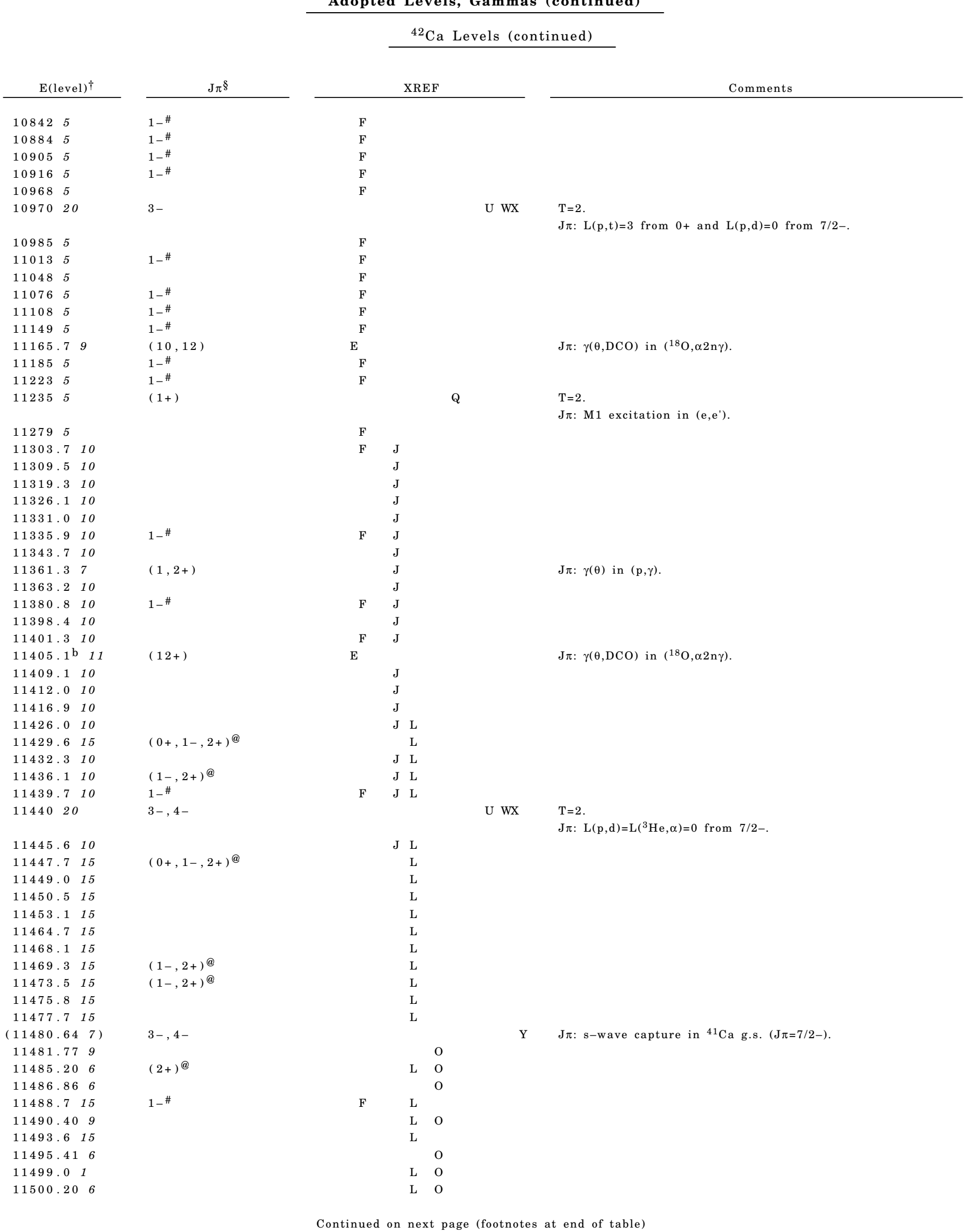


Adopted Levels, Gammas (continued)

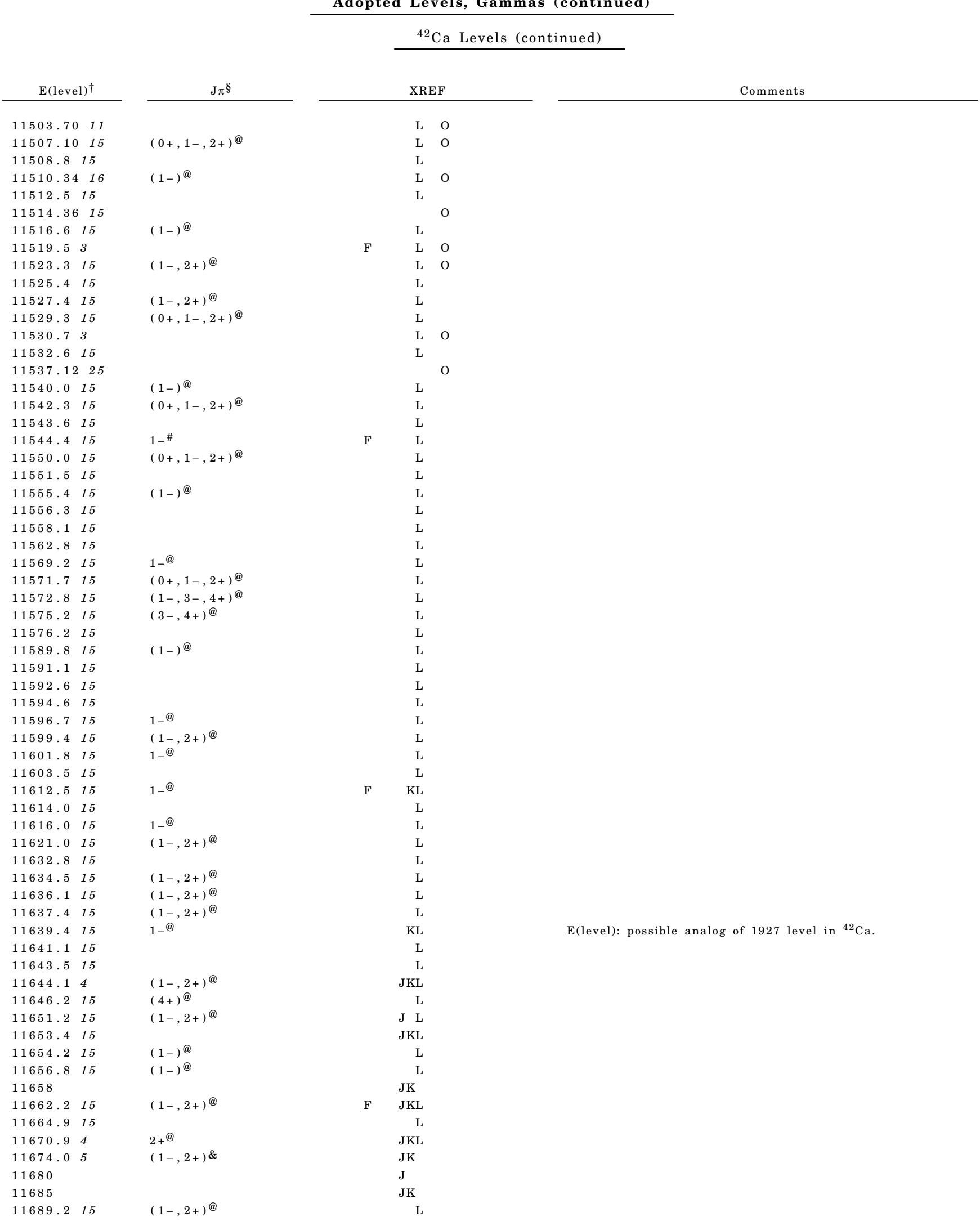


Adopted Levels, Gammas (continued)

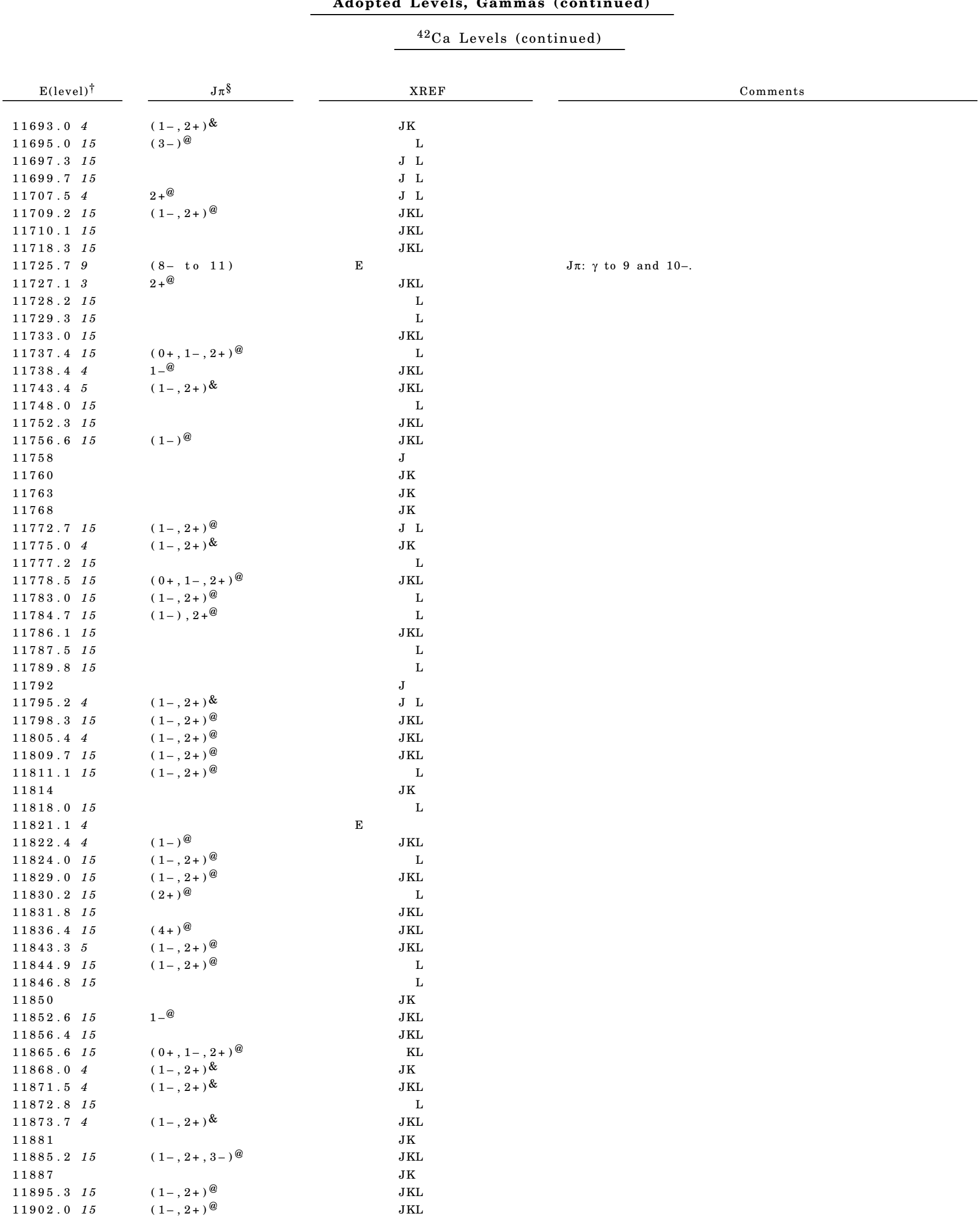


Adopted Levels, Gammas (continued)

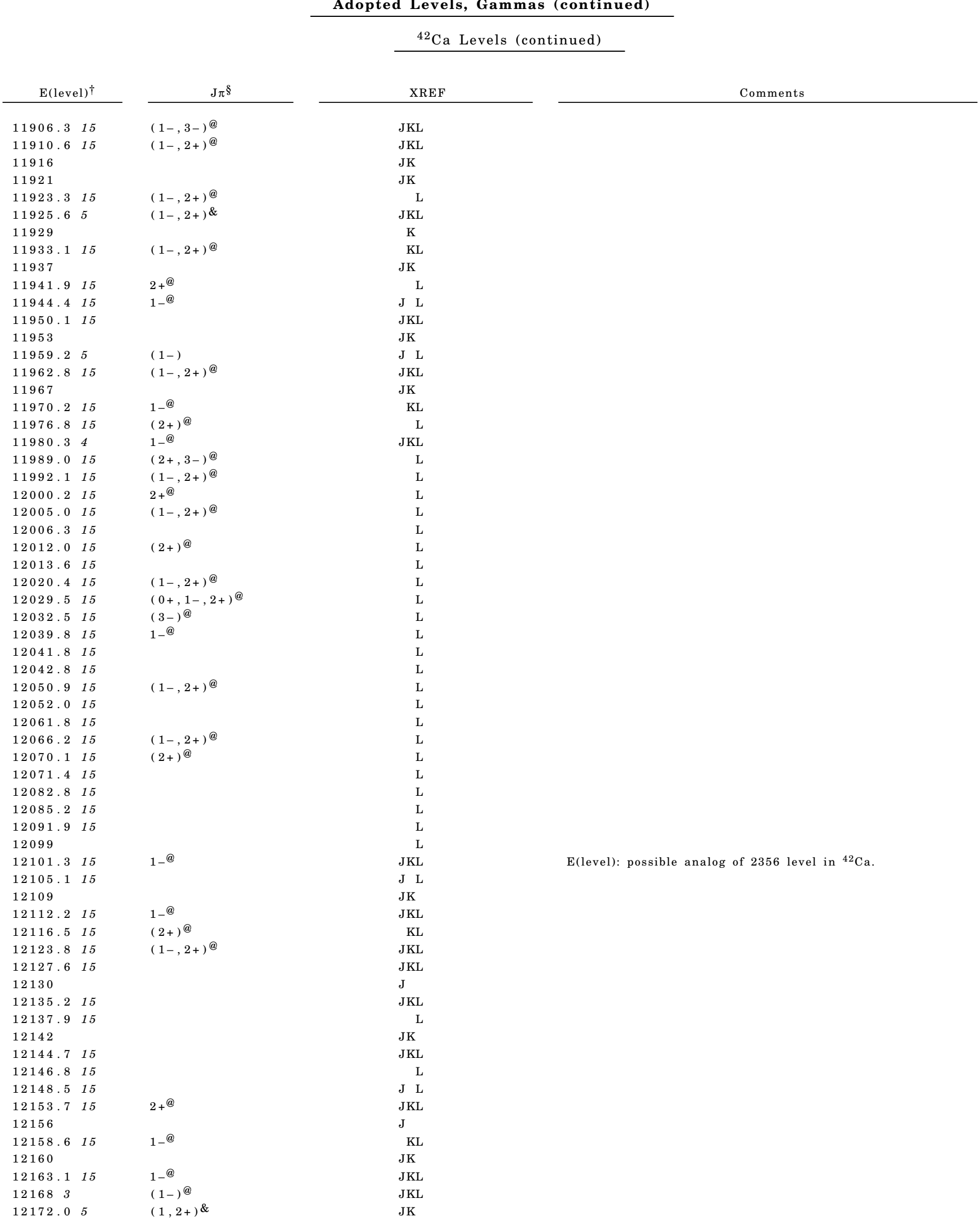


Adopted Levels, Gammas (continued)

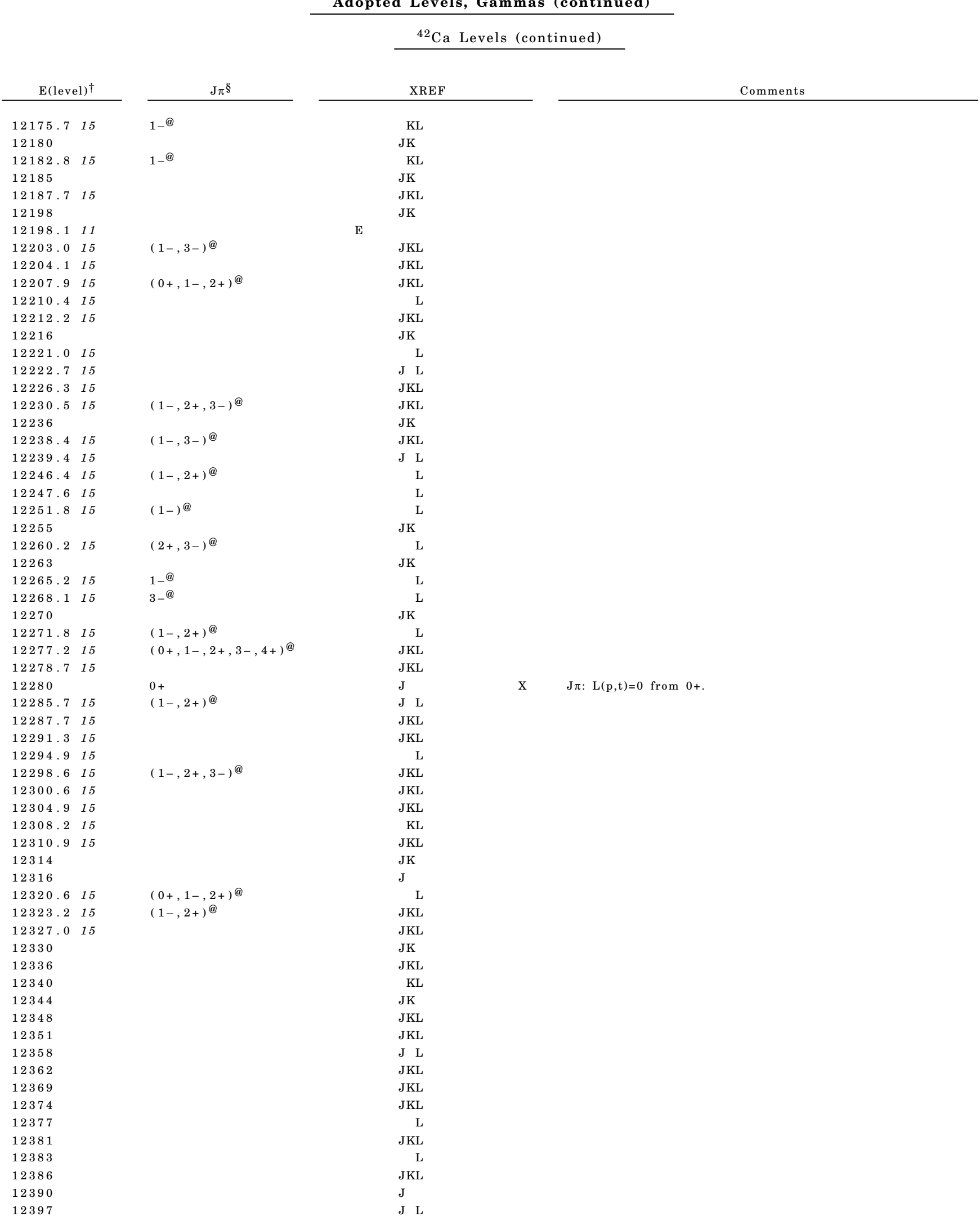


Adopted Levels, Gammas (continued)

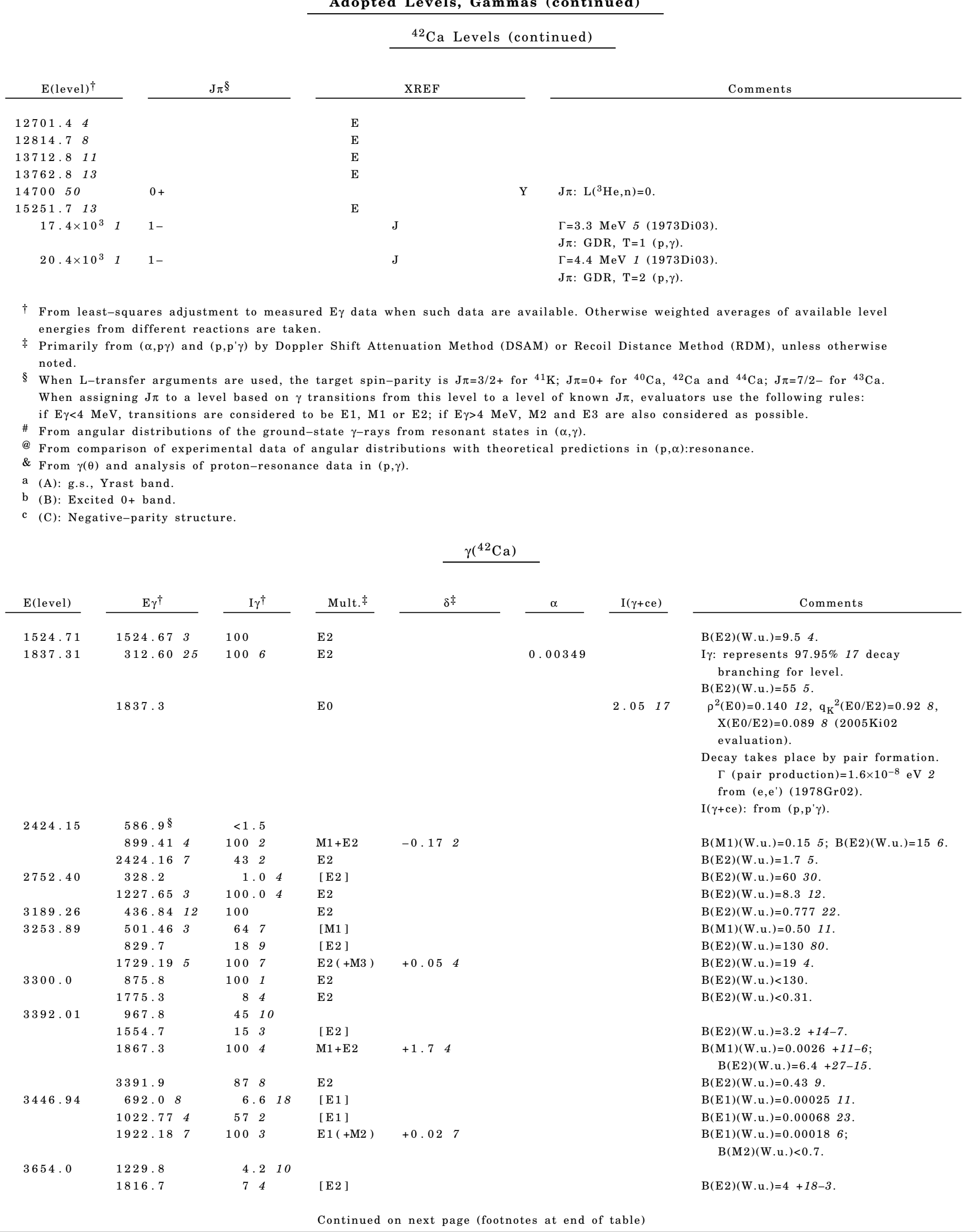


Adopted Levels, Gammas (continued)

\begin{tabular}{|c|c|c|c|c|c|c|}
\hline E(level) & $\mathrm{E} \gamma^{\dagger}$ & \multicolumn{2}{|c|}{$\mathrm{I} \gamma^{\dagger}$} & \multirow{3}{*}{$\begin{array}{l}\text { Mult. } \neq \\
\text { M1 (+E2) } \\
\text { E2 }\end{array}$} & $\delta^{\ddagger}$ & Comments \\
\hline \multirow[t]{2}{*}{3654.0} & 2129.2 & 100 & 3 & & $-0.06 \quad 17$ & $\mathrm{~B}(\mathrm{M} 1)(\mathrm{W} . \mathrm{u})=.0.03525 ; \mathrm{B}(\mathrm{E} 2)(\mathrm{W} . \mathrm{u})<0.55$. \\
\hline & 3653.8 & 22 & 210 & & & $\mathrm{~B}(\mathrm{E} 2)(\mathrm{W} . \mathrm{u})=0.3425$. \\
\hline \multirow[t]{3}{*}{3885.0} & 1460.8 & 4 & 2 & & & \\
\hline & 2047.6 & 93 & 3 & $\mathrm{E} 1$ & & \\
\hline & 3884.8 & 100 & 4 & $\mathrm{E} 1$ & & \\
\hline \multirow[t]{2}{*}{3954.39} & $507.45 \quad 3$ & 100 & 4 & $\mathrm{M} 1+\mathrm{E} 2$ & +0.115 & $\mathrm{~B}(\mathrm{M} 1)(\mathrm{W} . \mathrm{u})=.0.0424 ; \mathrm{B}(\mathrm{E} 2)(\mathrm{W} \cdot \mathrm{u})=.6+6-4$ \\
\hline & 1202.0 & 18 & 4 & $\mathrm{E} 1$ & & $\mathrm{~B}(\mathrm{E} 1)(\mathrm{W} \cdot \mathrm{u})=.1.5 \times 10^{-5} 4$ \\
\hline \multirow[t]{3}{*}{3999.66} & 1247.2 & 7 & 4 & & & \\
\hline & 1575.5 & 49 & 5 & & & \\
\hline & $2474.80 \quad 10$ & 100 & 5 & & & \\
\hline \multirow[t]{4}{*}{4047.0} & 600.1 & 22 & 5 & $\mathrm{M} 1+\mathrm{E} 2$ & $+0.21 \quad 12$ & $\mathrm{~B}(\mathrm{M} 1)(\mathrm{W} . \mathrm{u})=.0.073 ; \mathrm{B}(\mathrm{E} 2)(\mathrm{W} . \mathrm{u})=.30+70-25$. \\
\hline & 1294.6 & 29 & 8 & [ E 1 ] & & $\mathrm{B}(\mathrm{E} 1)(\mathrm{W} . \mathrm{u})=0.0002411$. \\
\hline & 1622.8 & 29 & 10 & [E1] & & $\mathrm{B}(\mathrm{E} 1)(\mathrm{W} . \mathrm{u})=0.000126$. \\
\hline & 2522.2 & 100 & 11 & [ E 1 ] & & $\mathrm{B}(\mathrm{E} 1)(\mathrm{W} . \mathrm{u})=0.000114$. \\
\hline \multirow[t]{3}{*}{4099.65} & 652.8 & & & & & \\
\hline & $910.37 \quad 15$ & 100 & 3 & $\mathrm{E} 1(+\mathrm{M} 2)$ & +0.042 & $\mathrm{~B}(\mathrm{E} 1)(\mathrm{W} . \mathrm{u})=$.0.0010224 . \\
\hline & $1347.26 \quad 14$ & 61 & 3 & $\mathrm{E} 1+\mathrm{M} 2$ & -0.094 & $\mathrm{~B}(\mathrm{E} 1)(\mathrm{W} . \mathrm{u})=0.000195$. \\
\hline \multirow[t]{3}{*}{4117.1} & 670.1 & 61 & 11 & & & \\
\hline & 1692.8 & 21 & 6 & & & \\
\hline & 2592.2 & 100 & 7 & & & \\
\hline \multirow[t]{2}{*}{4232.0} & 1807.8 & 27 & 5 & & & \\
\hline & 4231.8 & 100 & 5 & & & \\
\hline 4342.3 & 1918.1 & 100 & & & & \\
\hline 4354.0 & 399.6 & 9 & 2 & $\mathrm{M} 1+\mathrm{E} 2$ & $>0.09$ & $\delta:$ also, $<-0.09$ \\
\hline & & & & & & $\mathrm{B}(\mathrm{M} 1)(\mathrm{W} . \mathrm{u})<.0.046 ; \mathrm{B}(\mathrm{E} 2)(\mathrm{W} . \mathrm{u})>$.3.9 . \\
\hline & 907.0 & 53 & 9 & & & \\
\hline & 1100.1 & 20 & 5 & {$[\mathrm{E} 1]$} & & $\mathrm{B}(\mathrm{E} 1)(\mathrm{W} . \mathrm{u})=0.000103$. \\
\hline & 1601.6 & 100 & 9 & $\mathrm{E} 1$ & & $\mathrm{~B}(\mathrm{E} 1)(\mathrm{W} . \mathrm{u})=0.000163$. \\
\hline 4418.0 & 971.0 & 67 & 12 & & & \\
\hline & 1993.8 & 25 & 8 & & & \\
\hline & 2893.2 & 100 & 13 & & & \\
\hline 4443.0 & 1189.1 & 10 & 3 & & & \\
\hline & 1690.6 & 100 & 5 & & & \\
\hline & 2018.8 & 16 & 4 & & & \\
\hline 4448.8 & 794.8 & 34 & 6 & & & \\
\hline & 1001.8 & 37 & 9 & & & \\
\hline & 2611.4 & 49 & 11 & & & \\
\hline & 2924.0 & 100 & 17 & & & \\
\hline & 4448.5 & 66 & 14 & & & \\
\hline 4505.0 & 1113.0 & 34 & 6 & & & \\
\hline & 1251.1 & 15 & 4 & & & \\
\hline & 1752.6 & 64 & 11 & & & \\
\hline & 2980.2 & 100 & 13 & & & \\
\hline 4566.9 & 682.0 & 47 & 9 & & & \\
\hline & 2142.8 & 100 & 9 & & & \\
\hline & 3042.2 & 100 & 9 & & & $\mathrm{I} \gamma: \mathrm{I} \gamma(3043) / \mathrm{I} \gamma(2143)=78 / 44$ in $(\mathrm{p}, \gamma)$ \\
\hline & 4566 & & & & & $\mathrm{I} \gamma: \mathrm{I} \gamma(4566) / \mathrm{I} \gamma(2143)=100 / 44$ in $(\mathrm{p}, \gamma)$ \\
\hline 4690.06 & 2265.8 & 22 & 6 & & & \\
\hline & $3165.24 \quad 11$ & 100 & 6 & & & \\
\hline 4717.53 & $1463.7 \quad 3$ & 95 & 5 & [ E 2 ] & & $\mathrm{B}(\mathrm{E} 2)(\mathrm{W} \cdot \mathrm{u} \cdot)=48+30-13$ \\
\hline & 1525.5 & 35 & 7 & & & \\
\hline & 1965.24 & 100 & 3 & [E2 ] & & $\mathrm{B}(\mathrm{E} 2)(\mathrm{W} \cdot \mathrm{u})=.12+7-3$ \\
\hline 4759.71 & $2335.70 \quad 30$ & 57 & 29 & & & \\
\hline & 2922.5 & 43 & 14 & & & \\
\hline & 3235.1 & 86 & 29 & & & \\
\hline & 4759.6 & 100 & 29 & & & \\
\hline 4866.0 & 2441.8 & 100 & 9 & & & \\
\hline & 3341.2 & 22 & 9 & & & \\
\hline & 4865.7 & 22 & 9 & & & $\mathrm{I} \gamma: \mathrm{I} \gamma(4866) / \mathrm{I} \gamma(3341)=100 / 100$ in $(p, \gamma)$ \\
\hline 4897.0 & 779.0 & 8 & 1 & {$[\mathrm{E} 2]$} & & $\begin{array}{l}\text { I } \gamma \text { : branching most likely incorrect. } \\
\mathrm{B}(\mathrm{E} 2)(\mathrm{W} . \mathrm{u} .)=310150\end{array}$ \\
\hline & $797.8 \quad 3$ & 100 & 4 & $\mathrm{M} 1+\mathrm{E} 2$ & +0.148 & $\mathrm{~B}(\mathrm{M} 1)(\mathrm{W} \cdot \mathrm{u})=.0.74 ; \mathrm{B}(\mathrm{E} 2)(\mathrm{W} \cdot \mathrm{u})=.70+230-60$. \\
\hline & 1449.0 & 18 & 4 & $\mathrm{E} 2$ & & $\mathrm{~B}(\mathrm{E} 2)(\mathrm{W} . \mathrm{u})=3116$. \\
\hline
\end{tabular}


Adopted Levels, Gammas (continued)

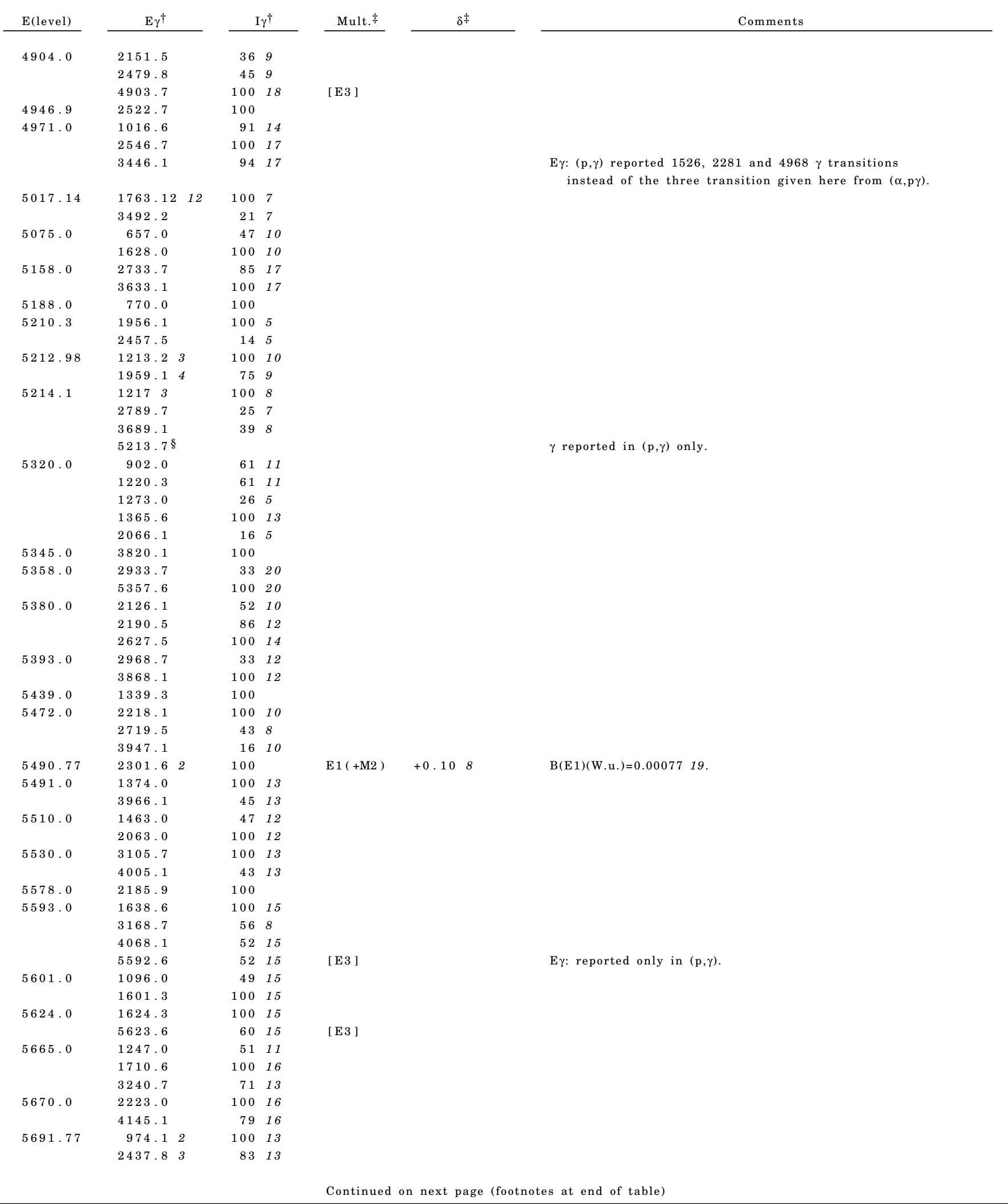


Adopted Levels, Gammas (continued)

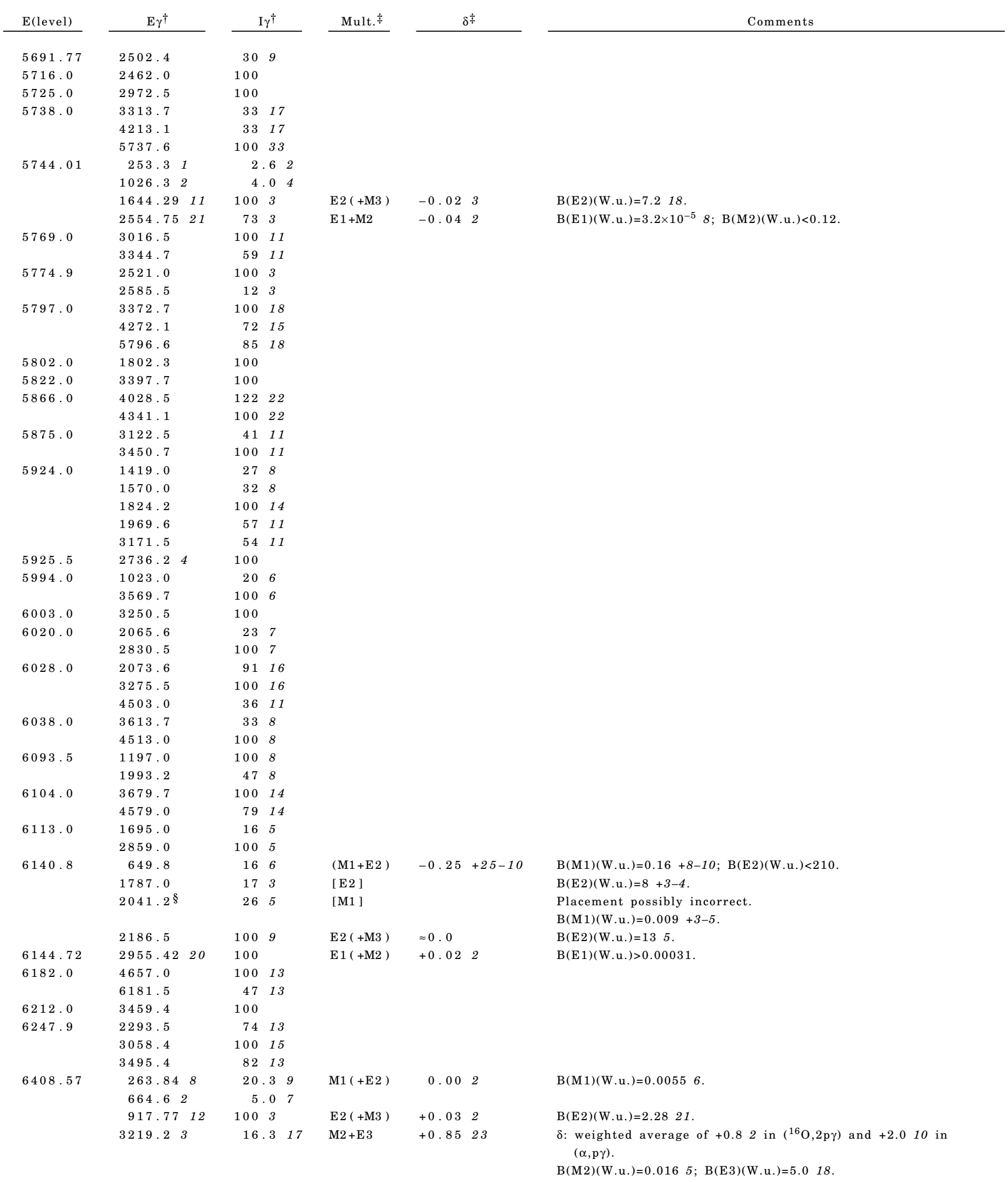


Adopted Levels, Gammas (continued)

\begin{tabular}{|c|c|c|c|c|c|c|c|}
\hline \multirow{2}{*}{$\begin{array}{l}\text { E(level) } \\
6516.0\end{array}$} & $\mathrm{E} \gamma^{\dagger}$ & \multicolumn{2}{|c|}{$\mathrm{I} \gamma^{\dagger}$} & \multirow[t]{4}{*}{ Mult. ${ }^{*}$} & \multicolumn{2}{|c|}{$\delta^{\ddagger}$} & Comments \\
\hline & 2630.9 & & & & & & $\mathrm{E} \gamma$ : from $\left({ }^{14} \mathrm{C},{ }^{12} \mathrm{C}\right)$ only. \\
\hline & 4091.6 & & & & & & $\mathrm{E} \gamma$ : from $\left({ }^{14} \mathrm{C},{ }^{12} \mathrm{C}\right)$ only. \\
\hline & 4991.0 & & & & & & $\mathrm{E} \gamma$ : from $\left({ }^{14} \mathrm{C},{ }^{12} \mathrm{C}\right)$ only. \\
\hline \multirow[t]{3}{*}{6541.8} & 1050.8 & 43 & 7 & & & & \\
\hline & 2442.2 & 29 & 7 & & & & \\
\hline & 3352.4 & 100 & 10 & & & & \\
\hline \multirow[t]{4}{*}{6553.72} & $145.12 \quad 10$ & 36 & $8 \quad 17$ & $\mathrm{M} 1(+\mathrm{E} 2)$ & 0.00 & 2 & $\mathrm{~B}(\mathrm{M} 1)(\mathrm{W} \cdot \mathrm{u})=0.0424$. \\
\hline & 409.12 & 3 & $0 \quad 5$ & & & & E $\gamma:$ reported only in $\left({ }^{18} \mathrm{O}, \alpha 2 \mathrm{n} \gamma\right)$ \\
\hline & $809.73 \quad 10$ & 100 & 2 & $\mathrm{E} 2(+\mathrm{M} 3)$ & -0.03 & 2 & $\mathrm{~B}(\mathrm{E} 2)(\mathrm{W} . \mathrm{u})=2.9623$. \\
\hline & $3364.4 \quad 3$ & 11 & 1 & [ E 3 ] & & & $\begin{array}{l}\text { E } \gamma: \text { reported only in }\left({ }^{18} \mathrm{O}, \alpha 2 \mathrm{n} \gamma\right) \\
\mathrm{B}(\mathrm{E} 3)(\mathrm{W} . \mathrm{u} .)=4.15\end{array}$ \\
\hline \multirow[t]{2}{*}{6584.7} & 840.7 & 100 & 11 & & & & \\
\hline & 1093.8 & 85 & 11 & & & & \\
\hline \multirow[t]{2}{*}{6636.30} & 1918.62 & 100 & 4 & & & & \\
\hline & 3447.14 & 100 & 3 & & & & \\
\hline 6674.8 & 3485.4 & 100 & & & & & \\
\hline \multirow[t]{3}{*}{6715.9} & 1505.0 & 100 & 13 & & & & \\
\hline & 1999.9 & 83 & 13 & & & & \\
\hline & 3525.4 & 68 & 13 & & & & \\
\hline \multirow[t]{3}{*}{6718.14} & 1505.12 & 100 & 9 & & & & \\
\hline & 2000.43 & 64 & 7 & & & & \\
\hline & 3528.85 & 52 & 5 & & & & \\
\hline \multirow[t]{2}{*}{6746.5} & 1850.0 & 100 & 18 & & & & \\
\hline & 2646.2 & 96 & 18 & & & & \\
\hline 6816.8 & 3627.4 & 100 & & & & & \\
\hline \multirow[t]{3}{*}{6895.8} & 970.5 & 32 & 6 & & & & \\
\hline & 1404.8 & 8 & 3 & & & & \\
\hline & 3706.4 & 100 & 6 & & & & \\
\hline 6940.2 & 1195.7 & 43 & 9 & & & & \\
\hline & 2043.9 & 100 & 13 & & & & \\
\hline & 2840.2 & 74 & 11 & & & & \\
\hline 6975.5 & 1483.8 & 36 & 7 & & & & \\
\hline & 2259.9 & 27 & 7 & & & & \\
\hline & 2875.2 & 60 & 9 & & & & \\
\hline & 3785.4 & 100 & 11 & & & & \\
\hline 7129.9 & 3940.4 & 100 & & & & & \\
\hline 7197.9 & 4008.4 & 100 & & & & & \\
\hline 7282.02 & $728.3 \quad 1$ & 30 & 3 & & & & \\
\hline & $873.5 \quad 2$ & 100 & 5 & & & & \\
\hline 7344.7 & 936.1 & 100 & & & & & \\
\hline 7360.6 & 1215.9 & 100 & & & & & \\
\hline 7368.46 & $814.70 \quad 19$ & 100 & 3 & $\mathrm{M} 1+\mathrm{E} 2$ & & & \\
\hline & 959.92 & 3 & 56 & [ E 2 ] & & & $\mathrm{B}(\mathrm{E} 2)(\mathrm{W} \cdot \mathrm{u})=.1.4+10-4$ \\
\hline 7388.8 & 4199.3 & 100 & & & & & \\
\hline 7415.87 & $779.6 \quad 2$ & 35 & 3 & & & & \\
\hline & 1723.93 & 30 & 5 & & & & \\
\hline & 2699.9 & 4 & $0 \quad 12$ & & & & \\
\hline & 4225.3 & 100 & 5 & & & & \\
\hline 7421.2 & 2704.9 & 33 & 5 & & & & \\
\hline & 4230.3 & 100 & 5 & & & & \\
\hline 7543.1 & 2645.9 & 100 & 19 & & & & \\
\hline & 2826.9 & 100 & 19 & & & & \\
\hline & 4352.3 & 63 & 19 & & & & \\
\hline 7562.5 & 2844.9 & 100 & & & & & \\
\hline 7634.03 & $1708.5 \quad 4$ & 51 & 9 & & & & \\
\hline & 1942.23 & 54 & 9 & & & & \\
\hline & $2916.3 \quad 3$ & 100 & 14 & & & & \\
\hline & 4444.3 & 80 & 11 & & & & \\
\hline 7696.8 & 4507.3 & 100 & & & & & \\
\hline 7726.5 & 3008.9 & 100 & & & & & \\
\hline 7750.66 & $382.20 \quad 8$ & 100 & & $\mathrm{M} 1(+\mathrm{E} 2)$ & +0.02 & 7 & \\
\hline 7758.0 & 1349.1 & 56 & 9 & & & & \\
\hline & 2013.6 & 75 & 13 & & & & \\
\hline
\end{tabular}


Adopted Levels, Gammas (continued)

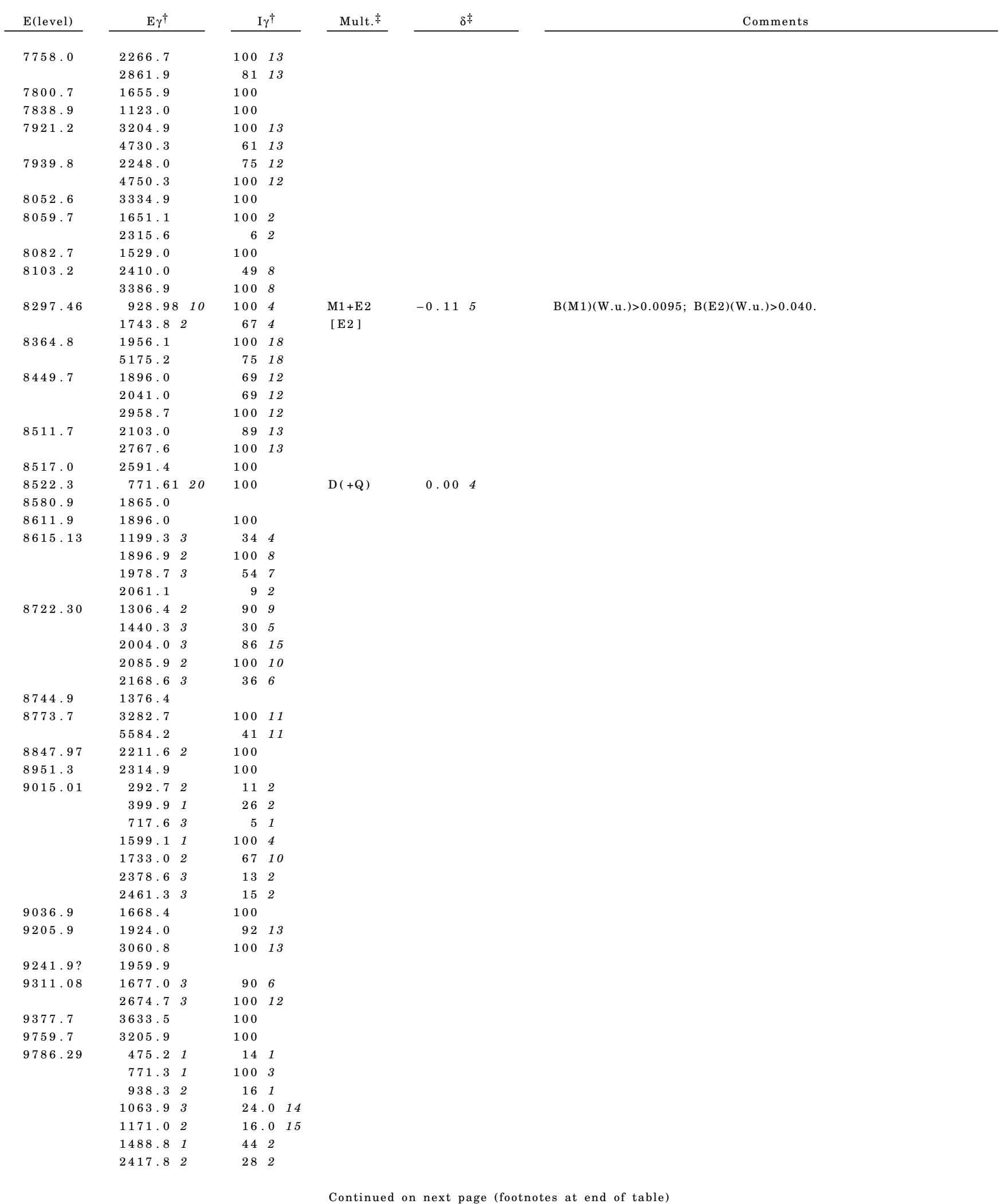


Adopted Levels, Gammas (continued)

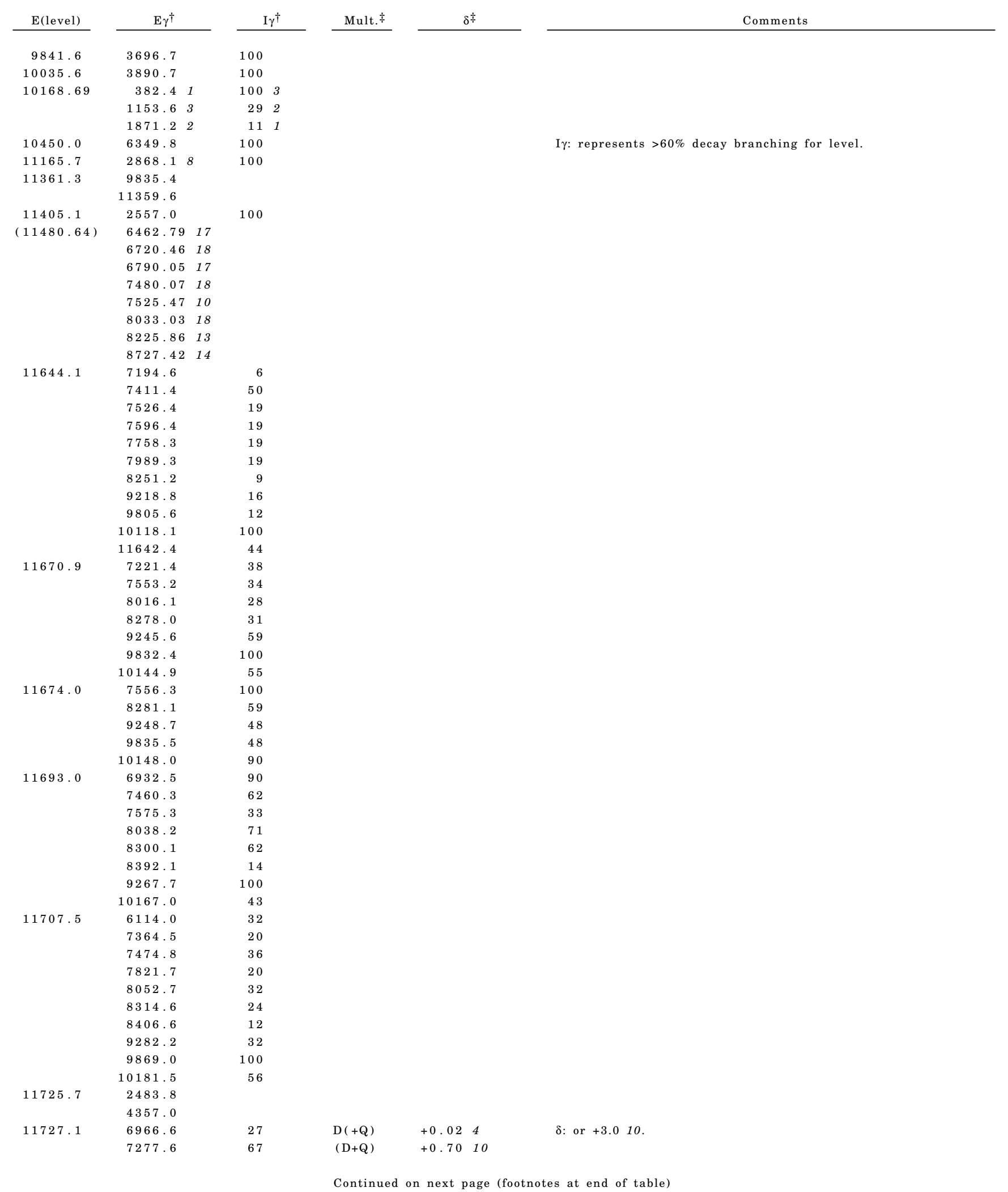


Adopted Levels, Gammas (continued)

\begin{tabular}{|c|c|c|c|c|c|c|c|}
\hline $\mathrm{E}($ level) & $\mathrm{E} \gamma^{\dagger}$ & & $\mathrm{I} \gamma$ & $\gamma^{\dagger}$ & Mult. $\neq$ & $\delta^{\ddagger}$ & Comments \\
\hline \multirow[t]{10}{*}{11727.1} & 7308.4 & & 27 & & & & \\
\hline & 7494.4 & & 47 & & $(D+Q)$ & $+0.30 \quad 15$ & $\delta:$ or +2.010 \\
\hline & 7609.4 & & 47 & & & & \\
\hline & 8072.3 & & 20 & & & & \\
\hline & 8334.2 & 2 & 40 & & $D+Q$ & $+3.7 \quad 1$ & \\
\hline & 8426.2 & & 40 & & & & \\
\hline & 9301.8 & 8 & 93 & & $D+Q$ & $+0.65 \quad 5$ & \\
\hline & 9888.5 & & 67 & & & & \\
\hline & 10201.1 & 1 & 93 & & $D+Q$ & $-0.20 \quad 5$ & \\
\hline & 11725.3 & 3 & 100 & & & & \\
\hline \multirow[t]{11}{*}{11738.4} & 6523.9 & 9 & 45 & & & & \\
\hline & 6833.8 & & 82 & & & & \\
\hline & 7288.9 & & 18 & & & & \\
\hline & 7505.7 & & 32 & & & & \\
\hline & 7620.7 & & 100 & & & & \\
\hline & 7852.6 & & 45 & & & & \\
\hline & 8083.6 & & 27 & & & & \\
\hline & 8345.5 & 5 & 50 & & & & \\
\hline & 9899.8 & 8 & 9 & & & & \\
\hline & 10212.4 & 4 & 23 & & & & \\
\hline & 11736.6 & 6 & 23 & & & & \\
\hline \multirow[t]{6}{*}{11743.4} & 7510.7 & 7 & 35 & & & & \\
\hline & 7625.7 & 7 & 29 & & & & \\
\hline & 8350.5 & 5 & 79 & & & & \\
\hline & 9904.8 & 8 & 26 & & & & \\
\hline & 10217.4 & 4 & 100 & & & & \\
\hline & 11741.6 & & 24 & & & & \\
\hline \multirow[t]{10}{*}{11775.0} & 7207.3 & & 33 & & & & \\
\hline & 7542.3 & & 29 & & & & \\
\hline & 7657.3 & 3 & 19 & & & & \\
\hline & 7774.6 & 6 & 76 & & & & \\
\hline & 7889.2 & 2 & 29 & & & & \\
\hline & 8327.2 & 2 & 100 & & & & \\
\hline & 8382.1 & 1 & 62 & & & & \\
\hline & 9349.7 & 7 & 76 & & & & \\
\hline & 10249.0 & 0 & 24 & & & & \\
\hline & 11773.2 & 2 & 29 & & & & \\
\hline \multirow[t]{7}{*}{11795.2} & 7677.4 & 4 & 50 & & & & \\
\hline & 8347.4 & & 25 & & & & \\
\hline & 8402.3 & & 100 & & & & \\
\hline & 9369.9 & 9 & 92 & & & & \\
\hline & 9956.6 & 6 & 46 & & & & \\
\hline & 10269.2 & 2 & 33 & & & & \\
\hline & 11793.4 & 4 & 71 & & & & \\
\hline \multirow[t]{10}{*}{11805.4} & 6211.9 & 9 & 86 & & & & \\
\hline & 7355.9 & 9 & 38 & & & & \\
\hline & 7386.7 & 7 & 52 & & & & \\
\hline & 7462.4 & 4 & 100 & & & & \\
\hline & 7757.6 & 6 & 71 & & & & \\
\hline & 8357.6 & 6 & 24 & & & & \\
\hline & 8412.5 & & 52 & & & & \\
\hline & 9380.1 & & 14 & & & & \\
\hline & 10279.3 & & 19 & & & & \\
\hline & 11803.6 & & 19 & & & & \\
\hline \multirow[t]{2}{*}{11821.1} & 1652.3 & 34 & 83 & 11 & & & \\
\hline & 2034.6 & 68 & 100 & 7 & & & \\
\hline \multirow[t]{7}{*}{11822.4} & 7589.7 & & 100 & & & & \\
\hline & 8167.5 & 5 & 56 & & & & \\
\hline & 8429.5 & 5 & 52 & & & & \\
\hline & 9397.1 & 1 & 48 & & & & \\
\hline & 9983.8 & & 36 & & & & \\
\hline & 10296.3 & & 92 & & & & \\
\hline & 11820.6 & & 16 & & & & \\
\hline
\end{tabular}


Adopted Levels, Gammas (continued)

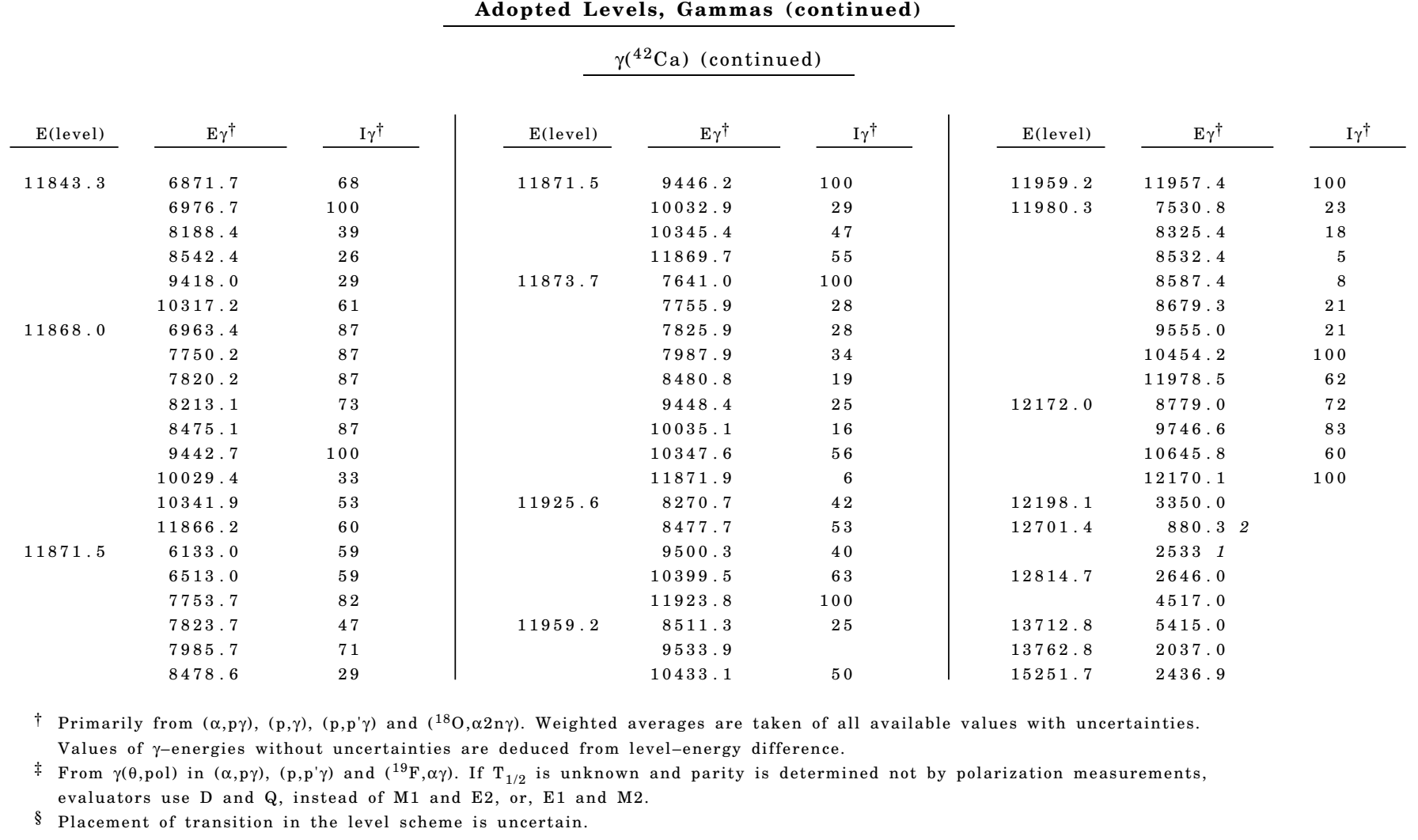

(A) g.s., Yrast band

(B) Excited 0+ band

(C) Negative-parity structure

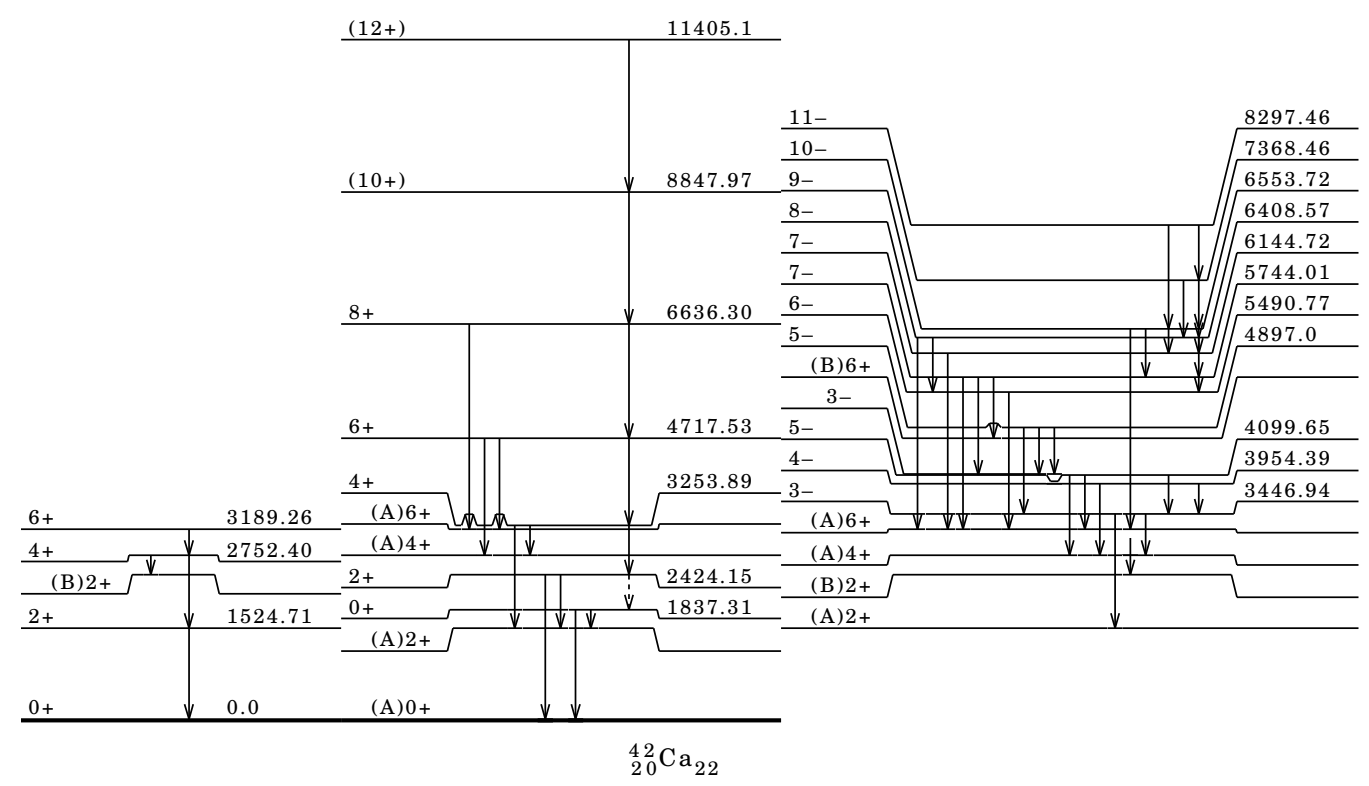




\section{${ }^{42} \mathrm{~K} \beta^{-}$Decay $(12.355 \mathrm{~h}) \quad 1970 \mathrm{Ka} 17$}

Parent ${ }^{42} \mathrm{~K}: \mathrm{E}=0 ; \mathrm{J} \pi=2-; \mathrm{T}_{1 / 2}=12.355$ h $7 ; \mathrm{Q}$ (g.s.) $=3525.2218 ; \% \beta^{-}$decay $=100$.

${ }^{42} \mathrm{~K}-\mathrm{J}, \mathrm{T}_{1 / 2}$ : From Adopted Levels of ${ }^{42} \mathrm{~K}$.

${ }^{42} \mathrm{~K}-\mathrm{Q}\left(\beta^{-}\right)$: From $2012 \mathrm{Wa} 38$.

$\gamma, \gamma \gamma: 1970 \mathrm{Ka} 17$. Others: 1966He11, 1965Mi09, 1964Ma05, 1962Pe19, 1961Mc03, 1960Ga06, 1959Mo17, 1959Ma27, 1956Po07, $1955 \mathrm{Em} 04,1954 \mathrm{Ca} 64,1953 \mathrm{Ka} 26,1947 \mathrm{Si} 08$.

$\% \mathrm{I} \gamma: 1990 \mathrm{Mi} 05,2004 \mathrm{HaZW}$. Others: 1959Ma27, 1955Em04, $1953 \mathrm{Ka} 26$.

$\gamma \gamma(\mathrm{t}): 1963 \mathrm{Bl} 09,1961 \mathrm{Si} 01$.

$\gamma \gamma(\theta)$ : 1966Az04, 1959As65 (also 1960As03), 1959Mo17, Arkiv Fysik 15, 303 (1959).

ß: 1989He11 (also 1986HeZY), 1987Na28, 1976Ra30, 1975Ra09, 1968Va06, 1968Da12, 1965Ch30, 1964Da16, 1956Po07, $1954 \mathrm{Ko} 55,1949 \mathrm{Sh} 26,1947 \mathrm{Si} 08$.

$\beta \gamma:$ 2001Si09, 1961Si01, 1953Ka26, $1947 \mathrm{Bl33}$.

Pair conversion, E0 strength: $1971 \mathrm{Be} 76$.

$\beta \gamma(\theta): 1975 \mathrm{Ve} 06,1961 \mathrm{St} 12,1953 \mathrm{Ha} 40,1951 \mathrm{St} 68,1950 \mathrm{Be} 60$.

$\beta \gamma(\operatorname{circ}$ pol, $\theta): 1968 \mathrm{He} 06,1968 \mathrm{Da} 12,1964 \mathrm{He} 10,1964 \mathrm{De} 09,1961 \mathrm{Da} 02$.

$\beta \gamma($ lin pol, $\theta)$ : $1953 \mathrm{Ha} 40$.

$\mathrm{T}_{1 / 2}\left({ }^{42} \mathrm{~K}\right.$ isotope): 2005Li66, 2004Un01, 2001Ko07, 1994Mi03, 1967Go21, 1962Me06. Others: 1984Ke14, 1982RuZV, 1963Ho17, $1962 \mathrm{Mo} 21,1959 \mathrm{Ma} 27,1957 \mathrm{Wr} 37,1953 \mathrm{Bu} 58,1953 \mathrm{Ka} 26,1947 \mathrm{Si} 08$.

Isotopic assignment: $1954 \mathrm{An} 25,1937 \mathrm{Hu} 01$.

\section{${ }^{42} \mathrm{Ca} \mathrm{Levels}$}

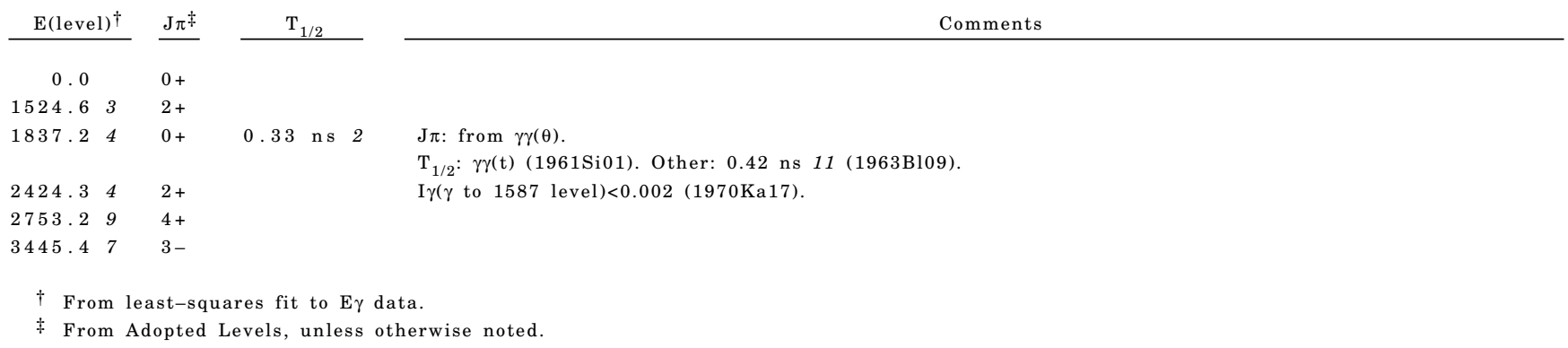

$\beta^{-}$radiations

\begin{tabular}{|c|c|c|c|c|}
\hline $\mathrm{E} \beta^{-}$ & E(level) & $\mathrm{I} \beta^{-\dagger}$ & & $\log f t$ \\
\hline$\left(\begin{array}{ll}79.8 & 8\end{array}\right)$ & 3445.4 & 0.07 & 1 & $4.98 \quad 7$ \\
\hline$\left(\begin{array}{l}1100.9 \\
5\end{array}\right)$ & 2424.3 & 0.05 & 1 & $9.05 \quad 9$ \\
\hline$\left(\begin{array}{l}1688.0 \\
5\end{array}\right)$ & 1837.2 & 0.34 & 3 & $9.92^{1 \mathrm{u}} \quad 4$ \\
\hline$(2000.6 \quad 4)$ & 1524.6 & 17.64 & 9 & $7.5501 \quad 23$ \\
\hline$\left(\begin{array}{lll}3525.22 & 18\end{array}\right)$ & 0.0 & 81.90 & 9 & $9.4768^{1 \mathrm{u}} \quad 6$ \\
\hline
\end{tabular}

$\mathrm{E} \beta^{-}: 198510$ (1956Po07), 1970 (1954Ko55).

I $\beta^{-}: 18$ (1954Ko55).

F-K plot first-forbidden unique shape (1956Po07,1954Ko55).

$\mathrm{E} \beta^{-}: 35246$ (1975Ra09), 35194 (1968Va06). Others: 1956Po07, $1954 \mathrm{Ko} 55$.

I $\beta^{-}: 82$ (1954Ko55).

$\dagger$ Absolute intensity per 100 decays.

$$
\gamma\left({ }^{42} \mathrm{Ca}\right)
$$

I $\gamma$ normalization: $\% \mathrm{I} \gamma(1524.7 \gamma)=18.089$ (1990Mi05). Others: 17.95 (1962Pe19), 18.45 (1959Ma27), 17.8 9 (1955Em04)

\begin{tabular}{|c|c|c|c|c|}
\hline $\mathrm{E} \gamma^{\dagger}$ & E(level) & $I \gamma^{\dagger \neq}$ & Mult. & $\alpha$ \\
\hline $312.60 \quad 25$ & 1837.2 & $1.86 \quad 11$ & [E2 ] & 0.00349 \\
\hline $692.0 \quad 8$ & 3445.4 & $0.018 \quad 4$ & & \\
\hline $899.7 \quad 4$ & 2424.3 & $0.285 \quad 14$ & & \\
\hline 1021.29 & 3445.4 & 0.1118 & & \\
\hline $1228.0 \quad 15$ & 2753.2 & $0.013 \quad 6$ & & \\
\hline 1524.63 & 1524.6 & 100 & & \\
\hline $1920.8 \quad 10$ & 3445.4 & $0.228 \quad 22$ & & \\
\hline $2424.3 \quad 7$ & 2424.3 & $0.110 \quad 16$ & & \\
\hline
\end{tabular}

Comments

$(313 \gamma)(1525 \gamma)(\theta): \mathrm{A}_{2}=+0.334, \mathrm{~A}_{4}=+1.075$ (1959As65). 
${ }^{42} \mathrm{~K} \beta^{-}$Decay $(12.355$ h) $1970 K a 17$ (continued)

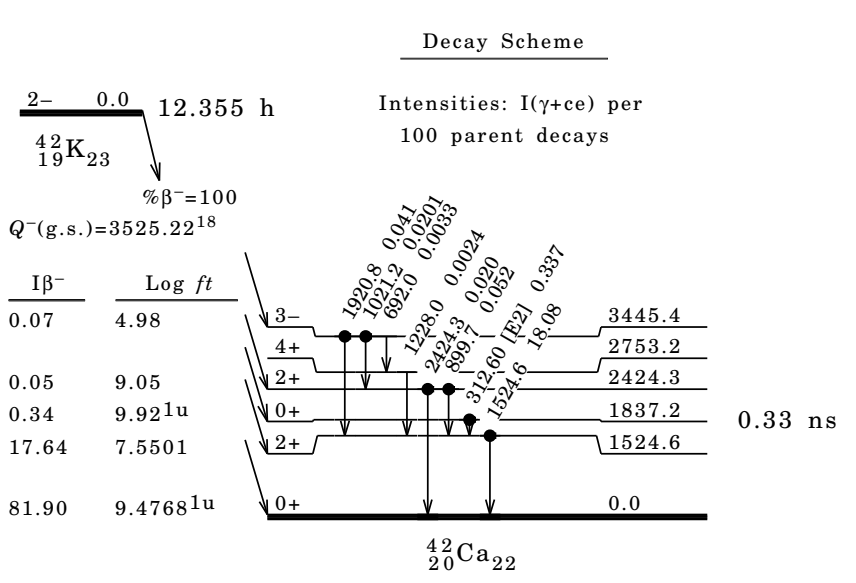

${ }^{42}$ Se $\varepsilon$ Decay $(680.79 \mathrm{~ms}) \quad 1985 \mathrm{Da04}, 1997 \mathrm{Ko65}, 1976 \mathrm{Wi08}$

Parent ${ }^{42} \mathrm{Sc}: \mathrm{E}=0 ; \mathrm{J} \pi=0+; \mathrm{T}_{1 / 2}=680.79 \mathrm{~ms} 28 ; \mathrm{Q}$ (g.s.) $=6426.1010 ; \% \varepsilon+\% \beta^{+}$decay $=100$.

${ }^{42} \mathrm{Sc}-\mathrm{J}, \mathrm{T}_{1 / 2}$ : From Adopted Levels of ${ }^{42} \mathrm{Sc}$. $2015 \mathrm{Ha} 07$ review gives $\mathrm{T}_{1 / 2}=680.72 \mathrm{~ms} 26$.

${ }^{42} \mathrm{Sc}-\mathrm{Q}(\varepsilon)$ : From 2012Wa38. $2015 \mathrm{Ha} 07$ review gives 6426.2830.

${ }^{42} \mathrm{Sc}$ decays mainly (>99\%) through $\beta^{+}$decay.

$\gamma: 1985 \mathrm{Da} 04,1980 \mathrm{Sa} 32,1977 \mathrm{In} 04,1971 \mathrm{Ke} 14,1971 \mathrm{Ga} 17,1957 \mathrm{Cl} 40$.

$\beta^{+}: 1961 \mathrm{Ja} 22,1957 \mathrm{Cl} 40,1955 \mathrm{Mo} 83$.

$\beta \gamma(\theta): 1974 \mathrm{HaYC}, 1973 \mathrm{KeZI}$

$\mathrm{T}_{1 / 2}\left({ }^{42} \mathrm{Sc}\right.$ g.s.): 1997Ko65, 1976Wi08 (also 1972Ha82), 1965Fr08, 1965Ne02, 1962Ja05, 1961Ja22, 1960Ja12, $1957 \mathrm{Cl} 40$, $1955 \mathrm{Mo} 83$.

Other: 2010StZY: deduced GT strength distributions for $\beta$-decay.

2015Ha07: review of superallowed decays; evaluated $\mathrm{Q}$ value, $\mathrm{T}_{1 / 2}$ branching ratios, ft value, isospin-symmetry-breaking corrections.

\section{${ }^{42} \mathrm{Ca}$ Levels}

\begin{tabular}{|c|c|}
\hline $\mathrm{E}(\mathrm{level})^{\dagger}$ & $\mathrm{J} \pi^{\dagger}$ \\
\hline 0.0 & $0+$ \\
\hline 1525 & $2+$ \\
\hline 1837 & $0+$ \\
\hline
\end{tabular}

$\dagger$ From Adopted Levels, energies are rounded values.

\begin{tabular}{|c|c|c|c|c|c|c|c|}
\hline \multirow{2}{*}{\multicolumn{2}{|c|}{$\mathrm{E} \varepsilon$}} & \multirow[b]{2}{*}{ E(level) } & \multirow{2}{*}{\multicolumn{2}{|c|}{$I \beta^{+\dagger}$}} & \multirow[b]{2}{*}{$\mathrm{I} \varepsilon^{\dagger}$} & \multicolumn{2}{|c|}{$\beta^{+}, \varepsilon$ Data } \\
\hline & & & & & & $\log f t$ & $\mathrm{I}\left(\varepsilon+\beta^{+}\right)^{\dagger}$ \\
\hline$(4589.10$ & $10)$ & 1837 & 0.0074 & 11 & $2.5 \times 10^{-5} \quad 4$ & $6.79 \quad 7$ & $0.0074 \quad 11$ \\
\hline ( 6426.10 & $10)$ & 0.0 & 99.8953 & 15 & $0.0972 \quad 10$ & $3.4845 \quad 2$ & $99.9925 \quad 11$ \\
\hline
\end{tabular}

E $\varepsilon$ : measured $\mathrm{E}\left(\beta^{+}\right)=4.8 \times 10^{3} 9(1957 \mathrm{Cl} 140)$. $0+$ to $0+$ superallowed $\beta$ transition. $\mathrm{I}\left(\varepsilon+\beta^{+}\right): 2015 \mathrm{Ha} 07$ review gives 99.994114

$\dagger$ Absolute intensity per 100 decays.

$$
\gamma\left({ }^{42} \mathrm{Ca}\right)
$$

\begin{tabular}{rllll}
\multicolumn{1}{r}{$\mathrm{E} \gamma$} & & $\mathrm{E}$ (level) & & $\mathrm{I} \gamma^{\dagger}$ \\
\cline { 5 - 5 } 313 & 1837 & & 0.0075 & 12 \\
1524 & 1525 & & 0.0075 & 12
\end{tabular}

Comments

0.00349

I $\gamma$ : assumed the same as for $1524.7 \gamma$.

I $\gamma$ : weighted average of $0.007012(1985 \mathrm{Da} 04), 0.010331(1980 \mathrm{Sa} 32), 0.007920$

(1977In04). Others: 0.002217 (1978De08, same laboratory as $1985 \mathrm{Da} 04), 1971 \mathrm{Ke} 14$.

$\dagger$ Absolute intensity per 100 decays. 
${ }^{42}$ Sc \& Decay $(680.79 \mathrm{~ms})$

1985Da04,1997Ko65,1976Wi08 (continued)

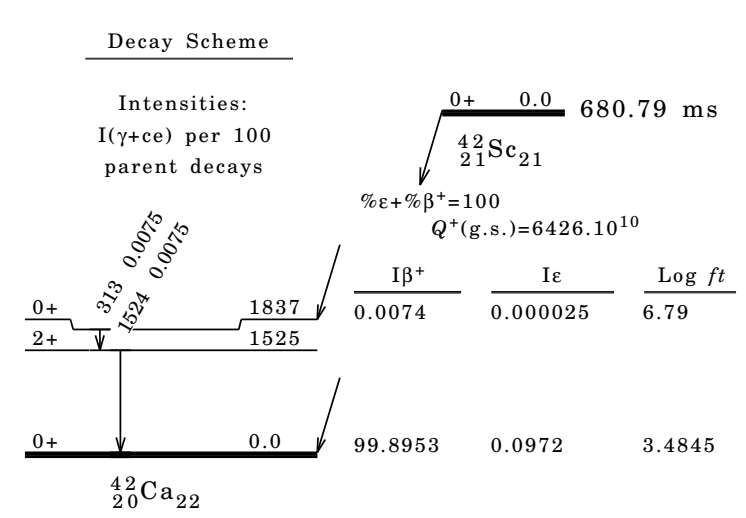




\section{${ }^{42}$ Sc $\varepsilon$ Decay $(61.7$ s $) \quad 1978 B e 61,1974 W i 14,1969 G a 27$}

Parent ${ }^{42} \mathrm{Sc}: \mathrm{E}=616.286 ; \mathrm{J} \pi=7+; \mathrm{T}_{1 / 2}=61.7$ s 4; Q(g.s.) $=6426.1010 ; \% \varepsilon+\% \beta^{+}$decay $=100$.

${ }^{42} \mathrm{Sc}-\mathrm{E}, \mathrm{J}, \mathrm{T}_{1 / 2}$ : From Adopted Levels of ${ }^{42} \mathrm{Sc}$.

${ }^{42} \mathrm{Sc}-\mathrm{Q}(\varepsilon)$ : From $2012 \mathrm{Wa} 38$.

${ }^{42} \mathrm{Sc}$ decays mainly (>99\%) through $\beta^{+}$decay.

$\gamma: 1969 \mathrm{Ga} 27,1965 \mathrm{Ne} 02,1963 \mathrm{Ro} 10$.

$\beta^{+}: 1965 \mathrm{Ne} 02,1963 \mathrm{Ro} 10$.

$\beta^{+} \gamma: 1969 \mathrm{Me} 12,1965 \mathrm{Ne} 02$.

$\beta \gamma(\mathrm{t}): 1969 \mathrm{Me} 12$.

$\mathrm{T}_{1 / 2}\left({ }^{42} \mathrm{Sc}\right.$ isomer): $1978 \mathrm{Be} 61,1974 \mathrm{Wi} 14,1965 \mathrm{Ne} 02,1963 \mathrm{Ro} 10$.

${ }^{42} \mathrm{Sc}$ isomer beam production in ${ }^{12} \mathrm{C}\left({ }^{40} \mathrm{Ca},{ }^{42} \mathrm{Sc}\right){ }^{10} \mathrm{~B}$ : $1994 \mathrm{Uz} 01,1994 \mathrm{Ke} 07$.

${ }^{42} \mathrm{Ca}$ Levels

\begin{tabular}{clll}
\multicolumn{2}{c}{ E(level) } & & $\mathrm{J} \pi^{\dagger}$ \\
\cline { 1 - 1 } 0.0 & & $0+$ \\
524.73 & 3 & & $2+$ \\
424.17 & 4 & & $2+$ \\
752.41 & 4 & & $4+$ \\
189.33 & 14 & & $6+$
\end{tabular}

$\dagger$ From Adopted Levels.

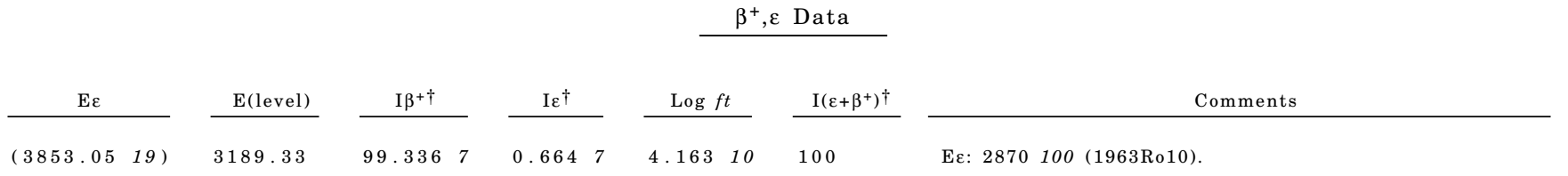

$\dagger$ Absolute intensity per 100 decays.

$\gamma\left({ }^{42} \mathrm{Ca}\right)$

I $\gamma$ normalization: $\mathrm{I}(\gamma+\mathrm{ce})(1524.7 \gamma+2424.1 \gamma)=100$

\begin{tabular}{|c|c|c|}
\hline $\mathrm{E} \gamma$ & E(level) & $\mathrm{I} \gamma \neq \S$ \\
\hline 328 & 2752.41 & $1.0 \quad 4$ \\
\hline $437.5^{\dagger}$ & 3189.33 & 100 \\
\hline 899 & 2424.17 & $0.70 \quad 30$ \\
\hline $1227.0^{\dagger}$ & 2752.41 & $99.0 \quad 4$ \\
\hline $1524.5^{\dagger}$ & 1524.73 & $99.70 \quad 12$ \\
\hline 2424 & 2424.17 & $0.30 \quad 12$ \\
\hline
\end{tabular}

$\dagger$ From 1969Ga27.

\# From level scheme using Adopted Branching and assuming $\mathrm{I} \gamma(437 \gamma)=100$.

$\S$ Absolute intensity per 100 decays.

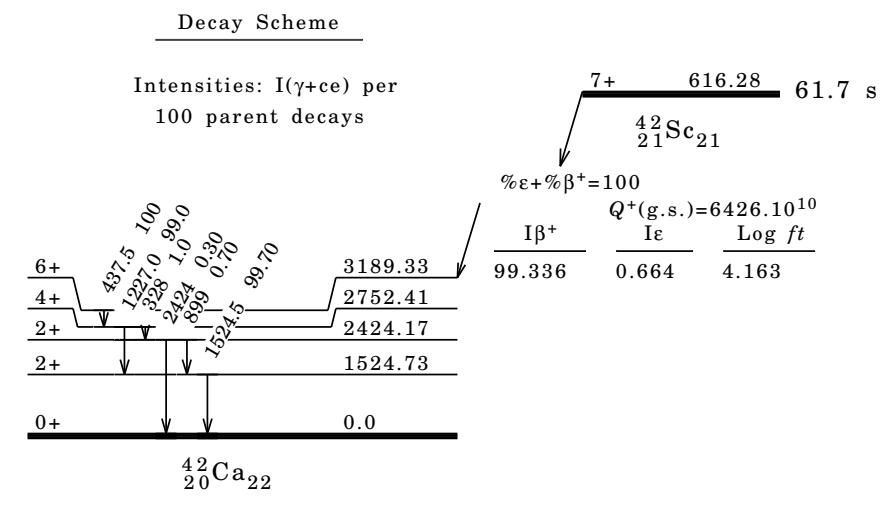




\section{${ }^{24} \mathrm{Mg}\left({ }^{24} \mathrm{Mg}, \alpha 2 \mathrm{p} \gamma\right) \quad 2008 \mathrm{Sa} 04$}

2008Sa04 (also 2008Sa44): E=91.72 MeV (for ON resonance), $92.62 \mathrm{MeV}$ (for OFF resonance) beam provided by Legnaro XTU Tandem accelerator. Target of a $40 \mu \mathrm{g} / \mathrm{cm}^{2}{ }^{24} \mathrm{Mg}$ film on a $15 \mu \mathrm{g} / \mathrm{cm}^{2}$ carbon backing. Charged particles were detected with the EUCLIDES detector composed of 40 silicon detectors $\Delta E-E$ and $\gamma$-rays were detected with the GASP array of $40 \mathrm{HPGe}$ detectors. Measured E $\gamma, \gamma \gamma$-coin, particle $\gamma$ coin. Deduced levels from the decay of a narrow $36+$ resonance in ${ }^{24} \mathrm{Mg}\left({ }^{24} \mathrm{Mg}, \mathrm{X}\right)$ system at $45.7 \mathrm{MeV}$ (c.m.) with $\Gamma=170 \mathrm{keV}$.

Placement of $\gamma$ rays from $2008 \mathrm{Sa} 04$ is based on Adopted Levels and Gammas of ${ }^{42} \mathrm{Ca}$. $2008 \mathrm{Sa} 04$ reported $24 \gamma$ rays from the decay of a $\mathrm{J} \pi=36+$ resonance in ${ }^{24} \mathrm{Mg}+{ }^{24} \mathrm{Mg}$ system.

${ }^{42} \mathrm{Ca}$ Levels

\begin{tabular}{|c|c|c|c|c|c|}
\hline E(level) & $\mathrm{J} \pi^{\dagger}$ & E(level) & $\mathrm{J} \pi^{\dagger}$ & E(level) & $\mathrm{J} \pi^{\dagger}$ \\
\hline 0.0 & $0+$ & 5491 & $6-$ & 7368 & $10-$ \\
\hline 1525 & $2+$ & 5744 & $7-$ & 8297 & $11-$ \\
\hline 2752 & $4+$ & 6145 & $7-$ & 9015 & $10+$ \\
\hline 3189 & $6+$ & 6409 & $8-$ & 9786 & $(9-, 11)$ \\
\hline 4100 & $5-$ & 6554 & $9-$ & 10169 & $(10,12+)$ \\
\hline 4718 & $6+$ & 7282 & $9-$ & & \\
\hline
\end{tabular}

$\dagger$ From Adopted Levels.

\begin{tabular}{|c|c|c|c|c|c|}
\hline $\mathrm{E} \gamma$ & E(level) & $\mathrm{E} \gamma$ & E(level) & $\mathrm{E} \gamma$ & E(level) \\
\hline 145 & 6554 & 810 & 6554 & 1525 & 1525 \\
\hline 253 & 5744 & 815 & 7368 & 1644 & 5744 \\
\hline 264 & 6409 & 874 & 7282 & 1733 & 9015 \\
\hline 382 & 10169 & 910 & 4100 & 1965 & 4718 \\
\hline 437 & 3189 & 918 & 6409 & 2301 & 5491 \\
\hline 665 & 6409 & 929 & 8297 & 2555 & 5744 \\
\hline 728 & 7282 & 1228 & 2752 & 2955 & 6145 \\
\hline 772 & 9786 & 1347 & 4100 & 3220 & 6409 \\
\hline
\end{tabular}

\section{${ }^{27} \mathrm{Al}\left({ }^{18} \mathrm{O}, 2 \mathrm{np} \gamma\right) \quad 1975 \mathrm{WaO4}$}

Includes ${ }^{26} \mathrm{Mg}\left({ }^{18} \mathrm{O}, 2 \mathrm{n} \gamma\right) \mathrm{E}=40 \mathrm{MeV}$ and ${ }^{28} \mathrm{Si}\left({ }^{19} \mathrm{~F}, \alpha \mathrm{p} \gamma\right) \mathrm{E}=45 \mathrm{MeV}$ from 19750101 , measured intensities.

1975 Wa04 (also 1975O101), 1974Po10: $\mathrm{E}=40 \mathrm{MeV}{ }^{18} \mathrm{O}$ beam was produced from the Brookhaven National Laboratory MP tandem Van de Graaff facility. a target of $250 \mu \mathrm{g} / \mathrm{cm}^{2}$ isotopically enriched ${ }^{27} \mathrm{Al}$ evaporated onto a thick W backing.

$\gamma$-rays were detected with two true coaxial Ge(Li) detectors. Measured E $\gamma, \mathrm{I} \gamma, \gamma \gamma, \gamma(\theta), \gamma\left(\mathrm{lin}\right.$ pol), $\mathrm{T}_{1 / 2}$ by recoil-distance method ( $R D M)$. Deduced levels, J $\pi$, mixing ratios.

\section{${ }^{42} \mathrm{Ca}$ Levels}

\begin{tabular}{|c|c|c|c|c|c|c|c|c|c|c|}
\hline $\mathrm{E}(\text { level })^{\dagger}$ & $\mathrm{J} \pi^{*}$ & $\mathrm{~T}_{1 / 2} \S$ & $\mathrm{E}(\text { level })^{\dagger}$ & $\mathrm{J} \pi^{\ddagger}$ & $\mathrm{T}_{1 / 2} \S$ & $\mathrm{E}(\text { level })^{\dagger}$ & $\mathrm{J} \pi \ddagger$ & \multicolumn{3}{|c|}{$\mathrm{T}_{1 / 2} \S$} \\
\hline 0.0 & $0+$ & & $3446.5 ? 6$ & $3-$ & & 6145.1125 & $7-$ & & & \\
\hline 1524.618 & $2+$ & & $3571.59 ? \quad 17$ & & & $6408.87 \quad 24$ & $8-$ & & & \\
\hline $2423.62 \quad 21$ & $2+$ & & $3953.5 \quad 12$ & $4-$ & & $6554.07 \quad 25$ & $9-$ & 42 & $\mathrm{ps}$ & \\
\hline $2752.29 \quad 12$ & $4+$ & $3.5 \mathrm{ps} 3$ & $4099.67 \quad 17$ & $5-$ & & $7368.8 \quad 3$ & $10-$ & 2 & $6 \mathrm{ps}$ & 11 \\
\hline $3189.32 \quad 14$ & $6+$ & & $5491.00 \quad 24$ & $6-$ & $<1.7 \mathrm{ps}$ & $8297.9 \quad 4$ & $11-$ & $<1$ & $7 \mathrm{ps}$ & \\
\hline $3254.3 \quad 10$ & $4+$ & & $5744.19 \quad 22$ & $7-$ & & & & & & \\
\hline
\end{tabular}

$\dagger$ From least-squares fit to E $\gamma$ data.

$\ddagger$ From Adopted Levels.

$\S$ From recoil-distance method (19750101). 


\begin{tabular}{ll}
${ }^{27} \mathrm{Al}\left({ }^{18} \mathrm{O}, 2 \mathrm{np} \gamma\right)$ & $1975 \mathrm{Wa04}$ (continued) \\
\hline & ${ }^{\left({ }^{42} \mathrm{Ca}\right)}$ \\
\hline
\end{tabular}

$\mathrm{A}_{2}, \mathrm{~A}_{4}$ and Pol values are from 1975OL01.

\begin{tabular}{|c|c|c|c|c|}
\hline $\mathrm{E} \gamma$ & E(level) & $\mathrm{I} \gamma$ & Mult. ${ }^{\dagger}$ & Comments \\
\hline 145 & 6554.07 & & & $\mathrm{I} \gamma(145) / \mathrm{I} \gamma(810)=24 \quad 4 / 76 \quad 4$ \\
\hline $263.74 \quad 15$ & 6408.87 & 4.7 & $\mathrm{D}+\mathrm{Q}$ & $\mathrm{A}_{2}=-0.182 ; \mathrm{A}_{4}=-0.143$ \\
\hline $382.24 \ddagger 10$ & $3571.59 ?$ & 2.8 & & $\begin{array}{l}\text { Placement considered uncertain (by the evaluators) since the level population } \\
\text { is tentative. } \\
\mathrm{A}_{2}=-0.4212 ; \mathrm{A}_{4}=0 \text {. } \\
\text { Pol=-0.52 } 15 \text {. }\end{array}$ \\
\hline $437.04 \quad 8$ & 3189.32 & 118.4 & $\mathrm{E} 2$ & $\begin{array}{l}\mathrm{A}_{2}=+0.232 ; \mathrm{A}_{4}=-0.152 \\
\mathrm{Pol}=+0.325\end{array}$ \\
\hline 502 & 3254.3 & & & \\
\hline 507 & 3953.5 & & & \\
\hline 694 & $3446.5 ?$ & & & \\
\hline $809.88 \quad 12$ & 6554.07 & $32.3 \quad 5$ & $\mathrm{E} 2$ & $\begin{array}{l}\mathrm{A}_{2}=+0.303 ; \mathrm{A}_{4}=-0.193 \\
\mathrm{Pol}=+0.378\end{array}$ \\
\hline $814.75 \quad 15$ & 7368.8 & 19.5 & M1 & $\begin{array}{l}\mathrm{A}_{2}=-0.306 ; \mathrm{A}_{4}=-0.024 \\
\mathrm{Pol}=-0.419\end{array}$ \\
\hline $898.99 \quad 20$ & 2423.62 & 4.99 & $\mathrm{D}$ & $\mathrm{A}_{2}=+0.1310 ; \mathrm{A}_{4}=+0.1115$ \\
\hline $910.45 \quad 15$ & 4099.67 & 19.2 & $\mathrm{E} 1$ & $\begin{array}{l}\mathrm{A}_{2}=-0.202 ; \mathrm{A}_{4}=-0.073 \\
\mathrm{Pol}=+0.199\end{array}$ \\
\hline $917.87 \quad 12$ & 6408.87 & 24.1 & $\mathrm{E} 2$ & $\begin{array}{l}\mathrm{A}_{2}=+0.315 ; \mathrm{A}_{4}=-0.175 \\
\mathrm{Pol}=+0.41 \quad 12\end{array}$ \\
\hline $929.10 \quad 25$ & 8297.9 & 6.9 & M1 & $\begin{array}{l}\mathrm{A}_{2}=-0.257 ; \mathrm{A}_{4}=0 \\
\text { Pol }=-0.6727\end{array}$ \\
\hline 1023 & $3446.5 ?$ & & & \\
\hline $1227.66 \quad 8$ & 2752.29 & & $\mathrm{E} 2$ & $\begin{array}{l}\mathrm{A}_{2}=+0.231 ; \mathrm{A}_{4}=-0.111 \\
\mathrm{Pol}=+0.296\end{array}$ \\
\hline $1347.24 \quad 20$ & 4099.67 & 10.2 & ( E1) & $\begin{array}{l}\mathrm{A}_{2}=-0.232 ; \mathrm{A}_{4}=0 \\
\mathrm{Pol}=+0.0525 \\
\mathrm{I} \gamma(910) / \mathrm{I} \gamma(1347)=612 / 332\end{array}$ \\
\hline $1524.58 \quad 8$ & 1524.61 & 222.5 & $\mathrm{E} 2$ & $\begin{array}{l}\mathrm{A}_{2}=+0.221 ; \mathrm{A}_{4}=-0.101 \\
\mathrm{Pol}=+0.379\end{array}$ \\
\hline 1644.5420 & 5744.19 & 22.2 & ( E 2$)$ & $\begin{array}{l}\mathrm{A}_{2}=+0.262 ; \mathrm{A}_{4}=-0.173 \\
\mathrm{Pol}=+0.2529\end{array}$ \\
\hline $1921.8 \quad 8$ & $3446.5 ?$ & 2.2 & $\mathrm{D}$ & $\mathrm{A}_{2}=-0.5514 ; \mathrm{A}_{4}=0$ \\
\hline $2301.68 \quad 25$ & 5491.00 & 32.3 & $\mathrm{E} 1$ & $\begin{array}{l}\mathrm{A}_{2}=+0.323 ; \mathrm{A}_{4}=-0.104 \\
\mathrm{Pol}=-0.3025\end{array}$ \\
\hline 2424 & 2423.62 & & & \\
\hline $2554.70 \quad 25$ & 5744.19 & 17.4 & ( E1) & $\begin{array}{l}\mathrm{A}_{2}=-0.2616 ; \mathrm{A}_{4}=0 \\
\mathrm{Pol}=-0.1730 \\
\mathrm{I} \gamma(1644) / \mathrm{I} \gamma(2556)=512 / 492\end{array}$ \\
\hline $2955.6 \quad 3$ & 6145.11 & 4.8 & $\mathrm{D}$ & $\mathrm{A}_{2}=-0.308 ; \mathrm{A}_{4}=0$ \\
\hline 3219.36 & 6408.87 & 4.5 & {$[\mathrm{M} 2+\mathrm{E} 3]$} & $\begin{array}{l}\mathrm{A}_{2}=+0.72 \quad 5 ; \mathrm{A}_{4}=0 \\
\mathrm{I} \gamma(264) / \mathrm{I} \gamma(918) / \mathrm{I} \gamma(3219)=17 \quad 3 / 70 \quad 2 / 132\end{array}$ \\
\hline
\end{tabular}

$\dagger$ From $\gamma\left(\right.$ lin pol) and $\mathrm{T}_{1 / 2}$ in 19750101 .

\# Placement of transition in the level scheme is uncertain. 


\section{${ }^{27} \mathrm{Al}\left({ }^{19} \mathrm{~F}, \alpha \gamma\right) \quad 1981 \mathrm{He} 20$}

1981He20 (also 1981He17): $\mathrm{E}=47-108 \mathrm{MeV}{ }^{19} \mathrm{~F}$ beam produced at the MP-Tandem at the MPI in Heidelberg. Main experiment

for ${ }^{42} \mathrm{Ca}$ at $\mathrm{E}=72 \mathrm{MEV}$. Targets of $1 \mathrm{mg} / \mathrm{cm}^{2} \mathrm{Al}$ on Au backings of $100 \mathrm{mg} / \mathrm{cm}^{2}$. $\gamma$-rays were detected with two Ge(Li) of

$26 \%$ relative efficiency, FWHM=1.9 keV and $2.1 \mathrm{keV}$. Measured E $\gamma, \mathrm{I} \gamma, \gamma \gamma, \gamma(\theta)$. Deduced levels, J $\pi, \gamma-\mathrm{ray}$ mixing

ratios.

Others: 1976Po03, 19750101: $\mathrm{E}=40 \mathrm{MeV}$. Measured cross sections.

\section{${ }^{42} \mathrm{Ca}$ Levels}

\begin{tabular}{|c|c|c|}
\hline $\mathrm{E}(\text { level })^{\dagger}$ & $J \pi^{\ddagger}$ & \\
\hline 0.0 & $0+$ & \\
\hline 1524.618 & $2+$ & \\
\hline $2752.29 \quad 12$ & $4+$ & \\
\hline $3189.37 \quad 19$ & $6+$ & \\
\hline $4099.6 \quad 7$ & $5-$ & \\
\hline 5491.18 & $6-$ & \\
\hline 5744.36 & $7-$ & \\
\hline 6145.18 & $7-$ & \\
\hline 6408.86 & $8-$ & \\
\hline 6553.86 & $9-$ & \\
\hline 7368.36 & $(10)-$ & \\
\hline $7750.5 \quad 6$ & $(11)-$ & \\
\hline 8297.16 & $(9)-$ & $\mathrm{J} \pi:(11)-$ in Adopted Levels \\
\hline 8522.17 & $\left(\begin{array}{ll}1 & 0\end{array}\right)$ & \\
\hline
\end{tabular}

$\dagger$ Deduced by the evaluators from $\mathrm{E} \gamma$ values.

$\ddagger$ From $\gamma(\theta)$ in $1981 \mathrm{He} 20$.

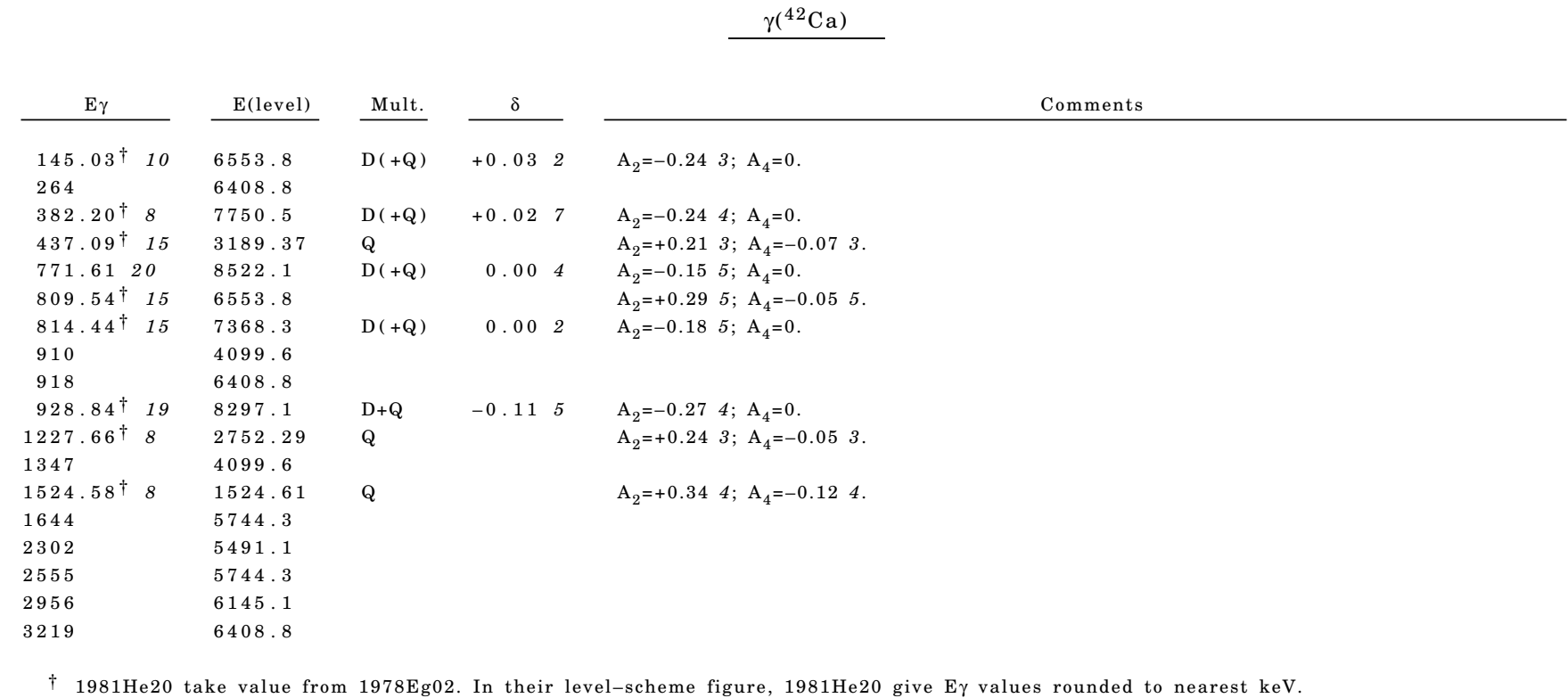




\section{${ }^{28} \mathrm{Si}\left({ }^{16} \mathrm{O}, 2 \mathrm{p} \gamma\right) \quad 1978 \mathrm{Eg} 02,1976 \mathrm{Ro06}$}

Includes ${ }^{12} \mathrm{C}\left({ }^{32} \mathrm{~S}, 2 \mathrm{p} \gamma\right)$ from $1987 \mathrm{TaZY}$.

1978Eg02: E=45 MeV ${ }^{16} \mathrm{O}$ beam was produced at the Utrecht EN tandem accelerator. A target of $250 \mu \mathrm{g} / \mathrm{cm}^{2}{ }^{28} \mathrm{Si}$ enriched to $99.91 \%$ on a $20 \mu \mathrm{m} \mathrm{Au} \mathrm{backing.} \gamma$-rays were detected with a NaI(Tl) Compton suppression spectrometer (CSS) and an array of three $\mathrm{Ge}(\mathrm{Li})$ detectors of about $25 \%$ efficiency. Measured E $\gamma, \mathrm{I} \gamma, \gamma \gamma, \gamma(\theta), \gamma($ lin pol). Deduced levels, $\mathrm{J} \pi$, mixing ratios.

1976Ro06: $\mathrm{E}=36-42 \mathrm{MeV}{ }^{16} \mathrm{O}$ beam was produced from the Oak Ridge tandem Van de Graaff accelerator. A natural target of $0.7 \mathrm{mg} / \mathrm{cm}^{2} \mathrm{Si}$ on a thick Pt backing. $\gamma$-rays were detected with Ge(Li) detectors. Measured E $\gamma, \mathrm{I} \gamma, \gamma \gamma, \gamma \gamma(\theta), \gamma(\theta)$. Deduced levels, J $\pi$, mixing ratios, $\gamma$-branchings, transition probabilities.

1975Wu01 (also 1974Li06): $\mathrm{E}=36.5-50 \mathrm{MeV}{ }^{16} \mathrm{O}$ beam was produced from from the Van de Graaff tandem accelerator of the

University of Cologne. A natural target of $150-200 \mu \mathrm{g} / \mathrm{cm}^{2}$ Si layer evaporated onto stretched target foils of $1 \mu \mathrm{m}$

$\mathrm{Au}$ or Ni. $\gamma$-rays were detected with $55-85 \mathrm{~cm}^{3} \mathrm{Ge}(\mathrm{Li})$ detectors, FWHM=3 $\mathrm{keV}$ at $1.33 \mathrm{MeV}$. Measured E $\gamma$, lifetimes by recoil-distance method.

1975Uh02: E=32.5 MeV. Measured $\gamma$ by $\gamma(\theta, \mathrm{H}, \mathrm{t})$.

1980Da22 (also 1983Da12): $\mathrm{E}=40 \mathrm{MeV}$. Measured average $\gamma$ multiplicity.

1987TaZY: ${ }^{12} \mathrm{C}\left({ }^{32} \mathrm{~S}, 2 \mathrm{p} \gamma\right) \mathrm{E}=75 \mathrm{MeV}$. Measured $\mathrm{g}$ factor of high-spin states

\section{${ }^{42} \mathrm{Ca}$ Levels}

\begin{tabular}{|c|c|c|c|}
\hline $\mathrm{E}(\text { level })^{\dagger}$ & $\mathrm{J} \pi^{\ddagger}$ & $\mathrm{T}_{1 / 2} \S$ & Comments \\
\hline 0.0 & $0+$ & & \\
\hline 1524.618 & $2+$ & & \\
\hline $2752.29 \quad 12$ & $4+$ & $2.63 \mathrm{ps}^{\#} 28$ & \\
\hline $3189.37 \quad 17$ & $6+$ & & \\
\hline $4099.58 \quad 17$ & $5-$ & $<0.7 \mathrm{p} \mathrm{s}$ & \\
\hline 5491.1923 & $6-$ & $<0.14 \mathrm{ps}^{@}$ & $\mathrm{~J} \pi: 5+$ in $1976 \mathrm{Ro} 06$. \\
\hline $5744.29 \quad 22$ & $7-$ & $10.5 \mathrm{ps} 10$ & $\mathrm{~J} \pi: 5-$ in $1976 \mathrm{Ro} 06$. \\
\hline 6144.9623 & $7-$ & & $\mathrm{J} \pi: 6+$ in $1976 \mathrm{Ro} 06$. \\
\hline 6408.7922 & $8-$ & $31.0 \mathrm{ps} 25$ & $\mathrm{~J} \pi: 7+$ in $1976 \mathrm{Ro} 06$. \\
\hline $6553.82 \quad 22$ & $9-$ & 44 p s 7 & $\mathrm{~J} \pi: 7-$ in $1976 \mathrm{Ro} 06$. \\
\hline $7368.3 \quad 3$ & $(8,10)-$ & 1.5 ps 8 & $\mathrm{~J} \pi: 6-, 8-$ in $1976 \mathrm{Ro} 06 ; 10-$ in Adopted Levels. \\
\hline $7750.5 \quad 3$ & $(7,9,11)-$ & $<2.1 \mathrm{ps}^{\&}$ & $\mathrm{~J} \pi: 5-$ to $9-$ in $1976 \mathrm{Ro} 06 ;(11)-$ in Adopted Levels. \\
\hline 8297.14 & $\left(\begin{array}{lll}7 & \text { to } & 11\end{array}\right)-$ & & $\mathrm{J} \pi: 11-$ in Adopted Levels. \\
\hline
\end{tabular}

$\dagger$ From least-squares fit to $\mathrm{E} \gamma$ data.

$\ddagger$ From $\gamma($ lin pol) and $\gamma(\theta)$ in $1978 \mathrm{Eg} 02$.

$\S$ From recoil-distance method (1975Wu01), unless otherwise stated.

\# From recoil-distance method (1974Li06).

(a) From DSA analysis $(1975 \mathrm{Wu} 01)$.

\& From line shape analysis (1978Eg02).

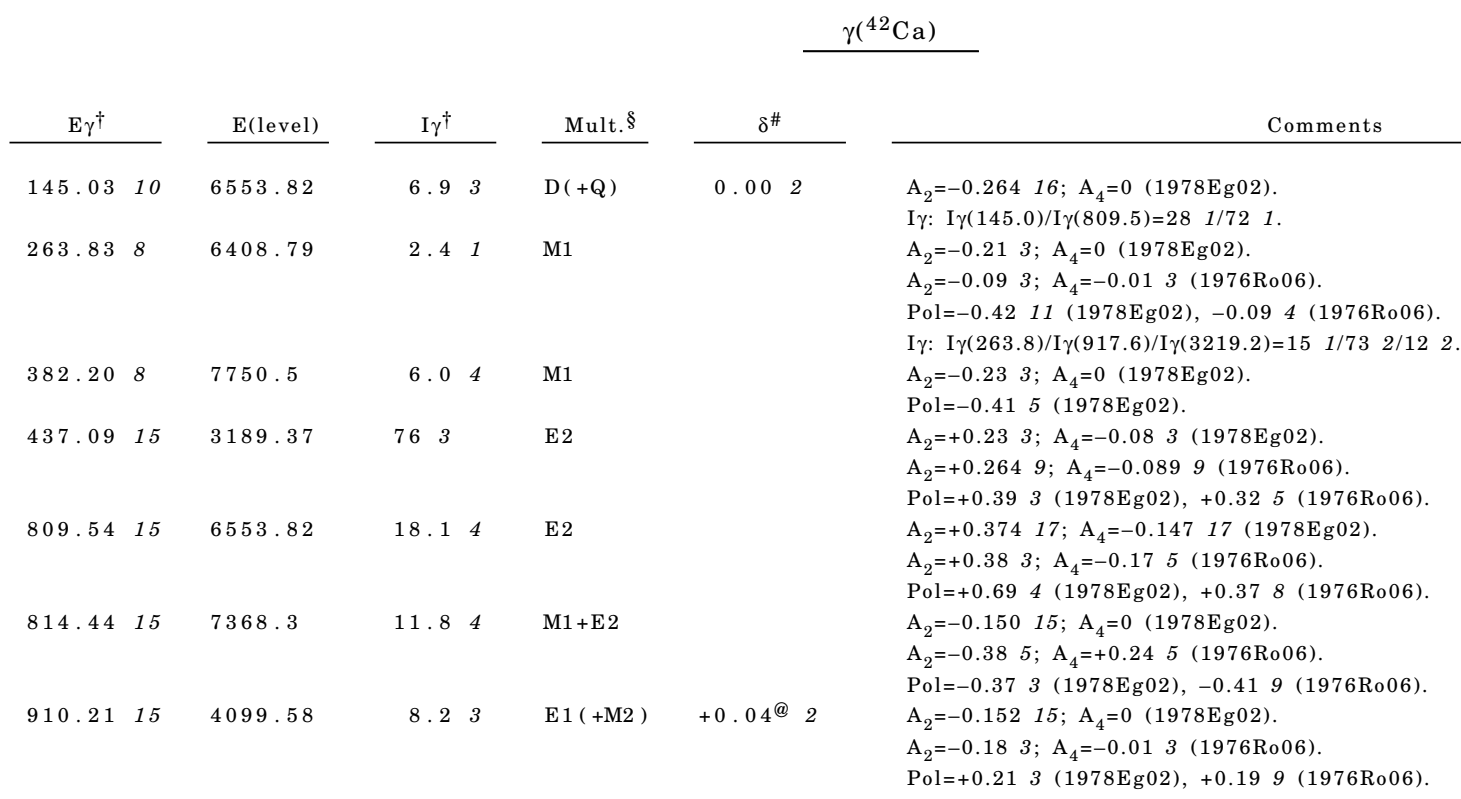




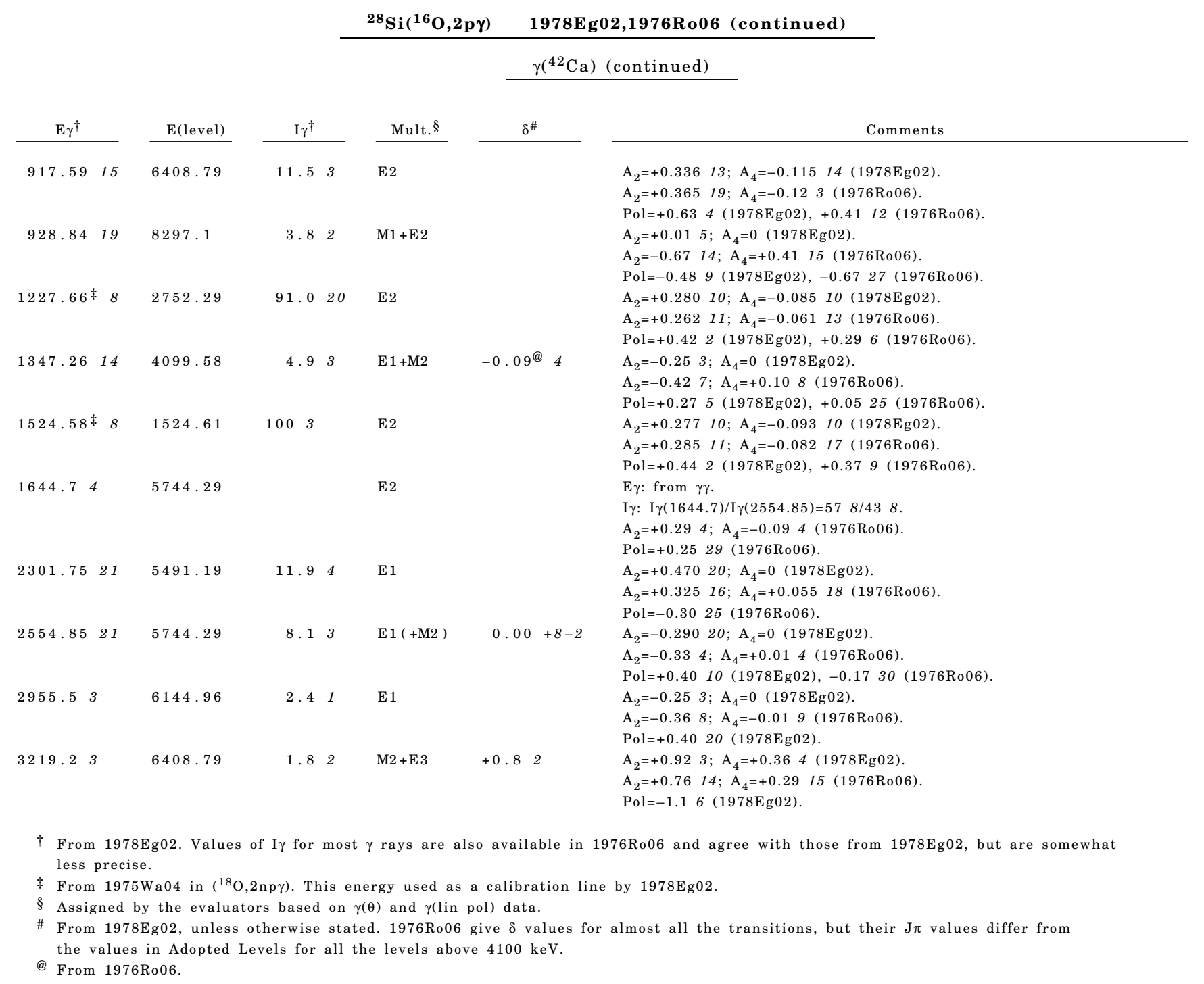

\section{${ }^{30} \mathrm{Si}\left({ }^{18} \mathrm{O}, \alpha 2 \mathrm{n} \gamma\right) \quad 2003 \mathrm{LaO4}$}

2003La04: E=68 MeV ${ }^{18} \mathrm{O}$ beam was produced from the VIVITRON accelerator at IReS Strasbourg. Target of a $800 \mu \mathrm{g} / \mathrm{cm}^{2}$ ${ }^{30} \mathrm{Si}$. $\gamma$-rays were detected with the high-efficiency EUROBALL IV germanium detector array in coincidence with the Recoil Filter Detector (RFD). Measured E $\gamma, \mathrm{I} \gamma, \gamma \gamma, \gamma \gamma(\theta)(\mathrm{DCO}), \gamma \gamma($ recoil) coin, $\gamma($ lin pol). Deduced levels, branchings,.

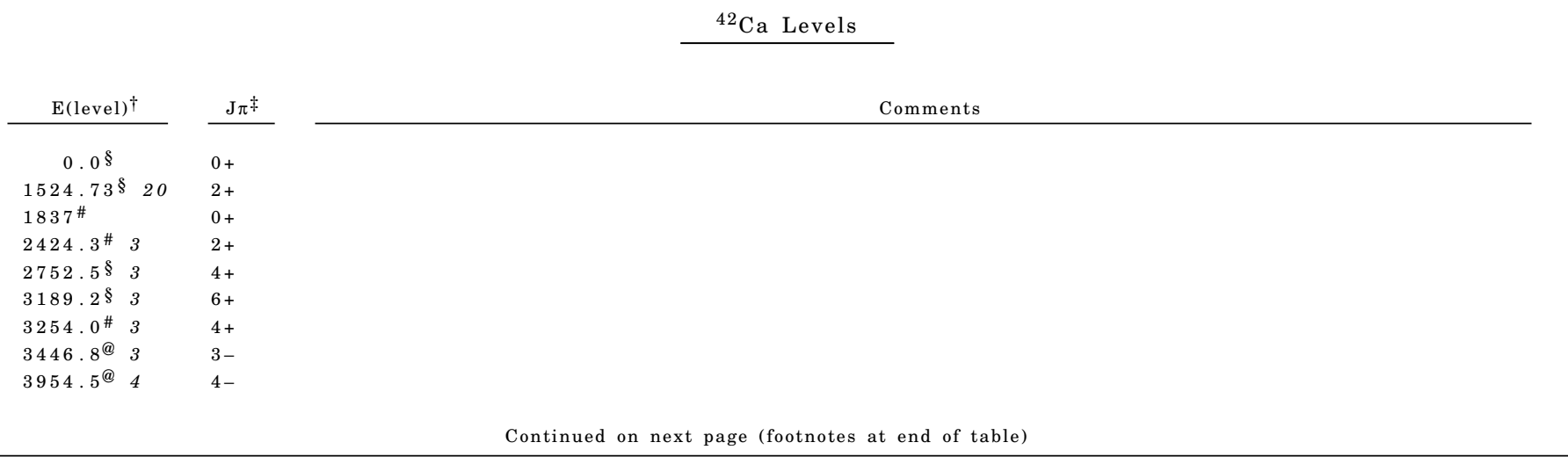


${ }^{30} \mathrm{Si}\left({ }^{18} \mathrm{O}, \alpha 2 \mathrm{n} \gamma\right) \quad 2003 \mathrm{La} 04$ (continued)

${ }^{42} \mathrm{Ca}$ Levels (continued)

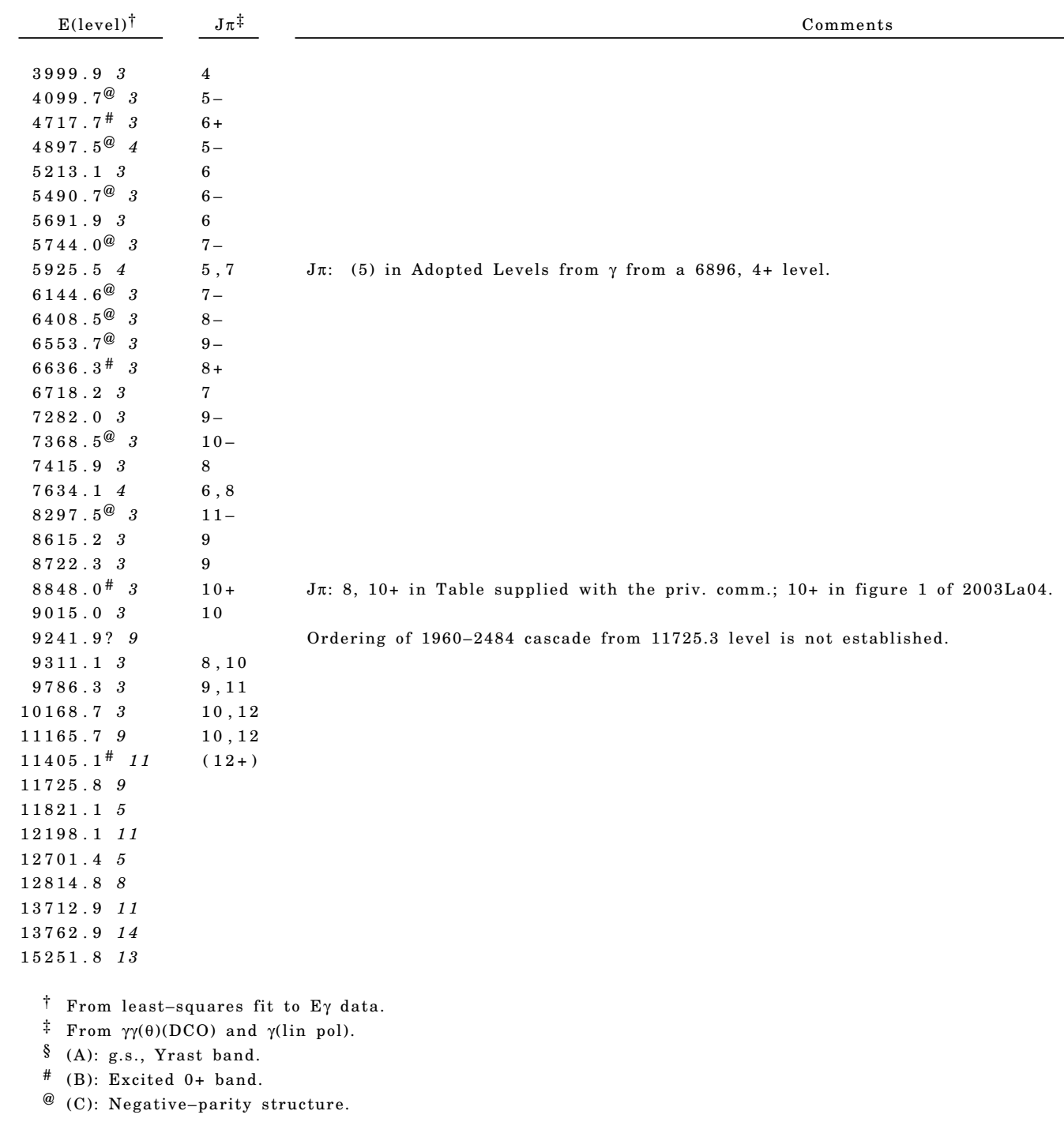

$\gamma\left({ }^{42} \mathrm{Ca}\right)$

\begin{tabular}{|c|c|c|c|c|c|}
\hline E(level) & $\mathrm{E} \gamma^{\dagger}$ & \multicolumn{2}{|c|}{$\mathrm{I} \gamma^{\dagger}$} & Mult. & Comments \\
\hline 1524.73 & 1524.72 & 100 & & & $\mathrm{DCO}=0.988$ \\
\hline 2424.3 & $899.5 \quad 3$ & & & & $\mathrm{DCO}=1.0018$ \\
\hline & $2424.2 \ddagger$ & & & & \\
\hline 2752.5 & 1227.72 & 100 & & & $\mathrm{DCO}=0.998$ \\
\hline 3189.2 & $436.7 \quad 1$ & 100 & & & $\mathrm{DCO}=1.0911$. \\
\hline \multirow[t]{3}{*}{3254.0} & 501.52 & 40 & 4 & & $\mathrm{DCO}=1.0216$ \\
\hline & $829.7 \ddagger$ & 5 & 1 & & \\
\hline & 1729.23 & 100 & 7 & & $\mathrm{DCO}=0.9616$. \\
\hline \multirow[t]{3}{*}{3446.8} & $694.4 \ddagger$ & & & & \\
\hline & $1022.5 \quad 3$ & 80 & 10 & & \\
\hline & $1922.0 \quad 3$ & 100 & 10 & & \\
\hline \multirow[t]{2}{*}{3954.5} & 507.72 & & & & $\mathrm{DCO}=2.25$. \\
\hline & $1202.0 \ddagger$ & & & & \\
\hline 3999.9 & $1575.6 \quad 3$ & 58 & 8 & & \\
\hline
\end{tabular}


${ }^{30} \mathrm{Si}\left({ }^{18} \mathrm{O}, \alpha 2 \mathrm{n} \gamma\right) \quad 2003 \mathrm{La} 04$ (continued)

$\gamma\left({ }^{42} \mathrm{Ca}\right)$ (continued)

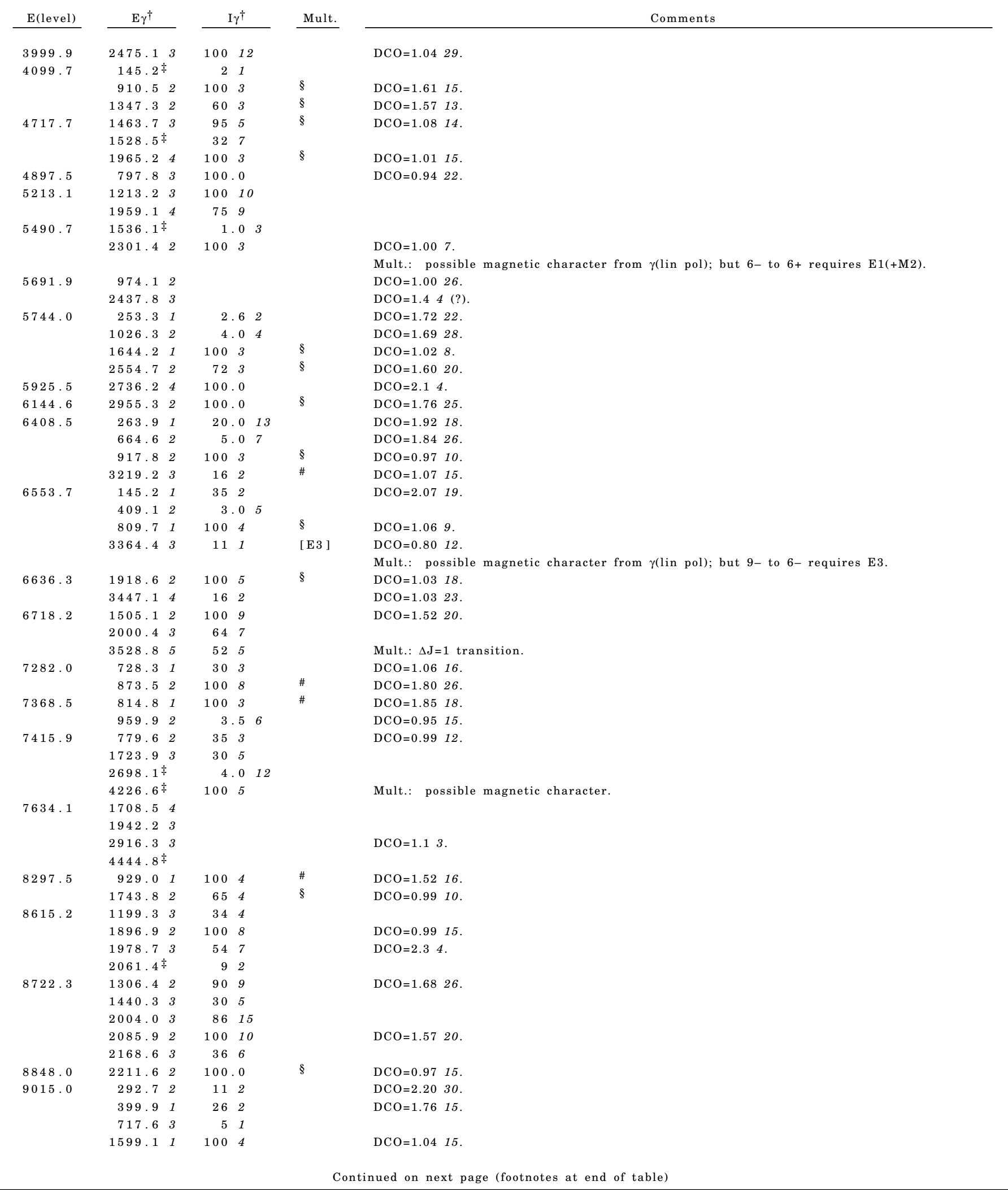


${ }^{30} \mathrm{Si}\left({ }^{18} \mathrm{O}, \alpha 2 \mathrm{n} \gamma\right) \quad 2003 \mathrm{La} 04$ (continued)

$\gamma\left({ }^{42} \mathrm{Ca}\right)$ (continued)

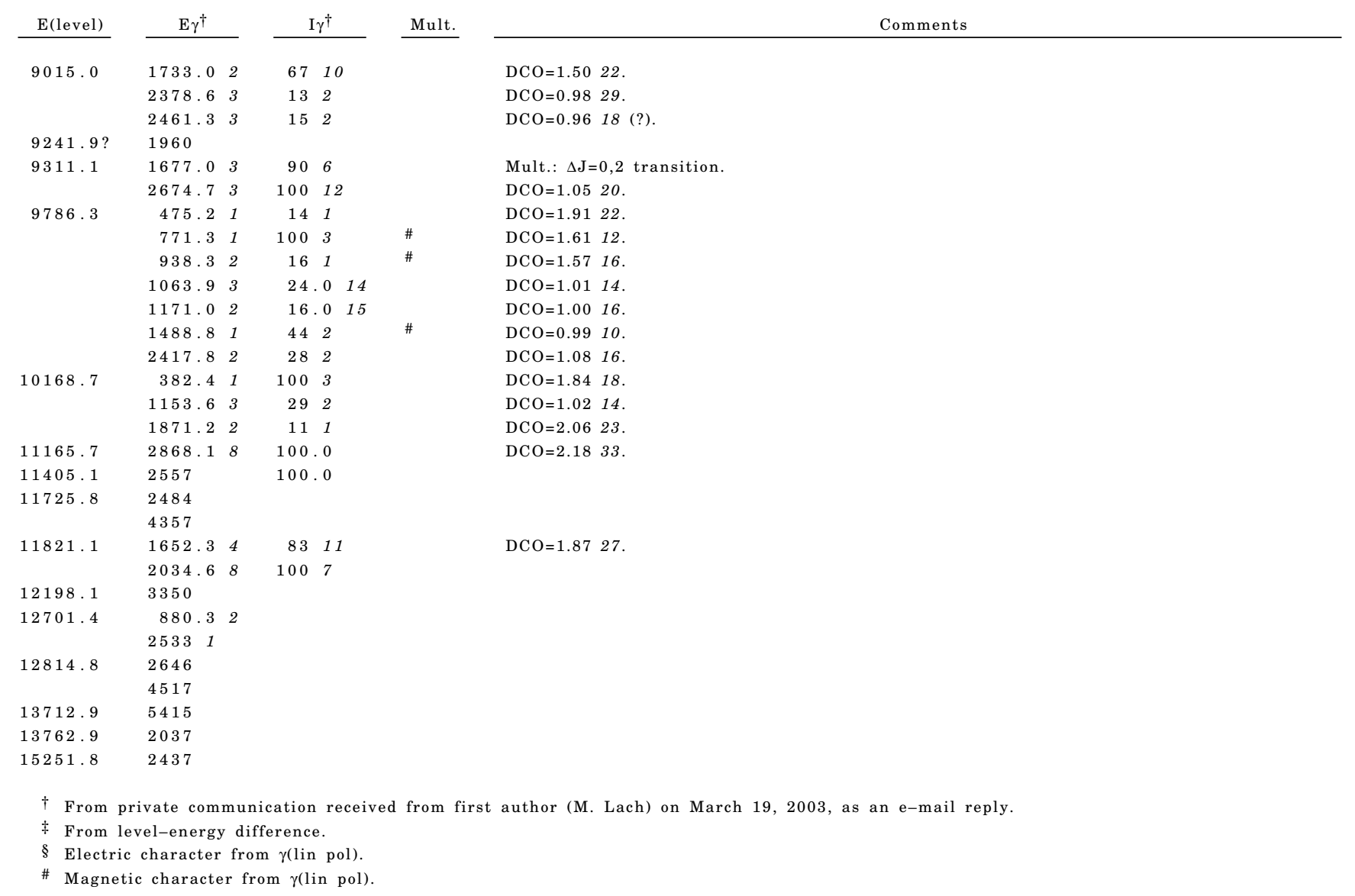

\section{${ }^{38} \operatorname{Ar}(\alpha, \gamma):$ Resonances $1967 \mathrm{Na10}$}

1967Na10: E=3-6 MeV alpha beam was produced from the Pennsylvania State University 6 MV Van de Graaff accelerator. Enriched ${ }^{38} \mathrm{Ar}$ target. $\gamma$-rays were detected with a NaI anti-coincidence spectrometer. Measured $\sigma(\theta)$. Deduced levels, J $\pi, \Gamma_{\gamma}$. Others:

1976Fo04,1974Fo04: $\mathrm{E}=6-15 \mathrm{MeV}$. Measured $\sigma(\theta)$ for the giant dipole resonances.

${ }^{42} \mathrm{Ca}$ Levels

All data are from $1967 \mathrm{Na} 10$.

\begin{tabular}{|c|c|c|c|c|c|c|c|c|c|c|c|c|}
\hline \multicolumn{2}{|c|}{$\mathrm{E}($ level $) \ddagger$} & \multirow{2}{*}{$\frac{\mathrm{J} \pi^{\dagger}}{}$} & \multicolumn{2}{|c|}{$(2 J+1) \Gamma_{\alpha} \Gamma_{\gamma} / \Gamma$} & \multicolumn{2}{|c|}{ Measured $\mathrm{E}_{\alpha}(\mathrm{lab})$} & \multicolumn{2}{|c|}{$\mathrm{E}($ level $) \ddagger$} & \multirow[t]{2}{*}{$\mathrm{J} \pi^{\dagger}$} & \multicolumn{2}{|c|}{$(2 \mathrm{~J}+1) \Gamma_{\alpha} \Gamma_{\gamma} / \Gamma$} & Measured $\mathrm{E}_{\alpha}$ (lab) \\
\hline 9115 & 5 & & 0.43 & $\mathrm{eV}$ & 3158 & 5 & 9757 & 5 & & 2.05 & $\mathrm{eV}$ & $3867 \quad 5$ \\
\hline 9191 & 5 & & 0.42 & $\mathrm{eV}$ & 3242 & 5 & 9784 & 5 & & 0.75 & $\mathrm{eV}$ & $3897 \quad 5$ \\
\hline 9280 & 5 & $1-$ & 1.49 & $\mathrm{eV}$ & 3340 & 5 & 9870 & 5 & & 1.44 & $\mathrm{eV}$ & $3992 \quad 5$ \\
\hline 9367 & 5 & & 0.78 & $\mathrm{eV}$ & 3436 & 5 & 9947 & 5 & & 1.74 & $\mathrm{eV}$ & $4077 \quad 5$ \\
\hline 9426 & 5 & & 1.48 & $\mathrm{eV}$ & 3501 & 5 & 10038 & 5 & & 0.62 & $\mathrm{eV}$ & $4178 \quad 5$ \\
\hline 9470 & 5 & & 0.83 & $\mathrm{eV}$ & 3551 & 5 & 10205 & 5 & & 0.85 & $\mathrm{eV}$ & $4362 \quad 5$ \\
\hline 9561 & 5 & & 0.76 & $\mathrm{eV}$ & 3651 & 5 & 10231 & 5 & & 0.57 & $\mathrm{eV}$ & $4391 \quad 5$ \\
\hline 9635 & 5 & & 0.53 & $\mathrm{eV}$ & 3732 & 5 & 10282 & 5 & $1-$ & 2.81 & $\mathrm{eV}$ & $4448 \quad 5$ \\
\hline 9672 & 5 & & 0.47 & $\mathrm{eV}$ & 3773 & 5 & 10314 & 5 & & 1.23 & $\mathrm{eV}$ & $4483 \quad 5$ \\
\hline 9699 & 5 & & 0.75 & $\mathrm{eV}$ & 3803 & 5 & 10358 & 5 & $1-$ & 2.23 & $\mathrm{eV}$ & $4532 \quad 5$ \\
\hline 9723 & 5 & & 0.75 & $\mathrm{eV}$ & 3830 & 5 & 10389 & 5 & & 0.99 & $\mathrm{eV}$ & $4566 \quad 5$ \\
\hline
\end{tabular}


${ }^{38} \operatorname{Ar}(\alpha, \gamma)$ : Resonances $1967 \mathrm{Na10}$ (continued)

\begin{tabular}{|c|c|c|c|c|c|c|c|c|c|c|c|c|}
\hline \multicolumn{2}{|c|}{ E(level) $\neq$} & \multirow{2}{*}{$\frac{\mathrm{J} \pi^{\dagger}}{}$} & \multicolumn{2}{|c|}{$(2 \mathrm{~J}+1) \Gamma_{\alpha} \Gamma_{\gamma} / \Gamma$} & \multicolumn{2}{|c|}{ Measured $E_{\alpha}$ (lab) } & \multicolumn{2}{|c|}{$\mathrm{E}($ level $) \ddagger$} & \multirow{2}{*}{$\frac{\mathrm{J} \pi^{\dagger}}{1-}$} & \multicolumn{2}{|c|}{$(2 \mathrm{~J}+1) \Gamma_{\alpha} \Gamma_{\gamma} / \Gamma$} & Measured $\mathrm{E}_{\alpha}(\mathrm{lab})$ \\
\hline 10453 & 5 & & 1.68 & $\mathrm{eV}$ & 4637 & 5 & 11013 & 5 & & 3.46 & $\mathrm{eV}$ & $5255 \quad 5$ \\
\hline 10500 & 5 & $1-$ & 2.13 & $\mathrm{eV}$ & 4688 & 5 & 11048 & 5 & & 1.28 & $\mathrm{eV}$ & $5294 \quad 5$ \\
\hline 10527 & 5 & & 0.50 & $\mathrm{eV}$ & 4718 & 5 & 11076 & 5 & $1-$ & 1.91 & $\mathrm{eV}$ & $5325 \quad 5$ \\
\hline 10561 & 5 & & 0.53 & $\mathrm{eV}$ & 4756 & 5 & 11108 & 5 & $1-$ & 1.68 & $\mathrm{eV}$ & 53615 \\
\hline 10588 & 5 & & 1.16 & $\mathrm{eV}$ & 4786 & 5 & 11149 & 5 & $1-$ & 2.51 & $\mathrm{eV}$ & $5406 \quad 5$ \\
\hline 10612 & 5 & & 0.83 & $\mathrm{eV}$ & 4812 & 5 & 11185 & 5 & $1-$ & 0.99 & $\mathrm{eV}$ & $5446 \quad 5$ \\
\hline 10633 & 5 & & 3.65 & $\mathrm{eV}$ & 4835 & 5 & 11223 & 5 & $1-$ & 2.63 & $\mathrm{eV}$ & $5488 \quad 5$ \\
\hline 10652 & 5 & & 0.45 & $\mathrm{eV}$ & 4857 & 5 & 11279 & 5 & & 1.05 & $\mathrm{eV}$ & $5550 \quad 5$ \\
\hline 10673 & 5 & $1-$ & 1. 32 & $\mathrm{eV}$ & 4880 & 5 & 11301 & 5 & & 1.21 & $\mathrm{eV}$ & $5574 \quad 5$ \\
\hline 10700 & 5 & & 0.98 & $\mathrm{eV}$ & 4910 & 5 & 11336 & 5 & $1-$ & 8.10 & $\mathrm{eV}$ & 56135 \\
\hline 10726 & 5 & $1-$ & 1.74 & $\mathrm{eV}$ & 4938 & 5 & 11378 & 5 & $1-$ & 2.43 & $\mathrm{eV}$ & $5659 \quad 5$ \\
\hline 10783 & 5 & $1-$ & 2.54 & $\mathrm{eV}$ & 5001 & 5 & 11399 & 5 & & 0.62 & $\mathrm{eV}$ & $5682 \quad 5$ \\
\hline 10805 & 5 & $1-$ & 4.29 & $\mathrm{eV}$ & 5026 & 5 & 11441 & 5 & $1-$ & 1.65 & $\mathrm{eV}$ & $5729 \quad 5$ \\
\hline 10842 & 5 & $1-$ & 5.37 & $\mathrm{eV}$ & 5067 & 5 & 11489 & 5 & $1-$ & 2.84 & $\mathrm{eV}$ & $5782 \quad 5$ \\
\hline 10884 & 5 & $1-$ & 2.89 & $\mathrm{eV}$ & 5113 & 5 & 11519 & 5 & & 0.66 & $\mathrm{eV}$ & $5815 \quad 5$ \\
\hline 10905 & 5 & $1-$ & 2.69 & $\mathrm{eV}$ & 5136 & 5 & 11545 & 5 & $1-$ & 1.59 & $\mathrm{eV}$ & $5843 \quad 5$ \\
\hline 10916 & 5 & $1-$ & 2.69 & $\mathrm{eV}$ & 5148 & 5 & 11612 & 5 & & 2.00 & $\mathrm{eV}$ & $5918 \quad 5$ \\
\hline 10968 & 5 & & 0.85 & $\mathrm{eV}$ & 5206 & 5 & 11661 & 5 & & 0.59 & $\mathrm{eV}$ & $5972 \quad 5$ \\
\hline 10985 & 5 & & 0.90 & $\mathrm{eV}$ & 5225 & 5 & & & & & & \\
\hline
\end{tabular}

$\dagger$ From angular distributions of the ground-state $\gamma$-rays from resonant states (1967Na10).

$\ddagger$ Excitation energy of resonance $=\mathrm{S}(\alpha)+\mathrm{E} \alpha(\mathrm{c} . \mathrm{m}$.) with $\mathrm{E} \alpha(\mathrm{c} . \mathrm{m}$.) deduced from $\mathrm{E} \alpha(\mathrm{lab}) . \mathrm{S}(\alpha)=6257.3425(2012 \mathrm{Wa} 38)$.

\section{${ }^{38} \mathrm{Ar}\left({ }^{6} \mathrm{Li}, \mathrm{d}\right) \quad 1978 \mathrm{Fo02}$}

Target ${ }^{38} \mathrm{Ar}$ g.s. $\mathrm{J} \pi=0+$.

1978Fo02: $\mathrm{E}=17.0 \mathrm{MeV}{ }^{6} \mathrm{Li}$ beam. 95\% enriched ${ }^{38} \mathrm{Ar}$ gas target. Reaction particles were momentum analyzed with a multi-range magnetic spectrograph. Measured $\sigma(\theta)$. Deduced levels, relative $\alpha$-strengths and major configurations for $0+$ states from DWBA calculations.

${ }^{42} \mathrm{Ca}$ Levels

All data are from 1978 Fo02.

\begin{tabular}{|c|c|c|c|c|c|c|c|}
\hline $\mathrm{E}$ (level) & $\mathrm{L}$ & Relative strength & $\mathrm{E}$ (level) & $\mathrm{L}$ & Relative strength & E(level) & Relative strength \\
\hline $0.0 \S$ & 0 & 1.00 & 3300 & 0 & 0.81 & $6020^{\#}$ & \\
\hline 1520 & & & 3650 & & & 6350 & \\
\hline 1840 & 0 & 0.22 & $4440^{\dagger}$ & & & 6520 末 & $0.13^{@}$ \\
\hline 2420 & & & $4450^{\dagger}$ & & & 6700 末 & $0.26^{@}$ \\
\hline 2750 & & & 5860 & & 0.31 & & \\
\hline
\end{tabular}

$\dagger$ Unresolved doublet.

$\ddagger \alpha$-unbound level.

$\S \mathrm{d} \sigma / \mathrm{d} \Omega\left(7.5^{\circ}\right)=3.8 \mu \mathrm{b} / \mathrm{sr}$.

\# Very weakly populated.

(a) DWBA cross section obtained by extrapolation. 


\section{${ }^{39} \mathbf{K}(\alpha, p) \quad 1995 T h 06$}

$\mathrm{J} \pi\left({ }^{39} \mathrm{~K}\right.$ g.s. $)=3 / 2+$.

1995Th06: E=25 MeV alpha beam was produced from the Niels Bohr Institute Tandem Van de Graaff Accelerator. A target of natural $\mathrm{KCl}$ enriched to $93.1 \%$ and $20 \mu \mathrm{g} / \mathrm{cm}^{2}$ in ${ }^{39} \mathrm{~K}$. Reaction products were momentum analyzed in a multigap magnetic spectrograph of the Elbek type and detected in nuclear emulsions, FWHM=30 keV. Measured $\sigma\left(E_{p}, \theta\right)$. Deduced levels, J $\pi$ from DWBA analysis.

1971Pe04: $\mathrm{E}=10 \mathrm{MeV}$. Measured $\sigma(\theta)$. Hauser-Feshbach calculations.

1955 Sc82: $\mathrm{E}=8.2 \mathrm{MeV}$. Eight groups reported up to $4100 \mathrm{keV}$.

$\begin{array}{crl}\text { Integrated cross } & \text { section }(\mathrm{mb}) & (1971 \mathrm{Pe} 04) \\ \text { Energy } & \text { cross } & \text { section } \\ 0 & 4.5 & 7 \\ 1520 & 14.8 & 22 \\ 1840 & 2.9 & 4 \\ 2430 & 11.0 & 16 \\ 2760 & 19.4 & 29 \\ 3200 & 18.5 & 37 \\ 3260 & 18.0 & 36 \\ 3390+3440 & 14.8 & 22 \\ 3680 & 9.6 & 14 \\ 3910 & 3.8 & 8 \\ 4260 & 4.7 & 7 \\ 4400 & 9.97 & 20 \\ 4810 & & \\ 5010 & & \end{array}$

${ }^{42} \mathrm{Ca} \mathrm{Levels}$

\begin{tabular}{|c|c|c|c|c|c|c|}
\hline $\mathrm{E}(\text { level })^{\dagger}$ & $\mathrm{J} \pi \ddagger$ & $\mathrm{d} \sigma / \mathrm{d} \Omega \mu \mathrm{b} / \mathrm{sr}^{\#}$ & $\mathrm{E}(\mathrm{le}$ & vel $)^{\dagger}$ & $\mathrm{J} \pi^{*}$ & $\mathrm{~d} \sigma / \mathrm{d} \Omega \mu \mathrm{b} / \mathrm{sr}^{\#}$ \\
\hline 0.0 & $0+f$ & 47 & 5618 & 7 & $3-\mathrm{b}$ & 286 \\
\hline $1524 \quad 4$ & $2+\mathrm{h}$ & 57 & 5669 & 7 & $3-\mathrm{c}$ & 229 \\
\hline 18364 & $0+\mathrm{f}$ & 27 & 5741 & 7 & $7-\mathrm{e}$ & 287 \\
\hline 24204 & $2+\mathrm{h}$ & 29 & 5791 & 7 & $3-b$ & 351 \\
\hline $2753 \quad 3$ & $4+i$ & 102 & 5866 & 7 & $0+, 2+f$ & 103 \\
\hline $3188 \quad 4$ & $6+\mathrm{i}$ & 123 & 5922 & 7 & $3-, 4-\mathrm{c}$ & 131 \\
\hline $3260 \S 40$ & & & 6026 & 5 & $1-$ to $6-d$ & 329 \\
\hline $3446^{\mathrm{k}} \quad 4$ & $3-\mathrm{c}$ & 152 & 6096 & 7 & $4+g$ & 260 \\
\hline $3680 \S 40$ & & & 6144 & 5 & $6-$ and $7-e$ & 641 \\
\hline $3953^{1} 3$ & $4-c$ & 339 & 6321 & 7 & $\mathrm{c}$ & 217 \\
\hline $4044 \quad 5$ & $3-\&$ & 97 & 6410 & 7 & $8-e$ & $219^{@}$ \\
\hline 41013 & $5-c$ & 384 & 6549 & 7 & $9-\mathrm{e}$ & 242 \\
\hline $4260 \S 40$ & & & 6587 & 7 & j & 339 \\
\hline $4425 \quad 4$ & $3-\mathrm{c}$ & 162 & 6737 & 7 & $\mathrm{f}$ & 174 \\
\hline $4691 \quad 4$ & $3-\mathrm{c}$ & 132 & 6779 & 7 & & $254^{@}$ \\
\hline $4894^{\mathrm{m}} 3$ & $5-c$ & 393 & 6931 & 7 & $\mathrm{f}$ & 190 \\
\hline $4960 \quad 5$ & $3-\mathrm{b}$ & 148 & 6975 & 7 & $\mathrm{j}$ & 218 \\
\hline $5073 \quad 5$ & $\leq 3-\mathrm{b}$ & 323 & 7031 & 7 & i & $251^{@}$ \\
\hline $5156 \quad 5$ & $3-\mathrm{c}$ & 131 & 7103 & 7 & $\mathrm{~b}$ & 206 \\
\hline $5316 \quad 5$ & $3-, 4-\mathrm{a}$ & 101 & 7153 & 7 & i & 209 \\
\hline $5387 \quad 5$ & $1-, 2-, 3-b$ & 419 & 7228 & 7 & $\mathrm{~b}$ & \\
\hline $5488 \quad 5$ & $6-\mathrm{d}$ & 589 & 7274 & 7 & $f$ & \\
\hline $5587 \quad 7$ & $3-\mathrm{c}$ & 70 & & & & \\
\hline
\end{tabular}

$\dagger$ Uncertainty of 3 to $7 \mathrm{keV}$ assigned (evaluators) based on a general statement by $1995 \mathrm{Th} 06$.

\# From comparisons of experimental $\sigma(\theta)$ data with DWBA calculations with a cluster form factor for a single particle transfer, as stated in comments for individual levels (1995Th06).

$\S$ From $1971 \mathrm{Pe} 04$ only.

\# At $17.5^{\circ}$, unless otherwise stated.

(a) At $22.5^{\circ}$.

\& For $\mathrm{p}_{1 / 2}$ transfer.

a For $\mathrm{p}_{3 / 2}$ transfer. 
${ }^{39} \mathrm{~K}(\alpha, p) \quad 1995 \mathrm{Th} 06$ (continued)

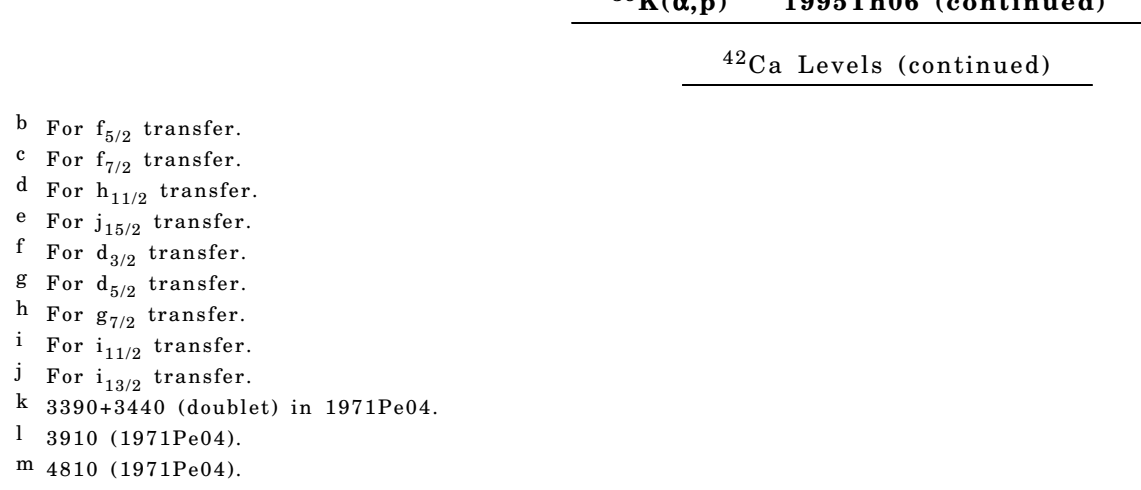

${ }^{39} \mathrm{~K}(\alpha, \mathrm{p} \gamma) \quad 1980 \mathrm{Ke01}$

Includes g-factor and $\mathrm{T}_{1 / 2}$ measurements in ${ }^{40} \mathrm{Ca}(\alpha, 2 \mathrm{p} \gamma)$.

1980Ke01 (also 1976Bi15, 1976Be13): E=15.14 MeV alpha beam was produced from the $7 \mathrm{MV}$ Van de Graaff accelerator of the University of Freiburg. A target of $140 \mu \mathrm{g} / \mathrm{cm}^{2}{ }^{39} \mathrm{~K}$ evaporated onto a $10 \mu \mathrm{g} / \mathrm{cm}^{2}$ carbon backing. Charged particles were detected with an $\Delta \mathrm{E}-\mathrm{E}$ telescope of two annular surface barrier detectors and $\gamma$-rays were detected with two $\mathrm{Ge}(\mathrm{Li})$ detectors of $22 \%$ efficiency. Measured $\mathrm{E} \gamma, \mathrm{I} \gamma, \mathrm{p}-\gamma$ coin. Lifetime measurement by DSA technique in 1976Bi15 and 1976Be13; $\gamma(\theta)$ in 1976Be13. Deduced levels, branchings, mixing ratios.

1978Pr02 (also 1976Pr15): E=9.5, 16.0 MeV. Measured $\gamma$ (lin pol), lifetimes by Doppler shift method. Also 1976Pr15 at $\mathrm{E}=9.5,11.5 \mathrm{MeV}$. Deduced levels, J $\pi$, branchings, mixing ratios.

1973Mc16: $\mathrm{E}=9 \mathrm{MeV}$. Measured $\mathrm{p}-\gamma$ coin, lifetime of 2750 level by DSA method.

1971Ha12: $\mathrm{E}=7.5 \mathrm{MeV}$. Measured $\mathrm{p} \gamma(\theta)$, lifetimes by DSA method.

1971AlZF: E=15 MeV. Measured $\gamma \gamma$ coin, $\gamma(\theta)$.

1970Ma39: $\mathrm{E}=10 \mathrm{MeV}$. Measured $\mathrm{p} \gamma(\theta, \mathrm{H}, \mathrm{t})$, deduced g factor for 3190 level.

1970Co32: E=8.3 MeV. Measured lifetime by recoil-distance method.

1969Ha02: $\mathrm{E}=7.5,9.0,10.6 \mathrm{MeV}$. Measured $\mathrm{p} \gamma(\mathrm{t})$ and Doppler-shift attenuation.

g-factor measurements of $3190,6+$ level using ${ }^{40} \mathrm{Ca}(\alpha, 2 \mathrm{p} \gamma)$ : 1975Yo02 ( $\left.\mathrm{E}=23 \mathrm{MeV}\right), 1971 \mathrm{No} 06$ (also 1972No09,1970No04) $(\mathrm{E}=25 \mathrm{MeV})$.

\section{${ }^{42} \mathrm{Ca}$ Levels}

\begin{tabular}{|c|c|c|c|c|}
\hline \multicolumn{2}{|c|}{$\mathrm{E}(\text { level })^{\dagger}$} & $\mathrm{J} \pi^{*}$ & $\mathrm{~T}_{1 / 2}$ & Comments \\
\hline 0 & 0 & $0+$ & & \\
\hline 1524 & 2 & $2+$ & $0.62 \mathrm{ps} 21$ & $\begin{array}{l}\mathrm{T}_{1 / 2}: \text { from } 1969 \mathrm{Ha} 02,1971 \mathrm{Ha} 12 \text {. Other: } 0.69 \mathrm{ps} 21 \text { (quoted by } 1971 \mathrm{Ha} 12 \text { from Carlsson } \\
(\text { priv comm)). }\end{array}$ \\
\hline 1837 & 2 & $0+$ & & \\
\hline 2423 & 3 & $2+$ & $0.114 \mathrm{ps} 30$ & $\begin{array}{l}\mathrm{T}_{1 / 2}: \text { average of } 0.11 \mathrm{ps} 3(1969 \mathrm{Ha} 02), 0.118 \mathrm{ps} 28(1971 \mathrm{Ha} 12) \text {. Other: } 0.125 \mathrm{ps} 21 \\
\text { (quoted by } 1971 \mathrm{Ha} 12 \text { from Carlsson (priv comm)). }\end{array}$ \\
\hline 2751 & 2 & $4+$ & $1.6 \mathrm{ps} 7$ & $\begin{array}{l}\mathrm{T}_{1 / 2}: \text { from } 1973 \mathrm{Mc} 16 \text {. Others: } 24 \mathrm{ps} 7(1969 \mathrm{Ha} 02), 1.3 \mathrm{ps}+14-7(1971 \mathrm{Ha} 12) \text {, } \\
2.0 \mathrm{ps}+14-7 \text { (quoted by } 1971 \mathrm{Ha} 12 \text { from Carlsson (priv comm)), } 8.0 \mathrm{ps} 17 \text { (1970Co32). }\end{array}$ \\
\hline 3190 & 2 & $6+$ & $5.30 \mathrm{~ns} 16$ & $\begin{array}{l}\mathrm{T}_{1 / 2}: \text { from } 1970 \mathrm{Ma} 39 . \text { Others: } 3.7 \mathrm{~ns} 5(1969 \mathrm{Ha} 02), 5.3 \mathrm{~ns} 3 \text { (quoted by } 1971 \mathrm{Ha} 12 \text { from } \\
\text { Carlsson (priv comm)), } 5.52 \mathrm{~ns} 15(1970 \mathrm{No} 04) \text { in }{ }^{40} \mathrm{Ca}(\alpha, 2 \mathrm{p} \gamma) .\end{array}$ \\
\hline 3253 & 2 & $4+$ & $0.118 \mathrm{ps} 21$ & $\begin{array}{l}\mathrm{g}=-0.423(1970 \mathrm{Ma} 39),-0.50+2-3(1971 \mathrm{No} 06,1972 \mathrm{No} 09),-0.41515(1975 \mathrm{Yo} 02) \\
\mathrm{T}_{1 / 2}: \text { average of } 0.10 \mathrm{ps} 3(1969 \mathrm{Ha} 02), 0.125 \mathrm{ps} 21(1971 \mathrm{Ha} 12) \text {. Other: } 0.17 \mathrm{ps} 3 \\
\quad \text { quoted by } 1971 \mathrm{Ha} 12 \text { from Carlsson (priv comm)). }\end{array}$ \\
\hline 3300 & 2 & $0+$ & & \\
\hline 3393 & 2 & $2+$ & $0.118 \mathrm{ps} 21$ & $\mathrm{~T}_{1 / 2}$ : from $1971 \mathrm{Ha} 12$. Other: $0.18 \mathrm{ps} 4$ (quoted by $1971 \mathrm{Ha} 12$ from Carlsson (priv comm)). \\
\hline 3446 & 6 & $3-$ & 0.23 ps 7 & $\begin{array}{l}\mathrm{T}_{1 / 2} \text { : average of } 0.25 \mathrm{ps} 7(1969 \mathrm{Ha} 02), 0.20 \mathrm{ps} 7(1971 \mathrm{Ha} 12) \text {. Other: } 0.28 \mathrm{ps} 7 \\
\text { (quoted by } 1971 \mathrm{Ha} 12 \text { from Carlsson (priv comm)). }\end{array}$ \\
\hline 3654 & 2 & $2+$ & 49 fs 35 & $\mathrm{~T}_{1 / 2}$ : from $1971 \mathrm{Ha} 12$. Other: 62 fs 28 (quoted by $1971 \mathrm{Ha} 12$ from Carlsson (priv comm)). \\
\hline 3884 & 2 & $1-$ & & \\
\hline 3953 & 2 & $4-$ & $3.26 \mathrm{ps} 21$ & $\mathrm{~T}_{1 / 2}:$ from $1976 \operatorname{Pr} 15$ \\
\hline 3998 & 2 & $4+$ & & \\
\hline 4048 & 2 & $3-$ & $0.17 \mathrm{ps} 5$ & $\mathrm{~T}_{1 / 2}:$ average of $0.17 \mathrm{ps} 5(1976 \operatorname{Pr} 15), 0.17 \mathrm{ps} 6$ (1976 Bi 15$)$ \\
\hline 4099 & 2 & $5-$ & $0.45 \mathrm{ps} 10$ & $\mathrm{~T}_{1 / 2}:$ average of $0.40 \mathrm{ps} 10(1976 \operatorname{Pr} 15), 0.51 \mathrm{ps}+10-8(1976 \mathrm{Bi} 15)$ \\
\hline 4117 & 2 & $3-$ & & \\
\hline 4232 & 2 & 1 & & \\
\hline 4355 & 2 & $4-$ & $0.47 \mathrm{ps} 7$ & $\mathrm{~T}_{1 / 2}:$ average of $0.53 \mathrm{ps} 7(1976 \operatorname{Pr} 15), 0.38 \mathrm{ps} 10$ (1976Bi15). \\
\hline 4420 & 2 & $3-$ & & \\
\hline
\end{tabular}




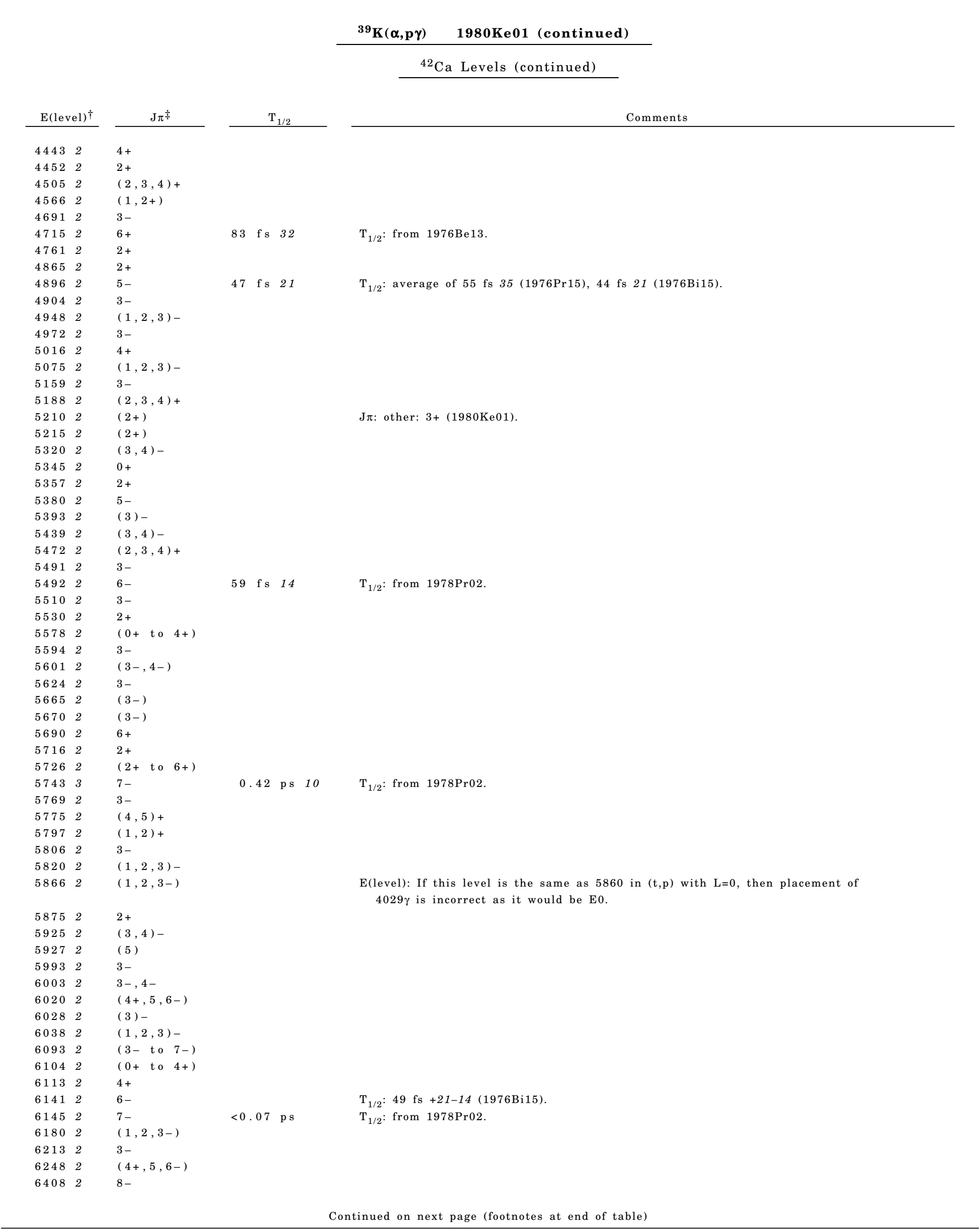




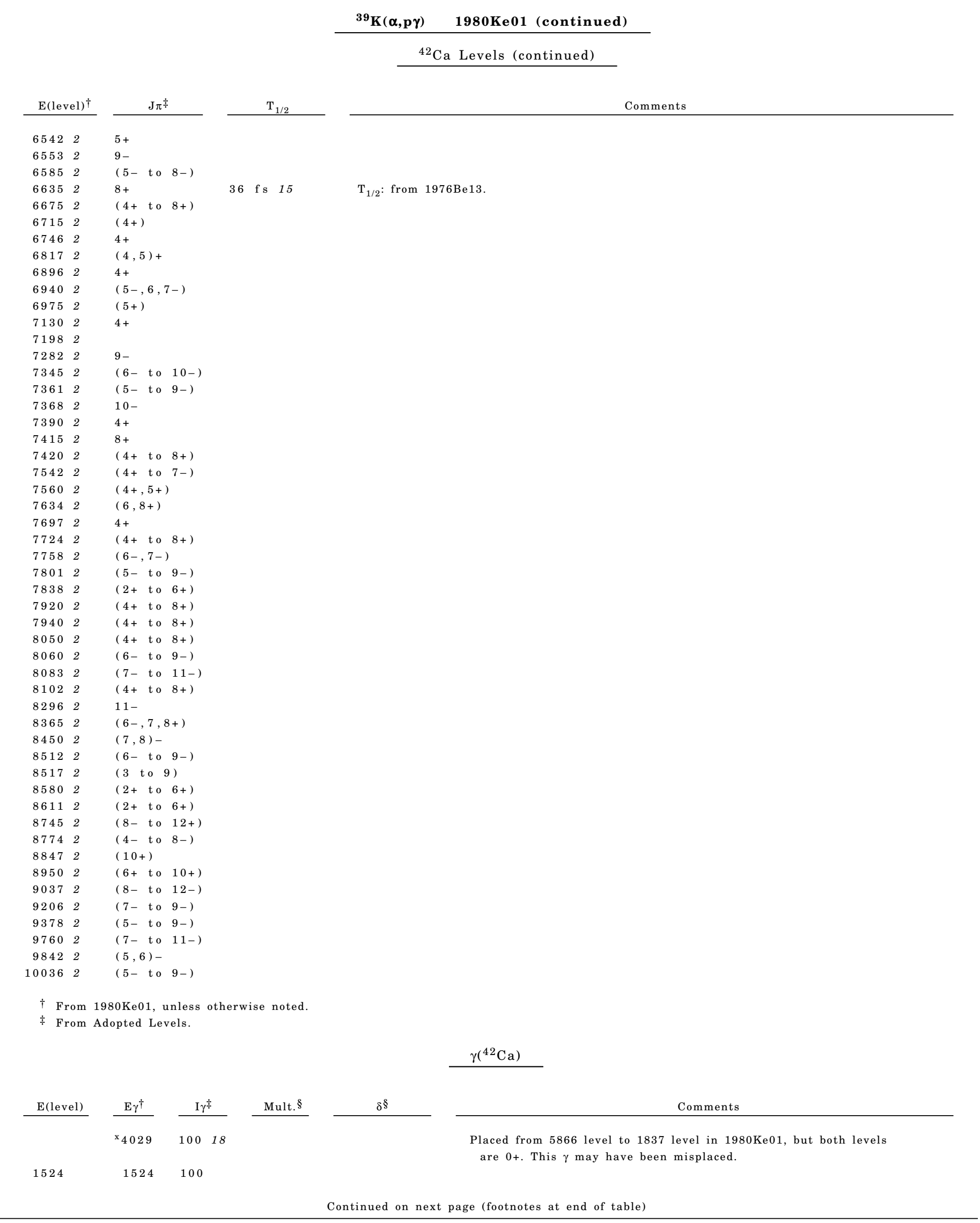




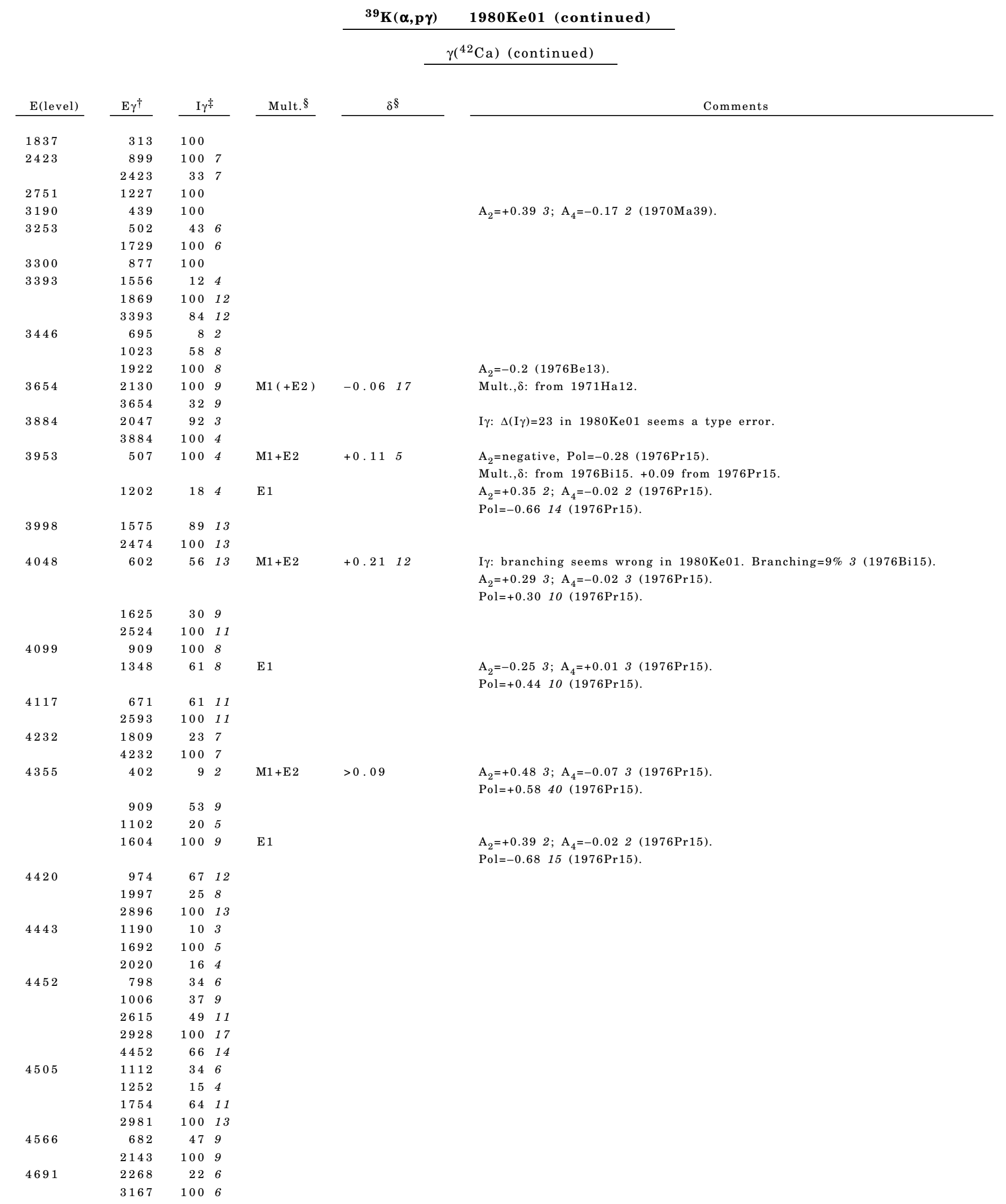




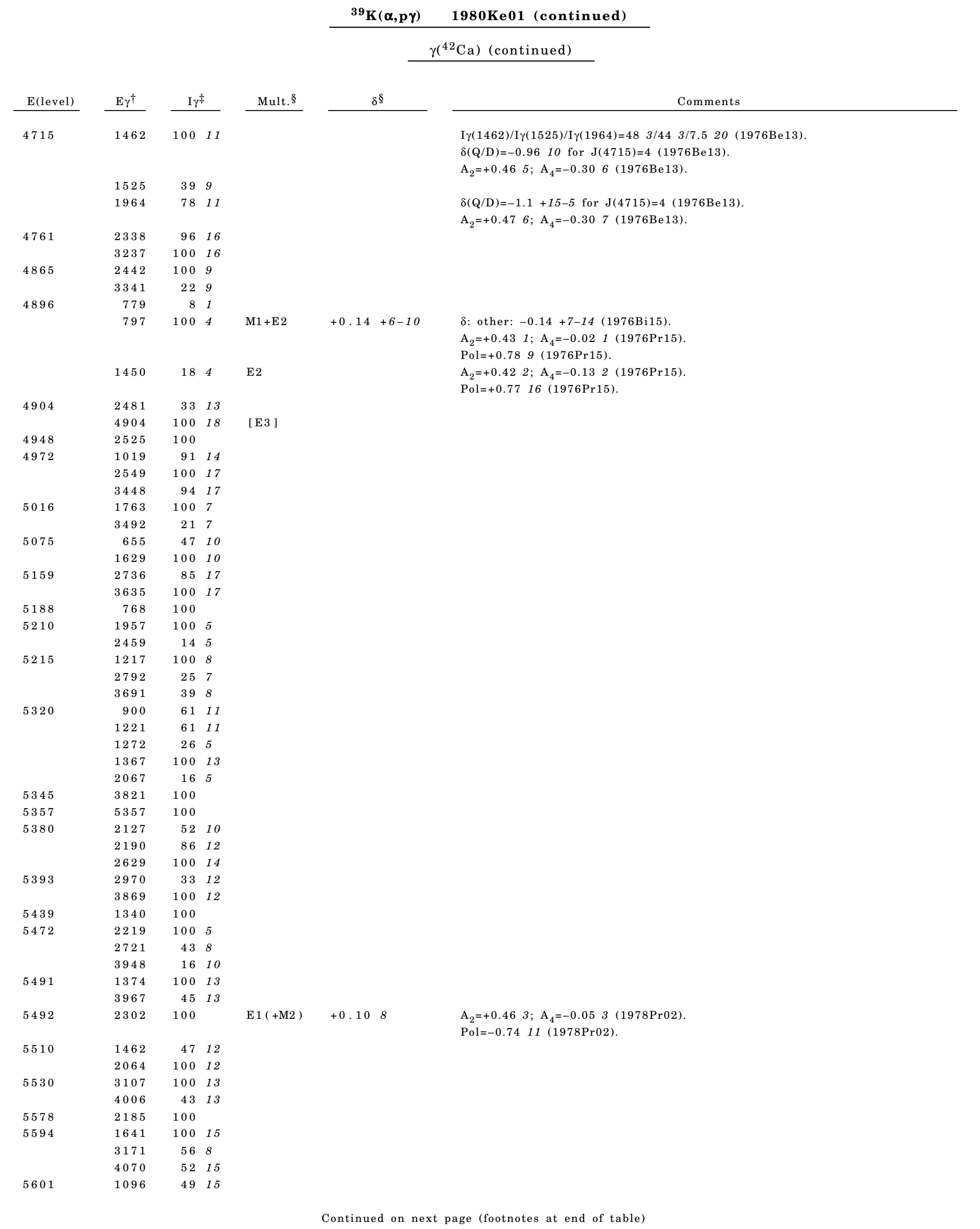


${ }^{39} \mathrm{~K}(\alpha, \mathrm{p} \gamma) \quad 1980 \mathrm{Ke} 01$ (continued)

$\gamma\left({ }^{42} \mathrm{Ca}\right)$ (continued)

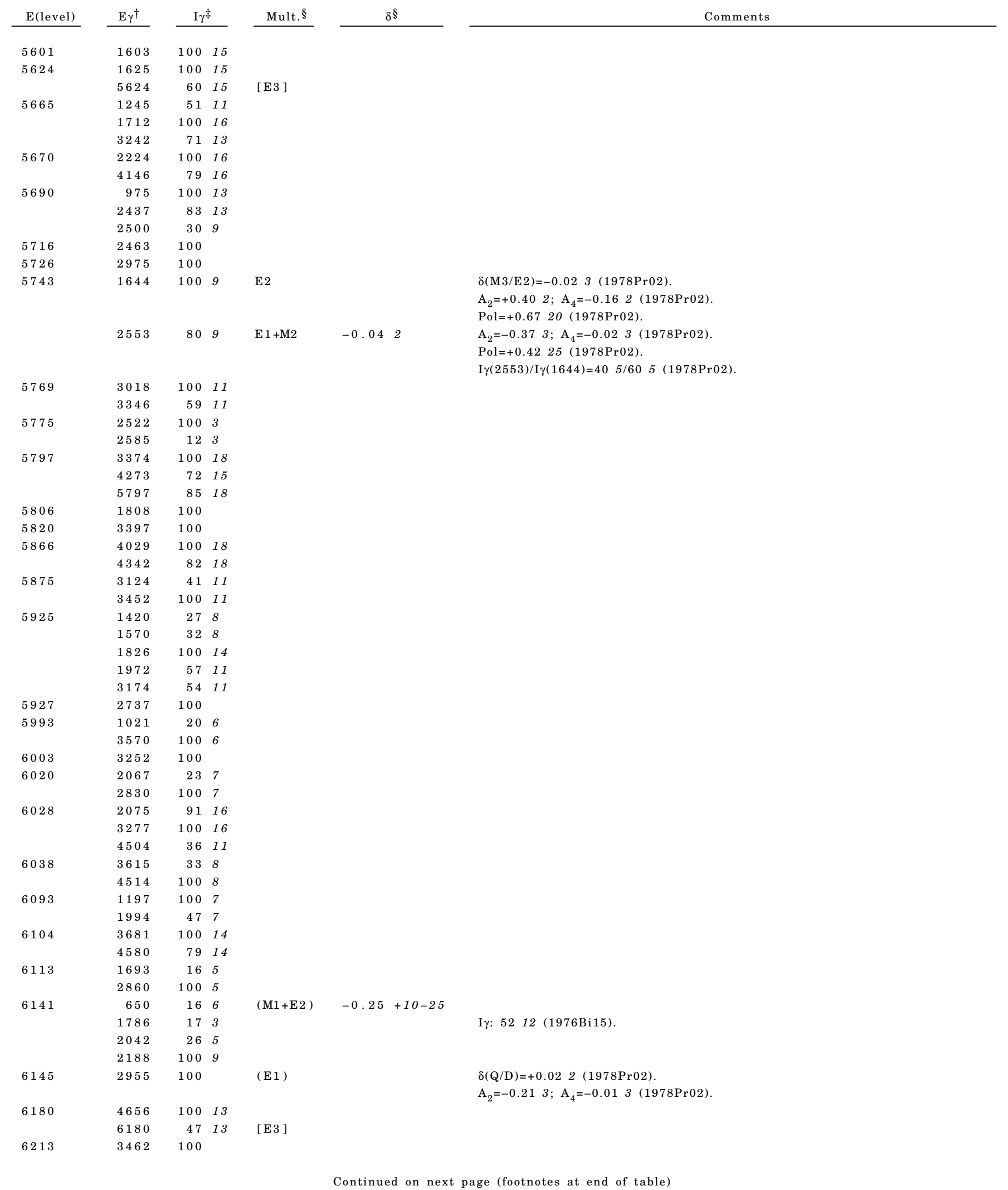




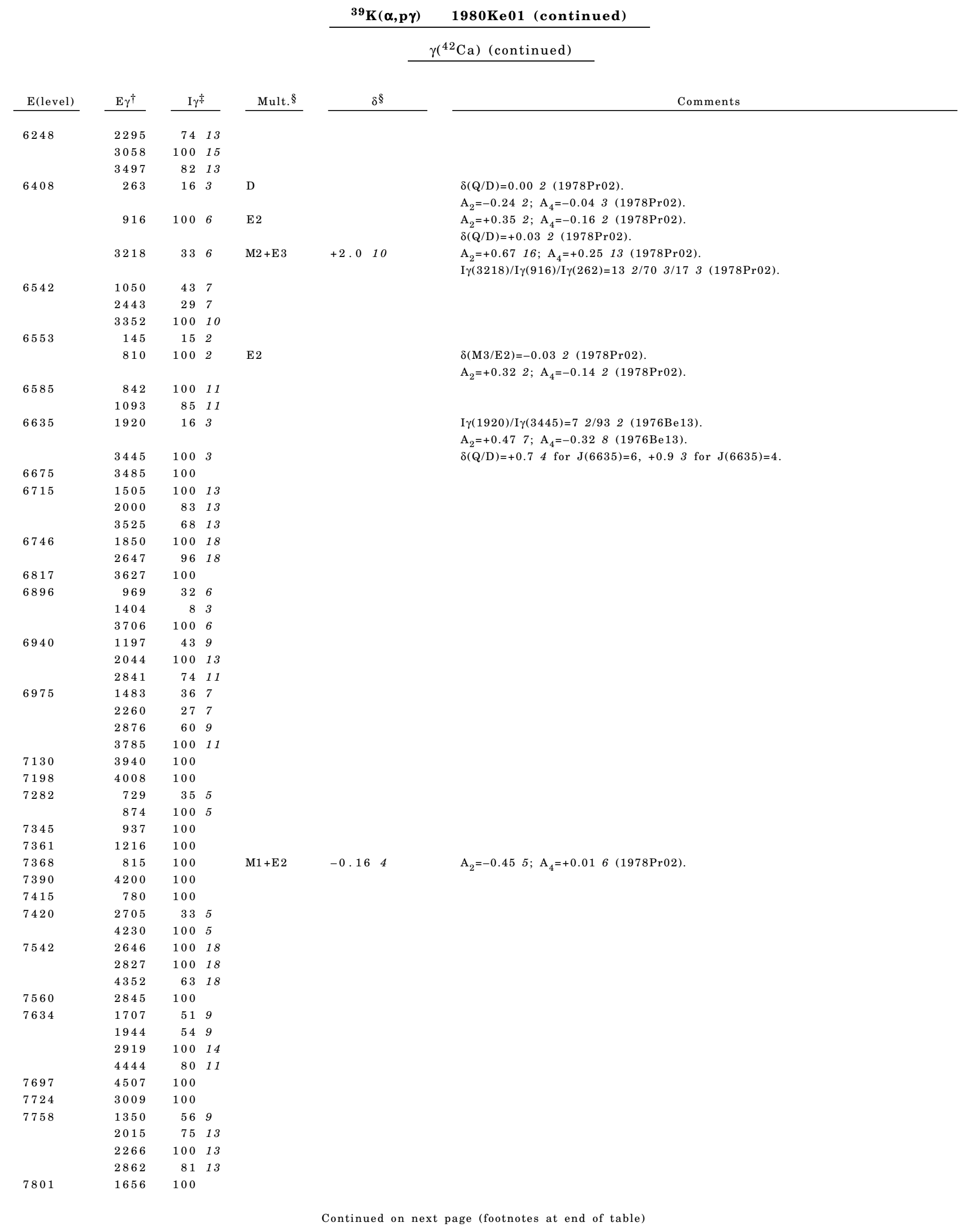




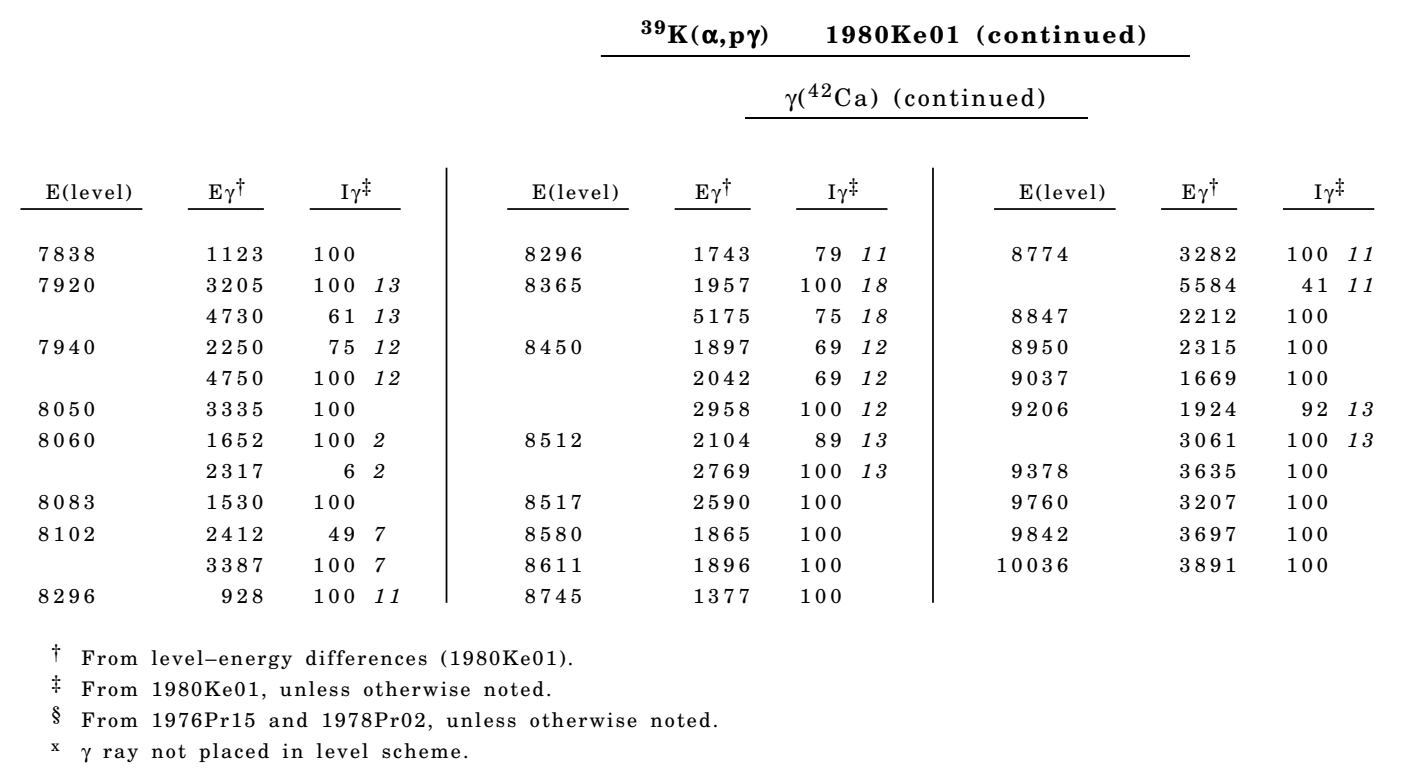

\section{${ }^{40} \mathrm{Ar}\left({ }^{3} \mathrm{He}, \mathrm{n}\right) \quad 1977 \mathrm{Bo16,1974Pe03}$}

Target ${ }^{40} \mathrm{Ar}$ g.s. $\mathrm{J} \pi=0+$.

1977Bo16: E=11.5 $\mathrm{MeV}{ }^{3} \mathrm{He}$ beam was produced at the CN Van de Graaff accelerator at the Hahn-Meitner Institute. Target of enriched ${ }^{40} \mathrm{Ar}$ gas. Neutrons were detected with 16 liquid scintillators and energies were determined by the time-of-flight (TOF) method with a flight path of $17.5 \mathrm{~m}$. FWHM=40-350 keV. Measured $\sigma(\theta)$. Deduced levels J $\pi$, L-tranfers from DWBA analysis.

1974Pe03: $\mathrm{E}=18.65 \mathrm{MeV}{ }^{3} \mathrm{He}$ beam was produced at the University of Michigan. A natural argon gas target. Neutrons were measured using a time-of-flight spectrometer, FWHM=320 keV. Measured cross sections at $0^{\circ}$ and $15^{\circ}$. Deduced levels. DWBA calculations.

Other:

1964Br13: $\mathrm{E}=25 \mathrm{MeV}$. Measured cross section at $0^{\circ}$. No levels identified.

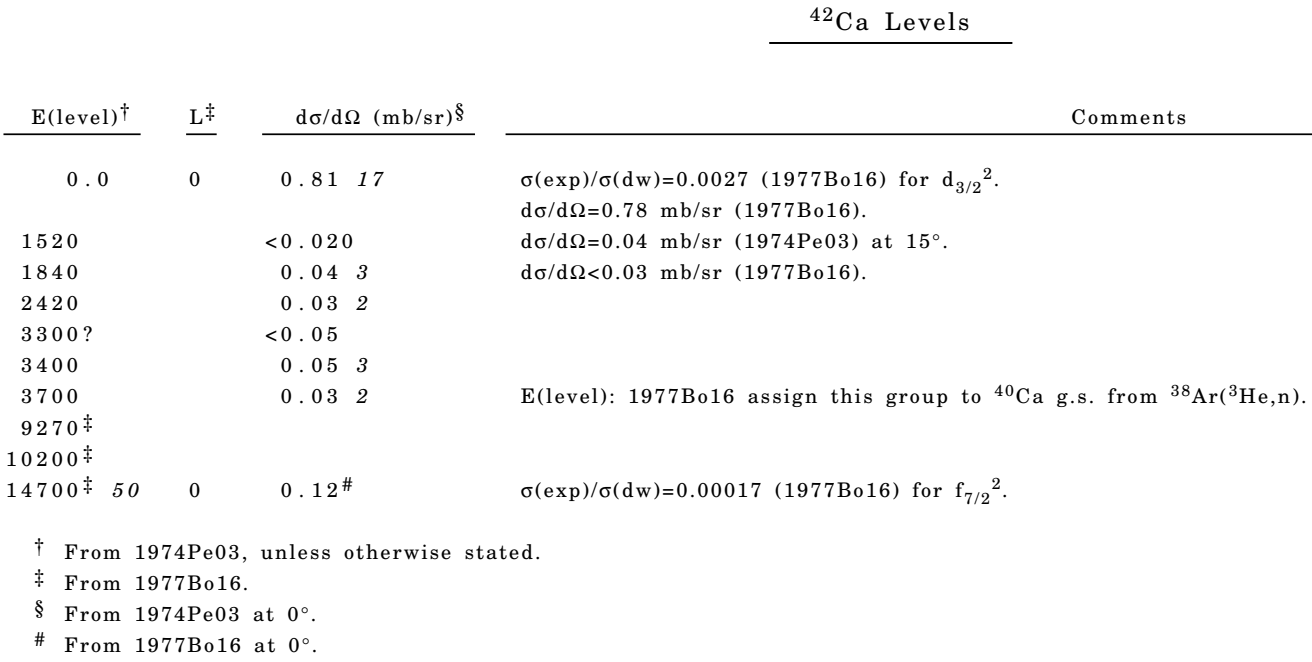


Target ${ }^{40} \mathrm{Ca}$ g.s. $\mathrm{J} \pi=0+$.

1967Bj06: $\mathrm{E}=10-12 \mathrm{MeV}$ triton beam was produced at the Aldermaston Tandem generator. $\mathrm{A}$ target of $\mathrm{CaCO}_{3}\left(97 \%\right.$ in $\left.{ }^{40} \mathrm{Ca}\right)$ evaporated onto a carbon film. Reaction products were momentum analyzed with a multi-angle, broad-range magnetic spectrograph and detected in nuclear emulsions, FWHM=15-20 keV. Measured $\sigma\left(E_{p}, \theta\right)$. Deduced levels, J $\pi$, L from DWBA analysis.

1967Wi15: E=7.5 MeV tritons were produced at the Los Alamos Vertical Van de Graaff Accelerator. A natural calcium target. Protons were detected by a $\Delta \mathrm{E}-\mathrm{E}$ solid-state detector telescope, FWHM=50 keV. Measured $\sigma(\theta)$. A total 28 levels reported, with $\mathrm{L}$ values for 19 groups.

1973Ca13: $\mathrm{E}=20 \mathrm{MeV}$. FWHM=20 keV. Measured $\sigma(\theta)$. Six $\mathrm{L}=0$ levels reported.

1964Mi06: $\mathrm{E}=7.2 \mathrm{MeV}$. Energies and L values of first five levels reported.

Others:

1977Is03: E=12 MeV. Measured form factor.

1997Be45: $\mathrm{E}=28,33,37.3 \mathrm{MeV}$. Measured $\sigma(\theta)$, deduced model parameters.

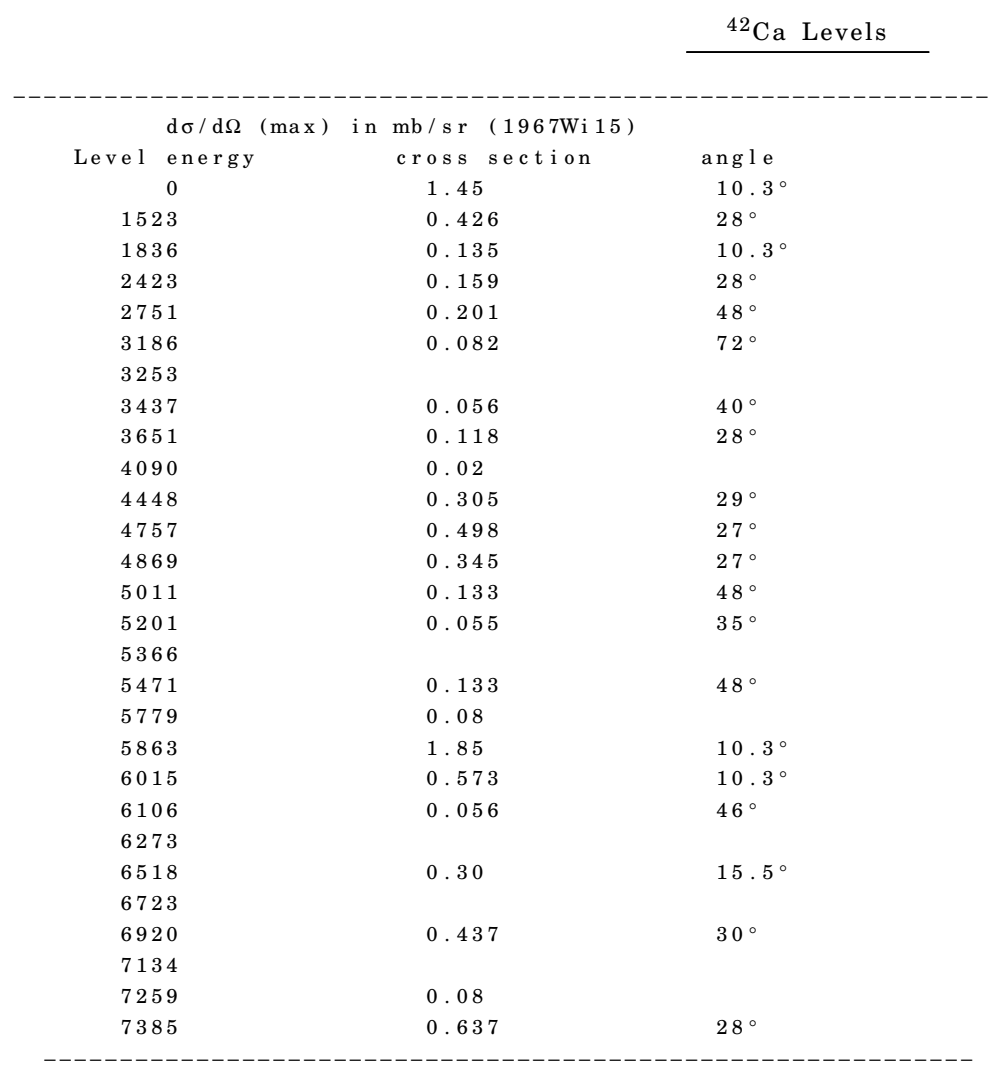

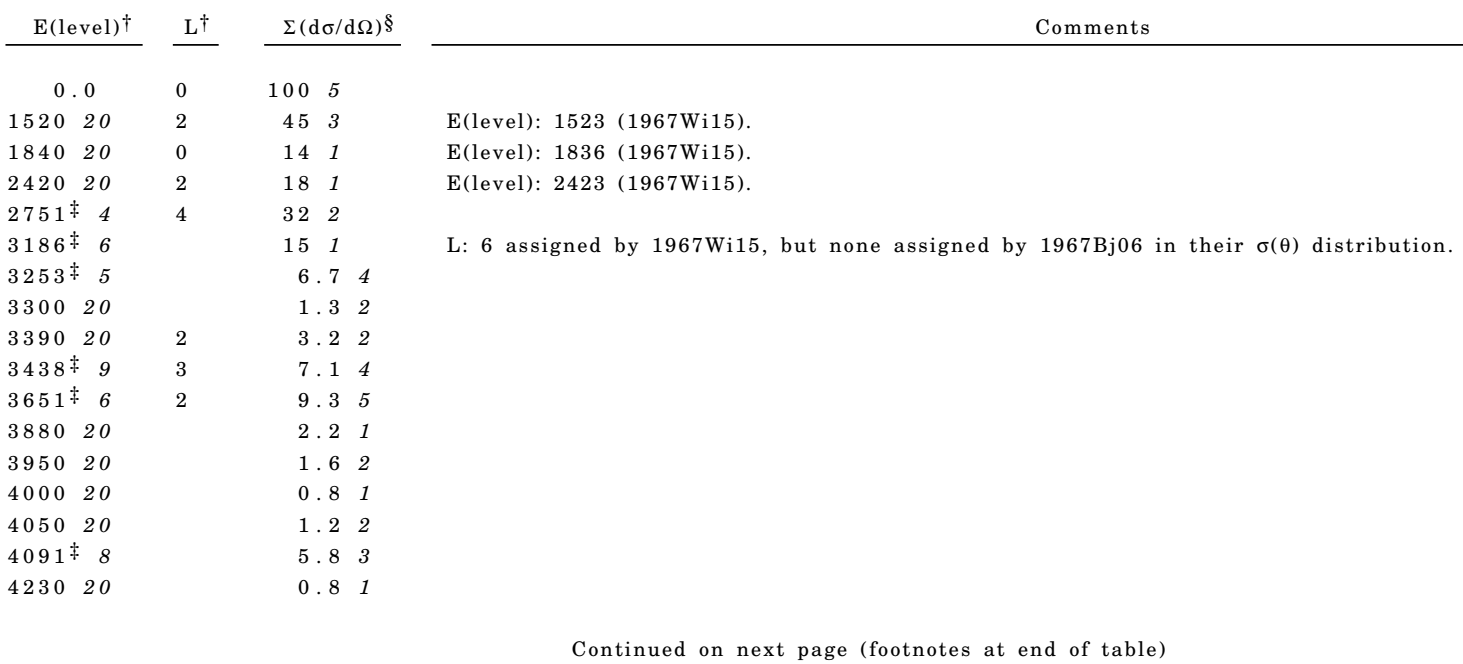


${ }^{40} \mathrm{Ca}(\mathrm{t}, \mathrm{p}) \quad 1967 \mathrm{Bj} 06,1967 \mathrm{Wi15}$ (continued)

${ }^{42} \mathrm{Ca}$ Levels (continued)

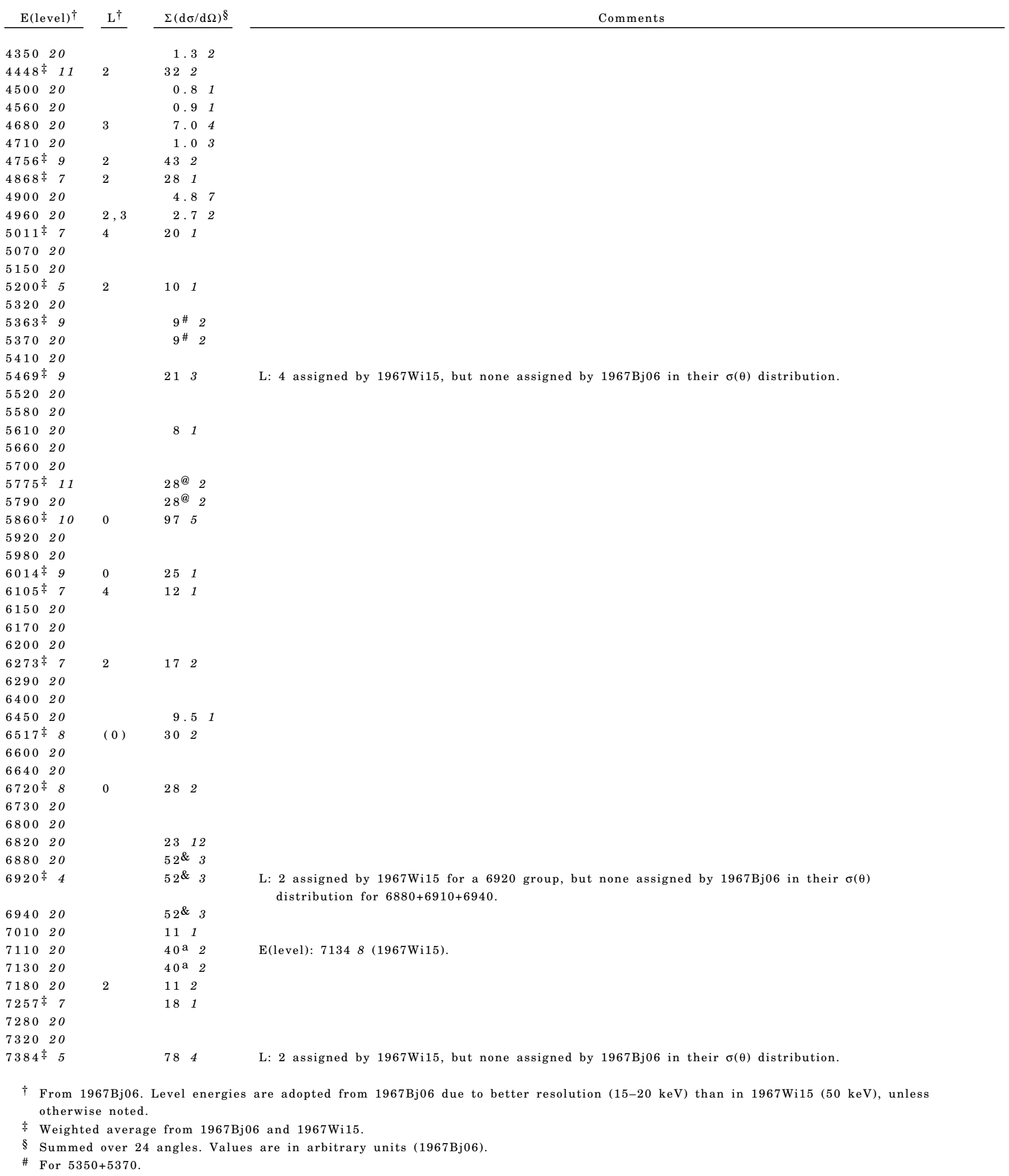


(a) For $5760+5790$.

\& For $6880+6910+6940$.

a For $7110+7130$

\title{
${ }^{40} \mathrm{Ca}(\mathrm{t}, \mathrm{p} \gamma) \quad 1977 \mathrm{LiZI}$
}

1977LiZI: $\mathrm{E}=10 \mathrm{MeV}$. Measured $\gamma(\theta)$ in coincidence with protons at $0^{\circ}$.

\author{
${ }^{42} \mathrm{Ca}$ Levels
}

\begin{tabular}{c} 
E(level) \\
\hline 0.0 \\
1520 \\
2420 \\
3890 \\
5850 \\
6020 \\
6520 \\
6700 \\
$6820^{\dagger}$
\end{tabular}

$(0) \ddagger$

$\dagger$ Strongly excited state.

$\ddagger$ From isotropic $\gamma(\theta)$.

\begin{tabular}{|c|c|c|c|c|c|}
\hline & & & & & $\gamma\left({ }^{42} \mathrm{Ca}\right)$ \\
\hline $\mathrm{E} \gamma^{\dagger}$ & E(level) & $\mathrm{E} \gamma^{\dagger}$ & E(level) & $\mathrm{E} \gamma^{\dagger}$ & $\mathrm{E}$ (level) \\
\hline 900 & 2420 & $2930 \ddagger$ & 6820 & $4400 \ddagger$ & 6820 \\
\hline 1520 & 1520 & 3430 & 5850 & 4500 & 6020 \\
\hline 1960 & 5850 & 3600 & 6020 & 5000 & 6520 \\
\hline 2130 & 6020 & 3890 & 3890 & 5180 & 6700 \\
\hline 2420 & 2420 & 4100 & 6520 & $5300 \ddagger$ & 6820 \\
\hline 2630 & 6520 & 4280 & 6700 & & \\
\hline 2810 & 6700 & 4330 & 5850 & & \\
\hline
\end{tabular}

\section{${ }^{40} \mathrm{Ca}\left(\alpha,{ }^{2} \mathrm{He}\right) \quad 1990 \mathrm{FiO7}, 1980 \mathrm{Va} 17$}

1990Fi07: E=55.7 MeV $\alpha$ beam was produced at the Bonn isochroneous cyclotron. A target of $40 \mathrm{Ca}$ enriched to $99.8 \%$. $2 \mathrm{He}$ is detected by measuring the two breakup protons in coincidence with a pair of vertically arranged $\Delta \mathrm{E}-\mathrm{E}$ detector telescopes, FWHM=200-300 keV. Measured $\sigma(\theta)$. Deduced levels, J $\pi$, L from DWBA analysis.

1980Va17: E=65 MeV $\alpha$ beam was produced at the AVF cyclotron of the KVI. A solid and self-supporting target of 40Ca. Break-up protons from the outgoing $2 \mathrm{He}$ were detected with two $\Delta \mathrm{E}-\mathrm{E}$ solid-state counter telescopes. Measured $\sigma(\theta)$ Deduced level, J $\pi$, L from DWBA analysis.

1978Ja10 (also 1978Ja22, 1980StZO): E=55 MeV. Measured pp coin, $\sigma(\theta)$. Deduced reaction mechanism. $6+$ state at 3190 is the most intensely populated state, whereas $0,1530,2750,7400$ and 9040 are very weak in the spectrum shown by $1978 \mathrm{Ja} 10$.

\section{${ }^{42} \mathrm{Ca}$ Levels}

\begin{tabular}{|c|c|c|c|}
\hline $\mathrm{E}(\text { level })^{\dagger}$ & $\mathrm{L}^{\dagger}$ & & Normalization constant $\mathrm{N}^{\dagger}$ \\
\hline $0.0 \S$ & 0 & 260 & 20 \\
\hline $1530 \S 50$ & 2 & 260 & 20 \\
\hline $2750 \S 50$ & 4 & 180 & 15 \\
\hline
\end{tabular}

Continued on next page (footnotes at end of table) 


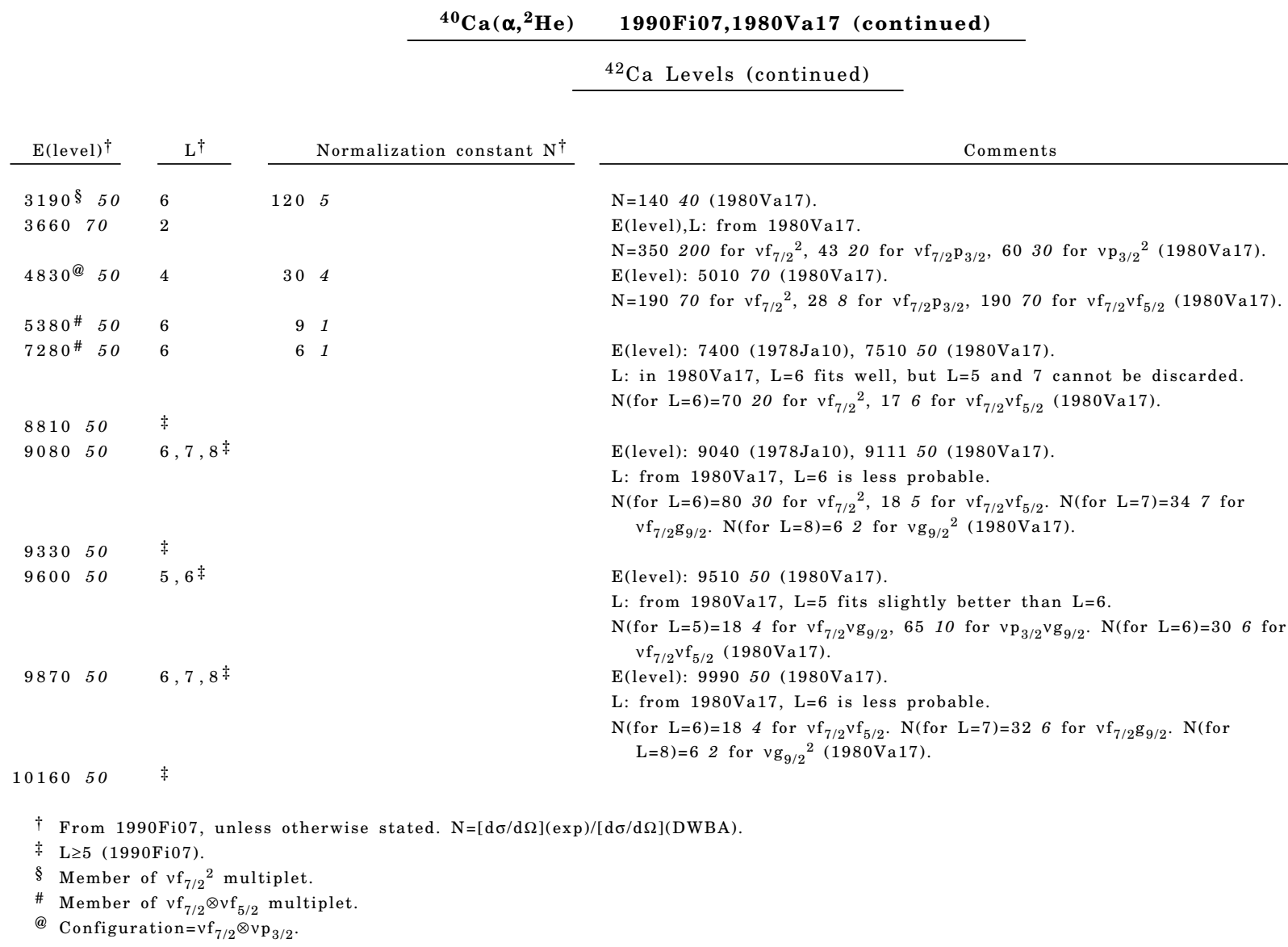

\section{${ }^{40} \mathrm{Ca}\left({ }^{14} \mathrm{C},{ }^{12} \mathrm{C}\right),\left({ }^{12} \mathrm{C},{ }^{10} \mathrm{C}\right),\left({ }^{10} \mathrm{~B},{ }^{8} \mathrm{~B}\right) \quad 1985 \mathrm{ViO} 1,1972 \mathrm{Sc} 21,1979 \mathrm{KuZQ}$}

Target ${ }^{40} \mathrm{Ca}$ g.s. $\mathrm{J} \pi=0+$.

1985Vi01: $\left({ }^{14} \mathrm{C},{ }^{12} \mathrm{C}\right) \mathrm{E}=64 \mathrm{MeV}{ }^{14} \mathrm{C}$ beam was produced from the Los Alamos three-state electrostatic accelerator. Target of ${ }^{40} \mathrm{Ca}$ on a carbon backing. Scattered particles were momentum analyzed with the Q3D spectrometer and detected in a $50 \mathrm{~cm}$ long gridded ionization counter, FWHM=250 keV. Measured $\sigma(\theta)$. Levels observed as multiplets up to 4800 . DWBA analysis.

1972Sc21,1974An36: $\left({ }^{12} \mathrm{C},{ }^{10} \mathrm{C}\right) \mathrm{E}=114 \mathrm{MeV}$. 3190, 6+ level reported.

1977 NaZS: $\left({ }^{12} \mathrm{C},{ }^{10} \mathrm{C}\right) \mathrm{E}=108 \mathrm{MeV}$. Measured $\sigma(\theta)$

1979KuZQ: $\left({ }^{10} \mathrm{~B},{ }^{8} \mathrm{~B}\right) \mathrm{E}=70 \mathrm{MeV}$, levels 1520,2750 and 3190 reported.

\section{${ }^{42} \mathrm{Ca}$ Levels}

\begin{tabular}{|c|c|c|c|}
\hline $\mathrm{E}(\text { level })^{\dagger}$ & $\mathrm{L}$ & $\mathrm{d} \sigma / \mathrm{d} \Omega(\max ) \mathrm{mb} / \mathrm{sr}^{\dagger}$ & Comm \\
\hline 0.0 & 0 & 0.3 & \\
\hline 1700 & & 0.3 & E(level): $1525+1837$. \\
\hline 2800 & & 0.4 & E(level): $2424+2752$ \\
\hline 3500 & & & E(level): multiplet of 7 levels from 3189 to 3886 \\
\hline 4800 & & 2.0 & E(level): multiplet of 10 levels from 4666 to 5015 . \\
\hline
\end{tabular}

$\dagger$ From $\left({ }^{14} \mathrm{C},{ }^{12} \mathrm{C}\right)(1985 \mathrm{Vi} 01)$ 


\section{${ }^{40} \mathrm{Ca}\left({ }^{96} \mathrm{Zr},{ }^{94} \mathrm{Zr}\right) \quad 2011 \mathrm{Co} 14$}

Two-neutron transfer channel in inverse kinematics.

2011Co14: $\mathrm{E}\left({ }^{96} \mathrm{Zr}\right)=275-330 \mathrm{MeV}$ from XTU-Tandem + ALPI at LNL, Legnaro. This energy is at or up to $25 \%$ below the Coulomb barrier, thus only the neutron transfer channels are relevant. Target $=\mathrm{CaF}_{2}, 50 \mu \mathrm{g} / \mathrm{cm}^{2}$ strip supported on a $15 \mu \mathrm{g} / \mathrm{cm}^{2}$ carbon backing. The Ca-like recoils were detected by PRISMA magnetic spectrometer. Mass identification was made from event-by-event reconstruction of the ion trajectory in the magnetic elements. Measured excitation functions. Deduced total kinetic energy loss (TKEL) distributions, differential cross sections and transfer probabilities. Comparison with semi-classical microscopic calculations.

\section{${ }^{42} \mathrm{Ca}$ Levels}

\begin{tabular}{|c|c|c|}
\hline E(level) & $\mathrm{J} \pi$ & Comments \\
\hline 0.0 & $0+$ & \\
\hline 5760 & $0+$ & $\begin{array}{l}\text { Transition to } 5.76 \mathrm{MeV}, 0+\text { state is much stronger than for g.s. The } 5.76 \mathrm{MeV} \text { state is dominated by two neutrons } \\
\text { in the } 2 \mathrm{p}_{3 / 2} \text { shell. }\end{array}$ \\
\hline
\end{tabular}

\section{${ }^{41} \mathrm{~K}(\mathrm{p}, \gamma) \quad 1971 \mathrm{Vi14}, 1967 \mathrm{An} 10,1963 \mathrm{Si} 13$}

1971Vi14: $\mathrm{E}=1.4-1.75 \mathrm{MeV}$. Measured $\gamma, \gamma(\theta), \gamma$ branching, resonances.

1967An10: $\mathrm{E}=1.86-2.17 \mathrm{MeV}$. A large number of resonances reported.

1963Si13: $\mathrm{E}=1060-1200 \mathrm{keV}$. Measured yield of $\gamma$ rays, $\gamma \gamma$ coin.

1986Se02: $\mathrm{E}=0.6$ to $4 \mathrm{MeV}$. Measured yields. Statistical-model analysis.

1973Di03 (also 1971DiZV): E=4-13 MeV. Measured $\gamma, \gamma(\theta)$. Deduced GDR structure and cross sections.

1967Lu08: $\mathrm{E}=1.4-1.75 \mathrm{MeV}$. A total of 74 resonances reported. Measured primary and secondary $\gamma$.

1966En04: $\mathrm{E}=0.3-2.1 \mathrm{MeV}$. Measured resonance strengths. Resonance at $\mathrm{E}(\mathrm{p})(\mathrm{lab})=1111$ measured.

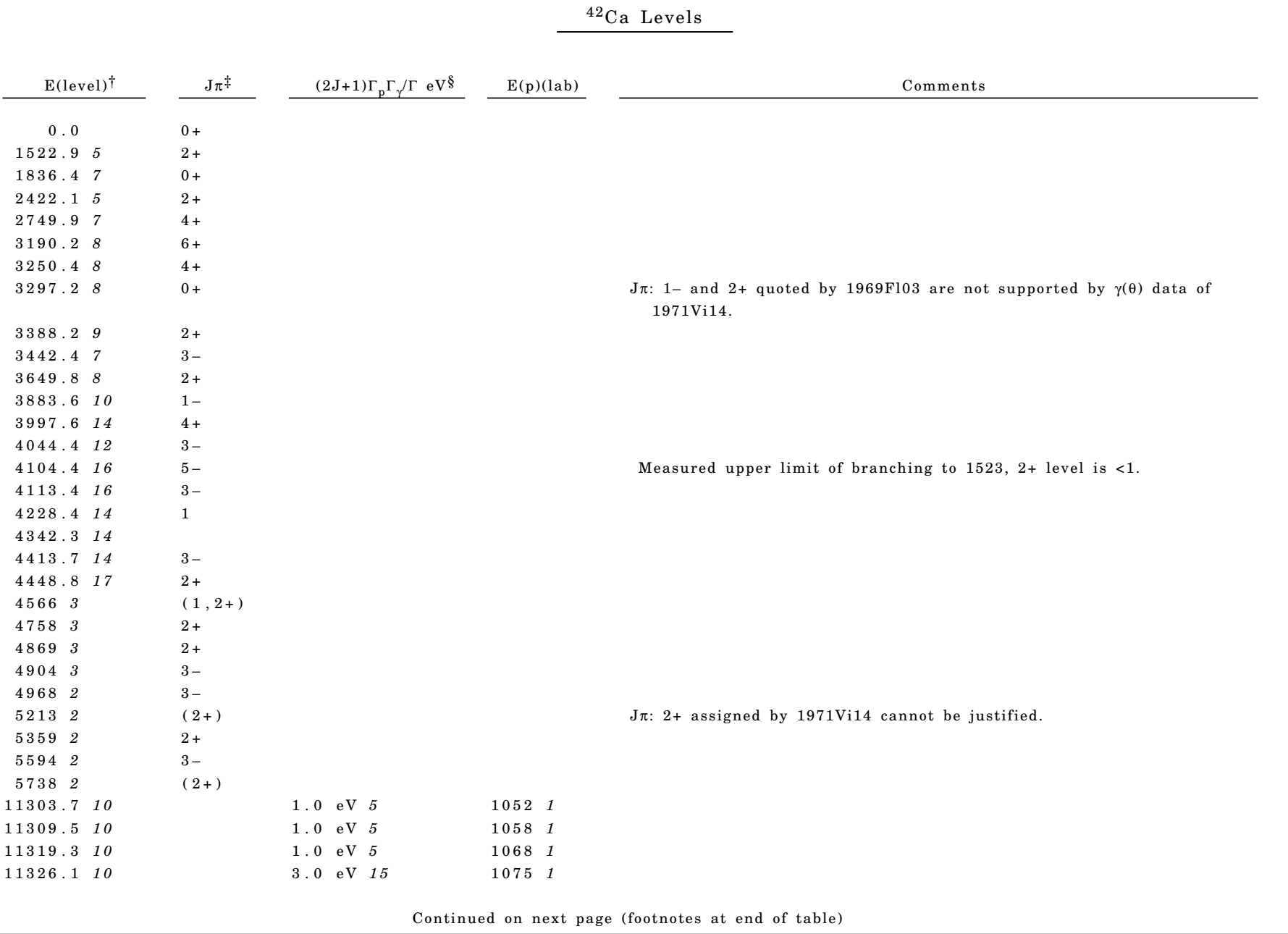


${ }^{41} \mathrm{~K}(\mathrm{p}, \gamma) \quad 1971 \mathrm{Vi14}, 1967 \mathrm{An} 10,1963 \mathrm{Si13}$ (continued)

${ }^{42} \mathrm{Ca}$ Levels (continued)

\begin{tabular}{|c|c|c|c|c|c|}
\hline \multicolumn{2}{|c|}{$\mathrm{E}(\text { level })^{\dagger}$} & \multirow[t]{2}{*}{$\mathrm{J} \pi^{\ddagger}$} & $(2 \mathrm{~J}+1) \Gamma_{\mathrm{p}} \Gamma_{\gamma} / \Gamma \mathrm{eV} \S$ & \multicolumn{2}{|c|}{$E(p)(l a b)$} \\
\hline 11331.0 & 10 & & $0.50 \quad \mathrm{eV} \quad 25$ & 1080 & 1 \\
\hline 11335.9 & 10 & & $1.0 \mathrm{eV} 5$ & 1085 & 1 \\
\hline 11343.7 & 10 & & $3.0 \mathrm{eV} \quad 15$ & 1093 & 1 \\
\hline 11361.3 & 10 & $(1,2+)$ & $8.5 \mathrm{eV} \quad 43$ & 1111 & 1 \\
\hline 11363.2 & 10 & & $7.0 \quad \mathrm{eV} \quad 35$ & 1113 & 1 \\
\hline 11380.8 & 10 & & $4.5 \quad \mathrm{eV} \quad 23$ & 1131 & 1 \\
\hline 11398.4 & 10 & & $1.5 \mathrm{eV} 8$ & 1149 & 1 \\
\hline 11401.3 & 10 & & $2.0 \quad \mathrm{eV} \quad 10$ & 1152 & 1 \\
\hline 11409.1 & 10 & & $7.0 \quad \mathrm{eV} \quad 35$ & 1160 & 1 \\
\hline 11412.0 & 10 & & $8.0 \mathrm{eV} \quad 40$ & 1163 & 1 \\
\hline 11416.9 & 10 & & $5.0 \mathrm{eV} \quad 25$ & 1168 & 1 \\
\hline 11425.7 & 10 & & $6.0 \mathrm{eV} \quad 30$ & 1177 & 1 \\
\hline 11432.5 & 10 & & $0.50 \quad \mathrm{eV} \quad 25$ & 1184 & 1 \\
\hline 11436.4 & 10 & & $1.0 \quad \mathrm{eV} \quad 5$ & 1188 & 1 \\
\hline 11439.4 & 10 & & $8.5 \mathrm{eV} \quad 43$ & 1191 & 1 \\
\hline 11446.2 & 10 & & $4.0 \mathrm{eV} 20$ & 1198 & 1 \\
\hline 11645 & & $(1-, 2+)$ & & 1402 & \\
\hline 11650 & & & & 1407 & \\
\hline 11654 & & & & 1411 & \\
\hline $11656^{\#}$ & & & & 1413 & \\
\hline 11658 & & & & 1415 & \\
\hline 11662 & & & & 1419 & \\
\hline 11668 & & $(1-, 2+)$ & & 1425 & \\
\hline 11674 & & $(1-, 2+)$ & & 1431 & \\
\hline $11680^{\#}$ & & & & 1438 & \\
\hline 11685 & & & & 1443 & \\
\hline 11693 & & $(1-, 2+)$ & & 1451 & \\
\hline 11698 & & & & 1456 & \\
\hline 11701 & & & & 1459 & \\
\hline 11706 & & $(1-, 2+)$ & & 1464 & \\
\hline 11709 ? \# & & & & 1467 & \\
\hline $11714 ?$ & & & & 1472 & \\
\hline $11719^{\#}$ & & & & 1477 & \\
\hline 11727 & & $2+$ & & 1486 & \\
\hline $11732^{\#}$ & & & & 1491 & \\
\hline 11740 & & $(1-, 2+)$ & & 1499 & \\
\hline 11743 & & $(1-, 2+)$ & & 1502 & \\
\hline $11751^{\#}$ & & & & 1510 & \\
\hline $11755^{\#}$ & & & & 1514 & \\
\hline $11758^{\#}$ & & & & 1517 & \\
\hline 11760 & & & & 1519 & \\
\hline 11763 & & & & 1523 & \\
\hline 11768 & & & & 1528 & \\
\hline $11772^{\#}$ & & & & 1532 & \\
\hline 11775 & & $(1-, 2+)$ & & 1535 & \\
\hline $11779^{\#}$ & & & & 1539 & \\
\hline $11783^{\#}$ & & & & 1543 & \\
\hline $11787^{\#}$ & & & & 1547 & \\
\hline 11792 & & & & 1552 & \\
\hline 11797 & & $(1-, 2+)$ & & 1557 & \\
\hline 11798 & & & & 1558 & \\
\hline 11805 & & $(1-, 2+)$ & & 1566 & \\
\hline $11809^{\#}$ & & & & 1570 & \\
\hline 11814 & & & & 1575 & \\
\hline 11823 & & $(1-, 2+)$ & & 1584 & \\
\hline $11829^{\#}$ & & & & 1590 & \\
\hline $11833^{\#}$ & & & & 1594 & \\
\hline 11836 & & & & 1597 & \\
\hline 11840 & & $(1-, 2+)$ & & 1601 & \\
\hline 11850 & & & & 1612 & \\
\hline 11853 & & & & 1615 & \\
\hline $11858^{\#}$ & & & & 1620 & \\
\hline 11868 & & $(1-, 2+)$ & & 1630 & \\
\hline
\end{tabular}

$\mathrm{J} \pi$ : the $(\mathrm{p}, \alpha)$ resonance at excitation $=11694 \mathrm{keV}$ has $\mathrm{J} \pi=3-$. 
${ }^{41} \mathrm{~K}(\mathrm{p}, \gamma) \quad 1971 \mathrm{Vi14}, 1967 \mathrm{An} 10,1963 \mathrm{Si13}$ (continued)

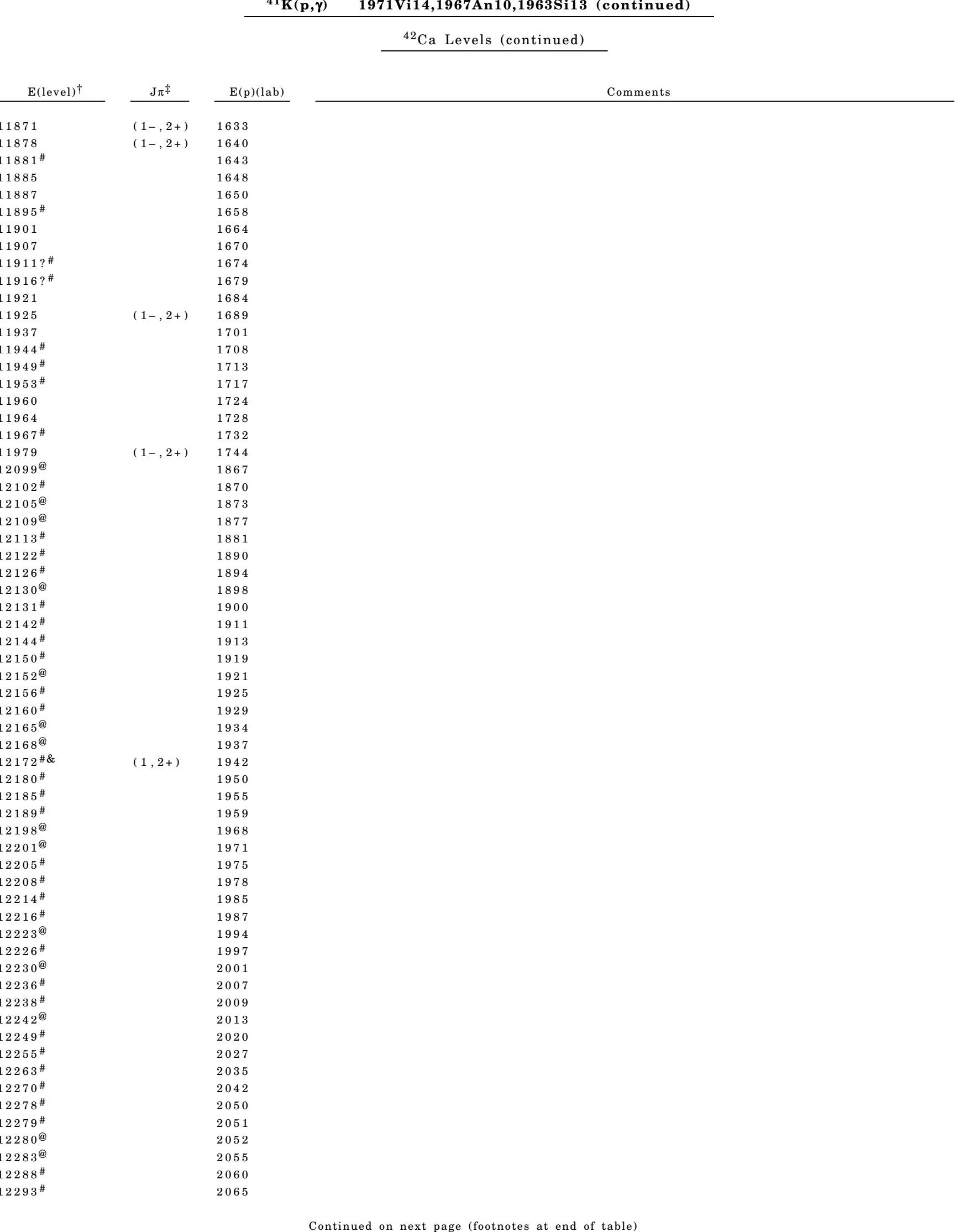


${ }^{41} \mathrm{~K}(\mathrm{p}, \gamma) \quad 1971 \mathrm{Vi14}, 1967 \mathrm{An} 10,1963 \mathrm{Si13}$ (continued)

${ }^{42} \mathrm{Ca}$ Levels (continued)

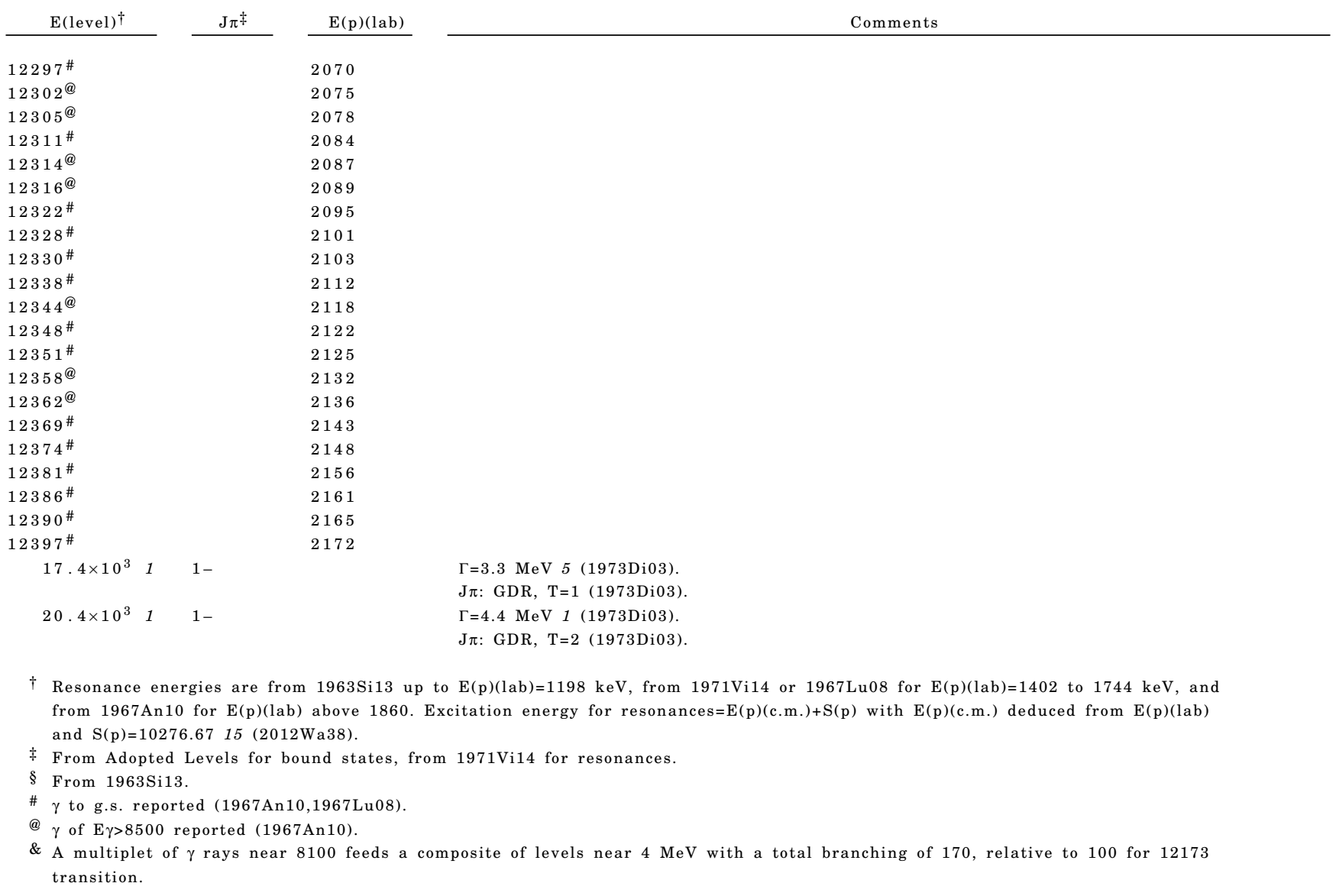
$\gamma\left({ }^{42} \mathrm{Ca}\right)$

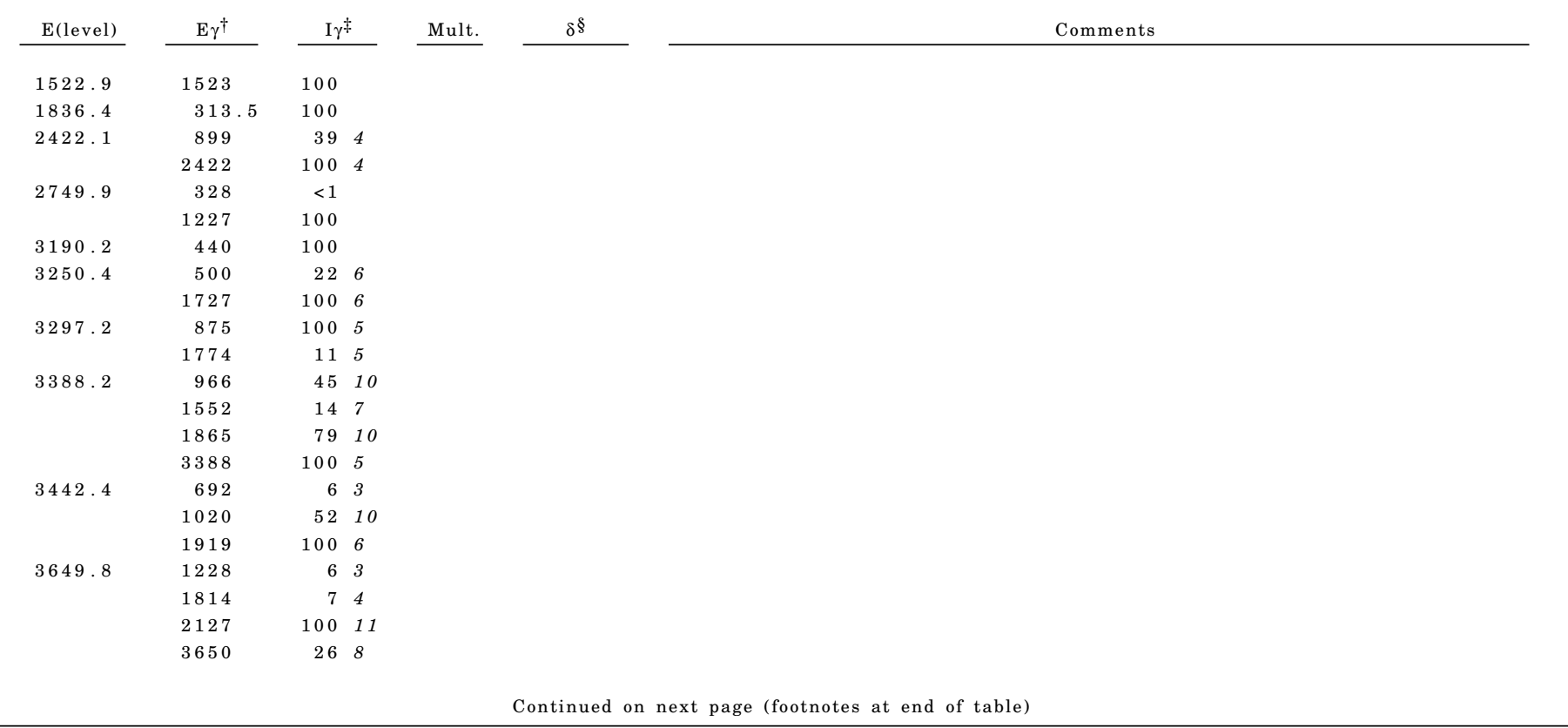


${ }^{41} \mathrm{~K}(\mathrm{p}, \gamma) \quad 1971 \mathrm{Vi14}, 1967 \mathrm{An} 10,1963 \mathrm{Si13}$ (continued)

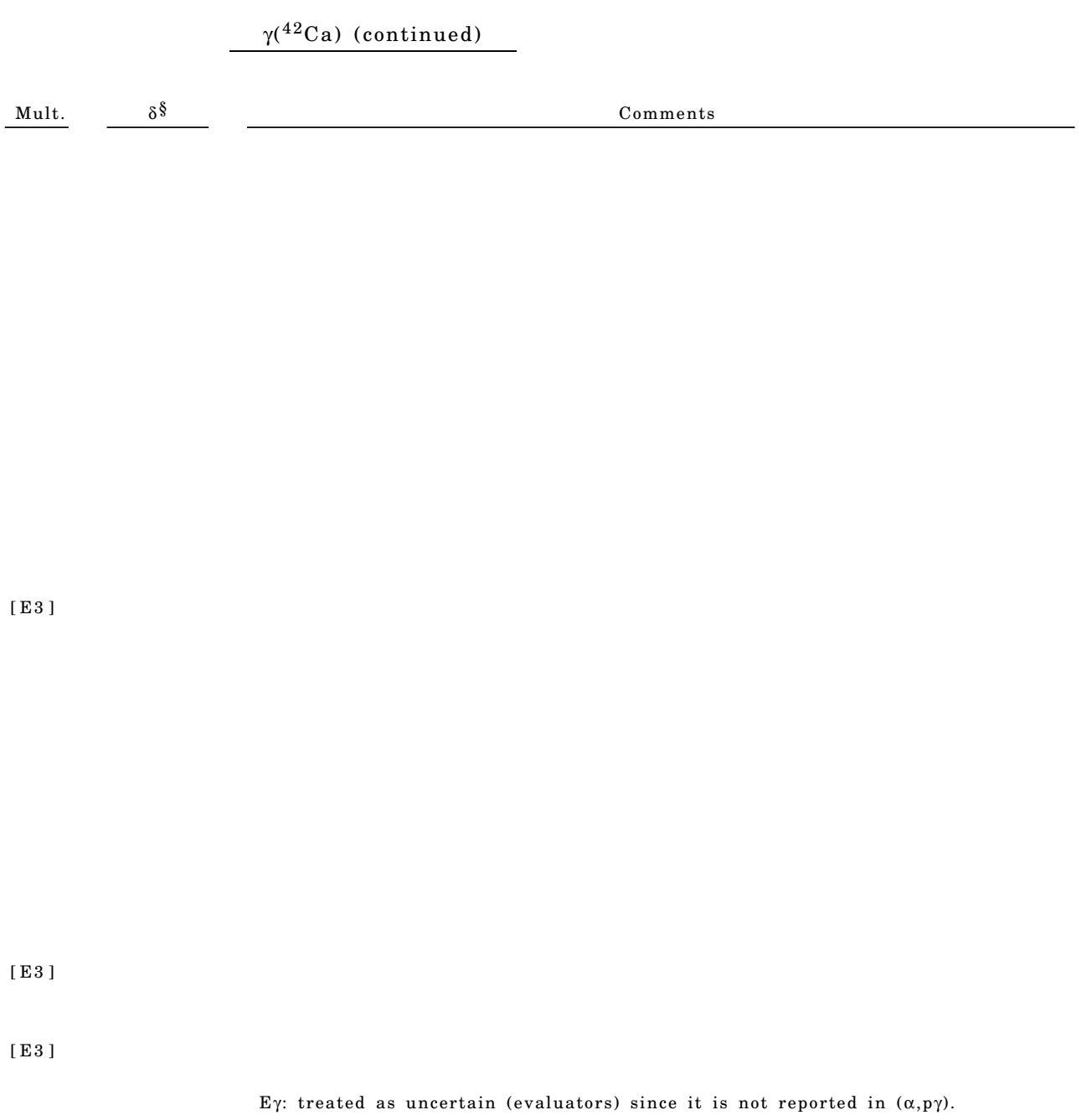

\begin{tabular}{|c|c|c|c|c|c|}
\hline E(level) & $E \gamma^{\dagger}$ & $I \gamma^{\ddagger}$ & & Mult. & $\delta^{\S}$ \\
\hline \multirow{2}{*}{3883.6} & 2048 & 43 & 6 & & \\
\hline & 3884 & 100 & 6 & & \\
\hline \multirow[t]{3}{*}{3997.6} & 1248 & 17 & 8 & & \\
\hline & 1576 & 50 & 8 & & \\
\hline & 2475 & 100 & 8 & & \\
\hline \multirow[t]{3}{*}{4044.4} & 602 & 23 & 8 & & \\
\hline & 1294 & 31 & 8 & & \\
\hline & 2521 & 100 & 15 & & \\
\hline \multirow[t]{3}{*}{4104.4} & 662 & 11 & 5 & & \\
\hline & 914 & 100 & 18 & & \\
\hline & 1354 & 71 & 11 & & \\
\hline \multirow[t]{2}{*}{4113.4} & 1691 & 23 & 7 & & \\
\hline & 2590 & 100 & 7 & & \\
\hline \multirow[t]{2}{*}{4228.4} & 1806 & 33 & 7 & & \\
\hline & 4228 & 100 & 7 & & \\
\hline 4342.3 & 1920 & 100 & & & \\
\hline \multirow[t]{2}{*}{4413.7} & 2891 & 85 & 11 & & \\
\hline & 4414 & 100 & 11 & [E3 ] & \\
\hline \multirow[t]{2}{*}{4448.8} & 2028 & 82 & 27 & & \\
\hline & 2925 & 100 & 27 & & \\
\hline \multirow[t]{3}{*}{4566} & 2144 & 44 & 22 & & \\
\hline & 3043 & 78 & 33 & & \\
\hline & 4566 & 100 & 33 & & \\
\hline \multirow[t]{4}{*}{4758} & 2336 & 57 & 29 & & \\
\hline & 2922 & 43 & 14 & & \\
\hline & 3235 & 86 & 29 & & \\
\hline & 4758 & 100 & 29 & & \\
\hline \multirow[t]{2}{*}{4869} & 3346 & 100 & 40 & & \\
\hline & 4869 & 100 & 40 & & \\
\hline \multirow[t]{3}{*}{4904} & 2154 & 36 & 9 & & \\
\hline & 2482 & 45 & 9 & & \\
\hline & 4904 & 100 & 18 & [E3 ] & \\
\hline \multirow[t]{3}{*}{4968} & 1526 & 40 & 20 & & \\
\hline & 2218 & 60 & 20 & & \\
\hline & 4968 & 100 & 20 & [E3 ] & \\
\hline \multirow[t]{2}{*}{5213} & 2791 & 82 & 27 & & \\
\hline & $5213^{\#}$ & 100 & 27 & & \\
\hline 5359 & 2937 & 33 & 20 & & \\
\hline & 5359 & 100 & 20 & & \\
\hline 5594 & 3172 & 57 & 22 & & \\
\hline & 3758 & 33 & 11 & [ E3 ] & \\
\hline & 4071 & 33 & 11 & & \\
\hline & 5594 & 100 & 33 & [ E3 ] & \\
\hline 5738 & 3316 & 33 & 17 & & \\
\hline & 4215 & 33 & 17 & & \\
\hline & 5738 & 100 & 33 & & \\
\hline 11361.3 & 9840 & & & & \\
\hline & 11362 & & & & \\
\hline 11645 & 7197 & 6 & & & \\
\hline & 7418 & 50 & & & \\
\hline & 7533 & 19 & & & \\
\hline & 7602 & 19 & & & \\
\hline & 7762 & 19 & & & \\
\hline & 7996 & 19 & & & \\
\hline & 8258 & 9 & & & \\
\hline & 9224 & 16 & & & \\
\hline & 9810 & 12 & & & \\
\hline & 10123 & 100 & & & \\
\hline & 11646 & 44 & & & \\
\hline 11668 & 7219 & 38 & & & \\
\hline & 7555 & 34 & & & \\
\hline & 8018 & 28 & & & \\
\hline & 8280 & 31 & & & \\
\hline
\end{tabular}

E $\gamma$ : treated as uncertain (evaluators) since it is not reported in $(\alpha, p \gamma)$. 
${ }^{41} \mathrm{~K}(\mathrm{p}, \gamma) \quad 1971 \mathrm{Vi14}, 1967 \mathrm{An} 10,1963 \mathrm{Si13}$ (continued)

$\gamma\left({ }^{42} \mathrm{Ca}\right)$ (continued)

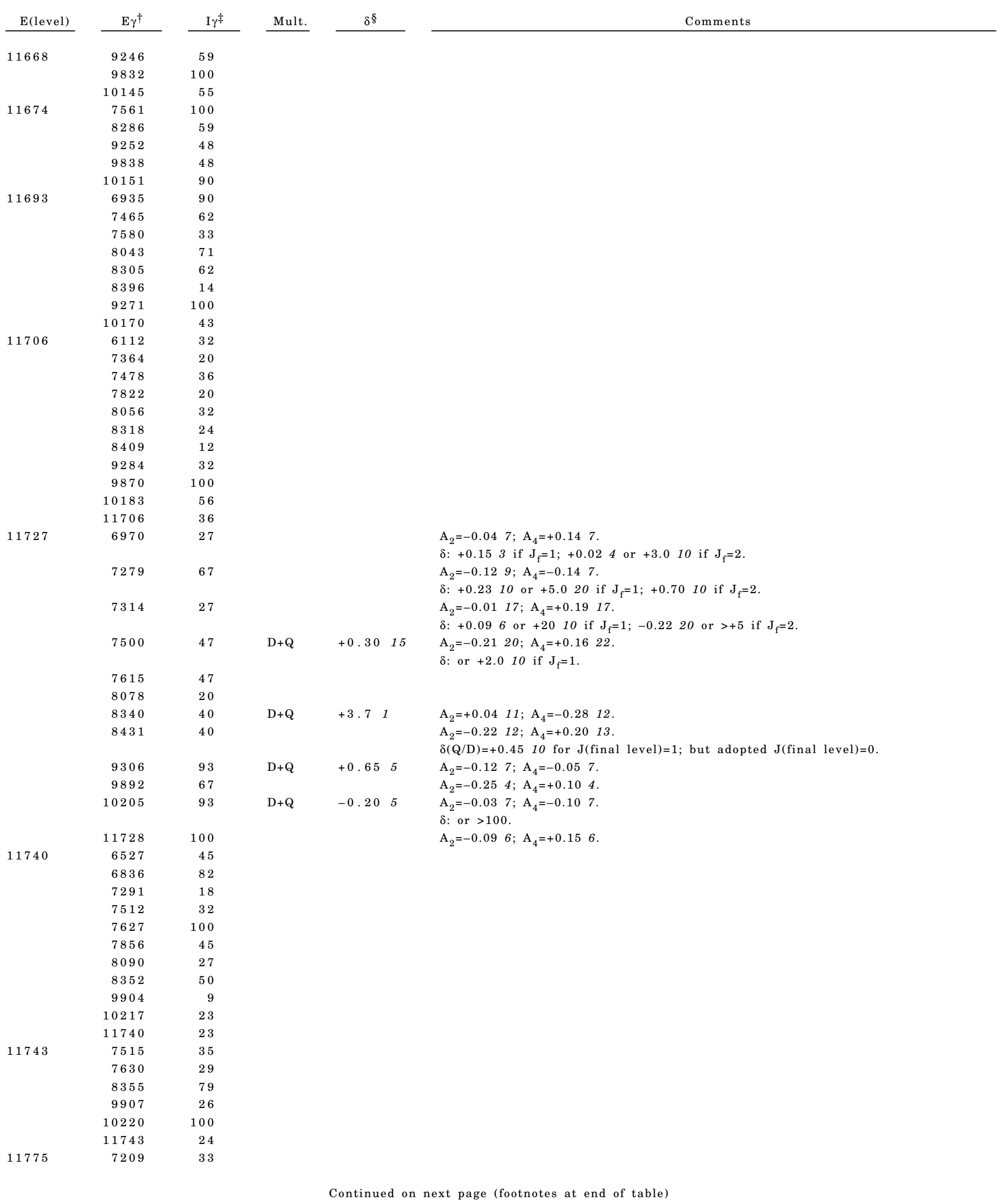


${ }^{41} \mathrm{~K}(\mathrm{p}, \gamma) \quad 1971 \mathrm{Vi14}, 1967 \mathrm{An} 10,1963 \mathrm{Si} 3$ (continued)

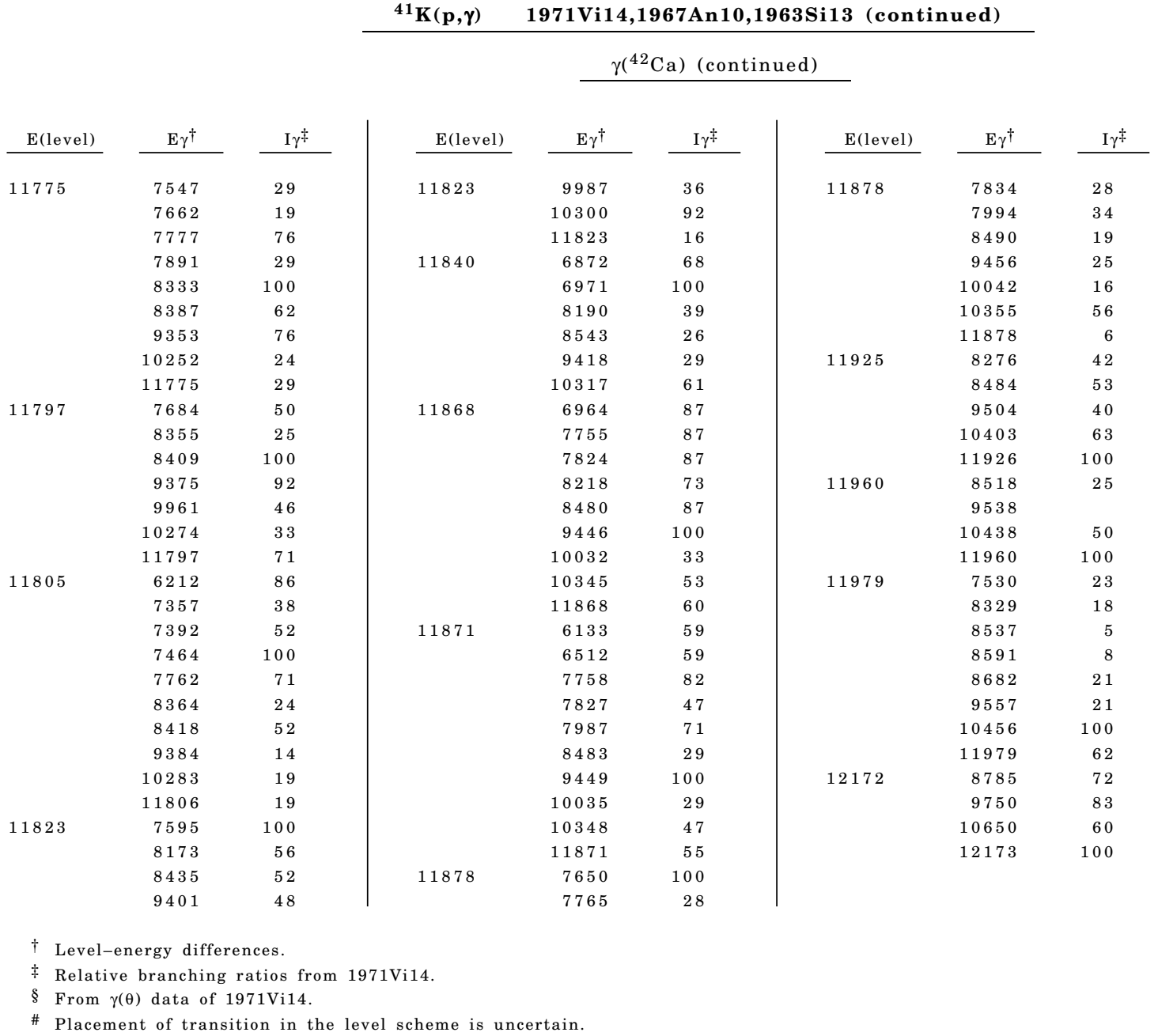

\section{${ }^{41} \mathrm{~K}(\mathrm{p}, \mathrm{n}),(\mathrm{p}, \mathrm{p}):$ Resonances $1967 \mathrm{An} 10,1967 \mathrm{Lu} 08,1970 \mathrm{De} 11$}

Includes resonances in ${ }^{42} \mathrm{Ca}$ from ${ }^{41} \mathrm{~K}(\mathrm{p}, \mathrm{p})$ (1970De11).

1967An10: E=1.86-2.17 MeV. A large number of resonances reported.

1967Lu08: $\mathrm{E}=1.40-1.74 \mathrm{MeV}$.

1970De11: E=1.35-1.96 MeV protons from the Utrecht $3 \mathrm{MV}$ Van de Graaff accelerator. 99.2\% enriched $41 \mathrm{~K}$ target. A BF 3 counter and Si detectors. Measured $\sigma\left(\mathrm{E}_{\mathrm{p}}, \theta\right)$. Deduced resonance energies, strengths, widths for a group of 14

resonances. Also deduced alpha width, see ${ }^{41} \mathrm{~K}(\mathrm{p}, \alpha)$ dataset.

$S_{n}$ and $S_{p}$ are neutron and proton resonance strengths, respectively.

${ }^{42} \mathrm{Ca}$ Levels

Resonance strength $\mathrm{S}=(2 \mathrm{~J}+1) \Gamma_{\mathrm{p}} \Gamma_{\mathrm{l}} / \Gamma$ from $1970 \mathrm{De} 11 . \Gamma_{\mathrm{l}}$ is $\Gamma_{\mathrm{n}}$ or $\Gamma_{\mathrm{p}}$.

\begin{tabular}{|c|c|c|c|c|c|c|c|}
\hline E(level) ${ }^{\dagger}$ & $\mathrm{J} \pi \ddagger$ & $E(p)(l a b)$ & $\mathrm{E}(\text { level })^{\dagger}$ & $E(p)(l a b)$ & E(level $)^{\dagger}$ & $\mathrm{J} \pi \neq$ & $E(p)(l a b)$ \\
\hline $11612.5 \S \quad 15$ & $1-$ & $1368.4 \quad 15$ & 11693 & 1451 & 11755 & & 1514 \\
\hline $11639.4^{\#} 15$ & $1-$ & $1395.9 \quad 15$ & 11709 & 1467 & 11760 & & 1519 \\
\hline 11645 & & 1402 & 11714 & 1472 & 11763 & & 1523 \\
\hline 11654 & & 1411 & 11719 & 1477 & 11768 & & 1528 \\
\hline 11658 & & 1415 & 11727 & 1486 & 11775 & & 1535 \\
\hline 11662 & & 1419 & 11732 & 1491 & 11779 & & 1539 \\
\hline 11668 & & 1425 & 11738 & 1497 & 11783 & & 1543 \\
\hline 11674 & & 1431 & 11743 & 1502 & 11787 & & 1547 \\
\hline 11688 & & 1446 & 11751 & 1510 & $11798.3^{@} 15$ & $(1-, 2+)$ & $1558.7 \quad 15$ \\
\hline
\end{tabular}




\section{${ }^{41} K(p, n),(p, p):$ Resonances 1967 An10,1967Lu08,1970De11 (continued)}

\begin{tabular}{|c|c|c|c|c|c|c|c|c|}
\hline \multirow[b]{3}{*}{$\mathrm{E}(\text { level })^{\dagger}$} & & \multicolumn{2}{|c|}{${ }^{41} K(p, n),(p, p):$ Resonances } & \multicolumn{5}{|c|}{ 1967An10,1967Lu08,1970De11 (continued) } \\
\hline & \multirow[b]{2}{*}{$\mathrm{J} \pi \ddagger$} & \multicolumn{5}{|c|}{${ }^{42} \mathrm{Ca}$ Levels (continued) } & \multirow[b]{2}{*}{$\mathrm{E}(\text { level })^{\dagger}$} & \multirow[b]{2}{*}{$E(p)(l a b)$} \\
\hline & & $E(p)(l a b)$ & $\mathrm{E}($ level) & & $\mathrm{J} \pi *$ & $E(p)(l a b)$ & & \\
\hline $11805.4^{\&} \quad 15$ & $(1-, 2+)$ & $1566.0 \quad 15$ & 11967 & & & 1732 & 12236 & 2007 \\
\hline $11811.1^{\mathrm{a}} \quad 15$ & $(1-, 2+)$ & $1571.8 \quad 15$ & $11970.2^{b}$ & 15 & $1-$ & $1734.8 \quad 15$ & 12238 & 2009 \\
\hline 11814 & & 1575 & $11980.3^{\mathrm{c}}$ & 15 & $1-$ & 1745.115 & 12255 & 2027 \\
\hline 11823 & & 1584 & $12101.3^{d}$ & 15 & $1-$ & $1869.1 \quad 15$ & 12263 & 2035 \\
\hline 11829 & & 1590 & 12109 & & & 1877 & 12270 & 2042 \\
\hline 11833 & & 1594 & $12112.2^{\mathrm{e}}$ & 15 & $1-$ & $1880.3 \quad 15$ & 12278 & 2050 \\
\hline 11836 & & 1597 & 12114 & & & 1882 & 12279 & 2051 \\
\hline 11840 & & 1601 & 12122 & & & 1890 & 12286 & 2058 \\
\hline 11850 & & 1612 & 12126 & & & 1894 & 12293 & 2065 \\
\hline 11855 & & 1617 & 12132 & & & 1901 & 12297 & 2070 \\
\hline 11858 & & 1620 & 12142 & & & 1911 & 12302 & 2075 \\
\hline 11865 & & 1627 & 12144 & & & 1913 & 12305 & 2078 \\
\hline 11868 & & 1630 & 12152 & & & 1921 & 12309 & 2082 \\
\hline 11871 & & 1633 & $12158.6^{\mathrm{f}}$ & 15 & $1-$ & $1927.8 \quad 15$ & 12311 & 2084 \\
\hline 11878 & & 1640 & 12160 & & & 1929 & 12314 & 2087 \\
\hline 11881 & & 1643 & $12163.1 \mathrm{~g}$ & 15 & $1-$ & $1932.4 \quad 15$ & 12322 & 2095 \\
\hline 11885 & & 1648 & $12168^{\mathrm{h}} \quad 3$ & & $(1-)$ & $1937 \quad 3$ & 12328 & 2101 \\
\hline 11887 & & 1650 & 12172 & & & 1942 & 12330 & 2103 \\
\hline 11895 & & 1658 & $12175.7^{\mathrm{i}}$ & 15 & $1-$ & $1945.3 \quad 15$ & 12336 & 2110 \\
\hline 11901 & & 1664 & 12180 & & & 1950 & 12340 & 2114 \\
\hline 11907 & & 1670 & $12182.8^{j}$ & 15 & $1-$ & $1952.6 \quad 15$ & 12344 & 2118 \\
\hline 11911 & & 1674 & 12185 & & & 1955 & 12348 & 2122 \\
\hline 11916 & & 1679 & 12189 & & & 1959 & 12351 & 2125 \\
\hline 11921 & & 1684 & 12198 & & & 1968 & 12362 & 2136 \\
\hline 11925 & & 1689 & 12201 & & & 1971 & 12369 & 2143 \\
\hline 11929 & & 1693 & 12205 & & & 1975 & 12374 & 2148 \\
\hline 11933 & & 1697 & 12208 & & & 1978 & 12381 & 2156 \\
\hline 11937 & & 1701 & 12214 & & & 1985 & 12386 & 2161 \\
\hline 11948 & & 1712 & 12216 & & & 1987 & & \\
\hline 11953 & & 1717 & 12226 & & & 1997 & & \\
\hline 11964 & & 1728 & 12230 & & & 2001 & & \\
\hline
\end{tabular}

$\dagger$ Excitation energy=S(p)+E(p)(c.m.) with E(p)(c.m.) deduced from E(p)(lab) and $\mathrm{S}(\mathrm{p})=10276.7167$ (2012Wa38). E(p)(lab) below $1.8 \mathrm{MeV}$ are from $1967 \mathrm{Lu} 08$, and above $1.8 \mathrm{MeV}$ from $1967 \mathrm{Lu} 08$; values with uncertainties are from $1970 \mathrm{De} 11$.

¥ From fits to experimental cross-section data (1970De11).

$\S \mathrm{S}_{\mathrm{n}}<5 \mathrm{eV}, \mathrm{S}_{\mathrm{p}}=465 \mathrm{eV} 240, \Gamma_{\mathrm{n}}<3 \mathrm{eV}, \Gamma_{\mathrm{p}}=160 \mathrm{eV} \mathrm{50}$, for J=1 (1970De11). No neutron emission observed.

\# $\mathrm{S}_{\mathrm{n}}<5 \mathrm{eV}, \mathrm{S}_{\mathrm{p}}=48 \mathrm{eV} 30, \Gamma_{\mathrm{n}}<4 \mathrm{eV}, \Gamma_{\mathrm{p}}=25 \mathrm{eV} \mathrm{10}$, for J=1 (1970De11). No neutron emission observed.

(9) $\mathrm{E}(\mathrm{p})(\mathrm{lab})=1557$ (1967Lu08). $\mathrm{S}_{\mathrm{n}}<5 \mathrm{eV}, \mathrm{S}_{\mathrm{p}}=400 \mathrm{eV} 200, \Gamma_{\mathrm{n}}<4 \mathrm{eV}$ for J=1 or 2; $\Gamma_{\mathrm{p}}=200 \mathrm{eV} 70$ for J=1, $120 \mathrm{eV} 40$ for J=2 (1970De11).

\& $\mathrm{E}(\mathrm{p})(\mathrm{lab})=1566$ (1967Lu08). $\mathrm{S}_{\mathrm{n}}=65 \mathrm{eV} \mathrm{33,} \mathrm{S}_{\mathrm{p}}=350 \mathrm{eV} \mathrm{175,} \Gamma_{\mathrm{n}}=26 \mathrm{eV} \mathrm{15}, \Gamma_{\mathrm{p}}=140 \mathrm{eV} 60$ for J=1; $\Gamma_{\mathrm{n}}=16 \mathrm{eV} 10, \Gamma_{\mathrm{p}}=85 \mathrm{eV} 40$ for J=2 (1970De11).

a $\mathrm{E}(\mathrm{p})(\mathrm{lab})=1570$ (1967Lu08). $\mathrm{S}_{\mathrm{n}}=30 \mathrm{eV} 15, \mathrm{~S}_{\mathrm{p}}=250 \mathrm{eV} 125, \Gamma_{\mathrm{n}}=11 \mathrm{eV} 7, \Gamma_{\mathrm{p}}=95$ eV 50 for J=1; $\Gamma_{\mathrm{n}}=6 \mathrm{eV} 4, \Gamma_{\mathrm{p}}=56 \mathrm{eV} 30$ for J=2 (1970De11).

b $\mathrm{S}_{\mathrm{n}}<5 \mathrm{eV}, \mathrm{S}_{\mathrm{p}}=100 \mathrm{eV} 50, \Gamma_{\mathrm{n}}<9, \Gamma_{\mathrm{p}}=120 \mathrm{eV} 30$ for $\mathrm{J}=1$ (1970De11).

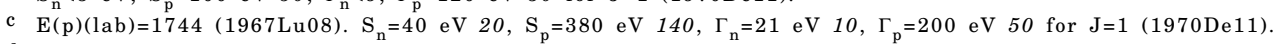

d $\mathrm{E}(\mathrm{p})(\mathrm{lab})=1867$ (1967An10). $\mathrm{S}_{\mathrm{n}}=200 \mathrm{eV} 100, \mathrm{~S}_{\mathrm{p}}=1700 \mathrm{eV} 850, \Gamma_{\mathrm{n}}=78 \mathrm{eV}$ 60, $\Gamma_{\mathrm{p}}=660 \mathrm{eV} 290$ for J=1 (1970De11).

e $\mathrm{E}(\mathrm{p})(\mathrm{lab})=1879(1967 \mathrm{An} 10) . \mathrm{S}_{\mathrm{n}}=150 \mathrm{eV} \mathrm{75}, \mathrm{S}_{\mathrm{p}}=2030 \mathrm{eV} 400, \Gamma_{\mathrm{n}}=55 \mathrm{eV} \mathrm{30}, \Gamma_{\mathrm{p}}=740 \mathrm{eV} \mathrm{80}$, for J=1 (1970De11).

f $\mathrm{E}(\mathrm{p})(\mathrm{lab})=1927$ (1967An10). $\mathrm{S}_{\mathrm{n}}<5 \mathrm{eV}, \mathrm{S}_{\mathrm{p}}=250 \mathrm{eV} 125, \Gamma_{\mathrm{n}}<5 \mathrm{eV}, \Gamma_{\mathrm{p}}=130 \mathrm{eV} 50$, for J=1 (1970De11).

g $\mathrm{E}(\mathrm{p})(\mathrm{lab})=1934$ (1967An10). $\mathrm{S}_{\mathrm{n}}=15 \mathrm{eV} \mathrm{8}, \mathrm{S}_{\mathrm{p}}=500 \mathrm{eV} \mathrm{250,} \Gamma_{\mathrm{n}}=7 \mathrm{eV} \mathrm{5,} \Gamma_{\mathrm{p}}=250 \mathrm{eV} 90$, for J=1 (1970De11).

$\mathrm{h} \quad \mathrm{E}(\mathrm{p})(\mathrm{lab})=1937$ (1967An10). $\mathrm{S}_{\mathrm{n}}=15 \mathrm{eV} 8, \mathrm{~S}_{\mathrm{p}}=300 \mathrm{eV} 150, \Gamma_{\mathrm{n}}<10 \mathrm{eV}, \Gamma_{\mathrm{p}}=100 \mathrm{eV} 50$, for J=1 (1970De11).

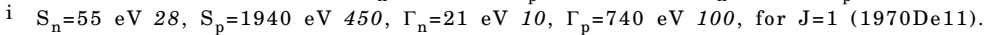

j $\mathrm{S}_{\mathrm{n}}<5 \mathrm{eV}, \mathrm{S}_{\mathrm{p}}=1210 \mathrm{eV} 250, \Gamma_{\mathrm{n}}<4 \mathrm{eV}, \Gamma_{\mathrm{p}}=440 \mathrm{eV} 50$ for J=1 (1970De11). 
${ }^{41} K(p, \alpha)$ : Resonances $1970 D e 10$

1970De10: E=1170-2100 keV proton beam was produced from the Utrecht $3 \mathrm{MV}$ Van de Graaff accelerator. A 99.2\% enriched

${ }^{41} \mathrm{~K}$ target on carbon backing. Emitted $\alpha$-particles were detected with Si detectors. Measured $\sigma\left(\mathrm{E}_{\mathrm{p}}, \theta\right)$. Deduced

resonance energies, $J \pi$.

1970De11: ${ }^{41} \mathrm{~K}(\mathrm{p}, \mathrm{n})$ and ${ }^{41} \mathrm{~K}(\mathrm{p}, \mathrm{p})$ resonances, $\mathrm{E}(\mathrm{p})=1350-1960 \mathrm{keV}$. Measured $\sigma(\theta)$. Deduced IAR strengths. 14 resonances reported.

1967An10: 51 resonances reported from $\mathrm{E}(\mathrm{p})(\mathrm{lab})=1867$ to 2172 .

1967Lu08: 48 resonances reported from $\mathrm{E}(\mathrm{p})(\mathrm{lab})=1402$ to 1744 .

1960Cl02: ${ }^{41} \mathrm{~K}(\mathrm{p}, \alpha) \mathrm{E}=1-3 \mathrm{MeV}$.

1950Ri59: ${ }^{41} \mathrm{~K}(\mathrm{p}, \mathrm{n}) \mathrm{E}=1.3$ to $3.5 \mathrm{MeV}$. Eight resonance peaks reported at $\mathrm{E}(\mathrm{p})(\mathrm{lab})=1600,1900,2240,2460,2600,2800$, 2960,3120 .

\section{${ }^{42} \mathrm{Ca}$ Levels}

$A_{2}, A_{4}$ and $A_{6}$ coefficients from angular distributions are from 1970De10.

\begin{tabular}{|c|c|c|c|c|c|}
\hline \multicolumn{2}{|c|}{$\mathrm{E}(\text { level })^{\dagger}$} & \multirow[t]{2}{*}{$\mathrm{J} \pi \ddagger$} & $(2 \mathrm{~J}+1) \Gamma_{\mathrm{p}} \Gamma_{\alpha} / \Gamma^{@}$ & $\mathrm{E}(\mathrm{p})(\mathrm{lab})$ & Comments \\
\hline 11426.8 & 15 & & $3.1 \mathrm{eV}$ & $1178.2 \quad 15$ & \\
\hline 11429.6 & 15 & $(0+, 1-, 2+)$ & $2.6 \mathrm{eV}$ & $1181.0 \quad 15$ & $\mathrm{~A}_{2}=+0.0313$ \\
\hline 11432.0 & 15 & & $5.6 \mathrm{eV}$ & $1183.5 \quad 15$ & \\
\hline 11435.5 & 15 & $1-, 2+$ & $2.8 \mathrm{eV}$ & 1187.115 & $\mathrm{~A}_{2}=-0.448, \mathrm{~A}_{4}=+0.0513, \mathrm{~A}_{6}=-0.0911$ \\
\hline 11440.4 & 15 & & $1.9 \mathrm{eV}$ & 1192.115 & \\
\hline 11444.3 & 15 & & $1.9 \mathrm{eV}$ & 1196.115 & \\
\hline 11447.7 & 15 & $0+, 1-, 2+$ & $6.8 \mathrm{eV}$ & 1199.615 & $\mathrm{~A}_{2}=-0.145, \mathrm{~A}_{4}=+0.057$. \\
\hline 11449.0 & 15 & & $10 \mathrm{eV}$ & $1200.9 \quad 15$ & \\
\hline 11450.5 & 15 & & $9.0 \mathrm{eV}$ & $1202.4 \quad 15$ & \\
\hline 11453.1 & 15 & & $1.9 \mathrm{eV}$ & 1205.115 & \\
\hline 11464.7 & 15 & & $0.7 \mathrm{eV}$ & $1217.0 \quad 15$ & \\
\hline 11468.1 & 15 & & $0.7 \mathrm{eV}$ & $1220.5 \quad 15$ & \\
\hline 11469.3 & 15 & $1-, 2+$ & $2.6 \mathrm{eV}$ & $1221.7 \quad 15$ & $\mathrm{~A}_{2}=-0.325, \mathrm{~A}_{4}=+0.137$ \\
\hline 11473.5 & 15 & $(1-, 2+)$ & $6.0 \mathrm{eV}$ & $1226.0 \quad 15$ & $\mathrm{~A}_{2}=-0.46 \quad 7, \mathrm{~A}_{4}=+0.1411, \mathrm{~A}_{6}=-0.1110$ \\
\hline 11475.8 & 15 & & $5.6 \mathrm{eV}$ & $1228.3 \quad 15$ & \\
\hline 11477.7 & 15 & & $3.5 \mathrm{eV}$ & $1230.3 \quad 15$ & \\
\hline 11484.4 & 15 & $(2+)$ & $7.4 \mathrm{eV}$ & $1237.2 \quad 15$ & $\mathrm{~A}_{2}=-0.405, \mathrm{~A}_{4}=+0.238, \mathrm{~A}_{6}=-0.117$ \\
\hline 11488.7 & 15 & $1-$ & $15 \mathrm{eV}$ & $1241.6 \quad 15$ & $\mathrm{~A}_{2}=-0.855, \mathrm{~A}_{4}=+0.117, \mathrm{~A}_{6}=-0.046$ \\
\hline 11490.3 & 15 & & $9.2 \mathrm{eV}$ & $1243.2 \quad 15$ & \\
\hline 11493.6 & 15 & & $2.1 \mathrm{eV}$ & $1246.6 \quad 15$ & \\
\hline 11499.4 & 15 & & $1.6 \mathrm{eV}$ & $1252.5 \quad 15$ & \\
\hline 11501.1 & 15 & & $2.4 \mathrm{eV}$ & 1254.315 & \\
\hline 11503.4 & 15 & & $2.8 \mathrm{eV}$ & $1256.6 \quad 15$ & \\
\hline 11505.4 & 15 & $0+, 1-, 2+$ & $2.7 \mathrm{eV}$ & $1258.7 \quad 15$ & $\mathrm{~A}_{2}=-0.115, \mathrm{~A}_{4}=+0.078, \mathrm{~A}_{6}=-0.097$ \\
\hline 11508.8 & 15 & & $2.4 \mathrm{eV}$ & $1262.2 \quad 15$ & \\
\hline 11510.9 & 15 & $(1-)$ & $7.1 \mathrm{eV}$ & 1264.315 & $\mathrm{~A}_{2}=-0.963, \mathrm{~A}_{4}=+0.155, \mathrm{~A}_{6}=-0.064$ \\
\hline 11512.5 & 15 & & $5.7 \mathrm{eV}$ & $1265.9 \quad 15$ & \\
\hline 11516.6 & 15 & $(1-)$ & $1.9 \mathrm{eV}$ & 1270.115 & $\mathrm{~A}_{2}=-0.767, \mathrm{~A}_{4}=-0.0511$ \\
\hline 11518.2 & 15 & & $2.9 \mathrm{eV}$ & $1271.8 \quad 15$ & \\
\hline 11523.3 & 15 & $(1-), 2+$ & $1.1 \mathrm{eV}$ & $1277.0 \quad 15$ & $\mathrm{~A}_{2}=+0.327, \mathrm{~A}_{4}=-0.219, \mathrm{~A}_{6}=+0.099$ \\
\hline 11525.4 & 15 & & $1.8 \mathrm{eV}$ & $1279.2 \quad 15$ & \\
\hline 11527.4 & 15 & $(1-, 2+)$ & $3.9 \mathrm{eV}$ & $1281.2 \quad 15$ & $\mathrm{~A}_{2}=+0.6618, \mathrm{~A}_{4}=-0.1420$ \\
\hline 11529.3 & 15 & $(0+), 1-, 2+$ & $10 \mathrm{eV}$ & $1283.2 \quad 15$ & $\mathrm{~A}_{2}=+0.038$ \\
\hline 11531.0 & 15 & & $6.9 \mathrm{eV}$ & $1284.9 \quad 15$ & \\
\hline 11532.6 & 15 & & $2.5 \mathrm{eV}$ & $1286.5 \quad 15$ & \\
\hline 11540.0 & 15 & $(1-)$ & $10 \mathrm{eV}$ & 1294.115 & $\mathrm{~A}_{2}=-0.717, \mathrm{~A}_{4}=+0.0711$ \\
\hline 11542.3 & 15 & $0+, 1-, 2+$ & $14 \mathrm{eV}$ & $1296.5 \quad 15$ & $\mathrm{~A}_{2}=-0.055, \mathrm{~A}_{4}=-0.037, \mathrm{~A}_{6}=+0.066$. \\
\hline 11543.6 & 15 & & $10 \mathrm{eV}$ & $1297.8 \quad 15$ & \\
\hline 11544.4 & 15 & & $18 \mathrm{eV}$ & $1298.6 \quad 15$ & \\
\hline 11550.0 & 15 & $0+, 1-, 2+$ & $2.2 \mathrm{eV}$ & $1304.4 \quad 15$ & $\mathrm{~A}_{2}==0.657, \mathrm{~A}_{4}=-0.048, \mathrm{~A}_{6}=+0.078$. \\
\hline 11551.5 & 15 & & $2.7 \mathrm{eV}$ & $1305.9 \quad 15$ & \\
\hline 11555.4 & 15 & $(1-)$ & $6.9 \mathrm{eV}$ & $1309.9 \quad 15$ & $\mathrm{~A}_{2}=-0.834, \mathrm{~A}_{4}=+0.136, \mathrm{~A}_{6}=-0.055$. \\
\hline 11556.3 & 15 & & $12 \mathrm{eV}$ & $1310.8 \quad 15$ & \\
\hline 11558.1 & 15 & & $15 \mathrm{eV}$ & $1312.7 \quad 15$ & \\
\hline 11562.8 & 15 & & $1.0 \mathrm{eV}$ & $1317.5 \quad 15$ & \\
\hline 11569.2 & 15 & $1-$ & $4.2 \mathrm{eV}$ & $1324.0 \quad 15$ & $\mathrm{~A}_{2}=-0.963, \mathrm{~A}_{4}=+0.086, \mathrm{~A}_{6}=-0.035$ \\
\hline 11571.7 & 15 & $0+, 1-, 2+$ & $3.3 \mathrm{eV}$ & $1326.6 \quad 15$ & $\mathrm{~A}_{2}=-0.069, \mathrm{~A}_{4}=-0.1512, \mathrm{~A}_{6}=+0.0810$ \\
\hline 11572.8 & 15 & $(1-, 3-, 4+)$ & $2.8 \mathrm{eV}$ & $1327.7 \quad 15$ & $\mathrm{~A}_{2}=+0.9534, \mathrm{~A}_{4}=-0.3435, \mathrm{~A}_{6}=+0.1830$ \\
\hline 11575.2 & 15 & $(3-, 4+)$ & $3.2 \mathrm{eV}$ & $1330.2 \quad 15$ & $\mathrm{~A}_{2}=+2.04, \mathrm{~A}_{4}=-0.1034, \mathrm{~A}_{6}=-0.3934$ \\
\hline 11576.2 & 15 & & $2.4 \mathrm{eV}$ & $1331.2 \quad 15$ & \\
\hline 11589.8 & 15 & $(1-)$ & $9.0 \mathrm{eV}$ & 1345.115 & $\mathrm{~A}_{2}=-1.023, \mathrm{~A}_{4}=+0.045, \mathrm{~A}_{6}=+0.044$. \\
\hline
\end{tabular}


${ }^{41} \mathrm{~K}(\mathrm{p}, \alpha)$ : Resonances $1970 \mathrm{De} 10$ (continued)

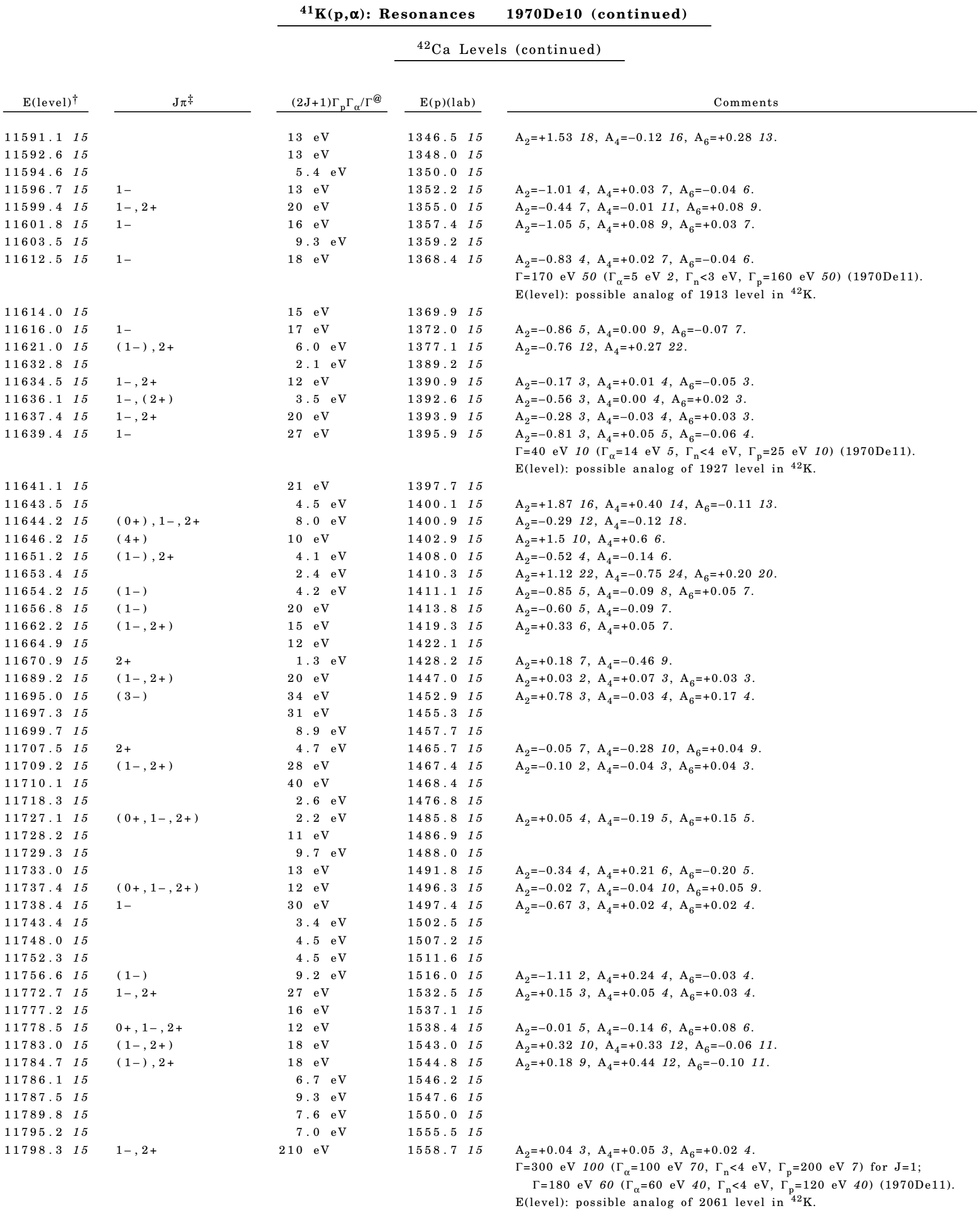


${ }^{41} K(p, \alpha):$ Resonances $1970 D e 10$ (continued)

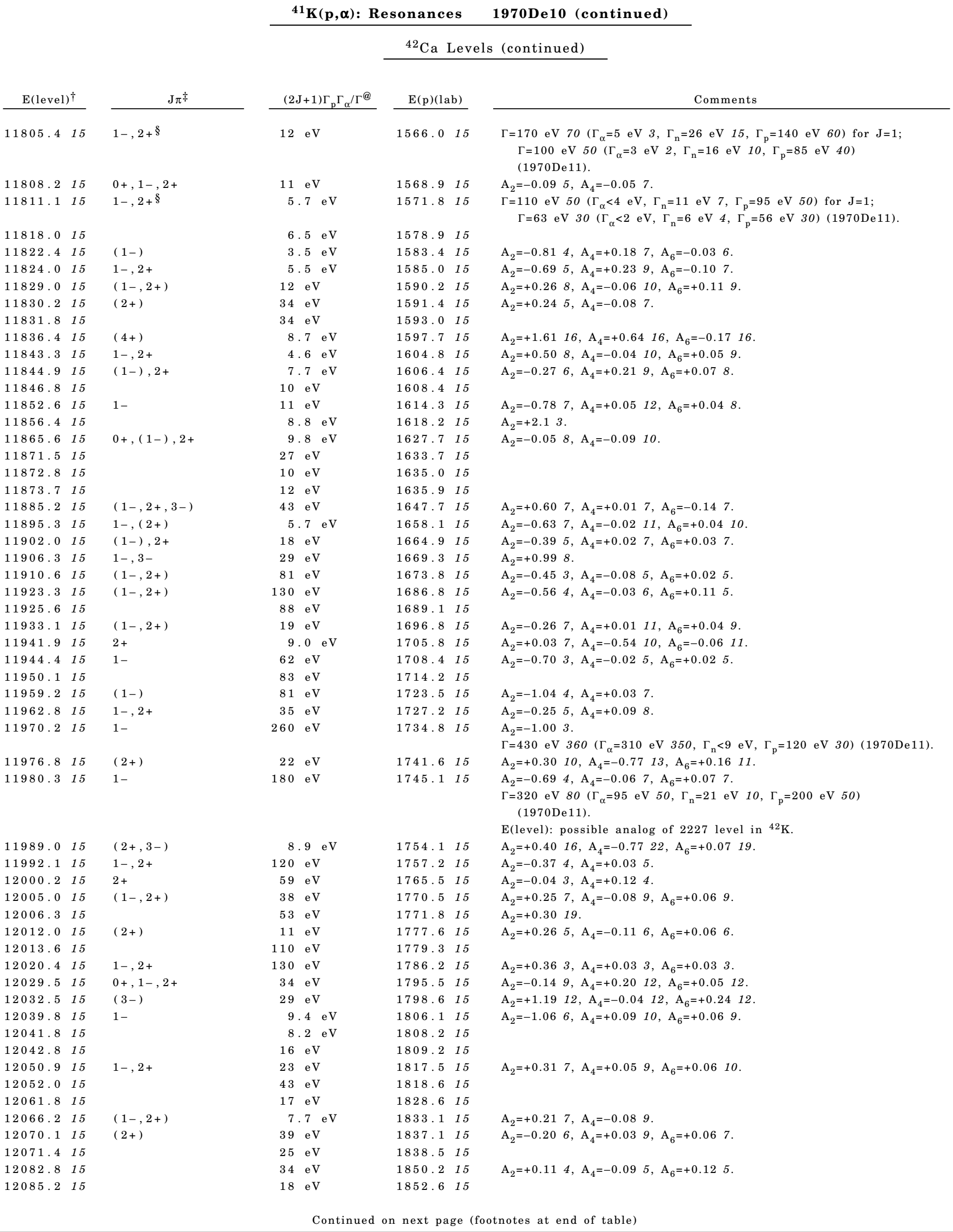


${ }^{41} \mathrm{~K}(\mathrm{p}, \boldsymbol{\alpha})$ : Resonances $1970 \mathrm{De} 10$ (continued)

\author{
${ }^{42} \mathrm{Ca}$ Levels (continued)
}

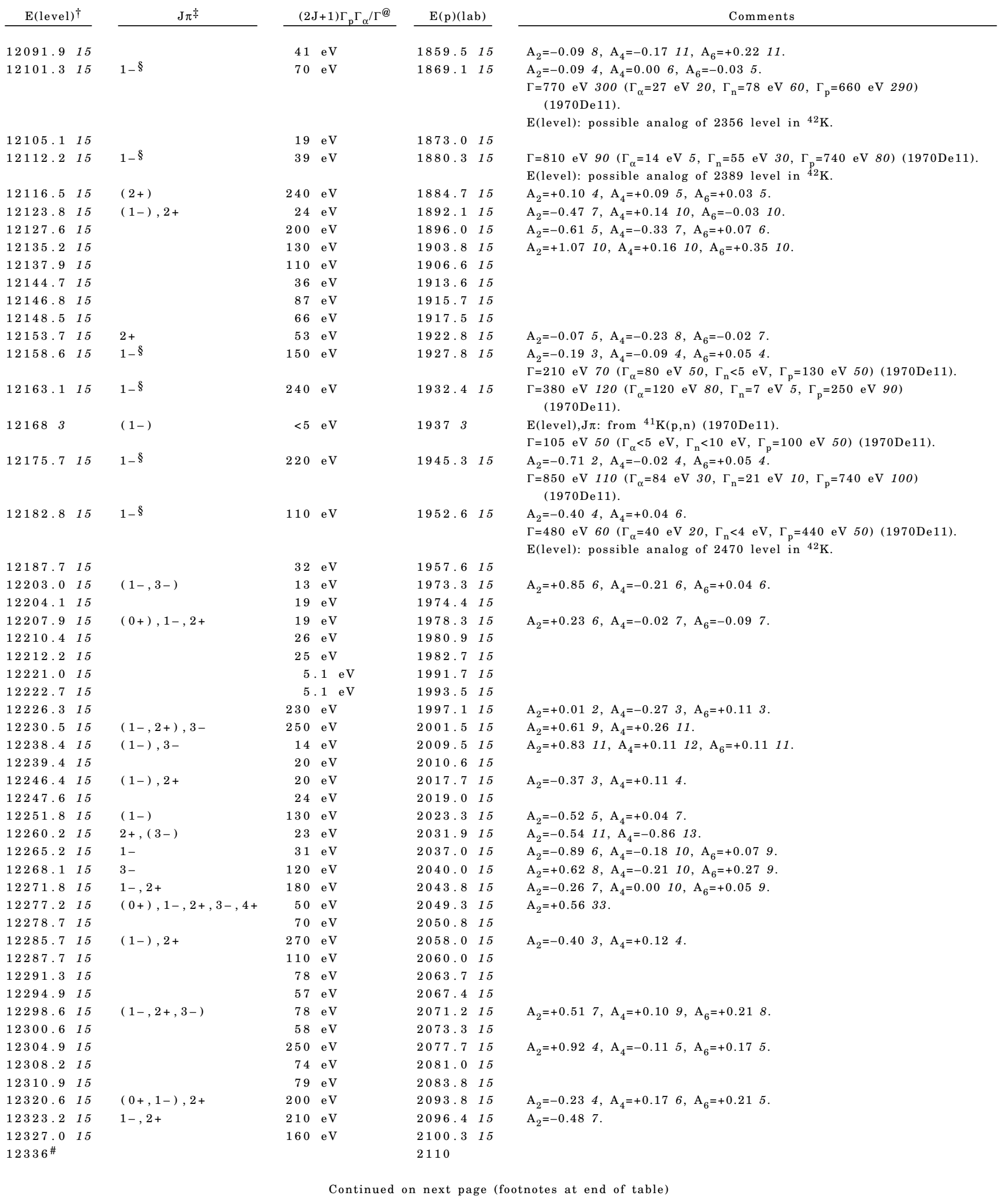


${ }^{41} \mathrm{~K}(\mathrm{p}, \alpha)$ : Resonances $1970 \mathrm{De} 10$ (continued)

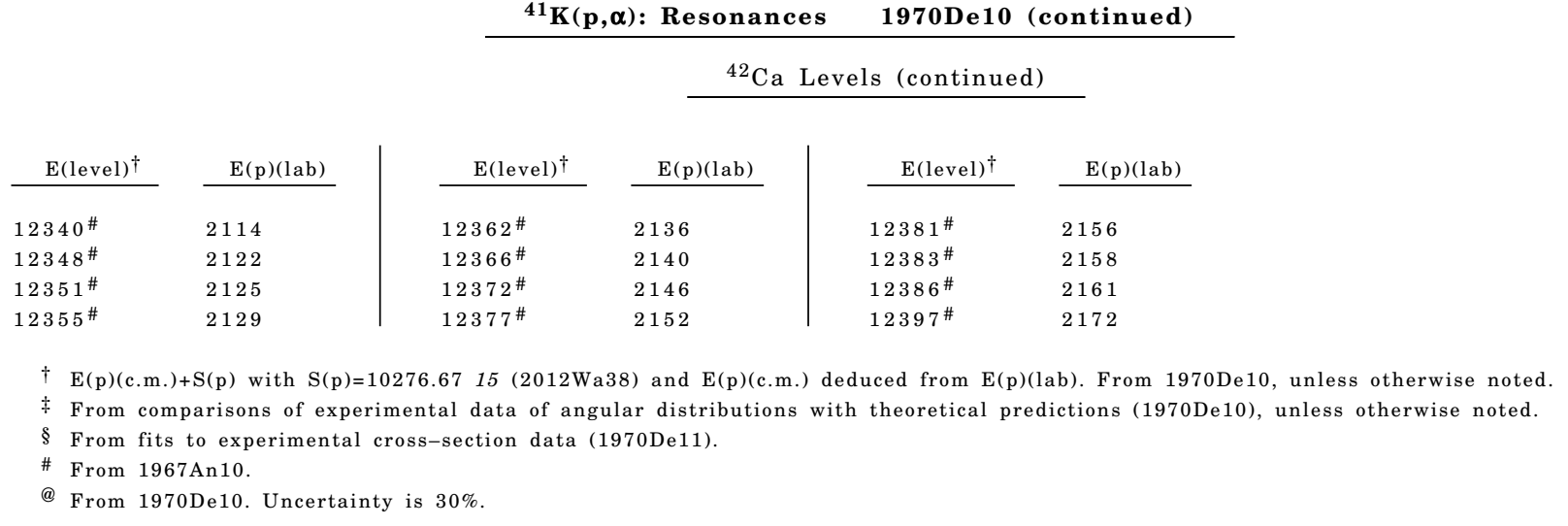

${ }^{41} \mathrm{~K}\left({ }^{3} \mathrm{He}, \mathrm{d}\right) \quad 1973 \mathrm{Ja17}, 1971 \mathrm{Pe04,1970Fo04}$

Target ${ }^{41} \mathrm{~K}$ g.s. $J \pi=3 / 2+$.

1973Ja17: $\mathrm{E}=23 \mathrm{MeV}{ }^{3} \mathrm{He}$ beam was produced at the Nuclear Structure Research Laboratory at the University of Rochester. Targets of KI (99\% enriched in $\left.{ }^{41} \mathrm{~K}\right)$ evaporated onto carbon backings. Deuterons were momentum analyzed with an Enge broad-range spectrograph and detected in nuclear emulsions, FWHM=9-14 keV. Measured $\sigma(\theta)$. Deduced levels, $J \pi, L-$ transfer, spectroscopic factors from DWBA analysis.

1971Pe04: E=10 MeV ${ }^{3} \mathrm{He}$ beam was produced from the CN Van de Graaff accelerator of Legnaro, Padova. Targets of KI (natural and $99.16 \%$ enriched in ${ }^{41} \mathrm{~K}$ ) evaporated onto carbon backings. Reaction products were detected with a $\Delta \mathrm{E}-\mathrm{E}$ telescope of surface-barrier detectors. Measured $\sigma(\theta)$. Deduced levels, $J \pi$, L-transfer, spectroscopic factors from DWBA analysis.

1970Fo04 (also 1968Fo05): $\mathrm{E}=11.0 \mathrm{MeV}$ beam was produced from the Liverpool University Tandem accelerator. Target of $300 \mu \mathrm{g} / \mathrm{cm}^{2} \mathrm{KI}\left(95.35 \%\right.$ enriched in $\left.{ }^{41} \mathrm{~K}\right)$ evaporated onto a thin carbon backing. Deuterons were measured with a magnetic spectrograph (14' to $\left.40^{\prime}\right)$ and a surface barrier detector telescope (35' to $\left.85^{\prime}\right)$. Measured $\sigma(\theta)$. Deduced levels, J $\pi$, L-tranfer, spectroscopic factors from DWBA analysis.

Other: $1968 \mathrm{Ly} 02$.

\section{${ }^{42} \mathrm{Ca}$ Levels}

Following $\mathrm{J} \pi$ values are implied by $\mathrm{L}$-transfers: 0 - to 3 - for $\mathrm{L}=1 ; 0+$ to $4+$ for $\mathrm{L}=2 ; 1-$ to $5-$ for $\mathrm{L}=3 ; 1-, 2-, 3-$ for $\mathrm{L}=1+3$.

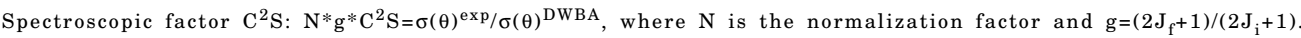

\begin{tabular}{|c|c|c|c|c|}
\hline \multicolumn{2}{|c|}{$\mathrm{E}(\text { level })^{\dagger}$} & $\mathrm{L}$ & $\left(2 \mathrm{~J}_{\mathrm{f}}+1\right) \mathrm{C}^{2} \mathrm{~S} \ddagger$ & Comment \\
\hline 0 & 5 & 2 & $2.22 \quad 21$ & $\left(2 \mathrm{~J}_{\mathrm{f}}+1\right) \mathrm{C}^{2} \mathrm{~S}: 2.90\left(1970 \mathrm{~F}_{004}\right), 2.50(1971 \mathrm{Pe} 04)$ \\
\hline 1522 & 5 & 2 & $0.25 \quad 6$ & $\left(2 \mathrm{~J}_{\mathrm{f}}+1\right) \mathrm{C}^{2} \mathrm{~S}:<0.20(1971 \mathrm{Pe} 04)$ \\
\hline 1832 & 5 & 2 & $0.39 \quad 7$ & $\left(2 \mathrm{~J}_{\mathrm{f}}+1\right) \mathrm{C}^{2} \mathrm{~S}:<0.39(1971 \mathrm{Pe} 04)$ \\
\hline 2418 & 5 & 2 & $0.37 \quad 6$ & $\left(2 \mathrm{~J}_{\mathrm{f}}+1\right) \mathrm{C}^{2} \mathrm{~S}:<0.24(1971 \mathrm{Pe} 04)$ \\
\hline $3297 ?$ & & & & Very weakly excited. \\
\hline 3444 & 5 & $1+3 \S$ & $0.06,1.06$ & $\left(2 \mathrm{~J}_{\mathrm{f}}+1\right) \mathrm{C}^{2} \mathrm{~S}: 2.11\left(1970 \mathrm{~F}_{0} 04\right), 2.31(1971 \mathrm{Pe} 04)$ for $\mathrm{L}=3$ \\
\hline 3880 & 5 & $1(+3)$ & $0.02,0.06$ & \\
\hline 3949 & 5 & 3 & $2.8 \quad 3$ & $\left(2 \mathrm{~J}_{\mathrm{f}}+1\right) \mathrm{C}^{2} \mathrm{~S}: 4.17\left(1970 \mathrm{Fo}_{0} 04\right), 4.59(1971 \mathrm{Pe} 04)$ \\
\hline 4044 & 5 & $1+3$ & $0.03,0.27$ & \\
\hline 4093 & 5 & 3 & $3.0 \quad 3$ & $\left(2 \mathrm{~J}_{\mathrm{f}}+1\right) \mathrm{C}^{2} \mathrm{~S}: 5.91\left(1970 \mathrm{~F}_{0} 04\right), 6.05(1971 \mathrm{Pe} 04)$ \\
\hline 4116 & 5 & ( 1 ) & 0.18 & \\
\hline 4225 & 5 & ( 1 ) & 0.02 & \\
\hline 4420 & 5 & $1+3 \S$ & $0.03,0.71$ & $\left(2 \mathrm{~J}_{\mathrm{f}}+1\right) \mathrm{C}^{2} \mathrm{~S}: 1.78\left(1970 \mathrm{Fo}_{0} 04\right), 1.40(1971 \mathrm{Pe} 04)$ for $\mathrm{L}=3$ \\
\hline 4685 & 5 & $1(+3) \#$ & $0.14,0.11$ & $\left(2 \mathrm{~J}_{\mathrm{f}}+1\right) \mathrm{C}^{2} \mathrm{~S}: 0.43\left(1970 \mathrm{~F}_{0} 04\right), 0.42(1971 \mathrm{Pe} 04)$ for $\mathrm{L}=1$ \\
\hline 4710 & 5 & & & \\
\hline 4896 & 5 & $1+3 \#$ & $0.18,0.40$ & $\left(2 \mathrm{~J}_{\mathrm{f}}+1\right) \mathrm{C}^{2} \mathrm{~S}: 0.61\left(1970 \mathrm{Fo}_{0} 04\right), 0.48(1971 \mathrm{Pe} 04)$ for $\mathrm{L}=1$ \\
\hline 4944 & 5 & $1(+3)$ & $0.06,0.07$ & \\
\hline 4969 & 5 & $1+3^{\#}$ & $0.09,0.23$ & $\left(2 \mathrm{~J}_{\mathrm{f}}+1\right) \mathrm{C}^{2} \mathrm{~S}: 0.12\left(1970 \mathrm{Fo}_{0} 04\right)$ for $\mathrm{L}=1$ \\
\hline 5075 & 5 & $1(+3) \#$ & $0.26,0.32$ & $\left(2 \mathrm{~J}_{\mathrm{f}}+1\right) \mathrm{C}^{2} \mathrm{~S}: 0.60\left(1970 \mathrm{Fo}_{0} 04\right), 0.48(1971 \mathrm{Pe} 04)$ for $\mathrm{L}=1$ \\
\hline 5156 & 5 & $1+3^{\#}$ & $0.07,0.12$ & $\left(2 \mathrm{~J}_{\mathrm{f}}+1\right) \mathrm{C}^{2} \mathrm{~S}: 0.17\left(1970 \mathrm{Fo}_{0} 04\right)$ for $\mathrm{L}=1$ \\
\hline 5205 & 5 & & & \\
\hline 5322 & 5 & 3 & $0.32 \quad 5$ & \\
\hline 5389 & 5 & $1(+3) \#$ & $0.10,0.12$ & $\left(2 \mathrm{~J}_{\mathrm{f}}+1\right) \mathrm{C}^{2} \mathrm{~S}: 0.35(1971 \mathrm{Pe} 04)$ for $\mathrm{L}=1$ \\
\hline 5410 & 5 & $1+3^{\#}$ & $0.06,0.15$ & $\left(2 \mathrm{~J}_{\mathrm{f}}+1\right) \mathrm{C}^{2} \mathrm{~S}: 0.33\left(1970 \mathrm{~F}_{0} 04\right)$ for $\mathrm{L}=1$ \\
\hline 5466 & 5 & 3 & $0.34 \quad 7$ & \\
\hline
\end{tabular}


${ }^{41} \mathrm{~K}\left({ }^{3} \mathrm{He}, \mathrm{d}\right) \quad 1973 \mathrm{Ja17}, 1971 \mathrm{Pe} 04,1970 \mathrm{FoO4}$ (continued)

${ }^{42} \mathrm{Ca}$ Levels (continued)

\begin{tabular}{|c|c|c|c|c|}
\hline \multicolumn{2}{|c|}{$\mathrm{E}(\text { level })^{\dagger}$} & $\mathrm{L}$ & $\left(2 \mathrm{~J}_{\mathrm{f}}+1\right) \mathrm{C}^{2} \mathrm{~S} \ddagger$ & Comments \\
\hline 5488 & 35 & $1+3^{\#}$ & $0.11,0.49$ & $\left(2 \mathrm{~J}_{\mathrm{f}}+1\right) \mathrm{C}^{2} \mathrm{~S}: 0.78(1971 \mathrm{Pe} 04)$ for $\mathrm{L}=1$ \\
\hline 5509 & 5 & $1+3^{\#}$ & $0.07,0.37$ & $\left(2 \mathrm{~J}_{\mathrm{f}}+1\right) \mathrm{C}^{2} \mathrm{~S}: 0.65\left(1970 \mathrm{~F}_{004}\right)$ for $\mathrm{L}=1$ \\
\hline 5587 & $7 \quad 5$ & $1+3$ & $0.06,0.27$ & \\
\hline 5622 & 5 & $1(+3) \#$ & $0.16,0.16$ & $\left(2 \mathrm{~J}_{\mathrm{f}}+1\right) \mathrm{C}^{2} \mathrm{~S}: 0.50\left(1970 \mathrm{Fo}_{0} 04\right), 0.44(1971 \mathrm{Pe} 04)$ for $\mathrm{L}=1$ \\
\hline 5665 & 5 & $1+3^{\#}$ & $0.07,0.30$ & $\left(2 \mathrm{~J}_{\mathrm{f}}+1\right) \mathrm{C}^{2} \mathrm{~S}: 0.29\left(1970 \mathrm{Fo}_{0} 04\right)$ for $\mathrm{L}=1$ \\
\hline 5720 & 5 & $(2,1+3)$ & & \\
\hline 5795 & 5 & 1 & 0.121 & $\left(2 \mathrm{~J}_{\mathrm{f}}+1\right) \mathrm{C}^{2} \mathrm{~S}: 0.36\left(1970 \mathrm{Fo}_{0} 04,1971 \mathrm{Pe} 04\right)$ \\
\hline 5826 & 5 & 1 & $0.04 \quad 1$ & \\
\hline 5877 & $7 \quad 5$ & $(2,1+3)$ & & \\
\hline 5918 & $8 \quad 5$ & $(1+3)$ & $0.02,0.07$ & \\
\hline 5975 & $5 \quad 5$ & 1 & $0.02 \quad 1$ & \\
\hline 6023 & 35 & 2 & $0.27 \quad 4$ & \\
\hline 6039 & 5 & $(1+3)$ & $0.03,0.13$ & \\
\hline 6100 & $0 \quad 5$ & $(1+3)$ & $0.01,0.24$ & \\
\hline 6158 & $8 \quad 5$ & $1+3$ & $0.07,0.23$ & \\
\hline 6191 & 15 & & & \\
\hline 6242 & 25 & $(1+3)$ & $0.05,0.19$ & \\
\hline$\dagger$ & From & 1973Ja17. Va & s from $1971 \mathrm{Pe}$ & and $1970 \mathrm{Fo} 04$ are in general agreement, but less accurate. \\
\hline$\ddagger$ & From & 1973Ja17. Va & s quoted by 19 & En 02 are adjusted upwards by $\approx 37 \%$, based on standard normalization factors in $1977 \mathrm{En} 02$ \\
\hline$\S$ & Pure & $\mathrm{L}=3$ in $1970 \mathrm{~F}$ & and/or $1971 \mathrm{Pe}$ & \\
\hline \# & Pure & $\mathrm{L}=1$ in $1970 \mathrm{~F}$ & and/or $1971 \mathrm{Pe}$ & \\
\hline
\end{tabular}

${ }^{41} \mathrm{~K}\left({ }^{3} \mathrm{He}, \mathrm{d} \gamma\right) \quad 1969 \mathrm{Te} 05$

Target ${ }^{41} \mathrm{~K}$ g.s. J $\pi=3 / 2+$.

1969Te05: E=18 MeV ${ }^{3} \mathrm{He}$ beam. Deuterons were detected with $\Delta \mathrm{E}-\mathrm{E}$ counter telescopes and $\gamma$-rays were detected with a NaI crystal. Measured $\gamma$, particle- $\gamma$ coin, particle- $\gamma(\theta)$. Deduced decay of isobaric-analog states.

${ }^{42} \mathrm{Ca}$ Levels

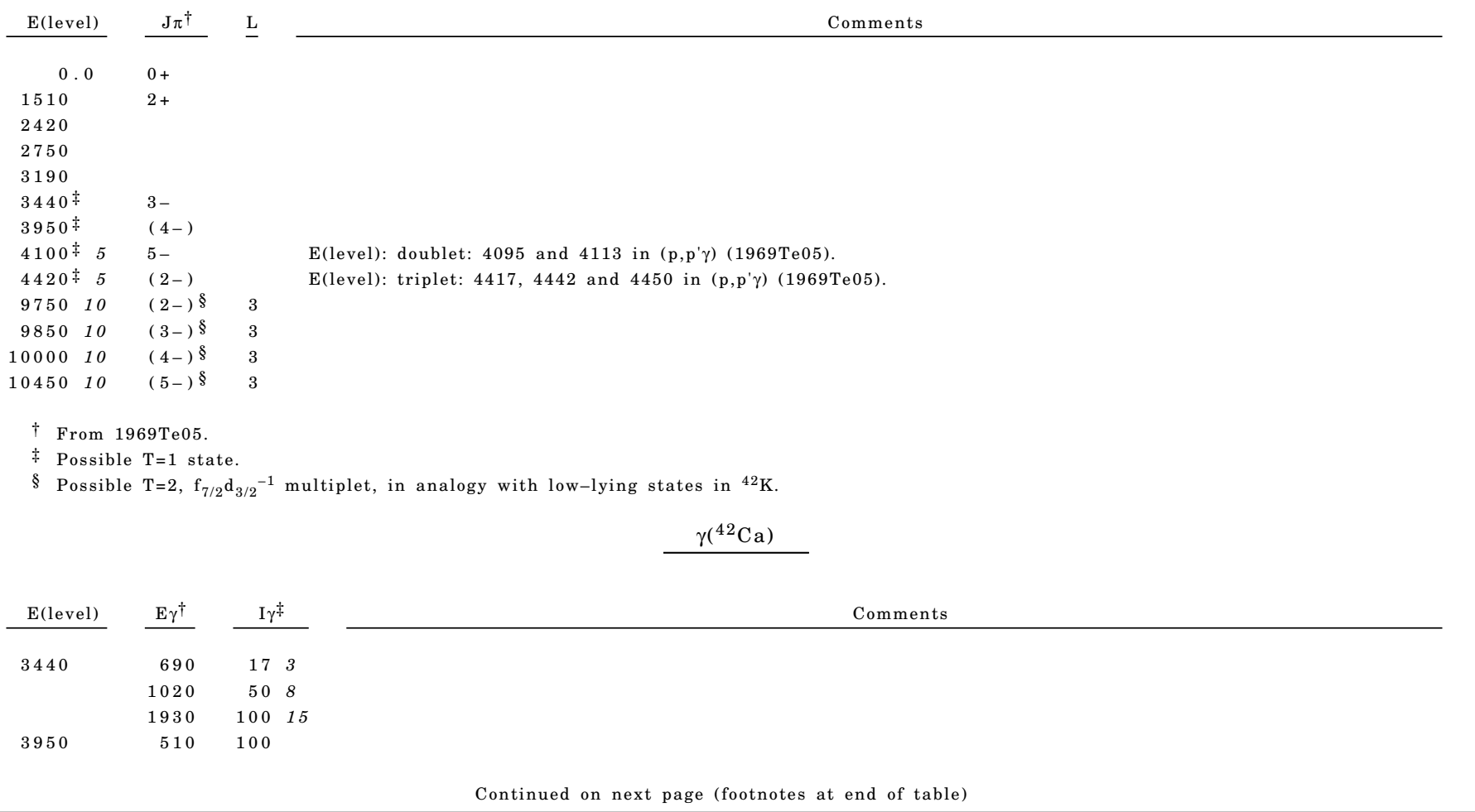


${ }^{41} \mathrm{~K}\left({ }^{3} \mathrm{He}, \mathrm{d} \gamma\right) \quad 1969 \mathrm{Te} 05$ (continued)

$\gamma\left({ }^{42} \mathrm{Ca}\right)$ (continued)

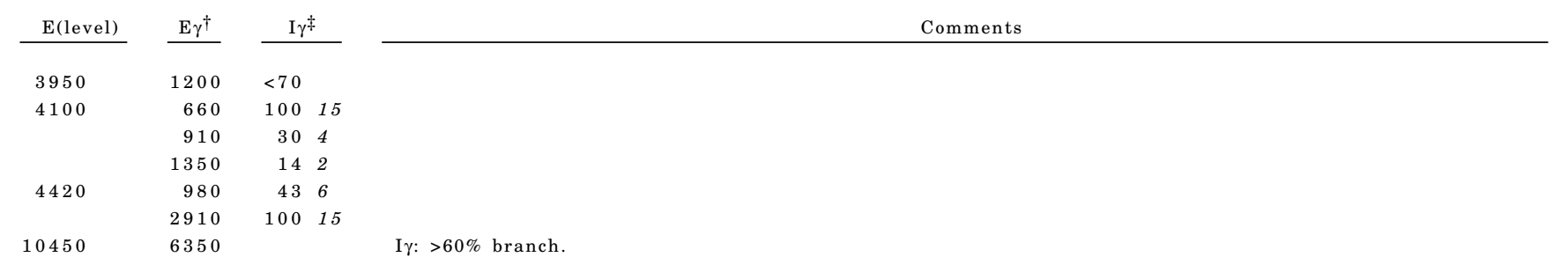

$\dagger$ From level-energy differences.

$\ddagger$ From 1969Te05.

\section{${ }^{41} \mathrm{Ca}(\mathrm{n}, \gamma)$ E=thermal 1989Ki11}

1989Ki11: thermal neutrons were produced from the Los Alamos Omega Reactor. Target of a $12 \mathrm{mg}^{\mathrm{CaCO}} \mathrm{C}_{3}$ enriched to $81.7 \%$ in ${ }^{41} \mathrm{Ca}$. $\gamma$-rays were detected with a $26 \mathrm{~cm}^{3} \mathrm{Ge}(\mathrm{Li})$ detector with a $\mathrm{NaI}(\mathrm{Tl})$ annulus operated in either a Compton-suppression mode or in a pair spectrometer mode.Measured E $\gamma$, I $\gamma$. Deduced neutron-separation energy. The authors report observing more than $250 \gamma$ rays in the range of $100 \mathrm{keV}$ to $10 \mathrm{MeV}$. But only selected cascades, relevant to the determination of the neutron-separation energy, are reported in the paper.

${ }^{42} \mathrm{Ca}$ Levels

\begin{tabular}{|c|c|c|c|c|c|}
\hline $\mathrm{E}(\text { level })^{\dagger}$ & $\mathrm{J} \pi \ddagger$ & $\mathrm{E}(\text { level })^{\dagger}$ & $\mathrm{J} \pi \neq$ & $\mathrm{E}(\text { level })^{\dagger}$ & $\mathrm{J} \pi \neq$ \\
\hline 0.0 & $0+$ & $3253.90 \quad 4$ & $4+$ & $4690.07 \quad 10$ & $3-$ \\
\hline $1524.73 \quad 3$ & $2+$ & $3446.96 \quad 4$ & $3-$ & $4759.71 \quad 16$ & $2+$ \\
\hline $2424.18 \quad 4$ & $2+$ & $3954.42 \quad 5$ & $4-$ & $5017.15 \quad 11$ & $4+$ \\
\hline $2752.42 \quad 4$ & $4+$ & $3999.67 \quad 9$ & $4+$ & ( 11480.65 & $3-, 4-\S$ \\
\hline
\end{tabular}

$\dagger$ From least-squares fit to $\mathrm{E} \gamma$ data.

¥ From Adopted Levels unless otherwise noted.

$\S$ s-wave capture in ${ }^{41} \mathrm{Ca}$ g.s. $(\mathrm{J} \pi=7 / 2-)$.

\begin{tabular}{|c|c|c|c|c|c|c|c|c|}
\hline \multirow{2}{*}{\multicolumn{2}{|c|}{$\mathrm{E} \gamma$}} & \multirow[b]{2}{*}{ E(level) } & & & \multicolumn{2}{|c|}{$\gamma\left({ }^{42} \mathrm{Ca}\right)$} & & \multirow[b]{2}{*}{ E(level) } \\
\hline & & & \multicolumn{2}{|l|}{$\mathrm{E} \gamma$} & $\mathrm{E}($ level) & \multicolumn{2}{|l|}{$\mathrm{E} \gamma$} & \\
\hline 501.46 & 3 & 3253.90 & 1763.12 & 12 & 5017.15 & 6720.46 & 18 & $(11480.65)$ \\
\hline 507.45 & 3 & 3954.42 & 1922.20 & 7 & 3446.96 & 6790.05 & 17 & $(11480.65)$ \\
\hline 899.42 & 3 & 2424.18 & 2335.70 & 30 & 4759.71 & 7480.07 & 18 & $(11480.65)$ \\
\hline 1022.77 & 3 & 3446.96 & 2424.17 & 6 & 2424.18 & 7525.47 & 10 & $(11480.65)$ \\
\hline 1227.65 & 3 & 2752.42 & 2474.80 & 10 & 3999.67 & 8033.03 & 18 & $(11480.65)$ \\
\hline 1524.68 & 3 & 1524.73 & 3165.24 & 11 & 4690.07 & 8225.86 & 13 & $(11480.65)$ \\
\hline 1729.19 & 5 & 3253.90 & 6462.79 & 17 & $(11480.65)$ & 8727.42 & 14 & $(11480.65)$ \\
\hline
\end{tabular}




\section{${ }^{41} \mathrm{Ca}(\mathrm{n}, \alpha):$ Resonances $2012 \mathrm{Ve} 01$}

2012Ve01: $\mathrm{E}=1$ to $80 \mathrm{keV}$ neutrons were produced via $(\gamma, \mathrm{n})$ and $(\gamma, \mathrm{F})$ at the gelina neutron time-of-flight facility of the Institute for Reference Materials and Measurement (rimm) in Geel (Belgium). Two flight paths for two different measurements: $8.5-\mathrm{m}$ and $30-\mathrm{m} . \alpha$ particles were detected with a Frisch gridded ionization chamber. Measured $\sigma\left(\mathrm{E}_{\mathrm{n}}\right)$, $\alpha$ yields. Deduced resonance energies, widths, strengths from R-Matrix analysis. $\mathrm{J} \pi\left({ }^{41} \mathrm{Ca}\right)$ g.s. $)=7 / 2-$.

\section{${ }^{42} \mathrm{Ca}$ Levels}

For $\Gamma_{\mathrm{n}} / \Gamma_{\mathrm{p}}, \omega_{\mathrm{p}}$ values were used from literature (reference 20 in 2012Ve01).

$\omega_{\alpha}=$ resonance strength.

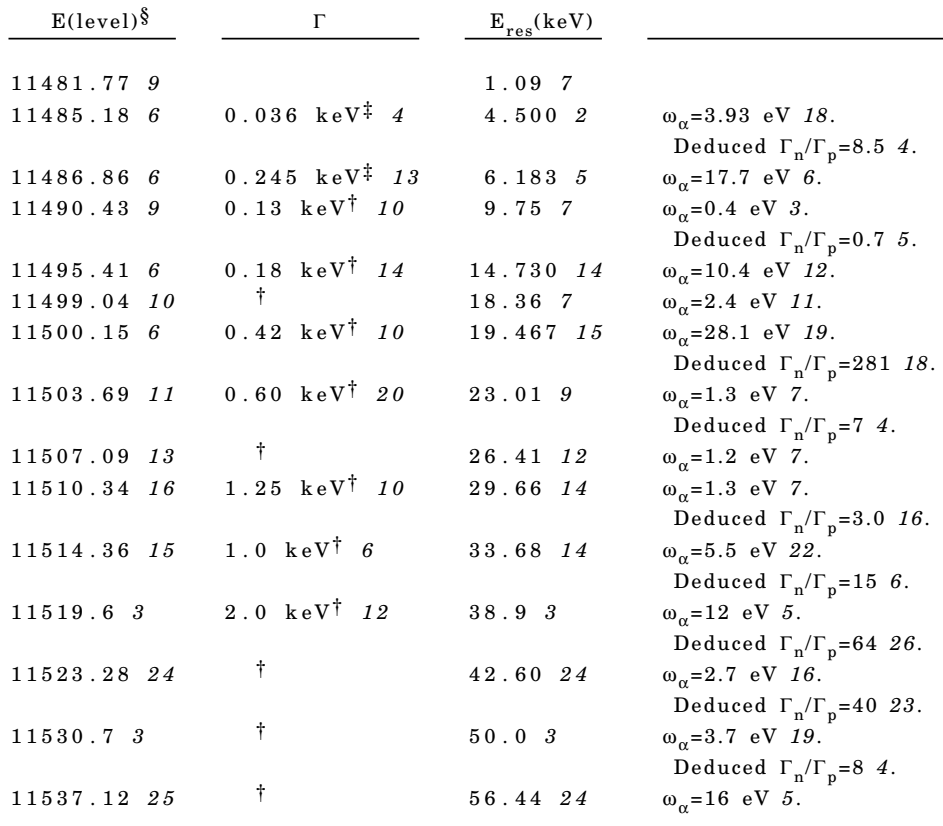

$\dagger$ From the measurement with the flight path=8.5 m and using Voigt fit method.

¥ From R-matrix analysis using SAMMY code.

$\S \mathrm{S}(\mathrm{n})\left({ }^{42} \mathrm{Ca}\right)=11480.67 \mathrm{keV} 6(2012 \mathrm{Wa} 38)$. The $\mathrm{E}(\mathrm{res})$ values listed in tables II and III are assumed to be in c.m. system.

\section{${ }^{41} \mathrm{Ca}(\mathrm{d}, \mathrm{p}),($ pol d,p) $\quad 1975 \mathrm{Ha} 14,1978 \mathrm{VoO4}$}

$\mathrm{J} \pi\left({ }^{41} \mathrm{Ca}\right.$ g.s. $)=7 / 2-$.

1975Ha14 (also 1972El12): (d,p) E=12 MeV deuteron beam was produced at the tandem accelerator of the Niels Bohr Institute. A $20 \mu \mathrm{g} / \mathrm{cm}^{2}$ target with a carbon backing $\left(81.8 \%\right.$ in $\left.{ }^{41} \mathrm{Ca}\right)$. Protons were momentum analyzed with a broad-range magnetic spectrograph, FWHM=15 keV. Measured $\sigma\left(E_{p}, \theta\right)$. Deduced levels, J $\pi$, L, spectroscopic factors from DWBA analysis.

1978Vo04 (also 1978Vo01): (pol d,p) E=11 MeV polarized deuteron beam was produced at the McMaster University FN tandem Van de Graaff accelerator. A $25 \mu \mathrm{g} / \mathrm{cm}^{2}$ thick target $(81.8 \%$ in $41 \mathrm{Ca})$ evaporated onto a carbon backing. Protons were momentum analyzed with a magnetic spectrograph and detected in nuclear emulsions, FWHM=15 keV. Measured $\sigma\left(E_{p}, \theta\right), A y(\theta)$. Deduced levels, J $\pi, L$, spectroscopic factors from DWBA analysis.

\begin{tabular}{|c|c|c|c|c|c|}
\hline Level energy & $\begin{array}{r}\sigma(\max ) \mu \mathrm{b} / \mathrm{s} \mathrm{r} \\
(1975 \mathrm{Ha} 1\end{array}$ & $\begin{array}{l}\text { ross section } \\
\theta(1 \mathrm{ab})\end{array}$ & $\begin{array}{l}\mathrm{data} \\
\sigma(\max )\end{array}$ & $\begin{array}{r}\mu \mathrm{b} / \mathrm{s} \mathrm{r} \\
(1978\end{array}$ & $\begin{array}{l}\theta(\mathrm{lab}) \\
04)\end{array}$ \\
\hline 0 & 105 & 35 & & 98 & 35 \\
\hline 1525 & 396 & 15 & & 448 & 15 \\
\hline 1836 & 22 & 30 & & 21 & 35 \\
\hline 2423 & 344 & 35 & & 346 & 30 \\
\hline 2752 & 1060 & 35 & & 054 & 35 \\
\hline 3187 & 2050 & 35 & & 243 & 35 \\
\hline 3251 & 266 & 35 & & 267 & 40 \\
\hline 3295 & 8 & 30 & & 12 & 35 \\
\hline
\end{tabular}


${ }^{41} \mathrm{Ca}(\mathrm{d}, \mathrm{p}),($ pol d,p) $1975 \mathrm{Ha14,1978Vo04}$ (continued)

3389

3441

3650

3888

3949

3999

4052

4103

4228

4356

4423

4448

4505

4566

4666

4691

4720

4760

4869

4899

4947

4977

5020

5084

5210

5325

5356

5384

5412

5442

5474

5533

5626

5669

5696

5778

5801

5926

5956

5988

6004

6021

6112

6138

6157

6184

6213

6250

6276

6304

6390

6426

6462

6495

6557

6572

6614

6653

6670

6717

6760

6785

6814

6884

6909

6937

6961

6987
60

10

$\begin{array}{rrr}15 & 2525 & 15 \\ 15 & 158 & 15 \\ 20 & & \end{array}$

1220

95

36
83

3950

160

6200

210

140

58
210

100

2660

150

100

87

80
4140

110
160

200

160

170

110

1010

160

100
460

460
94

94
190

590

130

340

220

450

320

300

510

40
10

10
160

350

39
2000
350

330

960

160

1920

200

10

15

25

15

5
30

30

5

15

15

5

15
15

15

5
15

15
10

5

5
15

5
5

5

5
10

15

15

5

5
5

5

5
10

5

5
10

5

5
10

10
10

5

10

5
10

10
10

10
5

5
15

20

30

10

15

10
15

15
10

10

10

10

10
1650

1277

10

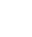

0

10

$\begin{array}{ll}4210 & 15\end{array}$

$6042 \quad 10$

$\begin{array}{rr}2804 & 15 \\ 158 & 15\end{array}$

$14530 \quad 15$

$1381 \quad 15$

$\begin{array}{ll}181 & 15\end{array}$

$617 \quad 15$

$360 \quad 15$

$408 \quad 10$

$282 \quad 10$

$232 \quad 10$

20

$794 \quad 15$

$2853 \quad 15$

$518 \quad 15$

$1072 \quad 15$
$2247 \quad 10$ 
${ }^{41} \mathrm{Ca}(\mathrm{d}, \mathrm{p}),($ pol d,p) $1975 \mathrm{Ha} 14,1978 \mathrm{VoO4}$ (continued)

7025

7041

7123

7160

7203

7221

7270

7348

7401

7422

7438

7468

7520

7571

7600

7643

7709

7760

7793
572

702

823

2184

1579

1534

2649

1272

1589

1309

4628
15

15

15

15

10

15

15

${ }^{42} \mathrm{Ca}$ Levels

\begin{tabular}{|c|c|c|c|c|c|}
\hline $\mathrm{E}(\mathrm{le}$ & vel $)^{\dagger}$ & $\mathrm{J} \pi$ & $L \S$ & $(2 \mathrm{~J}+1) \mathrm{S}^{\#}$ & Comments \\
\hline 0 . & 0 & & $3^{@ \&}$ & $0.91^{@}$ & $(2 \mathrm{~J}+1) \mathrm{S}: 1.0(1975 \mathrm{Ha} 14)$ \\
\hline 1525 & 5 & & $1+3^{@}$ & $0.24,2.7^{@}$ & $\begin{array}{l}\text { Spin of transferred neutron }=3 / 2+7 / 2(1978 \mathrm{Vo} 04) \\
(2 \mathrm{~J}+1) \mathrm{S}: 0.25,3.1(1975 \mathrm{Ha} 14)\end{array}$ \\
\hline 1836 & 5 & & $3^{@ \&}$ & $0.21^{@}$ & $(2 \mathrm{~J}+1) \mathrm{S}: 0.23(1975 \mathrm{Ha} 14)$ \\
\hline 2423 & 5 & & $3^{@ \&}$ & $3.14^{@}$ & $(2 \mathrm{~J}+1) \mathrm{S}: 3.4(1975 \mathrm{Ha} 14)$ \\
\hline 2752 & 5 & & $1+3^{@}$ & $0.32,8.25^{@}$ & $\begin{array}{l}\text { Spin of transferred neutron }=3 / 2+7 / 2\left(1978 \mathrm{~V}_{0} 04\right) \\
(2 \mathrm{~J}+1) \mathrm{S}: 0.35,9.5(1975 \mathrm{Ha} 14)\end{array}$ \\
\hline 3187 & 5 & & $3^{@ \&}$ & $17.1^{@}$ & $(2 \mathrm{~J}+1) \mathrm{S}: 20.0(1975 \mathrm{Ha} 14)$ \\
\hline 3251 & 5 & & $3^{@ \&}$ & $2.36^{@}$ & $\begin{array}{l}\mathrm{L}: 3(1978 \mathrm{Vo} 04) . \\
(2 \mathrm{~J}+1) \mathrm{S}: 2.4(\mathrm{~L}=3), 0.014(\mathrm{~L}=(1))(1975 \mathrm{Ha} 14) .\end{array}$ \\
\hline 3295 & 10 & & $3^{@}$ & $0.10^{@}$ & $(2 \mathrm{~J}+1) \mathrm{S}: 0.08(1975 \mathrm{Ha} 14)$ \\
\hline 3389 & & & $1^{@ \mathrm{a}}$ & $0.04^{@}$ & $\begin{array}{l}\mathrm{L}: 1+3(1975 \mathrm{Ha} 14) \\
(2 \mathrm{~J}+1) \mathrm{S}: 0.06(\mathrm{~L}=1), 0.05(\mathrm{~L}=3)(1975 \mathrm{Ha} 14)\end{array}$ \\
\hline 3441 & 10 & & & & \\
\hline 3650 & 10 & & $1+3^{@}$ & $0.25,0.42^{@}$ & $\begin{array}{l}\text { Spin of transferred neutron=3/2+7/2 }(1978 \mathrm{Vo} 04) \text {. } \\
(2 \mathrm{~J}+1) \mathrm{S}: 0.26,0.72(1975 \mathrm{Ha} 14)\end{array}$ \\
\hline 3888 & 10 & & & & \\
\hline 3949 & 10 & & $(0+2)$ & $0.006,0.13$ & \\
\hline 3999 & 10 & & & & \\
\hline 4052 & 10 & & $(0+2)$ & $0.011,0.10$ & \\
\hline 4103 & 10 & & & & \\
\hline 4228 & 10 & & & & \\
\hline 4356 & 10 & & $2(+0)$ & $0.35,0.001$ & \\
\hline 4423 & 10 & & $0+2$ & $0.024,0.32$ & \\
\hline 4448 & 10 & & $1^{@ a}$ & $1.54^{@}$ & $\begin{array}{l}\text { E(level): doublet. } \\
(2 \mathrm{~J}+1) \mathrm{S}: 1.7(1975 \mathrm{Ha} 14)\end{array}$ \\
\hline 4505 & 10 & & $1+3^{@}$ & $0.07,0.08^{@}$ & $\begin{array}{l}\text { Spin of transferred neutron }=(3 / 2+7 / 2)(1978 \mathrm{Vo} 04) \text {. } \\
(2 \mathrm{~J}+1) \mathrm{S}: 0.083,0.09(1975 \mathrm{Ha} 14) .\end{array}$ \\
\hline 4566 & 10 & & & & \\
\hline 4666 & 10 & & $0+2$ & $0.017,0.021$ & \\
\hline 4691 & 10 & & $0+2$ & $0.033,0.20$ & \\
\hline 4720 & 10 & & $0+2$ & $0.022,0.05$ & \\
\hline 4760 & 10 & & $1^{@ a}$ & $1.03^{@}$ & $(2 \mathrm{~J}+1) \mathrm{S}: 1.1(1975 \mathrm{Ha} 14)$ \\
\hline 4869 & 10 & & $1^{@ a}$ & $0.72^{@}$ & $\begin{array}{l}\mathrm{L}: 1(+3)(1975 \mathrm{Ha} 14) \\
(2 \mathrm{~J}+1) \mathrm{S}: 0.83(\mathrm{~L}=1), 0.51 \quad(\mathrm{~L}=3)(1975 \mathrm{Ha} 14)\end{array}$ \\
\hline 4899 & 10 & & $0+2$ & $0.023,0.29$ & \\
\hline 4947 & 10 & & ( 1 ) & 0.025 & \\
\hline 4977 & 10 & & 0 & 0.026 & \\
\hline 5020 & 10 & & $1^{@ a}$ & $2.43^{@}$ & $\begin{array}{l}\mathrm{L}: 1(+3)(1975 \mathrm{Ha} 14) \\
(2 \mathrm{~J}+1) \mathrm{S}: 2.6 \quad(\mathrm{~L}=1), 1.4 \quad(\mathrm{~L}=3)(1975 \mathrm{Ha} 14)\end{array}$ \\
\hline 5084 & 10 & & & & \\
\hline 5210 & 10 & & $1^{@ a}$ & $3.78^{@}$ & $(2 \mathrm{~J}+1) \mathrm{S}: 4.2(1975 \mathrm{Ha} 14)$ \\
\hline
\end{tabular}


${ }^{41} \mathrm{Ca}(\mathrm{d}, \mathrm{p}),($ pol d,p) $1975 \mathrm{Ha} 14,1978 \mathrm{VoO4}$ (continued)

\author{
${ }^{42} \mathrm{Ca} \mathrm{Levels} \mathrm{(continued)}$
}

\begin{tabular}{|c|c|c|c|c|c|c|}
\hline \multicolumn{2}{|c|}{$\mathrm{E}(\text { level })^{\dagger}$} & \multicolumn{2}{|c|}{$\mathrm{J} \pi$ * } & \multirow{2}{*}{$\frac{L \S}{0(+2)}$} & \multirow{2}{*}{$\frac{(2 \mathrm{~J}+1) \mathrm{S}^{\#}}{0.025,0.016}$} & Comments \\
\hline 5325 & 10 & & & & & \\
\hline 5356 & 10 & & & 1 & 0.083 & \\
\hline 5384 & 10 & & & $(0+2)$ & $0.005,0.04$ & \\
\hline 5412 & 10 & & & 0 & 0.022 & \\
\hline 5442 & 10 & & & 0 & 0.013 & \\
\hline 5474 & 10 & & & $1^{@ a}$ & 1.48@ & $\begin{array}{l}\mathrm{L}: 1(+3)(1975 \mathrm{Ha} 14) \\
(2 \mathrm{~J}+1) \mathrm{S}: 1.6(\mathrm{~L}=1), 1.0 \quad(\mathrm{~L}=3) \quad(1975 \mathrm{Ha} 14)\end{array}$ \\
\hline 5533 & 10 & & & $1^{@ \mathrm{a}}$ & $0.09^{@}$ & $(2 \mathrm{~J}+1) \mathrm{S}: 0.09(1975 \mathrm{Ha} 14)$ \\
\hline 5626 & 10 & & & $0(+2)$ & $0.011,0.021$ & \\
\hline 5669 & 10 & & & $0+2$ & $0.010,0.017$ & \\
\hline 5696 & 10 & & & & & \\
\hline 5778 & 10 & & & $1^{@ a}$ & $6.88^{@}$ & $(2 \mathrm{~J}+1) \mathrm{S}: 9.1(1975 \mathrm{Ha} 14)$ \\
\hline 5801 & 10 & & & & & \\
\hline 5926 & 10 & & & $0+2$ & $0.021,0.022$ & \\
\hline 5956 & 10 & & & $0+2$ & $0.023,0.021$ & \\
\hline 5988 & 10 & & & $0+2$ & $0.019,0.028$ & \\
\hline 6004 & 10 & & & $0+2$ & $0.015,0.071$ & \\
\hline 6021 & 10 & & & $0+2$ & $0.011,0.042$ & \\
\hline 6112 & 10 & & & $1^{@ \mathrm{a}}$ & $0.70^{@}$ & $\begin{array}{l}\mathrm{L}: 1+3(1975 \mathrm{Ha} 14) \\
(2 \mathrm{~J}+1) \mathrm{S}: 0.59(\mathrm{~L}=1), 0.57 \quad(\mathrm{~L}=3)(1975 \mathrm{Ha} 14)\end{array}$ \\
\hline 6138 & 10 & & & & & \\
\hline 6157 & 10 & & & $0+2$ & $0.019,0.040$ & \\
\hline 6184 & 10 & & & $(0+2)$ & $0.012,0.036$ & $\mathrm{~J} \pi:$ a dopted $\mathrm{J} \pi=(1,2+)$ \\
\hline 6213 & 10 & & & $0+2$ & $0.05,0.18$ & \\
\hline 6250 & 10 & & & $0+2$ & $0.010,0.030$ & \\
\hline 6276 & 10 & & & $1^{@ a}$ & $0.14^{@}$ & $(2 \mathrm{~J}+1) \mathrm{S}: 0.11(1975 \mathrm{Ha} 14)$ \\
\hline 6304 & 10 & $(2$ to & $5)+$ & $1^{@}$ & $0.33^{@}$ & $\begin{array}{l}\text { Spin of transferred neutron=(3/2) }(1978 \mathrm{Vo} 04) \text {. } \\
(2 \mathrm{~J}+1) \mathrm{S}: 0.33(1975 \mathrm{Ha} 14)\end{array}$ \\
\hline 6390 & 10 & & & $0(+2)$ & $0.014,0.006$ & \\
\hline 6426 & 10 & $(2 \mathrm{to}$ & $5)+$ & $1^{@ a}$ & $0.17^{@}$ & $(2 \mathrm{~J}+1) \mathrm{S}: 0.17(1975 \mathrm{Ha} 14)$ \\
\hline 6462 & 10 & & & $0+2$ & $0.023,0.071$ & \\
\hline 6495 & 10 & $(2$ to & $5)+$ & $1^{@ \mathrm{a}}$ & $0.26^{@}$ & $(2 \mathrm{~J}+1) \mathrm{S}: 0.22(1975 \mathrm{Ha} 14)$ \\
\hline 6557 & 15 & $(2 \mathrm{to}$ & $5)+$ & $1^{@ a}$ & $0.17^{@}$ & $(2 \mathrm{~J}+1) \mathrm{S}: 0.15(1975 \mathrm{Ha} 14)$ \\
\hline 6572 & 15 & $(2 \mathrm{to}$ & $5)+$ & $1^{@ a}$ & $0.15^{@}$ & $(2 \mathrm{~J}+1) \mathrm{S}: 0.16(1975 \mathrm{Ha} 14)$ \\
\hline 6614 & 15 & $3+, 4+$ & & $1^{@ b}$ & $0.34^{@}$ & $\begin{array}{l}\mathrm{L}: 1(+3)(1975 \mathrm{Ha} 14) \\
(2 \mathrm{~J}+1) \mathrm{S}: 0.30(\mathrm{~L}=1), 0.18 \quad(\mathrm{~L}=3)(1975 \mathrm{Ha} 14) .\end{array}$ \\
\hline 6653 & 15 & & & & & \\
\hline 6670 & 15 & & & & & \\
\hline 6717 & 15 & & & $(1+3)$ & $0.070,0.35$ & \\
\hline 6760 & 15 & $3+, 4+$ & & $1^{@}$ & $0.32^{@}$ & $\begin{array}{l}\text { Spin of transferred neutron=1/2+3/2 }(1978 \mathrm{Vo} 04) \text {. } \\
\mathrm{L}: 1(+3)(1975 \mathrm{Ha} 14) \text {. } \\
(2 \mathrm{~J}+1) \mathrm{S}: 0.18(\mathrm{~L}=1), 0.26(\mathrm{~L}=3)(1975 \mathrm{Ha} 14)\end{array}$ \\
\hline 6785 & 15 & & & & & \\
\hline 6814 & 15 & $\left(\begin{array}{ll}2 & \text { to }\end{array}\right.$ & $5)+$ & $1^{@ \mathrm{a}}$ & $1.17^{@}$ & $\begin{array}{l}\mathrm{L}: 1+3(1975 \mathrm{Ha} 14) \\
(2 \mathrm{~J}+1) \mathrm{S}: 1.0(\mathrm{~L}=1), 0.97 \quad(\mathrm{~L}=3)(1975 \mathrm{Ha} 14)\end{array}$ \\
\hline 6884 & 15 & $(2$ to & $5)+$ & $1^{@ \mathrm{a}}$ & $0.20 @$ & $(2 \mathrm{~J}+1) \mathrm{S}: 0.15(1975 \mathrm{Ha} 14)$ \\
\hline 6909 & 15 & $3+, 4+$ & & $1 @ \mathrm{~b}$ & $0.52^{@}$ & $(2 \mathrm{~J}+1) \mathrm{S}: 0.48(1975 \mathrm{Ha} 14)$ \\
\hline 6937 & 15 & & & $1+3$ & $0.058,0.14$ & \\
\hline 6961 & 15 & $3+, 4+$ & & $1^{@ b}$ & $1.06^{@}$ & $(2 \mathrm{~J}+1) \mathrm{S}: 0.97(1975 \mathrm{Ha} 14)$ \\
\hline 6987 & 15 & & & ( 1 ) & 0.075 & \\
\hline 7025 & 15 & $3+, 4+$ & & $1+3^{@}$ & $0.19,0.37^{@}$ & $\begin{array}{l}\text { Spin of transferred neutron=1/2+5/2 }(1978 \mathrm{Vo} 04) \text {. } \\
(2 \mathrm{~J}+1) \mathrm{S}: 0.20,0.33(1975 \mathrm{Ha} 14) .\end{array}$ \\
\hline 7041 & 15 & & & $(0+2)$ & $0.015,0.057$ & \\
\hline 7123 & 15 & $3+, 4+$ & & $1^{@}$ & $0.36^{@}$ & $\begin{array}{l}\text { Spin of transferred neutron=1/2+3/2 (1978Vo04). } \\
(2 \mathrm{~J}+1) \mathrm{S}: 0.31(1975 \mathrm{Ha} 14) \text {. }\end{array}$ \\
\hline 7160 & 15 & $3+, 4+$ & & $1^{@ b}$ & $0.38^{@}$ & $(2 \mathrm{~J}+1) \mathrm{S}: 0.35(1975 \mathrm{Ha} 14)$ \\
\hline 7203 & 15 & & & $(0+2)$ & $0.016,0.028$ & \\
\hline 7221 & 15 & & & $(0+2)$ & $0.019,0.026$ & \\
\hline 7270 & 15 & $3+, 4+$ & & $1^{\circledR \mathrm{b}}$ & $0.91^{@}$ & $(2 \mathrm{~J}+1) \mathrm{S}: 0.98(1975 \mathrm{Ha} 14)$ \\
\hline 7348 & 15 & $3+, 4+$ & & $1^{@ b}$ & $0.69^{@}$ & $(2 \mathrm{~J}+1) \mathrm{S}: 0.74(1975 \mathrm{Ha} 14)$ \\
\hline 7401 & 15 & $3+, 4+$ & & $1^{@ b}$ & $0.72^{@}$ & $(2 \mathrm{~J}+1) \mathrm{S}: 0.96(1975 \mathrm{Ha} 14)$ \\
\hline 7422 & 15 & & & & & \\
\hline 7438 & 15 & & & & & \\
\hline
\end{tabular}




\section{${ }^{41} \mathrm{Ca}(\mathrm{d}, \mathrm{p}),($ pol d,p) $1975 \mathrm{Ha14}, 1978 \mathrm{VoO4}$ (continued)}

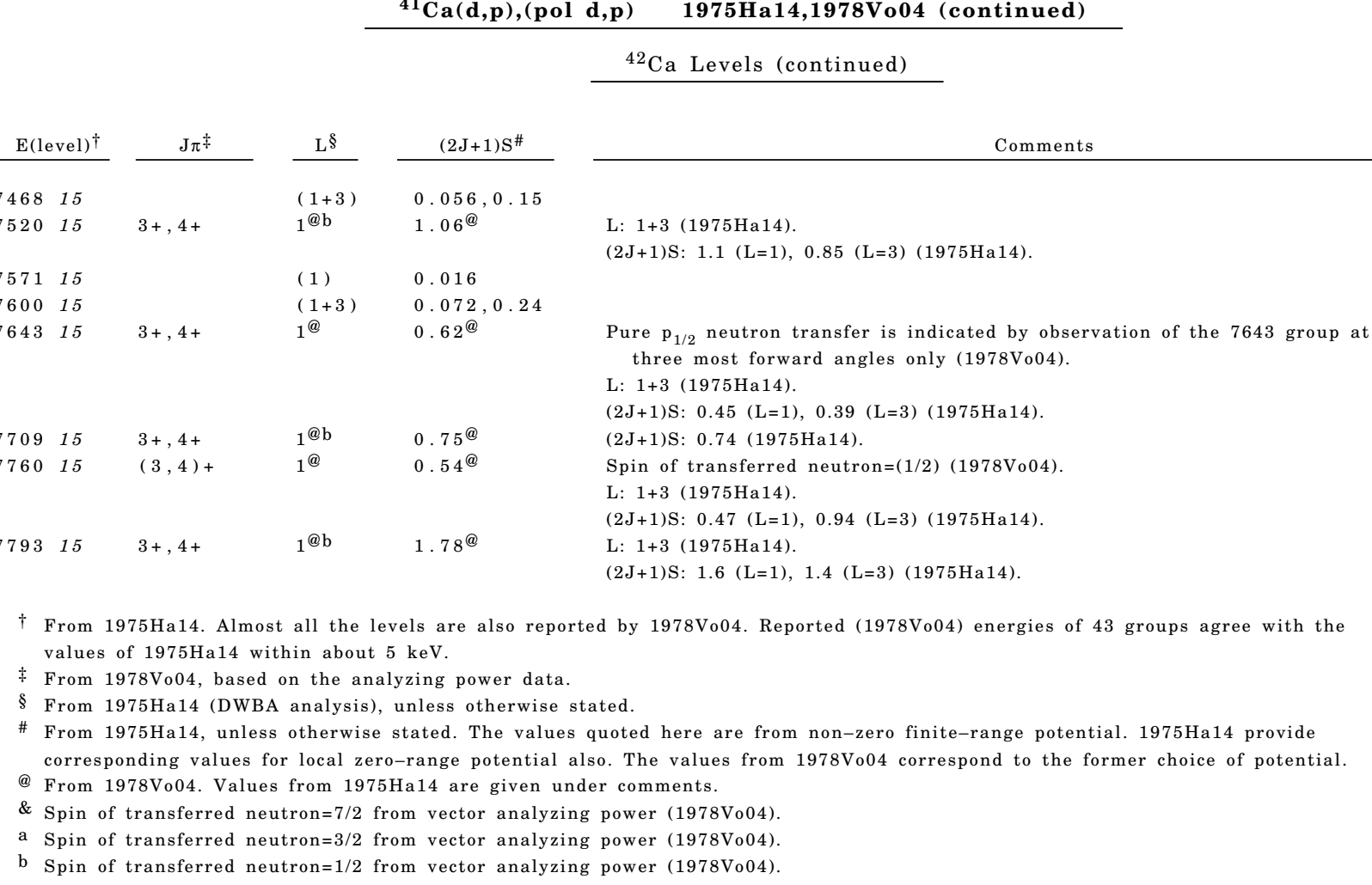

\section{${ }^{42} \mathrm{Ca}(\gamma, \gamma) \quad 1966 \mathrm{Me} 11$}

1966 Me11: $\mathrm{E}=1520 \mathrm{keV} \gamma$-rays from ${ }^{42} \mathrm{~K}$ radioactive source. Targets of natural and enriched $\mathrm{CaCO}_{3}\left(94.42 \%\right.$ in $\left.{ }^{42} \mathrm{Ca}\right)$. Scattered $\gamma$-rays were detected with a 3 by 3 in. NaI(Tl) detector. Measured cross section for resonant scattering. Deduced $\mathrm{T}_{1 / 2}$ from measured resonance width for the $1.52 \mathrm{MeV}$ level.

Other: $1972 \mathrm{KaXR}$.

\section{${ }^{42} \mathrm{Ca}$ Levels}

\begin{tabular}{|c|c|c|c|}
\hline E(level) & $\underline{\mathrm{J} \pi}{ }^{\dagger}$ & $\mathrm{T}_{1 / 2}$ & Comments \\
\hline 0.0 & $0+$ & & \\
\hline 1520 & $2+$ & $0.97 \mathrm{ps} 22$ & $\mathrm{~T}_{1 / 2}:$ from $\Gamma=0.00048 \mathrm{eV} 11$ (1966Me11). Other: $0.520 \mathrm{ps} 35$ (1972KaXR). \\
\hline$\dagger$ From & Adopted & Levels. & \\
\hline & & & $\gamma\left({ }^{42} \mathrm{Ca}\right)$ \\
\hline $\mathrm{E} \gamma$ & E(level) & & Comments \\
\hline
\end{tabular}

$1520 \quad 1520 \quad \mathrm{~B}(\mathrm{E} 2) \downarrow=0.007417$. 


\section{${ }^{42} \mathrm{Ca}\left(\mathbf{e}, \mathbf{e}^{\prime}\right) \quad 1989 \mathrm{It} 02$}

Target ${ }^{42} \mathrm{Ca}$ g.s. J $\pi=0+$.

1989It02 (also 1983It02,1981It02): $\mathrm{E}=62.5-250 \mathrm{MeV}$ electron beams were produced from the linear accelerators both at the Laboratory of Nuclear Science, Tohoku University and at the Accelerator Laboratory, University of Saskatchewan. Target of a $48.9 \mathrm{mg} / \mathrm{cm}^{2} 94.4 \%$ enriched ${ }^{42} \mathrm{Ca}$ metallic foil. Scattered electrons were measured by a hodoscope-type array of solid-state detectors or plastic scintillators mounted at the focal plane in a magnetic spectrometers. Measured $\sigma(\theta)$. Deduced levels, form factors, $J \pi, \beta(\lambda)$. Overall resolution is $0.15 \%$ for one experiment and $0.10 \%$ for the other. Comparisons of measured form factors with DWBA calculations. 1984Ra04: E=30-57.5 MeV. Measured cross sections, deduced isovector M2 transition strengths. FWHM=25-45 keV. 1980St17: E=39 MeV. Measured cross sections. Deduced M1 strength.

1978Gr02: $\mathrm{E}=31-67 \mathrm{MeV}$. Measured cross sections, deduced monopole (E0) excitation. FWHM $\approx 30 \mathrm{keV}$.

1971He08: $\mathrm{E}=198,250,300 \mathrm{MeV}$. Measured $\sigma(\theta)$.

1968Fr11: $\mathrm{E}=250 \mathrm{MeV}$. Measured cross section for elastic scattering.

\section{${ }^{42} \mathrm{Ca}$ Levels}

\begin{tabular}{|c|c|c|c|c|}
\hline $\mathrm{E}(\text { level })^{\dagger}$ & $\mathrm{J} \pi$ & $\mathrm{L}^{\dagger}$ & $\mathrm{B}(\mathrm{EL})^{\dagger}$ & Comments \\
\hline 0.0 & $0+$ & & & \\
\hline 1520 & $2+$ & 2 & $0.0418 \quad 15$ & Other $\mathrm{B}(\mathrm{E} 2)=0.032020(1971 \mathrm{He} 08)$. \\
\hline 1837 & & & & $\begin{array}{l}\text { E(level): from } 1978 \mathrm{Gr} 02 \text {, E0 excitation. } \\
\text { E0 matrix element }=5.24 \mathrm{fm}^{2} 39 \text { (1978Gr02). }\end{array}$ \\
\hline 2420 & $2+$ & 2 & $0.0118 \quad 12$ & \\
\hline 2750 & $4+$ & & & \\
\hline 3440 & $3-$ & 3 & $0.0136 \quad 6$ & Other $\mathrm{B}(\mathrm{E} 3)=0.00914(1971 \mathrm{He} 08)$ \\
\hline 4100 & $5-$ & 5 & 0.000119 & Other $\mathrm{B}(\mathrm{E} 5)=0.0000666(1971 \mathrm{He} 08)$. \\
\hline 4450 & $4+$ & & & \\
\hline 4690 & $3-$ & 3 & $0.00215 \quad 21$ & \\
\hline 4980 & $3-$ & 3 & $0.0010 \quad 1$ & \\
\hline 5520 & $3-$ & & & \\
\hline 5680 & $3-$ & & & \\
\hline 6100 & $(1-)$ & ( 1 ) & & $\begin{array}{l}\text { Assignment as isoscalar E1 transition is based on similarity of q-dependence of form } \\
\text { factor with isoscalar E1 transitions of } 5900 \text { and } 6950 \text { in }{ }^{40} \mathrm{Ca} \text {. }\end{array}$ \\
\hline 9750 & $(2-)$ & & & $\begin{array}{l}\mathrm{E}(\text { level }), \mathrm{J} \pi \text { : from } 1984 \mathrm{Ra} 04, \mathrm{M} 2 \text { excitation. } \\
\mathrm{B}(\mathrm{M} 2)=30 \mu_{\mathrm{n}}^{2} \mathrm{fm}^{2} 10(1984 \mathrm{Ra} 04)\end{array}$ \\
\hline 9770 & $(2+)$ & & $0.00032 \quad 12$ & $\mathrm{E}($ level),J $\pi, \mathrm{B}(\mathrm{EL})$ : from $1984 \mathrm{Ra} 04, \mathrm{E} 2$ excitation. \\
\hline 11235 & $(1+)$ & & & $\begin{array}{l}\text { E(level),J } \pi, B(E L) \text { : from } 1980 \text { St17, M1 excitation. } \\
B(M 1)=0.595 \text { (1980St17). }\end{array}$ \\
\hline
\end{tabular}




\section{${ }^{42} \mathrm{Ca}\left(\pi^{+}, \pi^{+\prime}\right),\left(\pi^{-}, \pi^{-\prime}\right) \quad 1984 \mathrm{BoO2}$}

1984Bo02, 1981Bo26: E=116.0, 180.0, 292.5 MeV pions were produced at the Clinton P. Anderson Meson Physics Facility (LAMPF). Scattered particles were analyzed with the epics spectrometer and detected by position-sensitive,

delay-line read-out drift chambers, FWHM=300 keV. Measured $\sigma(\theta)$. Deduced levels, J $\pi$, L from Distorted Wave Impulse Approximation (DWIA) and optical-model analysis.

1984KaZY: $\mathrm{E}=130,180,235,292 \mathrm{MeV}$. Measured $\sigma(\theta)$, deduced core-excitation role.

1999Ha66: $\mathrm{E}=164-292 \mathrm{MeV}$.

\section{${ }^{42} \mathrm{Ca}$ Levels}

All data are from 1984Bo02, unless otherwise noted.

\begin{tabular}{|c|c|c|}
\hline E(level) & $\mathrm{J} \pi \S$ & $\mathrm{L}$ \\
\hline 0.0 & $0+$ & 0 \\
\hline $1520^{\dagger}$ & $2+$ & 2 \\
\hline 2420 & $2+$ & 2 \\
\hline $3440 末$ & $3-$ & 3 \\
\hline 4104 & $5-$ & 5 \\
\hline 4680 & $3-$ & 3 \\
\hline 6300 & $(3-)$ & ( 3 ) \\
\hline
\end{tabular}

$\dagger$ E2 matrix elements (from microscopic model, in s.p.u.): 3.2222 (for neutron), 2.8021 (for proton) (1981Bo26).

† E3 matrix elements (from microscopic model, in s.p.u.): 3.5927 (for neutron), 4.0628 (for proton) (1981Bo26).

$\S$ From L-transfer.

\section{${ }^{42} \mathrm{Ca}\left(\mathbf{p}, \mathbf{p}^{\prime}\right) \quad 1968 \mathrm{Ba28}, 1971 \mathrm{Ma53,1970La22}$}

See also ${ }^{42} \mathrm{Ca}\left(\mathrm{p}, \mathrm{p}^{\prime} \gamma\right)$ dataset.

Inelastic and elastic scattering experiments:

1968Ba28: $\mathrm{E}=22.9 \mathrm{MeV}$ proton beam was produced at the University of Colorado 132 fixed-field alternating-gradient cyclotron. A self-supporting target of ${ }^{42} \mathrm{Ca}$ enriched to $93.7 \%$. Scattered protons were detected with a $3 \mathrm{~mm}$ lithium-drifted silicon detector, FWHM=100 $100 \mathrm{keV}$. Measured $\sigma\left(\mathrm{E}_{\mathrm{p}}, \theta\right)$. Deduced levels, L, deformation parameters from DWBA analysis.

1971Ma53: $\mathrm{E}=49.75 \mathrm{MeV}$ proton beam was produced at the proton linear accelerator at the Rutherford High Energy Laboratory, Chilton, Berkshire. A self-supporting target of $90 \%$ enriched ${ }^{42} \mathrm{Ca}, 2 \mathrm{mg} / \mathrm{cm}^{2}$ thick. Scattered protons were detected with an $n=1 / 2$ double-focusing magnetic spectrometer with an acoustic spark-chamber assembly, FWHM=65 keV. Measured $\sigma\left(E_{p}, \theta\right)$. Deduced levels, L, deformation parameters from DWBA analysis.

1970La22: (p, $\left.\mathrm{p}^{\prime} \gamma\right) \mathrm{E}=7.03 \mathrm{MeV}$ proton from the University of Liverpool $\mathrm{E}(\mathrm{n})$ tandem accelerator. A $200 \mu \mathrm{g} / \mathrm{cm}^{2}$ thick enriched ${ }^{42} \mathrm{Ca}$ target. Scattered protons were detected with an annular silicon surface barrier detected and $\gamma-$ rays were detected with NaI crystals. Measured scattered proton energies, E $\gamma, \mathrm{I} \gamma, \mathrm{p} \gamma(\theta)$. Deduced levels, J $\pi$, $\gamma$-branchings, mixing ratios.

1969Ko03: E=7.8 MeV. Measured scattered proton energies, lifetimes by Doppler-shift attenuation of $\gamma$ rays. FWHM (for proton spectra) $=120 \mathrm{keV}$.

1956Br08: $\mathrm{E}=6.5,7.0,7.4 \mathrm{MeV}$. A total of 14 groups reported up to $4043 \mathrm{keV}$.

1961Be19: E=3.6-6.3 MeV. E0 transition observed.

1976Al19: $\mathrm{E}=1.044 \mathrm{GeV}$. Measured $\sigma(\theta)$.

1976Ul01 (also 1976UlZZ): E=4.40 MeV. Measured pair spectra, deduced E0 strength.

1983Mi25, 1982Be32: (pol p,p') E=650 MeV. Measured $\sigma(\theta)$, DWIA analysis.

Elastic scattering experiments:

1986 Mc05 (also 1980NaZU): E=21-48.4 MeV. Measured $\sigma(\theta)$, deduced nuclear matter radii.

1981Ra02: ( $p o l \mathrm{p}, \mathrm{p}) \mathrm{E}=800 \mathrm{MeV}$. Measured $\sigma(\theta)$, analyzing powers.

1981No07: (pol p,p) E=65 MeV. Measured $\sigma(\theta)$, polarization.

1980Fa07: $\mathrm{E}=35.2 \mathrm{MeV}$. Measured $\sigma(\theta)$.

1979Ig01: (pol p,p) E=0.8 GeV. Measured $\sigma(\theta)$, $\operatorname{Ay}(\theta)$.

1971Ma17: $\mathrm{E}=49.3 \mathrm{MeV}$. Measured $\sigma(\theta)$.

1969Sm02: $\mathrm{E}=26.5 \mathrm{MeV}$.

${ }^{42} \mathrm{Ca}$ Levels

\begin{tabular}{|c|c|c|c|c|}
\hline $\mathrm{E}(\mathrm{le}$ & vel $)^{\dagger}$ & $\mathrm{L}^{\dagger}$ & $\beta_{\mathrm{L}}^{\#}$ & Comments \\
\hline \multicolumn{5}{|c|}{0.0} \\
\hline 1520 & 10 & 2 & 0.21 & $\mathrm{~B}(\mathrm{E} 2)($ W.u. $)=3.86(1971 \mathrm{Ma} 53), 6.410 \quad(1968 \mathrm{Ba} 28)$. \\
\hline 1850 & 10 & 0 & & \\
\hline 2430 & 10 & 2 & 0.07 & $\mathrm{~B}(\mathrm{E} 2)(\mathrm{W} . \mathrm{u})=.0.5910 \quad(1971 \mathrm{Ma} 53), 0.87 \quad 10 \quad(1968 \mathrm{Ba} 28)$ \\
\hline
\end{tabular}


${ }^{42} \mathrm{Ca}\left(\mathrm{p}, \mathrm{p}^{\prime}\right) \quad 1968 \mathrm{Ba} 28,1971 \mathrm{Ma53}, 1970 \mathrm{La} 2$ (continued)

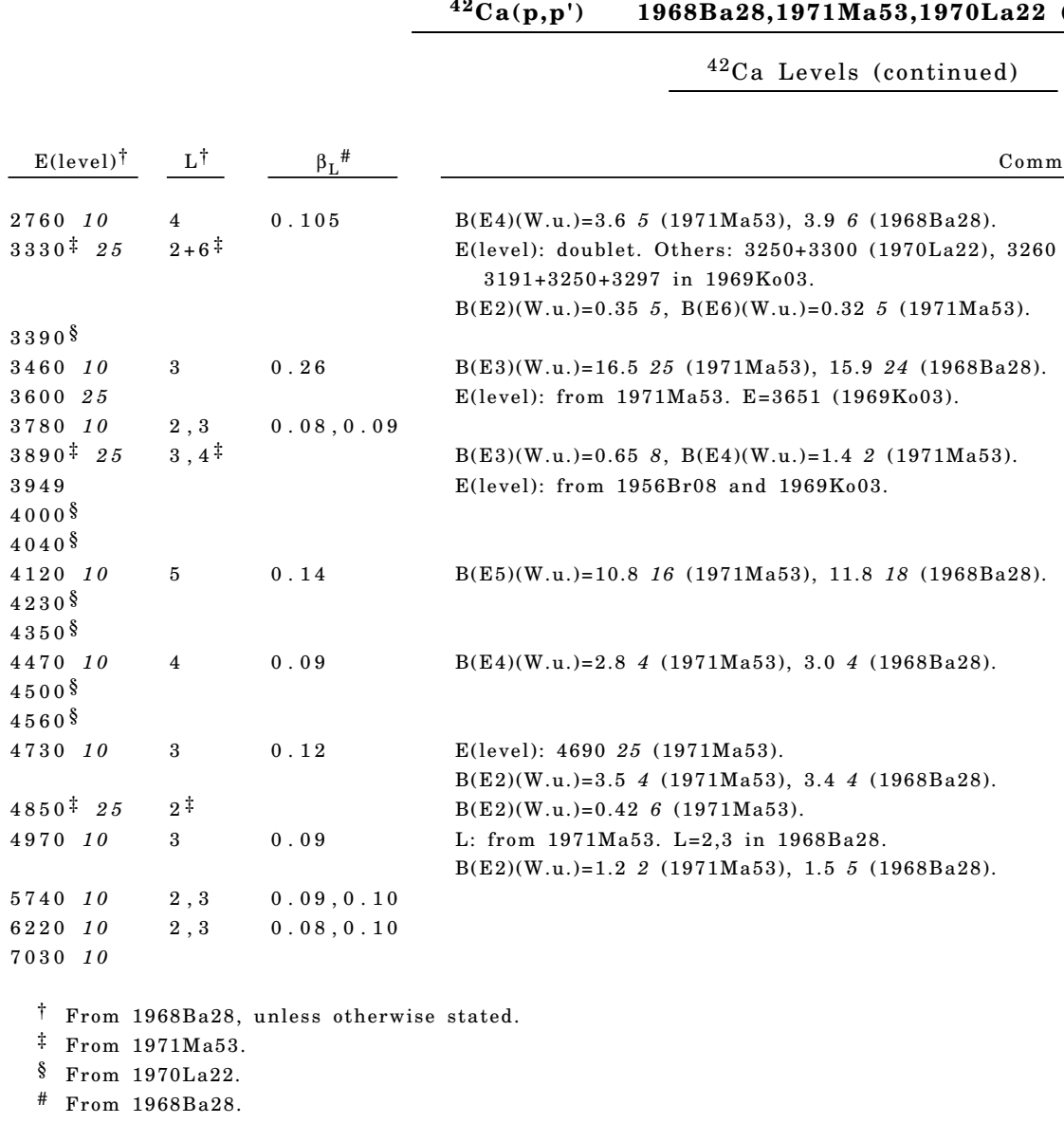

\section{${ }^{42} \mathrm{Ca}\left(p, p^{\prime} \gamma\right) \quad 1970 \mathrm{La22,1969Ko03}$}

See also ${ }^{42} \mathrm{Ca}\left(\mathrm{p}, \mathrm{p}^{\prime}\right)$.

1970La22: $\mathrm{E}=7.03 \mathrm{MeV}$ proton beam was produced at the University of Liverpool EN tandem accelerator. A target of 200 $\mu \mathrm{g} / \mathrm{cm}^{2}$ thick enriched ${ }^{42} \mathrm{Ca}$. Protons were detected in an annular silicon surface barrier detector and $\gamma$-rays were detected in NaI crystals. Measured E $\gamma, \mathrm{I} \gamma, \mathrm{E}(\mathrm{p}), \mathrm{p} \gamma(\theta)$. Deduced levels, J $\pi$, branching ratios, mixing ratios.

1969Ko03: $\mathrm{E}=7.8 \mathrm{MeV}$ proton beam was produced at the MIT cyclotron. A target of $1.19 \mathrm{mg} / \mathrm{cm}^{2} \mathrm{enriched}{ }^{42} \mathrm{Ca}(93.7 \%)$.

Scattered protons were detected by a solid state detector and $\gamma$-rays were detected with a $\mathrm{Ge}(\mathrm{Li})$ detector.

Measured E $\gamma$, I $\gamma$, lifetimes by Doppler-shift attenuation method. Deduced levels, branching ratios, transition strengths.

1969Sc31: $\mathrm{E}=6.5$ to $6.9 \mathrm{MeV}$. Measured $\mathrm{p} \gamma(\theta)$ for $\gamma$ rays from 2420 and 3666 levels.

1969Te05: E=12 MeV. Measured branching ratios from 3440, 3950, 4100 and 4420 levels.

1985Le17: $\mathrm{E}=5 \mathrm{MeV}$. Measured $\mathrm{p} \gamma$ coin, lifetime of 1836 level by electronic timing.

1976Ul01, 1961Be19 (also 1960Be07): E=4.4 MeV. Measured E0 electron-positron pairs and internal-conversion electrons corresponding to the g.s. E0 transition from $1840,0+$ state. 1971RoYQ: measured $\gamma$ rays.

1969Ho33: E=6.7, 7.7 MeV. Measured B(E1) ratios in decay of 3447, 3- level.

Others: 1985Ki07, 1984Pa15, 1984Pa16, $1982 \mathrm{Mi} 06$.

$$
{ }^{42} \mathrm{Ca} \text { Levels }
$$

\begin{tabular}{|c|c|c|}
\hline $\mathrm{E}(\text { level })^{\dagger}$ & $\mathrm{J} \pi \S$ & $\mathrm{T}_{1 / 2} \#$ \\
\hline 0.0 & $0+$ & \\
\hline 1523 & $2+$ & $1.11 \mathrm{ps} 21$ \\
\hline 1836 & $0+$ & $387 \mathrm{ps}^{@} 6$ \\
\hline 2422 & $2+$ & 0.21 ps 5 \\
\hline 2750 & $4+$ & $2.4 \mathrm{ps}+76-2$ \\
\hline
\end{tabular}


${ }^{42} \mathrm{Ca}\left(\mathrm{p}, \mathrm{p}^{\prime} \gamma\right) \quad 1970 \mathrm{La22}, 1969 \mathrm{Ko03}$ (continued)

${ }^{42} \mathrm{Ca}$ Levels (continued)

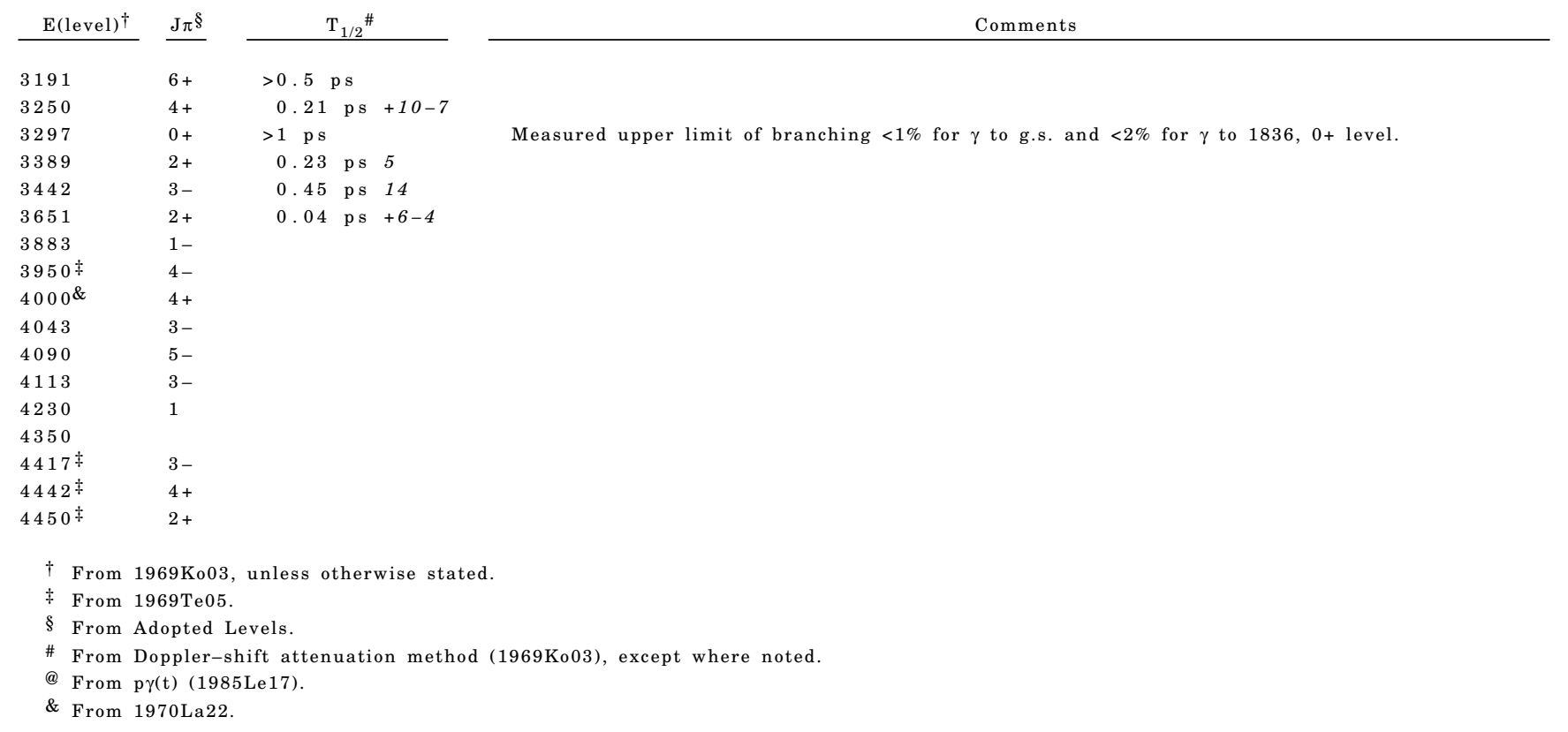

\& From 1970La22.

$\gamma\left({ }^{42} \mathrm{Ca}\right)$

$A_{2}$ and $A_{4}$ are from 1970La22.

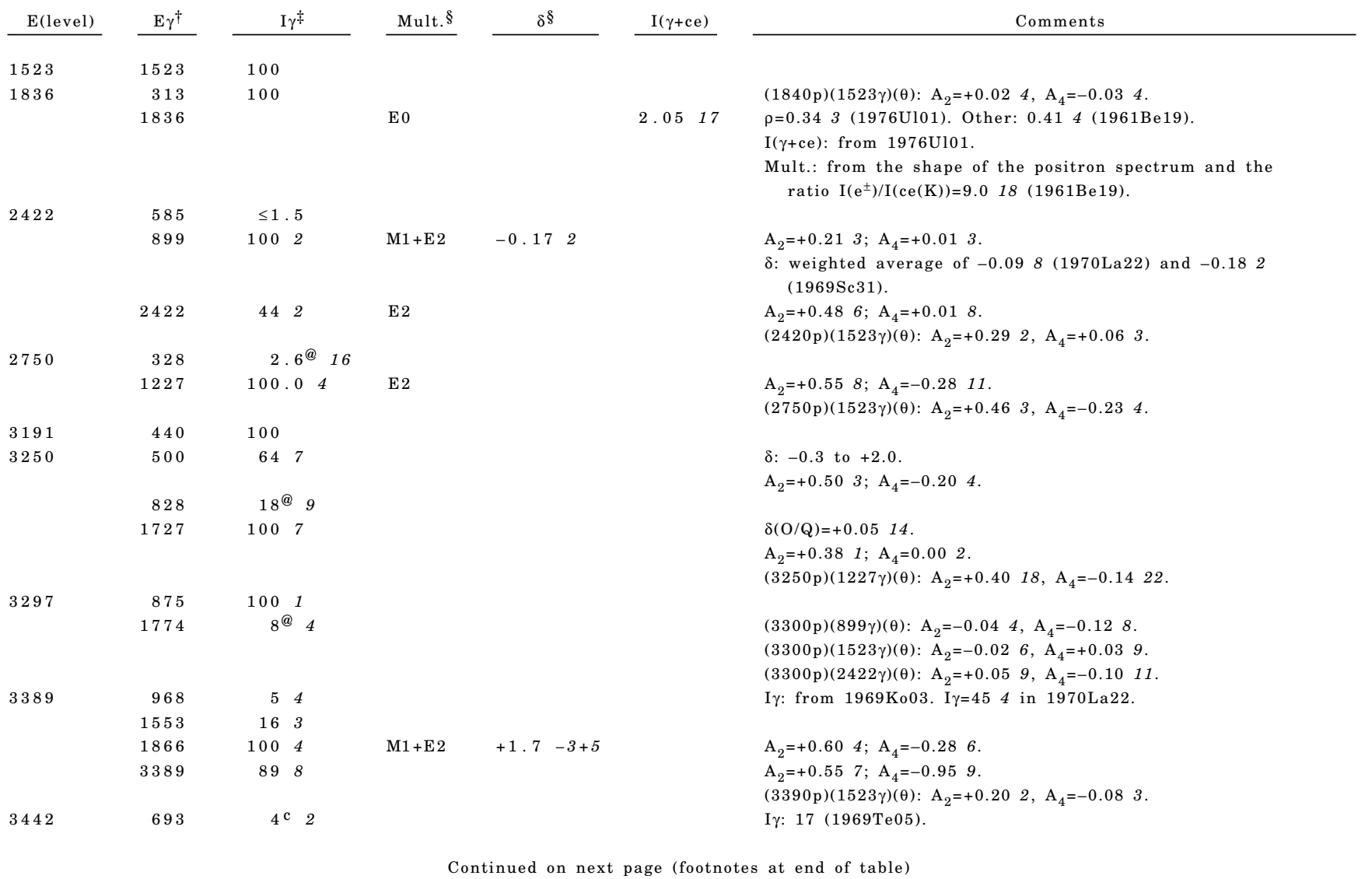




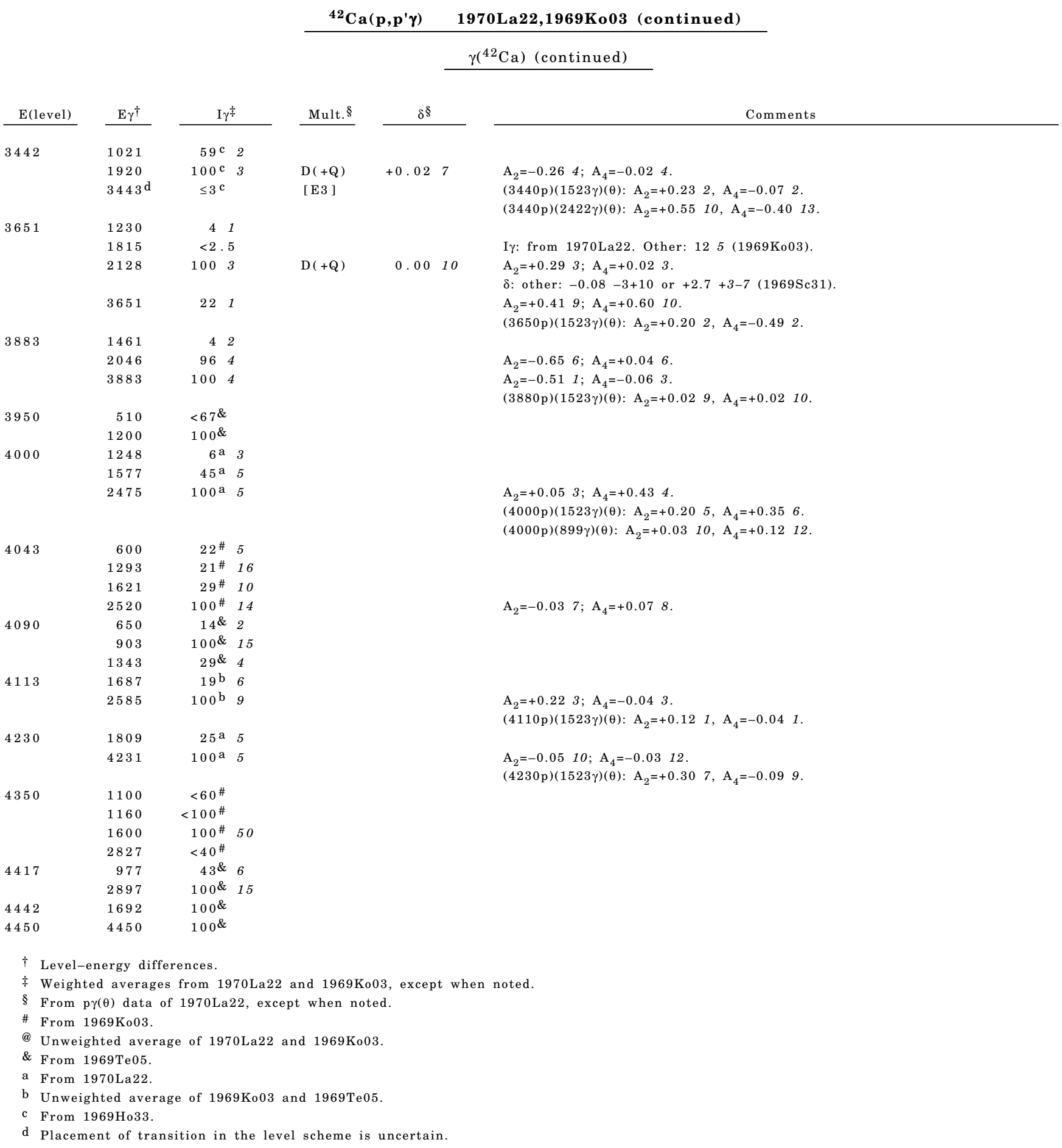




\section{${ }^{42} \mathrm{Ca}\left(\mathrm{d}, \mathrm{d}^{\prime}\right) \quad 1968 \mathrm{Ha} 31$}

1968Ha31: $\mathrm{E}=7.50 \mathrm{MeV}$ deuteron beam was produced from the ONR electrostatic generator. Target of $90.9 \%$ enriched ${ }^{42} \mathrm{Ca}$ on a carbon backing. Scattered particles were momentum analyzed with the MIT multi-range spectrograph and detected in nuclear emulsions. Measured $\sigma\left(\mathrm{E}_{\mathrm{d}}, \theta\right)$. Deduced levels, J $\pi$, L from the optical model analysis and distorted-wave (DW) analysis.

1968Be36: (d,d) E=7.0, 7.2 MeV. Measured $\sigma(\theta)$.

1970Br27: (d,d) E=12 MeV. Measured $\sigma(\theta)$. Deduced optical-model parameters.

1970Fi01: (d,d) E=11.8 MeV. Measured $\sigma(\theta)$. Deduced optical-model parameters.

\section{${ }^{42} \mathrm{Ca}$ Levels}

All data are from $1968 \mathrm{Ha} 31$.

\begin{tabular}{|c|c|c|c|}
\hline E(level) & $\mathrm{L}$ & $\mathrm{d} \sigma / \mathrm{d} \Omega(\max )(\mathrm{mb} / \mathrm{sr})$ & Comments \\
\hline 0.0 & 0 & & \\
\hline 15244 & 2 & 1.30 & $\beta_{2}=0.23$. \\
\hline 18355 & $(0)$ & 0.31 & \\
\hline $2423 \quad 6$ & & 0.20 & \\
\hline 27496 & & 0.10 & \\
\hline $3445 \quad 8$ & ( 3 ) & 0.25 & $\beta_{3}=0.17$ \\
\hline
\end{tabular}

${ }^{42} \mathrm{Ca}\left(\alpha, \alpha^{\prime}\right) \quad 1973 \mathrm{Ja} 17,1967 \mathrm{Li13}$

Includes $\left(\alpha, \alpha^{\prime} \gamma\right)$ from $1976 \mathrm{Br} 15$

Inelastic and elastic experiments: measured $\sigma(\theta)$ :

1973Ja17 (also 1973JaYN): $\mathrm{E}=28.5 \mathrm{MeV}$ alpha beam. A target of $\mathrm{CaCO}_{3}\left(94 \%\right.$ in ${ }^{42} \mathrm{Ca}, 90 \mu \mathrm{g} / \mathrm{cm}^{2}$ ) evaporated on a carbon foil. Scattered alpha particles were momentum analyzed with an Engel broad-range spectrograph and detected in nuclear emulsions, FWHM=25 keV. Measured $\sigma(\theta)$. Deduced levels, J $\pi$, L from DWBA analysis.

1967Li13: E=31 MeV alpha beam from the MIT cyclotron. A $1.19 \mathrm{mg} / \mathrm{cm}^{2}$ thick target of ${ }^{42} \mathrm{Ca}$ enriched to $93.7 \%$.

Scattered alpha particles were detected by a $500-\mu \mathrm{m}$ silicon surface barrier detector, FWHM=70 keV. Measured $\sigma(\theta)$.

Deduced levels, J $\pi$, L from DWBA analysis.

1966Pe16: E=42 MeV. Ten levels reported up to 5700 with $\mathrm{L}$ values for most of these levels which are in agreement with those from 1973Ja17 and $1967 \mathrm{Li} 13$.

1966Gr09: $\mathrm{E}=30.5 \mathrm{MeV}$.

1969Mo28: $\mathrm{E}=18-44 \mathrm{MeV}$

19720e01: $\mathrm{E}=24,29 \mathrm{MeV}$

1975Tr01: $\mathrm{E}=24-29 \mathrm{MeV}$.

1976Br15: $\left(\alpha, \alpha^{\prime} \gamma\right) \mathrm{E}=31 \mathrm{MeV}$. Measured lifetime of $3190,6+$ state by pulsed-beam electronic timing.

1979Ba14, 1977Al07: $\mathrm{E}=1.37 \mathrm{GeV}$.

1989Ai02: $\mathrm{E}=29.3-50.5 \mathrm{MeV}$.

Elastic scattering experiments: optical-model parameters deduced from $\sigma(\theta)$ measurements:

1980Gi02: $(\alpha, \alpha) \mathrm{E}=104 \mathrm{MeV}$.

1972St28: $(\alpha, \alpha) \mathrm{E}=40.7-72.3 \mathrm{MeV}$.

1971FuZT: $(\alpha, \alpha) \mathrm{E}=72,24 \mathrm{MeV}$

1970Fe02: $(\alpha, \alpha) \mathrm{E}=42 \mathrm{MeV}$.

1969Ga22: $(\alpha, \alpha) \mathrm{E}=18,22,24,29 \mathrm{MeV}$.

${ }^{42} \mathrm{Ca}$ Levels

\begin{tabular}{|c|c|c|c|c|}
\hline $\mathrm{E}(\text { level })^{\dagger}$ & $\mathrm{T}_{1 / 2}$ & $\mathrm{~L}^{\dagger}$ & $\beta_{\mathrm{L}}^{\S}$ & Comments \\
\hline $0.0 \ddagger$ & & $0 \neq$ & & \\
\hline 1520 \# & & $2 \ddagger$ & 0.19 & $\begin{array}{l}\beta_{2}=0.19(1975 \operatorname{Tr} 01), 0.16(1969 \mathrm{Mo} 28), 0.103 \text { (1966Gr09). } \\
B(\mathrm{E} 2)(\text { W.u. })=10.015 \text { (1967Li13). }\end{array}$ \\
\hline 1840 末 & & $0 \neq$ & & \\
\hline 2420 ま & & $2 \neq$ & 0.072 & $\begin{array}{l}\beta_{2}=0.092(1975 \operatorname{Tr} 01) \\
\mathrm{B}(\mathrm{E} 2)(\text { W.u. })=1.52(1967 \mathrm{Li} 13)\end{array}$ \\
\hline $2750 \quad 5$ & & 4 & 0.067 & $\begin{array}{l}\beta_{4}=0.107 \quad(1975 \operatorname{Tr} 01), 0.049(1969 \mathrm{Mo} 28) \\
B(\mathrm{E} 4)(\text { W.u. })=1.42(1967 \mathrm{Li} 13)\end{array}$ \\
\hline 31885 & $5.27 \mathrm{~ns} 14$ & 6 & & $\mathrm{~T}_{1 / 2}$ : from pulsed-beam electronic timing (1976Br15). \\
\hline $3250 \quad 5$ & & 4 & & \\
\hline $3299 \quad 5$ & & $(0)$ & & \\
\hline $3390 \quad 5$ & & 2 & & \\
\hline $3446 \quad 5$ & & 3 & 0.30 & $\beta_{3}$ from $1975 \operatorname{Tr} 01$ \\
\hline 3653 & & 2 & 0.051 & B(E2)(W.u.)=0.75 11 (1967Li13). \\
\hline
\end{tabular}


${ }^{42} \mathrm{Ca}\left(\alpha, \alpha^{\prime}\right) \quad 1973 J a 17,1967 L i 13$ (continued)

${ }^{42} \mathrm{Ca}$ Levels (continued)

\begin{tabular}{|c|c|c|c|}
\hline E(level) ${ }^{\dagger}$ & $\mathrm{L}^{\dagger}$ & $\beta_{L} \S$ & \\
\hline 38915 & 3 & & \\
\hline $4046 \quad 5$ & & & \\
\hline $4100 \quad 5$ & 5 & & \\
\hline $4446 \quad 5$ & 4 & 0.077 & $\mathrm{~B}(\mathrm{E} 4)(\mathrm{W} . \mathrm{u})=.1.84(1967 \mathrm{Li} 13)$ \\
\hline $4686 \quad 5$ & 3 & & \\
\hline $4760 \quad 5$ & ( 2 ) & & \\
\hline $4865 \quad 5$ & 2 & & \\
\hline $4902 \quad 5$ & 3 & & \\
\hline $4972 \quad 5$ & 3 & & \\
\hline 50175 & $(3,4)$ & & \\
\hline $5156 \quad 5$ & 3 & & \\
\hline $5205 \quad 5$ & 2 & & \\
\hline $5375 \quad 5$ & & & \\
\hline $5400 \ddagger$ & $(4) \ddagger$ & 0.036 & $\mathrm{~B}(\mathrm{E} 4)(\mathrm{W} . \mathrm{u})=$.0.41 (1967Li13). \\
\hline $5494 \quad 5$ & 3 & & \\
\hline $5527 \quad 5$ & 3 & & L: from $1967 \operatorname{Li} 13$. \\
\hline 55915 & ( 3 ) & & \\
\hline 56235 & ( 3 ) & & \\
\hline $5667 \quad 5$ & 3 & & \\
\hline $5693 \quad 5$ & $(4,5)$ & & \\
\hline $5720 \quad 5$ & ( 4 ) & & \\
\hline $5794 \quad 5$ & 3 & & \\
\hline $5866 \quad 5$ & 2 & & \\
\hline $5924 \quad 5$ & & & \\
\hline $6017 \quad 5$ & 2 & & \\
\hline $6050 \neq$ & $(3) \neq$ & & \\
\hline $6106 \quad 5$ & $(4)$ & & \\
\hline 61545 & 3 & & \\
\hline $6192 \quad 5$ & & & \\
\hline 62395 & 3 & & \\
\hline 6510 末 & $(4) \neq$ & 0.053 & $\mathrm{~B}(\mathrm{E} 4)(\mathrm{W} . \mathrm{u})=.0.92(1967 \mathrm{Li} 13)$. \\
\hline $6630^{\ddagger}$ & $(4) \neq$ & 0.048 & $\mathrm{~B}(\mathrm{E} 4)(\mathrm{W} . \mathrm{u})=.0.72(1967 \mathrm{Li} 13)$. \\
\hline 6950 & & & E(level): from 1967Li13. \\
\hline
\end{tabular}

$\dagger$ From 1973Ja17, unless otherwise stated.

$\ddagger$ From 1967Li13.

$\S$ From 1967Li13. 
Target ${ }^{42} \mathrm{Ca}$ g.s. J $\pi=0+$.

1982Re03: E=60 MeV ${ }^{16} \mathrm{O}$ beam was produced at the Argonne FN tandem. Target of 93.7\% enriched ${ }^{42} \mathrm{Ca}$. Outgoing particles were momentum analyzed with the Argonne split-pole magnetic spectrograph and detected in a position-sensitive ionization chamber in the focal plane. Measured $\sigma\left(\mathrm{E}\left({ }^{16} \mathrm{O}, \theta\right)\right.$. Deduced levels, $J \pi$, L, deformation lengths, $\mathrm{B}(\lambda)$ from DWBA analysis and coupled-channels analysis.

\section{${ }^{42} \mathrm{Ca} \mathrm{Levels}$}

\begin{tabular}{|c|c|c|}
\hline $\mathrm{E}(\text { level })^{\dagger}$ & $\mathrm{L}^{\ddagger}$ & Comments \\
\hline \multicolumn{3}{|l|}{0.0} \\
\hline 1525 & 2 & \\
\hline \multicolumn{3}{|l|}{1837} \\
\hline 2424 & 2 & \\
\hline 2752 & 4 & $\mathrm{~B}(\mathrm{E} 4) \uparrow=1.18 \times 10^{-4}$. \\
\hline \multicolumn{3}{|l|}{3254} \\
\hline 3447 & 3 & \\
\hline 4100 & 5 & \\
\hline 4449 & 2 & $\mathrm{~B}(\mathrm{E} 2) \uparrow=6.07 \times 10^{-3}$. \\
\hline 4690 & 3 & $\mathrm{~B}(\mathrm{E} 3) \uparrow=1.68 \times 10^{-3}$. \\
\hline 4971 & 3 & $\mathrm{~B}(\mathrm{E} 3) \uparrow=1.13 \times 10^{-3}$. \\
\hline
\end{tabular}

\section{Coulomb Excitation 2013Ha06,1973To07}

2013Ha06: $\left({ }^{42} \mathrm{Ca},{ }^{42} \mathrm{Ca} \gamma\right) \mathrm{E}=170 \mathrm{MeV}{ }^{42} \mathrm{Ca}$ beam was produced at the Laboratori Nazionali di Legnaro. A $1 \mathrm{mg} / \mathrm{cm}^{2} 208 \mathrm{~Pb}$ target. $\gamma$-rays were detected with the $\gamma$-ray spectrometer AGATA and charged particles were detected with the DANTE array. Measured E $\gamma$, I $\gamma, \gamma \gamma-c o i n$. Deduced levels, deformation parameters.

$1973 \mathrm{To} 07$ (also 1972To01): $\left({ }^{32} \mathrm{~S},{ }^{32} \mathrm{~S}^{\prime} \gamma\right) \mathrm{E}=60 \mathrm{MeV}$. Measured static quadrupole moment by reorientation method, $\gamma\left({ }^{32} \mathrm{~S}\right)(\theta)$.

${ }^{42} \mathrm{Ca}$ Levels

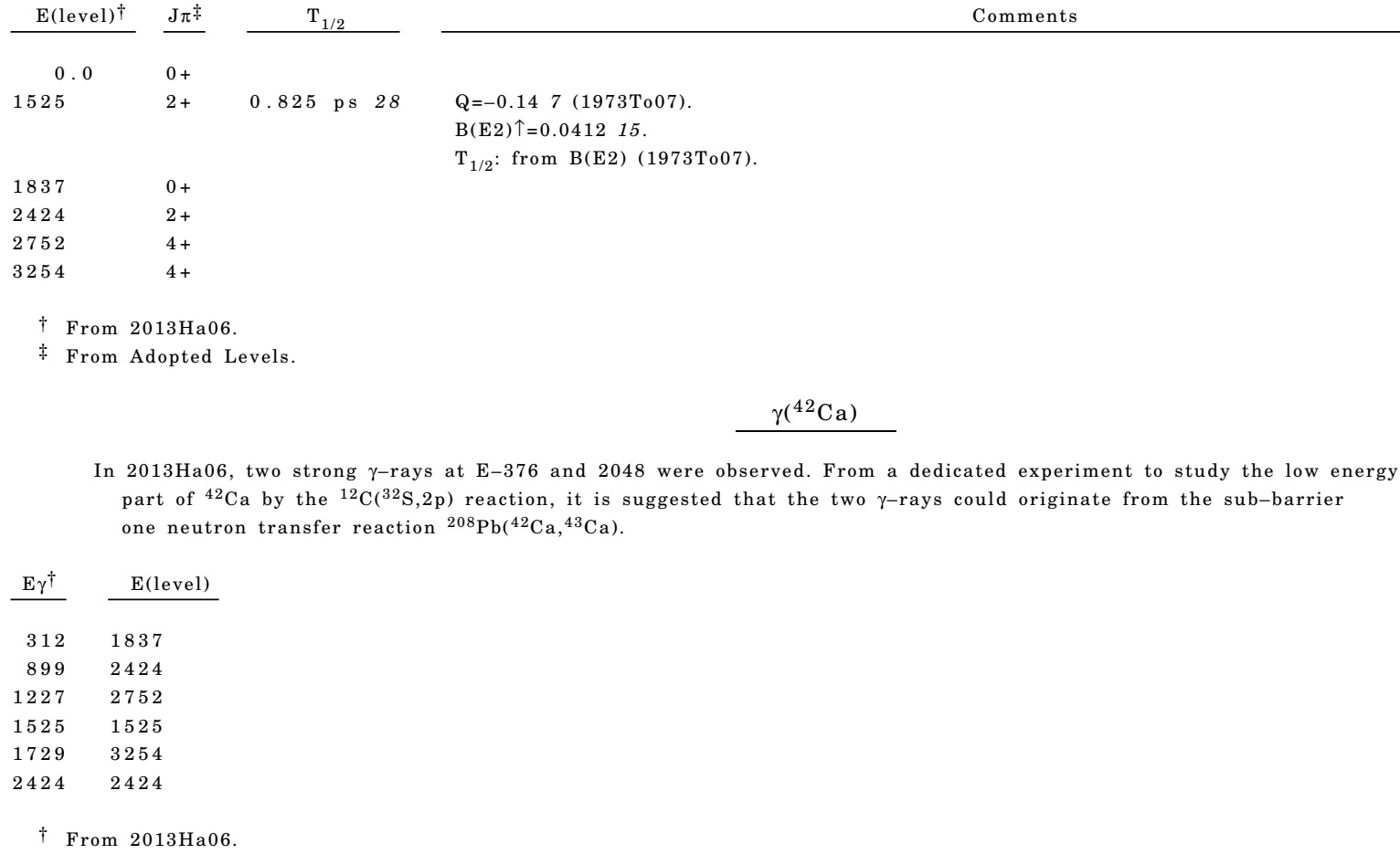

In $2013 \mathrm{Ha} 06$, two strong $\gamma$-rays at E-376 and 2048 were observed. From a dedicated experiment to study the low energy part of ${ }^{42} \mathrm{Ca}$ by the ${ }^{12} \mathrm{C}\left({ }^{32} \mathrm{~S}, 2 \mathrm{p}\right)$ reaction, it is suggested that the two $\gamma$-rays could originate from the sub-barrier one neutron transfer reaction ${ }^{208} \mathrm{~Pb}\left({ }^{42} \mathrm{Ca},{ }^{43} \mathrm{Ca}\right)$.

\begin{tabular}{|c|c|}
\hline $\mathrm{E} \gamma^{\dagger}$ & E(level) \\
\hline 312 & 1837 \\
\hline 899 & 2424 \\
\hline 1227 & 2752 \\
\hline 1525 & 1525 \\
\hline 1729 & 3254 \\
\hline 2424 & 2424 \\
\hline
\end{tabular}

$\dagger$ From $2013 \mathrm{Ha} 06$. 


\section{${ }^{43} \mathrm{Ca}(\mathrm{p}, \mathrm{d}) \quad 1973 \mathrm{Du} 02$}

Target ${ }^{43} \mathrm{Ca}$ g.s. J $\pi=7 / 2-$.

1973Du02: $\mathrm{E}=40 \mathrm{MeV}$ proton beam was produced at the Grenoble variable-energy cyclotron. Target of $1 \mathrm{mg} / \mathrm{cm}^{2}$ self-supported calcium. Reaction products were detected in a $\Delta \mathrm{E}-\mathrm{E}$ silicon counter telescope and a anti-coincidence counter, FWHM=80-100 keV. Measured $\sigma(\theta)$. Deduced levels, J $\pi$, L, spectroscopic factors from DWBA analysis.

\begin{tabular}{|c|c|c|c|c|c|c|c|c|}
\hline \multirow[b]{2}{*}{$\mathrm{E}$ (level) } & \multirow[b]{2}{*}{$\mathrm{L}$} & \multirow[b]{2}{*}{$\mathrm{C}^{2} \mathrm{~s}$} & \multirow[b]{2}{*}{ E(level) } & \multicolumn{2}{|c|}{${ }^{42} \mathrm{Ca}$ Levels } & \multirow[b]{2}{*}{ E(level) } & \multirow[b]{2}{*}{$\mathrm{L}$} & \multirow[b]{2}{*}{$\mathrm{C}^{2} \mathrm{~s}$} \\
\hline & & & & $\mathrm{L}$ & $\mathrm{C}^{2} \mathrm{~s}$ & & & \\
\hline 0.0 & 3 & 0.5 & 4100 & 2 & 0.24 & 9750 & 2 & $0.045 \S \quad 15$ \\
\hline 1520 & 3 & 0.15 & 4400 & 2 & 0.29 & 9850 & 2 & 0.10 \\
\hline 1840 & 3 & 0.04 & 4700 & $0+2$ & $0.06,0.045$ & 10010 & 2 & 0.17 \\
\hline 2420 & 3 & 0.11 & 4900 & 2 & 0.33 & 10430 & 2 & 0.23 \\
\hline 2750 & 3 & 0.42 & 5340 & 2 & 0.10 & 10970 & 0 & 0.10 \\
\hline 3200 & 3 & $0.66 \ddagger$ & 5790 & $0+2$ & $0.057,0.07$ & 11440 & 0 & 0.07 \\
\hline 3440 & $0+2$ & $0.18,0.20$ & 6030 & $(0+2)$ & $0.09,0.11$ & & & \\
\hline $3650^{\dagger}$ & ( 1 ) & & 6500 & $0+2$ & $0.12,0.07$ & & & \\
\hline
\end{tabular}

$\dagger$ Obscured by ${ }^{41} \mathrm{Ca}$ (g.s.).

¥ Corrected for ${ }^{43} \mathrm{Ca}$ contamination.

$\S$ Quoted (1973Du02) as >0.03, <0.06.

$\mathrm{J} \pi\left({ }^{43} \mathrm{Ca}\right.$ g.s. $\left.=7 / 2-\right)$.

1973Ja17 (also 1973JaYN): $\mathrm{E}=20 \mathrm{MeV}$ deuteron beam. A target of $\mathrm{CaCO}_{3}$ evaporated on a carbon foil, $81 \%$ enriched to ${ }^{43} \mathrm{Ca}, 60 \mu \mathrm{g} / \mathrm{cm}^{2}$ thick. Reaction products were momentum analyzed with an Enge broad-range spectrograph and detected in nuclear emulsions, FWHM=10-14 keV. Measured $\sigma\left(E_{t}, \theta\right)$. Deduced levels, J $\pi$, L, spectroscopic factors from DWBA analysis.

1969Yn01: E=22.6 MeV. FWHM=70 keV. Measured $\sigma(\theta)$, DWBA analysis. A total of 20 groups observed. All groups were assigned pure L transfers.

1964Bj02: E=8.522 MeV. FWHM $\approx 15 \mathrm{keV}$. Measured $\sigma(\theta)$, DWBA analysis. Five groups reported at $0,1532,2435,2764$ and 3195 .

The following groups reported by $1969 \mathrm{Yn} 01$ cannot be reconciled with those from 1973Ja17: $3580(\mathrm{~L}=0), 3780(\mathrm{~L}=1)$, $3920(\mathrm{~L}=2), 4230(\mathrm{~L}=2), 4310(\mathrm{~L}=2), 4560(\mathrm{~L}=(3)), 5670 \quad(\mathrm{~L}=0), 5870 \quad(\mathrm{~L}=(0))$.

\section{${ }^{42} \mathrm{Ca}$ Levels}

\begin{tabular}{|c|c|c|c|c|}
\hline \multicolumn{2}{|c|}{$\mathrm{E}(\text { level })^{\dagger}$} & \multirow{2}{*}{$\frac{\mathrm{L}^{\dagger}}{3}$} & \multicolumn{2}{|c|}{$\mathrm{C}^{2} \mathrm{~S}^{\dagger}$} \\
\hline 0 . & 0 & & 0.46 & 1 \\
\hline 1523 & 5 & 3 & 0.19 & 1 \\
\hline 1836 & 5 & 3 & 0.04 & \\
\hline 2425 & 5 & $3(+1)$ & 0.16 & 1 \\
\hline 2750 & 5 & $3(+1)$ & 0.59 & 2 \\
\hline 3188 & 5 & 3 & 0.87 & 3 \\
\hline 3253 & 5 & $3(+1)$ & 0.08 & 1 \\
\hline \multicolumn{5}{|l|}{$3297 ?$} \\
\hline 3393 & 5 & $1(+3)$ & 0.003 & \\
\hline 3446 & 5 & $0+2$ & 0.09 & 1 \\
\hline 3656 & 5 & 3 & 0.06 & 1 \\
\hline 3890 & 5 & $2(+0)$ & 0.02 , & 0.002 \\
\hline 3956 & 5 & $0(+2)$ & 0.002 &, 0.01 \\
\hline 4043 & 5 & $0+2$ & 0.01 , & 0.01 \\
\hline 4099 & 5 & 2 & 0.30 & 1 \\
\hline 4355 & 5 & 2 & 0.29 & 2 \\
\hline 4421 & 5 & 2 & 0.09 & 1 \\
\hline 4447 & 5 & $1+3$ & 0.02 & \\
\hline 4690 & 5 & $0+2$ & 0.04 & \\
\hline
\end{tabular}

Comments

$\mathrm{C}^{2} \mathrm{~S}: 0.50$ (1969Yn01), 0.75 (1964Bj02).

$\mathrm{C}^{2} \mathrm{~S}: 0.16$ (1969Yn01), 0.21 (1964Bj02).

$\mathrm{C}^{2} \mathrm{~S}: 0.05$ (1969Yn01).

$\mathrm{C}^{2} \mathrm{~S}$ : for $\mathrm{L}=3$. $\mathrm{C}^{2} \mathrm{~S}=0.004$ for $\mathrm{L}=1$.

$\mathrm{C}^{2} \mathrm{~S}$ : 0.11 (1969Yn01) for $\mathrm{L}=3,0.21$ (1964Bj02).

$\mathrm{C}^{2} \mathrm{~S}$ : for $\mathrm{L}=3$. $\mathrm{C}^{2} \mathrm{~S}=0.01$ for $\mathrm{L}=1$.

$\mathrm{C}^{2} \mathrm{~S}$ : 0.47 (1969Yn01) for $\mathrm{L}=3,0.75$ (1964Bj02).

$\mathrm{C}^{2} \mathrm{~S}$ : $20 \%$ of the observed strength due to ${ }^{43} \mathrm{Ca}$ g.s. from ${ }^{44} \mathrm{Ca}(\mathrm{d}, \mathrm{t})$ has been subtracted.

$\mathrm{C}^{2} \mathrm{~S}$ : 1.0 (1969Yn01), $1.08(1964 \mathrm{Bj} 02)$.

$\mathrm{C}^{2} \mathrm{~S}$ : for $\mathrm{L}=3$. $\mathrm{C}^{2} \mathrm{~S}=0.001$ for $\mathrm{L}=1$.

Very weakly excited.

$\mathrm{C}^{2} \mathrm{~S}$ : for $\mathrm{L}=1$. $\mathrm{C}^{2} \mathrm{~S}=0.011$ for $\mathrm{L}=3$.

$\mathrm{C}^{2} \mathrm{~S}$ : for $\mathrm{L}=0$. $\mathrm{C}^{2} \mathrm{~S}=0.142$ for $\mathrm{L}=2$.

$\mathrm{C}^{2} \mathrm{~S}$ : 0.09 (1969Yn01) for $\mathrm{L}=0$.

$\mathrm{C}^{2} \mathrm{~S}: 0.25$ (1969Yn01) for $\mathrm{L}=2,4020$ group

$\mathrm{C}^{2} \mathrm{~S}$ : 0.23 (1969Yn01) for $\mathrm{L}=2,4430$ group.

$\mathrm{C}^{2} \mathrm{~S}$ : for $\mathrm{L}=1 \mathrm{C}^{2} \mathrm{~S}=0.041$ for $\mathrm{L}=3$.

$\mathrm{C}^{2} \mathrm{~S}$ : for $\mathrm{L}=0 . \mathrm{C}^{2} \mathrm{~S}=0.061$ for $\mathrm{L}=2$.

$\mathrm{C}^{2} \mathrm{~S}$ : 0.04 (1969Yn01) for $\mathrm{L}=0,4720$ group. 
${ }^{43} \mathrm{Ca}(\mathrm{d}, \mathrm{t}) \quad 1973 \mathrm{Ja} 17$ (continued)

${ }^{42} \mathrm{Ca}$ Levels (continued)

\begin{tabular}{|c|c|c|c|c|}
\hline \multicolumn{2}{|c|}{$\mathrm{E}(\text { level })^{\dagger}$} & \multirow{2}{*}{$\frac{\mathrm{L}^{\dagger}}{2(+0)}$} & $\mathrm{C}^{2} \mathrm{~S}^{\dagger}$ & \\
\hline 4895 & 5 & & $0.16 \quad 3$ & $\mathrm{C}^{2} \mathrm{~S}$ : for $\mathrm{L}=2 . \mathrm{C}^{2} \mathrm{~S}=0.021$ for $\mathrm{L}=0$. \\
\hline 4966 & 5 & ( 2 ) & $0.18 \quad 1$ & $\begin{array}{l}\text { E(level): observed only at backward angles. } \\
\mathrm{C}^{2} \mathrm{~S}: 0.29(1969 \mathrm{Yn} 01) \text { for } \mathrm{L}=2,4970 \text { group. }\end{array}$ \\
\hline 5012 & 5 & $(1+3)$ & $0.08 \quad 2$ & $\mathrm{C}^{2} \mathrm{~S}$ : for $\mathrm{L}=3 . \mathrm{C}^{2} \mathrm{~S}=0.003$ for $\mathrm{L}=1$ \\
\hline 5189 & 5 & 3 & 0.131 & \\
\hline 5204 & 5 & $1+3$ & $0.08 \quad 2$ & $\mathrm{C}^{2} \mathrm{~S}$ : for $\mathrm{L}=3 . \mathrm{C}^{2} \mathrm{~S}=0.01$ for $\mathrm{L}=1$. \\
\hline 5321 & 5 & $0+2$ & 0.122 & $\mathrm{C}^{2} \mathrm{~S}$ : for $\mathrm{L}=2 . \mathrm{C}^{2} \mathrm{~S}=0.02$ for $\mathrm{L}=0$ \\
\hline 5377 & 5 & 2 & $0.05 \quad 1$ & $\mathrm{C}^{2} \mathrm{~S}: 0.17$ (1969Yn01) for $\mathrm{L}=2,5370$ group. \\
\hline 5403 & 5 & $2(+0)$ & $0.05 \quad 1$ & $\mathrm{C}^{2} \mathrm{~S}$ : for $\mathrm{L}=2 . \mathrm{C}^{2} \mathrm{~S}=0.004$ for $\mathrm{L}=0$ \\
\hline 5436 & 5 & $0(+2)$ & 0.01 & $\mathrm{C}^{2} \mathrm{~S}$ : for $\mathrm{L}=0 . \mathrm{C}^{2} \mathrm{~S}=0.021$ for $\mathrm{L}=2$. \\
\hline 5467 & 5 & $1+3$ & $0.01,0.04$ & \\
\hline 5488 & 5 & $3(+1)$ & $0.03,0.001$ & \\
\hline 5590 & 5 & $0+2$ & $0.08 \quad 1$ & $\mathrm{C}^{2} \mathrm{~S}$ : for $\mathrm{L}=0 . \mathrm{C}^{2} \mathrm{~S}=0.081$ for $\mathrm{L}=2$. \\
\hline 5767 & 5 & 2 & 0.172 & \\
\hline 5790 & 5 & 3 & 0.162 & \\
\hline 5923 & 5 & 2 & $0.06 \quad 1$ & \\
\hline 5985 & 5 & $0(+2)$ & 0.02 & $\mathrm{C}^{2} \mathrm{~S}$ : for $\mathrm{L}=0$. $\mathrm{C}^{2} \mathrm{~S}=0.031$ for $\mathrm{L}=2$. \\
\hline 5997 & 5 & $0(+2)$ & 0.03 & $\mathrm{C}^{2} \mathrm{~S}$ : for $\mathrm{L}=0 . \mathrm{C}^{2} \mathrm{~S}=0.021$ for $\mathrm{L}=2$. \\
\hline 6034 & 5 & $2(+0)$ & $0.04 \quad 1$ & $\mathrm{C}^{2} \mathrm{~S}$ : for $\mathrm{L}=2 . \mathrm{C}^{2} \mathrm{~S}=0.004$ for $\mathrm{L}=0$ \\
\hline 6098 & 5 & 2 & $0.06 \quad 1$ & \\
\hline 6162 & 5 & $(0+2)$ & $0.02 \quad 1$ & $C^{2} S$ : for $L=2 . C^{2} S=0.01$ for $L=0$ \\
\hline 6207 & 5 & $0(+2)$ & 0.05 & $\mathrm{C}^{2} \mathrm{~S}$ : for $\mathrm{L}=0$. $\mathrm{C}^{2} \mathrm{~S}=0.041$ for $\mathrm{L}=2$. \\
\hline 6235 & 5 & $0+2$ & $0.05 \quad 1$ & $\mathrm{C}^{2} \mathrm{~S}$ : for $\mathrm{L}=2 . \mathrm{C}^{2} \mathrm{~S}=0.002$ for $\mathrm{L}=0$ \\
\hline
\end{tabular}


$J \pi\left({ }^{43} \mathrm{Ca}\right.$ g.s. $)=7 / 2-$.

1969Ly02 (also 1967LyZY,1968Ly02): E=18 MeV ${ }^{3} \mathrm{He}$ beam was produced at the Heidelberg tandem. Targets made by evaporating $\mathrm{CaCO}_{3}$ onto a carbon backing. Reaction products were momentum analyzed with a broad-range magnetic spectrograph. Measured $\sigma\left(\mathrm{E}_{\alpha}, \theta\right)$. Deduced levels, J $\pi$, L and spectroscopic factors from DWBA analysis.

\begin{tabular}{|c|c|c|}
\hline \multicolumn{3}{|c|}{ Cross section data } \\
\hline Energy & $\begin{array}{r}\mathrm{d} \sigma / \mathrm{d} \Omega(\mathrm{n} \\
(\mathrm{mb} / \mathrm{s}\end{array}$ & $\begin{array}{l}\text { ax ) } \\
\text { r) }\end{array}$ \\
\hline 0 & 1.27 & 5 \\
\hline 1530 & 1.30 & 5 \\
\hline 1840 & 0.21 & 5 \\
\hline 2430 & 0.57 & 5 \\
\hline 2777 & 2.04 & 5 \\
\hline 3210 & 4.56 & 5 \\
\hline 3460 & 0.38 & 5 \\
\hline 3650 & 0.15 & 5 \\
\hline 4100 & 0.49 & 10 \\
\hline 4180 & 0.18 & 5 \\
\hline 4360 & 0.45 & 7 \\
\hline 4440 & 0.29 & 5 \\
\hline 4700 & 0.07 & 5 \\
\hline 4920 & 0.81 & 5 \\
\hline 5200 & 0.25 & 7 \\
\hline 5340 & 0.52 & 5 \\
\hline 5410 & 0.14 & 5 \\
\hline 5610 & 0.56 & 5 \\
\hline 5680 & 0.11 & 12 \\
\hline 5790 & 0.48 & 5 \\
\hline 6030 & 0.35 & 5 \\
\hline 6220 & 0.67 & 5 \\
\hline 6330 & 0.18 & 10 \\
\hline 6420 & 0.17 & 5 \\
\hline 6510 & 0.46 & 5 \\
\hline 6570 & 0.11 & 7 \\
\hline 6660 & 0.32 & 12 \\
\hline 6790 & 0.17 & 5 \\
\hline 7040 & 0.22 & 5 \\
\hline 7430 & 0.39 & 5 \\
\hline 7560 & 0.27 & 10 \\
\hline 8170 & 0.14 & 12 \\
\hline 8260 & 0.14 & 10 \\
\hline 8330 & 0.08 & 10 \\
\hline 8410 & 0.21 & 5 \\
\hline 8520 & 0.21 & 5 \\
\hline 8600 & 0.14 & 5 \\
\hline 8850 & 0.14 & 15 \\
\hline 9740 & 0.45 & 15 \\
\hline 9850 & 0.66 & 15 \\
\hline 10010 & 0.53 & 20 \\
\hline 10430 & 1.68 & 5 \\
\hline 10510 & 0.38 & 5 \\
\hline 10610 & 0.29 & 5 \\
\hline 10970 & 1.19 & 20 \\
\hline 11440 & 1.96 & 5 \\
\hline
\end{tabular}

${ }^{42} \mathrm{Ca}$ Levels

Spectroscopic factor $\left.\mathrm{C}^{2} \mathrm{~S}=(1 / \mathrm{N})\left[\sigma(\theta)^{\exp / \sigma(\theta)}\right)^{\mathrm{DWBA}}\right]$, where $\mathrm{n}$ is the normalization factor.

$J \pi$ values implied by $\mathrm{L}-$ transfers are: $3-, 4-$ for $\mathrm{L}=0$ or $\mathrm{L}=0+2 ; 2+$ to $5+$ for $\mathrm{L}=1 ; 1-$ to 6 - for $\mathrm{L}=2 ; 0+$ to $7+$ for $\mathrm{L}=3$.

\begin{tabular}{|c|c|c|}
\hline E(level) & $\mathrm{L}$ & $\mathrm{C}^{2} \mathrm{~S}$ \\
\hline 0.0 & 3 & 0.57 \\
\hline $1530 \quad 20$ & 3 & 0.17 \\
\hline $1840 \quad 20$ & 3 & 0.05 \\
\hline $2430 \quad 20$ & 3 & 0.18 \\
\hline $2770 \quad 20$ & 3 & 0.59 \\
\hline
\end{tabular}


${ }^{43} \mathrm{Ca}\left({ }^{3} \mathrm{He}, \alpha\right) \quad 1969 \mathrm{Ly} 02$ (continued)

\begin{tabular}{|c|c|c|c|c|c|c|c|c|c|}
\hline E(level & & $\mathrm{L}$ & $\mathrm{C}^{2} \mathrm{~S}$ & E(level) & $\mathrm{L}$ & $\mathrm{C}^{2} \mathrm{~S}$ & E(level) & $\mathrm{L}$ & $\mathrm{C}^{2} \mathrm{~S}$ \\
\hline $3210 \quad 2$ & 20 & 3 & 0.94 & $5790 \quad 20$ & $(0+2)$ & & $8330 \quad 20$ & & \\
\hline 34602 & 20 & $(0+2)$ & $0.48,0.05$ & $6030 \quad 20$ & & & $8410 \quad 20$ & & \\
\hline 36502 & 20 & (1) & 0.01 & $6220 \quad 20$ & $(0+2)$ & & $8520 \quad 20$ & & \\
\hline $4100 \quad 2$ & 20 & (2) & 0.43 & $6330 \quad 20$ & & & $8600 \quad 20$ & & \\
\hline $4180 \quad 2$ & 20 & (3) & 0.05 & $6420 \quad 20$ & & & $8850 \quad 20$ & & \\
\hline $4360 \quad 2$ & 20 & ( 3 ) & 0.12 & $6510 \quad 20$ & & & $9740 \quad 20$ & 2 & 0.32 \\
\hline 44402 & 20 & $(0+2)$ & $0.38,0.04$ & $6570 \quad 20$ & & & $9850 \quad 20$ & 2 & 0.43 \\
\hline 47002 & 20 & & & $6660 \quad 20$ & & & 1001020 & 2 & 0.50 \\
\hline 49202 & 20 & ( 3 ) & & $6790 \quad 20$ & & & 1043020 & 2 & 0.61 \\
\hline $5200 \quad 2$ & 20 & ( 3 ) & & $7040 \quad 20$ & & & 1051020 & & \\
\hline 53402 & 20 & $(0+2)$ & & $7430 \quad 20$ & & & 1061020 & & \\
\hline 54102 & 20 & $(0+2)$ & & $7560 \quad 20$ & & & $10970 \quad 20$ & & \\
\hline 56102 & 20 & $(0+2)$ & & $8170 \quad 20$ & & & 1144020 & 0 & 0.23 \\
\hline $5680 \quad 2$ & 20 & & & $8260 \quad 20$ & & & & & \\
\hline
\end{tabular}




\section{${ }^{44} \mathrm{Ca}(\mathrm{p}, \mathrm{t}) \quad 1977 \mathrm{SaZF}, 1973 \mathrm{Du} 02$}

1977SaZF: E=40 MeV. Measured $\sigma(\theta)$. Deduced levels, J $\pi$, L from DWBA analysis.

1973Du02: $\mathrm{E}=40 \mathrm{MeV}$ proton beam was produced at the Grenoble variable-energy cyclotron. Target of $1 \mathrm{mg} / \mathrm{cm}^{2}$ self-supported calcium. Reaction products were detected in a $\Delta \mathrm{E}-\mathrm{E}$ silicon counter telescope and a anti-coincidence counter, FWHM=80-100 keV. Measured $\sigma(\theta)$. Deduced levels, J $\pi$, L, spectroscopic factors from DWBA analysis.

1973Se01: E=52 MeV proton beam was produced at the Synchro-cyclotron at the Institute for Nuclear Studies in Japan. Targets of calcium carbonate on Mylar backings. Reaction products were momentum analyzed with a broad-range magnetic spectrograph and detected with 200 proportional counters in the focal plane, FWHM=70 keV. Measured $\sigma(\theta)$. Deduced levels, J $\pi$, L from DWBA analysis.

1970He23: E=20 MeV proton beam was produced at the Heidelberg Emperor Tandem. Target of enriched CaCO $\mathrm{C}_{3}$ a carbon backing. Reaction products were detected in a $\Delta \mathrm{E}-\mathrm{E}$ surface barrier counter telescope, FWHM=50-55 keV. Measured $\sigma(\theta)$. Deduced levels, J $\pi$, L from DWBA analysis. Levels reported up to $4960 \mathrm{keV}$.

1969Sm02: $\mathrm{E}=26.5 \mathrm{MeV}$ proton beam was produced at the University of Colorado cyclotron. Scattered particles were detected in a $\Delta \mathrm{E}-\mathrm{E}$ semiconductor detector telescope, FWHM=110 keV. Measured $\sigma(\theta)$. Deduced levels, J $\pi$, L from DWBA analysis. Levels reported up to $4700 \mathrm{keV}$.

Others:

1985Mi06: E=51.9 MeV. Measured ground-state transition strength.

1983Sa01: $\mathrm{E}=40 \mathrm{MeV}$. Measured ground-state transition strength.

\section{${ }^{42} \mathrm{Ca}$ Levels}

Levels up to $\mathrm{E}=5809$ are from $1977 \mathrm{SaZF}$ and from $1973 \mathrm{Du} 02$ after that, unless otherwise noted.

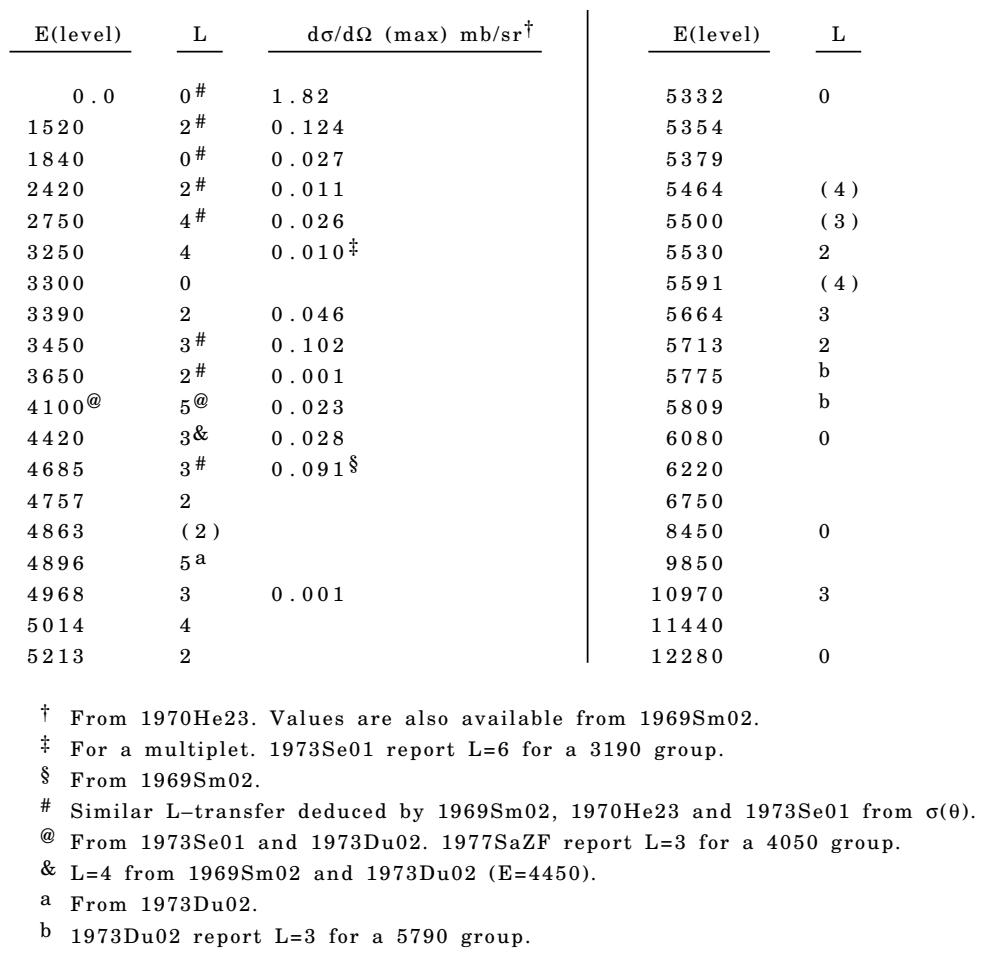




\section{${ }^{45} \mathrm{Sc}(\mathrm{p}, \alpha),(\operatorname{pol} \mathrm{p}, \alpha) \quad 1966 \mathrm{Ri11}, 2005 \mathrm{GuZW}$}

1966Ri11: $(p, \alpha)$ : E=8.5-9.4 MeV. Measured excitation functions and $\sigma(\theta)$. Lowest six excited states identified in the $\alpha$ spectrum. Estimated (by evaluators) $\mathrm{FWHM} \approx 60 \mathrm{keV}$.

2005GuZW: ( pol p, $\alpha), E=24.6 \mathrm{MeV}$; measured $\sigma(\theta)$, Ay $(\theta)$. Deduced configurations. Negative-parity $3-$, 4- and 5- states (homologous to $3 / 2+$ g.s. in ${ }^{41} \mathrm{~K}$ ) identified. No 2 - state seen.

Others:

1977Sc24: $\mathrm{E}=2.5-3.8 \mathrm{MeV}$. Measured $\sigma(\theta)$.

1962Sh29: $\mathrm{E}=17-18 \mathrm{MeV}$. Measured $\sigma(\theta)$.

${ }^{42} \mathrm{Ca}$ Levels

\begin{tabular}{|c|c|}
\hline $\mathrm{E}(\text { level })^{\dagger}$ & $\mathrm{J} \pi^{\dagger}$ \\
\hline $0.0 \ddagger$ & $0+$ \\
\hline 1525 末 & $2+$ \\
\hline 1837 末 & $0+$ \\
\hline 2424 末 & $2+$ \\
\hline 2752 末 & $4+$ \\
\hline 3190 末 & $6+$ \\
\hline 3254 末 & $4+$ \\
\hline $3954 \S$ & $4-\S$ \\
\hline $4100 \S$ & $5-\S$ \\
\hline $4117 \S$ & $3-\S$ \\
\hline
\end{tabular}

$\dagger$ From Adopted Levels, energies are rounded values.

$\ddagger$ Level population from 1966Ri11.

$\S$ Level population from $2005 \mathrm{GuZW}$; $J \pi$ value consistent with $\sigma(\theta)$ and analyzing powers.

\section{${ }^{46} \mathrm{Ti}\left(\mathrm{d},{ }^{6} \mathrm{Li}\right) \quad 1975 \mathrm{Me01}$}

Target ${ }^{46} \mathrm{Ti}$ g.s. J $\pi=0+$.

1975Me01: $\mathrm{E}=28 \mathrm{MeV}$ deuteron beam was produced at the Princeton AVF cyclotron. Target of $82.4 \%$ enriched ${ }^{46} \mathrm{Ti}$ on carbon backing. ${ }^{6} \mathrm{Li}$ particles were detected in a three-detector telescope of $\Delta \mathrm{E}(35 \mu \mathrm{m}), \mathrm{E}(500 \mu \mathrm{m})$, and anti-coincidence detectors, FWHM=100 keV. Measured $\sigma(\theta)$. Deduced levels, L, spectroscopic factors from DWBA analysis

${ }^{42} \mathrm{Ca} \mathrm{Levels}$

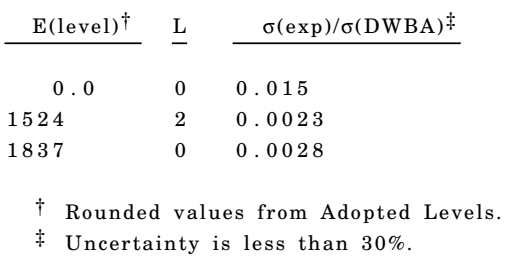




\section{${ }^{96} \mathrm{Zr}\left({ }^{40} \mathrm{Ca},{ }^{42} \mathrm{Ca} \gamma\right) \quad 2007 \mathrm{Sz} 05,2011 \mathrm{Co} 14$}

Includes ${ }^{208} \mathrm{~Pb}\left({ }^{40} \mathrm{Ca},{ }^{42} \mathrm{Ca}\right.$ ) from $2007 \mathrm{Co} 21$ (also $2005 \mathrm{Co} 25,2004 \mathrm{Sz} 05$ ).

Levels in ${ }^{42} \mathrm{Ca}$ populated by $2 \mathrm{n}$ transfer channel.

2007Sz05: E=152 $\mathrm{MeV}{ }^{40} \mathrm{Ca}$ beam was produced at the LNL Tandem. Target of $99.9 \%$ enriched ${ }^{96} \mathrm{Zr}$ in oxide form with a thickness of $150 \mu \mathrm{g} / \mathrm{cm}^{2}$ on a $20 \mu \mathrm{g} / \mathrm{cm}^{2}$ carbon backing. Projectile-like products were selected with the magnetic spectrometer PRISMA and $\gamma$-rays were detected with the CLARA array of 24 HPGe clover-type detectors.

2011Co14: the level scheme is further analyzed and discussed.

$2007 \mathrm{Co} 21$ (also $2005 \mathrm{Co} 25,2004 \mathrm{Sz} 05$ ): ${ }^{208} \mathrm{~Pb}\left({ }^{40} \mathrm{Ca},{ }^{42} \mathrm{Ca}\right), \mathrm{E}=225,236,250 \mathrm{MeV}$ using similar experimental arrangement as in 2007Sz05. Deduced 0+ excitation near $5.8 \mathrm{MeV}$.

All data listed here are from figure 8 in $2011 \mathrm{Co} 14$

${ }^{42} \mathrm{Ca}$ Levels

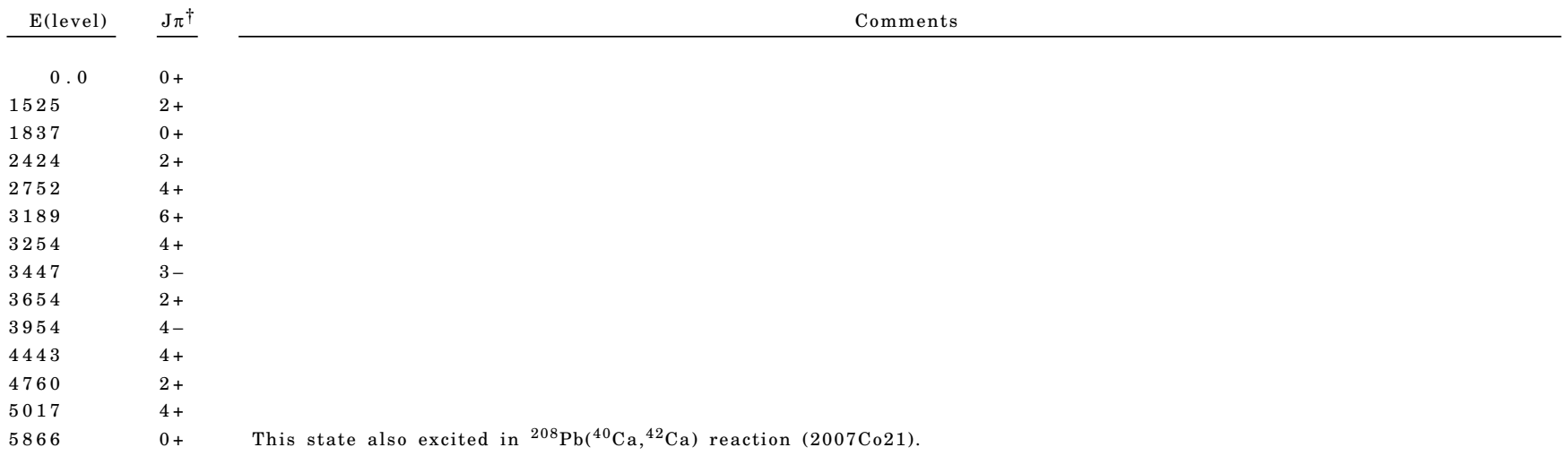

$\dagger$ From Adopted Levels.

$$
\gamma\left({ }^{42} \mathrm{Ca}\right)
$$

\begin{tabular}{|c|c|c|c|c|c|c|c|c|}
\hline $\mathrm{E} \gamma^{\dagger}$ & E(level) & $I \gamma^{\ddagger}$ & $\mathrm{E} \gamma^{\dagger}$ & E(level) & $I \gamma^{*}$ & $\mathrm{E} \gamma^{\dagger}$ & $\mathrm{E}$ (level) & $\mathrm{I} \gamma^{\ddagger}$ \\
\hline 313 & 1837 & 24 & 1227 & 2752 & 189 & 2129 & 3654 & 14 \\
\hline 437 & 3189 & 30 & 1525 & 1525 & 620 & 2424 & 2424 & 29 \\
\hline 502 & 3254 & 38 & 1691 & 4443 & 14 & 3235 & 4760 & 10 \\
\hline 507 & 3954 & 40 & 1729 & 3254 & 38 & 4340 & 5866 & 2 \\
\hline 899 & 2424 & 74 & 1763 & 5017 & 20 & & & \\
\hline 1023 & 3447 & 25 & 1922 & 3447 & 27 & & & \\
\hline
\end{tabular}

$\dagger$ Level-energy differences.

$\ddagger$ Reported intensities are based on branching ratios in Adopted Gammas. 


\section{Adopted Levels, Gammas}

$\mathrm{Q}\left(\beta^{-}\right)=-7016.4822 ; \mathrm{S}(\mathrm{n})=11550.0516 ; \mathrm{S}(\mathrm{p})=4272.23 \quad 10 ; \mathrm{Q}(\alpha)=-5745.3026 \quad 2012 \mathrm{Wa} 38$

$\mathrm{S}(2 \mathrm{n})=27740.428, \mathrm{~S}(2 \mathrm{p})=13163.5918(2012 \mathrm{Wa} 38)$

Identification and production of ms activity of ${ }^{42} \mathrm{Sc}$ by $1955 \mathrm{Mo} 83$ in ${ }^{39} \mathrm{~K}(\alpha, \mathrm{n})$ reaction at $18 \mathrm{MeV}$. The $62-\mathrm{s}$ isomer was identified much later by 1963 Ro 10 .

Mass measurements: $2009 \mathrm{Ku} 19,2006 \mathrm{Er} 08$.

\section{${ }^{42}$ Sc Levels}

In $\left({ }^{3} \mathrm{He}, \mathrm{t}\right)$ reaction total experimental $\mathrm{B}(\mathrm{GT})$ strength $=2.74(2015 \mathrm{Fu} 08)$ out of which $\approx 80 \%$ is concentrated in the 611 , $1+$ state.

All the isospin (T) assignments are from 1990En08 evaluation, see the arguments there for such assignments.

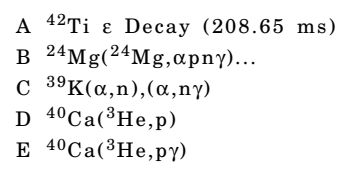

$\frac{\mathrm{E}(\text { level })^{\dagger}}{0.0 \&}-\frac{\mathrm{J} \pi \S}{0+}-$

$611.051^{\&} \quad 6 \quad 1+$

$616.28^{\mathrm{a}} \quad 6 \quad 7+$

$\begin{array}{lll}1490.97 \& & 10 & 3+ \\ 1510.36 & 11 & 5+ \\ 1586.32^{@} & 3 & 2+ \\ 1704 ? \quad 15 & & \\ 1844.84^{@} & 11 & 3(+) \\ & & \end{array}$

$1888.422 \quad 1+$

$2187.42 \quad 11 \quad 3+$
Cross Reference (XREF) Flags

$\begin{array}{ll}\mathrm{F} \quad{ }^{40} \mathrm{Ca}(\alpha, \mathrm{d}) & \mathrm{K}{ }^{42} \mathrm{Ca}\left({ }^{3} \mathrm{He}, \mathrm{t}\right) \\ \mathrm{G}{ }^{40} \mathrm{Ca}\left({ }^{12} \mathrm{C},{ }^{10} \mathrm{~B}\right) & \mathrm{L}{ }^{42} \mathrm{Ca}\left({ }^{6} \mathrm{Li},{ }^{6} \mathrm{He}\right) \\ \mathrm{H}{ }^{41} \mathrm{Ca}(\mathrm{p}, \gamma) & \mathrm{M} \mathrm{Pb}\left({ }^{16} \mathrm{O}, \mathrm{X} \gamma\right)\end{array}$

I ${ }^{41} \mathrm{Ca}\left({ }^{3} \mathrm{He}, \mathrm{d}\right)$

J ${ }^{42} \mathrm{Ca}(\mathrm{p}, \mathrm{n})$

Comments

XREF $\mathrm{T}_{1 / 2}^{\#}$

ABCDEF HIJKLM $680.79 \mathrm{~ms} 28$

$\% \varepsilon+\% \beta^{+}=100$.

$\mathrm{T}=1$.

IAS of ${ }^{42} \mathrm{Ca}$ g.s.

The $\mathrm{rms}$ charge radius $\left.\left(<\mathrm{r}^{2}\right\rangle\right)^{1 / 2}: 3.570 \mathrm{fm} 24(2013 \mathrm{An} 02$

evaluation). Other: $3.539 \mathrm{fm} 4$ (2006Ga47).

$\delta<\mathrm{r}^{2}>\left({ }^{45} \mathrm{Sc},{ }^{42} \mathrm{Sc}\right)=+0.172 \mathrm{fm}^{2} 31$ (stat) 136 (syst) $(2011 \mathrm{Av} 01)$

$\mathrm{J} \pi$ : superallowed $\beta$ decay to ${ }^{42} \mathrm{Ca}$ g.s. and from ${ }^{42} \mathrm{Ti}$ g.s.;

$\mathrm{L}\left({ }^{3} \mathrm{He}, \mathrm{t}\right)=0$.

$\mathrm{T}_{1 / 2}$ : weighted average of $680.67 \mathrm{~ms} 28$ (1997Ko65),

$683.0 \mathrm{~ms} 15$ (1965 $\mathrm{Fr} 08)$ and $681.0 \mathrm{~ms} 6(1976 \mathrm{Wi} 08)$. Others:

$684.5 \mathrm{~ms} 12(1972 \mathrm{Ha} 82), 650 \mathrm{~ms} 10(1965 \mathrm{Ne} 02), 720 \mathrm{~ms} 30$ (1960Mi09), $690 \mathrm{~ms} 20$ (1961Ja22), $680 \mathrm{~ms} 10$ (1957Cl40),

$620 \mathrm{~ms} 50$ (1955Mo83). In $2015 \mathrm{Ha} 07$ evaluation, $\mathrm{T}_{1 / 2}=680.72$ $\mathrm{ms} 26$.

XREF: K(615)

$\mathrm{T}=0$.

$\mathrm{J} \pi: \mathrm{L}\left({ }^{3} \mathrm{He}, \mathrm{p}\right)=0+2$ and $\gamma(\theta) ; \mathrm{L}\left({ }^{6} \mathrm{Li},{ }^{6} \mathrm{He}\right)=0$ and $\mathrm{J}\left({ }^{6} \mathrm{Li}\right)=1+$;

$\mathrm{L}\left({ }^{3} \mathrm{He}, \mathrm{t}\right)=0$.

XREF: K(624)

$\mathrm{T}=0$.

$\% \varepsilon+\% \beta^{+}=100$.

$\mathrm{J} \pi: \log f t=4.16$ to $6+; \mathrm{J}=7$ from $\mathrm{p} \gamma(\theta)$ in $\left({ }^{3} \mathrm{He}, \mathrm{p} \gamma\right) .7+$ is most likely from level systematics and shell model expectations.

$\mathrm{T}_{1 / 2}$; weighted average of $62.0 \mathrm{~s} 4$ (1963Ro10), $60.6 \mathrm{~s} 4$ (1965Ne02), $62.0 \mathrm{~s} 3$ (1974Wi14), and 62.2 s 9 (1978Be61).

Other: 68 s 8 (1994Uz01),.

$\mathrm{T}=0$.

$\mathrm{J} \pi: L\left({ }^{3} \mathrm{He}, \mathrm{d}\right)=1+3 ; \Delta \mathrm{J}=2 \gamma$ to $1+$ in $\left({ }^{3} \mathrm{He}, \mathrm{p} \gamma\right)$.

$\mathrm{T}=0$.

$\mathrm{J} \pi: \mathrm{L}(\alpha, \mathrm{d})=4 ; \gamma$ to $7+; \Delta \mathrm{J}=2 \gamma$ to $7+$ in $\left({ }^{3} \mathrm{He}, \mathrm{p} \gamma\right)$.

$\mathrm{T}=1$.

$\mathrm{J} \pi: L\left({ }^{3} \mathrm{He}, \mathrm{p}\right)=2 ; \Delta \mathrm{J}=1 \gamma$ to $1+$.

$\mathrm{E}\left(\right.$ level): level seen weakly in $\left({ }^{3} \mathrm{He}, \mathrm{p}\right)$ only.

$\mathrm{T}=0$.

$\mathrm{J} \pi: \Delta \mathrm{J}=1 \gamma$ to $2+; \Delta \mathrm{J}=2 \gamma$ to $1+$; weak $\gamma$ from $4+$; RUL

$\mathrm{T}_{1 / 2}: 0.7 \mathrm{ps}$ to $3.5 \mathrm{~ns}$.

$\mathrm{T}=1$.

$\mathrm{J} \pi: \mathrm{L}\left({ }^{3} \mathrm{He}, \mathrm{d}\right)=3$; isotropic $\gamma(\theta)$ to $1+$ allows $(0,1,2)+$. This level is the only candidate as isobaric analog of ${ }^{42} \mathrm{Ca}$ $(1837,0+)$.

$\mathrm{T}=0$.

$\mathrm{J} \pi: \mathrm{L}\left({ }^{3} \mathrm{He}, \mathrm{p}\right)=\mathrm{L}\left({ }^{3} \mathrm{He}, \mathrm{t}\right)=0 ; \Delta \mathrm{J}=1 \gamma$ to $0+$.

$\mathrm{T}_{1 / 2}$ : other: $42 \mathrm{fs} 21$.

$\mathrm{T}=0$.

$\mathrm{J} \pi: \quad \mathrm{L}(\alpha, \mathrm{d})=2 ; \quad \mathrm{L}\left({ }^{3} \mathrm{He}, \mathrm{d}\right)=1+3 ; \Delta \mathrm{J}=1 \gamma$ to $2+$. 
Adopted Levels, Gammas (continued)

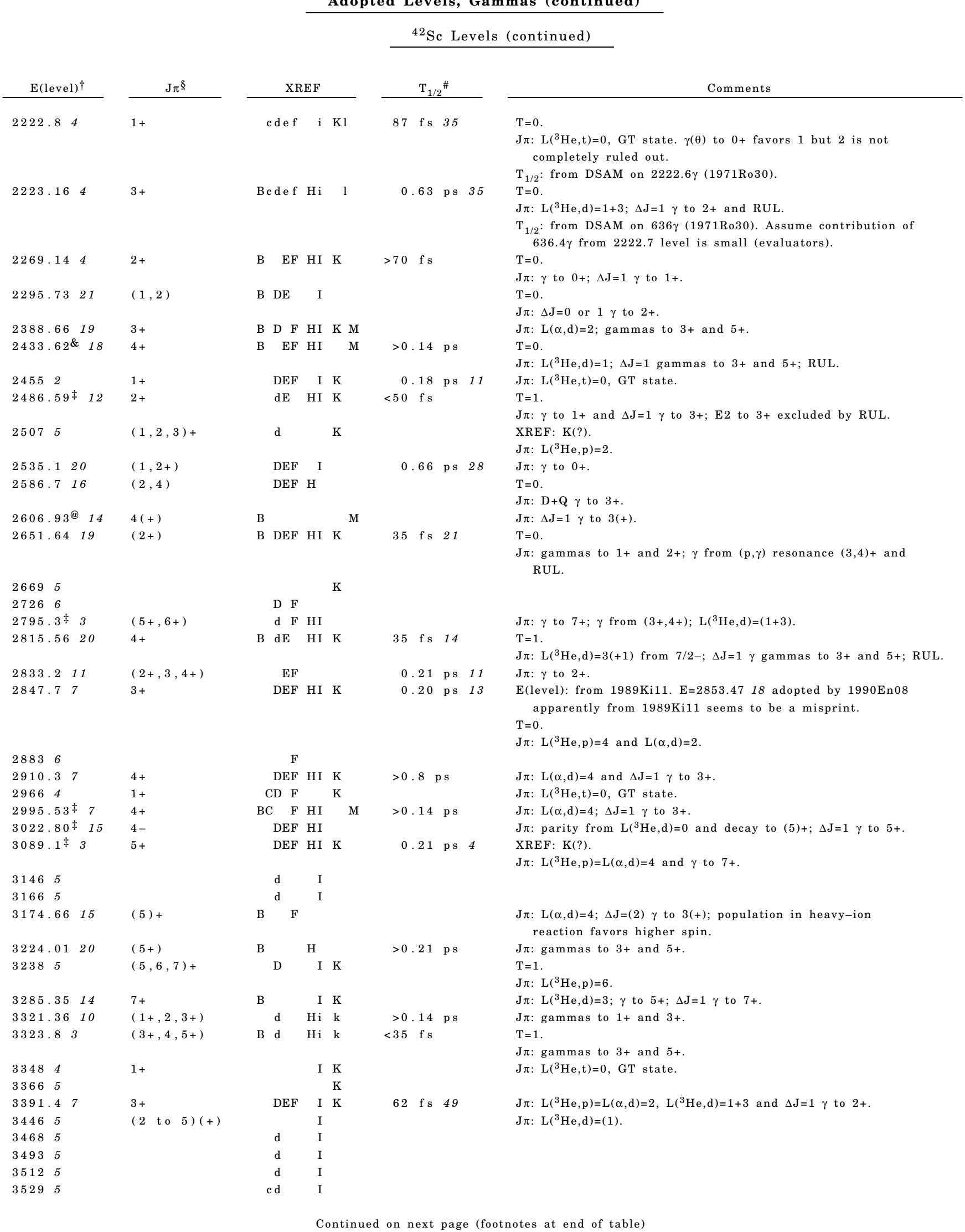


Adopted Levels, Gammas (continued)

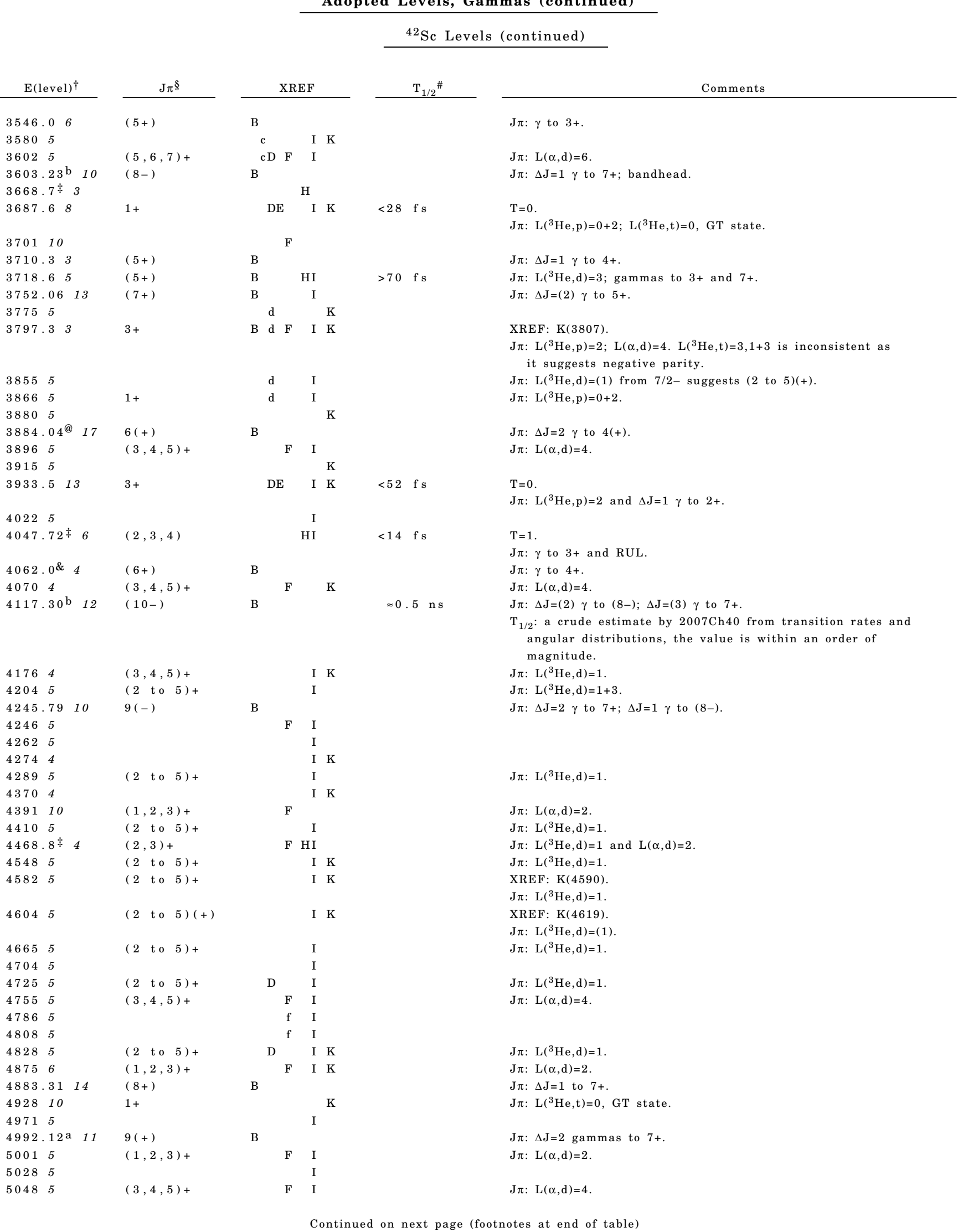


Adopted Levels, Gammas (continued)

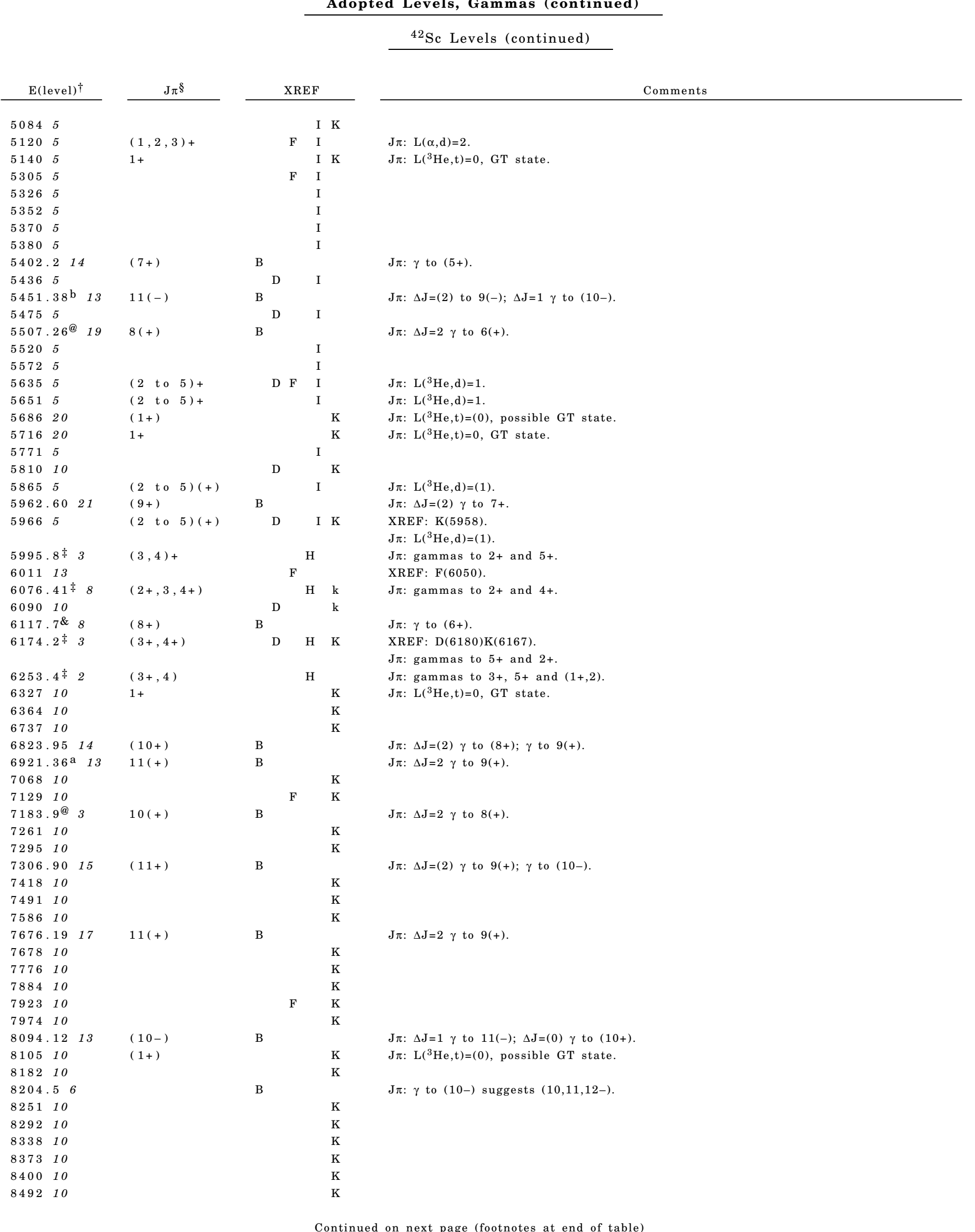


Adopted Levels, Gammas (continued)

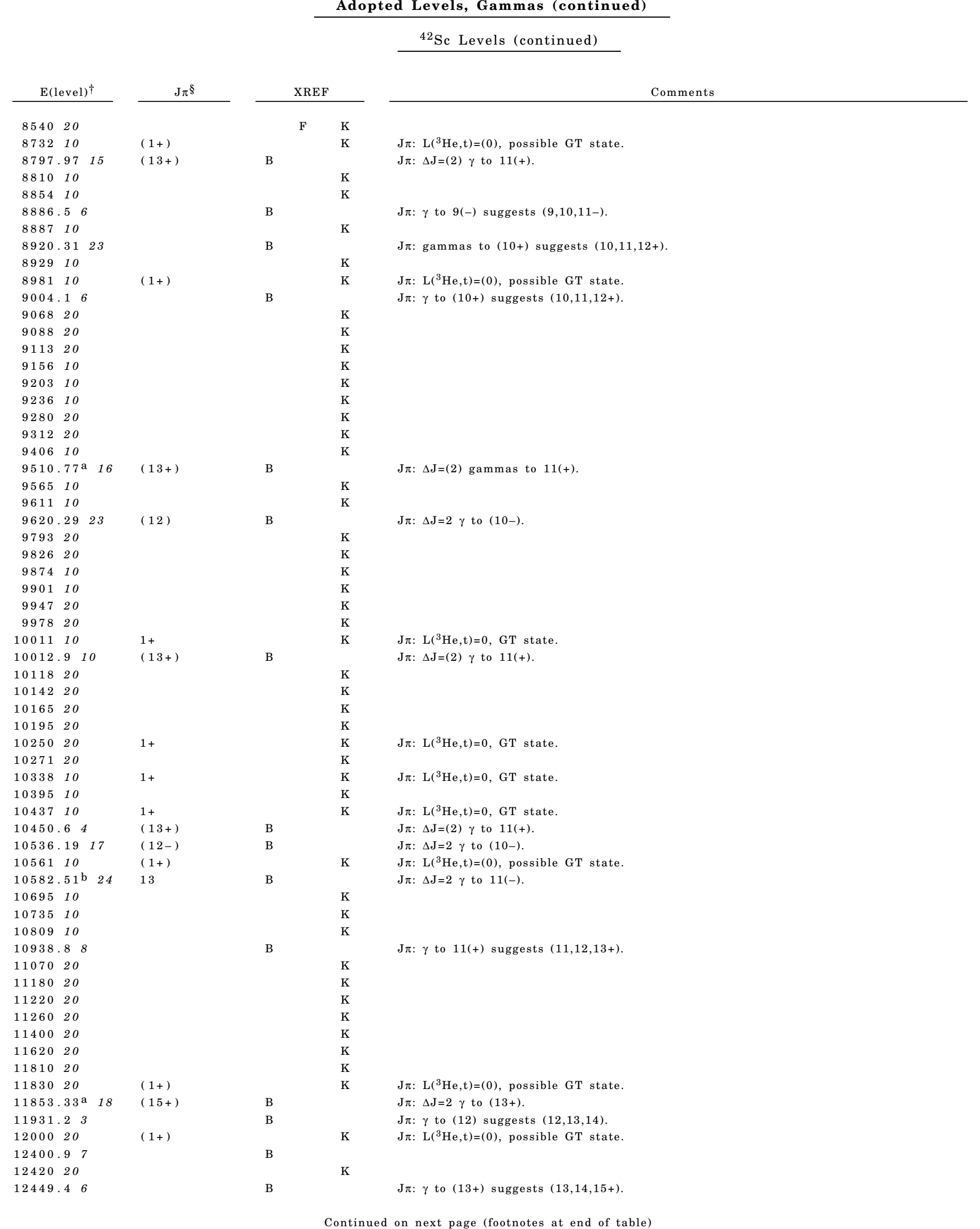


Adopted Levels, Gammas (continued)

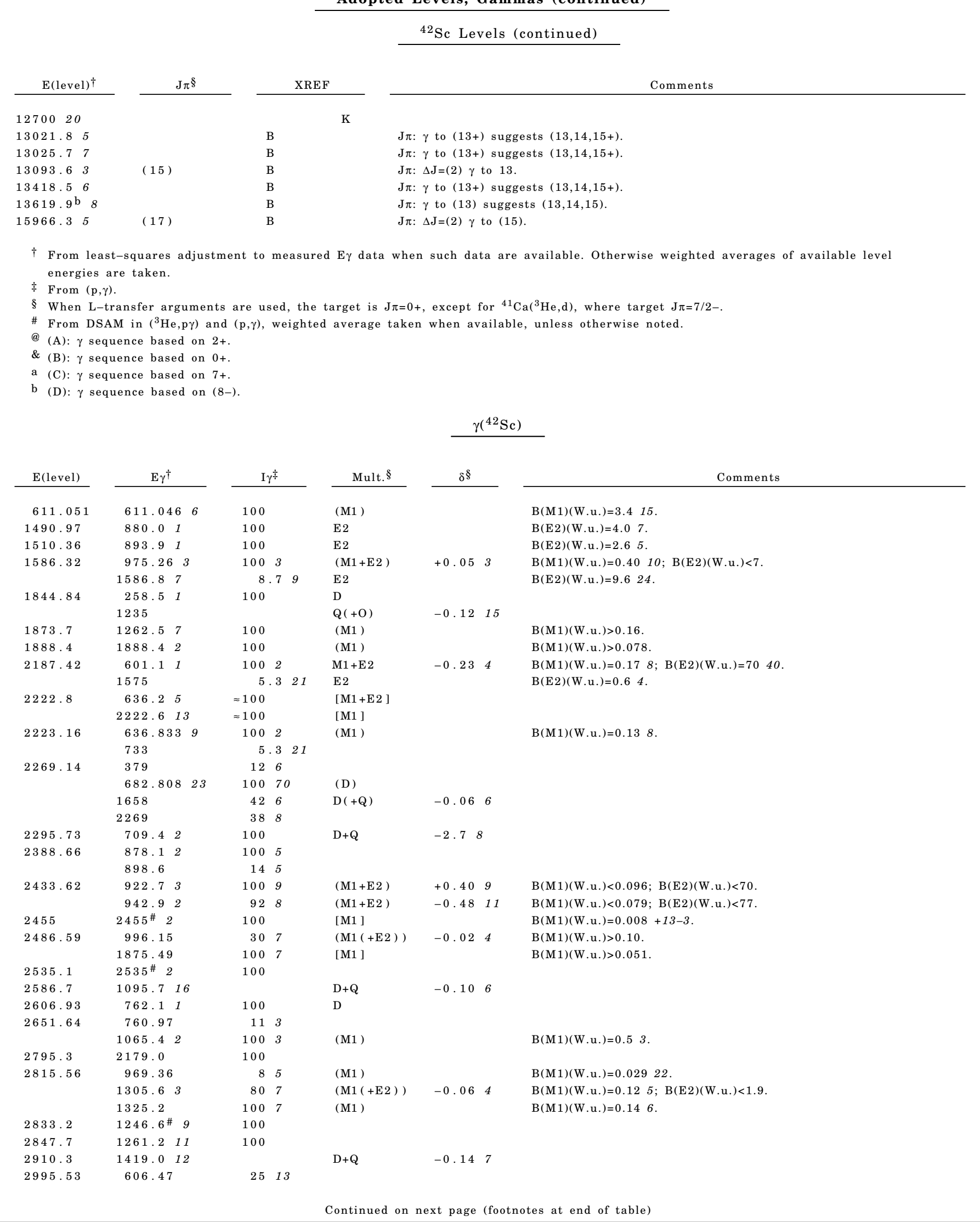


Adopted Levels, Gammas (continued)

\begin{tabular}{|c|c|c|c|c|c|c|c|}
\hline \multirow{2}{*}{$\begin{array}{r}\text { E(level) } \\
2995.53\end{array}$} & \multicolumn{2}{|l|}{$\mathrm{E} \gamma^{\dagger}$} & \multicolumn{2}{|c|}{$\mathrm{I} \gamma^{\ddagger}$} & \multirow{2}{*}{$\frac{\text { Mult. } \S}{\text { (M1) }}$} & $\delta \S$ & Comments \\
\hline & 772.6 & 1 & 100 & 5 & & & $\mathrm{~B}(\mathrm{M} 1)($ W.u. $)<0.27$. \\
\hline 3022.80 & 1512.67 & & 100 & & $\mathrm{D}(+\mathrm{Q})$ & $-0.03 \quad 8$ & \\
\hline 3089.1 & 2472.7 & & 100 & & [E 2 ] & & $\mathrm{B}(\mathrm{E} 2)(\mathrm{W} . \mathrm{u})=$.3.47 . \\
\hline 3174.66 & 1329.8 & 1 & 100 & & (Q) & & \\
\hline \multirow[t]{4}{*}{3224.01} & 408.6 & 2 & 100 & 9 & [M1 ] & & $\mathrm{B}(\mathrm{M} 1)$ (W.u. $)<0.50$. \\
\hline & 834.9 & 4 & 83 & 9 & [ E 2 ] & & $\mathrm{B}(\mathrm{E} 2)(\mathrm{W} . \mathrm{u})<210$. \\
\hline & 1000.8 & 3 & 67 & 6 & [ E 2 ] & & $\mathrm{B}(\mathrm{E} 2)(\mathrm{W} . \mathrm{u})<68$. \\
\hline & 1713 & & 55 & 6 & & & \\
\hline \multirow[t]{2}{*}{3285.35} & 1775.2 & 4 & 41 & 4 & & & \\
\hline & 2668.4 & 2 & 100 & 12 & $D+Q$ & & \\
\hline \multirow[t]{2}{*}{3321.36} & 1830.9 & & 100 & 20 & & & \\
\hline & 2710.22 & & 100 & 20 & & & \\
\hline \multirow[t]{2}{*}{3323.8} & 1813.9 & 6 & 100 & 9 & & & \\
\hline & 1832.6 & 4 & 82 & 9 & & & \\
\hline \multirow[t]{2}{*}{3391.4} & 575.9 & 12 & & & & & \\
\hline & 904.5 & 5 & 100 & & $\mathrm{D}+\mathrm{Q}$ & -0.115 & \\
\hline 3546.0 & 1358.6 & 6 & 100 & & & & \\
\hline 3603.23 & 2986.4 & 1 & 100 & & $\mathrm{D}$ & & \\
\hline \multirow[t]{2}{*}{3668.7} & 1235.4 & & & & & & \\
\hline & 1445.5 & & & & & & \\
\hline \multirow[t]{4}{*}{3687.6} & 1813.5 & 11 & $\approx 20$ & & [M1 ] & & E $\gamma:$ level-energy difference $=1814.2$. \\
\hline & & & & & & & $\mathrm{B}(\mathrm{M} 1)(\mathrm{W} . \mathrm{u})>0.020$. \\
\hline & 2101.2 & 10 & $\approx 100$ & & $(\mathrm{M} 1+\mathrm{E} 2)$ & $-0.07 \quad 4$ & $\mathrm{~B}(\mathrm{M} 1)(\mathrm{W} . \mathrm{u})>0.063$. \\
\hline & 36892 & & $\approx 13$ & & {$[\mathrm{M} 1]$} & & $\begin{array}{l}\text { E } \gamma: \text { level-energy difference }=3687.6 \text {. } \\
B(M 1)(\text { W.u. })>0.0015 \text {. }\end{array}$ \\
\hline 3710.3 & 894.7 & 2 & 100 & & $\mathrm{D}$ & & \\
\hline \multirow[t]{2}{*}{3718.6} & 723.0 & 3 & & & & & $\mathrm{E} \gamma, \mathrm{I} \gamma:$ only seen in $\left({ }^{24} \mathrm{Mg}, \alpha \mathrm{pn} \gamma\right)$ and $\left({ }^{24} \mathrm{Ne}\right.$, apng $)$. \\
\hline & 3102.9 & & & & & & E $\gamma, \mathrm{I} \gamma:$ only seen in $(p, \gamma)$ \\
\hline 3752.06 & 2241.5 & 1 & 100 & & $(\mathrm{Q})$ & & \\
\hline 3797.3 & 473.5 & 2 & 100 & 9 & & & \\
\hline & 1145.7 & 4 & 51 & 5 & & & \\
\hline 3884.04 & 1277.1 & 1 & 100 & & $\mathrm{Q}$ & & \\
\hline 3933.5 & 2347.1 & 13 & 100 & & $(\mathrm{M} 1+\mathrm{E} 2)$ & 1.25 & $\mathrm{~B}(\mathrm{M} 1)(\mathrm{W} . \mathrm{u})>.0.0068 ; \mathrm{B}(\mathrm{E} 2)(\mathrm{W} . \mathrm{u})>6.8$. \\
\hline 4047.72 & 1658.63 & & 100 & & & & $\begin{array}{l}\text { I } \gamma \text { : this } \gamma \text { is only about } 50 \% \text {, other } 50 \% \text { branching from } 4048 \\
\text { level is unaccounted. }\end{array}$ \\
\hline 4062.0 & 1628.3 & 3 & 100 & & & & \\
\hline 4117.30 & 514.0 & 1 & 100 & 3 & ( E2) & & $\mathrm{B}(\mathrm{E} 2)(\mathrm{W} . \mathrm{u}.) \approx 3.0$ \\
\hline & 3500.7 & 3 & 20 & 811 & (E3) & & $\mathrm{B}(\mathrm{E} 3)(\mathrm{W} . \mathrm{u}.) \approx 0.62$. \\
\hline 4245.79 & 642.2 & 1 & 34 & 216 & $\mathrm{D}+\mathrm{Q}$ & & \\
\hline & 3629.7 & 1 & 100 & 6 & $\mathrm{Q}$ & & \\
\hline 4468.8 & 1558.4 & & 100 & 20 & & & \\
\hline & 2882.4 & & 100 & 8 & & & \\
\hline 4883.31 & 1597.8 & 1 & 46 & 4 & & & \\
\hline & 4267.3 & 2 & 100 & 9 & $\mathrm{D}+\mathrm{Q}$ & & \\
\hline 4992.12 & 1239.8 & 2 & 4 & 93 & $\mathrm{Q}$ & & \\
\hline & 4375.8 & 1 & 100 & 8 & (Q) & & \\
\hline 5402.2 & 1856.1 & 12 & 100 & & & & \\
\hline 5451.38 & 1206.0 & 1 & 73 & $0 \quad 25$ & $(\mathrm{Q})$ & & \\
\hline & 1334.1 & 1 & 100 & 3 & $\mathrm{D}+\mathrm{Q}$ & & \\
\hline 5507.26 & 1623.2 & 1 & 100 & & $\mathrm{Q}$ & & \\
\hline 5962.60 & 2209.8 & 3 & 100 & 7 & (Q) & & \\
\hline & 5346.5 & 7 & 82 & 14 & & & \\
\hline 5995.8 & 1948.0 & & 38 & 13 & & & \\
\hline & 2771.9 & & 81 & 12 & & & \\
\hline & 2906.6 & & 19 & 6 & & & \\
\hline & 2972.9 & & $\approx 6$ & & & & \\
\hline & 3148.1 & & 25 & 6 & & & \\
\hline & 3180.3 & & 19 & 6 & & & \\
\hline & 3344.7 & & 25 & 6 & & & \\
\hline & 3509.1 & & 19 & 6 & & & \\
\hline & 3562.3 & & 19 & 6 & & & \\
\hline & 3772.5 & & 38 & 13 & & & \\
\hline & 3808.1 & & 12 & 6 & & & \\
\hline
\end{tabular}


Adopted Levels, Gammas (continued)

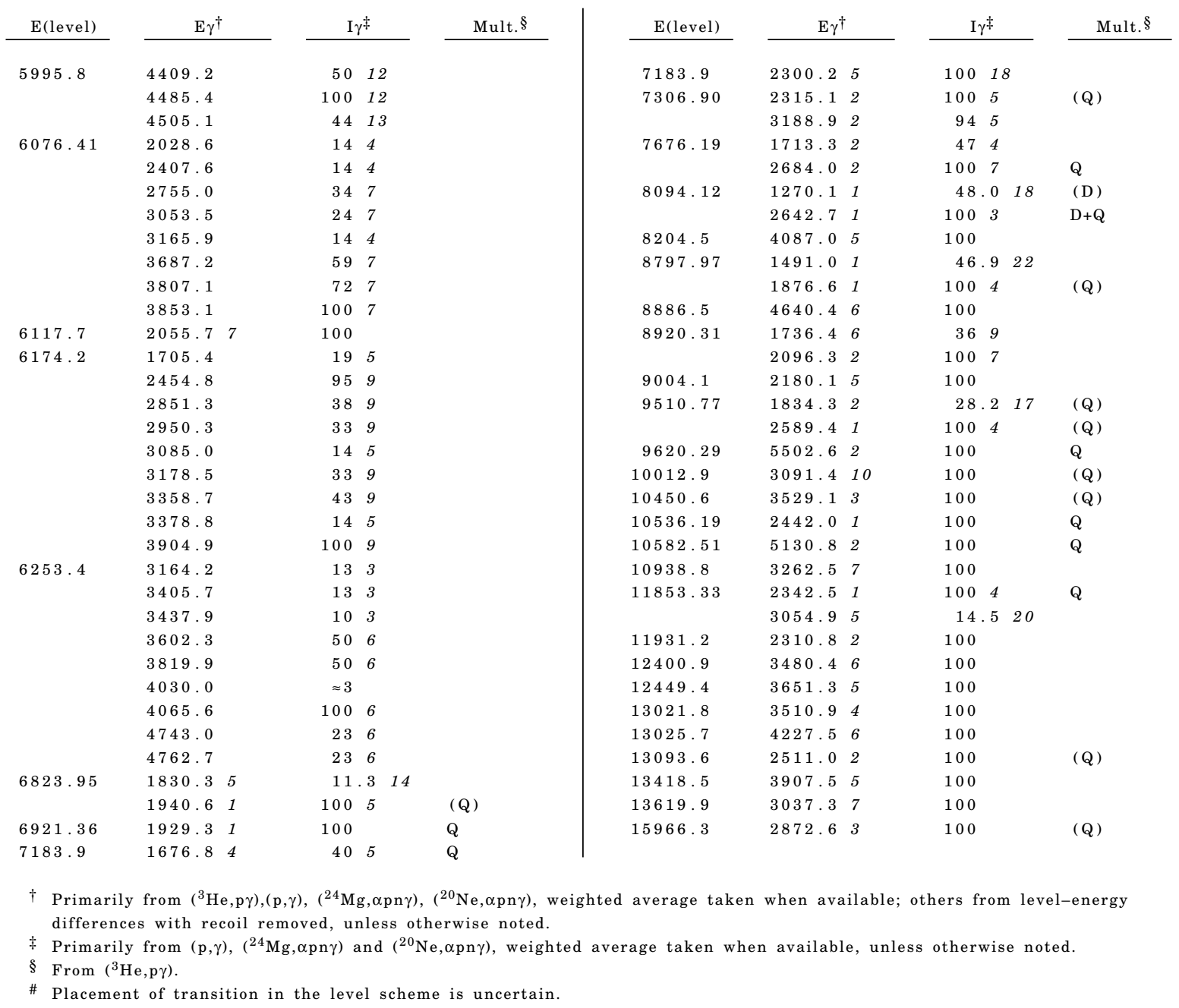


Adopted Levels, Gammas (continued)

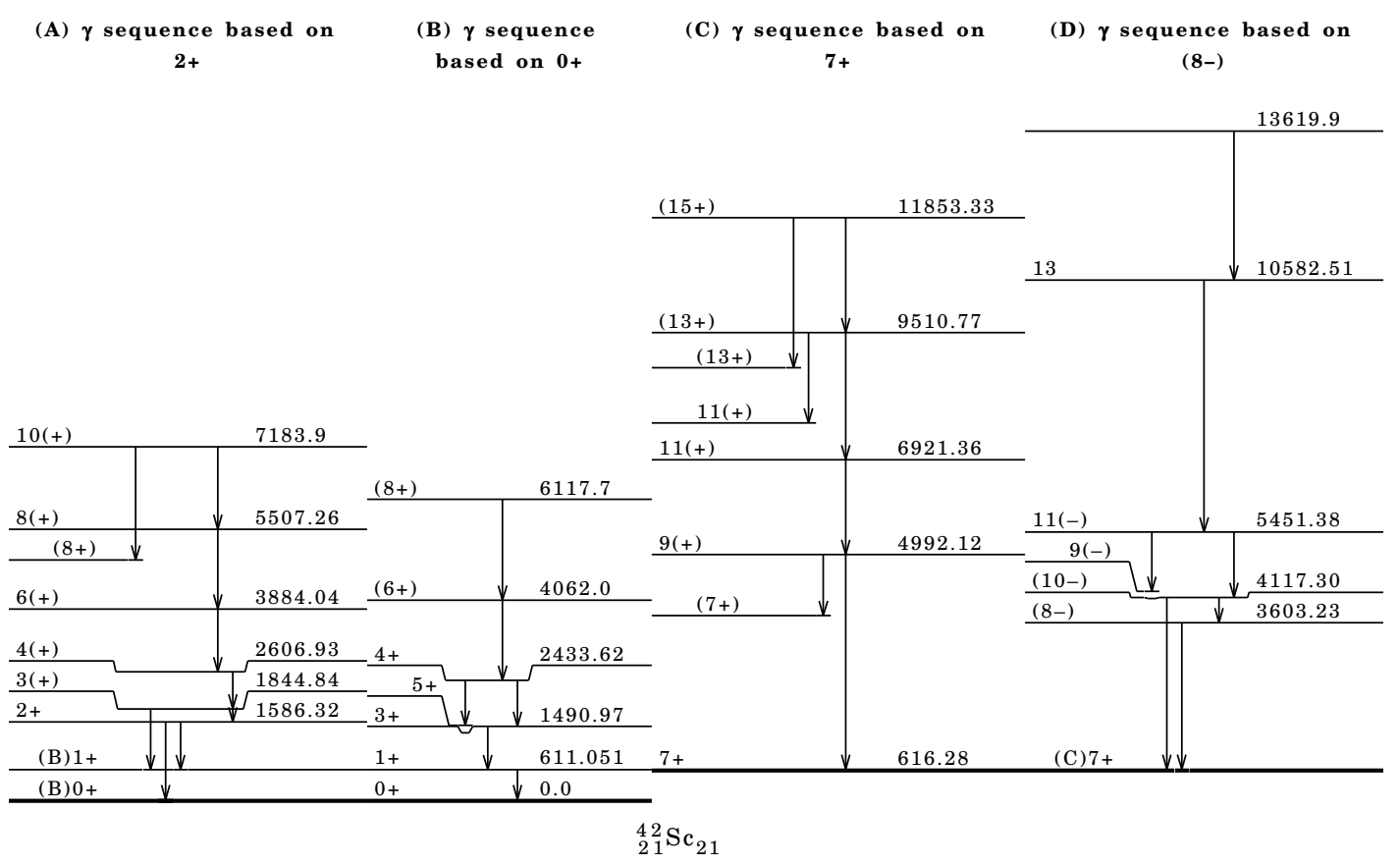


Parent ${ }^{42} \mathrm{Ti}: \mathrm{E}=0 ; \mathrm{J} \pi=0+; \mathrm{T}_{1 / 2}=208.65 \mathrm{~ms} 80 ; \mathrm{Q}$ (g.s.) $=7016.4822 ; \% \varepsilon+\% \beta^{+}$decay $=100$.

${ }^{42} \mathrm{Ti}-\mathrm{T}_{1 / 2}$ : From Adopted Levels of ${ }^{42} \mathrm{Ti} .2015 \mathrm{Ha} 07$ review gives $208.09 \mathrm{~ms} 55$.

${ }^{42} \mathrm{Ti}-\mathrm{Q}(\varepsilon)$ : From 2012Wa38. $2015 \mathrm{Ha} 07$ review gives 7016.8325 .

42 TI decays mainly (>99\%) through $\mathrm{B}+$ decay.

2015Mo01: ${ }^{42} \mathrm{Ti}$ ions were produced from fragmentation of $680 \mathrm{MeV} / \mathrm{nucleon}{ }^{58} \mathrm{Ni}$ beam with $400 \mathrm{mg} / \mathrm{cm}^{2}{ }^{9} \mathrm{Be}$ target using

SIS-18 synchrotron at GSI facility. Reaction fragments were separated in-flight using the fragment separator FRS.

The identification of nuclei was achieved by measurement of magnetic rigidity and velocity of fragments from

time-of-flight method. Separated ions were implanted in one of six double-sided silicon strip detectors (DSSSDs).

The $\beta$-decay signals were detected in the same DSSSD. Surrounding the implantation setup was the RISING array of 15

Euroball cluster detectors for $\gamma$ detection. Measured $\mathrm{E} \gamma, \mathrm{I} \gamma, \gamma \gamma$-coin, $\beta \gamma$-coin, and $\beta$-decay half-life. Deduced

levels, J, $\pi, \beta$ feedings, log $f t$ values, Gamow-Teller strengths. $2012 \mathrm{Fu} 02$ is an earlier report without any data.

1969Ga27: ${ }^{42} \mathrm{Ti}$ isotope was produced via ${ }^{40} \mathrm{Ca}\left({ }^{3} \mathrm{He}, \mathrm{n}\right)$ reaction using ${ }^{3} \mathrm{He}$ beam from the $5.5-\mathrm{MV}$ Van de Graaff at

Strasbourg. $\gamma$-rays were detected with $\mathrm{Ge}(\mathrm{Li})$ detectors (FWHM=4 keV at $1.22 \mathrm{MeV}$ ). Measured $\mathrm{E} \gamma, \mathrm{I} \gamma, \mathrm{T}_{1 / 2}$. Deduced levels, branchings.

1980HoZO: measured G, limit of delayed proton decay.

2009Ku 19: ${ }^{42} \mathrm{Ti}$ produced in ${ }^{40} \mathrm{Ca}\left({ }^{3} \mathrm{He}, \mathrm{n} \gamma\right) \mathrm{E}=17 \mathrm{MeV}$, beam from the Ion Guide Isotope Separator On-Line (IGISOL)

facility at the Accelerator Laboratory of the University of Jyvaskyla. Target of a $1.5 \mathrm{mg} / \mathrm{cm}^{2} \mathrm{natural} \mathrm{Ca}$. Measured

$\mathrm{E} \gamma, \beta \gamma$-coin, $\mathrm{T}_{1 / 2}$, mass differences using JYFLTRAP Penning-trap spectrometer. Measured $\mathrm{Q}(\varepsilon)=7016.83 \mathrm{keV} 25$.

2015Ha07: review of superallowed decays; evaluated $\mathrm{Q}$ value, $\mathrm{T}_{1 / 2}$ branching ratios, $f t$ value,

isospin-symmetry-breaking corrections.

Others:

$\mathrm{T}^{1 / 2}\left({ }^{42} \mathrm{Ti}\right), \gamma: 1972 \mathrm{Zi02}, 1969 \mathrm{Al} 12,1969 \mathrm{Ni} 03$.

$\mathrm{E} \beta^{+}: 1962 \mathrm{Ob} 03$.

A $22222(\mathrm{I} \gamma=1.24) \gamma$ reported by $1969 \mathrm{Ga} 27$ is omitted here since it is assigned (by $1980 \mathrm{HoZO})$ to ${ }^{1} \mathrm{H}(\mathrm{n}, \gamma)$ line related to background. Thus the 2222 level proposed by $1969 \mathrm{Ga} 27$ is also omitted.

$\% \varepsilon p<0.001$ (1980HoZO).

${ }^{42} \mathrm{Sc}$ Levels

\begin{tabular}{|c|c|c|}
\hline $\mathrm{E}$ (level) & $\mathrm{J} \pi^{\dagger}$ & $\mathrm{T}_{1 / 2}^{\dagger}$ \\
\hline 0.0 & $0+$ & $680.79 \mathrm{~ms} 28$ \\
\hline $611.0 \quad 1$ & $1+$ & \\
\hline 1888.42 & $1+$ & \\
\hline
\end{tabular}

\begin{tabular}{|c|c|c|c|c|c|c|}
\hline \multirow[b]{2}{*}{$\mathrm{E} \varepsilon$} & \multirow[b]{2}{*}{ E(level) } & \multirow[b]{2}{*}{$I \beta^{+\dagger}$} & \multirow[b]{2}{*}{$\mathrm{I} \varepsilon^{\dagger}$} & \multicolumn{3}{|c|}{$\beta^{+}, \varepsilon$ Data } \\
\hline & & & & $\log$ & $f t$ & $\mathrm{I}\left(\varepsilon+\beta^{+}\right)^{\dagger}$ \\
\hline$\left(\begin{array}{lll}5 & 128.1 & 3\end{array}\right)$ & 1888.4 & 0.416 & 0.00112 & 4.80 & 7 & 0.416 \\
\hline$(6405.48 \quad 25)$ & 611.0 & 564 & $0.065 \quad 4$ & 3.21 & 3 & $55.9 \quad 36$ \\
\hline$(7016.48 \quad 22)$ & 0.0 & $47.7 \quad 12$ & $0.0405 \quad 11$ & 3.495 & 11 & $47.7 \quad 12$ \\
\hline
\end{tabular}

$\dagger$ Absolute intensity per 100 decays. 
${ }^{42}$ Ti $\varepsilon$ Decay $(208.65 \mathrm{~ms}) \quad 2015 M o 01,1969 G a 27,1980 \mathrm{HoZO}$ (continued)

$$
\gamma\left({ }^{42} \mathrm{Sc}\right)
$$

I $\gamma$ normalization: From determination of number of $937-\mathrm{keV} \gamma$ rays emitted per ${ }^{54} \mathrm{Ni}$ decay (2015Mo01). Other: 0.5614 (1969Al12).

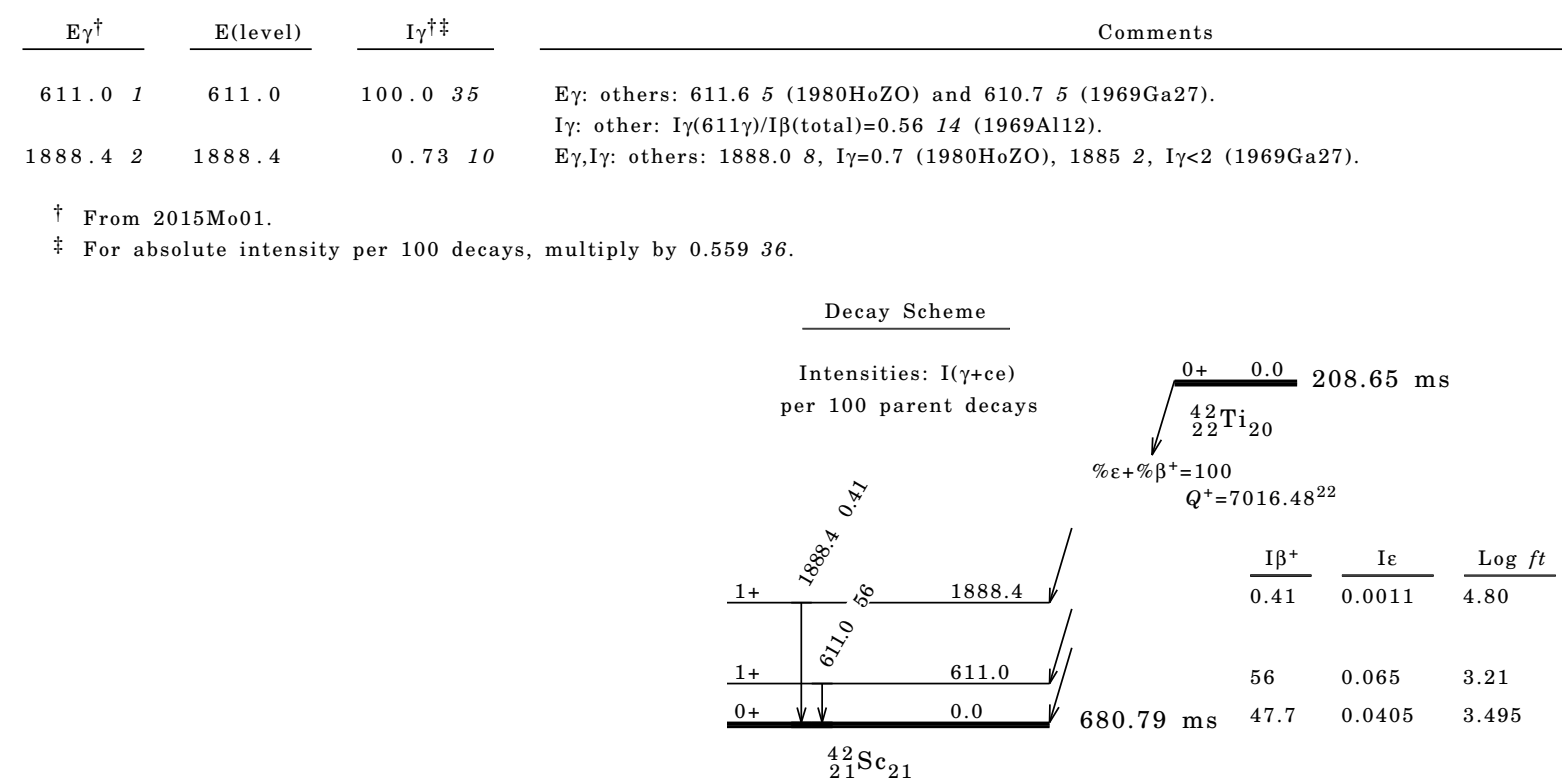

\section{${ }^{24} \mathrm{Mg}\left({ }^{24} \mathrm{Mg}, \alpha \operatorname{pn} \gamma\right),{ }^{28} \mathrm{Si}\left({ }^{20} \mathrm{Ne}, \alpha \mathrm{pn} \gamma\right) \quad 2007 \mathrm{Ch} 40$}

2007Ch40: $\mathrm{E}\left({ }^{24} \mathrm{Mg}\right)=94 \mathrm{MeV}$ beam was produced from Berkeley $88-\mathrm{Inch}$ cyclotron at LBN facility. Target of a $0.5 \mathrm{mg} / \mathrm{cm}^{2}$ self-supporting ${ }^{24} \mathrm{Mg}$ target. $\mathrm{E}\left({ }^{20} \mathrm{Ne}\right)=84 \mathrm{MeV}$ beam produced at the ATLAS accelerator at ANL facility. The $\gamma-\mathrm{rays}$ were detected by Gammasphere array of 102 Compton-suppressed HPGe detectors and particles were detected with an array of $95 \mathrm{CsI}(\mathrm{Tl})$ detectors with a $65 \%$ efficiency for detection $\alpha$ particles and $50 \%$ for protons. Measured E $\gamma$, $\mathrm{I} \gamma, \gamma \gamma, \gamma(\theta), \gamma \gamma(\theta)(\mathrm{DCO})$. Deduced levels, J $\pi$, branching ratios. ${ }^{28} \mathrm{Si}\left({ }^{20} \mathrm{Ne}, \alpha \mathrm{pn} \gamma\right)$. The $\gamma$-ray energies and angular distribution/ correlation coefficients are averages from the two experiments.

${ }^{42} \mathrm{Sc}$ Levels

\begin{tabular}{|c|c|c|c|}
\hline E(level $)^{\dagger}$ & $\mathrm{J} \pi \ddagger$ & $\mathrm{T}_{1 / 2}$ & Comments \\
\hline $0.0 \&$ & $0+$ & & \\
\hline $611.12^{\&} 10$ & $1+$ & & \\
\hline $616.9^{\mathrm{a}} 3$ & $7+$ & $61.7 \mathrm{~s} \S \quad 4$ & $\% \varepsilon+\% \beta^{+}=100$ \\
\hline $1491.10^{\&} 14$ & $3+$ & & \\
\hline $1510.92 \quad 24$ & $5+$ & & \\
\hline $1586.57^{@} 14$ & $2+$ & & \\
\hline $1845.07^{@} 17$ & $3(+)$ & & \\
\hline $2187.67 \quad 17$ & $3+$ & & \\
\hline $2223.50 \quad 17$ & $3+$ & & \\
\hline $2269.48 \quad 25$ & $(1+)$ & & \\
\hline $2295.98 \quad 25$ & & & \\
\hline 2389.23 & $3+$ & & \\
\hline $2433.89^{\&} 23$ & $4+$ & & \\
\hline $2607.19^{@} 20$ & $4(+)$ & & \\
\hline $2651.97 \quad 23$ & ( 2 ) & & \\
\hline $2816.22 \quad 21$ & $4+$ & & \\
\hline 2996.1120 & $(4)+$ & & \\
\hline $3174.90 \quad 20$ & $(5+)$ & & \\
\hline $3224.58 \quad 23$ & $(5+)$ & & \\
\hline $3286.2 \quad 3$ & $7+$ & & \\
\hline 3324.13 & & & \\
\hline
\end{tabular}


${ }^{24} \mathrm{Mg}\left({ }^{24} \mathrm{Mg}, \alpha \mathrm{pn} \gamma\right),{ }^{28} \mathrm{Si}\left({ }^{20} \mathrm{Ne}, \alpha \mathrm{pn} \gamma\right) \quad 2007 \mathrm{Ch} 40$ (continued)

${ }^{42} \mathrm{Sc}$ Levels (continued)

\begin{tabular}{|c|c|c|c|c|c|c|}
\hline $\mathrm{E}(\text { level })^{\dagger}$ & $\mathrm{J} \pi \ddagger$ & $\mathrm{T}_{1 / 2}$ & $\mathrm{E}(\text { level })^{\dagger}$ & $\mathrm{J} \pi \ddagger$ & $\mathrm{E}(\text { level })^{\dagger}$ & $\mathrm{J} \pi \ddagger$ \\
\hline $3546.3 \quad 7$ & $(5+)$ & & 5963.24 & $9+$ & 10013.511 & $(13+)$ \\
\hline $3603.9 \mathrm{~b} \quad 3$ & $8-$ & & $6118.0^{\&} 8$ & $(8+)$ & 10451.34 & $13+$ \\
\hline $3710.9 \quad 3$ & $(5+)$ & & $6824.8 \quad 3$ & $(10+)$ & $10537.0 \quad 3$ & $(12-)$ \\
\hline 3719.14 & $(5)+$ & & $6922.0^{\mathrm{a}} 3$ & $11+$ & $10583.3^{\mathrm{b}} 4$ & 13 \\
\hline $3752.6 \quad 3$ & $7+$ & & $7184.5^{@} 4$ & $10(+)$ & 10939.58 & \\
\hline $3797.6 \quad 4$ & $(3+)$ & & 7307.73 & $11+$ & $11854.0^{\mathrm{a}} 3$ & $15+$ \\
\hline $3884.31^{@} 22$ & $6(+)$ & & $7676.8 \quad 3$ & $11+$ & $11931.8 \quad 4$ & \\
\hline $4062.2^{\&} 4$ & $(6+)$ & & 8094.93 & $(10-)$ & $12401.7 \quad 7$ & \\
\hline $4118.0^{\mathrm{b}} \quad 3$ & $10-$ & $\approx 0.5 \mathrm{n} \mathrm{s}^{\#}$ & 8205.26 & & 12450.26 & \\
\hline $4246.2 \quad 3$ & $9-$ & & $8798.7 \quad 3$ & $13+$ & $13022.5 \quad 5$ & \\
\hline 4884.13 & $(8)+$ & & $8886.9 \quad 7$ & & $13026.4 \quad 7$ & \\
\hline $4992.7 \mathrm{a} \quad 3$ & $9+$ & & 8921.14 & & $13094.4 \quad 4$ & 15 \\
\hline $5402.4 \quad 14$ & $(7+)$ & & 9004.96 & & 13419.16 & \\
\hline $5452.1^{\mathrm{b}} 3$ & $11-$ & & $9511.4^{\mathrm{a}} 3$ & $13+$ & $13620.7 \mathrm{~b} \quad 8$ & \\
\hline $5507.55^{@} 24$ & $8(+)$ & & 9620.94 & 12 & 15967.15 & 17 \\
\hline
\end{tabular}

$\dagger$ From least squares fit to E $\gamma$ data. The normalized $\chi^{2}=5.3$ for the uncertainties as quoted by $2007 \mathrm{Ch} 40$. This value is much larger than the critical $\chi^{2}=1.8$. The uncertainties of the following $\gamma$ rays were increased by a factor of 2 to get an acceptable fit with normalized $\chi^{2}=2.7: 642.2,1830.3,2668.4,2986.4,3188.9,3629.7$. It should be noted that the uncertainties for level energies quoted in table IV of $2007 \mathrm{Ch} 40$ are much larger than those given here.

¥ As proposed by $2007 \mathrm{Ch} 40$, based on literature values for low-lying levels and $\gamma(\theta)$ and DCO data for higher levels. The assignments in Adopted Levels are the same, except that some are placed in parentheses there which, in the opinion of evaluators, still lack firm arguments.

$\S$ From Adopted Levels.

\# A crude estimate by $2007 \mathrm{Ch} 40$ from transition rates and angular distributions, the value is within an order of magnitude.

(a) (A) $\gamma$ cascade based on $2+$.

$\&$ (B): $\gamma$ cascade based on $0+$.

a (C): $\gamma$ cascade based on $7+$.

b (D): $\gamma$ cascade based on $8-$.

$\gamma\left({ }^{42} \mathrm{Sc}\right)$

The DCO values in $2007 \mathrm{Ch} 40$ are for $\approx 90^{\circ}$ ( range of $69.8^{\circ}-110.2^{\circ}$ ) and forward $/ \mathrm{backward}$ angles $\left(50.1^{\circ}-129.9^{\circ} \mathrm{range}\right.$ ). The gates are on $\Delta \mathrm{J}=1$, dipole transitions, unless otherwise stated. Expected values for $\Delta \mathrm{J}=1$, dipole gate are: 1.6 for $\Delta J=2$, quadrupole or $\Delta J=0$, dipole; 1.0 for $\Delta J=1$, dipole; 0.5 to 1.9 for $\Delta J=1$, dipole+quadrupole; 1.1 to 1.7 for $\Delta J=0$, dipole+quadrupole. Expected values for $\Delta J=2$, quadrupole gate are: 1.0 for $\Delta J=2$, quadrupole or $\Delta J=0$, dipole; 0.6 for $\Delta J=1$, dipole; 0.3 to 1.2 for $\Delta \mathrm{J}=1$, dipole+quadrupole; 0.6 to 1.1 for $\Delta \mathrm{J}=0$, dipole+quadrupole.

Values of $\mathrm{A}_{2}$ and $\mathrm{A}_{4}$ coefficients in $\gamma(\theta)$ and DCO ratios in $\gamma \gamma(\theta)$ are averages from the two experiments.

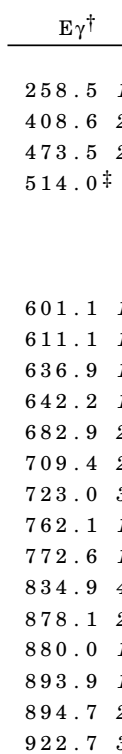

\begin{tabular}{|c|c|c|c|}
\hline E(level) & $\mathrm{I} \gamma(\mathrm{Mg}+\mathrm{Mg}) \S$ & Mult.@ & $\mathrm{I} \gamma(\mathrm{Si}+\mathrm{Ne})^{\#}$ \\
\hline 1845.07 & $18.0 \quad 6$ & D & $15.0 \quad 5$ \\
\hline 3224.58 & $2.29 \quad 24$ & & $2.09 \quad 19$ \\
\hline 3797.6 & $3.5 \quad 3$ & & $3.20 \quad 22$ \\
\hline 4118.0 & $89 \quad 3$ & $(\mathrm{Q})$ & $90 \quad 3$ \\
\hline 2187.67 & $4.4 \quad 3$ & D & $4.30 \quad 22$ \\
\hline 611.12 & 665 & D & 555 \\
\hline 2223.50 & 14.35 & D & 11.54 \\
\hline 4246.2 & 10.95 & $\mathrm{D}+\mathrm{Q}$ & 10.34 \\
\hline 2269.48 & $3.04 \quad 24$ & (D) & $2.72 \quad 19$ \\
\hline 2295.98 & $2.82 \quad 22$ & & $2.38 \quad 18$ \\
\hline 3719.1 & $1.74 \quad 17$ & & $1.46 \quad 12$ \\
\hline 2607.19 & 11.34 & D & 9.43 \\
\hline 2996.11 & $7.9 \quad 4$ & D & $6.0 \quad 3$ \\
\hline 3224.58 & $1.95 \quad 24$ & & $1.70 \quad 19$ \\
\hline 2389.2 & $8.0 \quad 5$ & & $6.7 \quad 4$ \\
\hline 1491.10 & 18.18 & & $15.6 \quad 6$ \\
\hline 1510.92 & 325 & $(\mathrm{Q}) \&$ & $32 \quad 5$ \\
\hline 3710.9 & $3.8 \quad 3$ & $\mathrm{D}$ & $3.7 \quad 3$ \\
\hline 2433.89 & $4.9 \quad 6$ & $\mathrm{D}^{\&}$ & $3.5 \quad 3$ \\
\hline
\end{tabular}

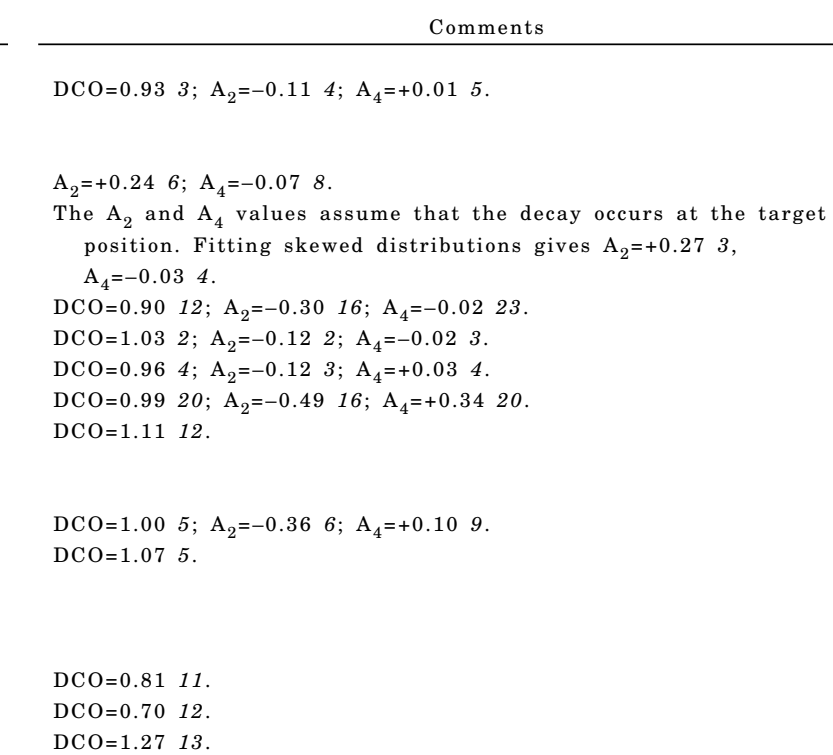

Continued on next page (footnotes at end of table) 


\begin{tabular}{|c|c|c|}
\hline $\mathrm{I} \gamma(\mathrm{Mg}+\mathrm{Mg})^{\S}$ & Mult.@ & $\mathrm{I} \gamma(\mathrm{Si}+\mathrm{Ne})^{\#}$ \\
\hline 4.13 & D & $3.47 \quad 21$ \\
\hline $42.7 \quad 14$ & D & $33.8 \quad 11$ \\
\hline $1.44 \quad 15$ & & $1.46 \quad 12$ \\
\hline $4.1 \quad 3$ & & $3.06 \quad 20$ \\
\hline $1.80 \quad 18$ & & $1.20 \quad 13$ \\
\hline $35.1 \quad 12$ & (Q) & $34.8 \quad 11$ \\
\hline $4.4 \quad 3$ & $\mathrm{Q}^{\&}$ & 3.5921 \\
\hline 13.25 & (D) & $10.5 \quad 4$ \\
\hline
\end{tabular}

$8.7 \quad 3$

$3.3 \quad 3$

$3.9 \quad 3$

5.13

48.115

$1.00 \quad 18$

$10.7 \quad 5$

2.14

9.27

6.43

3.54

$1.36 \quad 17$

$3.6 \quad 3$

1.64

5.78

2.13

$2.04 \quad 25$

$1.98 \quad 24$

6.64

$0.31 \quad 17$

22.88

66.922

18.08

$1.7 \quad 3$

$4.4 \quad 3$

$2.7 \quad 3$

$4.4 \quad 3$

12.96

3.46

5.13

12.36

15.26

20.28

7.04

23.48

27.59

163

$\begin{array}{rll}7.75 & \mathrm{Q} \\ 1.90 \quad 17 & (\mathrm{Q}) \& \\ 100 & 4 & \mathrm{D}\end{array}$

\section{$\mathrm{Q}$
$\mathrm{D}^{\&}$}

(Q)

$\mathrm{D}+\mathrm{Q}$

0.54

$7.0 \quad 3$

$2.58 \quad 22$

3. 3321

$3.92 \quad 20$

$45.0 \quad 14$

$0.90 \quad 13$

9.94

$2.7 \quad 3$

7.44

4.9120

3.23

1. $57 \quad 13$

$2.92 \quad 18$

1. $95 \quad 25$

6.46

1.6121

$1.88 \quad 17$

1. $98 \quad 19$

(Q)

(Q)

(Q)

(Q)

(Q)

(Q)

(Q)

$\mathrm{Q}$

Q

(Q)

$\mathrm{D}+\mathrm{Q}$

$\mathrm{D}+\mathrm{Q}$

(Q)

1004

D

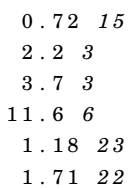

6.13

0. $33 \quad 12$

20.97

63.520

15.76

$1.66 \quad 22$

$4.06 \quad 22$

1. $96 \quad 20$

3. $30 \quad 22$

11.14

3.94

6. $3 \quad 3$

11.95

14.25

19.17

7.93

$22.8 \quad 8$

22.27

$14.7 \quad 18$

7.84

$2.34 \quad 14$ 1004
(Q)

0. $99 \quad 11$

2. $59 \quad 19$

$4.17 \quad 21$

$12.5 \quad 5$

1.394

1. $97 \quad 16$ $\begin{array}{llr}3037.3 & 7 & 13620.7 \\ 3054.9 & 5 & 11854.0 \\ 3091.4 & 10 & 10013.5 \\ 3188.9 & 2 & 7307.7 \\ 3262.5 & 7 & 10939.5 \\ 3480.4 & 6 & 12401.7\end{array}$
Comments

$\mathrm{DCO}=1.03 \quad 15$

$\mathrm{DCO}=0.982 ; \mathrm{A}_{2}=-0.122 ; \mathrm{A}_{4}=0.003$.

$\mathrm{A}_{2}=+0.367 ; \mathrm{A}_{4}=+0.029$.

$\mathrm{DCO}=1.24 \quad 17$.

$\mathrm{A}_{2}=+0.4010 ; \mathrm{A}_{4}=-0.1513$

Mult.: $\quad \Delta \mathrm{J}=(0)$, dipole transition.

$\mathrm{DCO}=1.6111 ; \mathrm{A}_{2}=+0.2911 ; \mathrm{A}_{4}=-0.1616$.

$\mathrm{DCO}=0.6410$

$\mathrm{DCO}=1.5711$

$\mathrm{A}_{2}=-0.387 ; \mathrm{A}_{4}=+0.259$.

$\mathrm{DCO}=1.5313 ; \mathrm{A}_{2}=+0.3912 ; \mathrm{A}_{4}=-0.0316$.

$\mathrm{DCO}=1.53$.

$\mathrm{A}_{2}=+0.326 ; \mathrm{A}_{4}=+0.029$.

$\mathrm{A}_{2}=+0.303 ; \mathrm{A}_{4}=0.003$.

$\mathrm{A}_{2}=+0.342 ; \mathrm{A}_{4}=-0.073$.

$\mathrm{A}_{2}=+0.347 ; \mathrm{A}_{4}=-0.1010$.

$\mathrm{A}_{2}=+0.5115 ; \mathrm{A}_{4}=0$.

$\mathrm{A}_{2}=+0.386 ; \mathrm{A}_{4}=-0.058$.

$\mathrm{A}_{2}=+0.496 ; \mathrm{A}_{4}=-0.059$.

$\mathrm{A}_{2}=+0.20 \quad 4 ; \mathrm{A}_{4}=-0.126$.

$\mathrm{A}_{2}=+0.249 ; \mathrm{A}_{4}=-0.2112$.

$\mathrm{A}_{2}=+0.4012 ; \mathrm{A}_{4}=-0.0417$.

$\mathrm{A}_{2}=+0.355 ; \mathrm{A}_{4}=-0.037$.

$\mathrm{A}_{2}=-0.367 ; \mathrm{A}_{4}=+0.169$.

$\mathrm{A}_{2}=+0.095 ; \mathrm{A}_{4}=-0.157$.

Mult.: $\Delta J=0$, dipole transition.

$\mathrm{A}_{2}=+0.249 ; \mathrm{A}_{4}=-0.2212$.

$\mathrm{DCO}=1.03$.

$\mathrm{A}_{2}=-0.367 ; \mathrm{A}_{4}=-0.0310$.

The $\mathrm{A}_{2}$ and $\mathrm{A}_{4}$ values assume that the decay occurs at the target position. Fitting skewed distributions gives $\mathrm{A}_{2}=-0.323$, $\mathrm{A}_{4}=-0.014$. 


\begin{tabular}{|c|c|c|c|c|c|c|c|}
\hline \multirow{3}{*}{\multicolumn{3}{|c|}{$\mathrm{E} \gamma^{\dagger}$}} & \multirow[b]{3}{*}{$\mathrm{E}($ level) } & \multirow[b]{3}{*}{$\mathrm{I} \gamma(\mathrm{Mg}+\mathrm{Mg}) \S$} & \multicolumn{3}{|c|}{${ }^{24} \mathrm{Mg}\left({ }^{24} \mathrm{Mg}, \alpha p n \gamma\right),{ }^{28} \mathrm{Si}\left({ }^{20} \mathrm{Ne}, \alpha p n \gamma\right)$} \\
\hline & & & & & \multirow[b]{2}{*}{ Mult. ${ }^{@}$} & $\gamma\left({ }^{42} \mathrm{~S}\right.$ & \multirow[b]{2}{*}{ Comments } \\
\hline & & & & & & $\mathrm{I} \gamma(\mathrm{Si}+\mathrm{Ne})^{\#}$ & \\
\hline 3500 & $0.7 \ddagger$ & & 4118.0 & $18.5 \quad 10$ & $(0)$ & 19.99 & $\begin{array}{l}\mathrm{A}_{2}=+0.53 \quad 6 ; \mathrm{A}_{4}=+0.088 . \\
\text { Mult.: } 2007 \mathrm{Ch} 40 \text { assign E3. } \\
\text { The } \mathrm{A}_{2} \text { and } \mathrm{A}_{4} \text { values assume that the decay occurs at the target } \\
\text { position. Fitting skewed distributions gives } \mathrm{A}_{2}=+0.595, \\
\mathrm{~A}_{4}=+0.137 \text {. }\end{array}$ \\
\hline 3510 & 0.9 & & 13022.5 & $3.32 \quad 24$ & & $2.94 \quad 17$ & \\
\hline 3529 & 9.1 & 3 & 10451.3 & $4.9 \quad 3$ & $(\mathrm{Q})$ & $5.6 \quad 3$ & $\mathrm{~A}_{2}=+0.4915 ; \mathrm{A}_{4}=-0.0623$ \\
\hline 3629 & 9.7 & 1 & 4246.2 & $31.9 \quad 18$ & $Q^{\&}$ & $32.8 \quad 15$ & $\begin{array}{l}\mathrm{DCO}=1.0414 . \\
\text { Mult.: } 2007 \mathrm{Ch} 40 \text { assign } \mathrm{M} 2 .\end{array}$ \\
\hline 3651 & 1.3 & 5 & 12450.2 & $1.84 \quad 19$ & & $2.04 \quad 15$ & \\
\hline 3907 & 7.5 & 5 & 13419.1 & $2.36 \quad 20$ & & $2.32 \quad 14$ & \\
\hline 4087 & 7.0 & 5 & 8205.2 & $2.67 \quad 24$ & & $3.14 \quad 18$ & \\
\hline 4227 & 7.5 & 6 & 13026.4 & $2.14 \quad 22$ & & $2.33 \quad 18$ & \\
\hline 4267 & 7.3 & 2 & 4884.1 & $20.0 \quad 18$ & $D+Q$ & $15.3 \quad 11$ & $\mathrm{~A}_{2}=-0.087 ; \mathrm{A}_{4}=+0.249$ \\
\hline 4375 & 5.8 & 1 & 4992.7 & 897 & (Q) & 756 & $\mathrm{~A}_{2}=+0.355 ; \mathrm{A}_{4}=+0.037$ \\
\hline 4640 & 0.4 & 6 & 8886.9 & $3.0 \quad 3$ & & $2.66 \quad 20$ & \\
\hline 5130 & 0.8 & 2 & 10583.3 & 11.24 & Q & $12.4 \quad 4$ & $\mathrm{~A}_{2}=+0.306 ; \mathrm{A}_{4}=-0.119$ \\
\hline 5346 & 6.5 & 7 & 5963.2 & 3.66 & & 4.05 & \\
\hline 5502 & 2.6 & 2 & 9620.9 & 8.54 & Q & 9.43 & $\mathrm{DCO}=1.83 ; \mathrm{A}_{2}=+0.2710 ; \mathrm{A}_{4}=+0.0413$ \\
\hline 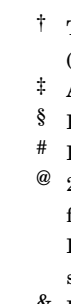 & $\begin{array}{l}\text { The } \\
\text { (max } \\
\text { Actu } \\
\text { Fron } \\
\text { Fron } \\
2007 \\
\text { for } \\
\text { Dipo } \\
\text { shor }\end{array}$ & $\begin{array}{l}\gamma-\mathrm{r} \\
\text { xim } \\
\text { ual } \\
\mathrm{m}^{2} \\
\mathrm{~m}^{28} \\
7 \mathrm{Ch} \\
\Delta \mathrm{J}= \\
\text { ole } \\
\mathrm{rt} \mathrm{li}\end{array}$ & 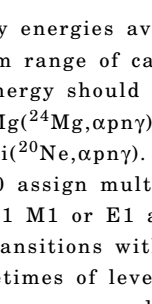 & $\begin{array}{l}\text { ges (by } 2007 \mathrm{C} \\
\text { ration curve), } \\
\text { slightly highe } \\
\text { larities for m } \\
\text { Q for } \Delta \mathrm{J}=2 \text {, } \\
\text { xpected M1 }\end{array}$ & $\begin{array}{l}\text { h40) from } \\
\text { systemati } \\
\text { r because }\end{array}$ & $\begin{array}{l}\text { two experim } \\
\text { acertainties ca } \\
\text { the shift caus }\end{array}$ & $\begin{array}{l}\text { based only on } J \pi \text { assignments. The evaluators assign mult=D } \\
\text { orting angular distribution/correlation data are available. } \\
\text { ponent. The mult=Q transitions are most likely E2 from expected }\end{array}$ \\
\hline
\end{tabular}

\section{${ }^{39} \mathrm{~K}(\alpha, \mathrm{n}),(\alpha, \mathrm{n} \gamma) \quad 1965 \mathrm{Ne} 02,1974 \mathrm{Br} 04,1972 \mathrm{Be} 50$}

1965Ne02: E=7.8-12 MeV alpha beam of 5-10 nA from the Florida State University Tandem Van de Graaff accelerator. Potassium targets of $\mathrm{KI}$ and $\mathrm{KBr}$ powder in disks of $2.5 \mathrm{~cm}$ diameter or evaporated onto tantalum backings. A $5.1 \mathrm{~cm}$ by $5.1 \mathrm{~cm}$ scintillator for detecting $\beta$ activity of the residual nucleus and a $7.6 \mathrm{~cm}$ by $7.6 \mathrm{~cm}$ NaI detector for $\gamma$-rays. Measured cross sections, $\beta+\gamma$ coin of decay of ${ }^{42} \mathrm{Sc}$ ground state and isomer activities, $\mathrm{T}_{1 / 2}$. Levels in ${ }^{42} \mathrm{Sc}$ deduced from observed thresholds.

1974Br04: $\mathrm{E}=10.4 \mathrm{MeV}$ alpha beam from the Stony Brook tandem Van de Graaff accelerator. Target of a $140 \mu \mathrm{g} / \mathrm{cm}^{2}$ natural KI. Ge(Li) detector. Measured E $\gamma$, I $\gamma$. Deduced levels, $\mathrm{T}_{1 / 2}$ using Recoil Distance Method (RDM) for levels at 1490 and $1511 \mathrm{keV}$.

1972Be50: $\mathrm{E}=15 \mathrm{MeV}$ alpha beam from the Stanford FN tandem Van de Graaff accelerator. Natural potassium target. A $\mathrm{Ge}(\mathrm{Li})$ detector for detecting $\gamma$-rays and a scintillator for neutrons. Measured $\mathrm{E} \gamma, \mathrm{n} \gamma$-coin. Deduced levels, $\mathrm{T}_{\mathrm{a} / 2}$ by RDM for levels at 1490 and $1511 \mathrm{keV}$.

1961Sm05: E=8.29 MeV alpha beam from Yale cyclotron. Natural potassium target. Neutrons were detected were detected by their proton recoils in nuclear emulsions. Measured neutron spectra. Neutron group corresponding to ${ }^{42} \mathrm{Sc}$ ground state is reported.

\section{${ }^{42}$ Sc Levels}

\begin{tabular}{|c|c|c|c|}
\hline $\mathrm{E}(\text { level })^{\dagger}$ & $\mathrm{J} \pi \ddagger$ & $\mathrm{T}_{1 / 2}$ & Comments \\
\hline 0.0 & $0+$ & $0.65 \mathrm{~s} \S 1$ & \\
\hline 611 & $1+$ & $60.6 \mathrm{~s} \S 4$ & $\mathrm{E}$ (level): 60030 in $1965 \mathrm{Ne} 02$. \\
\hline 616 & $7+$ & & $\mathrm{E}$ (level): 52620 in $1965 \mathrm{Ne} 02$ \\
\hline 1490 & $3+$ & 31 ps 9 & $\begin{array}{l}\mathrm{E}(\text { level): } 142030 \text { in } 1965 \mathrm{Ne} 02 . \\
\mathrm{T}_{1 / 2}: \text { Weighted average of } 33 \mathrm{ps} 9 \text { in } 1974 \mathrm{Br} 04 \text { and } 31 \mathrm{ps} 5 \text { in } 1972 \mathrm{Be} 50 \text {. } \\
\mathrm{E} \text { (level): } 134030 \text { in } 1965 \mathrm{Ne} 02 .\end{array}$ \\
\hline & & & $\mathrm{T}_{1 / 2}$ : Weighted average of $36 \mathrm{ps} 10$ in $1974 \mathrm{Br} 04$ and $51 \mathrm{ps} 8$ in $1972 \mathrm{Be} 50$. \\
\hline
\end{tabular}


${ }^{39} \mathrm{~K}(\alpha, \mathrm{n}),(\alpha, \mathrm{n} \gamma) \quad 1965 \mathrm{Ne02}, 1974 B \mathrm{Br}, 1972 B e 50$ (continued)

${ }^{42} \mathrm{Sc} \mathrm{Levels} \mathrm{(continued)}$

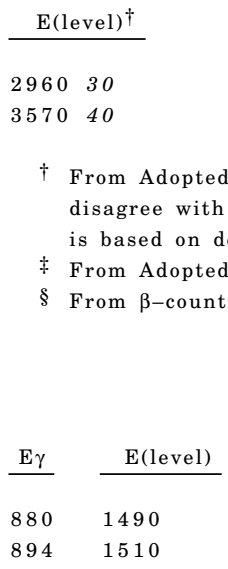




\section{${ }^{40} \mathrm{Ca}\left({ }^{3} \mathrm{He}, \mathrm{p}\right) \quad 1968 \mathrm{Pu} 04,1965 \mathrm{C} 101,1966 \mathrm{Zu01}$}

Target ${ }^{40} \mathrm{Ca} \mathrm{J} \pi=0+$.

1968Pu04: E=18 MeV ${ }^{3} \mathrm{He}$ beam was produced at the tandem Van de Graaff accelerator at Max-Planck-lnstitut Jur Kernphysik. Targets of natural calcium evaporated onto carbon backings. Proton were momentum analyzed using a broad-range magnetic spectrograph and detected by nuclear emulsions. Measured $\sigma\left(E_{p}, \theta\right)$. Deduced levels, J $\pi$, L from DWBA analysis.

1965Cl01: levels up to $3.6 \mathrm{MeV}$ excitation. Level energies from this work are quoted by $1971 \mathrm{Sh} 16$ as a private communication.

1966Zu01: $\mathrm{E}=10,12,15 \mathrm{MEV}$. Measured $\sigma\left(\mathrm{E}_{\mathrm{p}}, \theta\right)$. 17 groups reported up to $3.5 \mathrm{MeV}$ excitation energy.

1970Sc22: $\mathrm{E}=15,18 \mathrm{MeV}$. Measured $\sigma\left(\mathrm{E}_{\mathrm{p}}, \theta\right)$, DWBA analysis. L-transfers for $\mathrm{GS}(\mathrm{L}=0)$ and $1590(\mathrm{~L}=2)$ group

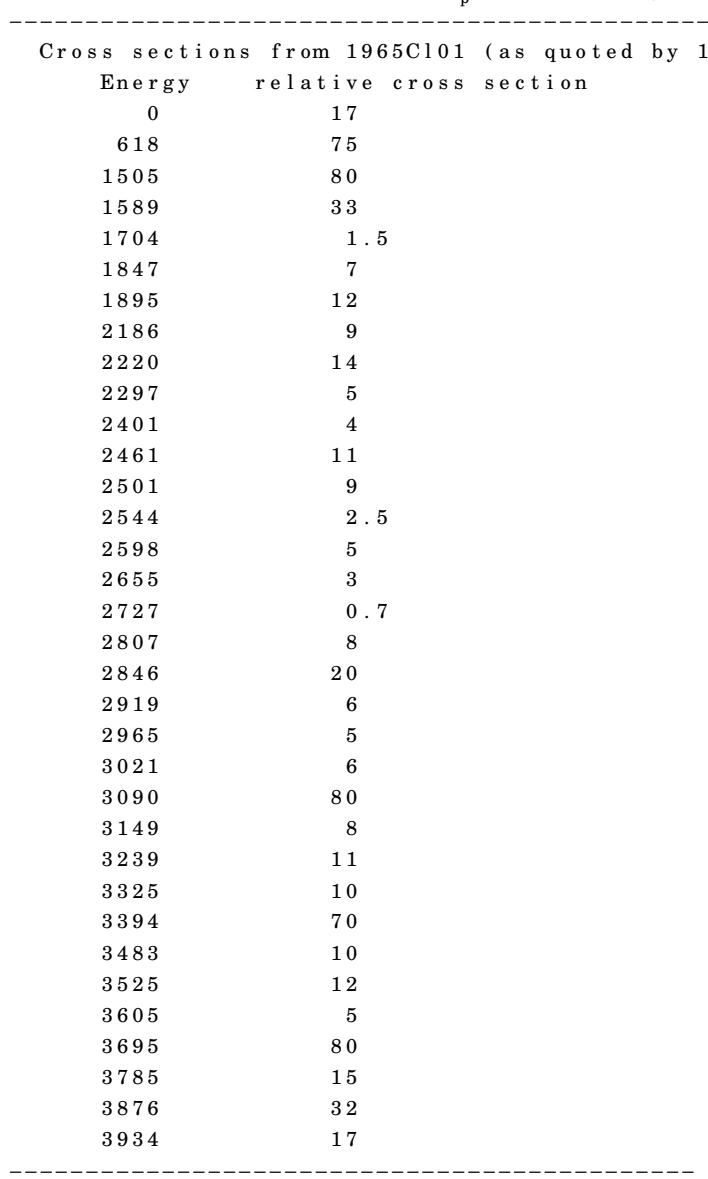

${ }^{42} \mathrm{Sc} \mathrm{Levels}$

\begin{tabular}{|c|c|c|c|c|c|}
\hline $\mathrm{E}(\text { level })^{\dagger}$ & $\mathrm{L}^{\dagger}$ & Relative intensity \& & $\mathrm{E}(\text { level })^{\dagger}$ & $\mathrm{L}^{\dagger}$ & Relative intensity \& \\
\hline 0.0 & 0 & $100^{\mathrm{a}}$ & $2655 * 15$ & & \\
\hline $615 \S 10$ & $0+2$ & $200^{\mathrm{a}}$ & $2727 ? \ddagger 15$ & & \\
\hline $1510 \# \quad 10$ & $2+4$ & 120 & $2807 \ddagger 15$ & & \\
\hline $1590 \quad 10$ & 2 & 45 & $2850 \quad 10$ & 4 & \\
\hline $1704 ? \ddagger 15$ & & & $2919 \ddagger 15$ & & \\
\hline $1847 \ddagger 15$ & & & $2965 \neq 15$ & & \\
\hline $1890 \quad 10$ & 0 & $17^{\mathrm{a}}$ & $3021 ? \ddagger 15$ & & \\
\hline $2186 \neq 15$ & & & $3100 \quad 10$ & 4 & \\
\hline 2220 ‡@ 15 & 2 & 17 & $3149 ? \ddagger 15$ & & \\
\hline $2297 \ddagger 15$ & & & $3250 \quad 10$ & 6 & \\
\hline $2401 \neq 15$ & & & $3330 \quad 10$ & & \\
\hline $2461 \neq 15$ & & & $3390 \quad 10$ & 2 & 130 \\
\hline $2500 \quad 10$ & 2 & 18 & $3483 \neq 15$ & & \\
\hline 2544 \# 15 & & & 3525 末 15 & & \\
\hline $2598 * 15$ & & & $3605 \neq 15$ & & \\
\hline
\end{tabular}

Continued on next page (footnotes at end of table) 
${ }^{40} \mathrm{Ca}\left({ }^{3} \mathrm{He}, \mathrm{p}\right) \quad 1968 \mathrm{Pu} 04,1965 \mathrm{Cl01}, 1966 \mathrm{Zu01}$ (continued)

\begin{tabular}{|c|c|c|c|c|c|c|}
\hline \multirow{3}{*}{\multicolumn{2}{|c|}{$\mathrm{E}(\text { level })^{\dagger}$}} & \multirow[b]{3}{*}{$\mathrm{L}^{\dagger}$} & \multirow[b]{3}{*}{ Relative intensity\& } & \multicolumn{3}{|c|}{$1968 \mathrm{Pu} 04,1965 \mathrm{Cl01}, 1966 \mathrm{Zu} 01$} \\
\hline & & & & \multicolumn{3}{|c|}{${ }^{42} \mathrm{Sc}$ Levels (continued) } \\
\hline & & & & \multicolumn{2}{|c|}{$\mathrm{E}(\text { level })^{\dagger}$} & Relative intensity \& \\
\hline 3690 & 10 & $0+2$ & $130^{\mathrm{a}}$ & 5480 & 10 & \\
\hline 3780 & 10 & 2 & 30 & 5640 & 10 & \\
\hline 3860 & 10 & $0+2$ & $38^{\mathrm{a}}$ & 5810 & 10 & $65^{\mathrm{a}}$ \\
\hline 3930 & 10 & 2 & 90 & 5970 & 10 & $30^{\mathrm{a}}$ \\
\hline 4720 & 10 & & 45 & 6090 & 10 & \\
\hline 4830 & 10 & & 35 & 6180 & 10 & \\
\hline 5440 & 10 & & & & & \\
\hline \multicolumn{7}{|c|}{$\dagger$ From $1968 \mathrm{Pu} 04$, unless otherwise stated. } \\
\hline \multicolumn{7}{|c|}{$\ddagger$ From $1965 \mathrm{Cl} 101$, as quoted by $1971 \mathrm{Sh} 16$. } \\
\hline \multicolumn{7}{|c|}{$\S$ doublet: $610+620$} \\
\hline \multicolumn{7}{|c|}{$\#$ doublet: $1500+1520$} \\
\hline \multicolumn{7}{|c|}{ @ $220010(1968 \mathrm{Pu} 04)$ is a composite of $2186+2220$} \\
\hline \multicolumn{7}{|c|}{ \& From $1968 \mathrm{Pu} 04$. Cross section for $\mathrm{L}=2$ at $15^{\circ}$, unless otherwise stated. } \\
\hline
\end{tabular}

\section{${ }^{40} \mathrm{Ca}\left({ }^{3} \mathrm{He}, \mathrm{p} \gamma\right) \quad 1971 \mathrm{Ro30,2007Sc26}$}

1971Ro30: $\mathrm{E}=8.4,8.6 \mathrm{MeV}{ }^{3} \mathrm{He}$ beam from the Utrecht MV tandem Van de Graaff accelerator. Targets of natural calcium metal on carbon foils. Protons were detected with two $1.5 \mathrm{~mm}$ Si surface-barrier detectors and $\gamma$-rays were detected with a Ge(Li) detector at 90'. Measured E $\gamma$, I $\gamma$, p $\gamma$-coin. Deduced levels, branching ratios, lifetimes by Doppler Shift Attenuation Method (DSAM).

2007Sc26: E=9 MeV beam from FN Tandem accelerator at Cologne university. Natural calcium target. Measured E $\gamma$, I $\gamma$, $\gamma \gamma, \gamma \gamma(\theta)$ using HORUS array comprised of nine HPGe detectors and a Euroball cluster detector. Two HPGe detectors were at $45^{\circ}$ to the beam axis, and two at $135^{\circ}$. The cluster Ge detector and five HPGe detectors were in a plane perpendicular to the beam axis.

Others:

2004Or03: $\mathrm{E}=4.2,5.1 \mathrm{MeV}$. Measured $\mathrm{E} \gamma, \mathrm{I} \gamma$, DSA.

1973Ha10: $\mathrm{E}=10.0-11.5 \mathrm{MeV}$. Targets of natural calcium on Ta backings. Protons were detected in annular silicon surface-barrier detectors and $\gamma$-rays in a Ge(Li) detector (FWHM=10 keV at $1.33 \mathrm{MeV}$ ). Measured E $\gamma$, p $\gamma-c o i n$. Deduced levels, lifetimes by DSAM.

1969Ni03: $\mathrm{E}=8.0-9.0 \mathrm{MeV}{ }^{3} \mathrm{He}$ beam from the University of Liverpool tandem accelerator. Natural calcium targets. Proton counter, $\mathrm{Ge}(\mathrm{Li})$ and $\mathrm{NaI}(\mathrm{Tl})$ detectors. Measured $\mathrm{E} \gamma, \mathrm{I} \gamma, \gamma(\theta), \gamma \gamma$-coin, p $\gamma$-coin. Deduced levels, branching ratios, mixing ratios and lifetimes by DSAM.

1974Br04: $\mathrm{E}=10.5 \mathrm{MeV}{ }^{3} \mathrm{He}$ beam from the Stony Brook tandem Van de Graaff accelerator. Target of a $50 \mu \mathrm{g} / \mathrm{cm}^{2} \mathrm{natural}$ $\mathrm{Ca}$ on a Au backing. Ge(Li) detector. Measured E $\gamma$, I $\gamma$. Deduced levels, $\mathrm{T}_{1 / 2}$ using Recoil Distance Method (RDM) for levels at 1490 and $1511 \mathrm{keV}$.

1970Fa09: $\mathrm{E}=10 \mathrm{MeV}$. Measured $\mathrm{E} \gamma, \mathrm{p} \gamma(\theta)$.

1970Ba26 (also 1970AnZZ): $\mathrm{E}=10,15 \mathrm{MeV}$. Measured $\mathrm{E} \gamma, \mathrm{p} \gamma(\theta)$. Deduced levels, J $\pi$, branching ratios, mixing ratios.

1966Zu01: E=10, 12, $15 \mathrm{MeV}$. Measured p $\gamma$-coin $\gamma(\theta)$.

\section{${ }^{42} \mathrm{Sc} \mathrm{Levels}$}

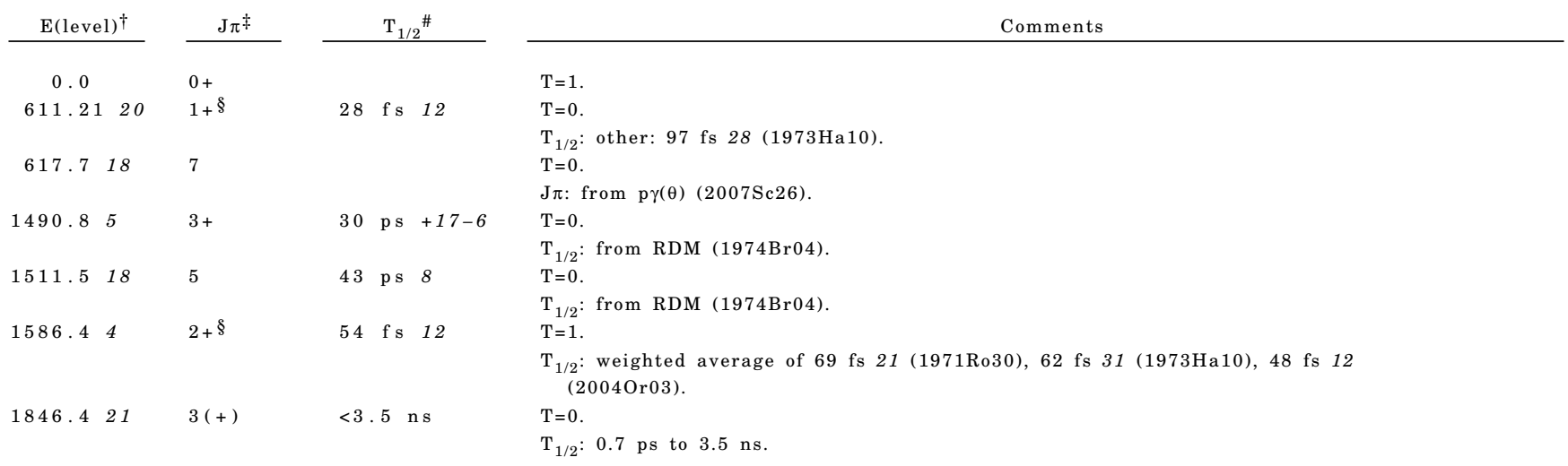


${ }^{40} \mathrm{Ca}\left({ }^{3} \mathrm{He}, \mathrm{p} \gamma\right) \quad 1971 \mathrm{Ro30}, 2007 \mathrm{Sc26}$ (continued)

${ }^{42} \mathrm{Sc}$ Levels (continued)

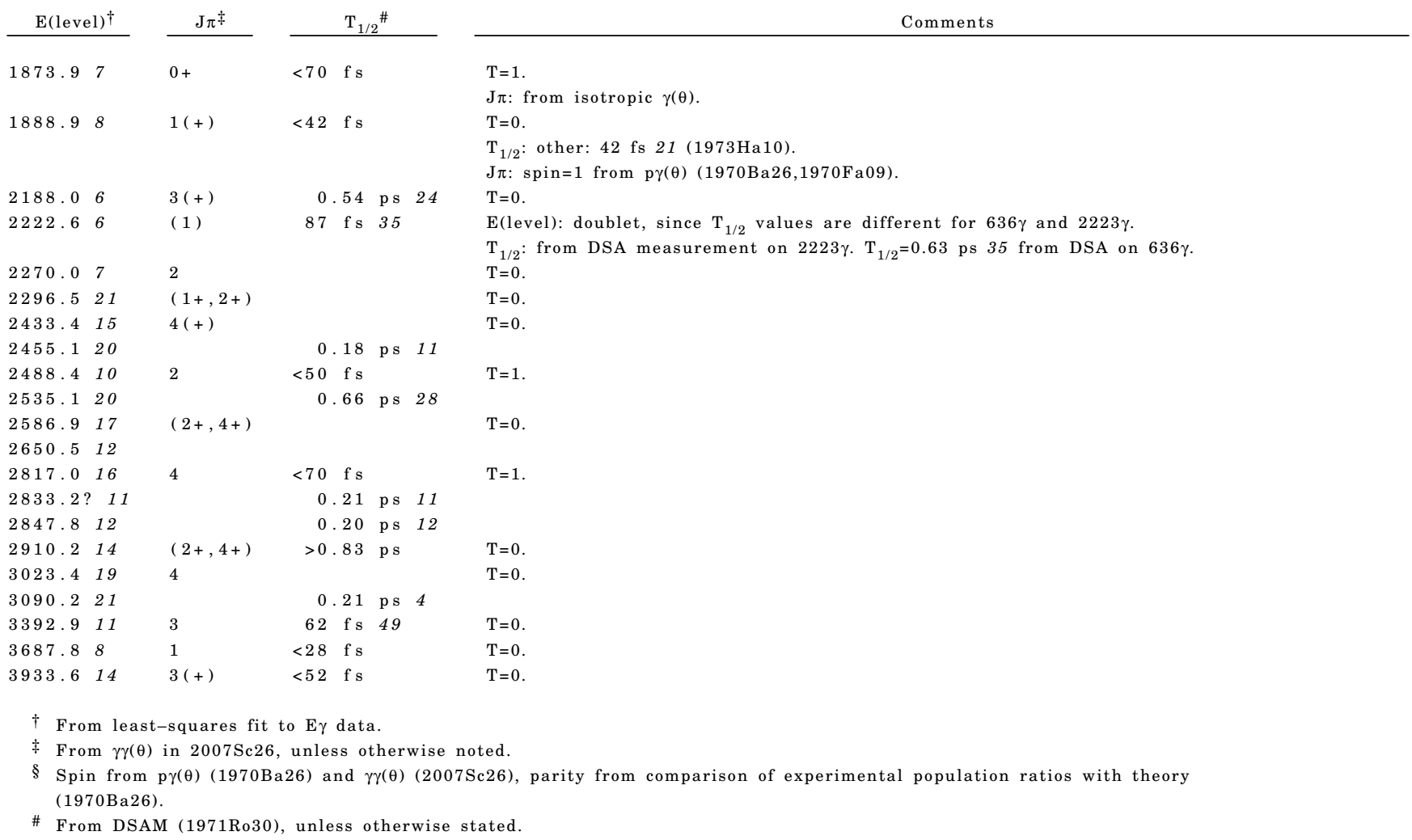

$\gamma\left({ }^{42} \mathrm{Sc}\right)$

Detailed $\gamma \gamma(\theta)$ plots are shown by $2007 \mathrm{Sc} 26$ for the following cascades with best fits indicated for spin-sequence and mixing ratios of both transitions in a cascade: 601-975, 709-975, 880-611, 905-1875, 923-894, 943-880,975-611, $996-880,1096-880,1235-611,1305-894,1325-880,1420-880,1513-894,1658-611,1875-611,2101-975,2347-975$.

A transition from 1880 doublet (composite of 1874 and 1889 levels here) to 1586 level (shown by $1969 \mathrm{Ni} 03$ and 1970Ba26) has been omitted here since it is not confirmed in any other study.

$\mathrm{A}_{2}$ and $\mathrm{A}_{4}$ are from $1969 \mathrm{Ni03}$ using $\mathrm{NaI}(\mathrm{Tl})$ detector.

\begin{tabular}{|c|c|c|c|c|c|c|c|}
\hline E(level) & $\mathrm{E} \gamma^{\dagger}$ & & $\mathrm{I} \gamma^{\dagger}$ & & Mult. ${ }^{@}$ & $\delta^{@}$ & \\
\hline 611.21 & 611.2 & 2 & 100 & & (M1) & & \\
\hline 1490.8 & 879.6 & 4 & 100 & & E 2 & & \\
\hline 1511.5 & 893.8 & 4 & 100 & & $(\mathrm{E} 2(+\mathrm{M} 3))$ & -0.07 & 5 \\
\hline \multirow[t]{2}{*}{1586.4} & 975.2 & 4 & $91^{\#}$ & 6 & $(\mathrm{M} 1+\mathrm{E} 2)$ & +0.05 & 3 \\
\hline & 1586.1 & 11 & $9 \#$ & 2 & & & \\
\hline 1846.4 & $\begin{array}{r}2602 \\
1235^{*}\end{array}$ & & 100 & & $(\mathrm{Q}(+\mathrm{O}))$ & -0.12 & 15 \\
\hline 1873.9 & 1262.5 & 7 & 100 & & $\mathrm{D}$ & & \\
\hline
\end{tabular}

Comments

$\mathrm{A}_{2}=-0.481 ; \mathrm{A}_{4}=-0.031$.

E $\gamma$ : weighted average of 611.55 from $1969 \mathrm{Ni03}$ and 611.22 from $1973 \mathrm{Ha} 10$.

Mult.: dipole from $\gamma(\theta)(1969 \mathrm{Ni} 03)$ and $\gamma \gamma(\theta)(2007 \mathrm{Sc} 26) ; \Delta \pi$ requires $M 1$.

$\mathrm{A}_{2}=+0.224 ; \mathrm{A}_{4}=-0.194(1969 \mathrm{Ni} 03)$.

E $\gamma$ : weighted average from $1971 \mathrm{Ro} 30,1973 \mathrm{Ha} 10$ and $1969 \mathrm{Ni} 03$.

Mult.: from $\gamma(\theta)(1969 \mathrm{Ni03})$ and RUL

$\delta: \delta(\mathrm{O} / \mathrm{Q})=-0.013(2007 \mathrm{Sc} 26),-0.0724(1970 \mathrm{Ba} 26),+0.397$ or $+0.257(1969 \mathrm{Ni} 03)$.

Mult.: $\gamma \gamma(\theta)(2007 \mathrm{Sc} 26)$ and RUL.

$\delta$ : or -0.1612 .

E $\gamma$ : weighted average from 1971Ro30, $1973 \mathrm{Ha} 10$ and $1969 \mathrm{Ni} 03$.

E $\gamma$ : weighted average from $1971 \mathrm{Ro} 30,1973 \mathrm{Ha} 10$ and $1969 \mathrm{Ni} 03$

Mult., $\delta$ : from 1970Ba26. Other: $\delta=0.00021$ (2007Sc26).

Mult.: $\gamma \gamma(\theta)(2007 \mathrm{Sc} 26)$

$\mathrm{A}_{2}=+0.033 ; \mathrm{A}_{4}=+0.043(1969 \mathrm{Ni0})$. 


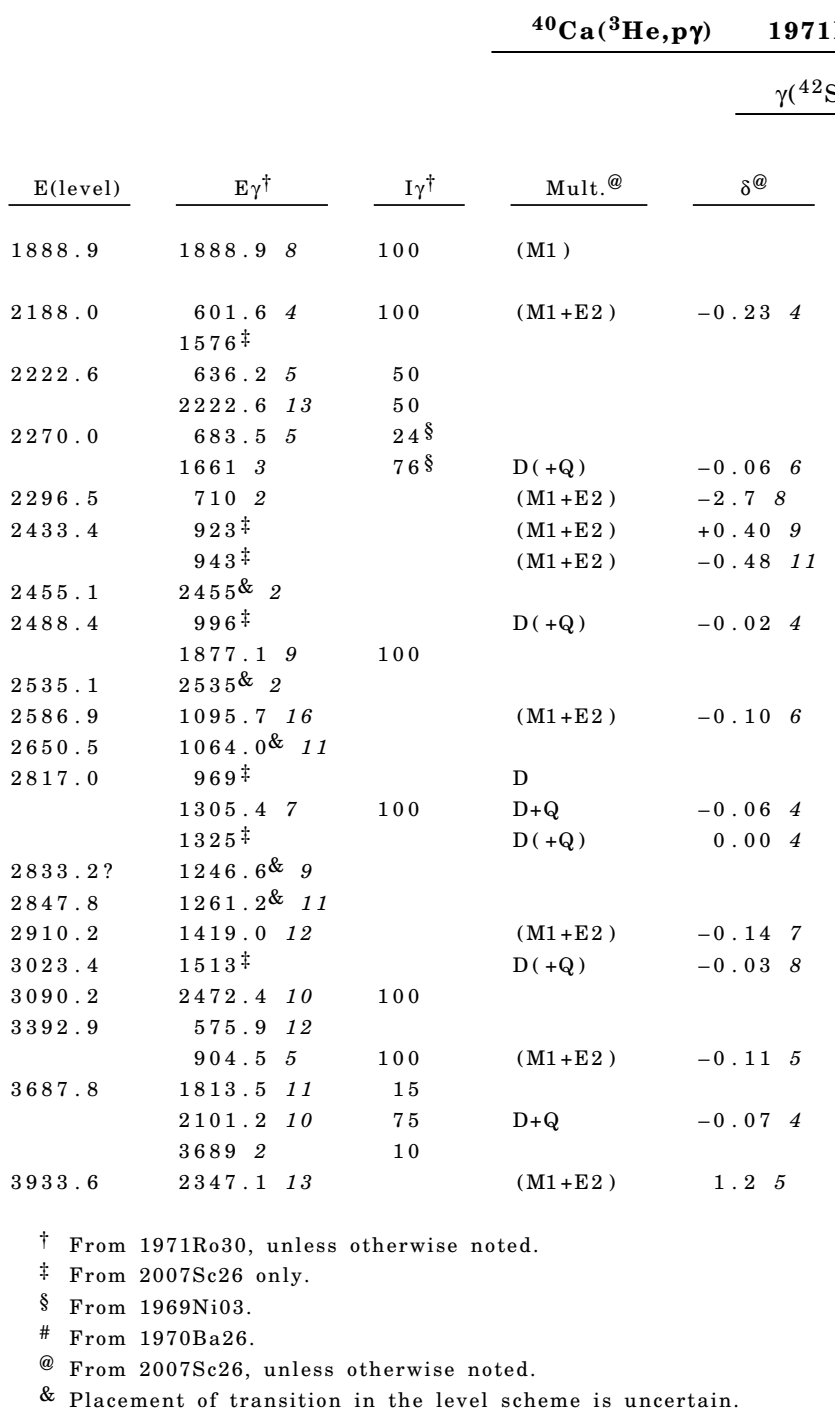




\section{${ }^{40} \mathrm{Ca}(\alpha, \mathrm{d}) \quad 1973 \mathrm{Th} 11,1977 \mathrm{Na25}$}

Target ${ }^{40} \mathrm{Ca} \mathrm{J} \pi=0+$.

1977Na25: $\mathrm{E}=40 \mathrm{MeV} \alpha$ beam was produced at the Michigan State University cyclotron. Target of $99.9 \%$ enriched metallic calcium onto a thin carbon backing. Deuterons were momentum analyzed with a split-pole magnetic spectrograph and detected with a position-sensitive resistive-wire proportional counter, energy resolution $\mathrm{FWHM}=50 \mathrm{keV}$. Measured $\sigma\left(\mathrm{E}_{\mathrm{d}}, \theta\right)$. Deduced levels, J $\pi$, L from DWBA analysis.

1973Th11: $\mathrm{E}=25.5 \mathrm{MeV} \alpha$ beam was produced at the University of Minnesota tandem generator. Targets of natural metallic calcium evaporated onto carbon or gold backings. Deuterons were analyzed with an Engel split-pole magnetic spectrometer and detected in an array of four position-sensitive surface-barrier counters in the focal plane, energy resolution FWHM $=10-17 \mathrm{keV}$. Measured $\sigma\left(\mathrm{E}_{\mathrm{d}}, \theta\right)$. Deduced levels, J $\pi$, L from DWBA analysis. 1994Fi01: $\mathrm{E}=55.7 \mathrm{MeV}$. Measured $\sigma\left(\mathrm{E}_{\mathrm{d}}, \theta\right)$, DWBA analysis. FWHM=120 keV.

1966Ri04: E=50 MeV. Five groups reported at 600, 1430, 2250, 3000 and 3555. FWHM $\approx 200 \mathrm{keV}$. 1966Ki10: four strong groups at $600,1440,2230$ and 3000. Others: 1967Be44, 1964Ri03.

\begin{tabular}{|c|c|}
\hline En e $\mathrm{r} g \mathrm{y}$ & $\sigma($ integral $)(1977 \mathrm{Na} 25)$ \\
\hline 618 & 294 \\
\hline 1514 & 145 \\
\hline 2182 & 3.8 \\
\hline 2384 & 4.4 \\
\hline 2844 & 2.8 \\
\hline 2918 & 2.5 \\
\hline 3001 & 3.8 \\
\hline 3091 & 34.5 \\
\hline 3182 & 4.4 \\
\hline 3307 & 3.7 \\
\hline 3395 & 15.5 \\
\hline 3607 & 28.0 \\
\hline 3794 & 4. 9 \\
\hline 3896 & 4.0 \\
\hline 4067 & 2.3 \\
\hline 4391 & 18.6 \\
\hline 4467 & 6.3 \\
\hline 4748 & 8.5 \\
\hline 4868 & 3.6 \\
\hline 4996 & 7.5 \\
\hline 5053 & 4.0 \\
\hline 5122 & 4.6 \\
\hline
\end{tabular}

\begin{tabular}{|c|c|c|c|c|c|}
\hline \multirow[b]{2}{*}{$\mathrm{E}(\text { level })^{\dagger}$} & \multirow[b]{2}{*}{$\mathrm{L}^{\ddagger}$} & \multirow[b]{2}{*}{ Relative intensity $\S$} & \multicolumn{3}{|c|}{${ }^{42} \mathrm{Sc}$ Levels } \\
\hline & & & $\mathrm{E}(\text { level })^{\dagger}$ & $\mathrm{L}^{\ddagger}$ & Relative intensity $\S$ \\
\hline 0.0 & & & $3024 @ 6$ & & 14.1 \\
\hline $611^{@} 6$ & & $740^{\&}$ & $3091 \quad 10$ & 4 & \\
\hline $619^{@} 6$ & 6 & $740^{\&}$ & $3182 \quad 10$ & 4 & \\
\hline $1491^{@ 6} 6$ & & 90 & $3307 \quad 10$ & 4 & \\
\hline $1511^{@} 6$ & 4 & 296 & $3395 \quad 10$ & 2 & \\
\hline $1846^{@} 6$ & & 3.3 & $3607 \quad 10$ & 6 & \\
\hline $1889^{@ 6} 6$ & & 5.8 & $3701 \quad 10$ & & \\
\hline $2189^{@} 6$ & 2 & 11.3 & $3794 \quad 10$ & 4 & \\
\hline $2223^{@} 6$ & & 12.1 & $3896 \quad 10$ & 4 & \\
\hline $2270^{@} 6$ & & 9.9 & $4067 \quad 10$ & 4 & \\
\hline $2390 @ 6$ & 2 & 13.8 & $4252 \quad 10$ & & \\
\hline $2439 @ 6$ & & 3.5 & $4391 \quad 10$ & 2 & \\
\hline $2459^{@} 6$ & & 4.2 & $4467 \quad 10$ & 2 & \\
\hline $2540^{@} 6$ & & 3.7 & $4748 \quad 10$ & 4 & \\
\hline $2588^{@ 6} 6$ & & 2.0 & $4800 \quad 10$ & & \\
\hline $2653^{@} 6$ & & 5.0 & $4868 \quad 10$ & 2 & \\
\hline $2726^{@} 6$ & & 3.1 & $4996 \quad 10$ & 2 & \\
\hline $2795^{@} 6$ & & 4.8 & $5053 \quad 10$ & 4 & \\
\hline $2832^{@} 6$ & & 4.7 & $5122 \quad 10$ & 2 & \\
\hline $2846^{@} 6$ & 2 & 6.8 & $5310^{\#} 30$ & & \\
\hline $2883^{@} 6$ & & 1.1 & $5630^{\#} 30$ & & \\
\hline $2914^{@} 6$ & 4 & 20.1 & $6050 \# 30$ & & \\
\hline $2964^{@} 6$ & & 4.6 & $7120 \# 30$ & & \\
\hline $2997^{@} 6$ & 4 & 5.2 & $7940 \# 30$ & & \\
\hline
\end{tabular}




\begin{tabular}{ll}
${ }^{40} \mathrm{Ca}(\boldsymbol{\alpha}, \mathrm{d})$ & $1973 \mathrm{Th} 11,1977 \mathrm{Na25}$ (continued) \\
\hline & ${ }^{42} \mathrm{Sc} \mathrm{Levels} \mathrm{(continued)}$ \\
\hline
\end{tabular}

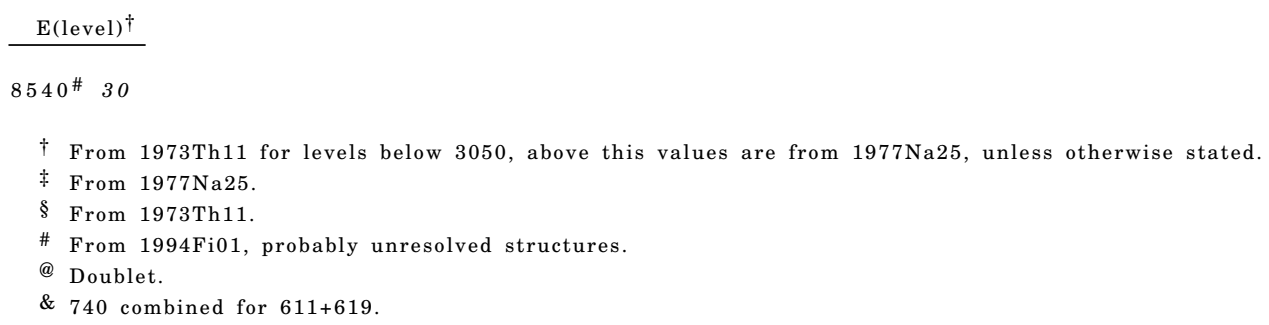

$\mathrm{E}($ level $)$

600

\section{${ }^{40} \mathrm{Ca}\left({ }^{12} \mathrm{C},{ }^{10} \mathrm{~B}\right) \quad 1972 \mathrm{Sc2} 1$}

1972Sc21(also 1974An36): E=114 MeV ${ }^{12} \mathrm{C}$ beam from the variable energy cyclotron of the Harwell Atomic Energy Research Establishment. Targets of $150 \mu \mathrm{g} / \mathrm{cm}^{2}$ calcium oxide on carbon backings. Emitted particles were detected and identified by $\Delta \mathrm{E}-\mathrm{E}$ telescopes. Measured $\sigma(\theta)$. Energy calibration accurate to $100 \mathrm{keV}$.

1994Uz01: ${ }^{12} \mathrm{C}\left({ }^{40} \mathrm{Ca},{ }^{42} \mathrm{Sc}\right){ }^{10} \mathrm{~B}, \mathrm{E}=30 \mathrm{MeV} /$ nucleon ${ }^{40} \mathrm{Ca}$ beam at GANIL. LISE 3 achromatic spectrometer. Silicon and Ge detectors. Production of ${ }^{42} \mathrm{Sc}$ isomeric beam $(\mathrm{Ex}=617 \mathrm{keV})$.

Other: $1966 \mathrm{Ch} 14$.

${ }^{42} \mathrm{Sc}$ Levels

E(level): assumed (by 1972Sc21) to be the $7+$ state (at 616 ).

\section{${ }^{41} \mathrm{Ca}(\mathrm{p}, \gamma) \quad 1989 \mathrm{Ki11}$}

1989Ki11: E=1.7-2.1 MeV proton beam was produced at the Utrecht $3 \mathrm{MV}$ Van de Graaff accelerator with currents of $20-40 \mu \mathrm{A}$ and energy spread of $200 \mathrm{eV}$ at $1 \mathrm{MeV}$. Targets of a $12 \mathrm{mg} \mathrm{CaCO}{ }_{3}$ enriched to 81.7 in ${ }^{41} \mathrm{Ca}$. Two close-end hyperpure Ge(HPGe) detectors for $\gamma$-ray detection, FWHM=1.72 and $1.81 \mathrm{keV}$ at $1.332 \mathrm{MeV}$. Measured E $\gamma$, I $\gamma$. Deduced levels, branching ratios, lifetimes by DSAM.

$\mathrm{S}(\mathrm{p})\left({ }^{42} \mathrm{Sc}\right)=4272.4015$ deduced from $\mathrm{E}_{\mathrm{x}}$ and $\mathrm{E}_{\mathrm{p}}(\mathrm{lab})$ of the $\mathrm{E}_{\mathrm{x}}=\mathrm{S}(\mathrm{p})+\mathrm{E}_{\mathrm{p}}(\mathrm{c} . \mathrm{m})=.6076.41 \mathrm{keV}$ resonance with $\mathrm{E}_{\mathrm{p}}$ (1989Ki11). $\mathrm{S}(\mathrm{p})=4272.2310$ from $2012 \mathrm{Wa} 38$.

\section{${ }^{42} \mathrm{Sc}$ Levels}

Resonance strength $=(2 \mathrm{~J}+1) \Gamma_{\mathrm{p}} \Gamma_{\gamma} / \Gamma(1989 \mathrm{Ki11})$.

\begin{tabular}{|c|c|c|}
\hline $\mathrm{E}(\text { level })^{\dagger}$ & $\mathrm{J} \pi \&$ & $\mathrm{~T}_{1 / 2}$ \\
\hline 0.0 & $0+$ & \\
\hline 611.0515 & $1+$ & \\
\hline $616.28 \quad 6$ & $7+a$ & \\
\hline $1490.43 \quad 4$ & $3+$ & \\
\hline $1510.10 \quad 6$ & $5+$ & \\
\hline 1586.312 & $2+$ & \\
\hline 1846 & $3(+)$ & \\
\hline 1874 & $0+$ & \\
\hline 1890 & $1+$ & \\
\hline $2187.54 \quad 5$ & $3+$ & \\
\hline $2223.15 \quad 3$ & $3+$ & $>0.21 \mathrm{p} \mathrm{s}$ \\
\hline $2269.13 \quad 3$ & $2+$ & $>70 \mathrm{fs}$ \\
\hline $2389.06 \quad 5$ & $3+$ & \\
\hline $2433.33 \quad 8$ & $4+$ & $>0.14 \mathrm{p} \mathrm{s}$ \\
\hline $2486.59 \quad 13$ & $2+$ & \\
\hline 2587 & $(2,4)$ & \\
\hline $2650.98 \quad 8$ & $(1+, 2)$ & $35 \mathrm{fs} 21$ \\
\hline $2795.3 \quad 3$ & $(5+, 6+) b$ & \\
\hline
\end{tabular}

Comments

Measured upper limit of branching $<2 \%$ for $\gamma$ to g.s.

Measured upper limit of branching $<2 \%$ for $\gamma$ to g.s.

Measured upper limit of branching $<2 \%$ for $\gamma$ to $1890,1+,<2 \%$ for $\gamma$ to $611,1+$, and $<4 \%$ to $616,7+$. 
${ }^{41} \mathrm{Ca}(\mathrm{p}, \gamma) \quad 1989 \mathrm{Ki11}$ (continued)

${ }^{42} \mathrm{Sc}$ Levels (continued)

\begin{tabular}{|c|c|c|c|c|}
\hline $\mathrm{E}(\text { level })^{\dagger}$ & $\mathrm{J} \pi \&$ & \multicolumn{2}{|l|}{$\mathrm{T}_{1 / 2}$} & Comments \\
\hline 2815.376 & $4+$ & $35 \mathrm{fs}$ & 14 & \\
\hline 2847.64 & $3+$ & & & \\
\hline $2910.4 \quad 4$ & $4+$ & & & \\
\hline $2995.53 \quad 7$ & $4+$ & $>0.14$ & $\mathrm{ps}$ & Measured upper limit of branching $<6 \%$ for $\gamma$ to $1890,1+$, and $<10 \%$ to $616,7+$. \\
\hline $3022.80 \quad 15$ & $4-$ & & & \\
\hline 3089.13 & $5+$ & $>0.14$ & $\mathrm{ps}$ & \\
\hline $3223.28 \quad 6$ & $(5+)$ & $>0.21$ & & Measured upper limit of branching $<3 \%$ for $\gamma$ to $1890,1+$, and $<4 \%$ to $611,1+$. \\
\hline $3321.36 \quad 10$ & $(1+, 2,3+)$ & $>0.14$ & $\mathrm{ps}$ & \\
\hline 3322.83 & $(3+, 4,5+)$ & $<35 \mathrm{fs}$ & & \\
\hline $3668.7 \quad 3$ & & & & \\
\hline 3719.34 & $(5)+$ & $>70 \mathrm{fs}$ & & \\
\hline 4047.726 & $(2,3,4)$ & $<14$ fs & & \\
\hline 4468.84 & $(2,3)+$ & & & \\
\hline $5995.8 \ddagger 3$ & $(3,4)+$ & & & \\
\hline $6076.41 \S \quad 8$ & $(2+, 3,4+)$ & & & \\
\hline $6174.2^{\#} 3$ & $(3,4)+$ & & & \\
\hline $6253.4 @ 2$ & $(3+, 4)$ & & & \\
\hline$\dagger$ From 198 & Ki11, deduced & rom $\gamma$ ener & rgies & \\
\hline$\ddagger \mathrm{E}(\mathrm{p})(\mathrm{lab})=$ & 765.8 3. Reso & nce streng & $\operatorname{gth}=$ & $\mathrm{eV} 8.21 \% 5$ of the decay occurs through unknown transitions. \\
\hline$\S E(p)(l a b)=$ & $848.4112 . \mathrm{Re}$ & nance stre & engt & $.7 \mathrm{eV} 2.6 \% 2$ of the decay occurs through unknown transitions. \\
\hline$\# \mathrm{E}(\mathrm{p})(\mathrm{lab})=$ & 948.6 3. Reso & nce streng & $\operatorname{gth}=$ & $\mathrm{eV} 14.18 \% 5$ of the decay occurs through unknown transitions. \\
\hline @ $\mathrm{E}(\mathrm{p})(\mathrm{lab})=$ & 029.8 3. Reso & nce streng & $\operatorname{gth}=$ & eV 4. $14 \% 5$ of the decay occurs through unknown transitions. \\
\hline $\begin{array}{l}\& \text { From Ado } \\
\text { a } 5+\text { and } 6\end{array}$ & $\begin{array}{l}\text { ed Levels. Fo } \\
\text { are not compl }\end{array}$ & $\begin{array}{l}\text { resonance } \\
\text { ely ruled }\end{array}$ & out & $\begin{array}{l}\text { assignments are based based on decay modes, assuming E1, M1 or E2 transitions. } \\
\text { t } 7+\text { is generally accepted from theoretical predictions. }\end{array}$ \\
\hline b $1989 \mathrm{Ki} 11$ & e $(5,6,7)$. & & 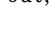 & \\
\hline
\end{tabular}

$\gamma\left({ }^{42} \mathrm{Sc}\right)$

\begin{tabular}{|c|c|c|c|c|}
\hline E(level) & $\mathrm{E} \gamma^{\dagger}$ & & & $\gamma^{\ddagger}$ \\
\hline 611.051 & 611.046 & 6 & & \\
\hline 1490.43 & 879.37 & & 100 & \\
\hline 1510.10 & 893.81 & & 100 & \\
\hline 1586.31 & 975.25 & 3 & 100 & 11 \\
\hline & 1586.28 & & 9 & $5 \quad 11$ \\
\hline 1846 & 260 & & & \\
\hline & 1235 & & & \\
\hline 1874 & 1263 & & 100 & \\
\hline 1890 & 1890 & & 100 & \\
\hline 2187.54 & 601.23 & & 95 & 2 \\
\hline & $677.4 \S$ & & $<2$ & \\
\hline & $697.1 \S$ & & $<2$ & \\
\hline & 1576.46 & & 5 & 2 \\
\hline 2223.15 & 636.833 & 9 & 95 & 2 \\
\hline & $713.0 \S$ & & $<2$ & \\
\hline & 732.71 & & 5 & 2 \\
\hline & $1612.1 \S$ & & $<2$ & \\
\hline 2269.13 & 379.13 & & 6 & 3 \\
\hline & 682.808 & 23 & 52 & 5 \\
\hline & $778.7 \S$ & & $<5$ & \\
\hline & 1658.04 & & 22 & 6 \\
\hline & 2269.06 & & 20 & 4 \\
\hline 2389.06 & $543.1 \S$ & & $<2$ & \\
\hline & $802.7 \S$ & & $<2$ & \\
\hline & 878.95 & & 88 & 4 \\
\hline & 898.62 & & 12 & 4 \\
\hline & $1778.0 \S$ & & $<4$ & \\
\hline 2433.33 & $847.0 \S$ & & $<2$ & \\
\hline & 923.22 & & 48 & 3 \\
\hline & 942.89 & & 52 & 3 \\
\hline 2486.59 & $596.6 \S$ & & $<4$ & \\
\hline
\end{tabular}

$\mathrm{I} \gamma<3$ for $\gamma$ to $1510 ; \mathrm{I} \gamma<1$ for $\gamma$ to 616

I $\gamma<3$ for $\gamma$ to 616 ; I $\gamma<4$ for $\gamma$ to g.s. 
${ }^{41} \mathrm{Ca}(\mathrm{p}, \gamma) \quad 1989 \mathrm{Ki11}$ (continued)

$\gamma\left({ }^{42} \mathrm{Sc}\right)$ (continued)

\begin{tabular}{|c|c|c|c|}
\hline \multirow{2}{*}{$\begin{array}{r}\text { E(level) } \\
2486.59\end{array}$} & \multirow{2}{*}{$\frac{\mathrm{E} \gamma^{\dagger}}{612.6{ }^{\S}}$} & \multicolumn{2}{|c|}{$\mathrm{I} \gamma^{\ddagger}$} \\
\hline & & $<5$ & \\
\hline & $640.6 \S$ & $<5$ & \\
\hline & $900.3 \S$ & $<4$ & \\
\hline & 996.15 & 23 & 5 \\
\hline & 1875.49 & 77 & 5 \\
\hline & $2486.5 \S$ & $<10$ & \\
\hline 2587 & 1097 & & \\
\hline \multirow[t]{7}{*}{2650.98} & 760.97 & 10 & 3 \\
\hline & $777.0 \S$ & $<3$ & \\
\hline & $805.0 \S$ & $<3$ & \\
\hline & 1064.66 & 90 & 3 \\
\hline & $1160.5 \S$ & $<3$ & \\
\hline & $2039.9 \S$ & $<3$ & \\
\hline & $2650.9 \S$ & $<3$ & \\
\hline 2795.3 & 2179.0 & 100 & \\
\hline \multirow[t]{5}{*}{2815.37} & $592.2^{\S}$ & $<2$ & \\
\hline & $627.8 \S$ & $<2$ & \\
\hline & 969.36 & 5 & 3 \\
\hline & 1305.25 & 61 & 3 \\
\hline & 1324.92 & 34 & 2 \\
\hline 2847.6 & 1261.3 & & \\
\hline 2910.4 & 1419.9 & & \\
\hline \multirow[t]{5}{*}{2995.53} & 606.47 & 20 & 10 \\
\hline & 772.37 & 80 & 10 \\
\hline & $808.0 \S$ & $<6$ & \\
\hline & $1149.5 \S$ & $<4$ & \\
\hline & $1485.4 \S$ & $<5$ & \\
\hline 3022.80 & 1512.67 & 100 & \\
\hline 3089.1 & 2472.7 & 100 & \\
\hline \multirow[t]{7}{*}{3223.28} & 834.21 & 41 & 3 \\
\hline & 1000.12 & 32 & 8 \\
\hline & $1035.7 \S$ & $<8$ & \\
\hline & $1377.3 \S$ & $<5$ & \\
\hline & 1713.14 & 27 & 3 \\
\hline & $1732.8 \S$ & $<6$ & \\
\hline & $2606.9 \S$ & $<2$ & \\
\hline \multirow[t]{9}{*}{3321.36} & $1098.2 \S$ & $<6$ & \\
\hline & $1133.8 \S$ & $<6$ & \\
\hline & $1431.3 \S$ & $<4$ & \\
\hline & $1447.3 \S$ & $<8$ & \\
\hline & $1475.3 \S$ & $<8$ & \\
\hline & $1811.2 \S$ & $<6$ & \\
\hline & 1830.89 & 50 & 10 \\
\hline & 2710.22 & 50 & 10 \\
\hline & $3321.2 \S$ & $<5$ & \\
\hline \multirow[t]{6}{*}{3322.8} & $1099.6 \S$ & $<3$ & \\
\hline & $1135.2 \S$ & $<8$ & \\
\hline & $1476.8 \S$ & $<3$ & \\
\hline & 1812.7 & 57 & 5 \\
\hline & 1832.3 & 43 & 5 \\
\hline & $2706.4 \S$ & $<4$ & \\
\hline \multirow[t]{2}{*}{3668.7} & 1235.4 & & \\
\hline & 1445.5 & & \\
\hline \multirow[t]{6}{*}{3719.3} & $1496.1 \S$ & $<2$ & \\
\hline & $1531.7 \S$ & $<7$ & \\
\hline & $1873.3 \S$ & $<4$ & \\
\hline & $2209.1 \S$ & $<4$ & \\
\hline & $2228.8 \S$ & $<6$ & \\
\hline & 3102.9 & 100 & \\
\hline 4047.72 & 1658.63 & $\approx 50$ & \\
\hline \multirow[t]{2}{*}{4468.8} & 1558.4 & 25 & 5 \\
\hline & 2882.4 & 25 & 2 \\
\hline 5995.8 & 1948.0 & 6 & 2 \\
\hline
\end{tabular}

Comments

I $\gamma<5$ for $\gamma$ to 616 and $\gamma$ to 1510

$\mathrm{I} \gamma<2$ for $\gamma$ to $616 ; \mathrm{I} \gamma<3$ for $\gamma$ to 1510.

I $\gamma<1$ for gammas to g.s., 611 and 616 ; I $\gamma<4$ for $\gamma$ to 1874 ; I $\gamma<2$ for $\gamma$ to 1890.

I $\gamma<3$ for $\gamma$ to g.s.; $\mathrm{I} \gamma<7$ for $\gamma$ to 1874 ; I $\gamma<2$ for $\gamma$ to 1890.

I $\gamma<4$ for $\gamma$ to g.s.; $\mathrm{I} \gamma<5$ for $\gamma$ to 1874

$\mathrm{I} \gamma<4$ for $\gamma$ to 616

I $\gamma<5$ for $\gamma$ to g.s.; $\mathrm{I} \gamma<4$ for $\gamma$ to 611 ; $\mathrm{I} \gamma<3$ for gammas to 1874 and 1890.

I $\gamma<2$ for gammas to g.s., 611,1590 and 1874 .

$50 \%$ of the decay from 4048 level proceeds through unknown transitions.

$50 \%$ of the decay from 4048 level proceeds through unknown transitions. 


$\frac{{ }^{41} \mathbf{C a}(\mathbf{p}, \boldsymbol{\gamma}) \quad 1989 \mathrm{Ki11} \text { (continued) }}{\gamma\left({ }^{42} \mathrm{Sc}\right)(\text { continued })}$

\begin{tabular}{|c|c|c|c|c|c|c|c|c|c|c|c|}
\hline $\mathrm{E}$ (level) & $\mathrm{E} \gamma^{\dagger}$ & & $\gamma^{\ddagger}$ & E(level) & $\mathrm{E} \gamma^{\dagger}$ & & $\gamma^{\ddagger}$ & E(level) & $\mathrm{E} \gamma^{\dagger}$ & & $\gamma^{\ddagger}$ \\
\hline \multirow[t]{13}{*}{5995.8} & 2772.4 & 13 & 2 & \multirow[t]{8}{*}{6076.41} & 2028.6 & 4 & 1 & \multirow[t]{4}{*}{6174.2} & 3178.5 & 7 & 2 \\
\hline & 2906.6 & 3 & 1 & & 2407.6 & 4 & 1 & & 3358.7 & 9 & 2 \\
\hline & 2972.9 & $\approx 1$ & & & 2755.0 & 10 & 2 & & 3378.8 & 3 & 1 \\
\hline & 3148.1 & 4 & 1 & & 3053.5 & 7 & 2 & & 3904.9 & 21 & 2 \\
\hline & 3180.3 & 3 & 1 & & 3165.9 & 4 & 1 & \multirow[t]{9}{*}{6253.4} & 3164.2 & 4 & 1 \\
\hline & 3344.7 & 4 & 1 & & 3687.2 & 17 & 2 & & 3405.7 & 4 & 1 \\
\hline & 3509.1 & 3 & 1 & & $3807.14 \quad 8$ & 21 & 2 & & 3437.9 & 3 & 1 \\
\hline & 3562.3 & 3 & 1 & & $3853.06 \quad 8$ & 29 & 2 & & 3602.3 & 15 & 2 \\
\hline & 3772.5 & 6 & 2 & \multirow[t]{5}{*}{6174.2} & 1705.4 & 4 & 1 & & 3819.9 & 15 & 2 \\
\hline & 3808.1 & 2 & 1 & & 2454.8 & 20 & 2 & & 4030.0 & $\approx 1$ & \\
\hline & 4409.2 & 8 & 2 & & 2851.3 & 8 & 2 & & 4065.6 & 30 & 2 \\
\hline & 4485.4 & 16 & 2 & & 2950.8 & 7 & 2 & & 4743.0 & 7 & 2 \\
\hline & 4505.1 & 7 & 2 & & 3085.0 & 3 & 1 & & 4762.7 & 7 & 2 \\
\hline
\end{tabular}

$\dagger$ Values without uncertainties are from level-energy differences.

$\ddagger$ Upper limits on branching ratio for transitions involving $\Delta \mathrm{J}>2$ are given under comments (1989Ki11).

$\S$ Placement of transition in the level scheme is uncertain.

\section{${ }^{41} \mathrm{Ca}\left({ }^{3} \mathrm{He}, \mathrm{d}\right) \quad 1979 \mathrm{VoO4}$}

Target ${ }^{41} \mathrm{Ca} \mathrm{J} \pi=7 / 2-$.

1979Vo04: $\mathrm{E}=20 \mathrm{MeV}{ }^{3} \mathrm{He}$ beam was produced from the Rochester MP tandem accelerator. Enriched target of $81.8 \%{ }^{41} \mathrm{Ca}$ and $18.1 \%{ }^{40} \mathrm{Ca}$ about $25 \mu \mathrm{g} / \mathrm{cm}^{2}$ on a carbon backing. Deuterons were momentum analyzed with a split-pole magnetic spectrometer and detected with nuclear emulsions, energy resolution FWHM $=20 \mathrm{keV}$. Measured $\sigma\left(\mathrm{E}_{\mathrm{d}}, \theta\right)$. Deduced levels, $\mathrm{J} \pi, \mathrm{L}$ and spectroscopic factors from DWBA analysis. Includes also ${ }^{41} \mathrm{Ca}\left({ }^{3} \mathrm{He}, \mathrm{d} \gamma\right)$

$\begin{array}{clrl}\text { Energy } & \mathrm{d} \sigma / \mathrm{d} \Omega(\mathrm{mb} / \mathrm{sr}) & \text { Energy } & \mathrm{d} \sigma / \mathrm{d} \Omega(\mathrm{mb} / \\ & & & \\ 611 & 0.17 & 4022 & 0.32 \\ 615 & 0.56 & 4044 & 0.13 \\ 1490 & 2.72 & 4175 & 0.53 \\ 1510 & 2.33 & 4204 & 1.44 \\ 1585 & 5.29 & 4245 & 0.17 \\ 1843 & 1.19 & 4262 & 0.10 \\ 1872 & 0.024 & 4276 & 0.078 \\ 1886 & 0.059 & 4289 & 0.34 \\ 2185 & 0.51 & 4370 & 0.083 \\ 2220 & 0.20 & 4410 & 0.72 \\ 2265 & 0.058 & 4469 & 8.51 \\ 2293 & 0.10 & 4548 & 2.23 \\ 2388 & 0.27 & 4582 & 1.22 \\ 2433 & 0.21 & 4604 & 0.57 \\ 2452 & 0.045 & 4665 & 0.48 \\ 2486 & 0.70 & 4704 & 0.19 \\ 2533 & 0.017 & 4727 & 0.86 \\ 2648 & 0.13 & 4760 & 0.44 \\ 2793 & 0.66 & 4786 & 0.24 \\ 2814 & 3.18 & 4808 & 0.22 \\ 2846 & 0.070 & 4827 & 1.14 \\ 2911 & 0.070 & 4876 & 0.16 \\ 2994 & 0.31 & 4971 & 0.060 \\ 3021 & 0.57 & 5003 & 0.16 \\ 3088 & 6.48 & 5028 & 0.26 \\ 3146 & 0.061 & 5045 & 0.066 \\ 3166 & 0.36 & 5084 & 1.94 \\ 3245 & 4.34 & 5120 & 4.08 \\ 3281 & 0.27 & 5140 & 0.57 \\ 3322 & 0.86 & 5303 & 0.058 \\ 3350 & 0.19 & 5326 & 0.45 \\ 3390 & 4.50 & 5352 & 0.67 \\ & & 5370 & 0.33\end{array}$


${ }^{41} \mathrm{Ca}\left({ }^{3} \mathrm{He}, \mathrm{d}\right) \quad 1979 V o 04$ (continued)

$\begin{array}{ll}3446 & 0.20 \\ 3468 & 0.14 \\ 3493 & 0.18 \\ 3512 & 0.079 \\ 3529 & 0.074 \\ 3577 & 0.046 \\ 3600 & 0.14 \\ 3686 & 0.33 \\ 3715 & 0.11 \\ 3754 & 0.19 \\ 3792 & 2.40 \\ 3855 & 0.077 \\ 3866 & 0.071 \\ 3896 & 0.25 \\ 3930 & 0.97\end{array}$

$5380 \quad 0.69$

$5434 \quad 0.46$

$5473 \quad 0.18$

$\begin{array}{ll}5520 & 0.35\end{array}$

$5572 \quad 0.76$

$5633 \quad 1.69$

$5651 \quad 4.18$

$\begin{array}{ll}5771 & 0.47\end{array}$

$5865 \quad 0.82$

$5964 \quad 0.77$

\begin{tabular}{|c|c|c|c|c|c|c|c|c|}
\hline E(level) & $\mathrm{L}$ & $(2 \mathrm{~J}+1) \mathrm{S}^{\dagger}$ & E(level) & $\mathrm{L}$ & $(2 \mathrm{~J}+1) \mathrm{S}^{\dagger}$ & E(level) & $\mathrm{L}$ & $(2 \mathrm{~J}+1) \mathrm{S}^{\dagger}$ \\
\hline 0.0 & 3 & 1.3 & $3350 \quad 5$ & & & $4665 \quad 5$ & 1 & 0.45 \\
\hline $611 \ddagger$ & $3 \ddagger$ & $4.0 \ddagger$ & $3390 \quad 5$ & $1+3$ & $2.4,2.9$ & $4704 \quad 5$ & (1) & 0.26 \\
\hline $615^{\ddagger} 5$ & $3 \ddagger$ & $19 \neq$ & $3446 \quad 5$ & (1) & 0.11 & $4727 \quad 5$ & 1 & 0.80 \\
\hline $1490 \quad 5$ & $1+3$ & $0.78,6.9$ & $3468 \quad 5$ & & & $4760 \quad 5$ & 3 & 1.4 \\
\hline $1510 \quad 5$ & $1+3$ & $2.3,11$ & $3493 \quad 5$ & (1) & 0.10 & $4786 \quad 5$ & & \\
\hline $1585 \quad 5$ & $1+3$ & $0.42,3.4$ & $3512 \quad 5$ & & & $4808 \quad 5$ & & \\
\hline 18435 & & & $3529 \quad 5$ & & & $4827 \quad 5$ & 1 & 1.3 \\
\hline $1872 \quad 5$ & 3 & 0.24 & $3577 ? \quad 5$ & & & $4876 \quad 5$ & & \\
\hline $1886 \quad 5$ & 3 & 0.34 & $3600 \quad 5$ & ( 3 ) & 0.45 & $4971 \quad 5$ & & \\
\hline $2185 \quad 5$ & $1+3$ & $0.26,0.46$ & $3686 \quad 5$ & 3 & 0.99 & $5003 \quad 5$ & ( 1 ) & 0.27 \\
\hline $2220 \quad 5$ & $1+3$ & $0.05,0.51$ & $3715 \quad 5$ & 3 & 0.28 & $5028 \quad 5$ & & \\
\hline $2265 \quad 5$ & ( 3 ) & 0.26 & $3754 \quad 5$ & $(1+3)$ & $0.05,0.19$ & $5045 \quad 5$ & & \\
\hline $2293 \quad 5$ & $(1+3)$ & $0.03,0.30$ & $3792 \quad 5$ & $1+3$ & $1.3,1.3$ & $5084 \quad 5$ & ( 1 ) & 3.0 \\
\hline $2388 \S 5$ & $3(+1)$ & $1.1,0.04$ & $3855 \quad 5$ & ( 1 ) & 0.06 & $5120 \quad 5$ & ( 1 ) & 6.3 \\
\hline $2433 \quad 5$ & 1 & 0.11 & $3866 \quad 5$ & & & $5140 \quad 5$ & & \\
\hline $2452 \quad 5$ & & & $3896 \quad 5$ & & & $5303 ? \quad 5$ & & \\
\hline $2486 \quad 5$ & 3 & 3.2 & $3930 \quad 5$ & & & $5326 \quad 5$ & & \\
\hline $2533 ? 5$ & & & $4022 \quad 5$ & & & $5352 \quad 5$ & & \\
\hline $2648 \quad 5$ & & & $4044 \quad 5$ & & & $5370 \quad 5$ & & \\
\hline $2793 \quad 5$ & $(1+3)$ & $0.27,1.5$ & $4175 \quad 5$ & 1 & 0.32 & $5380 \quad 5$ & & \\
\hline $2814 \quad 5$ & $3(+1)$ & $9.8,0.67$ & $4204 \quad 5$ & $1+3$ & $0.56,2.1$ & $5434 \quad 5$ & & \\
\hline $2846 \quad 5$ & & & $4245 \quad 5$ & & & $5473 \quad 5$ & & \\
\hline 29115 & & & $4262 ? \quad 5$ & & & $5520 \quad 5$ & & \\
\hline $2994 \quad 5$ & & & $4276 \quad 5$ & & & $5572 \quad 5$ & & \\
\hline 30215 & 0 & 0.29 & $4289 \quad 5$ & 1 & 0.26 & $5633 \quad 5$ & 1 & 3.4 \\
\hline $3088 \quad 5$ & $1+3$ & $3.5,4.2$ & $4370 \quad 5$ & & & 56515 & 1 & 7.7 \\
\hline $3146 ? \quad 5$ & & & $4410 \quad 5$ & 1 & 0.86 & $5771 \quad 5$ & & \\
\hline $3166 \quad 5$ & $(1+3)$ & $0.11,0.67$ & $4469 \quad 5$ & 1 & 7.9 & $5865 \quad 5$ & ( 1 ) & 2.6 \\
\hline $3245 \quad 5$ & 3 & 17 & $4548 \quad 5$ & 1 & 1.9 & $5964 \quad 5$ & ( 1 ) & 2.4 \\
\hline 32815 & 3 & 0.90 & $4582 \quad 5$ & 1 & 0.94 & & & \\
\hline $3322 \quad 5$ & $1+3$ & $0.13,2.4$ & $4604 \quad 5$ & (1) & 0.56 & & & \\
\hline
\end{tabular}

$\dagger 1979 \mathrm{Vo04}$ quote spectroscopic strengths $\mathrm{g}=\left[\left(2 \mathrm{~J}_{\mathrm{f}}+1\right) /\left(2 \mathrm{~J}_{\mathrm{i}}+1\right)\right] \mathrm{C}^{2} \mathrm{~S}$; where $\mathrm{J}_{\mathrm{i}}=7 / 2-$ and $\mathrm{C}^{2}=1 / 2$.

$\ddagger$ Doublet, spectroscopic factors isolated for two states from normalization to d $\gamma$-coin data in $\left({ }^{3} \mathrm{He}, \mathrm{d} \gamma\right)$.

$\S$ Doublet. 


\section{${ }^{42} \mathrm{Ca}(\mathrm{p}, \mathrm{n}) \quad 20020 \mathrm{Or} 05$}

2002Or05: E=35 MeV, measured neutron spectra by TOF, $\sigma$ at Cyclotron and Radioisotope Center, Tohoku University; deduced Gamow-Teller matrix elements.

${ }^{42} \mathrm{Sc} \mathrm{Levels}$

\begin{tabular}{|c|c|c|}
\hline E(level) & $\mathrm{J} \pi^{\dagger}$ & \\
\hline 0.0 & $0+$ & $\begin{array}{l}\text { IAS of }{ }^{42} \mathrm{Ca} \mathrm{g.s.} \\
\sigma=1.13 \mathrm{mb} / \mathrm{sr} .\end{array}$ \\
\hline 611 & $1+$ & $\begin{array}{l}\sigma=1.52 \mathrm{mb} / \mathrm{sr} . \\
\sigma(\text { Gamow-Teller }) / \sigma(\mathrm{IAS})=1.3510 . \\
\mathrm{B}(\mathrm{GT}) \text { strength }=2.64 \quad 15(2002 \mathrm{Or} 05) .\end{array}$ \\
\hline
\end{tabular}

$\dagger$ From Adopted Levels.

\section{${ }^{42} \mathrm{Ca}\left({ }^{3} \mathrm{He}, \mathrm{t}\right) \quad 2015 \mathrm{Fu} 08,1971 \mathrm{Sh} 16$}

2015Fu08 (also 2014Fu02): E=140 MeV/nucleon ${ }^{3} \mathrm{He}$ beam was produced at the $\mathrm{K}=400$ Ring Cyclotron at RCNP. Target was a self-supporting foil of $1.78 \mathrm{mg} / \mathrm{cm}^{2} 93.7 \%$ enriched in ${ }^{42} \mathrm{Ca}$. Reaction products were momentum analyzed with the Grand Raiden spectrometer $(\mathrm{FWHM}=29 \mathrm{keV})$ and detected with a focal-plane detector. Measured $\sigma\left(\mathrm{E}_{\mathrm{t}}, \theta\right)$ with five angle cuts $\left(\leq 0.5^{\circ}, 0.5^{\circ}-0.8^{\circ}, 0.8^{\circ}-1.2^{\circ}, 1.2^{\circ}-1.6^{\circ}, 1.6^{\circ}-2.0^{\circ}\right)$. Deduced levels, L-transfers, Gamow-Teller $\beta$ strengths. Comparisons with shell-model calculations, and with the data published earlier for ${ }^{46} \mathrm{~V}$, ${ }^{50} \mathrm{Mn}$, and ${ }^{54} \mathrm{Co}$ produced from the $\left({ }^{3} \mathrm{He}, \mathrm{t}\right)$ reaction. In $2014 \mathrm{Fu} 02$ only the ground state and levels at 611,1889 and 3688 are discussed. 2016Fu02, 2012Fu02, 2012St22, 2011St03 and 2007Ad27: conference papers which discuss the results of the same experiment as in $2015 \mathrm{Fu} 08$ and $2014 \mathrm{Fu} 02$ primary publications.

1971Sh16: $\mathrm{E}=26 \mathrm{MeV}{ }^{3} \mathrm{He}$ beam was produced from the University of Rochester MP Tandem. Target of $94.4 \%$ enriched ${ }^{42} \mathrm{Ca}$ about $30 \mu \mathrm{g} / \mathrm{cm}^{2}$ on a $10 \mu \mathrm{g} / \mathrm{cm}^{2}$ carbon backing. Tritons were momentum analyzed with the Split Pole Magnetic Spectrograph and detected with 100 micron Kodak NTB emulsion plates, energy resolution FWHM=9 keV. Measured $\sigma\left(E_{t}, \theta\right)$. Deduced levels, J $\pi$, L from DWBA analysis.

Others:

1989Va09: $\mathrm{E}=73 \mathrm{MeV}$. Measured $\sigma\left(\mathrm{E}_{\mathrm{t}}, \theta\right)$. Deduced effective projectile-nucleon force parameters. 1987Ko34: $\mathrm{E}=18.86 \mathrm{MeV}$. Measured triton energy differences.

1977Vo02: measured Q-values. Measured $\sigma\left(\mathrm{E}_{\mathrm{t}}, \theta\right)$.

1974Ha35: $\mathrm{E}=33 \mathrm{MeV}$.

1971Be29: E=24.6 MeV. Deduced Q value and Coulomb-displacement energy.

\section{${ }^{42} \mathrm{Sc}$ Levels}

The $\mathrm{B}(\mathrm{GT})$ strengths and count rates for levels are from $2015 \mathrm{Fu} 08$. Total experimental $\mathrm{B}(\mathrm{GT})$ strength=2.7 4 (2015Fu08) out of which $\approx 80 \%$ is concentrated in the $613,1+$ state

Total counts observed $(2015 \mathrm{Fu} 08)$ within $\theta=0^{\circ}$ to $0.5^{\circ}$ are given under comments.

\begin{tabular}{|c|c|c|c|c|}
\hline $\mathrm{E}(\text { level })^{\dagger}$ & $\mathrm{J} \pi \mathrm{b}$ & $\mathrm{L}$ & Relative $\mathrm{d} \sigma / \mathrm{d} \Omega^{\mathrm{c}}$ & Comments \\
\hline \multirow[t]{4}{*}{0.0} & $0+$ & 0 & $84^{\mathrm{f}}$ & $\mathrm{T}=1$ \\
\hline & & & & $\mathrm{E}$ (level): IAS of ${ }^{42} \mathrm{Ca}$ g.s. \\
\hline & & & & L: from $1971 \mathrm{Sh} 16$ and $2015 \mathrm{Fu} 08$. \\
\hline & & & & Counts $\left(0^{\circ}\right)=34563543$ \\
\hline \multirow[t]{5}{*}{$613^{\#} 4$} & $1+$ & $0 \&$ & $170^{\mathrm{d}}$ & $\mathrm{L}=0$ in $2015 \mathrm{Fu} 08 ; 2,1+3$ in $1971 \mathrm{Sh} 16$ \\
\hline & & & & E(level): 6124 (2015Fu08), 6155 (1971Sh16). \\
\hline & & & & $\mathrm{J} \pi$ : from $2014 \mathrm{Fu} 02,2015 \mathrm{Fu} 08,1971 \mathrm{Sh} 16$ \\
\hline & & & & $\begin{array}{l}\mathrm{B}(\mathrm{GT}) \text { strength }=2.17347 \text { for } 612 \text { level, which represents about } 80 \% \text { of the } \\
\text { total } \mathrm{B}(\mathrm{GT}) \text { strength in }{ }^{42} \mathrm{Sc} \text {. }\end{array}$ \\
\hline & & & & Counts $\left(0^{\circ}\right)=2410371069$ \\
\hline $624^{@} 5$ & & & $\leq 10^{\mathrm{i}}$ & $\mathrm{J} \pi: 7+(1971 \mathrm{Sh} 16)$ \\
\hline $1494^{\#} 4$ & & $2+4,3+5 \mathrm{a}$ & $68 \mathrm{f}$ & E(level): 14914 (2015Fu08), 14985 (1971Sh16). \\
\hline & & & & $J \pi: 3+(1971 \mathrm{Sh} 16)$ \\
\hline & & & & $\operatorname{Counts}\left(0^{\circ}\right)=10039156$ \\
\hline $1518^{@} 5$ & & $4+6,5+7$ a & $34 \mathrm{~g}$ & $\mathrm{~J} \pi: 5+(1971 \mathrm{Sh} 16)$ \\
\hline $1589^{\#} 4$ & & $2,1+3^{\mathrm{a}}$ & $29 \mathrm{~d}$ & E(level): $15864(2015 \mathrm{Fu} 08), 15935$ (1971Sh16). \\
\hline & & & & $J \pi: 2+(1971 \operatorname{Sh} 16)$ \\
\hline & & & & $\operatorname{Counts}\left(0^{\circ}\right)=66646$ \\
\hline $1844^{@} 5$ & & & $3.5^{\mathrm{g}}$ & $\mathrm{J} \pi:(3+, 4-)(1971 \mathrm{Sh} 16)$ \\
\hline
\end{tabular}


${ }^{42} \mathrm{Ca}\left({ }^{3} \mathrm{He}, \mathrm{t}\right) \quad 2015 \mathrm{Fu} 08,1971 \mathrm{Sh} 16$ (continued)

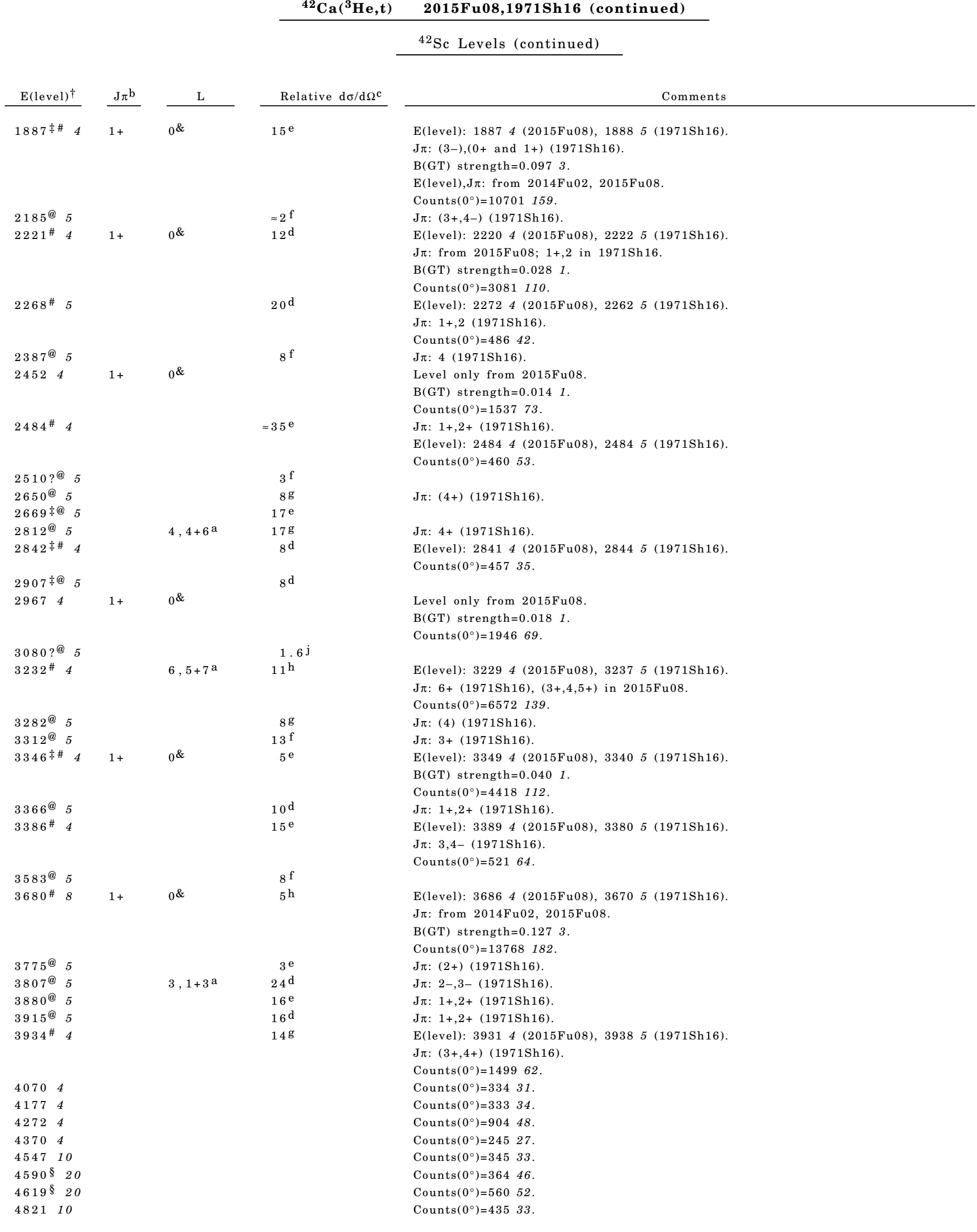




\begin{tabular}{cc}
${ }^{42} \mathrm{Ca}\left({ }^{3} \mathrm{He}, \mathrm{t}\right)$ & $2015 \mathrm{Fu} 08,1971 \mathrm{Sh} 16$ (continued) \\
\hline${ }^{42} \mathrm{Sc} \mathrm{Levels} \mathrm{(continued)}$ \\
\hline
\end{tabular}

\begin{tabular}{|c|c|c|c|c|}
\hline \multicolumn{2}{|c|}{$\mathrm{E}(\text { level })^{\dagger}$} & \multirow[t]{2}{*}{$\mathrm{J} \pi \mathrm{b}$} & \multirow[t]{2}{*}{$\mathrm{L}$} & Comments \\
\hline 4873 & 10 & & & $\operatorname{Counts}\left(0^{\circ}\right)=368086$ \\
\hline 4928 & 10 & $1+$ & 0 & $\begin{array}{l}\mathrm{B}(\mathrm{GT}) \text { strength }=0.0201 . \\
\text { Counts }\left(0^{\circ}\right)=2117 \quad 67 .\end{array}$ \\
\hline 5094 & 10 & & & Counts $\left(0^{\circ}\right)=53235$ \\
\hline 5143 & 10 & $1+$ & 0 & $\begin{array}{l}\mathrm{B}(\mathrm{GT}) \text { strength }=0.0211 . \\
\text { Counts }\left(0^{\circ}\right)=226966 .\end{array}$ \\
\hline $5686 \S$ & 20 & $(1+)$ & $(0)$ & $\begin{array}{l}\mathrm{B}(\mathrm{GT}) \text { strength }=0.0051 . \\
\text { Counts }\left(0^{\circ}\right)=48351 .\end{array}$ \\
\hline $5716 \S$ & 20 & $1+$ & 0 & $\begin{array}{l}\mathrm{B}(\mathrm{GT}) \text { strength }=0.0121 . \\
\text { Counts }\left(0^{\circ}\right)=122163 .\end{array}$ \\
\hline 5803 & 10 & & & $\operatorname{Counts}\left(0^{\circ}\right)=126864$ \\
\hline 5958 & 10 & & & Counts $\left(0^{\circ}\right)=57338$ \\
\hline 6007 & 10 & & & $\operatorname{Counts}\left(0^{\circ}\right)=180471$ \\
\hline 6078 & 10 & & & $\operatorname{Counts}\left(0^{\circ}\right)=51640$ \\
\hline 6167 & 10 & & & $\operatorname{Counts}\left(0^{\circ}\right)=108559$ \\
\hline 6327 & 10 & $1+$ & 0 & $\begin{array}{l}\mathrm{B}(\mathrm{GT}) \text { strength }=0.0181 . \\
\text { Counts }\left(0^{\circ}\right)=192470 .\end{array}$ \\
\hline 6364 & 10 & & & Counts $\left(0^{\circ}\right)=56757$ \\
\hline 6737 & 10 & & & Counts $\left(0^{\circ}\right)=110050$. \\
\hline 7068 & 10 & & & Counts $\left(0^{\circ}\right)=124158$. \\
\hline 7129 & 10 & & & Counts $\left(0^{\circ}\right)=476 \quad 62$ \\
\hline 7261 & 10 & & & Counts $\left(0^{\circ}\right)=876 \quad 78$ \\
\hline 7295 & 10 & & & $\operatorname{Counts}\left(0^{\circ}\right)=83057$ \\
\hline 7418 & 10 & & & $\operatorname{Counts}\left(0^{\circ}\right)=298883$. \\
\hline 7491 & 10 & & & $\operatorname{Counts}\left(0^{\circ}\right)=65357$ \\
\hline 7586 & 10 & & & $\operatorname{Counts}\left(0^{\circ}\right)=43037$ \\
\hline 7678 & 10 & & & $\operatorname{Counts}\left(0^{\circ}\right)=44138$ \\
\hline 7776 & 10 & & & Counts $\left(0^{\circ}\right)=209468$. \\
\hline 7884 & 10 & & & $\operatorname{Counts}\left(0^{\circ}\right)=54364$ \\
\hline 7923 & 10 & & & Counts $\left(0^{\circ}\right)=231376$. \\
\hline 7974 & 10 & & & $\operatorname{Counts}\left(0^{\circ}\right)=150362$ \\
\hline 8105 & 10 & $(1+)$ & $(0)$ & $\begin{array}{l}\mathrm{B}(\mathrm{GT}) \text { strength }=0.0041 . \\
\text { Counts }\left(0^{\circ}\right)=458 \quad 42 .\end{array}$ \\
\hline 8182 & 10 & & & $\operatorname{Counts}\left(0^{\circ}\right)=83570$ \\
\hline 8251 & 10 & & & Counts $\left(0^{\circ}\right)=115259$. \\
\hline 8292 & 10 & & & $\operatorname{Counts}\left(0^{\circ}\right)=52047$ \\
\hline 8338 & 10 & & & Counts $\left(0^{\circ}\right)=52249$ \\
\hline 8373 & 10 & & & $\operatorname{Counts}\left(0^{\circ}\right)=41865$ \\
\hline 8400 & 10 & & & Counts $\left(0^{\circ}\right)=123979$. \\
\hline 8492 & 10 & & & $\operatorname{Counts}\left(0^{\circ}\right)=43157$ \\
\hline $8540 \S$ & $\S 20$ & & & $\operatorname{Counts}\left(0^{\circ}\right)=749216$ \\
\hline 8732 & 10 & $(1+)$ & $(0)$ & $\begin{array}{l}\mathrm{B}(\mathrm{GT}) \text { strength }=0.007 \quad 1 . \\
\text { Counts }\left(0^{\circ}\right)=754 \quad 47 .\end{array}$ \\
\hline 8810 & 10 & & & $\operatorname{Counts}\left(0^{\circ}\right)=149260$ \\
\hline 8854 & 10 & & & $\operatorname{Counts}\left(0^{\circ}\right)=102062$. \\
\hline 8887 & 10 & & & Counts $\left(0^{\circ}\right)=1710 \quad 72$ \\
\hline 8929 & 10 & & & $\operatorname{Counts}\left(0^{\circ}\right)=153780$ \\
\hline 8981 & 10 & $(1+)$ & $(0)$ & $\begin{array}{l}\mathrm{B}(\mathrm{GT}) \text { strength }=0.0081 . \\
\text { Counts }\left(0^{\circ}\right)=801116 .\end{array}$ \\
\hline $9068 \S$ & $\S 20$ & & & $\operatorname{Counts}\left(0^{\circ}\right)=549164$ \\
\hline $9088 \S$ & $\S 20$ & & & Counts $\left(0^{\circ}\right)=792149$. \\
\hline $9113 \S$ & $\S 20$ & & & Counts $\left(0^{\circ}\right)=491100$. \\
\hline 9156 & 10 & & & $\operatorname{Counts}\left(0^{\circ}\right)=64348$ \\
\hline 9203 & 10 & & & $\operatorname{Counts}\left(0^{\circ}\right)=129166$ \\
\hline 9236 & 10 & & & Counts $\left(0^{\circ}\right)=104463$. \\
\hline $9280 \S$ & $\S 20$ & & & $\operatorname{Counts}\left(0^{\circ}\right)=55156$ \\
\hline $9312 \S$ & $\S 20$ & & & $\operatorname{Counts}\left(0^{\circ}\right)=110664$. \\
\hline 9406 & 10 & & & Counts $\left(0^{\circ}\right)=91667$ \\
\hline 9565 & 10 & & & $\operatorname{Counts}\left(0^{\circ}\right)=52447$ \\
\hline 9611 & 10 & & & $\operatorname{Counts}\left(0^{\circ}\right)=65149$ \\
\hline $9793 \S$ & $\S 20$ & & & Counts $\left(0^{\circ}\right)=85864$ \\
\hline $9826 \S$ & $\S 20$ & & & $\operatorname{Counts}\left(0^{\circ}\right)=1124 \quad 69$ \\
\hline 9874 & 10 & & & $\operatorname{Counts}\left(0^{\circ}\right)=62075$ \\
\hline
\end{tabular}


${ }^{42} \mathrm{Ca}\left({ }^{3} \mathrm{He}, \mathrm{t}\right) \quad 2015 \mathrm{Fu} 08,1971 \mathrm{Sh} 16$ (continued)

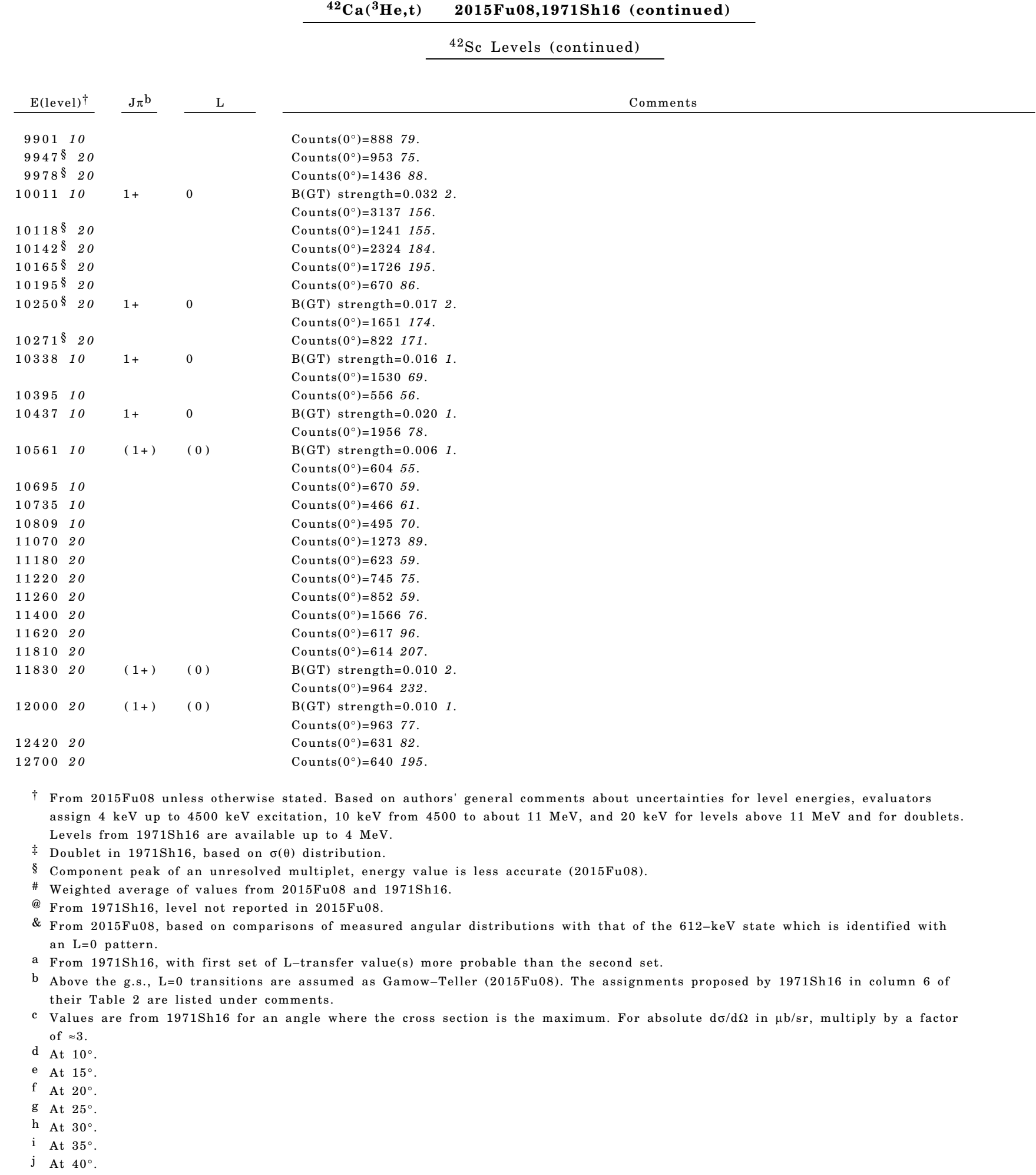




\section{${ }^{42} \mathrm{Ca}\left({ }^{6} \mathrm{Li},{ }^{6} \mathrm{He}\right) \quad 1990 \mathrm{Mo13}, 1975 \mathrm{WhO1}$}

1990Mo13: $\mathrm{E}=156 \mathrm{MeV}{ }^{6} \mathrm{Li}$ beam was produced at the Karlsruhe Isochronous Cyclotron KIZ. Target of a $2.7 \mathrm{mg} / \mathrm{cm}^{2}$ self-supporting ${ }^{42} \mathrm{Ca}$ foil (enriched to $87.7 \%$ ). Reaction products were momentum analyzed with the QQDS magnetic spectrograph "little John" and detected by position sensitive proportional counter, an ionization chamber and a plastic scintillator. Measured cross section at $0^{\circ}, \sigma(\theta)$. Deduced levels. DWBA analysis.

1975Wh01 (also 1974Wh07): E=34 MeV beam was produced at the Argonne National Laboratory (ANL). Reaction products were analyzed with the ANL Engel split-pole spectrograph and detected by two $4.5 \mathrm{~cm}$ long Si surface barrier position-sensitive detectors. Measured $\sigma(\theta)$. DWBA analysis.

${ }^{42}$ Sc Levels

$\underline{\mathrm{E}(\text { level })} \quad \underline{\mathrm{J} \pi^{\ddagger}} \quad \underline{\mathrm{L}} \quad \underline{\sigma(\mathrm{mb} / \mathrm{sr})^{\dagger}}$

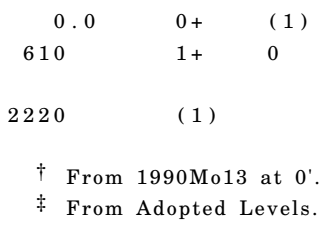

Comments

L: implied from $\sigma(\theta)(1975 \mathrm{Wh} 01)$

$\mathrm{L}$ : from $1990 \mathrm{Mo13}$. $\mathrm{L}=90 \%(\mathrm{~L}=0)+10 \%(\mathrm{~L}=6)$ for $1+$ and $7+\operatorname{doublet}(1975 \mathrm{Wh} 01)$.

$\mathrm{d} \sigma / \mathrm{d} \Omega=30 \mu \mathrm{b} / \mathrm{sr}$ for first maximum at $10^{\circ}(1975 \mathrm{Wh} 01)$.

\section{$\operatorname{Pb}\left({ }^{16} \mathrm{O}, \mathrm{X} \gamma\right) \quad 2006 \mathrm{MoZS}$}

2006MoZS: E $=60 \mathrm{MeV}{ }^{16} \mathrm{O}$ beam was produced at JAEA. A target of $11 \mathrm{mg} / \mathrm{cm}^{2} \mathrm{PbS}$. $\gamma$-rays were detected by the GEMINI-II array. Measured E $\gamma$, I $\gamma, \gamma \gamma$-coin. Deduced levels.

${ }^{42}$ Sc Levels

\begin{tabular}{|c|c|c|c|c|c|}
\hline E(level) & $\mathrm{J} \pi^{\dagger}$ & E(level) & $\mathrm{J} \pi^{\dagger}$ & E(level) & $\mathrm{J} \pi^{\dagger}$ \\
\hline 0.0 & $0+$ & 1512 & $5+$ & $2391 ?$ & $3+$ \\
\hline 611 & $1+$ & 1586 & $2+$ & 2435 & $4+$ \\
\hline 618 & $7+$ & 1845 & $3(+)$ & $2606 ?$ & $4(+)$ \\
\hline 1490 & $3+$ & 2223 & $3+$ & $2995 ?$ & $4+$ \\
\hline
\end{tabular}

$\dagger$ From Adopted Levels.

\begin{tabular}{|c|c|c|c|c|c|}
\hline $\mathrm{E} \gamma$ & E(level) & $\mathrm{E} \gamma$ & E(level) & $\mathrm{E} \gamma$ & E(level) \\
\hline 259 & 1845 & $761^{\dagger}$ & $2606 ?$ & 894 & 1512 \\
\hline $606^{\dagger}$ & $2995 ?$ & $772^{\dagger}$ & $2995 ?$ & 923 & 2435 \\
\hline 611 & 611 & 879 & 1490 & 975 & 1586 \\
\hline 637 & 2223 & & 2391 ? & & \\
\hline
\end{tabular}

$\dagger$ Placement of transition in the level scheme is uncertain. 


\section{Adopted Levels, Gammas}

$\mathrm{Q}\left(\beta^{-}\right)=-17490 \mathrm{SY} ; \mathrm{S}(\mathrm{n})=17478 \quad 28 ; \mathrm{S}(\mathrm{p})=3751.22 \quad 27 ; \mathrm{Q}(\alpha)=-5471.132012 \mathrm{Wa} 38$.

Estimated uncertainty for $Q\left(\beta^{-}\right)=300$ (syst,2012Wa38).

$\mathrm{S}(2 \mathrm{n})=32400160, \mathrm{~S}(2 \mathrm{p})=4836.2028, \mathrm{Q}(\varepsilon \mathrm{p})=2744.2524(2012 \mathrm{Wa} 38)$.

Identification and production of ${ }^{42} \mathrm{TI}$ nuclide by $1962 \mathrm{Ob} 03$ using ${ }^{40} \mathrm{Ca}\left({ }^{3} \mathrm{He}, \mathrm{n}\right)$ which measured a half-life of $0.25 \mathrm{~s} 4$.

2009Ku 19: ${ }^{42} \mathrm{Ti}$ produced in ${ }^{40} \mathrm{Ca}\left({ }^{3} \mathrm{He}, \mathrm{n} \gamma\right) \mathrm{E}=17 \mathrm{MeV}$, beam from the Ion Guide Isotope Separator On-Line (IGISOL)

facility at the Accelerator Laboratory of the University of Jyvaskyla. Target of a $1.5 \mathrm{mg} / \mathrm{cm}^{2} \mathrm{natural} \mathrm{Ca}$. Measured

$\mathrm{E} \gamma, \beta \gamma$-coin, $\mathrm{T}_{1 / 2}$, mass differences using JYFLTRAP Penning-trap spectrometer.

\section{${ }^{42} \mathrm{Ti}$ Levels}

\section{Cross Reference (XREF) Flags}
A ${ }^{43} \mathrm{Cr}$ ep Decay $(21.2 \mathrm{~ms})$
B ${ }^{45} \mathrm{Fe} \varepsilon 3 \mathrm{p}$ Decay $(2.45 \mathrm{~ms})$
D ${ }^{40} \mathrm{Ca}\left({ }^{3} \mathrm{He}, \mathrm{n} \gamma\right)$
C ${ }^{40} \mathrm{Ca}\left({ }^{3} \mathrm{He}, \mathrm{n}\right)$
E ${ }^{40} \mathrm{Ca}\left({ }^{12} \mathrm{C},{ }^{10} \mathrm{Be}\right)$

$\frac{\mathrm{E}(\text { level })^{\dagger}}{0.0} \frac{\mathrm{J} \pi}{0+} \frac{\mathrm{XREF}}{\mathrm{ABCD} \mathrm{F}} \frac{\mathrm{T}_{1 / 2} \S}{208.65 \mathrm{~ms} 80}$

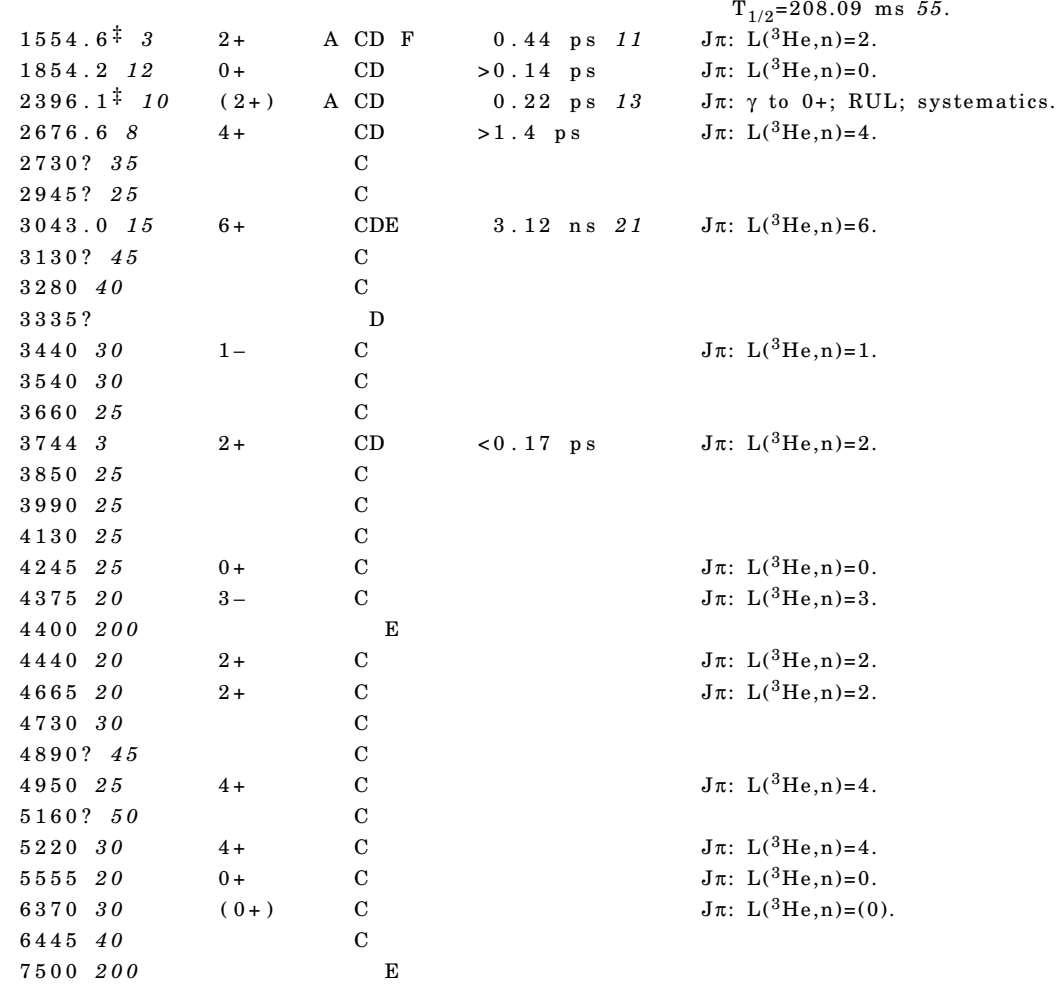

$\% \varepsilon+\% \beta^{+}=100$.

$\mathrm{T}_{1 / 2}$ : weighted average of $211.7 \mathrm{~ms} 19(2015 \mathrm{Mo} 01$, from analysis of $\beta$-decay and correlated implantations), $209.5 \mathrm{~ms} 52(2015 \mathrm{Mo} 01$, from the analysis of $\gamma$-ray data), $208.14 \mathrm{~ms} 45(2009 \mathrm{Ku} 19$, also $2011 \mathrm{KuZY}$, from decay timing of positrons emitted by a pure ${ }^{42} \mathrm{Ti}$ source deposited on a mylar tape and counted by a $4 \pi$ cylindrical plastic scintillator, source production used Penning-trap system; uncertainty increased by evaluators by a factor of 2 ), $230 \mathrm{~ms} 50$ (1972Zi02, $\beta$ counting), $202 \mathrm{~ms} 5$ (1969Ga27, $\gamma$ counting), and $200 \mathrm{~ms} 20(1969 \mathrm{Ni03}, \gamma$ counting), $250 \mathrm{~ms} 40$ (1962Ob03). Other: $173 \mathrm{~ms} 14$ (1969Al12, $\beta$ counting) seems discrepant as compared to all the other values. $2015 \mathrm{Ha} 07$ review gives $\mathrm{T}_{1 / 2}=208.09 \mathrm{~ms} 55$

$\dagger$ From $\left({ }^{3} \mathrm{He}, \mathrm{n} \gamma\right)$ and $\left({ }^{3} \mathrm{He}, \mathrm{n}\right)$.

$\ddagger$ From $\mathrm{E} \gamma$ in ${ }^{43} \mathrm{Cr}$ ecp decay.

$\S$ From DSAM in $\left({ }^{3} \mathrm{He}, \mathrm{n} \gamma\right)$, unless otherwise noted. 
Adopted Levels, Gammas (continued)

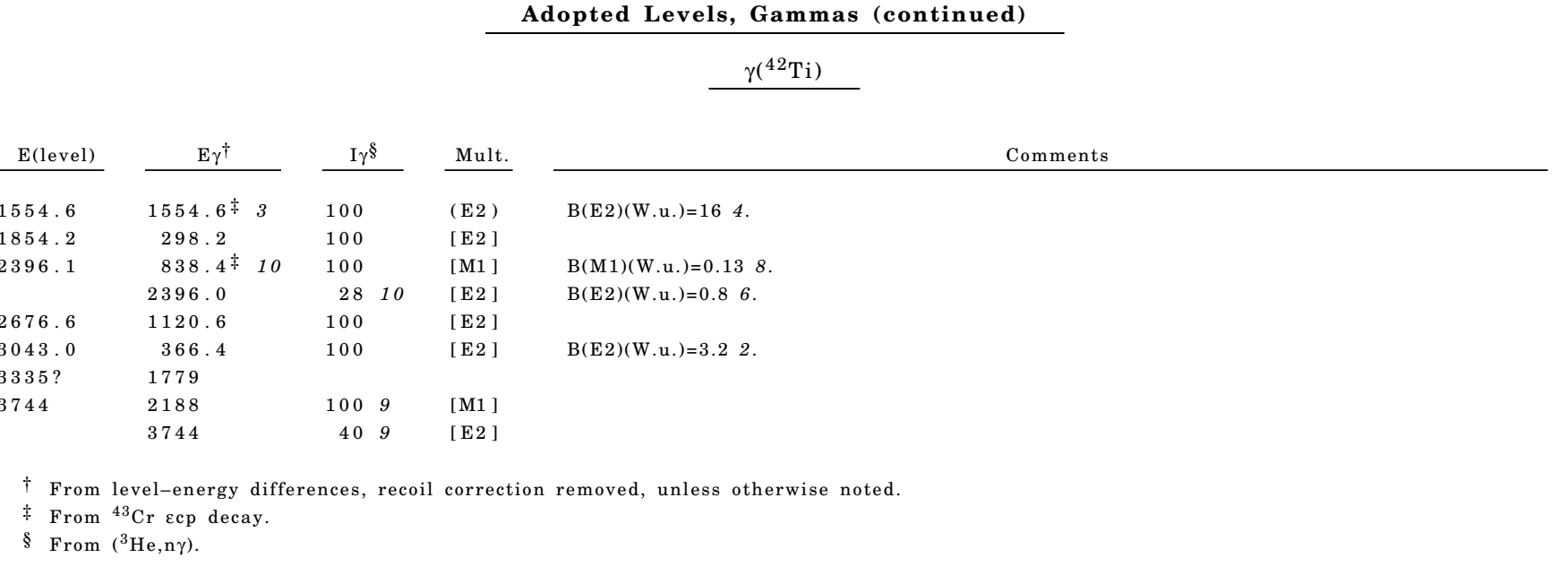




\section{${ }^{43}$ Cr Ep Decay $(21.2 \mathrm{~ms}) \quad 2007$ Do17,2011Po01,2012Au08}

Parent ${ }^{43} \mathrm{Cr}: \mathrm{E}=0 ; \mathrm{J} \pi=(3 / 2+) ; \mathrm{T}_{1 / 2}=21.2 \mathrm{~ms} 7$; Q(g.s. $)=15520$ syst; \%عp decay=79.3 30 .

${ }^{43} \mathrm{Cr}-\mathrm{Q}(\varepsilon \mathrm{p}): 15520400$ (syst,2012Wa38).

${ }^{43} \mathrm{Cr}-\mathrm{J}, \mathrm{T}_{1 / 2}$ : From ${ }^{43} \mathrm{Ar}$ Adopted Levels in the ENSDF database (March 2015 update).

${ }^{43} \mathrm{Cr}-\% \varepsilon p$ decay: \%عp $=79.330$, deduced by $2012 \mathrm{Au} 08$ from relative $\varepsilon$ p branching $87.1 \% 25$ and total proton branching $91.0 \% 23$ (weighted average of $92.5 \% 28$ (2007Do17) and 88\% 4 (2011Po01). Others: \%عp=23 6 (1992Bo37), 28 1 (2007Do17, from proton spectrum), 814 (2011Po01).

2007Do17: ${ }^{43} \mathrm{Cr}$ was produced by the fragmentation of a $74.5 \mathrm{MeV} / \mathrm{nucleon}{ }^{58} \mathrm{Ni}$ beam on a $250 \mathrm{mg} / \mathrm{cm}^{2} \mathrm{natural} \mathrm{nickel}$ target at SISSE/LISE 3 facility in GANIL. Fragments were selected by the ALPHA-LISE 3 separator and identified by TOF and energy-loss with two micro-channel plate (MCP) detectors and a detection setup consisting of silicon and germanium detectors. Double-sided silicon-strip detectors (DSSSD) and a thick Si(Li) detector were used to detect implanted events, charged particles and $\beta$ particles. The $\gamma$ rays were detected by four Ge detectors. Measured Ep, $\mathrm{Ip}, \mathrm{E} \gamma, \mathrm{I} \gamma, \mathrm{p} \gamma$-coin, $\mathrm{T}_{1 / 2}$. Deduced levels, proton decay branching ratios.

2011Po01: ${ }^{43} \mathrm{Cr}$ was produced by the fragmentation of a $161 \mathrm{MeV} / \mathrm{nucleon}{ }^{58} \mathrm{Ni}$ beam on an $800 \mathrm{mg} / \mathrm{cm}^{2} \mathrm{natural} \mathrm{nickel}$ target at NSCL. Fragments were selected using the A1900 separator and identified by time-of-flight (TOF) and energy-loss. Measured Ep, Ip, $\mathrm{T}_{1 / 2}$. Deduced proton decay branching ratios.

2012Au08: ${ }^{43} \mathrm{Cr}$ nuclei produced in the reaction $\mathrm{Ni}\left({ }^{58} \mathrm{Ni}, \mathrm{X}\right)$ using the LISE3 separator at GANIL. ${ }^{43} \mathrm{Cr}$ ions were separated, identified and then implanted onto the time projection chamber (TPC). Decays were detected in a time-projection chamber (TPC), where signals from four gas electron multipliers (GEM) detected in a two-dimensional strip detector combined with drift-time analysis were used to reconstruct the tracks of the particles in three dimensions. Characterization of the TCP was done with the $\beta^{+} p$ decay of $52 \mathrm{Ni}$ with reference to proton energies and branching ratios. Measured energy loss, decay event counts, angular correlation between two protons. Deduced branching ratio. Implantation and decay events were time correlated. Simulations were performed taking account different ratios of the decay energy shared between the two protons. 180 events were recorded for ${ }^{43} \mathrm{Cr} \quad \beta^{+} 2 p$ emission. Correlations observed show that the $\beta^{+} 2 p$ emission favor a sequential emission.

1992Bo37: ${ }^{43} \mathrm{Cr}$ was produced by the fragmentation of a $69 \mathrm{MeV} / \mathrm{nucleon}{ }^{58} \mathrm{Ni}$ beam on a $150 \mathrm{mg} / \mathrm{cm}^{2} \mathrm{natural} \mathrm{nickel}$ target in GANIL. Measured Ep, Ip, $\mathrm{T}_{1 / 2}$. Deduced levels, proton decay branching ratios.

2001Gi01: ${ }^{43} \mathrm{Cr}$ was produced by the fragmentation of a $74.5 \mathrm{MeV} / \mathrm{nucleon}{ }^{58} \mathrm{Ni}$ on a $230.6 \mathrm{mg} / \mathrm{cm}^{2} \mathrm{natural} \mathrm{nickel} \mathrm{target}$ in GANIL. Measured Ep, Ip, E $\gamma, \mathrm{I} \gamma, \mathrm{T}_{1 / 2}$. Deduced levels, branching ratios.

\section{${ }^{42} \mathrm{Ti}$ Levels}

\begin{tabular}{|c|c|}
\hline $\mathrm{E}$ (level) & $\mathrm{J} \pi^{\dagger}$ \\
\hline 0.0 & $0+$ \\
\hline 1554.63 & $2+$ \\
\hline $2393.0 \quad 11$ & $(2+$ \\
\hline
\end{tabular}

$\mathrm{x}$
$\mathrm{E}$ (level): $\mathrm{x} \approx 5.2 \mathrm{MeV}$ or $6.6 \mathrm{MeV}$ (2012Au08). From simulations studies and in comparison with the experimental results, 2012Au08 show that two protons do not share equally the 2p decay energy of $4363 \mathrm{keV} 19$ (2007Do17) and are emitted in sequence. A ratio of $34 \%-66 \%$ between the two protons is in good agreement with experimental data in $2012 \mathrm{Au} 08$. In addition the angular correlation measured between the two protons in $2012 \mathrm{Au} 08$ shows an isotropic distribution of the relative angle between the two protons which is a signature of sequential emission. $2012 \mathrm{Au} 08$ conclude that $\beta^{+}$-delayed emission of protons proceeds in a sequential process via an intermediate state in ${ }^{42} \mathrm{Ti}$ at either 5.2 or $6.6 \mathrm{MeV}$ in ${ }^{42} \mathrm{Ti}$. As the level density is quite high in this energy region, no definite level energy can be assigned.

$\dagger$ From Adopted Levels.

\begin{tabular}{|c|c|c|}
\hline$E(p)^{\ddagger}$ & \multicolumn{2}{|c|}{$\mathrm{I}(\mathrm{p})^{\dagger @}$} \\
\hline $1014 \quad 17$ & 0.6 & 1 \\
\hline $1605 \quad 18$ & 2.1 & 11 \\
\hline $1818 \quad 15$ & 7.1 & 12 \\
\hline $2232 \quad 50$ & 4.7 & 7 \\
\hline $2765 \quad 19$ & 1.2 & 4 \\
\hline $3148 \quad 16$ & 3.4 & 7 \\
\hline $3382 \S \quad 25$ & 1.0 & 4 \\
\hline $3835 \# \quad 90$ & 3.0 & 14 \\
\hline $4680 \quad 26$ & 4.5 & 8 \\
\hline
\end{tabular}

$\dagger$ For intensity per 100 decays, multiply by 0.79330 .

$\ddagger$ Weighted average of 2007 Do 17 and $2001 \mathrm{Gi} 01$, unless otherwise noted.

$\S$ From 2007Do17 only.

\# Unweighted average of 374427 from 2007Do17 and 392624 E from 2001Gi01.

(@) From 2007Do17. 


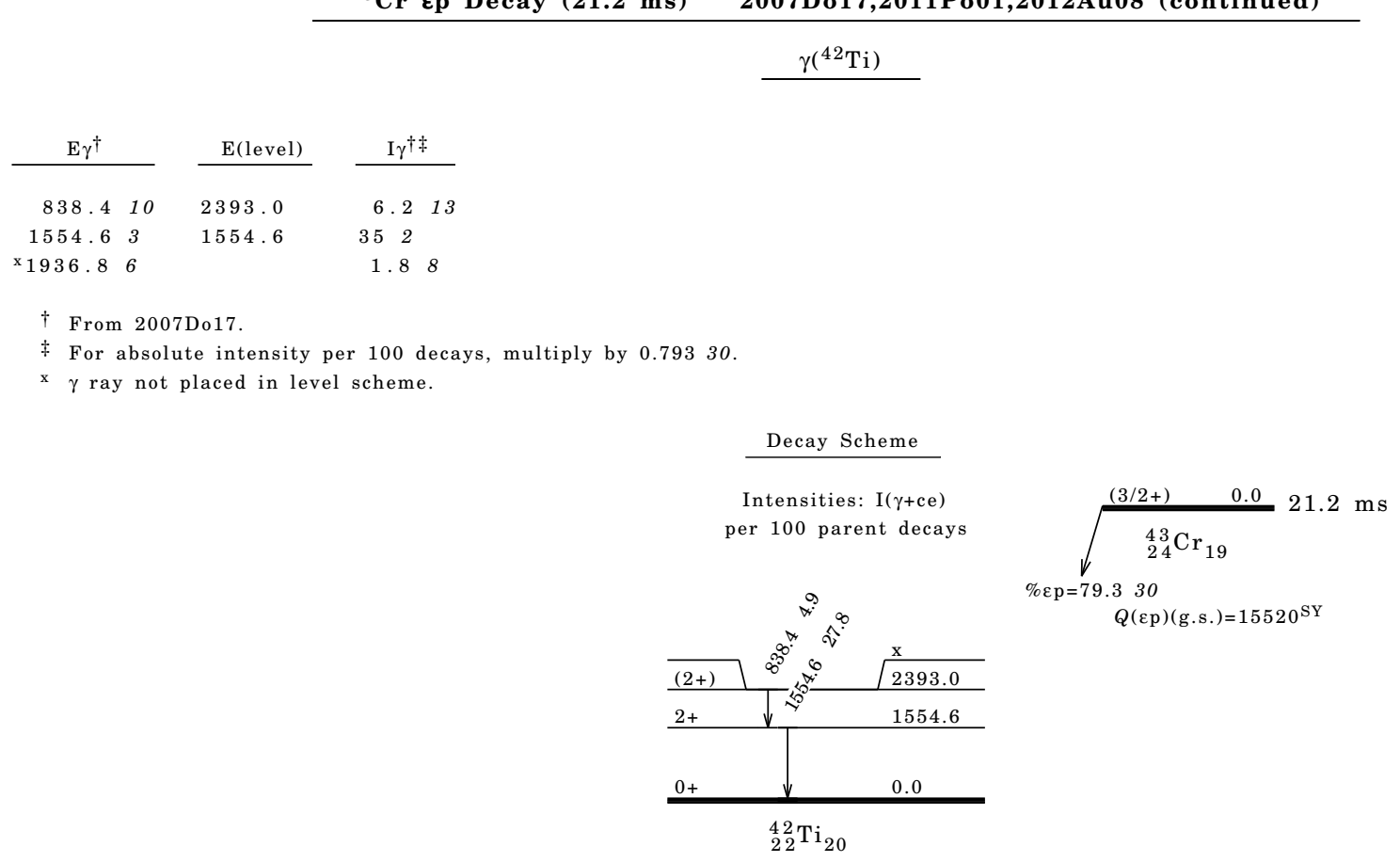

\section{${ }^{45}$ Fe $\varepsilon 3 p$ Decay $(2.45 \mathrm{~ms}) \quad 2007 \mathrm{Mi36,2007Mi40,2009Mi29}$}

Parent ${ }^{45} \mathrm{Fe}: \mathrm{E}=0 ; \mathrm{J} \pi=(3 / 2+) ; \mathrm{T}_{1 / 2}=2.45 \mathrm{~ms} 23 ; \mathrm{Q}$ (g.s.)=16670 syst; \%ع3p decay=3.3 15 .

${ }^{45} \mathrm{Fe}-\mathrm{J}$ : From Adopted Levels of ${ }^{45} \mathrm{Fe}$ in the ENSDF database (Oct 2007 update).

${ }^{45} \mathrm{Fe}-\mathrm{T}_{1 / 2}$ : Weighted average of $4.7 \mathrm{~ms}+34-14(2002 \mathrm{Gi09}$, earlier value of $6.0 \mathrm{~ms}+17-3$ in $2001 \mathrm{Gi02}$, GANIL); 3.2 $\mathrm{ms}+26-10$ (2002Pf02, GSI); $1.6 \mathrm{~ms}+5-3$ (2005Do20, GANIL); $2.6 \mathrm{~ms} 2$ (2007Mi40,2007Mi36, 2009Mi29, NSCL-MSU); 3.6 $\mathrm{ms}+16-8(2012 \mathrm{Au} 08,2007 \mathrm{Gi} 10$, GANIL). Others: $2.5 \mathrm{~ms} 2$ (2008Bl03, review paper, average of four values in literature); $2.56 \mathrm{~ms} 14$ (2012Au08, average of their value with two previous values in literature).

${ }^{45} \mathrm{Fe}-\mathrm{Q}(\varepsilon)$ : Deduced by the evaluators from masses taken from $2012 \mathrm{Wa} 38$, estimated $\Delta \mathrm{Q}=400$.

${ }^{45} \mathrm{Fe}-\% \varepsilon 3 p$ decay: $\% 2 p=704(2007 \mathrm{Mi} 36,2007 \mathrm{Mi} 40,2009 \mathrm{Mi} 29), \% \varepsilon+\% \beta^{+}=30 \quad 4, \% \beta^{+} \mathrm{p}=193, \% \beta^{+} 2 \mathrm{p}=7.820, \% \beta^{+} 3 \mathrm{p}=3.3 \quad 15(2007 \mathrm{Mi} 36$, $2007 \mathrm{Mi} 40,2009 \mathrm{Mi} 29)$. The $\% \varepsilon+\% \beta^{+}$branch=30 4 assumes that all such $\varepsilon+\beta^{+}$decay events are followed by delayed proton decays. Others: $\% 2 \mathrm{p}=78+14-22$ (2012Au08), 5710 (2005Do20), 70-80 (2002Gi09), $\approx 80$ (2002Pf02).

First observation of $\beta$-delayed three proton channel by $2007 \mathrm{Mi} 36$.

2007Mi36, 2007Mi40, 2009Mi29 (also 2008Mi03): ${ }^{45} \mathrm{Fe}$ was produced by the fragmentation of a $161 \mathrm{MeV} / \mathrm{nucleon} 58 \mathrm{Ni}$ beam on a $800 \mathrm{mg} / \mathrm{cm}^{2}$ natural nickel target at National Superconducting Cyclotron laboratory (NSCL) at MSU. Fragments were separated using the A1900 fragment separator and identified in-flight by time-of-flight (TOF) and energy-loss with a plastic scintillator and a thin silicon detector. Silicon detector was used to measured time-of-flight and $\delta$ E. Identified ions stopped in a gaseous detector-the Optical Time Projection Chamber (OTPC) for measuring emitted protons. Measured decay branches, $\mathrm{T}_{1 / 2}$. Out of a total of 125 observed decays of ${ }^{45} \mathrm{Fe}, 87$ events were assigned to direct $2 p$ decay, 38 to $\varepsilon+\beta^{+}$decay followed by proton emission. Out of $38 \varepsilon+\beta^{+}$decay events, 24 were of $\beta^{+} P$ type, ten of $\beta^{+} 2 \mathrm{p}$ and four of $\beta^{+} 3 \mathrm{P}$ type.

2012Au08: measured half-life of ${ }^{45} \mathrm{Fe}$ decay and $2 \mathrm{p}$-decay branching ratio at GANIL.

\section{${ }^{42} \mathrm{Ti}$ Levels}

$\begin{array}{ll}\frac{\mathrm{E}(\text { level })}{0.0} & \frac{\mathrm{J} \pi}{0}\end{array}$


Target ${ }^{40} \mathrm{Ca} \mathrm{J} \pi=0+$.

1977Bo16: $\mathrm{E}=13 \mathrm{MeV}{ }^{3} \mathrm{He}$ beam was produced from the CN Van de Graaff accelerator at the Hahn-Meitner-Institut. A 196 $\mu \mathrm{g} / \mathrm{cm}^{2}$ target of natural ${ }^{40} \mathrm{Ca}$ layer on $0.2 \mathrm{~mm}$ tungsten backing. Neutrons were detected in 16 detectors between 0 ' and $82.5^{\prime}$ at a flight path of $17.5 \mathrm{~m}$ (energy resolution $\mathrm{FWHM}=40-350 \mathrm{keV}$ ). Measured $\sigma\left(\mathrm{E}_{\mathrm{n}}, \theta\right)$. Deduced levels, L, J $\pi$ from DWBA analysis of the data.

1974Al14: $\mathrm{E}=15 \mathrm{MeV}{ }^{3} \mathrm{He}$ beam was produced from the University of Rochester pulsed beam facility. Targets were prepared by evaporating natural calcium onto gold or tantalum backings. Neutrons were detected by scintillators at a flight path of $4 \mathrm{~m}(\mathrm{FWHM}=300 \mathrm{keV})$. Measured $\sigma\left(\mathrm{E}_{\mathrm{n}}, \theta\right)$. Deduced levels, J $\pi$, L from DWBA analysis.

1974Ev02: E=18 MeV beam was produced from the Munich MP tandem accelerator. Target of enriched ${ }^{40} \mathrm{Ca}$. Neutrons were detected by scintillators. Measured $\sigma\left(\mathrm{E}_{\mathrm{n}}, \theta\right)$. Deduced levels, J $\pi$, L from DWBA analysis.

1968Sh09: $\mathrm{E}=8.5-10 \mathrm{MeV}{ }^{3} \mathrm{He}$ beam was produced from the Van de Graaff accelerator at the California Institute of Technology. Natural ${ }^{40} \mathrm{Ca}$ target. Neutrons were detected with the ONR-CIT pulsed-beam neutron spectrometer (FWHM=70-300 keV). Measured $\sigma\left(\mathrm{E}_{\mathrm{n}} \theta\right)$. Deduced levels, J $\pi$, L from DWBA analysis.

1968Ha09: E=6.5 MeV beam was produced from the $5.5 \mathrm{MeV}$ Van de Graaff accelerator at the Hahn-Meitner-Institut. Measured $\sigma\left(\mathrm{E}_{\mathrm{n}}\right)$. Deduced levels.

1964Br13: $\mathrm{E}=24.4 \mathrm{MeV}$. Measured $\sigma\left(\mathrm{E}_{\mathrm{n}}\right)$.

1994NaZY: E $=50 \mathrm{MeV}$. Measured $\sigma\left(\mathrm{E}_{\mathrm{n}}, \theta\right)$. Deduced levels, J $\pi$ from DWBA analysis.

\begin{tabular}{|c|c|c|c|}
\hline \multirow[t]{2}{*}{ Level } & \multicolumn{2}{|c|}{$\mathrm{d} \sigma / \mathrm{d} \Omega\left(\theta_{\max }\right)(\mathrm{mb} / \mathrm{sr})$} & \multirow[b]{2}{*}{$1974 \mathrm{Ev} 02$} \\
\hline & 1977 Bo 16 & $1974 \mathrm{Al} 14$ & \\
\hline 0 & $2.01 \quad\left(0^{\circ}\right)$ & $3.2\left(0^{\circ}\right)$ & $3.2\left(0^{\circ}\right)$ \\
\hline 1555 & $0.51\left(25^{\circ}\right)$ & $0.78 \quad\left(20^{\circ}\right)$ & $0.75\left(20^{\circ}\right)$ \\
\hline 1860 & $0.01 \quad\left(0^{\circ}\right)$ & $0.2 \quad\left(0^{\circ}\right)$ & $0.12 \quad\left(0^{\circ}\right)$ \\
\hline 2355 & $0.08 \quad\left(25^{\circ}\right)$ & $<0.2\left(20^{\circ}\right)$ & \\
\hline 2670 & $0.32\left(0^{\circ}\right)$ & $0.28 \quad\left(0^{\circ}\right)$ & $0.50 \quad\left(0^{\circ}\right)$ \\
\hline 2945 & & & $0.18\left(10^{\circ}\right)$ \\
\hline 3060 & $0.09\left(55^{\circ}\right)$ & $0.1 \quad\left(30^{\circ}\right)$ & \\
\hline 3440 & $0.11\left(10^{\circ}\right)$ & $0.1\left(<15^{\circ}\right)$ & \\
\hline 3745 & $0.37\left(25^{\circ}\right)$ & $0.55\left(20^{\circ}\right)$ & $0.42\left(20^{\circ}\right)$ \\
\hline 4245 & $0.14 \quad\left(0^{\circ}\right)$ & & \\
\hline 4375 & $0.70 \quad\left(30^{\circ}\right)$ & $0.40 \quad\left(40^{\circ}\right)$ & $1.3\left(20^{\circ}\right)$ \\
\hline 4665 & $0.14\left(25^{\circ}\right)$ & & \\
\hline 4950 & $0.12 \quad\left(0^{\circ}\right)$ & & \\
\hline 5220 & $0.12\left(0^{\circ}\right)$ & & \\
\hline 5555 & $1.03\left(0^{\circ}\right)$ & $1.5\left(0^{\circ}\right)$ & $2.2\left(0^{\circ}\right)$ \\
\hline 6445 & & $1.4\left(5^{\circ}\right)$ & $2.2\left(5^{\circ}\right)$ \\
\hline
\end{tabular}

${ }^{42} \mathrm{Ti}$ Levels

\begin{tabular}{|c|c|c|c|c|c|}
\hline $\mathrm{E}(\text { level })^{\dagger}$ & $\mathrm{L}$ & $\mathrm{d} \sigma(\exp ) / \mathrm{d} \sigma(\mathrm{DWBA})(1974 \mathrm{Al14})$ & $\mathrm{E}(\text { level })^{\dagger}$ & $\mathrm{L}$ & $\mathrm{d} \sigma(\exp ) / \mathrm{d} \sigma(\mathrm{DWBA})(1974 \mathrm{Al} 14)$ \\
\hline 0.0 & 0 & 1 & $3990 \quad 25$ & & \\
\hline $1555 \quad 20$ & 2 & 0.85 & $4130 \quad 25$ & & \\
\hline $1860 \quad 20$ & 0 & 0.07 & $4245 \quad 25$ & $0 \S$ & \\
\hline $2355 \quad 25$ & & & $4375 \quad 20$ & $3 \S$ & \\
\hline $2670 \quad 30$ & $4^{\#}$ & 0.42 & $4440 \quad 20$ & $2^{@}$ & 0.66 \\
\hline $2730 \quad 35$ & & & $4665 \quad 20$ & 2 & \\
\hline $2945 \quad 25$ & & & $4730 \quad 30$ & & \\
\hline $3060 \quad 20$ & 6 & $\leq 0.2$ & $4890 ? \ddagger 45$ & & \\
\hline $3130 ? \ddagger 45$ & & & $4950 \quad 25$ & 4 & \\
\hline $3280 \quad 40$ & & & $5160 ? \ddagger 50$ & & \\
\hline $3440 \quad 30$ & $1 \S$ & & $5220 \quad 30$ & 4 & \\
\hline $3540 \quad 30$ & & & $5555 \quad 20$ & 0 & 0.85 \\
\hline $3660 \quad 25$ & & & $6370 \quad 30$ & $(0)$ & 0.75 \\
\hline $3745 \quad 20$ & 2 & 0.65 & $6445 \quad 40$ & & \\
\hline $3850 \quad 25$ & & & & & \\
\hline
\end{tabular}

$\dagger$ Weighted averages from 1968Sh09, 1974Al14, 1974Ev02 and 1977Bo16.

$\ddagger$ Tentative level from 1968 Sh09 only.

$\S$ From 1977Bo16.

\# Other: 0+4 (1974Al14).

(a) Other: $2+4(1974 \mathrm{Al} 14)$. 


\section{${ }^{40} \mathrm{Ca}\left({ }^{3} \mathrm{He}, \mathrm{n} \gamma\right) \quad 1973 \mathrm{Co38}, 1973 \mathrm{Ha} 10$}

1973Co38: $\mathrm{E}=8.0 \mathrm{MeV}$ beam was produced at the Oxford University tandem Van de Graaff accelerator. Target of about 1 $\mathrm{mg} / \mathrm{cm}^{2}$ natural Ca metal (99.7\% purity) evaporated onto a $10 \mathrm{mg} / \mathrm{cm}^{2}$ gold foil. Neutrons were detected by the Oxford high efficiency neutron detector and $\gamma$-rays were detected by a true coaxial Ge(Li) detector Measured $\sigma\left(\mathrm{E}_{\mathrm{n}}\right)$, $\mathrm{E} \gamma$. Deduced levels, $\mathrm{T}_{1 / 2}$ by DSAM.

1973Ha10: E=10.0-11.5 MeV. Targets of 200-300 $\mu \mathrm{g} / \mathrm{cm}^{2}$ natural calcium evaporated on Ta backings. Neutrons were detected in a $5.1-\mathrm{cm}$-diam by $7.6-\mathrm{cm}$-long liquid scintillator and $\gamma$-rays were detected by a $55 \mathrm{~cm}^{3} \mathrm{Ge}(\mathrm{Li})$ detector (FWHM=4.5 keV at $1.28 \mathrm{MeV}$ ). Measured $\mathrm{n} \gamma, \mathrm{n} \gamma \gamma$-coin. Deduced levels, $\mathrm{T}_{1 / 2}$ by DSAM.

1977Br20 (1971BrYK), 1975Ku17: $\mathrm{E}=10,12 \mathrm{MeV}$. Measured $\mathrm{T}_{1 / 2}$ by $\gamma(\mathrm{t})$.

1971Bo23: $\mathrm{E}=9-10 \mathrm{MeV}$. Measured $\mathrm{E} \gamma, \gamma \gamma-\operatorname{coin}, \mathrm{T}_{1 / 2}$ by DSAM.

1971FoZV: $\mathrm{E}=6,6.5,10 \mathrm{MeV}$. Measured $\mathrm{n} \gamma$-coin, $\mathrm{p} \gamma(\theta), \mathrm{T}_{1 / 2}$ by DSAM.

1969Ni03: $\mathrm{E}=8.0-9.0 \mathrm{MeV}$. Measured $\mathrm{E} \gamma, \gamma(\theta), \mathrm{T}_{1 / 2}$.

${ }^{42} \mathrm{Ti}$ Levels

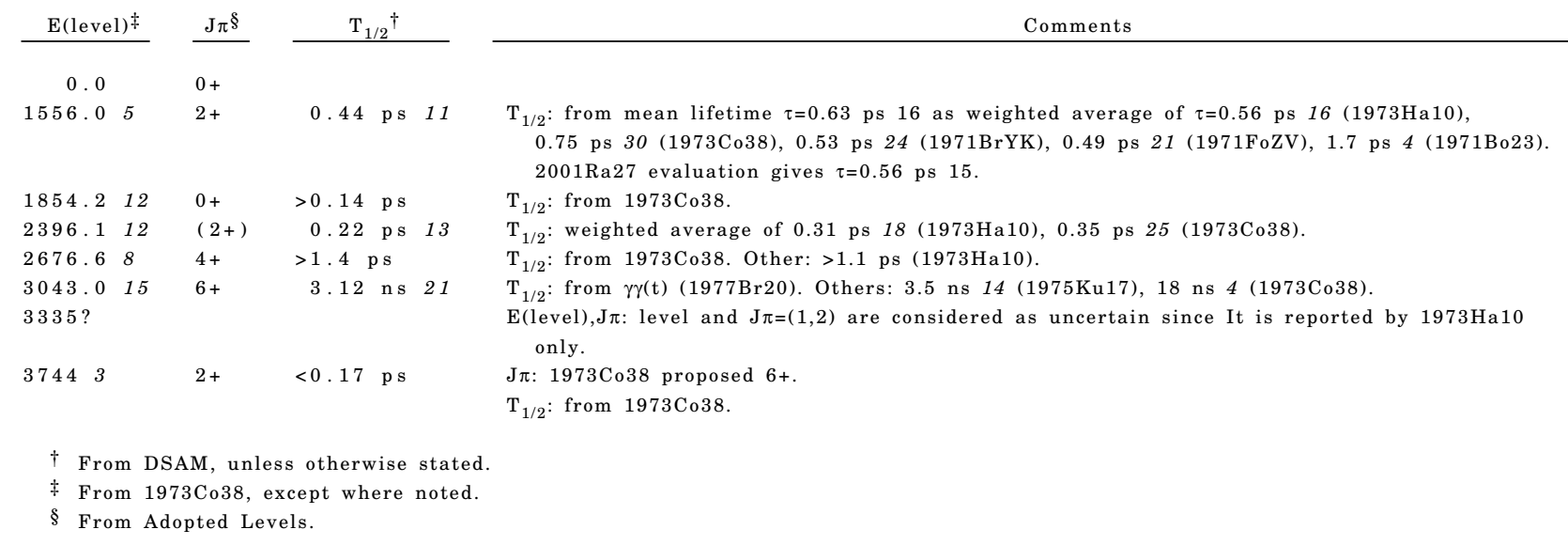

\begin{tabular}{|c|c|c|}
\hline E(level) & $\mathrm{E} \gamma^{\dagger \S}$ & $\mathrm{I} \gamma$ \\
\hline 1556.0 & 1556.0 & \\
\hline 1854.2 & 298.2 & \\
\hline \multirow[t]{2}{*}{2396.1} & 840.1 & 100 \\
\hline & 2396.0 & $28 \div 10$ \\
\hline 2676.6 & 1120.6 & \\
\hline 3043.0 & 366.4 & \\
\hline $3335 ?$ & 1779 & \\
\hline \multirow[t]{2}{*}{3744} & 2188 & $100 \quad 9$ \\
\hline & 3744 & $40 \S 9$ \\
\hline
\end{tabular}

$\dagger$ From level-energy differences.

† Average of 37 (1973Co38) and 18 (1973Co38).

$\S$ From $1973 \mathrm{Ha} 10$. 


\section{${ }^{40} \mathrm{Ca}\left({ }^{12} \mathrm{C},{ }^{10} \mathrm{Be}\right) \quad 1988 \mathrm{Kr} 11$}

1988Kr11(also 1988Me10): E=480 MeV ${ }^{12} \mathrm{C}$ beam at GANIL. Measured $\sigma(\mathrm{E}, \theta)$. Deduced levels, J $\pi$ from DWBA analysis.

Comparisons with shell model calculations.

1972Sc21: E=114 MeV. Measured charged-particle spectra.

${ }^{42} \mathrm{Ti}$ Levels

\begin{tabular}{|c|c|c|}
\hline $\mathrm{E}($ level) & $\mathrm{J} \pi^{\dagger}$ & \\
\hline 3040200 & $(6+)$ & E(level): 3050100 (1972Sc21). \\
\hline $4400 \quad 200$ & $(4+)$ & \\
\hline $7500 \quad 200$ & $(6+)$ & \\
\hline
\end{tabular}

Comments

${ }^{42} \mathrm{Ca}\left(\pi^{+}, \pi^{-}\right) \quad 1992 L e 16,1991 \mathrm{WiO3}, 1990 \mathrm{WeO5}$

1992Le16: E=25-65 MeV. Measured $\sigma(\theta)$.

1991Wi03: $\mathrm{E}=300-500 \mathrm{MeV}$. Measured $\sigma(\theta)$.

$1990 \mathrm{We} 05$ (also 1988We02): $\mathrm{E}=35 \mathrm{keV}$. Measured $\sigma(\theta)$

1984Se04 (also 1984KaZY,1981SeZZ): $\mathrm{E}=125-300 \mathrm{MeV}$. Measured $\sigma(\theta)$.

1993Wa30: $\mathrm{E}<300 \mathrm{MeV}$. Analyzed $\sigma(\theta)$ data.

${ }^{42} \mathrm{Ti}$ Levels

E(level)

0.0

1500 


\title{
Adopted Levels
}

$\mathrm{Q}\left(\beta^{-}\right)=-13860 S Y ; \mathrm{S}(\mathrm{n})=15890 S Y ; \mathrm{S}(\mathrm{p})=-790 \mathrm{SY} ; \mathrm{Q}(\alpha)=-5500 \quad S Y \quad 2012 \mathrm{Wa} 38$.

Estimated uncertainties (syst,2012Wa38): $\Delta \mathrm{Q}\left(\beta^{-}\right)=500, \Delta \mathrm{Q}(\alpha)=360, \Delta \mathrm{S}(\mathrm{n})=420, \Delta \mathrm{SP}=300 \quad(2012 \mathrm{Wa} 38)$.

$\mathrm{S}(2 \mathrm{n})=35650500, \mathrm{~S}(2 \mathrm{p})=1670300, \mathrm{Q}(\varepsilon \mathrm{p})=13730300 \quad($ syst, $2012 \mathrm{Wa} 38)$.

Identification and production by $1993 \mathrm{BoZO}$ in $\mathrm{Ni}\left({ }^{58} \mathrm{Ni}, \mathrm{X}\right) \mathrm{E}=69 \mathrm{MeV} /$ nucleon; measured fragment spectra (1992Bo37,1993BoZO).

Others: 2000BlZZ, 2001Gi02, 2001Gi01

\author{
${ }^{42} \mathrm{~V}$ Levels
}

\begin{tabular}{|c|c|c|}
\hline $\mathrm{E}$ (level) & $\mathrm{T}_{1 / 2}$ & Comments \\
\hline $0.0 ?$ & $<55 \mathrm{n} \mathrm{s}$ & $\% \mathrm{p}=?$ \\
\hline
\end{tabular}




\section{Adopted Levels}

$\mathrm{S}(\mathrm{n})=20340 C A ; \mathrm{S}(\mathrm{p})=1240 \mathrm{~S} Y ; \mathrm{Q}(\alpha)=-6850 \mathrm{SY} \quad 2012 \mathrm{Wa} 38,1997 \mathrm{Mo} 25$

Estimated uncertainties (syst,2012Wa38): $\Delta \mathrm{S}(\mathrm{p})=\Delta \mathrm{Q}(\alpha)=500$.

$\mathrm{S}(\mathrm{n})$ from $1997 \mathrm{Mo} 25 ; \mathrm{S}(\mathrm{p})$ and $\mathrm{Q}(\alpha)$ from $2012 \mathrm{Wa} 38$.

$\mathrm{S}(2 \mathrm{p})=-510430, \mathrm{Q}(\varepsilon \mathrm{p})=14650400$ (syst,2012Wa38). Theoretical $\mathrm{S}(2 \mathrm{n})=38750$

1996B121: ${ }^{42} \mathrm{Cr}$ identified from ${ }^{9} \mathrm{Be}\left({ }^{58} \mathrm{Ni}, \mathrm{X}\right) \mathrm{E}=600 \mathrm{MeV} /$ nucleon; measured fragment charge vs mass to charge ratio at GANIL facility.

$2001 \mathrm{Gi} 01$ (also 2001Gi02,2002Ch28,2000B101,1999B108): $\mathrm{Ni}\left({ }^{58} \mathrm{Ni}, \mathrm{X}\right)$ at $74 \mathrm{MeV} / \mathrm{nucleon}$ at GANIL facility. Measured cross section, half-life and particle spectra.

2007Do17: fragmentation reaction used to produce ${ }^{42} \mathrm{Cr}$ isotope at SISSE/LISE 3 facility in GANIL. Primary beam:

${ }^{58} \mathrm{Ni}^{26+}$ at $74.5 \mathrm{MeV} /$ nucleon; target=natural Ni. Fragment separator=ALPHA-LISE3. Fragment identification by energy loss, residual energy and time-of- flight measurements using two micro-channel plate (MCP) detectors and Si detectors. Double-sided silicon-strip detectors (DSSSD) and a thick Si(Li) detector were used to detect implanted events, charged particles and $\beta$ particles. The $\gamma$ rays were detected by four Ge detectors. Coincidences measured between charged particles and $\gamma$ rays.

${ }^{42} \mathrm{Cr}$ is a possible candidate for $2 \mathrm{p}$ decay. Theoretical calculations of this decay mode: $2003 \mathrm{Gr} 24,2001 \mathrm{Gr} 29$, $1996 \mathrm{Na} 03,1991 \mathrm{Br} 06,1988 \mathrm{Go} 21,1988 \mathrm{Go} 24$.

${ }^{42} \mathrm{Cr}$ Levels

$\mathrm{E}\left(\right.$ level) $\quad \mathrm{J} \pi \quad \mathrm{T}_{1 / 2}$

$0.0 \quad 0+\quad 13.3 \mathrm{~ms} 10$
Comments

$\% \varepsilon+\% \beta^{+} \approx 100 ; \% \varepsilon p=94.450 \quad(2007 \mathrm{Do} 17)$.

$\mathrm{T}_{1 / 2}$ : measured by time correlation of implantation events due to ${ }^{42} \mathrm{Cr}$ and subsequent emission of protons and $\gamma$ rays (2007Do17). Previous result from the same group: $13.4 \mathrm{~ms}^{36-24}$ (2001Gi01).

Total proton branching ratio is from time spectrum of events with energy $>900 \mathrm{keV}$ in the charged-particle spectrum. Possible small contributions from delayed- $\alpha$ and delayed-2p decays were ignored.

No evidence was found for $2 p$ decay $(2001 \mathrm{Gi01})$ where a $1.9 \mathrm{MeV}$ peak in the charged-particle spectrum was ascribed to delayed proton decay.

${ }^{42} \mathrm{Cr}$ decays by $\beta^{+}+\varepsilon$ to ${ }^{42} \mathrm{~V}$ whose g.s. is unbound towards proton emission, thus each $\beta$ decay is followed by a proton decay to ${ }^{41} \mathrm{Ti}$. 


\section{REFERENCES FOR A=42}

1937 Hu01 D.G.Hurst, H.Walke - Phys.Rev. 51, 1033 (1937)

1947 Bl33 E.Bleuler, W.Zunti - Helv.Phys.Acta 20,195 (1947)

1947Si 08 K.Siegbahn - Arkiv Mat.Astron.Fysik 34B, No.4 (1947)

1949 Sh26 F.B.Shull, E.Feenberg - Phys.Rev. 75, 1768 (1949)

1950 Be 60 J.R.Beyster, M.L.Wiedenbeck - Phys.Rev. 79, 728 (1950)

1950 Ri 59 H.T.Richards, R.V.Smith, C.P.Browne - Phys.Rev. 80, 524 (1950)

$1950 \mathrm{Sa} 03 \quad$ V.L.Sailor - Phys.Rev. 77, 794 (1950)

1951 Si 25 W.K.Sinclair, A.F.Holloway - Nature 167, 365 (1951)

1951 St 68 D.T.Stevenson, M.Deutsch - Phys.Rev. 84, 1071 (1951)

$1952 \mathrm{Ka} 44 \quad$ S.Katcoff - Phys.Rev. 87, 886 (1952)

1953 Bu58 P.R.J.Burch - Nature 172, 361 (1953)

$1953 \mathrm{Ha} 40$ D.R.Hamilton, A.Lemonick, F.M.Pipkin - Phys.Rev. 92, 1191 (1953)

1953 Ka 26 B.Kahn, W.S.Lyon - Phys.Rev. 91, 1212 (1953)

1954 An 25 G.Andersson - Phil.Mag. 45, 621 (1954)

$1954 \mathrm{Ca} 64 \quad$ U.Cappeller, R.Klingelhofer - Z.Naturforsch. 9a, 1052 (1954)

1954 Ko 55 L.Koerts, A.Schwarzschild, R.Gold, C.S.Wu - Phys.Rev. 95, 612 (1954)

1955 Em0 4 E.W.Emery, N.Veall - Proc.Phys.Soc.(London) 68A, 346 (1955); Erratum Proc.Phys.Soc.(London) 72 , 675 (1958)

1955 Mo 83 H.Morinaga - Phys.Rev. 100, 431 (1955)

$1955 \mathrm{Sc} 82$ J.P.Schiffer - Phys.Rev. 97, 428 (1955)

$1956 \mathrm{Br} 08$ C.M.Braams - Thesis, University of Utrecht (1956)

1956 Po0 $\quad$ A.V.Pohm, R.C.Waddell, E.N.Jensen - Phys.Rev. 101, 1315 (1956)

$1957 \mathrm{Cl} 40$ J.A.R.Cloutier, A.Henrikson - Can.J.Phys. 35, 1190 (1957)

1957 Wr 37 H.W.Wright, E.I.Wyatt, S.A.Reynolds, W.S.Lyon, T.H.Handley - Nuclear Sci.and Eng. 2, 427 (1957)

$1958 \mathrm{MoZZ}$ W.H.Moore, J.W.Kelley, H.A.Enge - Priv.Comm. (1958)

1959As 65 I.Asplund, T.Wiedling - Phys.Rev. 116, 741 (1959)

1959 Ma 27 J.Mackin, D.Love - J.Inorg.Nuclear Chem. 10, 17 (1959)

1959 Mo 17 H.Morinaga, N.Mutsuro, M.Sugawara - Phys.Rev. 114, 1146 (1959)

1960As03 I.Asplund, T.Wiedling - Arkiv Fysik 16, 483A (1960)

1960 Be 07 N.Benczer-Koller, M.Nessin, T.H.Kruse - Bull.Am.Phys.Soc. 5, No.4, 248, 16 (1960)

$1960 \mathrm{Cl} 02$ R.L.Clarke, E.Almqvist, E.B.Paul - Nuclear Phys. 14, 472 (1960)

$1960 \mathrm{Ga06}$ C.Gatrousis, C.E.Crouthamel - J.Inorg.Nuclear Chem. 13, 13 (1960)

$1960 \mathrm{Ja} 12$ J.Janecke - Z.Naturforsch. 15a, 593 (1960)

1960 Mi 09 J.H.Miller,III - Thesis, Princeton University (1960); NYO-2959 (1960)

1961 Be 19 N.Benczer-Koller, M.Nessin, T.H.Kruse - Phys.Rev. 123, 262 (1961)

1961 Da02 H.Daniel, M.Kuntze, O.Mehling - Z.Naturforsch. 16a, 1118 (1961)

$1961 \mathrm{Ja} 07 \quad$ N.Jarmie, M.G.Silbert - Phys.Rev. 123, 909 (1961)

1961 Ja2 2 J.Janecke, H.Jung - Z.Phys. 165, 94 (1961)

$1961 \mathrm{Mc} 03$ J.D.McCullen, J.J.Kraushaar - Phys.Rev. 122, 555 (1961)

1961 Si 01 P.C.Simms, N.Benczer-Koller, C.S.Wu - Phys.Rev. 121, 1169 (1961)

1961 Sm0 5 A.M.Smith, F.E.Steigert - Phys.Rev. 122, 1527 (1961)

1961 St 12 R.M.Steffen - Phys.Rev. 123, 1787 (1961)

$1962 \mathrm{Ja} 05$ J.Janecke - Nuclear Phys. 30, 328 (1962)

$1962 \mathrm{Me} 06 \quad$ J.S.Merritt, J.G.V.Taylor - Can.J.Phys. 40, 1044 (1962)

1962 Mo 21 J.E.Monahan, S.Raboy, C.C.Trail - Nuclear Instr.Methods 17, 225 (1962)

19620 Ob03 J.D.Oberholtzer - Diss.Abst.Int. 23, 3942 (1962)

1962 Pe19 B.Persson - Acta Radiol. 58, 192 (1962); Nuclear Sci.Abstr. 16, 3670, Abstr.28041 (1962)

1962 Sh29 R.Sherr - Proc.Conf.Direct Interactions and Nucl.Reaction Mechanisms, Padua, Italy, E.Clementel, C.Villi, Eds., Gordon and Breach, New York, p.1025 (1962)

1963 Bl09 D.Bloess, F.Munnich - Z.Naturforsch. 18, 671 (1963)

1963 Ho 17 S.Hontzeas, L.Yaffe - Can.J.Chem. 41, 2194 (1963)

1963 Ro 10 P.C.Rogers, G.E.Gordon - Phys.Rev. 129, 2653 (1963)

1963 Si 13 L.Simons, K.E.Nysten, E.Spring, L.Kald, H.Jungner, P.Holmberg, I.Forsblom - Phys.Letters 7 , 344 (1963)

1964 Bj02 J.H.Bjerregaard, H.R.Blieden, O.Hansen, G.Sidenius, G.R.Satchler - Phys. Rev. 136, B1348 (1964)

1964 Br13 H.C.Bryant, J.G.Beery, E.R.Flynn, W.T.Leland - Nucl. Phys. 53, 97 (1964)

1964 Da 16 H.Daniel, G.T.Kaschl, H.Schmitt, K.Springer - Phys.Rev. 136, B1240 (1964)

1964De09 P.de saintignon, M.Chabre, P.Depommier - Compt.Rend. 258, 884 (1964)

1964 He $10 \quad$ R.Hess, F.C.Roehmer - Helv.Phys.Acta 37, 217 (1964)

1964 Ho 31 M.Honda, D.Lal - Nucl.Phys. 51, 363 (1964)

$1964 \mathrm{Ma} 05 \quad$ K.W.Marlow - Nucl.Phys. 51, 684 (1964)

1964 Mi 06 R.Middleton, D.J.Pullen - Nucl.Phys. 51, 77 (1964)

1964Rio3 E.J-M.Rivet, J.Cerny, B.G.Harvey, R.H.Pehl, J.Haag - UCRL-11213, p.71 (1964)

$1965 \mathrm{Ch} 30$ P.Charoenkwan - Nucl.Instrum.Methods 34, 93 (1965)

$1965 \mathrm{Cl} 01$ D.Cline, H.E.Gove, B.Cujec - Bull.Am.Phys.Soc. 10, No.1, 25, BC3 (1965)

1965 Fr08 J.M.Freeman, G.Murray, W.E.Burcham - Phys.Letters 17, 317 (1965)

1965 Mi 09 K.Miyano - J.Phys.Soc.Japan 20, 2094 (1965)

$1965 \mathrm{Ne} 02$ J.W.Nelson, J.D.Oberholtzer, H.S.Plendl - Nucl.Phys. 62, 434(1965)

1965St09 R.W.Stoenner, O.A.Schaeffer, S.Katcoff - Science 148, 1325 (1965)

1966Az04 T.Azuma, Y.Sato - Ann.Rep.Radiat.Cent.Osaka Prefect. 7, 18 (1966) 


\section{REFERENCES FOR A=42 (CONTINUED)}

1966 Ch14 J.Chwaszczewska, L.Freindl, W.Karcz, W.Przyborski, M.Slapa - Nukleonika 11, 359 (1966)

1966 En04 G.A.P.Engelbertink, P.M.Endt - Nucl.Phys. 88, 12(1966)

1966 Gro9 C.R.Gruhn, N.S.Wall - Nucl.Phys. 81, 161(1966)

1966 He 11 R.L.Heath, J.E.Cline - IDO-17188 (June 1966)

1966 Ki 10 D.-H.Kim - J.Phys.Soc.Japan 21, $2445(1966)$

$1966 \mathrm{Me} 11$ F.R.Metzger, G.K.Tandon - Phys.Rev. 148, 1133 (1966)

1966 Pe 16 R.J.Peterson - Thesis, Univ.Washington (1966)

1966Ri 04 E.Rivet, R.H.Pehl, J.Cerny, B.G.Harvey - Phys.Rev. 141, 1021 (1966)

1966Ri11 A.Richter, A.Bamberger, P.Von Brentano, T.Mayer-Kuckuk, W.Von Witsch - Z.Naturforsch. 21a, 1002 (1966)

1966 Zu01 R.W.Zurmuhle, C.M.Fou, L.W.Swenson - Nucl.Phys. 80, 259(1966)

1967 An 10 A.Anttila - Ann.Acad.Sci.Fennicae: Ser.A VI, No.247 (1967)

1967 Be 44 J.Benisz, K.Grotowski, L.Freindl, W.Karcz, A.Jasielska, T.Panek, S.Wiktor - Acta Phys.Polon. 31, 299 (1967)

1967 Bj06 J.H.Bjerregaard, O.Hansen, O.Nathan, R.Chapman, S.Hinds, R.Middleton - Nucl.Phys. A103, 33 (1967); Erratum Nucl.Phys. A139, 710 (1969)

1967 Go 21 I.W.Goodier - Intern.J.Appl.Radiation Isotopes 18, 334 (1967)

1967 Ha 28 J.A.Harvey, C.C.Weitkamp, E.C.Campbell, G.G.Slaughter - Intern.Conf.Nucl.Structure, Tokyo, p.147(1967)

$1967 \mathrm{Li} 13$ E.P.Lippincott, A.M.Bernstein - Phys.Rev. 163, 1170 (1967)

1967 Lu08 A.Luukko - Ann.Acad.Sci.Fennicae, Ser.A VI, No.249 (1967)

1967 LyZY U.Lynen - Thesis, Heidelberg Univ. (1967)

1967 Na $10 \quad$ C.W.Nahm, T.T.Thwaites - Nucl.Phys. A103, 503(1967)

1967 Wi 15 D.C.Williams, J.D.Knight, W.T.Leland - Phys.Rev. 164, 1419 (1967)

1968 Ba 28 J.C.Bane, J.J.Kraushaar, B.W.Ridley, M.M.Stautberg - Nucl.Phys. A116, 580(1968)

1968 Be 36 T.A.Belote, W.E.Dorenbusch, J.Rapaport - Nucl.Phys. A120, 401 (1968)

1968Da12 H.Daniel, W.Collin, M.Kuntze, S.Margulies, B.Martin, O.Mehling, P.Schmidlin, H.Schmitt - Nucl.Phys. A118, 689 (1968)

1968 Fo05 J.S.Forster, K.Bearpark, J.L.Hutton, J.F.Sharpey-Schafer - Phys.Letters 27B, 285 (1968)

1968 Fr11 R.F.Frosch, R.Hofstadter, J.S.McCarthy, G.K.Noldeke, K.J.van Oostrum, M.R.Yearian, B.C.Clark, R.Herman, D.G.Ravenhall - Phys.Rev. 174, 1380 (1968)

1968 Ha 09 M.Hagen, K.H.Maier, R.Michaelsen - Phys.Letters 26B, 432 (1968)

1968 Ha 31 O.Hansen, T.A.Belote, W.E.Dorenbusch - Nucl.Phys. A118, 41 (1968)

$1968 \mathrm{He} 06 \quad$ R.Hess - Helv.Phys.Acta 41, 77 (1968)

1968 Ly02 U.Lynen, R.Santo, R.Stock, R.Bock - Proc.Symp.Direct Reactions with 33e, Tokyo (1967), K.Matsuda, H.Kamitsubo, Eds.,

IPCR Cyclotron Progr.Rept., Suppl.1, p.147 (1968)

1968 Pu04 F.Puhlhofer - Nucl.Phys. A116, 516(1968)

1968 Sh09 M.H.Shapiro - Nucl.Phys. A114, 401(1968)

1968Va06 J.van Klinken, F.Pleiter, H.T.Dijkstra - Nucl.Phys. A112, 372(1968)

$1969 \mathrm{Al} 12$ A.M.Aldridge, K.W.Kemper, H.S.Plendl - Phys.Letters 30B, 165 (1969)

1969 Ch20 Y.W.Chan, V.W.Cohen, H.B.Silsbee - Phys.Rev. 184, 1102 (1969)

1969 Fl03 B.H.Flowers, L.D.Skouras - Nucl.Phys. A136, 353 (1969)

1969 Ga22 G.Gaul, H.Ludecke, R.Santo, H.Schmeing, R.Stock - Nucl.Phys. A137, 177 (1969)

1969 Ga2 7 A.Gallmann, E.Aslanides, F.Jundt, E.Jacobs - Phys.Rev. 186, 1160 (1969)

$1969 \mathrm{Ha} 02$ R.Hartmann, K.P.Lieb, H.Ropke - Nucl.Phys. A123, 437(1969)

1969 Ho 33 R.N.Horoshko, H.L.Scott, D.M.Van Patter - Phys.Rev. 185, 1470 (1969)

1969Ko03 W.J.Kossler, J.Winkler, C.D.Kavaloski - Phys.Rev. 177, 1725(1969)

1969 Ly02 U.Lynen, H.Oeschler, R.Santo, R.Stock - Nucl.Phys. A127, 343 (1969)

$1969 \mathrm{Me} 12$ R.A.Mendelson, R.T.Carpenter - Phys.Rev. 181, 1552 (1969)

1969 Mo 28 C.G.Morgan, D.F.Jackson - Phys.Rev. 188, 1758 (1969)

1969 Ni 03 F.M.Nicholas, N.Lawley, I.G.Main, M.F.Thomas, P.J.Twin - Nucl.Phys. A124, 97(1969)

1969 Sc31 H.L.Scott, R.N.Horoshko, D.M.Van Patter - Nucl.Instr.Methods 70, 320 (1969)

1969 Sm02 S.M.Smith, A.M.Bernstein - Nucl.Phys. A125, 339(1969)

1969 Te05 A.Tellez, R.Ballini, J.Delaunay, J.P.Fouan - Phys.Letters 29B, 655 (1969)

1969 Yn 01 J.L.Yntema - Phys.Rev. 186, 1144 (1969)

1970 AnZZ G.P.Anastassiou - Thesis, Univ.Penn (1970); Diss.Abst.Int. 31B, 3627 (1970)

1970 Ba 26 D.P.Balamuth, G.P.Anastassiou, R.W.Zurmuhle - Phys.Rev. C2, 215 (1970)

1970 Br 27 G.Brown, A.Denning, A.E.MacGregor - Nucl.Phys. A153, 145 (1970)

1970 Co32 S.Cochavi, D.B.Fossan, S.H.Henson, D.E.Alburger, E.K.Warburton - Phys.Rev. C2, 2241 (1970)

1970 De $10 \quad$ R.J.de Meijer, J.M.M.van Gasteren - Nucl.Phys. A148, 62 (1970)

1970 De 11 R.J.de Meijer, T.F.Risselada, H.G.Berendsen - Nucl.Phys. A148, 77 (1970)

$1970 \mathrm{Fa} 09$ H.U.Fahrbach, W.G.Alberts, K.H.Bodenmiller, S.Buhl - Z.Phys. 235, 265 (1970)

1970 Fe 02 B.Fernandez, J.S.Blair - Phys.Rev. C1, 523 (1970)

1970 Fi 01 W.Fitz, J.Heger, R.Santo, S.Wenneis - Nucl.Phys. A143, 113 (1970)

1970 Fo04 J.S.Forster, K.Bearpark, J.L.Hutton, J.F.Sharpey-Schafer - Nucl.Phys. A150, 30 (1970)

1970 Fr 10 R.M.Freeman, A.Gallmann - Nucl.Phys. A156, 305 (1970)

1970 He 23 H.Hefele, U.Lynen, R.Santo - Nucl.Phys. A157, 93 (1970)

1970 Jo04 L.V.Johnson, T.J.Kennett - Can.J.Phys. 48, 1109 (1970)

1970 Ka 17 K.Kawade, H.Yamamoto, K.Yoshikawa, K.Iizawa, I.Kitamura, S.Amemiya, T.Katoh, Y.Yoshizawa - J.Phys.Soc.Jap. 29, 43 (1970)

1970 La 22 N.Lawley, N.Dawson, G.D.Jones, I.G.Main, P.J.Mulhern, R.D.Symes, M.F.Thomas - Nucl.Phys. A159, 385 (1970)

1970 Ma 39 M.Marmor, S.Cochavi, D.B.Fossan - Phys.Rev.Lett. 25, 1033 (1970) 


\section{REFERENCES FOR A=42 (CONTINUED)}

$1970 \mathrm{No} 04$ $1970 \mathrm{Sc} 22$ $1970 \mathrm{StZZ}$ $1971 \mathrm{AlZF}$

1971 Ar 32 $1971 \mathrm{Be} 29$ $1971 \mathrm{Be} 76$

$1971 \mathrm{Bo} 23$ $1971 \mathrm{BrYK}$

$1971 \mathrm{DiZV}$

$1971 \mathrm{FoZV}$

$1971 \mathrm{FuZT}$

$1971 \mathrm{Ga} 17$

$1971 \mathrm{Ha} 12$

$1971 \mathrm{He} 08$

$1971 \mathrm{Ke} 14$

$1971 \mathrm{Ma} 17$

$1971 \mathrm{Ma} 53$

$1971 \mathrm{No} 06$

$1971 \mathrm{Pe} 04$

$1971 \mathrm{Ra} 35$

$1971 \mathrm{Ro} 30$

$1971 \mathrm{RoYQ}$

$1971 \mathrm{Sh} 16$

$1971 \mathrm{SiYI}$

$1971 \mathrm{Vi} 14$

$1972 \mathrm{Be} 50$

$1972 \mathrm{El} 12$

$1972 \mathrm{Ha} 82$

$1972 \mathrm{KaXR}$

$1972 \mathrm{Ki} 24$

1972 No 09

19720 e 01

$1972 \mathrm{Sc} 21$

$1972 \mathrm{Sp} 04$

$1972 \mathrm{St} 28$

1972 To 01

$1972 \mathrm{Zi} 02$

$1973 \mathrm{BaXX}$

$1973 \mathrm{BrXY}$

$1973 \mathrm{Ca} 13$

$1973 \mathrm{Co} 38$

$1973 \mathrm{CoZG}$

1973 D i 03

$1973 \mathrm{Du} 02$

1973 EnVA

$1973 \mathrm{Ha} 10$

$1973 \mathrm{~J} \mathrm{a} 17$

$1973 \mathrm{JaYN}$

$1973 \mathrm{KeZ} \mathrm{I}$

$1973 \mathrm{LeYI}$

$1973 \mathrm{Mc} 16$

$1973 \mathrm{Mo} 13$

1973 Pr 10

$1973 \mathrm{Se} 01$

$1973 \mathrm{Si} 32$ $1973 \mathrm{Th} 11$

1973 To 07

$1974 \mathrm{Al} 14$

T.Nomura, C.Gil, H.Saito, T.Yamazaki, M.Ishihara - Phys.Rev.Lett. 25, 1342 (1970)

W.Schlegel, D.Schmitt, R.Santo, F.Puhlhofer - Nucl.Phys. A153, 502 (1970)

E.P.Stergakos - Thesis, Virginia Poly. (1970); Diss.Abst.Int. 31B, 3638 (1970)

N.G.Alenius, S.E.Arnell, O.Skeppstedt, E.Wallander, Z.P.Sawa - Proc.Top.Conf.Structure 1f $7 / 2$ Nuclei, Legnaro, Padova, R.A.Ricci, Ed., Editrice Compositori, Bologna, p.343 (1971)

A.G.Artukh, V.V.Avdeichikov, G.F.Gridnev, V.L.Mikheev, V.V.Volkov, J.Wilczynski - Nucl.Phys. A176, 284 (1971)

F.D.Becchetti, D.Dehnhard, T.G.Dzubay - Nucl.Phys. A168, 151 (1971)

B.N.Belyaev, S.S.Vasilenko, D.M.Kaminker - Izv.Akad.Nauk SSSR, Ser.Fiz. 35 , 806 (1971); Bull.Acad.Sci.USSR, Phys.Ser. 35, $742(1972)$

M.Bocciolini, P.Sona, N.Taccetti - Lett.Nuovo Cim. 1, 695 (1971)

B.A.Brown, M.Marmor, D.B.Fossan - Proc.Topical Conf.Struct.of $1 \mathrm{f}_{7} /{ }_{2}$ Nuclei, R.A.Ricci, Ed., Editrice Compositori, Bologna, p.123 (1971)

E.M.Diener - Thesis, SUNY(Stony Brook) (1971); Diss.Abst.Int. 31B, 6818 (1971)

J.S.Forster, G.C.Ball, W.G.Davies - Bull.Amer.Phys.Soc. 16, No.4, 555, EE 8 (1971)

G.F.Fulop - Thesis, New York Univ. (1971); Diss.Abst.Int. 32B, 4125 (1972)

G.T.Garvey, K.W.Jones, D.Schwalm, E.K.Warburton - Phys.Lett. 35B, 563 (1971)

R.Hartmann, H.Grawe - Nucl.Phys. A164, 209 (1971)

J.Heisenberg, J.S.McCarthy, I.Sick - Nucl.Phys. A164, 353 (1971)

H.J.Kennedy, J.D.McCullen - Phys.Rev.Lett. 27, 198 (1971)

G.S.Mani, D.T.Jones, D.Jacques - Nucl.Phys. A165, 384 (1971)

G.S.Mani, D.Jacques - Nucl.Phys. A177, 448 (1971)

T.Nomura, T.Yamazaki, S.Nagamiya, T.Katou - Phys.Rev.Lett. 27, 523 (1971)

F.Pellegrini, F.Gentilin, I.Filosofo - Phys.Rev. C4, 112 (1971)

J.Rapaport, W.E.Dorenbusch, T.A.Belote - Nucl.Phys. A177, 307 (1971)

N.R.Roberson, G.van Middelkoop - Nucl.Phys. A176, 577 (1971)

H.Ronsin - FRNC TH 83 (1971)

R.Sherr, T.S.Bhatia, D.Cline, J.J.Schwartz - Ann.Phys.(N.Y.) 66, 548 (1971)

U.N.Singh - Thesis, State Univ. New York(Albany) (1971); Diss.Abst.Int. 33B, 2753 (1971)

M.Vitasalo - Comment.Phys.-Math. 41, 319 (1971)

M.C.Bertin, G.J.Kumbartzki, R.G.Hirko - Nucl.Phys. A192, 524 (1972)

C.Ellegaard, J.R.Lien, O.Nathan, G.Sletten, F.Ingebretsen, E.Osnes, P.O.Tjom, O.Hansen, R.Stock - Phys.Lett. 40B, $641(1972)$

J.C.Hardy, D.E.Alburger - Phys.Lett. 42B, 341 (1972)

D.K.Kaipov, Y. G.Kosyak, D. N.Smirin, Y.K.Shubny - Program and Theses, Proc. 22 nd Ann.Conf.Nucl.Spectrosc.Struct.At.Nuclei, Kiev, p.48 (1972)

J.I.Kim, E.Gryntakis - Radiochim.Acta 17, 191 (1972)

T.Nomura, T.Yamazaki, S.Nagamiya, T.Katou - J.Phys.Soc.Jap. 33, 286 (1972)

H.Oeschler, H.Schroter, H.Fuchs, L.Baum, G.Gaul, H.Ludecke, R.Santo, R.Stock - Phys.Rev.Lett. 28, 694 (1972)

D.K.Scott, P.N.Hudson, P.S.Fisher, C.U.Cardinal, N.Anyas-Weiss, A.D.Panagiotou, P.J.Ellis - Phys.Rev.Lett. 28, 1659 (1972)

D.J.Spyr, R.T.Carpenter - Phys.Rev. C5, 1576 (1972)

R.Stock, G.Gaul, R.Santo, M.Bernas, B.Harvey, D.Hendrie, J.Mahoney, J.Sherman, J.Steyaert, M.Zisman - Phys.Rev. C6, 1226 (1972)

C.W.Towsley, D.Cline, R.N.Horoshko - Phys.Rev.Lett. 28, 368 (1972)

J.Zioni, A.A.Jaffe, E.Friedman, N.Haik, R.Schectman, D.Nir - Nucl.Phys. A181, 465 (1972)

T.T.Bardin, J.A.Becker, T.R.Fisher, B.A.Watson - USNDC-7, p.125 (1973)

T.H.Braid, J.L.Yntema - ANL-8035, p.106 (1973)

R.F.Casten, E.R.Flynn, J.D.Garrett, S.Orbesen, O.Hansen - Phys.Lett. 43B, 473 (1973)

A.J.Cox, J.M.G.Caraca, B.Schlenk, R.D.Gill, H.J.Rose - Nucl.Phys. A217, 400 (1973)

V.W.Cohen - Bull.Am.Phys.Soc. 18, No.4, 727, KO9 (1973)

E.M.Diener, J.F.Amann, P.Paul, J.D.Vergados - Phys.Rev. C7, 705 (1973)

Y.Dupont, P.Martin, M.Chabre - Phys.Rev. C7, 637 (1973)

P.M.Endt, C.Van der Leun - Nucl.Phys. A214, 1 (1973); Erratum Nucl.Phys. A248, 153 (1975)

R.Hartmann, H.Grawe, K.Kandler - Nucl.Phys. A203, 401 (1973)

A.Jamshidi, W.P.Alford - Phys.Rev. C8, 1782 (1973)

A.Jamshidi - Thesis, Univ.Rochester (1973); Diss.Abst.Int. 34B, 809 (1973)

H.J.Kennedy - Thesis, Univ.Arizona (1973); Diss.Abst.Int. 34B, 1219 (1973)

S.T.Lee - Thesis, Virginia Poly. (1973); Diss.Abst.Int. 34B, 1689 (1973)

J.D.McCullen, D.J.Donahue - Phys.Rev. C8, 1406 (1973)

H.P.Morsch, H.Breuer - Nucl.Phys. A208, 255 (1973)

J.G.Pronko, R.E.McDonald - Phys.Rev. C7, 1061 (1973)

K.K.Seth, H.Ohnuma, T.Suehiro, S.Yamada, S.Takeda - Phys.Rev.Lett. 30, 132 (1973); Erratum Phys.Rev.Lett. 30 , 250 (1973)

U.N.Singh, H.I.Liou, G.Hacken, M.Slagowitz, F.Rahn, J.Rainwater, W.Makofske, J.B.Garg - Phys.Rev. C8, 1833 (1973)

M.F.Thomas, L.D.Skouras - J.Phys.(London) A6, 1763 (1973)

C.W.Towsley, D.Cline, R.N.Horoshko - Nucl.Phys. A204, 574 (1973)

W.P.Alford, R.A.Lindgren, D.Elmore, R.N.Boyd - Phys.Rev. C10, 1013 (1974) 


\section{REFERENCES FOR A=42 (CONTINUED)}

1974 An36 N.Anyas-Weiss, J.C.Cornell, P.S.Fisher, P.N.Hudson, A.Menchaca-Rocha, D.J.Millener, A.D.Panagiotou, D.K.Scott, D.Strottman, D.M.Brink, B.Buck, P.J.Ellis, T.Engeland - Phys.Rep. 12, 201 (1974)

1974 Bro4 B.A.Brown, D.B.Fossan, J.M.McDonald, K.A.Snover - Phys.Rev. C9, 1033 (1974)

1974 Ev02 D.Evers, W.Assmann, K.Rudolph, S.J.Skorka, P.Sperr - Nucl.Phys. A230, 109 (1974)

1974 Fi 01 T.R.Fisher, T.T.Bardin, J.A.Becker, B.A.Watson - Phys.Rev. C9, 598 (1974)

1974 Fo04 G.S.Foote, D.Branford, D.C.Weisser, N.Shikazono, F.C.P.Huang - J.Phys.(London) A7, L4 (1974)

1974 Fr 10 N.Frascaria, J.P.Didelez, J.P.Garron, E.Gerlic, J.C.Roynette - Phys.Rev. C10, 1422 (1974)

1974 Ha 35 J.C.Hardy, G.C.Ball, J.S.Geiger, R.L.Graham, J.A.Macdonald, H.Schmeing - Phys.Rev.Lett. 33, 320 (1974)

1974 HaYC J.C.Hardy, H.R.Andrews, G.C.Ball, J.S.Geiger, R.L.Graham, J.A.Macdonald, H.Schmeing, I.S.TownerProc.Inter.Conf.Nucl.Struct.Spectrosc., Amsterdam, Vol.1, p.147 (1974)

1974 Le08 S.T.Lee, D.D.Long - Can.J.Phys. 52, 772 (1974)

1974 Li $06 \quad$ K.P.Lieb, M.Uhrmacher - Z.Phys. 267, 399 (1974)

$1974 \mathrm{Pe} 03$ J.F.Petersen, W.C.Parkinson - Phys.Lett. 49B, 425 (1974)

1974 Po10 A.R.Poletti, B.A.Brown, D.B.Fossan, P.Gorodetzky, J.J.Kolata, J.W.Olness, E.K.Warburton - Phys.Rev. C10, 997 (1974)

1974 WAYT Reference Field Missing

1974 Wh 07 W.R.Wharton, P.T.Debevec - Phys.Lett. 51B, 451 (1974)

1974 Wi 14 D.H.Wilkinson, D.E.Alburger - Phys.Rev. C10, 1993 (1974)

1975 BrZB T.H.Braid, J.L.Yntema, H.T.Fortune, L.R.Medsker - ANL-75-75, p.82 (1975)

1975 Eg01 H.H.Eggenhuisen, L.P.Ekstrom, G.A.P.Engelbertink, J.Mondria, M.A.van Driel, J.A.J.Hermans - Nucl.Phys. A246, 231 (1975)

1975 Fl08 E.R.Flynn, O.Hansen, R.F.Casten, J.D.Garrett, F.Ajzenberg-Selove - Nucl.Phys. A246, 117 (1975)

1975 Ha 14 O.Hansen, J.R.Lien, O.Nathan, A.Sperduto, P.O.Tjom - Nucl.Phys. A243, 100 (1975)

$1975 \mathrm{Ku} 17$ W.Kutschera, B.A.Brown, H.Ikezoe, G.D.Sprouse, Y.Yamazaki, Y.Yoshida, T.Nomura, H.Ohnuma - Phys.Rev. C12, 813 (1975)

$1975 \mathrm{Me} 01$ L.R.Medsker, S.C.Headley, H.T.Fortune, J.R.Duray - Phys.Rev. C11, 100 (1975)

19750101 J.W.Olness, A.H.Lumpkin, J.J.Kolata, E.K.Warburton, J.S.Kim, Y.K.Lee - Phys.Rev. C11, 110 (1975)

1975 Ra 09 C.N.Rao, B.M.Rao, P.M.Rao, K.Venkata Reddy - Phys.Rev. C11, 1735 (1975)

1975 Tr01 W.Trombik, K.A.Eberhard, J.S.Eck - Phys.Rev. C11, 685 (1975)

1975 Uh 02 M.Uhrmacher, A.Gelberg, F.Brandolini, A.M.Kleinfeld, K.P.Lieb - Phys.Lett. 56B, 247 (1975)

1975 Ve 06 B.Vema Reddy, M.L.Narasimha Raju, V.Seshagiri Rao, D.L.Sastry - J.Phys.(London) G1, 956 (1975)

1975 Wa 04 E.K.Warburton, J.J.Kolata, J.W.Olness - Phys.Rev. C11, 700 (1975)

1975 Wh 01 W.R.Wharton, P.T.Debevec - Phys.Rev. C11, 1963 (1975)

1975WiZG K.H.Wilcox, N.A.Jelley, R.B.Weisenmiller, J.Cerny - LBL-4000, Nucl.Sci.Div.,1974 Ann.Rept., p.92 (1975)

1975 Wu 01 N.Wust, M.Uhrmacher, J.Dauk, K.P.Lieb, A.M.Kleinfeld - J.Phys.(London) G1, 57 (1975)

1975 Yo02 L.E.Young, R.Brenn, S.K.Bhattacherjee, D.B.Fossan, G.D.Sprouse - Phys.Rev.Lett. 35, 497 (1975)

1976Al19 G.D.Alkhazov, T.Bauer, R.Beurtey, A.Boudard, G.Bruge, A.Chaumeaux, P.Couvert, G.Cvijanovich, H.H.Duhm, J.M.Fontaine, D.Garreta, A.V.Kulikov, D.Legrand, J.C.Lugol, J.Saudinos, J.Thirion, A.A.Vorobyov - Nucl.Phys. A274, 443 (1976)

$1976 \mathrm{Be} 13$

$1976 \mathrm{Bi} 15$

$1976 \mathrm{Br} 15$

$1976 \mathrm{De} 24$

$1976 \mathrm{~F}$ o 04

$1976 \mathrm{P}_{0} 03$

$1976 \operatorname{Pr} 15$

$1976 \mathrm{Ra} 30$

P.Betz, E.Bitterwolf, B.Busshardt, H.Ropke - Z.Phys. A276, 295 (1976)

E.Bitterwolf, H.Ropke, P.Betz - J.Phys.(London) G2, L171 (1976)

J.Britz, A.Chevallier, J.Chevallier, B.Haas - Nucl.Phys. A262, 189 (1976)

R.M.del Vecchio, R.T.Kouzes, R.Sherr - Nucl.Phys. A265, 220 (1976)

G.S.Foote, D.Branford, D.C.Weisser, N.Shikazono, R.A.I.Bell, F.C.P.Huang - Nucl.Phys. A263, 349 (1976)

A.R.Poletti, E.K.Warburton, J.W.Olness, J.J.Kolata, P.Gorodetzky - Phys.Rev. C13, 1180 (1976)

H.G.Price, G.D.Jones, P.R.G.Lornie, M.R.Nixon, P.J.Nolan, P.J.Twin - J.Phys.(London) G2, L177 (1976)

B.Mallikarjuna Rao, C.Narasimha Rao, P.Mallikharjuna Rao, R.Mathews, K.Venkata Reddy - Curr.Sci.(India) 45, 319 (1976)

1976Ro06 R.L.Robinson, H.J.Kim, J.B.McGrory, G.J.Smith, W.T.Milner, R.O.Sayer, J.C.Wells, Jr., J.Lin - Phys.Rev. C13, 1922 (1976)

1976 Ul01 M.Ulrickson, W.Hartwig, N.Benczer-Koller, J.R.MacDonald, J.W.Tape - Phys.Rev. C13, 536 (1976)

1976 UlZZ M.A.Ulrickson - Thesis, Rutgers Univ. (1975); Diss.Abst.Int. 36B, 5117 (1976)

1976 Wi 08 D.H.Wilkinson, D.E.Alburger - Phys.Rev. C13, 2517 (1976)

1977 Al07 G.D.Alkhazov, T.Bauer, R.Bertini, L.Bimbot, O.Bing, A.Boudard, G.Bruge, H.Catz, A.Chaumeaux, P.Couvert, J.M.Fontaine, F.Hibou, G.J.Igo, J.C.Lugol, M.Matoba - Nucl.Phys. A280, 365 (1977)

1977 Bo 16 W.Bohne, K.D.Buchs, H.Fuchs, K.Grabisch, D.Hilscher, U.Jahnke, H.Kluge, T.G.Masterson, H.Morgenstern - Nucl.Phys. A284, 14 (1977)

1977 Br $20 \quad$ B.A.Brown, C.Jachcinski, G.D.Sprouse - Phys.Rev. C16, 480 (1977)

$1977 \mathrm{Ek01}$ L.P.Ekstrom, H.H.Eggenhuisen, G.A.P.Engelbertink, J.A.J.Hermans, H.J.M.Aarts - Nucl.Phys. A283, 157 (1977)

1977 En02 P.M.Endt - At.Data Nucl.Data Tables 19, 23 (1977)

1977 In04 P.D.Ingalls, J.C.Overley, H.S.Wilson - Nucl.Phys. A293, 117 (1977)

1977 Is 03 M.Ismail - J.Phys.(Paris) 38, 897 (1977)

1977 LiZI J.M.Lind, D.P.Balamuth, K.C.Young,Jr. - Bull.Amer.Phys.Soc. 22, No.8, 1024, DD7 (1977)

$1977 \mathrm{Na} 25$ H.Nann, W.S.Chien, A.Saha, B.H.Wildenthal - Nucl.Phys. A292, 195 (1977)

1977 NaZS K.G.Nair, C.W.Towsley, K.Nagatani, H.Voit - Bull.Am.Phys.Soc. 22, No.4, 592, GF2 (1977)

1977 Pa 24 M.Paul, A.Marinov, J.Burde, C.Drory, J.Lichtenstadt, S.Mordechai, E.Navon - Nucl.Phys. A289, 94 (1977)

$1977 \mathrm{SaZF}$ A.Saha, K.K.Seth, H.Nann - Proc.Intern.Conf.Nuclear Structure, Tokyo, Vol.1, p.233 (1977)

1977 Sc24 J.S.Schweitzer, Z.E.Switkowski, R.M.Wieland - Nucl.Phys. A287, 344 (1977)

1977 Voo2 H.Vonach, P.Glassel, E.Huenges, P.Maier-Komor, H.Rosler, H.J.Scheerer, H.Paul, D.Semrad - Nucl.Phys. A278, 189 (1977)

1978 Be 61 J.A.Becker, R.A.Chalmers, B.A.Watson, D.H.Wilkinson - Nucl.Instrum.Methods 155, 211 (1978) 


\section{REFERENCES FOR A=42 (CONTINUED)}

1978 De 08 R.M.DelVecchio, W.W.Daehnick - Phys.Rev. C17, 1809 (1978)

1978 Eg02 H.H.Eggenhuisen, L.P.Ekstrom, G.A.P.Engelbertink, H.J.M.Aarts - Nucl.Phys. A305, 245 (1978)

1978 Eno2 P.M.Endt, C.van der Leun - Nucl.Phys. A310, 1 (1978)

1978 Fo02 H.T.Fortune, R.R.Betts, J.N.Bishop, M.N.I.Al-Jadir, R.Middleton - Nucl.Phys. A294, 208 (1978)

1978 Gr02 H.D.Graf, H.Feldmeier, P.Manakos, A.Richter, E.Spamer, D.Strottman - Nucl.Phys. A295, 319 (1978)

1978Ja10 R.Jahn, D.P.Stahel, G.J.Wozniak, R.J.de Meijer, J.Cerny - Phys.Rev. C18, 9 (1978)

1978Ja22 R.Jahn, D.P.Stahel, G.J.Wozniak, R.J.de Meijer, J.Cerny - Fizika, Suppl. 10, 33 (1978)

1978 Li 27 J.Lichtenstadt, A.Marinov, M.Paul, J.Burde, S.Mordechai - Nucl.Phys. A311, 61 (1978)

1978 Pro2 H.G.Price, G.D.Jones, P.R.G.Lornie, P.J.Twin, R.Wadsworth - J.Phys.(London) G4, 707 (1978)

1978Vo01 P.B.Vold, D.Cline, R.N.Boyd, H.Clement, W.P.Alford, J.A.Kuehner - Phys.Lett. 72B, 311 (1978)

1978 Vo04 P.B.Vold, D.Cline, R.N.Boyd, H.Clement, W.P.Alford, J.A.Kuehner - Nucl.Phys. A302, 12 (1978)

1979 Au03 P.Auger, T.H.Chiang, J.Galin, B.Gatty, D.Guerreau, E.Nolte, J.Pouthas, X.Tarrago, J.Girard - Z.Phys. A289, 255 (1979)

1979 Ba14 T.S.Bauer, R.Beurtey, A.Boudard, G.Bruge, H.Catz, P.Couvert, J.L.Escudie, J.M.Fontaine, M.Garcon, J.C.Lugol, M.Matoba, S.Platchkov, M.Rouger, Y.Terrien - Phys.Rev. C19, 1438 (1979)

1979 Ig01 G.Igo, G.S.Adams, T.S.Bauer, G.Pauletta, C.A.Whitten, Jr., A.Wriekat, G.W.Hoffmann, G.S.Blanpied, W.R.Coker,

C.Harvey, R.P.Liljestrand, L.Ray, J.E.Spencer, H.A.Thiessen, C.Glashausser, N.M.Hintz, M.A.Oothoudt, H.Nann,

K.K.Seth, B.E.Wood, D.K.McDaniels, M.Gazzaly - Phys.Lett. 81B, 151 (1979)

$1979 \mathrm{KuZQ}$

S.Kubono, J.C.Sens, P.Bond, J.Bronson, K.Nagatani - Bull.Am.Phys.Soc. 24, No.7, 842, FB7 (1979)

1979 Vo04 P.B.Vold, D.Cline, M.J.A.De Voigt, O.Hansen, O.Nathan - Nucl.Phys. A321, 109 (1979)

1979 We10 G.D.Westfall, T.J.M.Symons, D.E.Greiner, H.H.Heckman, P.J.Lindstrom, J.Mahoney, A.C.Shotter, D.K.Scott, H.J.Crawford, C.McParland, T.C.Awes, C.K.Gelbke, J.M.Kidd - Phys.Rev.Lett. 43, 1859 (1979)

1980 Da 22 R.Daniel, P.A.Butler, A.D.Irving, P.J.Nolan, J.F.Sharpey-Schafer - J.Phys.(Paris), Colloq.C-10, 155 (1980)

1980Fa07 E.Fabrici, S.Micheletti, M.Pignanelli, F.G.Resmini, R.De Leo, G.D'Erasmo, A.Pantaleo - Phys.Rev. C21, 844 (1980)

1980 Gio2 H.J.Gils, E.Friedman, H.Rebel, J.Buschmann, S.Zagromski, H.Klewe-Nebenius, B.Neumann, R.Peel, G.Bechtold Phys.Rev. C21, 1239 (1980)

1980 HoZO J.Honkanen, J.Aysto, M.Kortelahti, K.Eskola, A.Hautojarvi - JYFL Ann.Rept., 1980, p.28 (1980)

$1980 \mathrm{Ke} 01$ T.Kern, P.Betz, E.Bitterwolf, F.Glatz, H.Ropke - Z.Phys. A294, 51 (1980)

1980 NaZU T.N.Nasr - Diss.Abst.Int. 41B, 1814 (1980)

1980 Sa 32 A.M.Sandorfi, C.J.Lister, D.E.Alburger, E.K.Warburton - Phys.Rev. C22, 2213 (1980)

1980St17 W.Steffen, H.-D.Graf, W.Gross, D.Meuer, A.Richter, E.Spamer, O.Titze, W.Knupfer - Phys.Lett. B95, 23 (1980)

$1980 \mathrm{StZO} \quad$ D.P.Stahel - Diss.Abst.Int. 41B, 247 (1980)

1980 Va 17 J.Van Driel, R.Kamermans, R.J.De Meijer - Nucl.Phys. A350, 109 (1980)

1981 Bo 26 K.G.Boyer, W.B.Cottingame, L.E.Smith, S.J.Greene, C.F.Moore, J.S.McCarthy, R.C.Minehart, J.F.Davis, G.R.Burleson, G.Blanpied, C.A.Goulding, H.A.Thiessen, C.L.Morris - Phys.Rev. C24, 598 (1981)

1981 He 17 P.Herges, H.V.Klapdor, T.Oda - Phys.Lett. 106B, 167 (1981)

1981 He 20 P.Herges, H.V.Klapdor, T.Oda - Nucl.Phys. A372, 253 (1981)

1981 HuZT A.Huck, G.Klotz, A.Knipper, C.Miehe, C.Richard-Serre, G.Walter - Proc.Int.Conf.Nuclei Far from Stability, Helsingor, Denmark, Vol.2, p.378 (1981); CERN-81-09 (1981)

1981 I t 02 K.Itoh, Y.M.Shin, T.Saito, Y.Torizuka - Phys.Rev. C24, 1969 (1981)

1981 No07 T.Noro, H.Sakaguchi, M.Nakamura, K.Hatanaka, F.Ohtani, H.Sakamoto, S.Kobayashi - Nucl.Phys. A366, 189 (1981)

1981 Ra 02 L.Ray, G.W.Hoffmann, M.Barlett, J.McGill, J.Amann, G.Adams, G.Pauletta, M.Gazzaly, G.S.Blanpied - Phys.Rev. C23, $828(1981)$

1981SeZZ K.K.Seth, M.Kaletka, S.G.Iversen, A.Saha, D.Barlow, D.Smith, M.Bosko - Bull.Am.Phys.Soc. 26, No.4, 607, HG11 (1981)

1981 Vo04 B.Vosicki, T.Bjornstad, L.C.Carraz, J.Heinemeier, H.L.Ravn - Nucl.Instrum.Methods 186, 307 (1981)

1982 Ba5 5 A.M.Baxter, A.M.Mcdonald, P.G.Ikossi, J.A.Kuehner - Nucl.Phys. A390, 29 (1982)

1982 Be 32 A.M.Bernstein, R.A.Miskimen, B.Quinn, S.A.Wood, M.V.Hynes, G.S.Blanpied, B.G.Ritchie, V.R.Brown - Phys.Rev.Lett. 49, 451 (1982)

$1982 \mathrm{Mi} 06 \quad$ L.W.Mitchell, M.R.Anderson, S.R.Kennett, D.G.Sargood - Nucl.Phys. A380, 318 (1982)

1982 Re03 K.E.Rehm, W.Henning, J.R.Erskine, D.G.Kovar, M.H.Macfarlane, S.C.Pieper, M.Rhoades-Brown - Phys.Rev. C 25 , 1915 (1982)

$1982 \mathrm{RuZV} \quad$ A.R.Rutledge, L.V.Smith, J.S.Merritt - NBS-SP-626, p.5 (1982)

1982 To02 F.Touchard, P.Guimbal, S.Buttgenbach, R.Klapisch, M.De Saint Simon, J.M.Serre, C.Thibault, H.T.Duong, P.Juncar, S.Liberman, J.Pinard, J.L.Vialle - Phys.Lett. 108B, 169 (1982)

1983 Da12 R.Daniel, J.D.Burrows, P.A.Butler, J.R.Cresswell, A.D.Irving, D.J.G.Love, T.P.Morrison, P.J.Nolan, J.F.Sharpey-Schafer - J.Phys.(London) G9, 947 (1983)

1983 I t 02 K.Itoh, Y.M.Shin - Phys.Rev. C28, 2505 (1983)

1983 Mi 25 R.A.Miskimen, A.M.Bernstein, B.Quinn, S.A.Wood, M.V.Hynes, G.S.Blanpied, B.G.Ritchie, V.R.Brown - Phys.Lett. 131B, 26 (1983)

1983 Sa01 A.Saha, K.K.Seth, H.Nann - Phys.Rev. C27, 405 (1983)

1984Bo02 K.G.Boyer, W.J.Braithwaite, W.B.Cottingame, S.J.Greene, L.E.Smith, C.F.Moore, C.L.Morris, H.A.Thiessen, G.S.Blanpied, G.R.Burleson, J.F.Davis, J.S.McCarthy, R.C.Minehart, C.A.Goulding - Phys.Rev. C29, 182 (1984)

$1984 \mathrm{KaZY} \quad$ M.O.Kaletka - Diss.Abst.Int. 44B, 3446 (1984)

1984 Ke 14 G.Kennedy - Nucl.Instrum.Methods 224, 207 (1984)

$1984 \mathrm{Ma} 40$ R.L.Macklin - Nucl.Sci.Eng. 88, 129 (1984)

1984 Pa15 A.Passoja, P.Tikkanen, A.Krasznahorkay, Z.Gacsi, T.Kibedi, T.Fenyes - ATOMKI Kozlem. 26, 75 (1984)

1984 Pa16 A.Passoja, P.Tikkanen, A.Krasznahorkay, Z.Gacsi, T.Kibedi, T.Fenyes - Nucl.Instrum.Methods 223, 96 (1984)

1984Ra04 C.Rangacharyulu, W.Steffen, A.Richter, E.Spamer, O.Titze - Phys.Lett. 135B, 29 (1984) 


\section{REFERENCES FOR A=42 (CONTINUED)}

1984Se04 K.K.Seth, M.Kaletka, S.Iversen, A.Saha, D.Barlow, D.Smith, L.C.Liu - Phys.Rev.Lett. 52, 894 (1984)

$1985 \mathrm{Aj} 03$ F.Ajzenberg-Selove, R.E.Brown, E.R.Flynn, J.W.Sunier - Phys.Rev. C32, 756 (1985)

1985 Da04 W.W.Daehnick, R.D.Rosa - Phys.Rev. C31, 1499 (1985)

1985 Ki 07 A.Z.Kiss, E.Koltay, B.Nyako, E.Somorjai, A.Anttila, J.Raisanen - J.Radioanal.Nucl.Chem. 89, 123 (1985)

1985 Kr06 B.Krusche, Ch.Winter, K.P.Lieb, P.Hungerford, H.H.Schmidt, T.Von Egidy, H.J.Scheerer, S.A.Kerr, H.G.Borner Nucl.Phys. A439, 219 (1985)

1985 Le 17 P.M.Lewis, A.R.Poletti, M.J.Savage, C.L.Woods - Nucl.Phys. A443, 210 (1985)

1985 Mi 06 K.Miura, Y.Hiratate, T.Shoji, T.Suehiro, H.Yamaguchi, Y.Ishizaki - Nucl.Phys. A436, 221 (1985)

1985Vi01 F.Videbaek, O.Hansen, B.S.Nilsson, E.R.Flynn, J.C.Peng - Nucl.Phys. A433, 441 (1985)

$1986 \mathrm{HeZY}$ D.W.Hetherington - Diss.Abst.Int. 46, 3897 (1986); Thesis, Univ.McGill (1984)

$1986 \mathrm{Mc05}$ R.H.McCamis, T.N.Nasr, J.Birchall, N.E.Davison, W.T.H.van Oers, P.J.T.Verheijen, R.F.Carlson, A.J.Cox, B.C.Clark, E.D.Cooper, S.Hama, R.L.Mercer - Phys.Rev. C33, 1624 (1986)

1986 Se 02 M.E.Sevior, L.W.Mitchell, C.I.W.Tingwell, D.G.Sargood - Nucl.Phys. A454, 128 (1986)

1987 Ko 34 V.T.Koslowsky, J.C.Hardy, E.Hagberg, R.E.Azuma, G.C.Ball, E.T.H.Clifford, W.G.Davies, H.Schmeing, U.J.Schrewe, K.S.Sharma - Nucl.Phys. A472, 419 (1987)

$1987 \mathrm{Na} 28$ Nathu Ram, M.K.Mehta - Indian J.Phys. 61A, 489 (1987)

1987TaZY R.Tanczyn, A.Pakou, D.Barker, N.Benczer-Koller, G.Kumbartzki, G.Lenner, A.Pique - Bull.Am.Phys.Soc. 32, No. 8, 1568, DC3 (1987)

1988Br02 C.Brendel, P.von Neumann-Cosel, A.Richter, G.Schrieder, H.Lenske, H.H.Wolter, J.Carter, D.Schull - Nucl.Phys. A477, 162 (1988)

1988 Go 21 V.I.Goldanskii - Phys.Lett. 212B, 11 (1988)

1988Go24 V.I.Goldansky - Pisma Zh.Eksp.Teor.Fiz. 48, 3 (1988); JETP Lett.(USSR) 48, 1 (1988)

1988 Kr11 L.Kraus, A.Boucenna, I.Linck, B.Lott, R.Rebmeister, N.Schulz, J.C.Sens, M.C.Mermaz, B.Berthier, R.Lucas, J.Gastebois, A.Gillibert, A.Miczaika, E.Tomasi-Gustafsson, C.Grunberg - Phys.Rev. C37, 2529 (1988)

$1988 \mathrm{Me} 10 \quad$ M.C.Mermaz - Rev.Roum.Phys. 33, 739 (1988)

198 8We 02 Z.Weinfeld, E.Piasetzky, H.W.Baer, R.L.Burman, M.J.Leitch, C.L.Morris, D.H.Wright, S.H.Rokni, J.R.Comfort Phys.Rev. C37, 902 (1988)

1989Ai02 S.Ya.Aisina, K.A.Kuterbekov, N.N.Pavlova, A.V.Yushkov- Izv.Akad.Nauk SSSR, Ser.Fiz. 53, 37 (1989); Bull.Acad.Sci.USSR, Phys.Ser. 53, No.1, 35 (1989)

1989 He 11 D.W.Hetherington, A.Alousi, R.B.Moore - Nucl.Phys. A494, 1 (1989)

1989 I t 02 K.Itoh, Y.M.Shin, W.J.Gerace, Y.Torizuka - Nucl.Phys. A492, 426 (1989)

1989 Ki 11 S.W.Kikstra, C.van der Leun, S.Raman, E.T.Jurney, I.S.Towner - Nucl.Phys. A496, 429 (1989)

1989 Le16 M.Lewitowicz, Yu.E.Penionzhkevich, A.G.Artukh, A.M.Kalinin, V.V.Kamanin, S.M.Lukyanov, Nguyen Hoai Chau,

A.C.Mueller, D.Guillemaud-Mueller, R.Anne, D.Bazin, C.Detraz, D.Guerreau, M.G.Saint-Laurent, V.Borrel, J.C.Jacmart, F.Pougheon, A.Richard, W.D.Schmidt-Ott - Nucl.Phys. A496, 477 (1989)

1989Mi 03 Ch.Miehe, Ph.Dessagne, P.Baumann, A.Huck, G.Klotz, A.Knipper, G.Walter, G.Marguier - Phys.Rev. C39, 992 (1989)

1989 MuZU A.C.Mueller, M.Lewitowicz, R.Anne, A.G.Artukh, D.Bazin, V.Borrel, C.Detraz, D.Guerreau, D.Guillemaud-Mueller, J.C.Jacmart, D.X.Jiang, A.M.Kalinin, V.V.Kamanin, S.M.Lukyanov, H.C.Nguyen, Yu.E.Penionzhkevich, F.Pougheon, A.Richard, M.G.Saint-Laurent, W.D.Schmidt-Ott - Univ.Paris, Inst.Phys.Nucl., 1988 Ann.Rept., p.25 (1989)

1989Ra06 S.Raman, S.Kahane, R.M.Moon, J.A.Fernandez-Baca, J.L.Zarestky, J.E.Lynn, J.W.Richardson, Jr. - Phys.Rev. C39, 1297 (1989)

1989Va09 S.Y.van der Werf, S.Brandenburg, P.Grasdijk, W.A.Sterrenburg, M.N.Harakeh, M.B.Greenfield, B.A.Brown, M.Fujiwara Nucl.Phys. A496, 305 (1989)

1990 En08 P.M.Endt - Nucl.Phys. A521, 1 (1990); Errata and Addenda Nucl.Phys. A529, 763 (1991); Errata Nucl.Phys. A564, 609 (1993)

1990 Fi 07 U.Fister, R.Jahn, P.von Neumann-Cosel, P.Schenk, T.K.Trelle, D.Wenzel, U.Wienands - Phys.Rev. C42, 2375 (1990)

1990 Le03 M.Lewitowicz, R.Anne, A.G.Artukh, D.Bazin, A.V.Belozyorov, P.Bricault, C.Detraz, D.Guillemaud-Mueller, J.C.Jacmart, E.Kashy, A.Latimier, S.M.Lukyanov, A.C.Mueller, Yu.E.Penionzhkevich, F.Pougheon, A.Richard, W.D.Schmidt-Ott, Y.Zhang - Z.Phys. A335, 117 (1990)

1990 Mi 05 H.Miyahara, S.Kitaori, Y.Nozue, T.Watanabe - Nucl.Instrum.Methods Phys.Res. A286, 519 (1990)

1990 Mo13 M.Moosburger, E.Aschenauer, H.Dennert, W.Eyrich, A.Lehmann, R.Rudeloff, H.Schlosser, H.Wirth, H.J.Gils, H.Rebel, S.Zagromski - Phys.Rev. C41, 2925 (1990)

1990 Tu01 X.L.Tu, X.G.Zhou, D.J.Vieira, J.M.Wouters, Z.Y.Zhou, H.L.Seifert, V.G.Lind - Z.Phys. A337, 361 (1990)

1990Vo07 P.Von Neumann-Cosel, A.Richter, H.-J.Schmidt-Brucken, G.Schrieder, H.Lenske, H.H.Wolter, J.Carter, R.Jahn, B.Kohlmeyer, D.Schull - Nucl.Phys. A516, 385 (1990)

1990 We 05 Z.Weinfeld, E.Piasetzky, M.J.Leitch, H.W.Baer, C.S.Mishra, J.R.Comfort, J.Tinsley, D.H.Wright - Phys.Lett. 237B, 33 (1990)

1991 Bro6 B.A.Brown - Phys.Rev. C43, R1513 (1991); Erratum Phys.Rev. C44, 924 (1991)

1991 Wa 19 E.K.Warburton - Phys.Rev. C44, 268 (1991)

199 1Wi03 A.L.Williams, J.A.McGill, C.L.Morris, G.R.Burleson, J.A.Faucett, D.S.Oakley, M.Burlein, H.T.Fortune, J.M.O'Donnell, G.P.Kahrimanis, C.F.Moore - Phys.Rev. C43, 766 (1991)

1991 Zh24 X.G.Zhou, X.L.Tu, J.M.Wouters, D.J.Vieira, K.E.G.Lobner, H.L.Seifert, Z.Y.Zhou, G.W.Butler - Phys.Lett. 260B, 285 (1991)

1992Bo37 V.Borrel, R.Anne, D.Bazin, C.Borcea, G.G.Chubarian, R.Del Moral, C.Detraz, S.Dogny, J.P.Dufour, L.Faux, A.Fleury, L.K.Fifield, D.Guillemaud-Mueller, F.Hubert, E.Kashy, M.Lewitowicz, C.Marchand, A.C.Mueller, F.Pougheon, M.S.Pravikoff, M.G.Saint-Laurent, O.Sorlin - Z.Phys. A344, 135 (1992)

1992 Le16 M.Leitch, H.W.Baer, A.Klein, C.S.Mishra, Z.Weinfeld, E.Piasetzky, Y.Ezra, R.Weiss, C.Allgower, J.R.Comfort, J.Tinsley, D.H.Wright - Phys.Lett. 294B, 157 (1992) 


\section{REFERENCES FOR A=42 (CONTINUED)}

1993BoZO V.Borrel, S.Dogny, D.Guillemaud-Mueller, A.C.Mueller, F.Pougheon, O.Sorlin, R.Anne, C.Borcea, L.K.Fifield, M.Lewitowicz, M.G.Saint-Laurent, D.Bazin, R.Del Moral, J.P.Dufour, L.Faux, A.Fleury, F.Hubert, C.Marchand, M.S.Pravikoff, C.Detraz, E.Kashy, G.G.Chubarian - Proc.6th Intern.Conf.on Nuclei Far from Stability + 9th Intern.Conf.on Atomic Masses and Fundamental Constants, Bernkastel-Kues, Germany, 19-24 July, 1992, R.Neugart, A.Wohr, Eds., p.317 (1993)

1993 Wa $30 \quad$ G.J.Wagner - Acta Phys.Pol. B24, 1641 (1993)

1994Fi 01 U.Fister, R.Jahn, P.von Neumann-Cosel, P.Schenk, T.K.Trelle, D.Wenzel, U.Wienands - Nucl.Phys. A569, 421 (1994)

1994Ke07 H.Keller, V.Borrel, D.Guillemaud-Mueller, A.C.Mueller, F.Pougheon, O.Sorlin, P.Baumann, F.Didierjean, A.Huck, A.Knipper, G.Walter, R.Anne, D.Bazin, M.Lewitowicz, M.G.Saint-Laurent, G.Marguier - Z.Phys. A348, 67 (1994)

$1994 \mathrm{Mi} 03$ E.P.Mignonsin - Appl.Radiat.Isot. 45, 17 (1994)

1994 NaZY A.Narita, T.Tohei, T.Nakagawa, T.Matsumoto, Y.Fujii, H.Orihara, K.Ishii, A.Terakawa, M.Hosaka, S.Miyamoto, G.Zhong, Y.Ishimaru, K.Ito, K.Abe, T.Suehiro, K.Miura, T.Inomata, H.Ohnuma - Cyclotron Rad.Center, Tohoku Univ., Ann.Rept., p.16 (1994)

1994 Uz01 J.L.Uzureau, P.Delbourgo-Salvador, T.Ethvignot, J.E.Sauvestre, J.Trochon, B.Bonnereau, M.Szmigiel, R.Anne, D.Bazin, J.M.Corre, M.Lewitowicz, M.G.St Laurent, R.Bimbot, H.Keller, A.C.Mueller, O.Sorlin - Phys.Lett. 331B, 280 (1994)

1995So03 O.Sorlin, D.Guillemaud-Mueller, R.Anne, L.Axelsson, D.Bazin, W.Bohmer, V.Borrel, Y.Jading, H.Keller, K.-L.Kratz, M.Lewitowicz, S.M.Lukyanov, T.Mehren, A.C.Mueller, Yu.E.Penionzhkevich, F.Pougheon, M.G.Saint-Laurent, V.S.Salamatin, S.Shoedder, A.Wohr - Nucl.Phys. A583, 763c (1995)

$1995 \mathrm{Th} 06$ $1996 \mathrm{~B} 121$

T.F.Thorsteinsen, K.Nybo, G.Lovhoiden - Phys.Scr. 52, 498 (1995)

B.Blank, S.Czajkowski, F.Davi, R.Del Moral, J.P.Dufour, A.Fleury, C.Marchand, M.S.Pravikoff, J.Benlliure, F.Boue, R.Collatz, A.Heinz, M.Hellstrom, Z.Hu, E.Roeckl, M.Shibata, K.Summerer, Z.Janas, M.Karny, M.Pfutzner, M.Lewitowicz - Phys.Rev.Lett. 77, 2893 (1996)

1996Na03 W.Nazarewicz, J.Dobaczewski, T.R.Werner, J.A.Maruhn, P.-G.Reinhard, K.Rutz, C.R.Chinn, A.S.Umar, M.R.Strayer Phys.Rev. C53, 740 (1996)

1996Sc31 H.Scheit, T.Glasmacher, B.A.Brown, J.A.Brown, P.D.Cottle, P.G.Hansen, R.Harkewicz, M.Hellstrom, R.W.Ibbotson, J.K.Jewell, K.W.Kemper, D.J.Morrissey, M.Steiner, P.Thirolf, M.Thoennessen - Phys.Rev.Lett. 77, 3967 (1996)

1997 Be45 M.B.Becha, C.O.Blyth, C.N.Pinder, N.M.Clarke, R.P.Ward, P.R.Hayes, K.I.Pearce, D.L.Watson, A.Ghazarian, M.D.Cohler, I.J.Thompson, M.A.Nagarajan - Phys.Rev. C56, 1960 (1997)

1997 Ko 65 V.T.Koslowsky, E.Hagberg, J.C.Hardy, G.Savard, H.Schmeing, K.S.Sharma, X.J.Sun - Nucl.Instrum.Methods Phys.Res. A401, 289 (1997)

1997 Mo 25 P.Moller, J.R.Nix, K.-L.Kratz - At.Data Nucl.Data Tables 66, 131 (1997)

1997 Wa 25 S.Wan, P.Reiter, J.Cub, H.Emling, J.Gerl, R.Schubart, D.Schwalm, for the CB-LAND-FRS Collaboration - Z.Phys. A358, 213 (1997)

$1998 \mathrm{En} 04$ P.M.Endt - Nucl.Phys. A633, 1 (1998)

1998 Mo16 M.Moralles, P.Janker, H.Leitz, K.E.G.Lobner, K.Rudolph, F.J.Schindler, H.-G.Thies, T.Winkelmann, P.R.Pascholati, B.A.Brown - Phys.Rev. C58, 739 (1998)

1998 WiZV J.A.Winger, H.H.Yousif, W.C.Ma, V.Ravikumar, Y.-W.Lui, S.K.Phillips, R.B.Piercey, P.F.Mantica, B.Pritychenko, R.M.Ronningen, M.Steiner - Proc.Conf on Exotic Nuclei and Atomic Masses, Bellaire, Michigan, June 23-27, 1998, p.606 (1998); AIP Conf.Proc. 455 (1998)

1998WiZX J.A.Winger, P.F.Mantica, R.M.Ronningen - Proc.Intern.Conf.Fission and Properties of Neutron-Rich Nuclei, Sanibel Island, Florida, November 1997, J.H.Hamilton, A.V.Ramayya, Eds., p.311 (1998)

1999Aio2 N.Aissaoui, N.Added, N.Carlin, G.M.Crawley, S.Danczyk, J.Finck, M.M.de Moura, D.Hirata, D.J.Morrissey, S.J.Sanders, J.Stasko, M.Steiner, A.A.P.Suaide, E.M.Szanto, A.Szanto de Toledo, M.Thoennessen, J.A.Winger - Phys.Rev. C60, 034614 (1999)

1999 B 108 B.Blank - J.Phys.(London) G25, 629 (1999)

1999 Ha 66 E.Hai, S.Soheli, Md.A.Rahman, H.M.Sen Gupta - Nuovo Cim. 112A, 1049 (1999)

1999YoZW K.Yoneda, H.Sakurai, N.Aoi, N.Fukuda, T.Gomi, E.Ideguchi, N.Imai, H.Iwasaki, T.Kubo, Z.Liu, S.M.Lukyanov, T.Nakamura, M.Notani, H.Ogawa, Y.E.Penionzhkevich, W.-D.Schmidt-Ott, S.Shimoura, E.Sokol, Y.X.Watanabe, A.Yoshida, X.Zhou, M.Ishihara - RIKEN-98, p.78 (1999)

2000 Bl01 B.Blank, M.Chartier, S.Czajkowski, J.Giovinazzo, M.S.Pravikoff, J.-C.Thomas, G.de France, F.de Oliveira Santos, M.Lewitowicz, C.Borcea, R.Grzywacz, Z.Janas, M.Pfutzner - Phys.Rev.Lett. 84, 1116 (2000)

2000 BlzZ B.Blank, C.Borcea, M.Chartier, S.Czajkowski, G.de France, J.Giovinazzo, R.Grzywacz, Z.Janas, M.Lewitowicz, F.de Oliveira Santos, M.Pfutzner, M.S.Pravikoff, J.C.Thomas - Proc.Inter.Workshop PINGST 2000, Selected Topics on N=Z Nuclei, Lund, Sweden, June 6-10, 2000, D.Rudolph, M.Hellstrom, Eds., p.2 (2000)

$2000 \mathrm{Mu} 17$ P.Muller, B.A.Bushaw, W.Nortershauser, K.Wendt - Eur.Phys.J. D 12, $33(2000)$

2000 Sa 21 F.Sarazin, H.Savajols, W.Mittig, F.Nowacki, N.A.Orr, Z.Ren, P.Roussel-Chomaz, G.Auger, D.Baiborodin, A.V.Belozyorov, C.Borcea, E.Caurier, Z.Dlouhy, A.Gillibert, A.S.Lalleman, M.Lewitowicz, S.M.Lukyanov, F.De Oliveira, Y.E.Penionzhkevich, D.Ridikas, H.Sakurai, O.Tarasov, A.de Vismes - Phys.Rev.Lett. 84, 5062 (2000)

2001 Gi01 J.Giovinazzo, B.Blank, C.Borcea, M.Chartier, S.Czajkowski, G.de France, R.Grzywacz, Z.Janas, M.Lewitowicz, F.de Oliveira Santos, M.Pfutzner, M.S.Pravikoff, J.C.Thomas - Eur.Phys.J. A 10, 73 (2001)

$2001 G i 02$ J.Giovinazzo, B.Blank, C.Borcea, M.Chartier, S.Czajkowski, G.de France, R.Grzywacz, Z.Janas, M.Lewitowicz, F.de Oliveira Santos, M.Pfutzner, M.S.Pravikoff, J.C.Thomas - Nucl.Phys. A685, 127c (2001)

2001 Gr29 L.V.Grigorenko, R.C.Johnson, I.G.Mukha, I.J.Thompson, M.V.Zhukov - Phys.Rev. C64, 054002 (2001)

2001 He 29 F.Herfurth, J.Dilling, A.Kellerbauer, G.Audi, D.Beck, G.Bollen, H.-J.Kluge, D.Lunney, R.B.Moore, C.Scheidenberger, S.Schwarz, G.Sikler, J.Szerypo, and the ISOLDE Collaboration - Phys.Rev.Lett. 87, 142501 (2001)

2001 Ko 07 Y.Kojima, M.Shibata, H.Uno, K.Kawade, A.Taniguchi, Y.Kawase, K.Shizuma - Nucl.Instrum.Methods Phys.Res. A458, 656 (2001)

2001 Ra 27 S.Raman, C.W.Nestor, P.Tikkanen - At.Data Nucl.Data Tables 78, 1 (2001) 


\section{REFERENCES FOR A=42 (CONTINUED)}

2001 Sa72 F.Sarazin, H.Savajols, W.Mittig, F.Nowacki, N.A.Orr, Z.Ren, P.Roussel-Chomaz, G.Auger, D.Baiborodin, A.V.Belozyorov, C.Borcea, E.Caurier, Z.Dlouhy, A.Gillibert, A.S.Lalleman, M.Lewitowicz, S.M.Lukyanov, F.de Oliveira, Y.E.Penionzhkevich, D.Ridikas, O.Tarasov, H.Sakurai, A.De Vismes - Hyperfine Interactions 132, 147 (2001)

$2001 \mathrm{Sc} 01$ H.Scheit, F.Marechal, T.Glasmacher, E.Bauge, Y.Blumenfeld, J.P.Delaroche, M.Girod, R.W.Ibbotson, K.W.Kemper, J.Libert, B.Pritychenko, T.Suomijarvi - Phys.Rev. C63, 014604 (2001)

$2001 \mathrm{Si} 09$ D.Simoes, M.F.Koskinas, M.S.Dias - Appl.Radiat.Isot. 54, 443 (2001)

$2001 \mathrm{~S}$ i 10 B.Singh, J.A.Cameron - Nucl.Data Sheets 92, 1 (2001)

$2002 \mathrm{Az} 01 \quad$ F.Azaiez - Nucl.Phys. A704, 37c (2002)

$2002 \mathrm{Az} 02$ F.Azaiez, M.Belleguic, D.Sohler, M.Stanoiu, Zs.Dombradi, O.Sorlin, J.Timar, F.Amorini, D.Baiborodin, A.Bauchet, F.Becker, C.Borcea, C.Bourgeois, Z.Dlouhy, C.Donzaud, J.Duprat, D.Guillemaud-Mueller, F.Ibrahim, M.J.Lopez, R.Lucas, S.M.Lukyanov, V.Maslov, J.Mrazek, C.Moore, F.Nowacki, B.M.Nyako, Yu.-E.Penionzhkevich, M.G.Saint-Laurent, F.Sarazin, J.A.Scarpaci, G.Sletten, C.Stodel, M.Taylor, C.Theisen, G.Voltolini - Eur.Phys.J. A 15, 93 (2002)

2002 Ch28 M.Chartier, B.Blank, S.Czajkowski, J.Giovinazzo, M.S.Pravikoff, J.-C.Thomas, G.de France, F.de Oliveira Santos, M.Lewitowicz, C.Borcea, R.Grzywacz, Z.Janas, M.Pfutzner - Nucl.Phys. A701, 433c (2002)

2002 Gi09 J.Giovinazzo, B.Blank, M.Chartier, S.Czajkowski, A.Fleury, M.J.Lopez Jimenez, M.S.Pravikoff, J.-C.Thomas, F.de Oliveira Santos, M.Lewitowicz, V.Maslov, M.Stanoiu, R.Grzywacz, M.Pfutzner, C.Borcea, B.A.Brown - Phys.Rev.Lett. 89, 102501 (2002)

$2002 \mathrm{Gu} 08$ D.Guillemaud-Mueller - Eur.Phys.J. A 13, 63 (2002)

$20020 r 05$ H.Orihara, A.Terakawa, K.Itoh, H.Suzuki, K.Kumagai, Y.Kikuchi, G.C.Jon, K.Ishii, T.Niizeki, H.Sagawa, H.Ohnuma Phys.Lett. 539B, $40(2002)$

2002 Pfo2 M.Pfutzner, E.Badura, C.Bingham, B.Blank, M.Chartier, H.Geissel, J.Giovinazzo, L.V.Grigorenko, R.Grzywacz, M.Hellstrom, Z.Janas, J.Kurcewicz, A.S.Lalleman, C.Mazzocchi, I.Mukha, G.Munzenberg, C.Plettner, E.Roeckl, K.P.Rykaczewski, K.Schmidt, R.S.Simon, M.Stanoiu, J.-C.Thomas - Eur.Phys.J. A 14, 279 (2002)

2002 So 14 D.Sohler, Zs.Dombradi, J.Timar, O.Sorlin, F.Azaiez, F.Amorini, M.Belleguic, C.Bourgeois, C.Donzaud, J.Duprat, D.Guillemaud-Mueller, F.Ibrahim, J.A.Scarpaci, M.Stanoiu, M.J.Lopez, M.G.Saint-Laurent, F.Becker, F.Sarazin, C.Stodel, G.Voltolini, S.M.Lukyanov, V.Maslov, Yu.-E.Penionzhkevich, M.Girod, S.Peru, F.Nowacki, G.Sletten, R.Lucas, C.Theisen, D.Baiborodin, Z.Dlouhy, J.Mrazek, C.Borcea, A.Bauchet, C.J.Moore, M.J.Taylor - Phys.Rev. C 66, 054302 (2002)

2003 Gr22 S.Grevy, J.Mrazek, J.C.Angelique, P.Baumann, C.Borcea, A.Buta, G.Canchel, W.Catford, S.Courtin, J.M.Daugas, F.De Oliveira, P.Dessagne, Z.Dlouhy, A.Knipper, K.L.Kratz, F.R.Lecolley, J.L.Lecouey, G.Lehrsenneau, M.Lewitowicz, E.Lienard, S.Lukyanov, F.Marechal, C.Miehe, F.Negoita, N.A.Orr, D.Pantelica, Y.Penionzhkevich, J.Peter, B.Pfeiffer, S.Pietri, E.Poirier, O.Sorlin, M.Stanoiu, C.Stodel, C.Timis - Nucl.Phys. A722, 424c (2003)

2003 Gr 24 L.V.Grigorenko, M.V.Zhukov - Phys.Rev. C 68, 054005 (2003)

2003 La04 M.Lach, J.Styczen, W.Meczynski, P.Bednarczyk, A.Bracco, J.Grebosz, A.Maj, J.C.Merdinger, N.Schulz, M.B.Smith, K.M.Spohr, J.P.Vivien, M.Zieblinski - Eur.Phys.J. A 16, 309 (2003)

2003 Mo09 P.Moller, B.Pfeiffer, K.-L.Kratz - Phys.Rev. C 67, 055802 (2003)

2003 Sc21 S.Schielke, D.Hohn, K.-H.Speidel, O.Kenn, J.Leske, N.Gemein, M.Offer, J.Gerber, P.Maier-Komor, O.Zell, Y.Y.Sharon, L.Zamick - Phys.Lett. B 571, 29 (2003)

2004 Gr20 S.Grevy, J.C.Angelique, P.Baumann, C.Borcea, A.Buta, G.Canchel, W.N.Catford, S.Courtin, J.M.Daugas, F.de Oliveira, P.Dessagne, Z.Dlouhy, A.Knipper, K.L.Kratz, F.R.Lecolley, J.L.Lecouey, G.Lehrsenneau, M.Lewitowicz, E.Lienard, S.Lukyanov, F.Marechal, C.Miehe, J.Mrazek, F.Negoita, N.A.Orr, D.Pantelica, Y.Penionzhkevich, J.Peter, B.Pfeiffer, S.Pietri, E.Poirier, O.Sorlin, M.Stanoiu, I.Stefan, C.Stodel, C.Timis - Phys.Lett. B 594, 252 (2004)

2004 Gr 28 S.Grevy, J.C.Angelique, P.Baumann, C.Borcea, A.Buta, G.Canchel, W.N.Catford, S.Courtin, J.M.Daugas, F.De Oliveira, P.Dessagne, Z.Dlouhy, A.Knipper, K.L.Kratz, F.R.Lecolley, J.L.Lecouey, G.Lehrsenneau, M.Lewitowicz, E.Lienard, S.Lukyanov, F.Marechal, C.Miehe, J.Mrazek, F.Negoita, N.A.Orr, D.Pantelica, Y.Penionzhkevich, J.Peter, B.Pfeiffer, S.Pietri, E.Poirier, O.Sorlin, M.Stanoiu, I.Stefan, C.Stodel, C.Timis - Nucl.Phys. A746, 145c (2004)

$2004 \mathrm{HaZW}$ H.Hayashi, O.Suematsu, M.Shibata, Y.Kojima, A.Taniguchi, K.Kawade - INDC(JPN)-192/U (JAERI-Conf 2004-005), p.207 $(2004)$

$20040 r 03$ J.N.Orce, P.Petkov, C.J.McKay, S.N.Choudry, S.R.Lesher, M.Mynk, D.Bandyopadhyay, S.W.Yates, M.T.McEllistrem Phys.Rev. C 70, $014314(2004)$

2004 Szo5 S.Szilner, L.Corradi, F.Haas, G.Pollarolo, S.Beghini, B.R.Behera, E.Caurier, E.Fioretto, A.Gadea, A.Latina, G.Montagnoli, F.Nowacki, F.Scarlassara, A.M.Stefanini, M.Trotta, A.M.Vinodkumar, Y.W.Wu - Eur.Phys.J. A 21, 87 (2004)

2004 Un 01 M.P.Unterweger, R.M.Lindstrom - Appl.Radiat.Isot. 60, 325 (2004)

2005 B 133 K.Blaum, W.Geithner, J.Lassen, P.Lievens, K.Marinova, R.Neugart - Hyperfine Interactions 162, 101 (2005)

2005 Co25 L.Corradi, A.M.Stefanini, S.Szilner, S.Beghini, B.R.Behera, E.Farnea, A.Gadea, E.Fioretto, F.Haas, A.Latina, N.Marginean, G.Montagnoli, G.Pollarolo, F.Scarlassara, M.Trotta, C.Ur, and the PRISMA-CLARA Collaboration Eur.Phys.J. A 25, Supplement 1, 427 (2005)

2005 Do20 C.Dossat, A.Bey, B.Blank, G.Canchel, A.Fleury, J.Giovinazzo, I.Matea, F.de Oliveira Santos, G.Georgiev, S.Grevy, I.Stefan, J.C.Thomas, N.Adimi, C.Borcea, D.Cortina Gil, M.Caamano, M.Stanoiu, F.Aksouh, B.A.Brown, L.V.Grigorenko Phys.Rev. C 72, 054315 (2005)

2005 Fr19 J.Fridmann, I.Wiedenhover, A.Gade, L.T.Baby, D.Bazin, B.A.Brown, C.M.Campbell, J.M.Cook, P.D.Cottle, E.Diffenderfer, D.-C.Dinca, T.Glasmacher, P.G.Hansen, K.W.Kemper, J.L.Lecouey, W.F.Mueller, H.Olliver, E.Rodriguez-Vieitez, J.R.Terry, J.A.Tostevin, K.Yoneda - Nature(London) 435, 922 (2005)

2005 GuZW P.Guazoni, L.Zetta, F.Della Vedova, S.M.Lenzi, A.Vitturi, G.Graw, R.Hertenberger, H.-F.Wirth, M.JaskolaMaier-Leibnitz-Laboratorium 2004 Ann.Rept., p.7 (2005) 


\section{REFERENCES FOR A=42 (CONTINUED)}

2005 IdZZ E.Ideguchi, M.Niikura, C.Ishida, T.Fukuchi, H.Baba, N.Hokoiwa, H.Iwasaki, T.Koike, T.Komatsubara, T.Kubo, M.Kurokawa, S.Michimasa, K.Miyakawa, K.Morimoto, T.Ohnishi, S.Ota, A.Ozawa, S.Shimoura, T.Suda, M.Tamaki, I.Tanihata, Y.Wakabayashi, K.Yoshida, B.Cederwall - Proc.Nuclei at the Limits, Argonne, Illinois, D.Seweryniak and T.L.Khoo, eds., p.136 (2005); AIP Conf. Proc 764 (2005)

2005 Ki 02 T.Kibedi, R.H.Spear - At.Data Nucl.Data Tables 89, 77 (2005)

2005 Li 66 R.M.Lindstrom, M.Blaauw, M.P.Unterweger - J.Radioanal.Nucl.Chem. 263, 311 (2005)

2006 Er08 T.Eronen, V.Elomaa, U.Hager, J.Hakala, A.Jokinen, A.Kankainen, I.Moore, H.Penttila, S.Rahaman, J.Rissanen, A.Saastamoinen, T.Sonoda, J.Aysto, J.C.Hardy, V.S.Kolhinen - Phys.Rev.Lett. 97, 232501 (2006)

2006 Fr13 J.Fridmann, I.Wiedenhover, A.Gade, L.T.Baby, D.Bazin, B.A.Brown, C.M.Campbell, J.M.Cook, P.D.Cottle, E.Diffenderfer, D.-C.Dinca, T.Glasmacher, P.G.Hansen, K.W.Kemper, J.L.Lecouey, W.F.Mueller, E.Rodriguez-Vieitez, J.R.Terry, J.A.Tostevin, K.Yoneda, H.Zwahlen - Phys.Rev. C 74, 034313 (2006)

2006 Ga4 7 Y.Gangrsky, K.Marinova, S.Zemlyanoi, M.Avgoulea, J.Billowes, P.Campbell, B.Cheal, B.Tordoff, M.Bissell, D.H.Forest, M.Gardner, G.Tungate, J.Huikari, H.Penttila, J.Aysto - Hyperfine Interactions 171, 209 (2006)

$2006 \mathrm{GrZZ}$ S.Grevy, B.Bastin, Zs.Dombradi, D.Sohler, N.L.Achouri, J.C.Angelique, F.Azaiez, D.Baiborodin, R.Borcea, C.Bourgeois, A.Buta, A.Burger, R.Chapman, J.C.Dalouzy, A.Drouard, Z.Elekes, S.Franchoo, S.Iacob, B.Laurent, M.Lazar, X.Liang, E.Lienard, J.Mrazek, L.Nalpas, F.Negoita, N.A.Orr, Y.Penionshkevitch, Zs.Podolyak, F.Pougheon, P.Roussel-Chomaz, O.Sorlin, M.Stanoiu, I.Stefan, M.-G.Saint-Laurent, F.Nowacki, A.Poves - Atomki 2005 Ann.Rept., p.13 (2006)

2006 Kh08 A.Khouaja, A.C.C.Villari, M.Benjelloun, D.Hirata, G.Auger, H.Savajols, W.Mittig, P.Roussel-Chomaz, N.A.Orr, M.G.Saint-Laurent, S.Pita, A.Gillibert, M.Chartier, C.E.Demonchy, L.Giot, D.Baiborodin, Y.Penionzhkevich, W.N.Catford, A.Lepine-Szily, Z.Dlouhy - Nucl.Phys. A780, 1 (2006)

2006 MoZS T.Morikawa, H.Kusakari, M.Oshima, Y.Toh, M.Koizumi, A.Kimura, A.Osa, Y.Hatsukawa, K.Furutaka, J.Katakura, M.Nakamura, M.Sugawara, K.Sagara - JAEA-Review 2006-029, p.21 (2006)

2006 MuZX S.F.Mughabghab - Atlas of Neutron Resonances, 5ed., Resonance Parameters and Thermal Cross Sections, Z = 1-100, Elsevier, Amsterdam (2006)

2006 Ro34 B.T.Roeder, K.W.Kemper, N.Aoi, D.Bazin, M.Bowen, C.M.Campbell, J.M.Cook, D.-C.Dinca, A.Gade, T.Glasmacher, H.Iwasaki, S.Kanno, T.Motobayashi, W.F.Mueller, T.Nakamura, H.Sakurai, H.Suzuki, S.Takeuchi, J.R.Terry, K.Yoneda, H.Zwahlen - Phys.Rev. C 74, 034602 (2006)

$2006 \mathrm{Sp01}$ K.-H.Speidel, S.Schielke, J.Leske, J.Gerber, P.Maier-Komor, S.J.Q.Robinson, Y.Y.Sharon, L.Zamick - Phys.Lett. B 632, 207 (2006)

2006 Wi $10 \quad$ J.A.Winger, P.F.Mantica, R.M.Ronningen - Phys.Rev. C 73, 044318 (2006)

2007Ad27 T.Adachi, Y.Fujita, P.von Brentano, G.P.A.Berg, C.Fransen, D.De Frenne, H.Fujita, K.Fujita, K.Hatanaka, M.Honma, E.Jacobs, J.Kamiya, K.Kawase, T.Mizusaki, K.Nakanishi, A.Negret, T.Otsuka, N.Pietralla, L.Popescu, Y.Sakemi, Y.Shimbara, Y.Shimizu, Y.Tameshige, A.Tamii, M.Uchida, T.Wakasa, M.Yosoi, K.O.Zell - Nucl.Phys. A788, 70c (2007)

2007 Ba 47 B.Bastin, S.Grevy, D.Sohler, O.Sorlin, Zs.Dombradi, N.L.Achouri, J.C.Angelique, F.Azaiez, D.Baiborodin, R.Borcea, C.Bourgeois, A.Buta, A.Burger, R.Chapman, J.C.Dalouzy, Z.Dlouhy, A.Drouard, Z.Elekes, S.Franchoo, S.Iacob, B.Laurent, M.Lazar, X.Liang, E.Lienard, J.Mrazek, L.Nalpas, F.Negoita, N.A.Orr, Y.Penionzhkevich, Zs.Podolyak, F.Pougheon, P.Roussel-Chomaz, M.G.Saint-Laurent, M.Stanoiu, I.Stefan, F.Nowacki, A.Poves - Phys.Rev.Lett. 99, 022503 (2007)

2007 Ba71 T.Baumann, A.M.Amthor, D.Bazin, B.A.Brown, C.M.Folden III, A.Gade, T.N.Ginter, M.Hausmann, M.Matos, D.J.Morrissey, M.Portillo, A.Schiller, B.M.Sherrill, A.Stolz, O.B.Tarasov, M.Thoennessen - Nature(London) 449, 1022 (2007)

2007 Ch40 C.J.Chiara, M.Devlin, E.Ideguchi, D.R.LaFosse, F.Lerma, W.Reviol, S.K.Ryu, D.G.Sarantites, O.L.Pechenaya, C.Baktash, A.Galindo-Uribarri, M.P.Carpenter, R.V.F.Janssens, T.Lauritsen, C.J.Lister, P.Reiter, D.Seweryniak, P.Fallon, A.Gorgen, A.O.Macchiavelli, D.Rudolph, G.Stoitcheva, W.E.Ormand - Phys.Rev. C 75, 054305 (2007); Publisher's note Phys. Rev. C 75, 059904 (2007)

2007 Co 21 L.Corradi - Nucl.Phys. A787, 160c (2007)

2007 Do17 C.Dossat, N.Adimi, F.Aksouh, F.Becker, A.Bey, B.Blank, C.Borcea, R.Borcea, A.Boston, M.Caamano, G.Canchel, M.Chartier, D.Cortina, S.Czajkowski, G.de France, F.de Oliveira Santos, A.Fleury, G.Georgiev, J.Giovinazzo, S.Grevy, R.Grzywacz, M.Hellstrom, M.Honma, Z.Janas, D.Karamanis, J.Kurcewicz, M.Lewitowicz, M.J.Lopez Jimenez, C.Mazzocchi, I.Matea, V.Maslov, P.Mayet, C.Moore, M.Pfutzner, M.S.Pravikoff, M.Stanoiu, I.Stefan, J.C.Thomas - Nucl.Phys. A792, 18 (2007)

2007Gi10 J.Giovinazzo, B.Blank, C.Borcea, G.Canchel, J.-C.Dalouzy, C.E.Demonchy, F.de Oliveira Santos, C.Dossat, S.Grevy, L.Hay, J.Huikari, S.Leblanc, I.Matea, J.-L.Pedroza, L.Perrot, J.Pibernat, L.Serani, C.Stodel, J.-C.Thomas Phys.Rev.Lett. 99, 102501 (2007)

2007 Ju03 B.Jurado, H.Savjols, W.Mittig, N.A.Orr, P.Roussel-Chomaz, D.Baiborodin, W.N.Catford, M.Chartier, C.E.Demonchy, Z.Dlouhy, A.Gillibert, L.Giot, A.Khouaja, A.Lepine-Szily, S.Lukyanov, J.Mrazek, Y.E.Penionzhkevich, S.Pita, M.Rousseau, A.C.Villari - Phys.Lett. B 649, 43 (2007)

2007 Mi 36 K.Miernik, W.Dominik, Z.Janas, M.Pfutzner, C.R.Bingham, H.Czyrkowski, M.Cwiok, I.G.Darby, R.Dabrowski, T.Ginter, R.Grzywacz, M.Karny, A.Korgul, W.Kusmierz, S.N.Liddick, M.Rajabali, K.Rykaczewski, A.Stolz - Phys.Rev. C 76 , 041304 (2007)

2007 Mi 40 K.Miernik, W.Dominik, Z.Janas, M.Pfutzner, L.Grigorenko, C.R.Bingham, H.Czyrkowski, M.Cwiok, I.G.Darby, R.Dabrowski, T.Ginter, R.Grzywacz, M.Karny, A.Korgul, W.Kusmierz, S.N.Liddick, M.Rajabali, K.Rykaczewski, A.Stolz Phys.Rev.Lett. 99, 192501 (2007)

2007 Sc26 C.Scholl, P.Petkov, V.Werner, A.Linnemann, T.Adachi, A.Dewald, A.Fitzler, C.Fransen, Y.Fujita, J.Jolie, K.Langanke, A.F.Lisetskiy, G.Martinez-Pinedo, D.Mucher, J.N.Orce, N.Pietralla, N.Warr, K.O.Zell, P.von Brentano - Phys.Rev. C $75,064321(2007)$ 


\section{REFERENCES FOR A=42 (CONTINUED)}

2007Sz05 S.Szilner, C.A.Ur, L.Corradi, N.Marginean, G.Pollarolo, A.M.Stefanini, S.Beghini, B.R.Behera, E.Fioretto, A.Gadea, B.Guiot, A.Latina, P.Mason, G.Montagnoli, F.Scarlassara, M.Trotta, G.de Angelis, F.Della Vedova, E.Farnea, F.Haas, S.Lenzi, S.Lunardi, R.Marginean, R.Menegazzo, D.R.Napoli, M.Nespolo, I.V.Pokrovsky, F.Recchia, M.Romoli, M.-D.Salsac, N.Soic, J.J.Valiente-Dobon - Phys.Rev. C 76, 024604 (2007)

$2008 \mathrm{BeZH}$ N.Benczer-Koller - Proc.of the 9 th Internat. Spring Seminar on Nuclear Physics: Changing Facets of Nuclear Structure, Vico Equense, Italy, May 20-24 2007, A.Covello, Ed., World Scientific, Singapore, p.49 (2008)

2008 B 01 K.Blaum, W.Geithner, J.Lassen, P.Lievens, K.Marinova, R.Neugart - Nucl.Phys. A799, 30 (2008)

2008 B 103 B.Blank, M.Ploszajczak - Rep.Prog.Phys. 71, 046301 (2008)

2008 Mi 03 K.Miernik, W.Dominik, Z.Janas, M.Pfutzner, L.Grigorenko, C.Bingham, H.Czyrkowski, M.Cwiok, I.Darby, R.Dabrowski, T.Ginter, R.Grzywacz, M.Karny, A.Korgul, W.Kusmierz, S.Liddick, M.Rajabali, K.Rykaczewski, A.Stolz - Acta Phys.Pol. B39, 477 (2008)

2008 Sa04 M.-D.Salsac, F.Haas, S.Courtin, A.Algora, C.Beck, S.Beghini, B.R.Behera, R.Chapman, L.Corradi, Z.Dombradi, E.Farnea, E.Fioretto, A.Gadea, D.G.Jenkins, A.Latina, D.Lebhertz, S.Lenzi, X.Liang, N.Marginean, G.Montagnoli, D.Napoli, P.Papka, I.Pokrovski, G.Pollarolo, M.Rousseau, E.Sahin, A.Sanchez i Zafra, F.Scarlassara, D.Sohler, A.M.Stefanini, S.Szilner, M.Trotta, C.Ur, F.Della Vedova, Z.M.Wang, K.T.Wiedemann - Nucl.Phys. A801, 1 (2008)

$2008 \mathrm{Sa} 44$ M.-D.Salsac, F.Haas - Int.J.Mod.Phys. E17, 2029 (2008)

2009 Co21 N.J.Costiris, E.Mavrommatis, K.A.Gernoth, J.W.Clark - Phys.Rev. C 80, 044332 (2009)

2009 Ku19 T.Kurtukian Nieto, J.Souin, T.Eronen, L.Audirac, J.Aysto, B.Blank, V.-V.Elomaa, J.Giovinazzo, U.Hager, J.Hakala, A.Jokinen, A.Kankainen, P.Karvonen, T.Kessler, I.D.Moore, H.Penttila, S.Rahaman, M.Reponen, S.Rinta-Antila, J.Rissanen, A.Saastamoinen, T.Sonoda, C.Weber - Phys.Rev. C 80, 035502 (2009)

2009 Mi 29 K.Miernik, W.Dominik, Z.Janas, M.Pfutzner, L.Grigorenko, C.Bingham, H.Czyrkowski, M.Cwiok, I.G.Darby, R.Dabrowski, T.Ginter, R.Grzywacz, M.Karny, A.Korgul, W.Kusmierz, S.N.Liddick, M.Rajabali, K.Rykaczewski, A.Stolz - Eur.Phys.J. A $42,431(2009)$

2009 No01 F.Nowacki, A.Poves - Phys.Rev. C 79, 014310 (2009)

2009 Ri12 R.Ringle, C.Bachelet, M.Block, G.Bollen, M.Facina, C.M.Folden III, C.Guenaut, A.A.Kwiatkowski, D.J.Morrissey, G.K.Pang, A.M.Prinke, J.Savory, P.Schury, S.Schwarz, C.S.Sumithrarachchi - Phys.Rev. C 80, 064321 (2009)

2009Zi01 M.Zielinska, A.Gorgen, E.Clement, J.-P.Delaroche, M.Girod, W.Korten, A.Burger, W.Catford, C.Dossat, J.Iwanicki, J.Libert, J.Ljungvall, P.J.Napiorkowski, A.Obertelli, D.Pietak, R.Rodriguez-Guzman, G.Sletten, J.Srebrny, Ch.Theisen, K.Wrzosek - Phys.Rev. C 80, 014317 (2009)

2010 Ga15 L.Gaudefroy - Phys.Rev. C 81, 064329 (2010)

2010 Ha 33 K.Hagino, M.T.Win, Y.Nakagawa - Int.J.Mod.Phys. E19, 2552 (2010)

$2010 \mathrm{Sm} 02$ N.A.Smirnova, B.Bally, K.Heyde, F.Nowacki, K.Sieja - Phys.Lett. B 686, 109 (2010)

2010 StZY L.Stuhl, T.Adachi, A.Algora, M.Csatlos, J.Deaven, E.Estevez, H.Fujita, Y.Fujita, C.Guess, J.Gulyas, K.Hatanaka,

K.Hirota, O.Hooi Jim, D.Ishikawa, A.Krasznahorkay, H.Matsubara, R.Meharchand, F.Molina, H.Okamura, G.Perdikakis, B.Rubio, C.Scholl, T.Suzuki, G.Susoy, A.Tamii, J.Thies, R.Zegers, J.Zenihiro - ATOMKI 2009 Ann.Rept., p.30 (2010)

2011Av01 M.Avgoulea, Yu.P.Gangrsky, K.P.Marinova, S.G.Zemlyanoi, S.Fritzsche, D.Iablonskyi, C.Barbieri, E.C.Simpson, P.D.Stevenson, J.Billowes, P.Campbell, B.Cheal, B.Tordoff, M.L.Bissell, D.H.Forest, M.D.Gardner, G.Tungate, J.Huikari, A.Nieminen, H.Penttila, J.Aysto - J.Phys.(London) G38, 025104 (2011)

2011 Co14 L.Corradi, S.Szilner, G.Pollarolo, G.Colo, P.Mason, E.Farnea, E.Fioretto, A.Gadea, F.Haas, D.Jelavic-Malenica, N.Marginean, C.Michelagnoli, G.Montagnoli, D.Montanari, F.Scarlassara, N.Soic, A.M.Stefanini, C.A.Ur, J.J.Valiente-Dobon - Phys.Rev. C 84, 034603 (2011)

$2011 \mathrm{Fi} 11 \quad$ R.B.Firestone, Zs.Revay - Radiochim.Acta 1S, 305 (2011)

$2011 \mathrm{Ka} 03$ K.Kaneko, Y.Sun, T.Mizusaki, M.Hasegawa - Phys.Rev. C 83, 014320 (2011)

$2011 \mathrm{KuZY}$ T.Kurtukian-Nieto, for the NEX group of CENBG - Proc.of the 4th Inter.Conf.Proton Emitting Nuclei and Related Topics (PROCON 2011), Bordeaux, France, 6-10 June 2011, LB.Blank Ed. p.171 (2011); AIP Conf.Proc.1409 (2011)

2011 Li 47 Z.P.Li, J.M.Yao, D.Vretenar, T.Niksic, H.Chen, J.Meng - Phys.Rev. C 84, 054304 (2011)

2011 Po01 M.Pomorski, K.Miernik, W.Dominik, Z.Janas, M.Pfutzner, C.R.Bingham, H.Czyrkowski, M.Cwiok, I.G.Darby, R.Dabrowski, T.Ginter, R.Grzywacz, M.Karny, A.Korgul, W.Kusmierz, S.N.Liddick, M.Rajabali, K.Rykaczewski, A.Stolz - Phys.Rev. C 83,014306 (2011)

2011 St03 L.Stuhl, A.Krasznahorkay, M.Csatlos, T.Adachi, A.Algora, J.Deaven, E.Estevez, H.Fujita, Y.Fujita, C.Guess, J.Gulyas, K.Hatanaka, K.Hirota, H.J.Ong, D.Ishikawa, H.Matsubara, R.Meharchand, F.Molina, H.Okamura, G.Perdikakis, B.Rubio, C.Scholl, T.Suzuki, G.Susoy, A.Tamii, J.Thies, R.Zegers, J.Zenihiro - Acta Phys.Pol. B42, 667 (2011)

$2011 \mathrm{Sz} 02$ S.Szilner, L.Corradi, F.Haas, D.Lebhertz, G.Pollarolo, C.A.Ur, L.Angus, S.Beghini, M.Bouhelal, R.Chapman, E.Caurier, S.Courtin, E.Farnea, E.Fioretto, A.Gadea, A.Goasduff, D.Jelavic-Malenica, V.Kumar, S.Lunardi, N.Marginean, P.Mason, D.Mengoni, G.Montagnoli, F.Nowacki, F.Recchia, E.Sahin, M.-D.Salsac, F.Scarlassara, R.Silvestri, J.F.Smith, N.Soic, A.M.Stefanini, J.J.Valiente-Dobon - Phys.Rev. C 84, 014325 (2011)

$2012 \mathrm{Au} 07$ G.Audi, F.G.Kondev, M.Wang, B.Pfeiffer, X.Sun, J.Blachot, M.MacCormick - Chin.Phys.C 36 , 1157 (2012)

2012 Au08 L.Audirac, P.Ascher, B.Blank, C.Borcea, B.A.Brown, G.Canchel, C.E.Demonchy, F.de Oliveira Santos, C.Dossat, J.Giovinazzo, S.Grevy, L.Hay, J.Huikari, S.Leblanc, I.Matea, J.-L.Pedroza, L.Perrot, J.Pibernat, L.Serani, C.Stodel, J.-C.Thomas - Eur.Phys.J. A 48, 179 (2012)

2012 Ca 13 M.A.Caprio, F.Q.Luo, K.Cai, V.Hellemans, Ch.Constantinou - Phys.Rev. C 85, 034324 (2012)

$2012 \mathrm{Ca} 27$ B.G.Carlsson, J.Toivanen, A.Pastore - Phys.Rev. C 86, 014307 (2012)

2012 Fu02 Y.Fujita, H.Fujita, B.Rubio, W.Gelletly, B.Blank - Acta Phys.Pol. B43, 153 (2012)

2012 Ha 26 G.Hagen, M.Hjorth-Jensen, G.R.Jansen, R.Machleidt, T.Papenbrock - Phys.Rev.Lett. 109, 032502 (2012)

2012 St 22 L.Stuhl, A.Krasznahorkay, M.Csatlos, T.Marketin, E.Litvinova, T.Adachi, A.Algora, J.Daeven, E.Estevez, H.Fujita, Y.Fujita, C.Guess, J.Gulyas, K.Hatanaka, K.Hirota, H.J.Ong, D.Ishikawa, H.Matsubara, R.Meharchand, F.Molina, H.Okamura, G.Perdikakis, B.Rubio, C.Scholl, T.Suzuki, G.Susoy, A.Tamii, J.Thies, R.Zegers, J.Zenihiro J.Phys.:Conf.Ser. 381, 012096 (2012) 


\section{REFERENCES FOR A=42 (CONTINUED)}

2012 Ta20 S.Takeuchi, M.Matsushita, N.Aoi, P.Doornenbal, K.Li, T.Motobayashi, H.Scheit, D.Steppenbeck, H.Wang, H.Baba, D.Bazin, L.Caceres, H.Crawford, P.Fallon, R.Gernhauser, J.Gibelin, S.Go, S.Grevy, C.Hinke, C.R.Hoffman, R.Hughes, E.Ideguchi, D.Jenkins, N.Kobayashi, Y.Kondo, R.Krucken, T.Le Bleis, J.Lee, G.Lee, A.Matta, S.Michimasa, T.Nakamura, S.Ota, M.Petri, T.Sako, H.Sakurai, S.Shimoura, K.Steiger, K.Takahashi, M.Takechi, Y.Togano, R.Winkler, K.Yoneda Phys.Rev.Lett. 109, 182501 (2012)

2012 Ve 01 S.Vermote, C.Wagemans, L.De Smet, C.Lampoudis, J.Van Gils - Phys.Rev. C 85, 015803 (2012)

2012 Wa 38 M.Wang, G.Audi, A.H.Wapstra, F.G.Kondev, M.MacCormick, X.Xu, B.Pfeiffer - Chin.Phys.C 36, 1603 (2012)

2013 Ano2 I.Angeli, K.P.Marinova - At.Data Nucl.Data Tables 99, 69 (2013)

2013 Fi 01 R.B.Firestone, M.Krticka, Zs.Revay, L.Szentmiklosi, T.Belgya - Phys.Rev. C 87, 024605 (2013)

2013 Ha 06 K.Hadynska-Klek, P.J.Napiorkowski, A.Maj, F.Azaiez, M.Kicinska-Habior, J.J.Valiente-Dobon, G.de Angelis, T.Abraham, G.Anil Kumar, B.-Q.Arnes, D.Bazzacco, M.Bellato, D.Bortolato, P.Bednarczyk, G.Benzoni, L.Berti, B.Birkenbach, B.Bruyneel, S.Brambilla, F.Camera, J.Chavas, M.Ciemala, P.Cocconi, P.Coleman-Smith, A.Colombo, A.Corsi, F.C.L.Crespi, D.M.Cullen, A.Czermak, P.Desesquelles, B.Dulny, J.Eberth, E.Farnea, B.Fornal, S.Franchoo, A.Gadea, A.Giaz, A.Gottardo, X.Grave, J.Grebosz, M.Gulmini, T.Habermann, R.Isocrate, J.Iwanicki, G.Jaworski, A.Jungclaus, N.Karkour, M.Kmiecik, D.Karpinski, M.Kisielinski, N.Kondratyev, A.Korichi, M.Komorowska, M.Kowalczyk, W.Korten, M.Krzysiek, G.Lehaut, S.Leoni, A.Lopez-Martens, S.Lunardi, G.Maron, K.Mazurek, R.Menegazzo, D.Mengoni, E.Merchan, W.Meczynski, C.Michelagnoli, J.Mierzejewski, B.Million, P.Molini, S.Myalski, D.R.Napoli, R.Nicolini, M.Niikura, A.Obertelli, S.F.Ozmen, M.Palacz, A.Pullia, G.Rampazzo, F.Recchia, N.Redon, P.Reiter, D.Rosso, K.Rusek, E.Sahin, M.-D.Salsac, P.-A.Soderstrom, J.Srebrny, I.Stefan, O.Stezowski, J.Styczen, Ch.Theisen, N.Toniolo, C.A.Ur, V.Vandone, R.Wadsworth, B.Wasilewska, A.Wiens, K.Wrzosek-Lipska, M.Zielinska, M.Zieblinski - Acta Phys.Pol. B44, 617 (2013)

$2013 \mathrm{StZZ}$ $2013 \mathrm{Sz} 01$

$2013 \mathrm{Sz} 02$

$2014 \mathrm{Fu} 02$

N.J.Stone - REPT INDC(NDS)-0650 (2013)

S.Szilner, L.Corradi, G.Pollarolo, E.Fioretto, A.M.Stefanini, G.de Angelis, J.J.Valiente-Dobon, E.Farnea, S.Lunardi, D.Mengoni, G.Montagnoli, D.Montanari, F.Recchia, F.Scarlassara, C.A.Ur, T.Mijatovic, D.Jelavic Malenica, N.Soic, S.Courtin, F.Haas, A.Goasduff, A.Gadea, N.M.Marginean, M.-D.Salsac - Acta Phys.Pol. B44, 417 (2013)

S.Szilner, L.Corradi, F.Haas, G.Pollarolo, L.Angus, S.Beghini, M.Bouhelal, R.Chapman, E.Caurier, S.Courtin, E.Farnea, E.Fioretto, A.Gadea, A.Goasduff, D.Jelavic-Malenica, V.Kumar, S.Lunardi, N.Marginean, D.Mengoni, T.Mijatovic, G.Montagnoli, F.Recchia, E.Sahin, M.-D.Salsac, F.Scarlassara, J.F.Smith, N.Soic, A.M.Stefanini, C.A.Ur, J.J.Valiente-Dobon - Phys.Rev. C 87, 054322 (2013)

Y.Fujita, H.Fujita, T.Adachi, C.L.Bai, A.Algora, G.P.A.Berg, P.von Brentano, G.Colo, M.Csatlos, J.M.Deaven, E.Estevez Aguado, C.Fransen, D.De Frenne, K.Fujita, E.Ganioglu, C.J.Guess, J.Gulyas, K.Hatanaka, K.Hirota, M.Honma, D.Ishikawa, E.Jacobs, A.Krasznahorkay, H.Matsubara, K.Matsuyanagi, R.Meharchand, F.Molina, K.Muto, K.Nakanishi, A.Negret, H.Okamura, H.J.Ong, T.Otsuka, N.Pietralla, G.Perdikakis, L.Popescu, B.Rubio, H.Sagawa, P.Sarriguren, C.Scholl, Y.Shimbara, Y.Shimizu, G.Susoy, T.Suzuki, Y.Tameshige, A.Tamii, J.H.Thies, M.Uchida, T.Wakasa, M.Yosoi, R.G.T.Zegers, K.O.Zell, J.Zenihiro - Phys.Rev.Lett. 112, 112502 (2014)

2014 Kr04 K.Kreim, M.L.Bissell, J.Papuga, K.Blaum, M.De Rydt, R.F.Garcia Ruiz, S.Goriely, H.Heylen, M.Kowalska, R.Neugart, G.Neyens, W.Nortershauser, M.M.Rajabali, R.Sanchez Alarcon, H.H.Stroke, D.T.Yordanov - Phys.Lett. B 731 , 97 (2014)

J.Papuga, M.L.Bissell, K.Kreim, C.Barbieri, K.Blaum, M.De Rydt, T.Duguet, R.F.Garcia Ruiz, H.Heylen, M.Kowalska, R.Neugart, G.Neyens, W.Nortershauser, M.M.Rajabali, R.Sanchez, N.Smirnova, V.Soma, D.T.Yordanov - Phys.Rev. C 90, 034321 (2014)

$2014 \mathrm{~Pa} 45$

$2014 \mathrm{StZZ}$

$2015 \mathrm{Fu} 08$

N.J.Stone - REPT INDC(NDS)-0658 (2014)

Y.Fujita, H.Fujita, T.Adachi, G.Susoy, A.Algora, C.L.Bai, G.Colo, M.Csatlos, J.M.Deaven, E.Estevez Aguado, C.J.Guess, J.Gulyas, K.Hatanaka, K.Hirota, M.Honma, D.Ishikawa, A.Krasznahorkay, H.Matsubara, R.Meharchand, F.Molina, H.Nakada, H.Okamura, H.J.Ong, T.Otsuka, G.Perdikakis, B.Rubio, H.Sagawa, P.Sarriguren, C.Scholl, Y.Shimbara, E.J.Stephenson, T.Suzuki, A.Tamii, J.H.Thies, K.Yoshida, R.G.T.Zegers, J.Zenihiro - Phys.Rev. C 91, $064316(2015)$

2015 Go2 4 C.Gorges, K.Blaum, N.Frommgen, C.Geppert, M.Hammen, S.Kaufmann, J.Kramer, A.Krieger, R.Neugart, R.Sanchez, W.Nortershauser - J.Phys.(London) B48, 245008 (2015)

$2015 \mathrm{Ha} 07 \quad$ J.C.Hardy, I.S.Towner - Phys.Rev. C 91, 025501 (2015)

2015 Mo 01 F.Molina, B.Rubio, Y.Fujita, W.Gelletly, J.Agramunt, A.Algora, J.Benlliure, P.Boutachkov, L.Caceres, R.B.Cakirli, E.Casarejos, C.Domingo-Pardo, P.Doornenbal, A.Gadea, E.Ganioglu, M.Gascon, H.Geissel, J.Gerl, M.Gorska, J.Grebosz, R.Hoischen, R.Kumar, N.Kurz, I.Kojouharov, L.Amon Susam, H.Matsubara, A.I.Morales, Y.Oktem, D.Pauwels, D.Perez-Loureiro, S.Pietri, Zs.Podolyak, W.Prokopowicz, D.Rudolph, H.Schaffner, S.J.Steer, J.L.Tain, A.Tamii, S.Tashenov, J.J.Valiente-Dobon, S.Verma, H-J.Wollersheim - Phys.Rev. C 91, 014301 (2015)

2015 Xu14 X.Xu, M.Wang, Y.-H.Zhang, H.-S.Xu, P.Shuai, X.-L.Tu, Y.A.Litvinov, X.-H.Zhou, B.-H.Sun, Y.-J.Yuan, J.-W.Xia, J.-C.Yang, K.Blaum, R.-J.Chen, X.-C.Chen, C.-Y.Fu, Z.Ge, Z.-G.Hu, W.-J.Huang, D.-W.Liu, Y.-H.Lam, X.-W.Ma, R.-S.Mao, T.Uesaka, G.-Q.Xiao, Y.-M.Xing, T.Yamaguchi, Y.Yamaguchi, Q.Zeng, X.-L.Yan, H.-W.Zhao, T.-C.Zhao, W.Zhang, W.-L.Zhan - Chin.Phys.C 39, 104001 (2015)

2016 Fu02 Y.Fujita, B.Rubio, F.Molina, T.Adachi, H.Fujita, B.Blank, E.Ganioglu, W.Gelletly, S.E.A.Orrigo - Acta Phys.Pol. B47, 867 (2016)

$2016 \mathrm{Li} 07 \quad$ Z.P.Li, T.Niksic, D.Vretenar - J.Phys.(London) G43, 024005 (2016)

$2016 \mathrm{Ma} 12$ T.Marketin, L.Huther, G.Martinez-Pinedo - Phys.Rev. C 93, 025805 (2016)

2016 Wo 02 J.L.Wood, K.Heyde - J.Phys.(London) G43, 020402 (2016) 